\title{
Solar Photovoltaic Applications Seminar: Design, Installation and Operation of Small, Stand-Alone Photovoltaic Power Systems
}

\section{MASTER}

July 1980

Prepared for:

U.S. Department of Energy

Assistant Secretary for Conservation and Solar Energy

Office of Solar Applications for Buildings

Under Contract No. AC01-77CS32522 


\section{DISCLAIMER}

This report was prepared as an account of work sponsored by an agency of the United States Government. Neither the United States Government nor any agency Thereof, nor any of their employees, makes any warranty, express or implied, or assumes any legal liability or responsibility for the accuracy, completeness, or usefulness of any information, apparatus, product, or process disclosed, or represents that its use would not infringe privately owned rights. Reference herein to any specific commercial product, process, or service by trade name, trademark, manufacturer, or otherwise does not necessarily constitute or imply its endorsement, recommendation, or favoring by the United States Government or any agency thereof. The views and opinions of authors expressed herein do not necessarily state or reflect those of the United States Government or any agency thereof. 


\section{DISCLAIMER}

Portions of this document may be illegible in electronic image products. Images are produced from the best available original document. 


\section{NOTICE}

This report was prepared as an account of work sponsored by the United States Government. Neither the United States nor the United States Department of Energy, nor any of their employees, makes any warranty, express or implied, or assumes any legal liability or responsibility for the accuracy, completeness, or usefulness of any information, apparatus, product, or process disclosed, or represents that ite use would not infringe privately owned rights. Feference herein to any specific commercial product, process, or service by trade name, mark, manufacturer, or otherwise, does not necessarily constitute or imply its endorsement, recommendation, or favoring by the United Statec Govarnment or anv ageney thereof. The views and opinions of authors expressed herein do not necessarily state or reflect those of the United States Government or any agency thereof.

Available from:

National Technical Information Service (NTIS)

U.S. Department of Commerce

5285 Port Royal Road

Springfield, Virginia 22161

Price: Printed copy: $\$ 19.00$

Microfiche: $\$ 4.00$ 


\section{Solar Photovoltaic Applications Seminar: Design, Installation and Operation of Small, Stand-Alone Photovoltaic Power Systems}

July 1980

Prepared for:

U.S. Department of Energy

Assistant Secretary for Conservation and Solar Energy

Office of Solar Applications for Buildings

Washington, D.C. 20585

Prepared by:

PRC Energy Analysis Company

McLean, Virginia 22102

Under Contract No. AC01-77CS32522

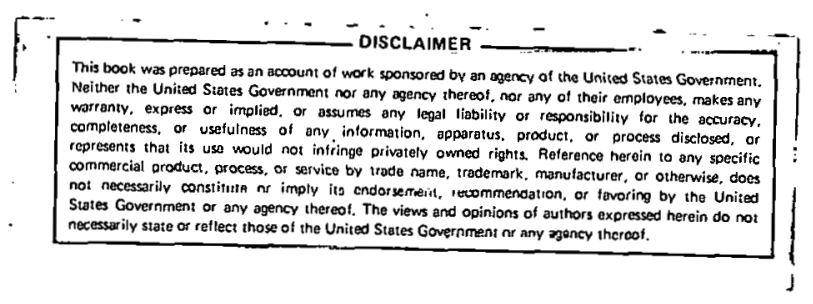


The Department of Energy (DOE) Act of 1978-Civilian Applications (Public Law 95-238, Section 208) authorized $\$ 12,000,000$ in FY 1978 to acquire 1 ifecycle cost-effective photovoltaic systems for Federal facilities. That program was greatly augmented by the establishment of the Federal Photovoltaic Utilization Program (FPUP) under Title V, Part 4, of the National Energy Conservation Policy Act (Publ ic Law 95-619) which authorized the appropriation of an additional $\$ 98,000,000$ for FY 1981 .

As indicated in P.L. 95-619, the key objectives of FPUP are:

(1) To accelerate the growth of a commercially viable and cooperative industry to make photovoltaic solar electric systems available to the general public as an option in order to reduce national consumption of fossil fuel.

(2) To reduce fossil fuel costs to the Federal government.

(3) Stimulate the general use within the Federal government of methods for the minimization of 1 ife-cycle costs.

(4) To develop performance data on the program.

To achieve the objectives established by Congress, the FPUP must stimulate industry and market development. It must support the applications that have been identified as having significant market potential in the private domestic arena and in foreign countries as well as in the Federal agencies.

The FPUP is being conducted in five cycles, depending upon funds availability, as summarized in Table 1.

Table 1. FPUP Application Cycles

\begin{tabular}{|c|c|c|l|}
\hline $\begin{array}{c}\text { Fiscal } \\
\text { Year }\end{array}$ & Cycle & $\begin{array}{c}\text { Major } \\
\text { Applications }\end{array}$ & $\begin{array}{c}\text { Cost } \\
\text { Effective }\end{array}$ \\
\hline 1978 & I & Small Remote & Now \\
\hline 1979 & II & Small Remote & Intermediate Remote \\
\hline 1980 & II & Intermediate Remote & Early 1980's \\
\hline 1981 & V & $\begin{array}{l}\text { Residential } \\
\text { Selected Intermediate } \\
\text { Grid Connected }\end{array}$ & Mid 1980's on \\
\hline
\end{tabular}


To date, FPUP has funded more than 3,000 photovoltaic applications in thirteen Federal departments ( 1 isted below) at a cost of $\$ 23,000,000$. Every state is represented by applications, except North Dakota. There are also applications in the West Indies, the Pacific Islands and Europe.

The Advisory Committee established in P.L. 95-619 has been chartered and members named. A rule for the monitoring and assessment of systems installed under the program was published in final form on November 7, 1979.

The program will be closely coordinated with the activities and experiments underway under the Solar Photovoltaic Energy Research Development and Demonstration Act of 1978 (Public Law 95-590). Technical support will be provided to the agencies that will draw on the technical expertise gained both by industry and DOE field centers in prior applications. This seminar material is part of that technical support.

\section{Participaing Federal Agencies}

Department of Agriculture Department of Commerce Department of Defense Department of tnergy Department of Interior Department of State
Department of Transportation Department of Treasury Environmental Protection Agency General Services Administration Health and Human Services Administration National Aeronautics and Space Administration Tennessee Valley Authority 


\section{PREFACE}

This seminar material was developed primarily to provide solar photovoltaic (PV) applied engineering technology to the Federal community. An introduction to photoconductivity, semiconductors, and solar photovoltaic cells is included along with a demonstration of specific applications and application identification.

The seminar details general systems design and incorporates most known information from industry, academia, and Government concerning small solar cell power system design engineering, presented in a practical and applied manner. Solar PV power system applications involve classical direct electrical energy conversion and electric power system analysis and synthesis. Presentations and examples involve a variety of disciplines including structural analysis, electric power and load analysis, reliability, sizing and optimization; and, installation, operation and maintenance.

Four specific system designs are demonstrated: water pumping, domestic uses, navigational and aircraft aids, and telecommunications. All of the applications discussed are for small power requirement. (under 2 kilowatts), stand-alone systems to be used in remote locations. Also presented are practical Tessons gained from currently installed and operating systems, problems at sites and their resolution, a logical progression through each major phase of system acquisition, as well as thorough design reviews for each application.

A11 PV system applications discussed are solar powered. The radioisotope powered photovoltaic system is not considered, except as a secondary possibility for supplemental energy. A distinguishing characteristic of these small PV power systems is that they can be stand-alone systems. The utility gridconnected application is a secondary possibility for required supplementa? energy. 


\section{TABLE OF CONTENTS}

Section

Page

BACKGROUND

PREFACE

1.0 INTRODUCTION

1.1 Photovoltaic Cells $1-1$

1.2 Components of the Photovoltaic System $1-4$

1.3 Supply/Load Interaction

2.0 PHOTOVOLTAIC ARRAYS

2.1 Background

2.2 Solar Cell Characteristics

$2-1$

2.3 Array Performance Characteristics

2.4 Cell Degradation

2.5 Operational Considerations

2.6 Loading Considerations

$2-13$

2.7 Cell Type Trade-Offs

$2-14$

2.8 Array Trade-Offs

$2-14$

2.9 Environmental Effects

\subsection{STRUCTURAL ANALYSIS}

3.1 Introduction

3.2 Structural Loads

3.3 Building Codes

3.4 BOCA Code

3.5 Example of the Use of the BOCA Code

3.6 Available Structures

3.7 Thermal Design

3.8 Frost, Snow, and lce 


\section{TABLE OF CONTENTS}

Continued

Section

Page

4.0 BATTERY STORAGE - 4-1

4.1 Energy Storage . . 2 4-1

4.2 Terminology $\quad: \quad 4-1$

4.3 Lead-Acid Battery . $\quad$ 4-4

4.4 Types of Lead-Acid Batteries $\quad 4-4$

4.5 Failure Mechanisms of Lead-Acid Batteries $\quad$ 4-6

4.6 Discharge and Recharge Characteristics 4-8

4.7 Failure Mechanisms of Nickel-Cadmium Batteries 4-13

4.8 Recharging Multiple Battery Strings 4-14

4.9 Safety Problems . 4- 4 . 4 . 4

4.10 Summary 4-15

5.0 POWER CONDITIONING ? . 5-1

5.1 Power Conditioning 5-1

5.2 Self-Regulated PV Power Systems 5-1

5.3 Voltage Regulated PV Power Systems . 5-3

5.4 Electronic Switching Regulators $\quad$ 5-6

5.5 DC/AC Inverters . . . . . . . . .

5.5.1 Solid State Inverters

5.5.2 Inverter Technology

5.6 Inverter Output Filters

6.0 ELECTRIC POWER LOAD ANALYSIS AND SYNTHESIS 6-1

6.1 Introduction 6-1

6.2 Estimating the Load Demand 6-1

6.3. Load-Reduction Strategies 6-4

6.4 DC Versus' AC Power 6-6

6.4.1 C.Inssification of l.ogd

6.4.2 Classification of Controls.

6.4.3 Appliance Listing 


\section{TABLE OF CONTENTS}

Continued

$\underline{\text { Section }}$

Page

6.4.4 Operation on DC Voltage

6.4.5 Conclusions

6.5 Load/Power System Interactions

6.5.1 AC Motors

6.5.2 DC Motors

6.5.3 Incandescent Lights

6.5.4 Fluorescent Lights

6.5.5 Electronics

6.5.6 Batteries

6.5.7 DC/AC Inverters

6.5.8 Conclusion

6.6 Load Analysis Lab

$6+9$

6.7 Electrical Power Factor Correction

7.0 BACKUP SYSTEMS

$7-1$

7.1 Introduction

$7-1$

7.2 Causes of Power Loss

$7-1$

7.3 Potential Backup Systems

$7-3$

7.4 Appropriateness of Various Backup Systems

$7-4$

7.5 Integration of the Backup into the System

$7-5$

7.6 Conclusion

8.0 PHOTOVOLTAIC SYSTEM DESION

8.1 Introduction

8.2 Quick Sizing of Components

8-2

8.3 Insoluliun Computation

8.3.1 Introduction

8.3.2 Variability of Insolation

8.3.3 Measurements of Insolation

8.3.4 Values of $\bar{K}_{T}$ 


\section{TABLE OF CONTENTS}

Continued

Section

Page

8.3.5 Insolation on Tilted Surfaces

8.3.6 Computational Procedure

8.3.7 Computation of Insolation on the Average Day

8.3.8 Computation of Average Insolation

8.3.9 Conclusion

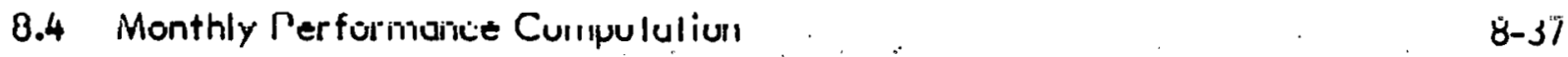

8.5 Component Sizing 8-39

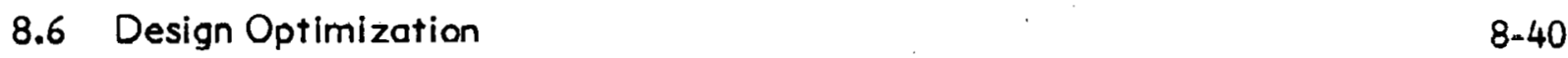

9.0 PHOTOVOLTAIC SYSTEM COMPONENTS 9-1

9.1 Introduction 9. 9-1

9.2 Available Components 9.. 9-1

9.3 Solar Panels $9-1$

9.4 DC Regulators _ _ . . . . . . . . . . $\quad 9-3$

9.5 Batteries , , , 9-3

9.6 DC/AC Inverters $\quad \ldots \quad . \quad$. .

9.7 AC/DC Converters $\quad \because \quad \therefore \quad \therefore \quad \cdots-7$

9.8 Monitoring Instrumentation $\quad 9-7$

9.9 Component Manufacturers $\quad 9-8$

10.0 MODEL SYSTEMS SPECIFICATIOYS _.

10.1 Introduction 10-1

10.2 General Photovoltaic (PV) System Kequirements 10-2

10.3 System Requirements 10-3

10.4 PV Module Specifications : 10-4

10.4.1 Design Requirements

10.4.1.1 Performance Measurement Definitions

10.4.1.2 Electrical Design Requirements

10.4.1.3 Mechanical Design Requirements

10.4.1.4 Environmental Design Requirements

10.4.1.5 Optical Design R̈equirements 


\section{TABLE OF CONTENTS}

Continued

Section

Page

10.4.2 Qualification Requirements

10.4.2.1 Performance Characterization Requirements

10.4.2.2 Design Qualification Test Requirements '.....

10.4.2.3 Module Acceptance Requirements

11.0 INSTALLATION, OPERATION AND MAINTENANCE

$11-1$

11.1 Introduction

$11-1$

11.2 Operation and Maintenance

$11-1$

11.3 Maintenance and Remote Monitoring with Telemetry

$11-2$

11.4 Manufacturers Supplement

$11-4$

Appendix 11.A: Performance Measurement Procedures

$11-10$

Appendix 11.B: Test Procedures

$11-16$

12.0 PERFORMANCE CHARACTERISTICS OF TYPICAL SOLAR CELL MODULES

12.1 Introduction

12.2. Construction/Manufacturing Data

12.3. Typical Characteristics

12.4 Electrical Performance

12.4.1 Electrical Characteristics

12.4.2 Conclusion

12.5 Generally Accepted DC and AC Equivalent Circuits

12.5.1 Interesting Observations

12.5.2 Conclusions

12.6 Factors Degrading Performance

12.7 Shadowed, Fractured, or Damaged Solor Cells

12.7.1 Single Solar Celi

12.7.2 Solar Array Circuit

12.7.3 Conclusions

12.8 Operational Considerations

12.8.1 Safety/Maintenance Requirements

12.8.2 Grovinding Requirements 


\title{
TABLE OF CONTENTS
}

\author{
Continued.
}

\section{Section}

Page

12.9 Electro-Magnetic Interference (Conducted and Radiated)

12.9.1 Two Conditions

12.9.2 Lightning Protection

12.10 Load Considerations

13.11 Power Utilization Considerations

12.11.1 Maximum Power Point. Tracker

12.11.2 Observations

12.12 Summary of the Key Solar Array Performance Parameters as Applied to Power Conditioning

12.13 List of Reference Documents

\subsection{SPECIFIC SYSTEM DESIGNS AND INSTALLED SYSTEMS}

13.2 General System Considerations and Checklists

13.3 Specific System Designs

13.3.1 National Electrical Code

13.3.2 NV Water Pumping Systems

13.3.3 Domestic PV Power

13.3.4 Navigational and Aircraft Aids

13.3.5 Telecommunications

13.4 Practical Lessons Learned from Installed Systems

\subsection{ADDENDUM}

(.........

14.1 Phatovoltaic System RFP Recommended Addendum

14.2 Purchase Description - 5500 Watt Array

14.3 Rejection Criteria for JPL LSSA Modules 


\section{SECTIONI \\ INTRODUCTION}

The subject matter of this seminar is solar photovoltaic (PV) power systems, which are stand-alone systems. This section will enable the seminar participant to: describe the PV process and the equivalent circuit; plot a typical current-voltage (I-V) curve under various insolations and temperatures (to determine the electric power profile); list the array-to-cell component nomenclature; sketch block diagrams; and, show load/array interactions on an $1-V$ plot. The radioisotope powered PV system is not considered.

\section{I.I SOLAR (PV) CELLS}

Solar radiation, as it impinges upon the earth, has the properties of both energy waves and particles. The wave property is associated with both the light spectrum detectable with glass prisms, heat, and electromagnetic radiation. "The wave and particle properties are associated with the production of electricity by PV cells (semiconductors). In brief, the energy particles (photons) penetrate the two-layered PV device (solar cell). The top (n layer) contains many free electrons. The bottom ( $p$ layer) contains many highly mobile positive charges called holes. Photons of high enough energy pass through the thin $n$ layer and are absorbed by atoms in the $p$ layer. Electrons are energized and hole-electron pairs are created. The electrons wander around in the $p$ layer until recaptured by holes. If the electrons enter the junction between the $n$ and $p$ layers, they get caught in the crysta! electric field charge differential near the $p-n$ junction and are drawn into the $n$ layer. Hence, electrons gather in the upper layer and the region becomes negatively charged. Because the electrons have migrated from the $p$ region, the $p$ region becomes positively charged. Therefore, a voltage develops across the junction as though it were a capacitor. If wires are connected to the top and bottom of the cell, a current will flow through the wire (see exhibit 1.1). As a result of the photon tombardment, the solar cell (semiconductor) ac is as an electron source. This entire process is termed photoconductivity. The photon is defined as a quantum of electromagnetic energy having both particle and wave behavior. It has no charge or mass but possesses momentum and carries the energy of light. The frequencies of the photon radiation equal the speed of light divided by the photon wavelengths. The energy of the radiation equals the frequency times a constant.

PV CELL

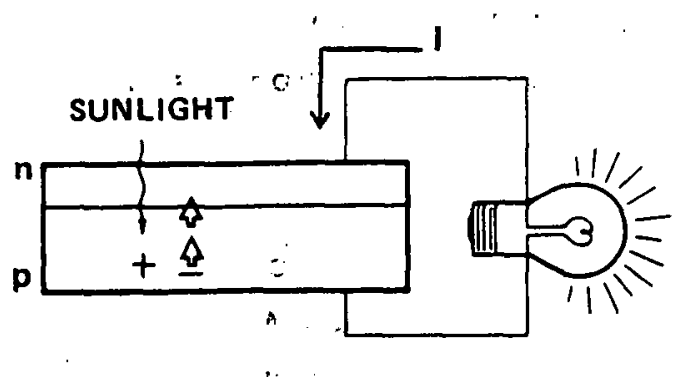

Exhibit 1.1 Solar Photoconductivity of the p-n Junction Semiconductor

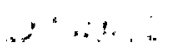


Exhibit 1.2 shows an equivalent circuit for one cell. Due to photoconductivity, a current source is contained within the circuit. The current source is "shorted" (paralleled) by a diode (which represents the $p-n$ junction) with a current that opposes the photon-induced current. This semiconductor junction dark current is a function of the semiconductor reverse saturation current and $p-n$ junction voltage. The dark current is always present, even for an un-illuminated cell, which is typical of semiconductors. In addition, there is a series resistance resulting from the bulk electrical resistance of the cell material, as well as the series resistance of the electrical leads and the joint between the leads and the cell material. A shunt (parallel) resistance also is present, simulating the recombination of electrons and holes that occurs within the material before the electrons can leave the cell for load current. The unilluminated current is also referred to as the dark current. $1=1$ + $I_{D}$ where $I_{L}=$ illuminated current and $I_{D}=$ unilluminated current, and 1 = cell output
current.

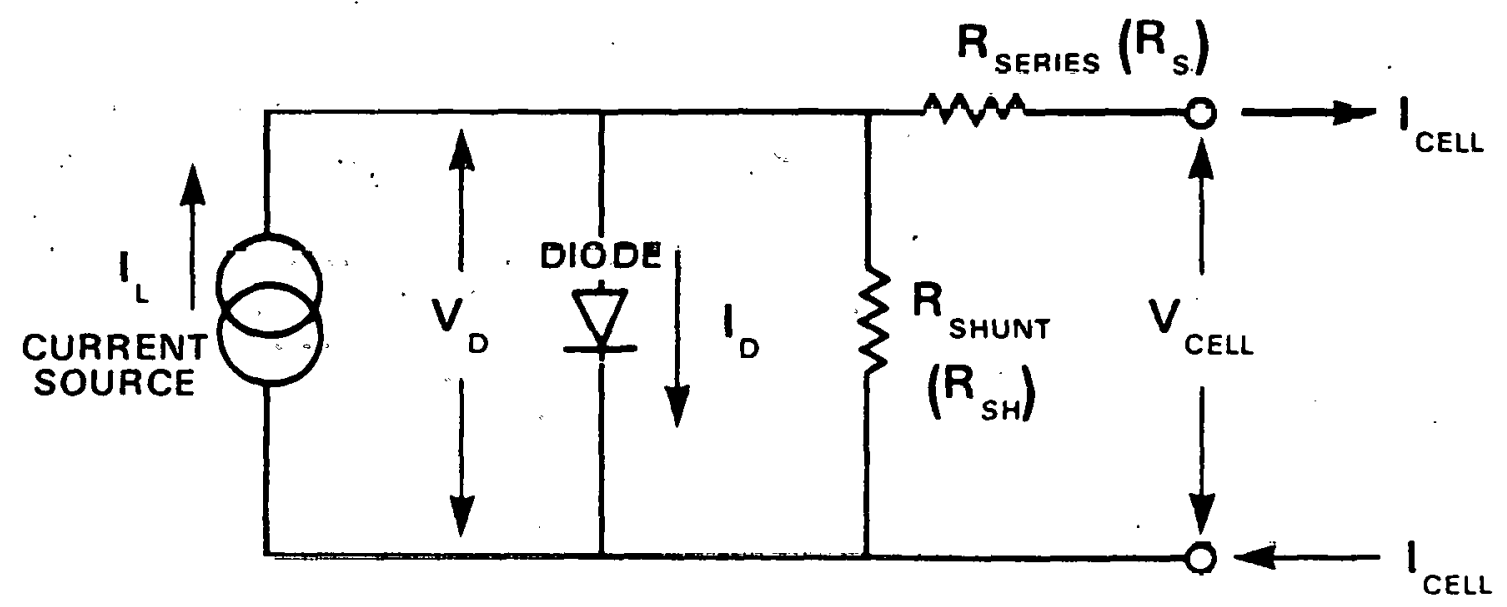

Exhibit 1.2 Equivalent Circuit for Solar Cells.

Exhibit 1.3 illustrates the voltage-current relationship for a single cell. The cell output is shown in the upper left. The power: output of the cell is equal to the product of the current and voltage; the maximum-power point is indicated as $P M$ in the exhibit. The upper right curve shows the variation of output current and voltage with insolation (solar flux). An insolation intensity of $100 \mathrm{~mW} / \mathrm{cm}^{2}$ corresponds to a clear day at sea level. The curve shows that the short-circuit current is proportional to the insolation, but that the open-circuit voltage is insensitive to insolation. The effects of temperature are illustrated on the curve on the lower left. The maximum output power decreases approximately 0.5 percent per degree (C) temperature increase. This effect is illustrated on the lower right. Unlike conventional generators, the PV cell current decreases for increases in cell voltage. 


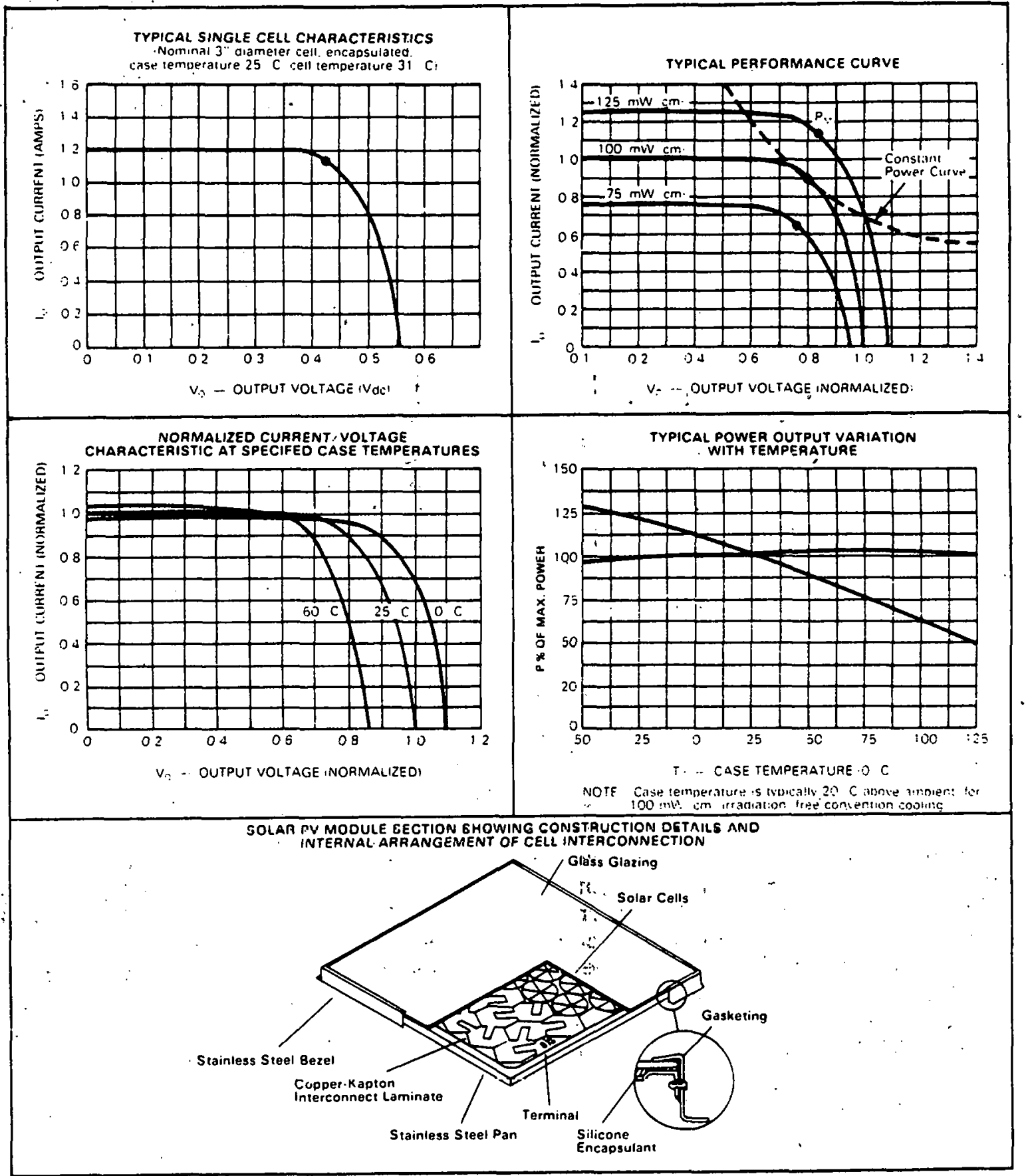

Exhibit 1.3 The Relationship Between Current and Voltage for Typical PV Cells. 


\subsection{COMPONENTS OF THE PV SYSTEM}

A PV array is composed of many subarrays, panels, modules, and cells in various series, parallel, star, and delta combinations. Environmental effects are usually mitigated by designing solar cell packages that "control" the near-term effect of the environment. Present-day construction encases the solar cells behind tempered, low-iron-content glass, backed up by high temperature silicone rubber. compounds and a back supporting member such as a fiberglass sheet as illustrated in exhibit 1.4. The solar PV power system is a standalone system but can incorporate back-up power. One-axis and two-axis tracking collectors are not considered in this seminar, and usually involve the mounting of PV modules at the foci of concentrators. These collectors are being studied in the solar heating/cooling, solar thermal power system, and solar total energy system programs.

Exhibit 1.5 shows a PV array and component parts. Individual cells are wired together and mounted to produce a module. The individual cells within the module are wired together to produce the required output voltage and current. These cells are usually placed in a compound of silicone rubber because it has good thermal expansion and weathering capabilities. Currently, these modules are covered with glass, plastic, or a silicon-rubber compound to provide further protection to the cells. They are then wired together to form a panel of adequate electrical and structural size. Panels are usually installed side-by-side. The structure is tied together and electrically and structurally integrated to form subarrays. Subarrays are then arranged, usually one behind the other (although a side-by-side arrangement is possible), to form arrays. Exhibit 1.6 shows network connections for balanced loads and generators. The summation of current at any node equals zero, and the summation of voltage in any loop equals zero, according to the Kirchhoff circuit laws of the conservation of energyccharge. Voltage equals energy per unit charge, and current equals charge per unit time. The final array configuration is heavily dependent on the cell I-V characteristics.

Based on application and load requirements, the terminals of the array are connected to various "power conditioning". equipment. This power conditioning equipment is designed to convert the direct current (DC) produced by the array to a more suitable type (alternating current ( $A C$ ), if $A C$ voltage is required) and various duty cycles of power. Exhibit 1.7 shows the basic components of the PV system. The power conditioning equipment also integrates battery storaqe and supplementgl energy sources (if required) with the PV arrny and Innd. The system is regulated to maintain system voltage, adequate battery storage, and frequency for $A C$ loads, continuously.

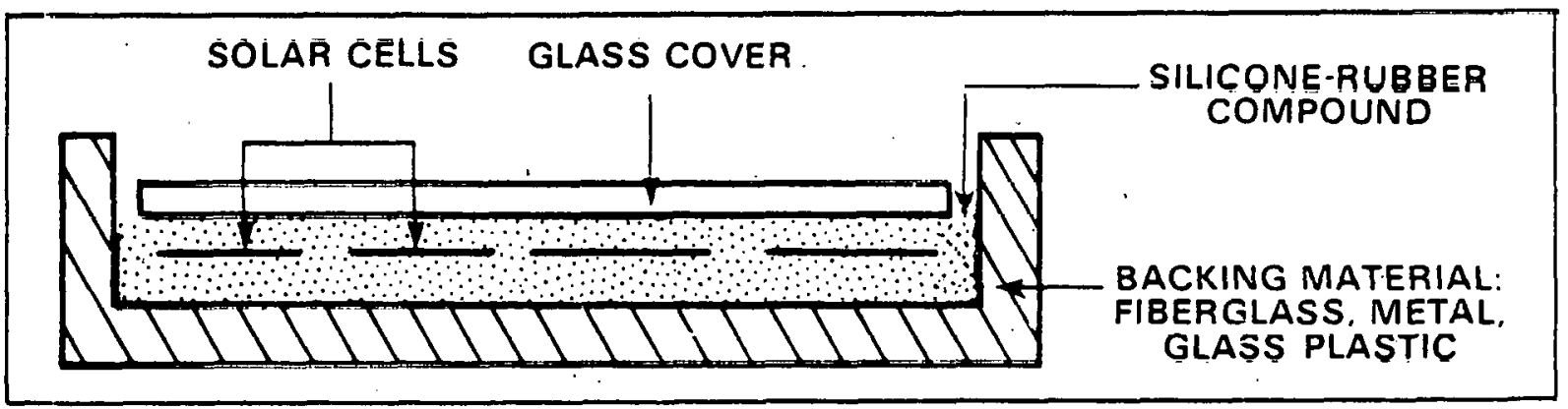

Exhibit 1.4 Cross-Section of a Photovoltaic Module. 


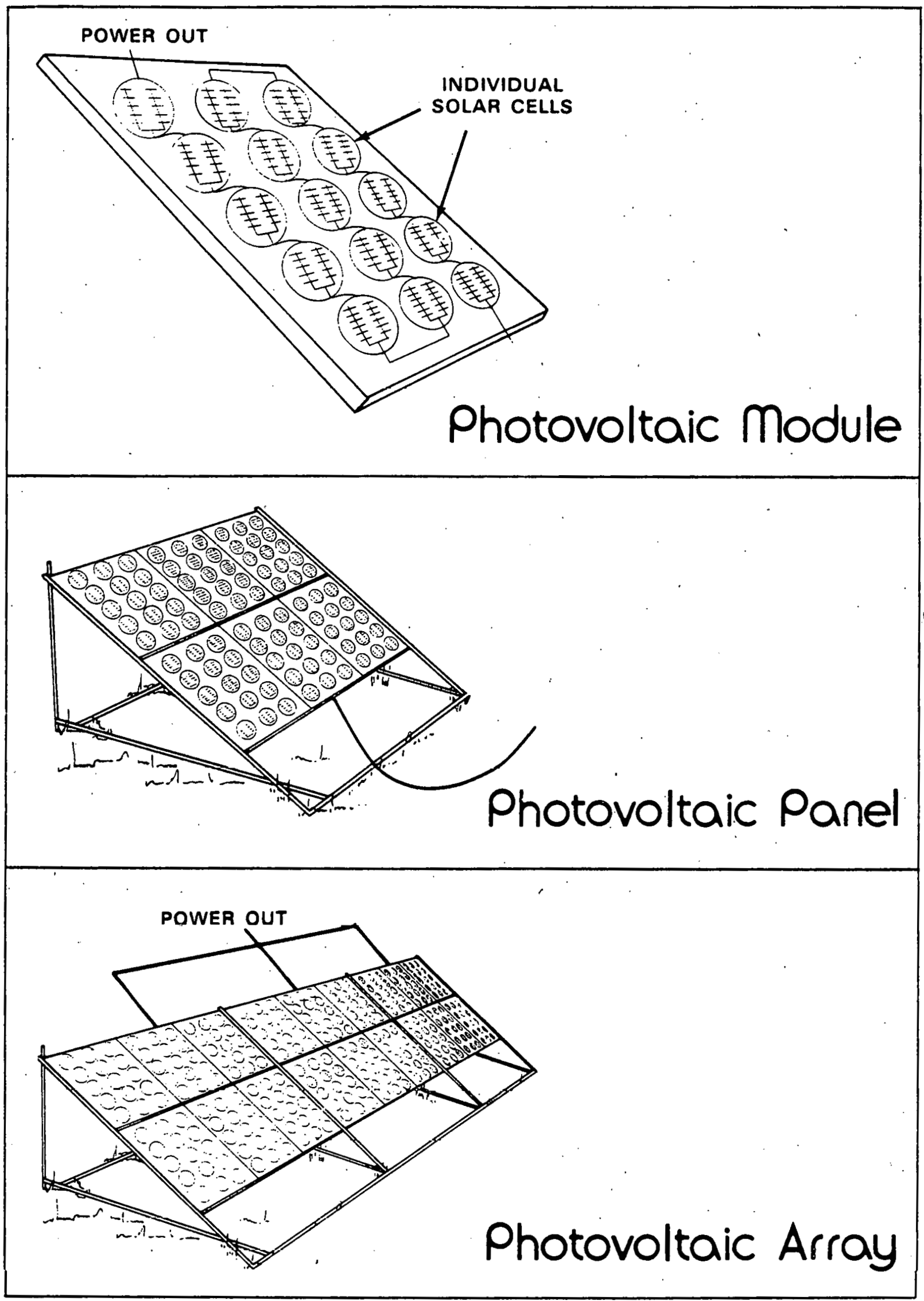

Exhibit 1.5 Solar Cell Array Components. 


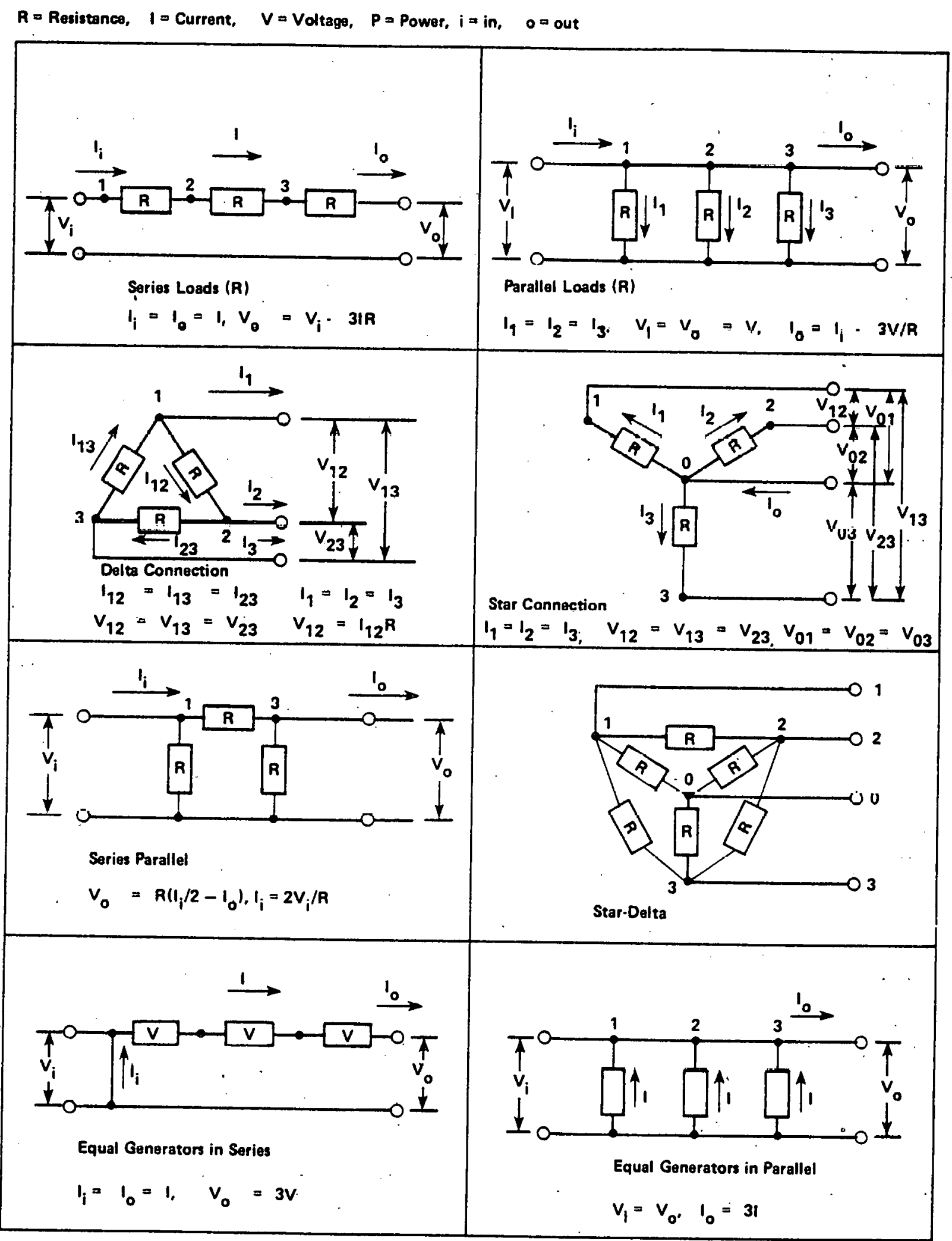

$V=$ IR: Chms Law, $R_{t}=R_{1}+R_{2}+R_{3}$ : Sories Load Law $1 / R_{t}=1 / R_{1}+1 / R_{2}+1 / R_{3}: \quad$ Parallel Load Law.

Exhibit 1.6 Balanced Network Connections 
A power inverter is an element of the power conditioner and is an electrical device that bidirectionally switches the polarity of the array output direct current at a desired frequency (usually 60 cycle) for AC applications (see exhibit 1.8). The operation of the electronic switching on-off devices produces an alternating current "square wave" effect that is highly undesirable, so a filter is employed to smooth out the square edges. This sinusoidal conditioned power can then be used in place of utility power for conventional AC electricity or in conjunction with utility power for grid connected applications. The amplitude of the voltage wave is adjusted to match the effective voltage required. For a 60 cycle per second frequency $(60 \mathrm{Hertz})$ sine wave, 170 volts peak $A C$ produces the equivalent of 120 volts DC. The DC equivalent is the r.m.s. value (root-mean-square), the effective value.

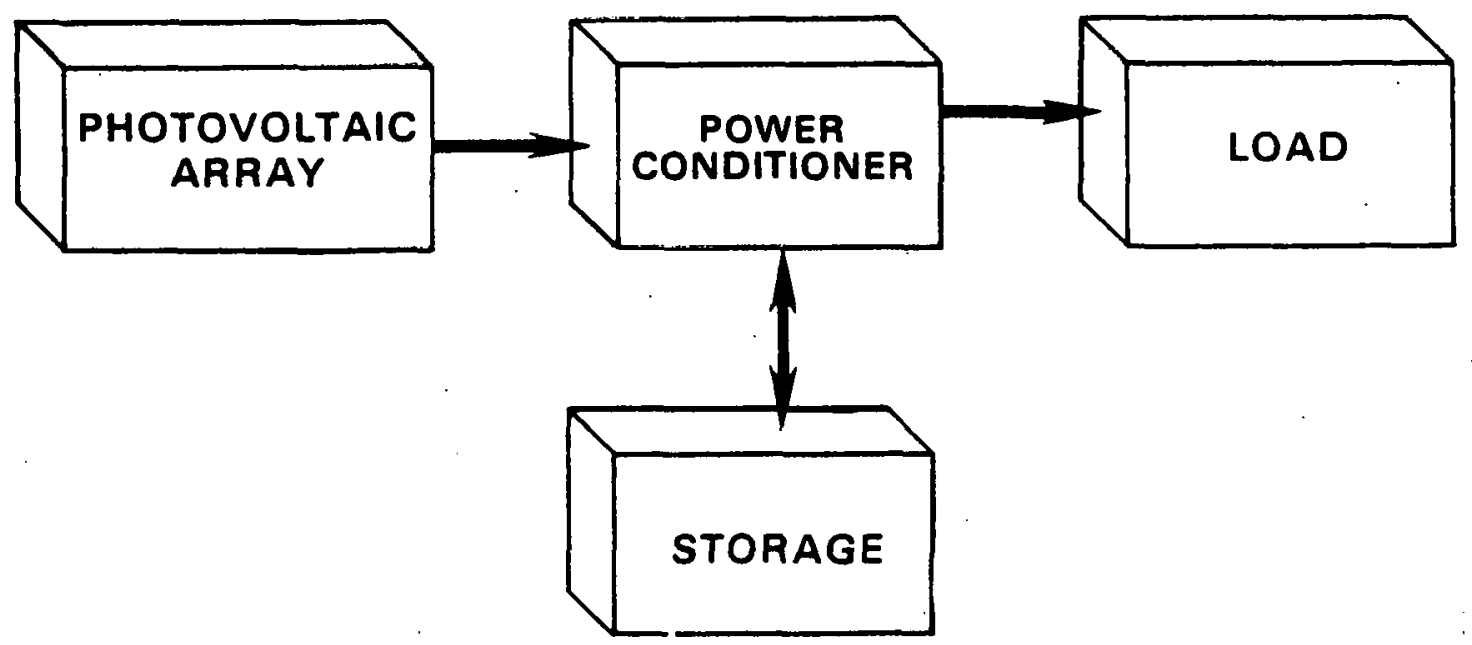

Exhibit 1.7 Basic Components of the Photovoltaic System.

Energy storage is necessary for solar-produced power during sunset, night, sunrise, inclement weather, or a series of cloudy days. The best-suited and most common storage device is the electrical storage battery. Batteries are inherently DC devices and, for some opplications, the array and a battery storage system can be employed to provide power directly to a load without a power inverter (exhibit 1.9). Applications of such systems , include DC motors for irrigation of farm land, providing power to DC-operated radio equipment, and applications where DC transmission and/or distribution are required or feasible.

Systems designed for $A C$ loads can use storage batteries in various ways. Exhibit 1.10 illustrates the addition of a DC/AC power inverter to the system shown in exhibit 1.9.

Exhibit 1.11 is a different design for using storage batteries for AC loads. The system in exhibit 1.10 can be improved by adding a converter, and a smaller power inverter (exhibit 1, (1). This retrofit would be made if the load requirements are reduced drastically when stored energy is used (e.g., nighttime minimal load). A low voltage array can supply a high voltage load while charging batteries at an intermediate voltage. 
THE DC/AC INVERTEF:

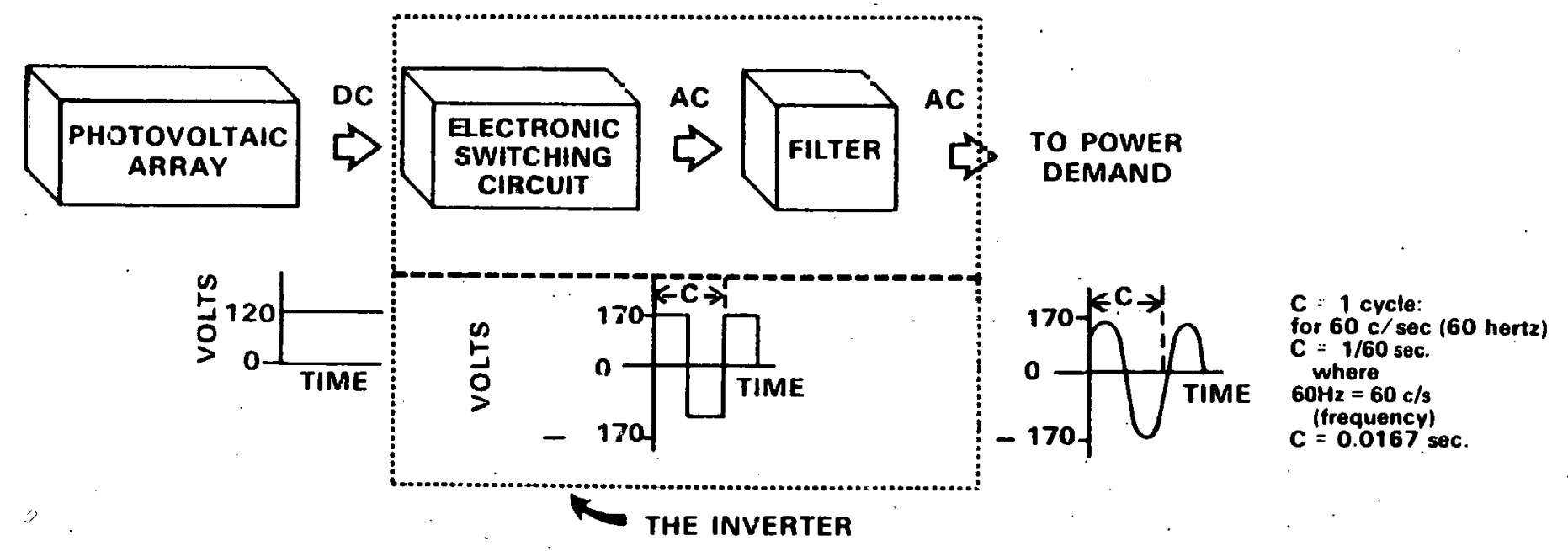

Exhibit 1-8 Operation of the Basic Power Inverter (DC/AC) 


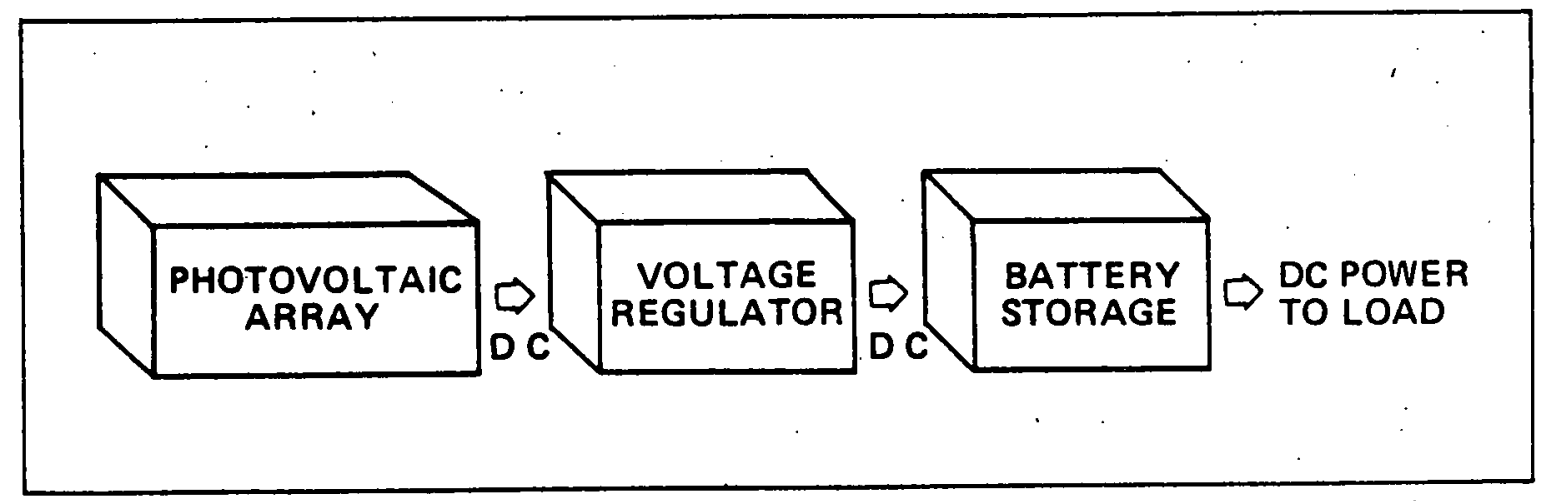

Exhibit 1.9 Supplying DC with Battery Storage.

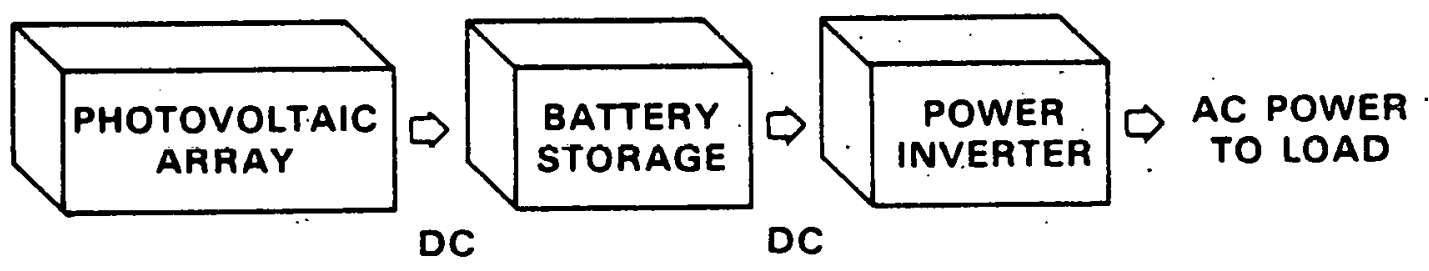

Exhibit 1.10 Supplying AC with Battery Storage. 


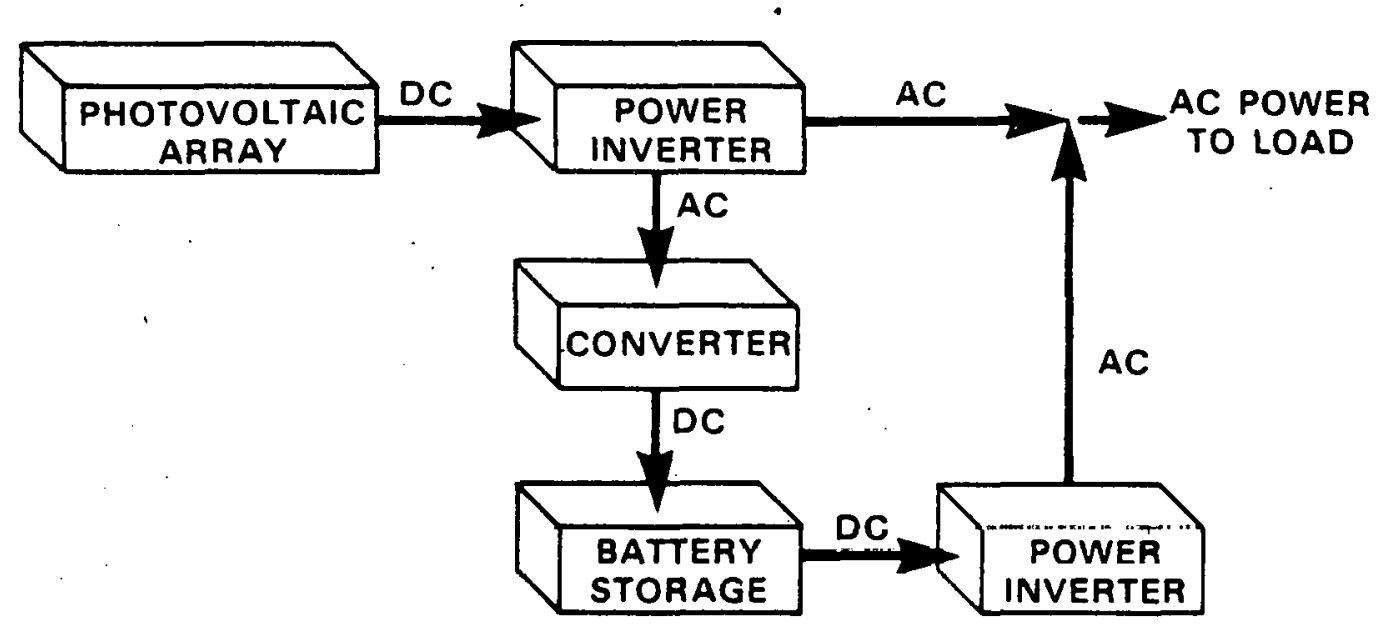

IF INVERTER \#1 IS 20\% EFFICIENT AT NIGHT LOAD, USE OF ONLY ONE INVERTER GIVES $1.9 \%$ TO $2.7 \%$ EFFICIENCY AT NIGHT.

Exhibit 1.11 Supplying AC with Battery Storage for Low Night Demands.

\begin{tabular}{|r|r|}
\hline DIRECT & STORED \\
\hline $9.0 \%$ & $5.3 \%$ \\
$13.2 \%$ & $10.3 \%$ \\
\hline
\end{tabular}

EFFICIENCIES

\subsection{SUPPLY/LOAD INTTERACTION}

Exhibit 1.12 illustrates the current-voltage relationship of cells under various illuminations. With decreasing (or increasing) amounts of illumination, the solar cell maximum power point "moves" for a constant lood. The cell power ouput $(P=V \times 1)$ is maximized, where $P=$ power, $V=$ voltage, $I$ = current, Isc = short circuit current, and Voc = open circuit voltage. The rectangular area of the $\mid-V$ plot is the cell electric power for a given operating point. The $I$ intercept is Isc and the $V$ intercept is $V o c$ for $V=O$ and $I=0$, respectively, for the curves.

The design load locus is a line $(y=m x+b)$ where $y=$ current, $m=a$ constant $(1 / R), x=$ voltage, and $b=0$. Isc is a linear function of intensity (insolation) and Voc is a logarithmic function of intensity. Varying the lood to produce optimal conditions results in maximum power being transferred from the array to the load. While the actual load may not change in value, the system can compensate for a shift in the load by either adding or decreasing the power going into storage. A distinguishing characteristic of PV cells is that current decreases for increases in cell voltage. Resistance $(R)$ equals voltage/current, where one ohm equals one volt per ampere. 


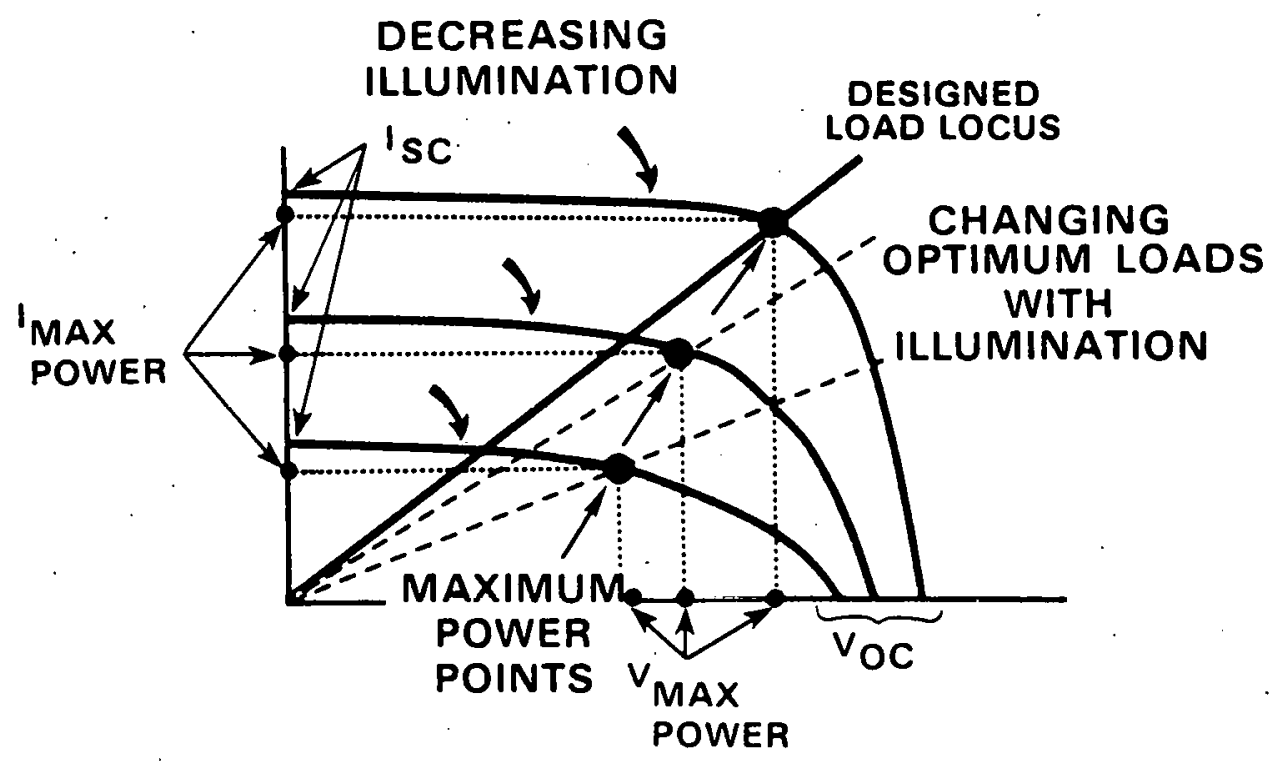

Exhibit 1.12 I-V Relationship of PV Cells with Decreasing Illumination. 
Exhibit 1.13 shows the system illustration in Exhibit 1.11 with the addition of a maximum power tracker and a reference solar cell. The maximum power tracker adjusts the effective load on the photovoltaic array, using the signal from the reference solar cell. This compensates for a change in power due to a change in illumination.

The system shown in Exhibit 1.13 is considered to be an effective system for supplying power to a varying load under varying solar (insolation) conditions. Also shown in the figure is a backup system which can be utilized as a supplement or to supply power when prolonged periods of lack of sunlight completely deplete the storage capability of the photovoltaic system. Energy storage and backup power are regulated to cause an optimal effective load condition.

$P I=P a+P s+P b$ where $P I$ equals effective load power, $P a$ equals array power, $P s$ equals storage power, and $\mathrm{Pb}$ equals backup power. Ps becomes negátive when the battery subsystem is being charged.

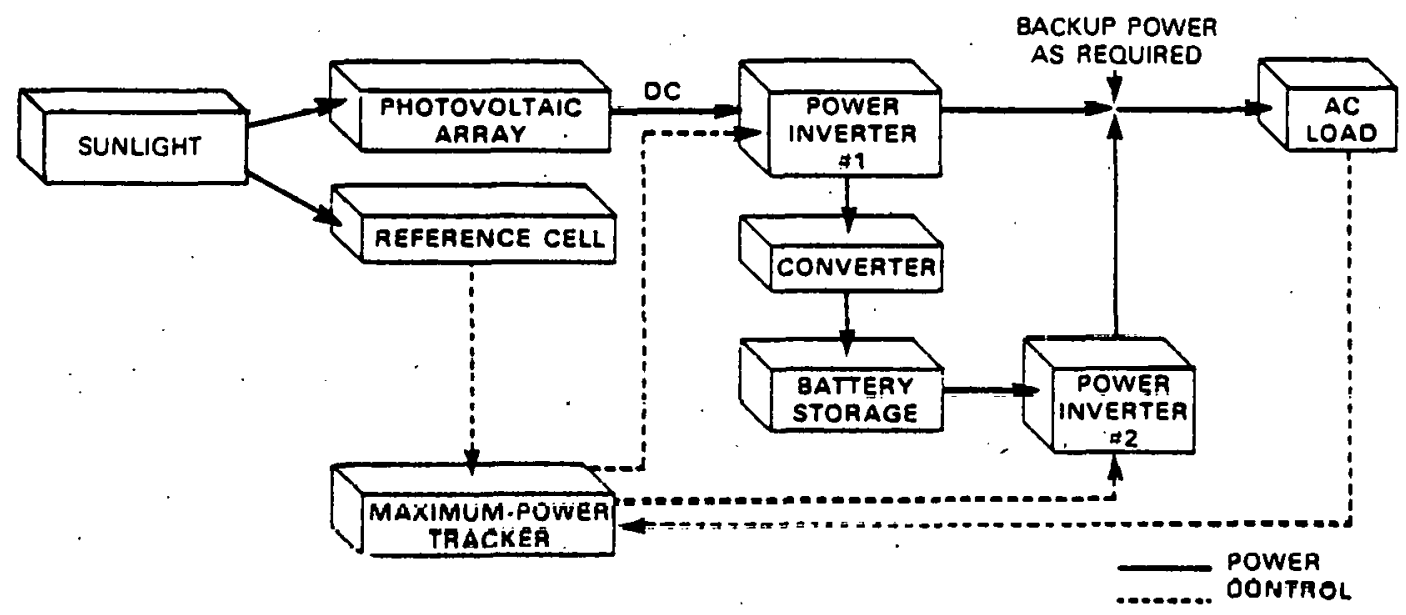

Exhiblt 1.13 Maximum Power Tracking 


\section{SECTION 2 \\ PHOTOVOLTAIC ARRAYS}

\subsection{BACKGROUND}

Solar cells are the basic unit of a photovoltaic power system and convert sunlight directly into DC electricity. The incident photons are absorbed in the solar cells and produce a flow of electricity when an electrical load is connected to the solar cell. Solar cells are a form of solid state diode and can be made from various semiconductor materials. However, the preponderance of experience is with silicon. Photoconductivity of semiconductors is the fundamental principle of solar cell electricity, where conductivity is the reciprocal of resistivity. Resistance equals resistivity $\times$ length/cross-sectional area.

The use of silicon solar cells in outer space dates back to 1958 with the launch of Vanguard 1. Hundreds of silicon-solar-cell-powered spacecraft have a few thousand years of cumulative operational experience in space. No space flight has ended because of solar cell failure. In addition to space applications, many remote terrestrial power applications, such as communication repeater stations, have been operated by private and governmental organizations for up to 10 years at power levels from 1 to several hundred watts. Historically, silicon solar cells have been in general use on Earth since 1955 when the Bell Telephone Laboratories successfully powered telephone amplifiers in field tests. RCA was involved also.

\subsection{SOLAR CELL CHARACTERISTICS}

Solar cells are fabricated from high purity single-crystal, silicon wafers which are doped with the necessary trace elements to form a semiconductor material. Doping increases the number of charge carriers. An $n-p$ diode junction is formed by diffusing $c$ second element, such as phosphorous or arsenic, into boron containing silicon wafers to form a 0.001 in. layer of doped material on the illuminated surface of the cell. The designation $n-p$ refers to negative and positive charge carriers. The contact of the doped and undoped layers (the n-p junction) produces an electrical field within the silicon. When the solar cell is exposed to sunlight, the photons (light energy from the sun) are absorbed by the silicon and energize electrons within the crystal. The built-in electrical field separates the electrons from the parent material and gives rise to an electrical potential of approximately 0.5 volts. To access this voltage, electrical contacts are added to the front and rear surfaces of the cell as shown in Exhibit 2.l. The contact on the front (illuminated) surface is characteristically a finger-like grid pattern designed to maximize current gathering while minimizing shadowing of the surface. An anti-reflective coating is also sometimes added to the surface of the cell to minimize surface reflections and increase the energy absorbed.

Typical solar cells have efficiencies of 10 to 15 percent and, therefore, generate approximately $12.5 \mathrm{~W}$ per square foot $\left(\mathrm{ft}^{2}\right)$ of area under peak, midday. solar illumination (maximum insolation equals $100 \mathrm{~W}$ per square foot of air). The cell voltage is a function of the cell materials only and is independent of cell size. The current from a cell is a function of the incident radiation and is directly proportional to the illuminated surface area. For space applications, power per unit area is very important, so cells are cut into squares or rectangles to achieve high packing factors. When crystals are "grown," the manufacturing process results in a silicon ingot, cylindrical in shape. For terrestrial applications where cost is most important, solar cells are generally circular so maximum use can be made of the silicon crystal's cylindrical shape. 
Exhibit 2.2 shows how the I-V output of a typical cell varies with insolation. The current density is used for the current axis and is designated $\mathrm{J}$. The open circuit voltage, as determined by the cell junction, varies only slightly with illumination level, but decreases at a rate of approximately $2 \mathrm{mV} /{ }^{\circ} \mathrm{C}$ with increasing temperature. The maximum power output from the cell occurs at the knee of "maximum power point" of the $1-V$ curve. The maximum power is typically about 0.7 times the product of open-circuit voltage and short-circuit current. (The factor 0.7 is called the "fill factor.") The short-circuit current is nearly independent of temperature and is directly proportional to the solar illumination level (insolation). The basic relationships used are Ohm's law: $V=\mathbb{R}$, where $R$ is resistance; $J$ is the current density, $J=\mid / A$, where $A$ is the area, and $P=V \times I$, where $P$ is the power. Also, voltage equals energy per unit charge and current equals charge per unit time. A solar cell is a current generator and its output is proportional to the exposed area and the sunlight intensity on the area. The current density (current per unit area) for a given insolation level is a good indicator of solar cell capability.

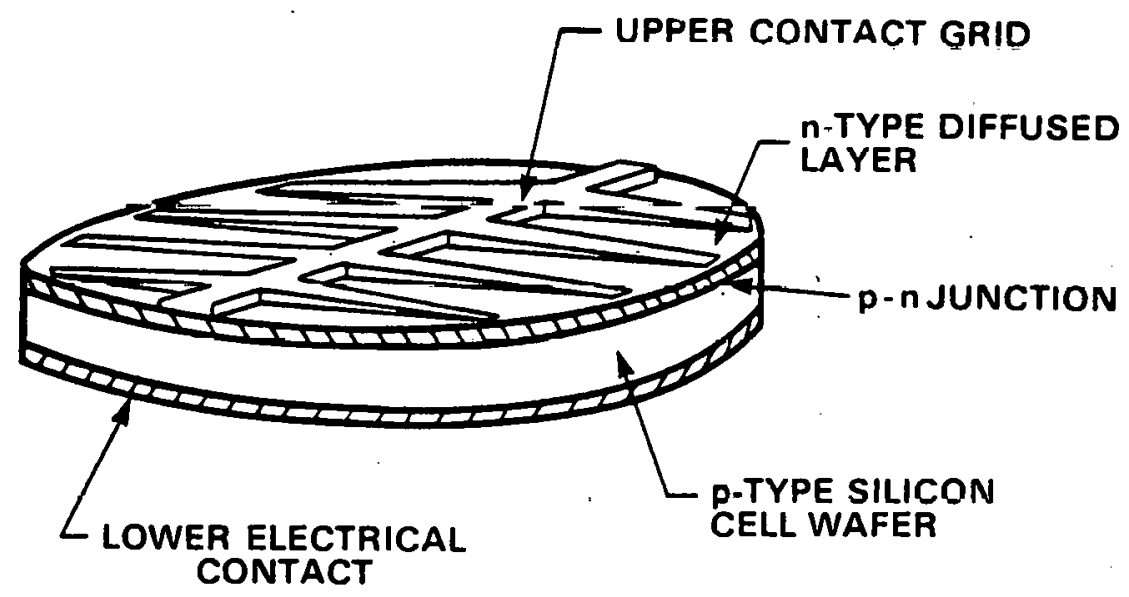

Exhlbit 2.1 Typlcal Terrestrial Solar Cell Structure. 


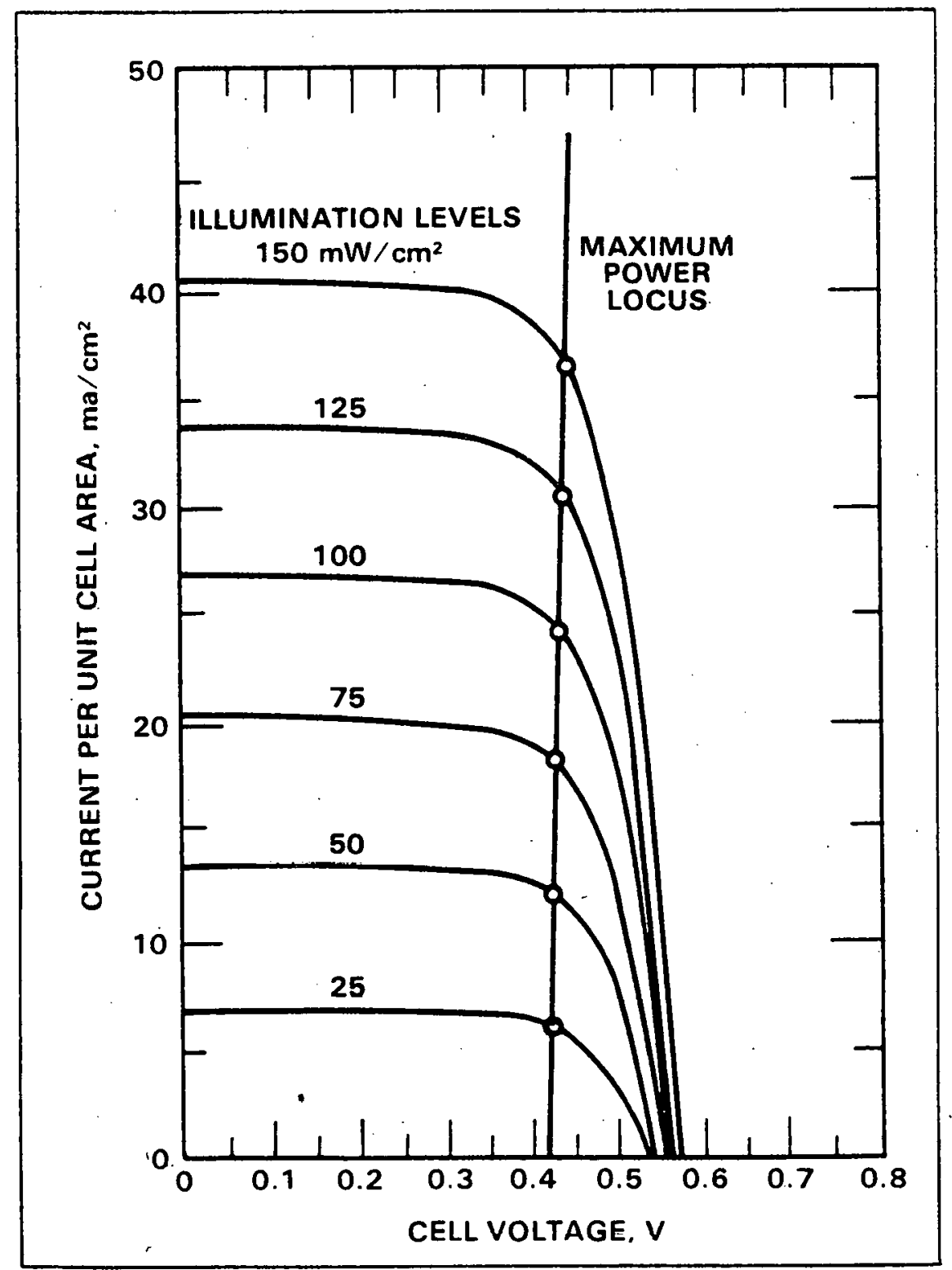

Exhibit 2.2 Current Density vs. Voltage for Different Levels of Cell Illumination

$$
J=1 / \text { area, } \operatorname{ImA}=0.001 \text { Amp }
$$




\subsection{ARRAY PERFORMANCE CHARACTERISTICS}

To achieve higher voltage and power levels, individual solar cells are combined in series and parallel like storage battery cells. The smallest grouping of cells, a solar cell module, consists of a string of series-connected solar cells as well as the encapsulating material needed to support the cells and provide protection from the environment. Typical solar cell modules provide about 5 peak $\mathrm{W} / \mathrm{ft}^{2}$ of module area and weigh from 0.1 to 1.0 $\mathrm{Ib} / \mathrm{W}$. To achieve larger power levels, modules are grouped into "solar panels" or "subarrays" with sizes close to $4 \mathrm{ft}^{2}$. Panel efficiencies range from 10 to 12 percent.

To achieve the needed voltage and power levels, solar arrays are constructed by combining solar panels. The term solar array is generally reserved for the complete array of cells, modules, and panels, independent of the size of the total installation. For an installation with a single module, the module would be referred to as the solar array. The same would be true if the array consisted of I solar panel or I subarray.

One of the primary advantages of solar cell power is the reliability and design flexibility resulting from the extreme modularity of solar arrays. The modularity allows designs to be easily scaled up and down without requiring requalification of the basic power generation unit. Units also can be added or subtracted to match changing power requirements. In the event of a module failure, the series/parallel redundancy of the system will allow the system to remain operable with only a minor loss of power. The extreme modularity combined with the nearly infinite shelf life of spare modules also greatly simplifies parts inventory and maintenance operations.

Electrical circuit principles state that voltages in series add and in parallel are equivalent to the value of the lowest voltage. Likewise, currents in parallel add, while in series are equivalent to the lowest current produced (refer to exhibit 2.3). Current sources in parallel are summed at a constant system voltage to determine the total system current, and voltage sources in series are summed at a constant system current to determine the total system voltage. Generally, current sources in series are equivalent and voltage sources in parallel are equivalent.

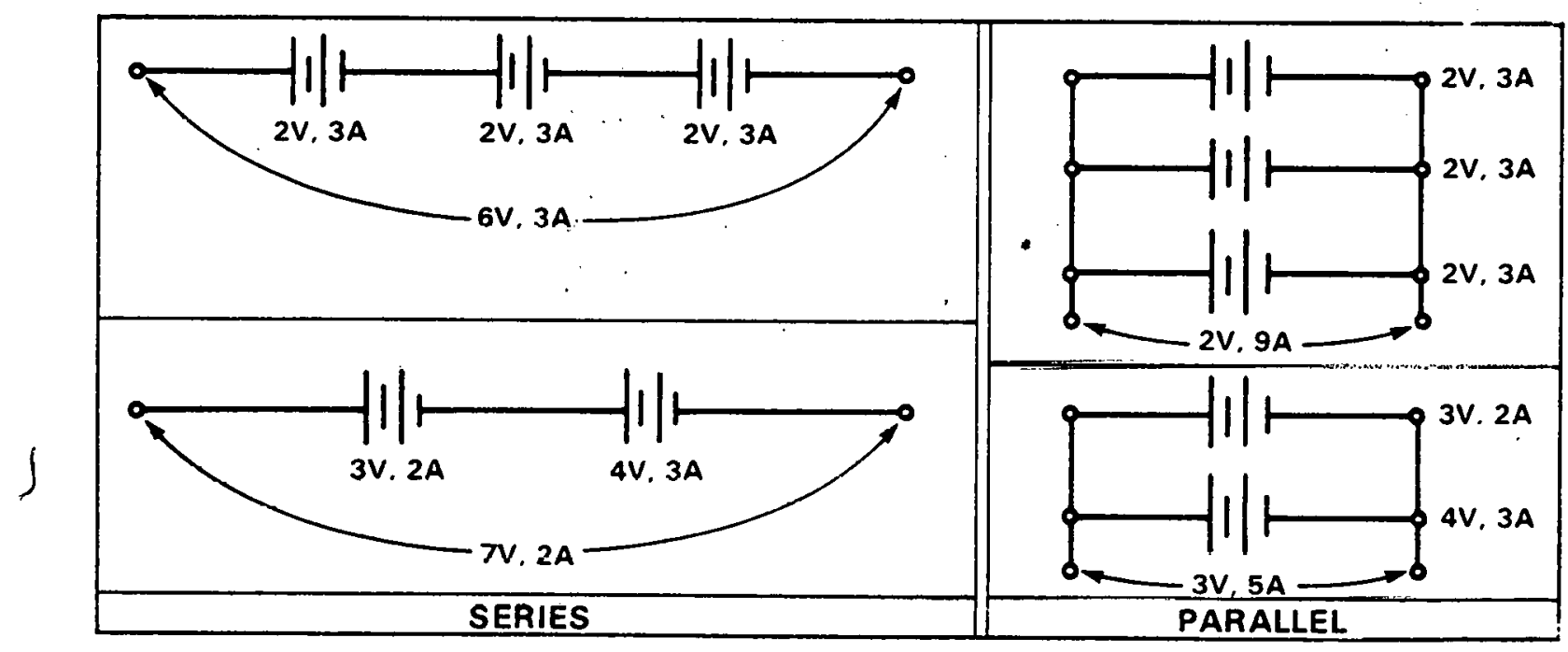

Exhibit 2.3 Basic Series-Parallel Relationships. 
To compute the I-V characteristics' of, a solar array one must, therefore, sum the currents of all the cells (or modules) at each voltage (for a parallel connection of cells) minus the losses of the interconnects. Exhibit 2.4 shows such a computation. No interconnect losses are assumed. It should be noted that only for identical cells is the maximum power available from the array equal to the sum of the maximum power available from the three individual cells. For cells in series, the sum of all individual cell (or module) voltages minus the voltage drop across the interconnects must be computed at each current level to produce the array. IV curve. It should be noted that these curves must be computed at a given insolation level and at a given temperature.

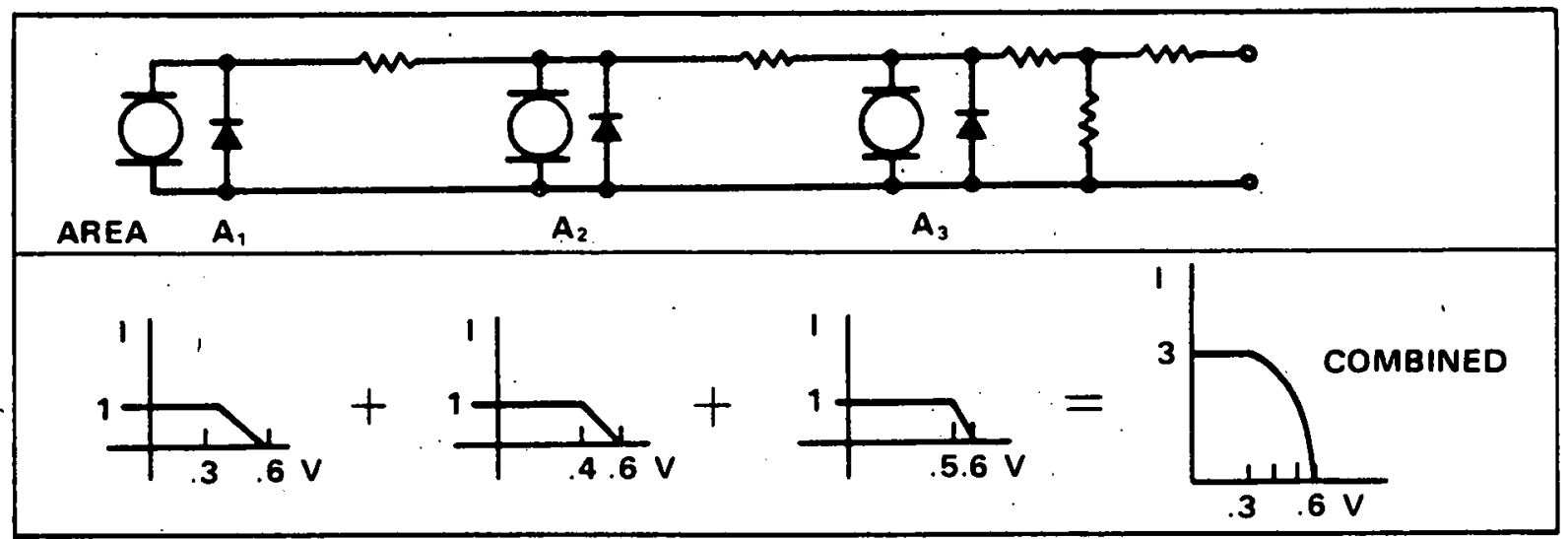

Exhibit 2.4 I-V Characteristics of Unmatched Cells in Parallel.

The effect due to shading (or damaging) a portion of an array depends on whether the cells in that array are in series or parallel. If the cells are connected in series (see exhibit 2.5), the output current of the array will be limited by the current of the single shaded cell, which causes a dramatic decrease in output power. The parallel combination, however, suffers a decrease in current equal to the current of a single cell (see exhibit 2-6). For example; there are two panels of ten cells each, one cell is connected in series, the other 9 in parallel. Each cell is operating at about 0.45 volts and 1 amp, therefore, the power output of each panel is 4.5 watts $(10 \times 0.45 \times 1)$. If a single cell is completely shaded in both panels, the power output of the series panel connected array will drop to almost zero while the parallel connected panel array will output 4.05 watts (if each parallel string is protected by a diode from back biasing), by the computation 4.50 watts -0.45 watts $=4.05$ watts. Corrosion and cell breakage manifest themselves as increased series resistance, possibly approaching infinity (open circuit). A series network of solar cells would be rendered inoperable due to this type of failure compared to a partial decrease in power in a parallel network. 
- SERIES-CONNECTED CELLS (PARTIALLY SHADOWED OR DAMAGED)

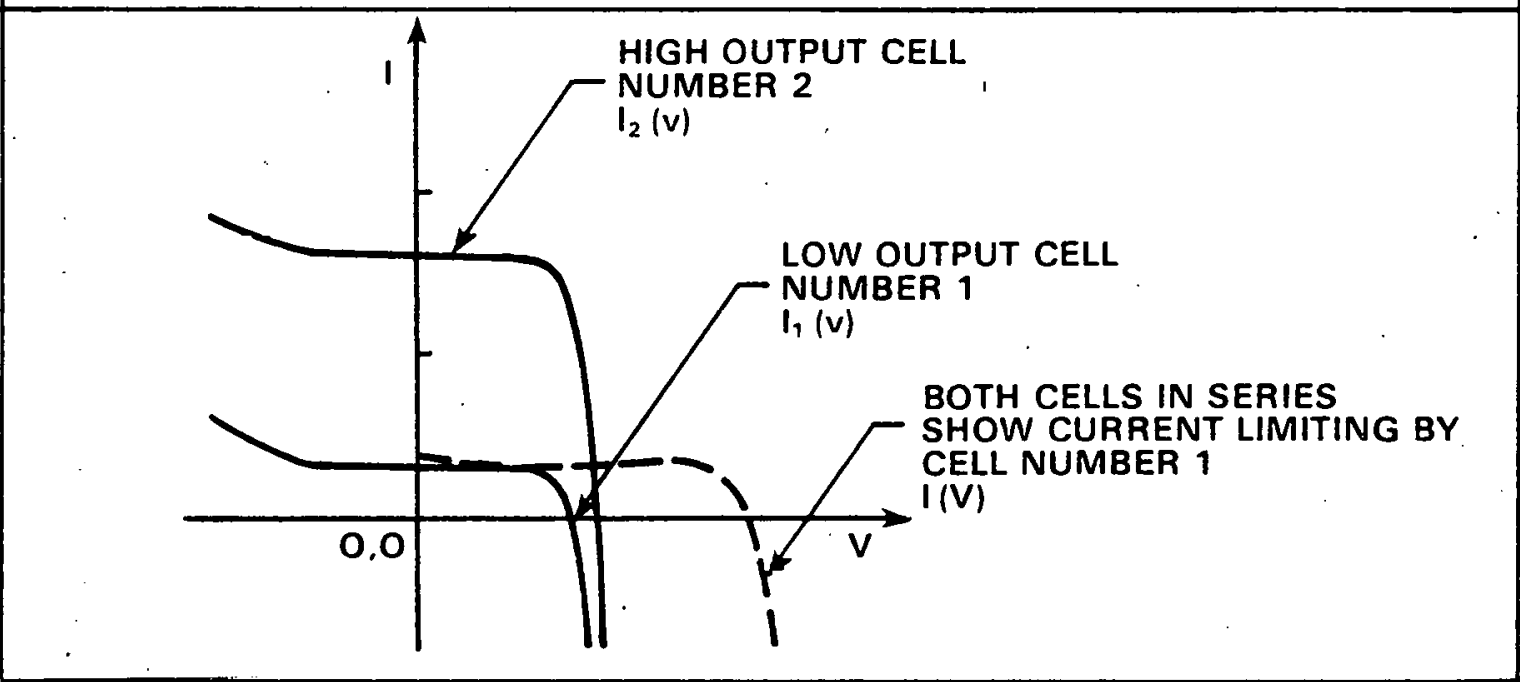

Exhibit 2.5 Shadowed Solar Cells.

- electrical CHARACTERISTICS OF A 2-SOLAR CELl MOdULE WITH ONE CELL TOTALLY OR PARTIALLY SHADOWED.

PARALLEL-CONNECTED CELLS

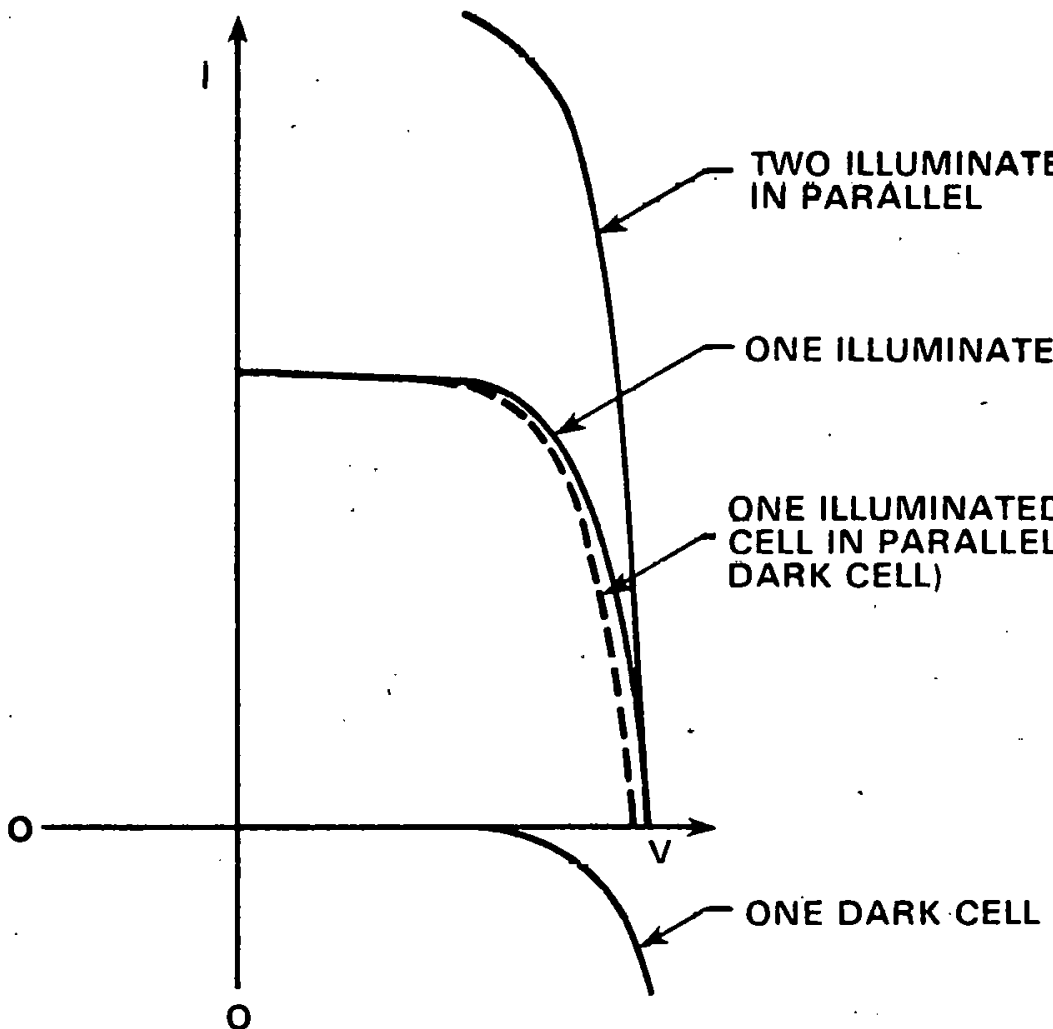

Exhibit 2.6 Shadowed Solar Cells. 
Were it not for the cost of carrying high currents and of inverting DC voltages to $A C$ voltage, designers would always arrange the cells in parallel. Cells are usually placed in series to meet a minimum adequate system voltage, and then multiple strings of cells are paralleled to attain the required system current. Keeping the system voltage low reduces the possibility of injury due to electrical shock. The alternative to large strings of series connected cells, when high system voltages are required, would be to utilize a DC-AC inverter to transform the voltage up to desired levels by use of electromagnetic induction from $A C$ voltage in a transformer. The tradeoff would be the added inefficiency of the DC$A C$ inverter as compared to the higher reliability of the paralleled cells (assuming the probability of inverter failure is smaller than the probability of a failure of a cell in the series string). DC amplifiers can also be used. For an increase in voltage there is a corresponding decrease in current due to the law of the conservation of energy and since power equals voltage $x$ current.

Environmental effects are usually mitigated by designing solar cell packages which "control" the near-term effect of the environment. Present-day construction encases the solar cells behind tempered, low-iron-content glass; backed up by high temperature silicone rubber compounds, and a back supporting member such as a fiberglass sheet (refer to Exhibit 2.7)

\subsection{CELL DEGRADATION}

Solar photovoltaic cells, particularly silicon cells, exhibit an extremely stable element that has been only slightly ( 1 in $10^{8}$ atoms) doped to produce the desired photovoltaic effect. The contacts, which are placed on both sides of the solar cell to collect the produced current, will also exhibit a life time of greater than 20 years if protected from the environment. When this protection or isolation from the environment breaks down, degradotion will take place, causing poorer system performance and ultimately a shorter life time for the system.

Solar cell degradation, therefore, is due primarily to the effects of temperature and humidity and to the corrosion effects of the surrounding environment. As shown in exhibit 2.7 , local humidity and oxygen will tend to oxidize (corrode) the contacts and interconnects of the cell. This corrosion manifests itself in the form of increased series resistance to the flow of available current, resulting in power losses and finally, when advanced corrosion is present, to an open circuit. Many cell manufacturers now use redundant interconnects to help mitigate this problem.

The glass transmits the incident solar energy (low-iron-content glass absorbs less than $1 \%$ of the usable incoming radiation) to the cell while protecting the cell from major shocks (i.e., hail) and from the environment. The silicone rubber serves to cushion the cell from any minor shock, as well as providing a transfer medium for heat dissipation. The thermo/mechanical characteristics of this material are usually matched as closely as possible to that of the cell to minimize stress caused by thermal cycling. The backing material supports the package for structural strength and rigidity, and provides moisturepermeation protection (with the addition of Tedlar or a similar material) from the environment. 


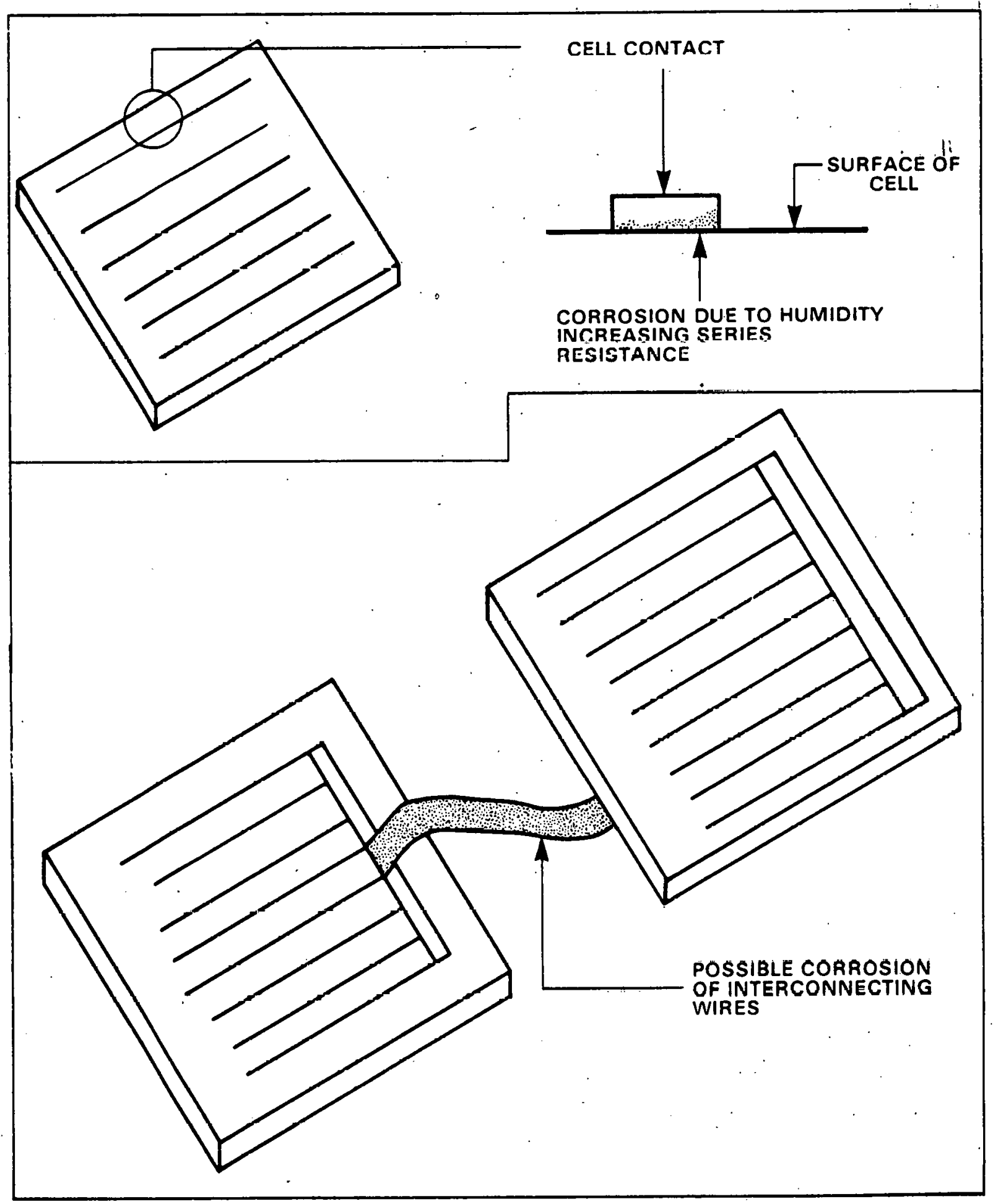

Exhibit 2.7 Corrosion Effects on Solar Cells. 
The front encasing material employed also has an effect on the output-power degradation over the system's life. Field data collected on the effects of soiling on array performance show that one can expect at least 2-8\% ouput-power degradation due to soiling if glass is used as an encapsulant, at least $6-20 \%$ if a semi-flexible silicone conformal coating is used, and a minimum of $8-20 \%$ if room temperature vulcanizing (TRV) rubber is used. The silicon-based compounds will also suffer transmission degradation due to the effects of ultraviolet light. Transmission losses due to these effects can grow to $10 \%$, over several years, before stabilizing.

- Temperature effects exhibited by the environment contribute to the cells' degradation in two major ways. First, thermal cycling causes expansion and contraction of the cell at rates different than the host material that the cell may be encased in. The resulting physical stress may cause hair-line cracks to develop which can result in total loss of cell output. The second aspect of temperature degradation centers around the fact that power output from the cell will decrease approximately $0.4 \%$ per ${ }^{\circ} \mathrm{C}$ of increasing temperature.

Modes of failure and degradation are summarized in Exhibit 2.8A and Exhibit 2.8B (compiled by JPL).

\subsection{OPERATIONAL CONSIDERATIONS}

The solar array as a power source must be compatible with the load. Such factors as grounding, safety and maintenance, electro-magnetic interference, and lightning protection must be considered during the system design, as well as power processing for primary and parasitic loads and power conditioning.

Center-tap grouping of the solar array electrical current via a resistor is recommended. A short in the array can then be detected via a sensing circuit of two resistors and an ammeter (see exhibit 2.9). If a short occurs anywhere on the array, current will flow through the ammeter causing a deflection of the meter's indicator needle. The current flow through the meter can also be utilized to energize switches and remove the defective array from the system. 


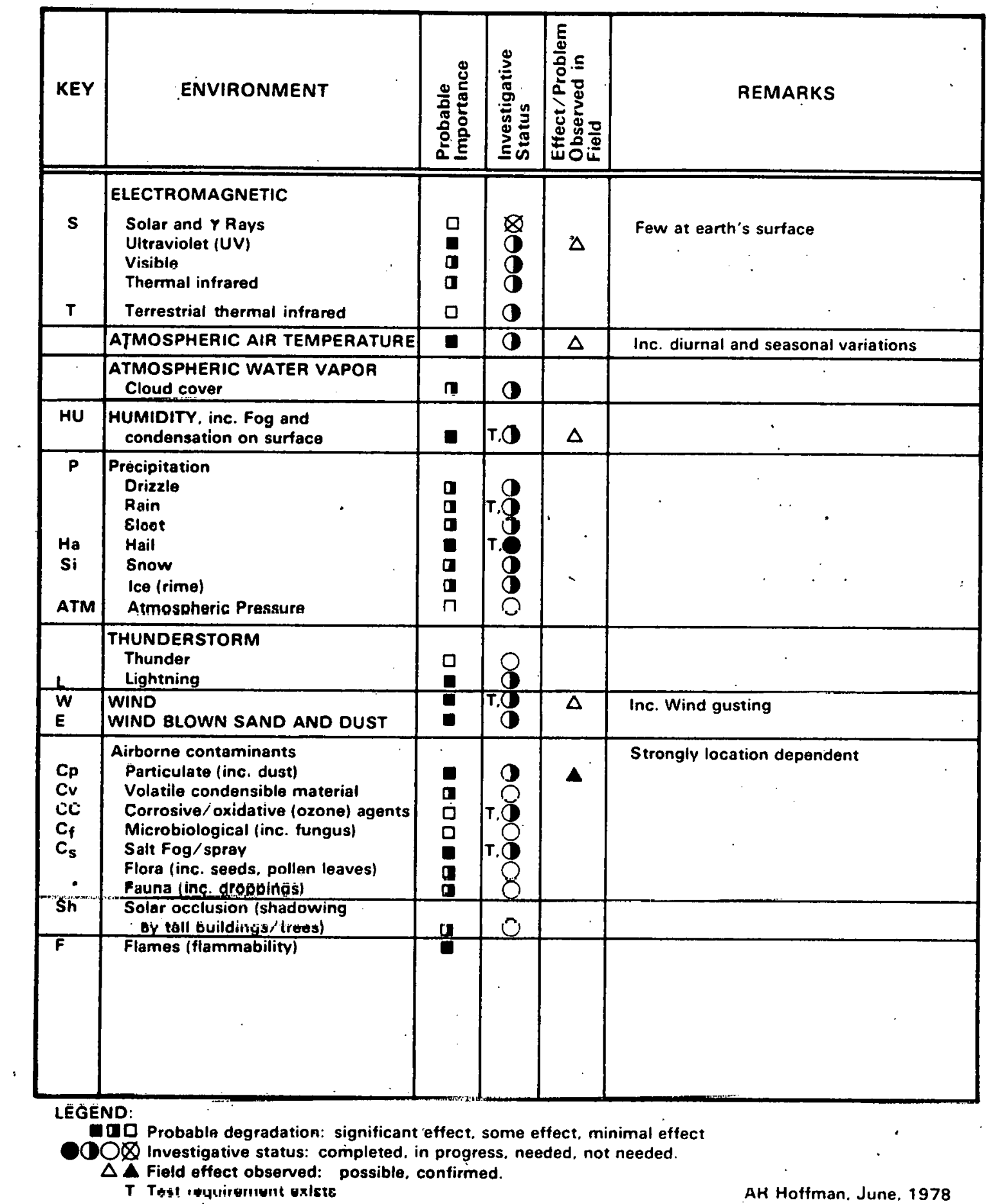

Exhibit 2.8A Degradation and Failure Mechanisms from Environment. 


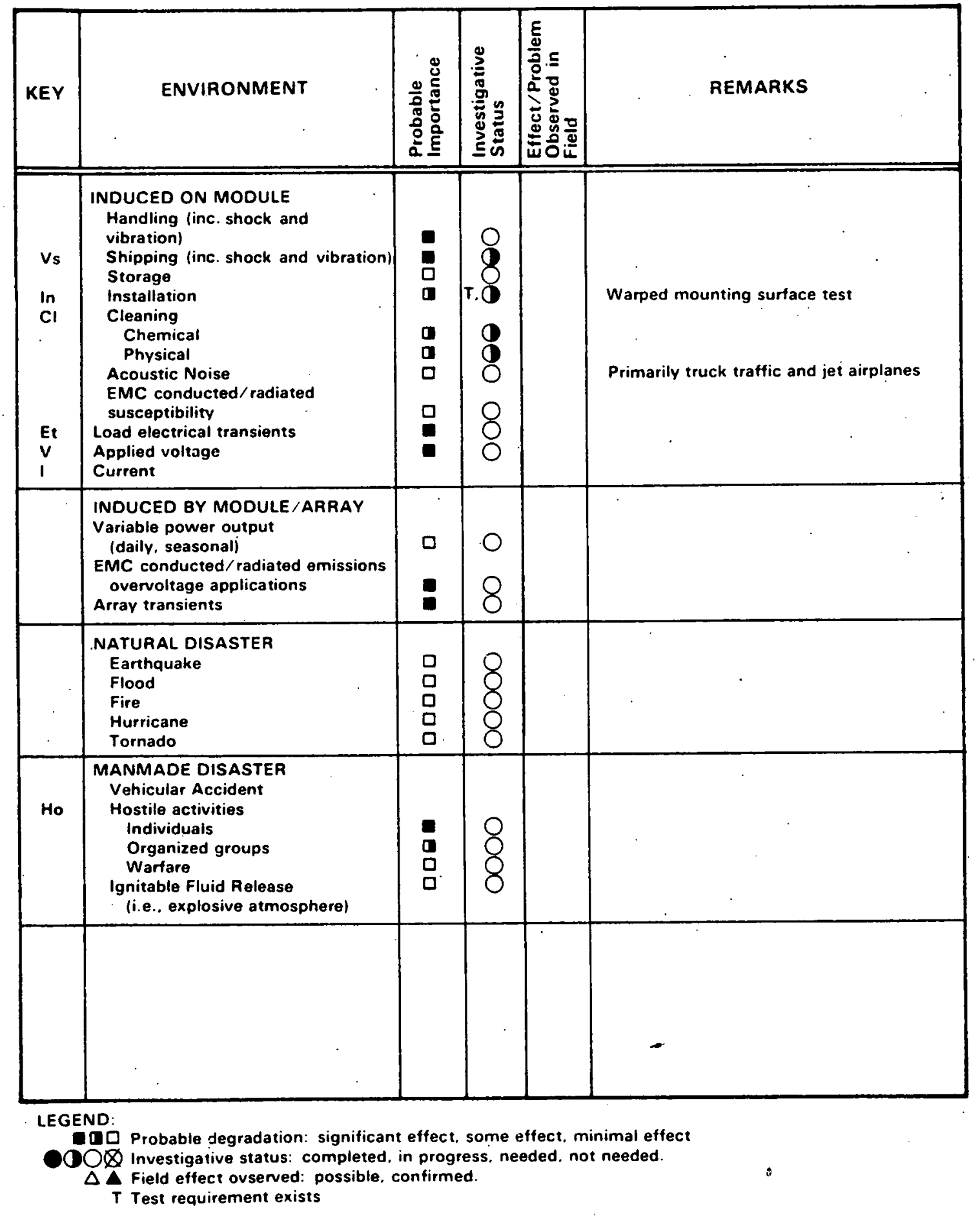

Exhibit 2.8B Degradation and Failure Mechanisms from Handling and/Operations. 


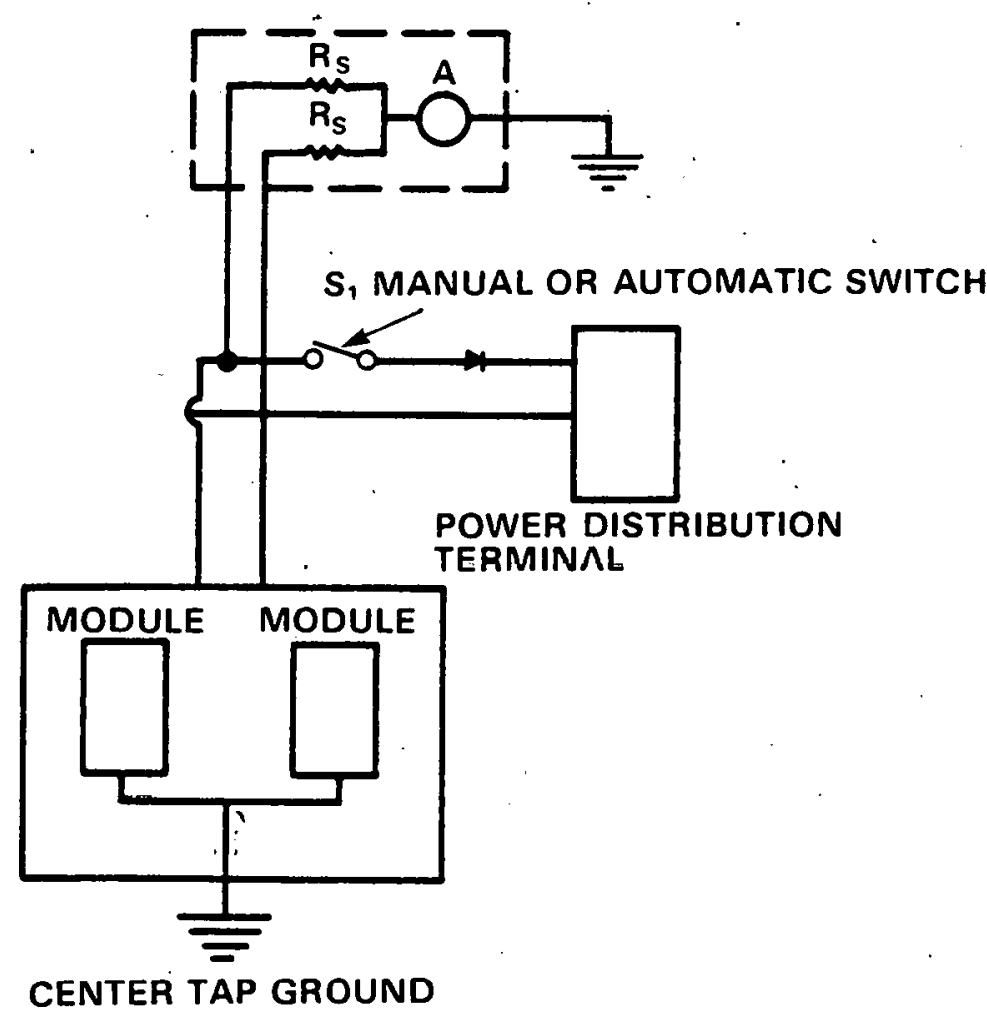

Exhibit 2.9 Terrestrial Solar Array Grounding and Safety Protection.

Because the array is an active power source when illuminated, care must be taken during servicing and maintenance. In the interest of safety, the array voltage should be no higher than 50-60 volts (as recommended by the National Electrical Code). If a higher voltage is required, switches should be installed between the cell modules at 50-60 volt intervals. These switches can then be disengaged, during maintenance, to limit the voltage anywhere on the array.

The electrical circuitry of the PV array, when acting as a power source, will radiate an electromagnetic $(E-M)$ field. This internally generated interference can be limited by arranging the array wiring to cancel the generated E-M field. Electronic switching voltage regulators or inverters, if included in the PV systems, can cause a voltage ripple to be fed back into the array circuitry. The E-M interference generated, and any problems that it might create, should be estimated. Electric fields are primarily of three types: Capacitance Effect, Faraday Effect, and the Hall Effect. The resultant electric field is the summation of components present. "The magnetic field of conductors carrying current is estimated by Ampere's Law.

Photovoltaic arrays are exposed to the environment and are, therefore, vulnerable to Ilghtning strikes. The benefits of providing lightning protection in the form of lightning rods and arresters will depend on the location of the system and should be investigated. 


\subsection{LOADNG CONSIDERATIONS}

The photovoltaic array in reality is neither a constant current nor a constant voltage source; therefore, specific analyses must be conducted to optimize its use as a power source. The combination of cells in series and parallel arrangements results in a specific maximum power output (voltage and current) under design operating conditions (i.e., insolation, temperature, loads, etc.). Unlike a battery, which will provide extremely large amounts of current at a relatively constant voltage (as required by the load), a photovoltaic array cannot provide more power than induced by the insolation. The result of overloading an array is essentially to cause a demand current greater than that of the maximum power point (refer to exhibit 2.10) and thereby to reduce the power output from the array. For this reason, photovoltaic systems not incorporating battery storage cannot be used effectively to

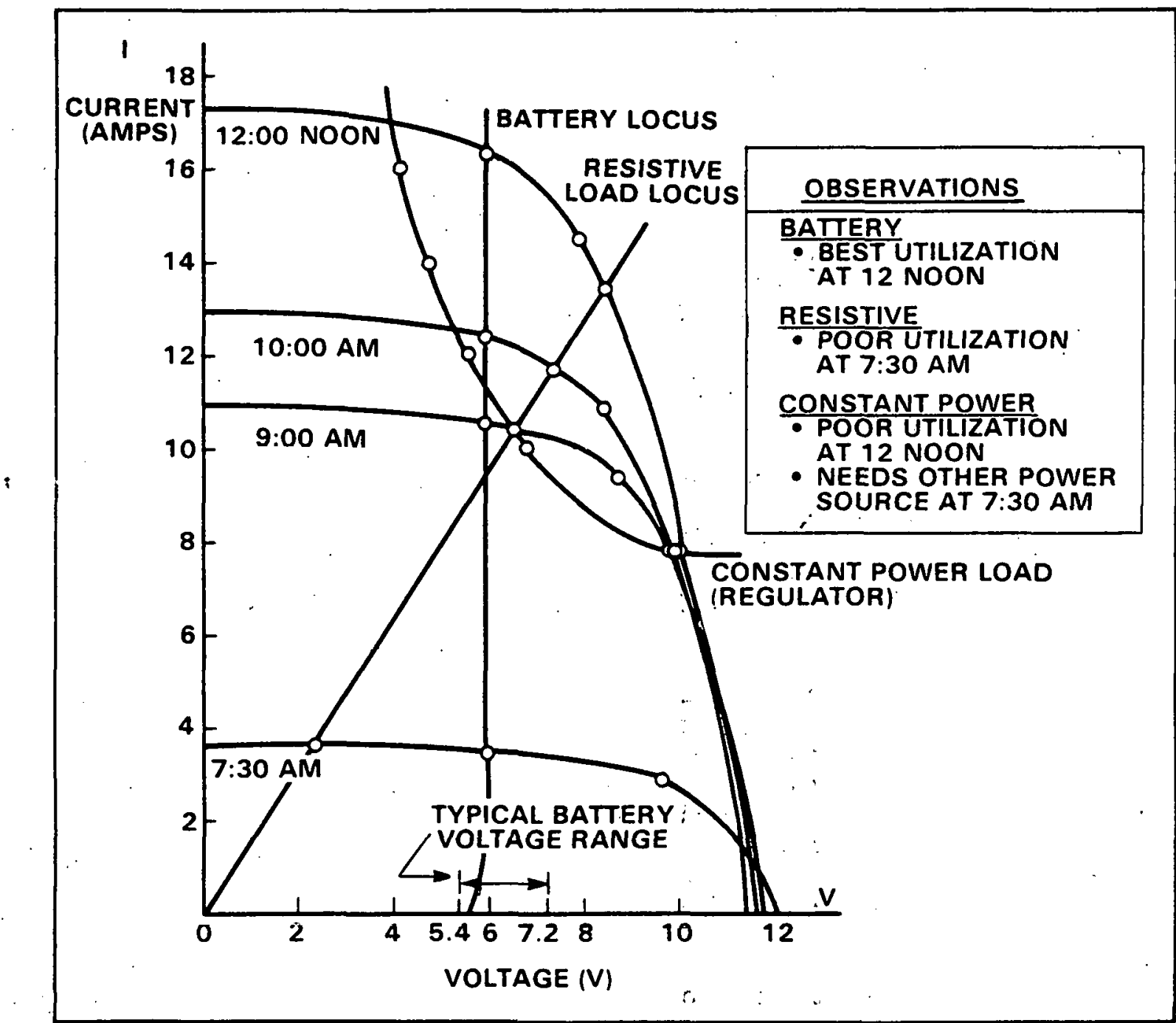

Exhibit 2.10 Power Utilization Con sideration s-One-Day Operation in Terrestrial Operations. 
supply power for short high peak demands. Adding loads in parallel decreases system resistance and increases system current even though individual locd current may not decrease. If enough loads are added in parallel, then individual load current may decrease and resistive losses throughout the system will increase. The net effect is voltage and power loss due to high current resistance loss, where power loss $=1^{2} \times R$ and vol tage loss $=1$ $x R$. $I$ is current, and $R$ is resistance. The power of the load equals $V^{2} / R$ load where $V$ is the system voltage. A decrease in system voltage magnifies the power decrease by the square of voltage.

\subsection{CELL TYPE TRADE-OFFS}

Photovoltaic cells, like transistors and diodes, can be fabricated from various materials which exhibit the proper electrical characteristics under controlled doping. Currently, the semi-conductor industry relies on silicon and germanium as host materials in transistors, diodes, and integrated circuits. The PV industry presently utilizes silicon as the primary host material. Two manufacturers are producing cells made from codmium sulfide. Due to the thinfilm nature of cadmium sulfide cells, much less material is needed for their construction than is needed for a typical silicon cell. This inherent advantage promises lower costs per watt, once efficiency and manufacturing problems are overcome.

Every manufacturer of solar cells has what he believes to be a "proprietary" technique or encasement design which make his cells and orrays superior. For example, some manufacturers now market solar arrays which feature stainless steel or thick glass frames, instead of aluminum frames, to reduce corrosion effects in marine environments. The technique used to apply the current collector grids on the solar cells varies from manufacturer to manufacturer. Some silk-screen the grids on, while others use vapor deposition techniques which they feel will aid in reducing both series resistance and delamination effects. Before selecting an array for a given application, trade-offs, in both quality and cost, must be evaluated.

\subsection{ARRAY TRADE-OFFS}

Presently available on the market as off-the-shelf products are solar photovoltaic irudules of varlous physical sizes, producing various outputs, made of various materials (casings). Trade-offs associated with the selection of an array (sub-array, $f$ mel, module, and cell) lie with the specific application of the system and the resulting erivironment in which the system is to operate, including the availability of back-up systems or the possibility of on electrical grid-connersted nppliration. The distance from a distribution or transmission line could be a factor.

For example, a photovoltaic array which is to provide power to a small diresit-riurrent refrigerator on top of a mountain requires that the orray be lightweight, small in physical size, and transportable. Under these conditions (assuming there is enough insolation for the application), an array may be chosen which folds on a central axis, uses a silicon rubber compound to reduce weight, and which contains "high density" cells, which are higher efficiency photovoltaic cells designed to cover more of the available area of the encasement (refer to exhibit 2.11). 
The orray shown in exhibit 2.11 was optimized for a specific application at a specific location. The same array shown could not provide 20-year lifetime and power if placed on an ocean bouy. In the marine environment, consideration must be given to salt-water corrosion, wind, and currents which prohibit physical stabilization and corrosive effects of bird droppings. A more suitable array might be as shown in Exhibit 2.12.

In contrast to the array shown in exhibit 2.11 , the array shown in exhibit 2.12 is designed to be rugged, highly efficient under all conditions of motion, and resistant to a marine environment. The module would more than likely be covered with tempered glass and sealed at the edges of the panel, and the casing would be steel instead of lighter weight aluminum. The cells can be either round or high-density hex-sided, depending on the available area on the buoy and the power requirements. Costs also play a role as the high density modules are more expansive than the standard round cell designs.

In conclusion, selection of any one solar array is mainly dependent on the power requirements, operational environment, and cost associated with the system.

\subsection{ENVIRONMENTAL EFFECTS}

As discussed earlier, the environment in which the photovoltaic system is to operate plays a major role in the overall performance, lifetime, and initial cost of the system.

The combination of site remoteness and the environment must be examined, if the system is to last for many years, since remote sites will suffer from infrequent maintenance.

Environmental effects, such as temperature cycling, freezing, high humidity, fog, snow, wind, etc., result in specific system performance (or lack of performance) and, therefore, should be included in the calculation and resulting selection of a specific system. A chart summarizing the operating characteristics and capabilities of available PV modules is contained in Section 9. 


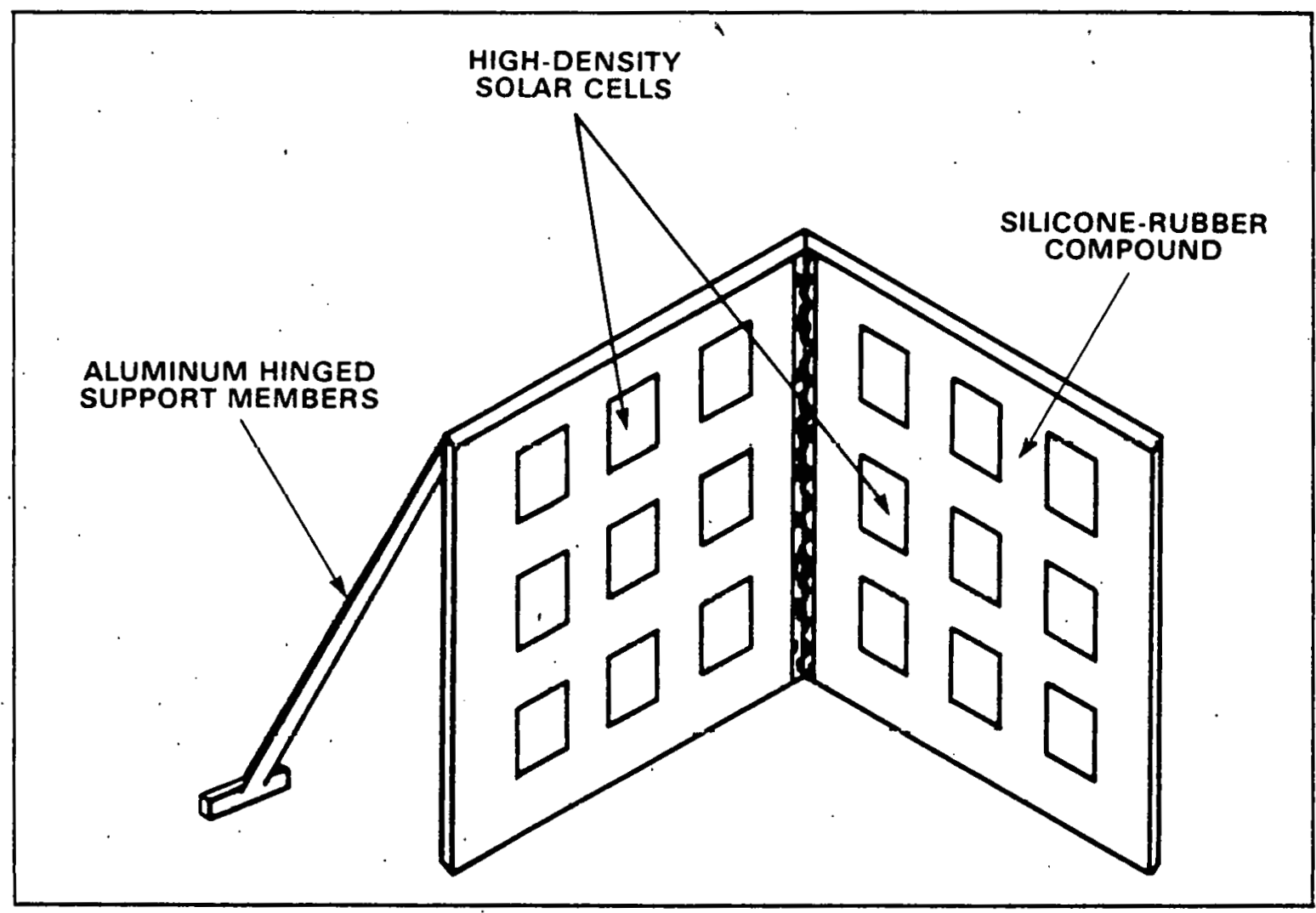

Exhibit 2.11 A Simple Array Design Incorporating Lightweight Design, Portability, and High Density Solar Cells.

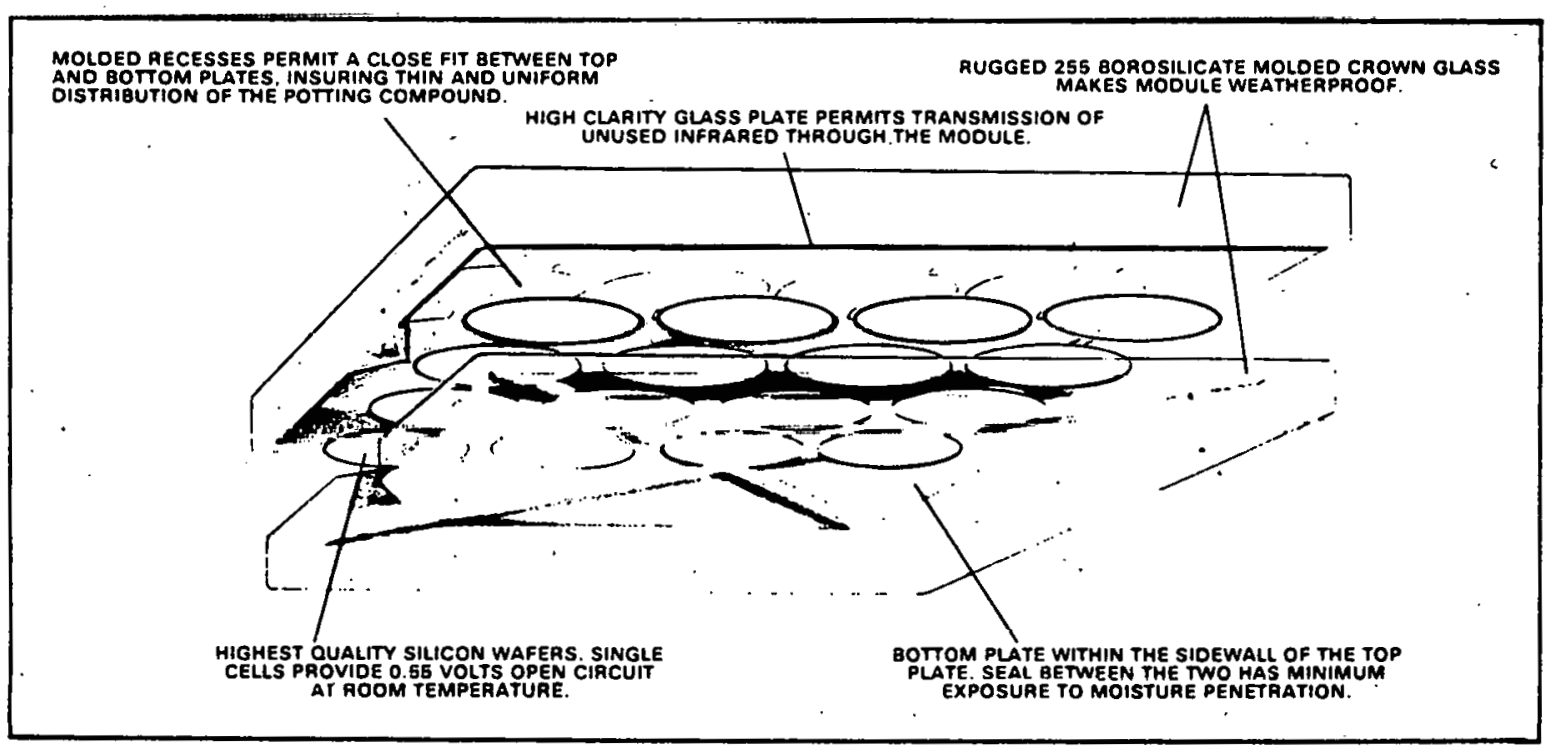

Exhibit 2.12 PV Array Used to Power Buoy Lights. 


\section{SECTION 3 \\ STRUCTURAL ANALYSIS}

\section{I INTRODUCTION}

What has been dealt with up until now is the PV electrical system and its design. The PV system's structure represents approximately 3 percent of the total cost of present systems. In future systems, when the cell costs drop to 50 cents/peak $W$, the structure will represent approximately 20 percent of the total system cost. In both cases, the structure must be provided and will influence the usefulness of the application. In this section, an introduction is provided to the various building codes applicable to commercial structures. One code will be discussed in detail, so the reader will be able to calculate, at least for preliminary evaluation, the size and complexity of the supporting framework. The structural analysis involves the civil engineering of the solar power system; and thus is comprehensive, rigorous, extensive, and involves environmental engineering, engineering law, engineering administration, and civil works codes.

\subsection{STRUCTURAL LOADS}

Structural loads are tabulated in exhibit 3.1. They consist of dead loads:--the weight of the panel, and the structure--as well as various live loads: maintenance crews, wind, snow, hail, ice, settlement, seismic loads, and deflection-induced loads. Typical values of the various loads are also shown. Wind and snow are both of the same order of magnitude and, for solar cells, represent a much greater load than the weight of the panel. The other loads are usually less significant; many are not even considered in some of the commonly used building codes.

\subsection{BUILDING CODES}

Exhibit 3.2 shows the common building codes applicable to solar cell systems. The Building Officials and Code Administrator International, Inc. (BOCA), the Uniform Building Code (UBC), and Standard Building Codes are applied extensively in building design. They are called model codes because they include all the elements important to a code, but do not include some of the local idiosyncracies. Each locality has its own building code and many simply adopt one of the model codes. BOCA has been adopted by many of the Atlantic communities and the UBC is common in the West. The Standard Building Code is so common in the South that it is frequently called the "southern building code." The American National Standards Institute (ANSI) also has a building code (ANSI A58.1), similar to the previous three, but it treats the wind loads more extensively than the others.

\begin{tabular}{|c|l|}
\hline & \\
\hline Dead Load & $5 \mathrm{lb} / \mathrm{ft}^{2}$ \\
\hline Live Loads & \\
\hline Maintenance & Approximate Pressure \\
Snow & $12 \mathrm{lb} / \mathrm{ft}^{2}$ \\
Wind & $20 \mathrm{lb} / \mathrm{tt}^{7}$ \\
\hline Seismic & $25 \mathrm{lb} / \mathrm{ft}^{2}$ \\
\hline Hail & $0.3^{*} \mathrm{Wt}^{2}$ \\
\hline Ice & Glass limit \\
\hline Settlement & Wire limit \\
\hline Deflection & $2^{\prime \prime} / 50^{\prime}$ \\
& $1 \%$ of span \\
\hline
\end{tabular}

Exhibit 3.1 Structural Loads (Pressure is given as pounds per square foot) 


\begin{tabular}{|c|}
\hline $\begin{array}{l}\text { Building OHicials and Code Administrator International, Inc.: } \\
\text { BocA Code-Adopted mostly in Eastern U.S. and by the } \\
\text { International Conference of Building Officials }\end{array}$ \\
\hline Uniform Building Code: UBC-Adopted mostly in Western U. S. \\
\hline $\begin{array}{l}\text { Southern Building Code Congress } \\
\text { Standard Building Code: SBC-Adopted mostly in Southarn U. S. }\end{array}$ \\
\hline $\begin{array}{l}\text { American National Standards Institute } \\
\text { ANSI-ANSI A58.1 }\end{array}$ \\
\hline $\begin{array}{l}\text { National Bureau of Standards } \\
\begin{array}{l}\text { NBS-NBS IR 76.1187: Interim Performance Criteria for Solar Heating and Cooling } \\
\text { Systems in Commercial Buildings }\end{array}\end{array}$ \\
\hline $\begin{array}{l}\text { HUD Minimum Property Standards (Department of Housing and Urben Development) } \\
\qquad \begin{array}{l}4900.1 \text { : One and Two family dwellings } \\
4910.1: \text { Multifamily dwellings } \\
\text { 4080.1: Càretrwe liuuslaty } \\
4930.1 \text { : Solar Heating and Domestic Hot Water }\end{array}\end{array}$ \\
\hline
\end{tabular}

\section{Exhibit 3.2 Building Codes.}

The National Bureau of Standards (NBS), at the request of ERDA (now DOE), examined the various codes as they might be applied to solar power systems. The result was NBSIR $76-$ 1187. This code is not widely accepted. Seismic, hail, ice, settlement and deflection specifications, ignored in many of the other codes, are included in the NBS code. At ERDA's request, the Housing and Urban Development (HUD) Minimum Property Standards were modified for solar energy systems. The NBS standard, the most complete code, frequently references the HUD and UBC codes and must be used in conjunction with these codes.

A comparison of the structural requirernents as defined by the various codes for one particular location (Albany, New York) is presented in exhibits 3.3 and 3.4. The first section defines the minimum roof load for roofs at various angles. The code assumes the structures will be occupied. Some code administrators consider these minima to pertain to snow only. Others presume they opply to all categories of loads and are, therefore, applicable to the slant area, not the horizontally projected aren.

The snow loads should be applied to the horizontally projected area, as specified in the Southern code. Factors should then be applied to allow for the fact that the snow will not accumulate on a steeply slanted surface. Allowance must also be made for drifting. When dealing with unoccupied structures, the UBC avoids these complexities by calling only for the use of the minimum roof load. The variation in the snow load requirement is substantial among the codes.

The wind load is also not computed in the same manner for all codes. The Southern and ANSI codes call for an allowance 'for suction in the leeward side, and allow snme load reduction for tllted surfaces. The other codes treat the array the same as vertical signs. The codes are ambiguous as to the surface to which the load must be applied, not specifying whether it should be the vertical projection or the slant area, which are possibilities. If the horizontal thrust-the major effect of the wind-is being computed, there is no difference. However, the codes do not include the evaluation of the vertical force. To be safe, the wind load should be on the panel (slant) area. 


\begin{tabular}{|c|c|c|c|c|c|c|}
\hline \multicolumn{7}{|c|}{$\begin{array}{l}\text { Design Load for } 12^{\prime} \text { Solar Panels In Albany, N.Y. } \\
\qquad \begin{array}{c}\text { Slope }=57.5^{\circ} \\
\text { Spacing }=30^{\circ}\end{array}\end{array}$} \\
\hline ITEM $\quad$ CODE & Southern & UBC & BOCA & ANSI & NBS & HUD \\
\hline $\begin{array}{l}\text { Minimum Roof Load } \\
18^{\circ} \\
45^{\circ} \\
45^{\circ}\end{array}$ & $\begin{array}{l}\text { (due to people, sn } \\
20 \mathrm{lb} / \mathrm{ft}^{2} \\
16 \mathrm{lb} / \mathrm{ft}^{2} \\
12 \mathrm{lb} / \mathrm{ft}^{2}\end{array}$ & $\begin{array}{l}\text { vetc.) } \\
20 \mathrm{lb} / \mathrm{ft}^{2} \\
16 \mathrm{lb} / \mathrm{ft}^{2} \\
12 \mathrm{lb} / \mathrm{ft}^{2}\end{array}$ & $\begin{array}{l}20 \mathrm{lb} / \mathrm{ft}^{2} \\
16 \mathrm{lb} / \mathrm{ft}^{2} \\
12 \mathrm{lb} / \mathrm{tt}^{2} \\
\end{array}$ & $\begin{array}{l}20 \mathrm{lb} / \mathrm{ft}^{2} \\
16 \mathrm{Jb} / \mathrm{ft}^{2} \\
12 \mathrm{lb} / \mathrm{ft}^{2}\end{array}$ & & \\
\hline $\begin{array}{l}\text { Snow Load } \\
\text { Basis } \\
\text { Horizontal } \\
\text { Sloped (a-30 } \\
\text { Static } \\
\text { or Drifting } \\
\text { Avg. Depth } \\
\end{array}$ & $\begin{array}{l}\text { Horizontal Proj. } \\
30 \mathrm{lb} / \mathrm{ft}^{2} \\
0^{\circ} \\
\times 0.55 \\
\times 2 \\
\times 0.68 \\
\end{array}$ & $\begin{array}{l}- \\
- \\
- \\
- \\
\end{array}$ & $\begin{array}{l}-\overline{25} \mathrm{lb} / \mathrm{ft}^{2} \\
\times 0.55 \\
\times 2 \\
\times 0.68 \\
\end{array}$ & $\begin{array}{l}25 \mathrm{lb} / \mathrm{ft}^{2} \\
\times 0.55 \\
\times 2 \\
\times 0.68 \\
\end{array}$ & & Use ANSI \\
\hline Design Load & $34.0 \mathrm{lb} / \mathrm{ft}^{2}$ & $12 \mathrm{lb} / \mathrm{ft}^{2}$ & $34.0 \mathrm{lb} / \mathrm{ft}^{2}$ & $34.0 \mathrm{lb} / \mathrm{ft}^{2}$ & & \\
\hline $\begin{array}{c}\text { Wind Load } \\
\text { Basis }\end{array}$ & No Shielding & & $\begin{array}{l}\text { Can be } \\
\text { Shielded }\end{array}$ & $\begin{array}{l}25 \mathrm{yr} \\
\text { No Shielding } \\
\end{array}$ & $\cdot$ & Use ANSI \\
\hline $\begin{array}{l}\text { Front Load } \\
\text { Speed } \\
\text { Pressure } \\
\text { Tilt/Suction } \\
\end{array}$ & $\begin{array}{r}85 \mathrm{MPH} \\
14 \mathrm{Ib} / \mathrm{ft}^{2} \\
\times \quad 1.25 \\
\end{array}$ & $20 \mathrm{lb} / \mathrm{ft}^{2}$ & $\begin{array}{l}15 \mathrm{lb} / \mathrm{ft}^{2} \\
-\end{array}$ & $\begin{array}{c}70 \mathrm{MPH} \\
19 \mathrm{lb} / \mathrm{ft}^{2} \\
\times \quad 2 \\
\end{array}$ & & \\
\hline Design Load & $17.5 \mathrm{lb} / \mathrm{ft}^{2}$ & $20 \mathrm{lb} / \mathrm{ft}^{2}$ & $15 \mathrm{lb} / \mathrm{ft}^{2}$ & $38 \mathrm{lb} / \mathrm{ft}^{2}$ & & \\
\hline $\begin{array}{l}\text { Rear Load } \\
\text { Pressure } \\
\text { Tilt/Suction } \\
\end{array}$ & - & $\begin{array}{l}20 \\
\times \quad 1.25 \\
\end{array}$ & - & $\begin{array}{r}19 \\
\times 2 \\
\end{array}$ & & \\
\hline Design Load & - & $25 \mathrm{lb} / \mathrm{ft}^{2}$ & - & $38 \mathrm{lb} / \mathrm{ft}^{2}$ & & \\
\hline $\begin{array}{l}\text { Seismic } \\
\text { Lateral Load, } \\
V= \\
\text { ZIC SWW }\end{array}$ & $\begin{array}{c}\text { Use } \\
\text { ANSI }\end{array}$ & $\begin{array}{c}Z \leq Z^{*} W t \\
I C_{p} S \leq 1\end{array}$ & $\begin{array}{l}\text { Not. } \\
\text { Severe }\end{array}$ & $\begin{array}{l}C_{p}^{*} Z^{*} W t \\
Z \leqslant 1\end{array}$ & $\begin{array}{c}\text { in } \\
\text { Doubt. } \\
\text { use } \\
C_{p}=1\end{array}$ & $\begin{array}{c}\text { Use UBC } \\
\text { with } C_{p} \times 0.30\end{array}$ \\
\hline Hail & & & & & $\begin{array}{l}\text { Map. } \\
\text { Table }\end{array}$ & $\begin{array}{l}\text { Same as } \\
\text { NBS }\end{array}$ \\
\hline $\begin{array}{l}\text { Tce } \\
\text { On Wires }\end{array}$ & . & & .1 & & $\begin{array}{l}\text { Man. } \\
\text { Table }\end{array}$ & \\
\hline $\begin{array}{l}\text { Deflection } \\
\text { Thermal } \\
\text { Moisture } \\
\text { Settlement } \\
\text { Ground Uplift } \\
\text { Under Load"• }\end{array}$ & . & . & $\begin{array}{l}3 \\
\cdots \\
n \\
n\end{array}$ & $\therefore$ & $\begin{array}{l} \\
- \\
2 " \\
0.9 \times 4 \\
8<\frac{1.25}{180}\end{array}$ & $(1.5+0.2 \dot{D} / L)$ \\
\hline
\end{tabular}

- Calls for use of HUD, model code, or local code

$* S=$ Span, $D=$ Dead Luad, $L$ = Live Lood, 8 - Deflection in Incline

Pressure is given in pounds per square foot.

Wind speed is given in miles per hour.

\section{Exhibit 3.3 Comparison of Codes.}




\subsection{USE OF THE BOCA CODE}

The purpose of this section on structural analysis is to provide at least one method for estimating the loads on the structural members and structural system required to support the solar-cell array. It is not a complete course in the application of the codes. In fact, such a course would include local variations in the code and, therefore, would be far more extensive than the few codes listed in exhibit 3.2. The BOCA code is considered because it is the most easily applied. However, some of the variations used by other codes will be indicated.

\subsubsection{WIND LOADS}

Signs must be designed to withstand wind loads; occupied buildings are not. For signs that are ground mounted, top within 50 feet of the ground, the wind exerts a 15 pound per square foot $\left(\mathrm{lb} / \mathrm{ft}^{2}\right)$ force on the sign under maximum conditions. If the sign top is more than 50 feet high from the ground, the load is $20 \mathrm{lb} / \mathrm{ft}^{2}$. If the sign is roof mounted, the load is $30 \mathrm{lb} / \mathrm{ft}^{2}$. Wind loading from front and back is to be treated the same. Most signs are vertical, so the code does not include tilt effects. Therefore, it seems necessary to design for the loads just mentioned as applied to the slant (array) area and not its projection for vertical mounting.

An example using projections is given in exhibit 3.4. A 60-fmt wide, 100-foot long collector is mounted at a $30^{\circ}$. tilt to the horizontal, so its highest point is 30 feet from the ground. Since this is less than $50 \mathrm{feet}$, the design wind load is $15 \mathrm{lb} / \mathrm{ft}^{2}$. The lateral force is $15 \mathrm{lb} / \mathrm{ft}^{2}$ multiplied by the array area of $(60 \times 100) \mathrm{ft}^{2}$ and multiplied by the verticai component projection factor (sine of $30^{\circ}$ ): the horizontal force is $45,000 \mathrm{lb}$. The vertical force is computed similarly, except the vertical force is computed as multiplied by the cosine of $30^{\circ}$, so the vertical load is 77,940 nounds, using the horizontal projection factor. Pressure equals weight per unit area and force equals pressure $x$ area.

While other codes make a distinction as to the region of the country in which the sign, or collector, is mounted, the BOCA code does not. Exhibit 3.5 shows the wind loads on buildings as specified in the ANSI code. The loads are for vertical surfaces placed 30 feet above ground level. The regional variation is seen to he significant. For heighte other than 30 feet, the table in exhibit 3.6 is used.

The effects of tilt (as defined in the Southern Code) further alter the wind loads (see exhibit 3.7). If the southern code were applied in Albany, New York, the force on a vertical sign would be $15 \mathrm{lb} / \mathrm{ft}^{2}$, in agreement with BOCA, except this load would be multiplied by 1.5 to account for suction on the leeward side. In Norfolk, Virginia, the load would be 24 $\mathrm{lb} / \mathrm{ft}^{2}$, multiplied by 1.5 to account for suction on the rear surface, for a total of $36 \mathrm{lb} / \mathrm{ft}^{2}$.

None of the codes allows for the effect of rows of collectors. Some data were obtained on saw-tooth roofs and are presented at the hnttom of exhibit 3.7. For the $45^{\circ}$ isnssclcos roof, the force on the first slope is only. 0.3 times the map values, and only a suction is felt on the other slopes (negative factors). On more tilted surfaces, a higher force is felt on the first slope, but higher suction is felt on the others. No code allows for this type of wind loading. An isosceles triangle has two equal angles. 


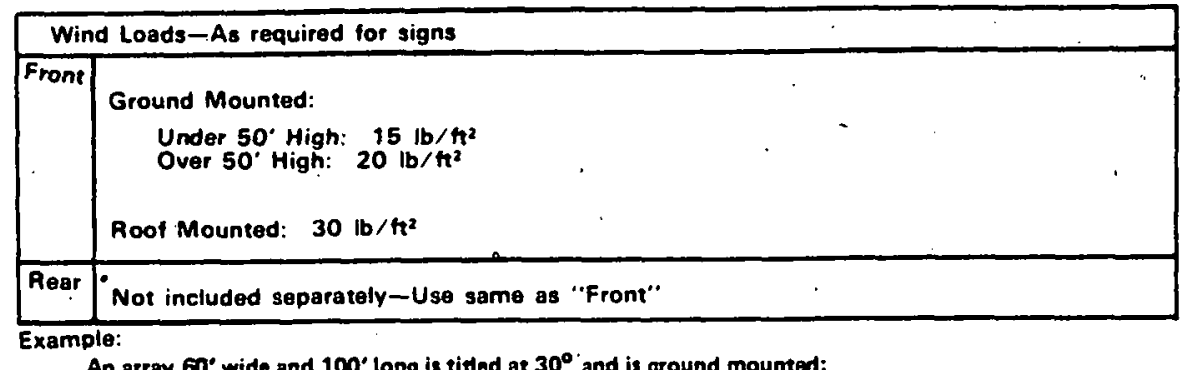

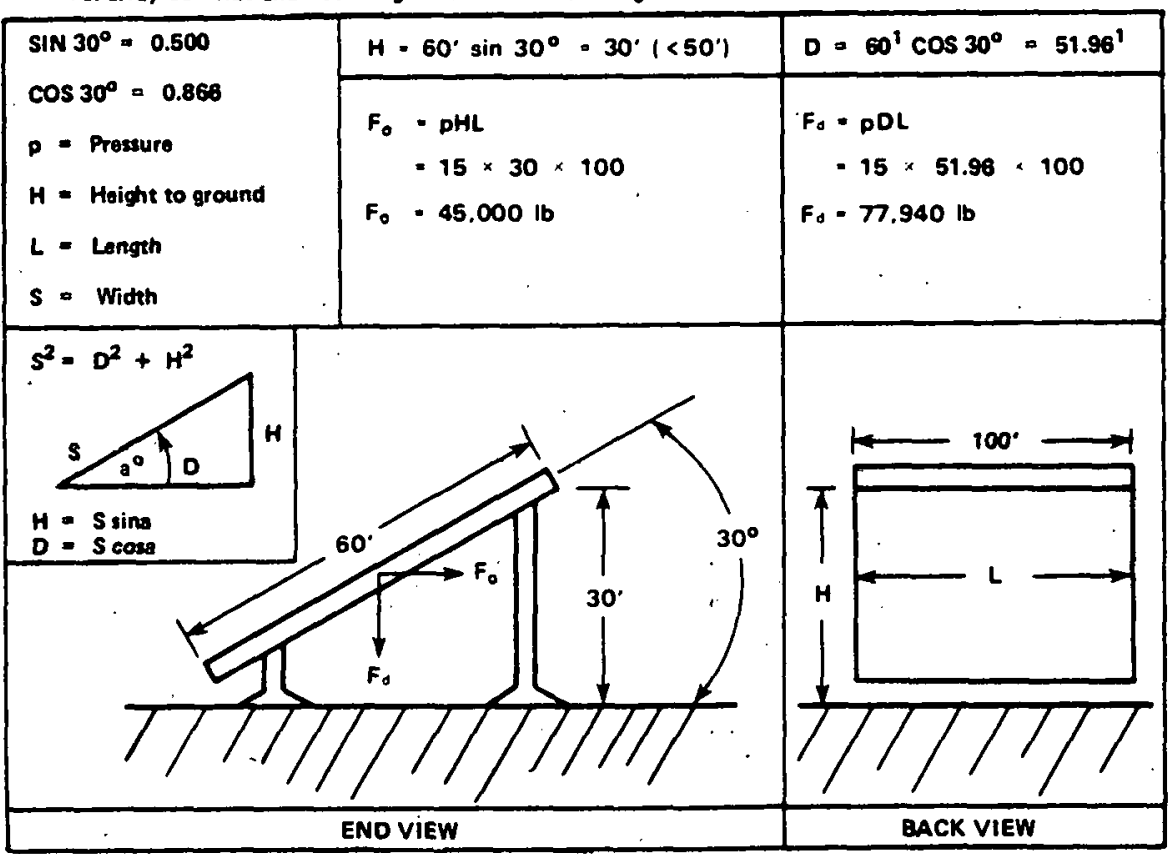

\section{Exhibit 3.4 BOCA - A Typical Model Code (Pressure: pounds per square foot)}

Only the UBC and ANSI codes distinguish between winds from the front and winds from the rear. The wind loads, as specified by various codes, are seen to vary by a factor of greater than 2:1. HUD calls for use-of ANSI, the largest load; NBS calls for the use of HUD.

The seismic loads are all in agreement with the ANSI code, although BOCA states that seismic loads are not as severe as wind or snow loads and need not be considered under normal circumstances. Hail, ice, and deflection-induced loads are included only in the NBS and HUD codes. The ice loads pertain only to the icing of wires. The deflection limit is not a stress limit, but an aesthetic limit. 


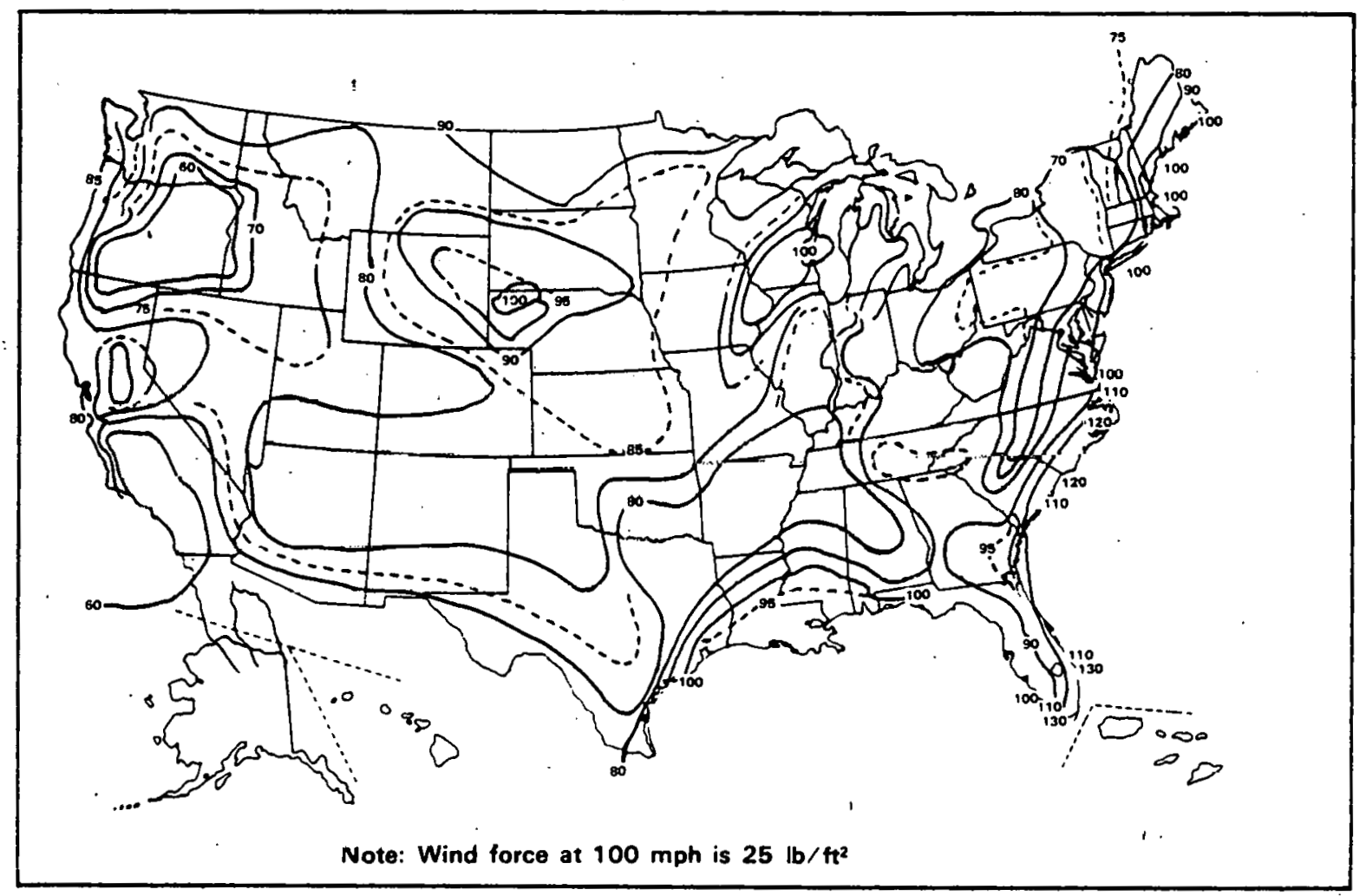

Exhibit 3.5 Wind Loads-Some Complications Due tn Region.

Fastest mile wind velocity at $30 \mathrm{ft}$ above ornund, Sô-yf mean recurrence

\begin{tabular}{|r|c|}
\hline HEIGHT & PRESSURE FORCE/MAP PRESSURE \\
\hline $30^{\prime}$ & 1.0 \\
$50^{\circ}$ & 1.3 \\
$100^{\circ}$ & 18 \\
$150^{\circ}$ & 2.1 \\
\hline
\end{tabular}

Exhibit 3.6 Wind Loads-Some Complications Due to Height. 


\subsubsection{SNOW LOADS}

The BOCA requirements for snow loads are shown in exhibit 3.8. The loads are taken on the horizontal projection of the surface with an additional correction for tilted surfaces, because not as much snow sticks to these as to horizontal surfaces. (Rime ice, a frozen mist, can form on surfaces in some parts of the country; especially in mountainous areas. Rime ice will stick to vertical surfaces in thicknesses up to several feet. If rime ice is important at the location of interest, special design procedures must be used, in conformance with local codes). The correction for tilt is shown in exhibit 3.9. The factor of 0.8 is applied even for a horizontal surface, which is the basis for the map.

The code makes additional allowances for drifting near vertical surfaces. There is no mention made of drifting near titled surfaces. The author's' suggestion is to use the vertical projection of the solar collector and the drift angle of $27^{\circ}$ as deduced from the tables in the code. Exhibit 3.10 shows the geometry of the drift. The map is based on $10 \mathrm{lb} / \mathrm{ft}^{3}$ for the density of the snow, so this density can be used to estimate the force on the collector due to drifting. The average depth of the snow on the collector is given by the equation on the figure. The load, in $\mathrm{lb} / \mathrm{ft}^{2}$, is the product of the average depth in feet and the snow density. Despite the snow loads just computed, the design load should not be less than the minimum loads as shown in exhibit 3.11, where pressure equals weight per unit area and density equals weight per unit volume. Density $x$ depth equals pressure.

\subsubsection{SEISMIC LOADS}

According to the BOCA code, the seismic loads are not as severe as wind and snow on signs, so seismic loads need not be considered (see exhibit 3.12). ANSI calls for lateral loads due to seismic effects that are equal to the dead load multiplied by two factors, $Z$ and $C$. The value of $Z$ depends on the region of the United States $\left(0.25\right.$ for the middle Atlant $P_{c}$ states and 1.0 for the most severe conditions in California). The same map of $Z$ is given in the UBC and Southern codes. A reasonable value for $C_{p}$ is 0.3 (see exhibit 3.13 ).

\begin{tabular}{|c|c|c|c|}
\hline \multirow[t]{2}{*}{ TILT } & \multicolumn{2}{|c|}{$\begin{array}{l}\text { NORMAL PRESSURE } \\
\text { (SOUTHERN CODE) }\end{array}$} & \multirow{2}{*}{$\begin{array}{l}\text { NORMAL PRESSURE } \\
\text { PER TYPICAL DATA } \\
\text { TOTAL }\end{array}$} \\
\hline & FRONT & REAR & \\
\hline $\begin{array}{r}70-90^{\circ} \\
60-70^{\circ} \\
\end{array}$ & $\begin{array}{l}0.80 \\
0.65 \\
\end{array}$ & $\begin{array}{r}+0.70 \\
+0.70 \\
\end{array}$ & 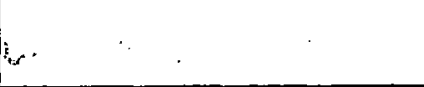 \\
\hline $\begin{array}{l}50-60^{\circ} \\
40-50^{\circ} \\
30-40^{\circ}\end{array}$ & $\begin{array}{r}0.55 \\
0.25 \\
-0.25 \\
\end{array}$ & $\begin{array}{l}+0.70 \\
+0.70 \\
+0.70 \\
\end{array}$ & \\
\hline $\begin{array}{l}20-30^{\circ} \\
10-20^{\circ}\end{array}$ & $\begin{array}{l}-0.75 \\
-0.93\end{array}$ & $\begin{array}{l}+0.70 \\
+0.70\end{array}$ & $\begin{array}{l}1.3 \\
1.2\end{array}$ \\
\hline & $\begin{array}{l}\text { a: } \\
\text { b: } \\
\text { c: }\end{array}$ & & $\begin{array}{r}0.3 \\
-0.6 \\
-0.6 \\
\end{array}$ \\
\hline $60^{\circ} 30^{\circ}$ & $\begin{array}{l}\text { a: } \\
\text { b: } \\
\text { c: }\end{array}$ & r & $\begin{array}{r}0.6 \\
-0.7 \\
-0.7 \\
\end{array}$ \\
\hline
\end{tabular}

Exhibit 3.7 Wind Loads-Some Complications Due to Tilt. 


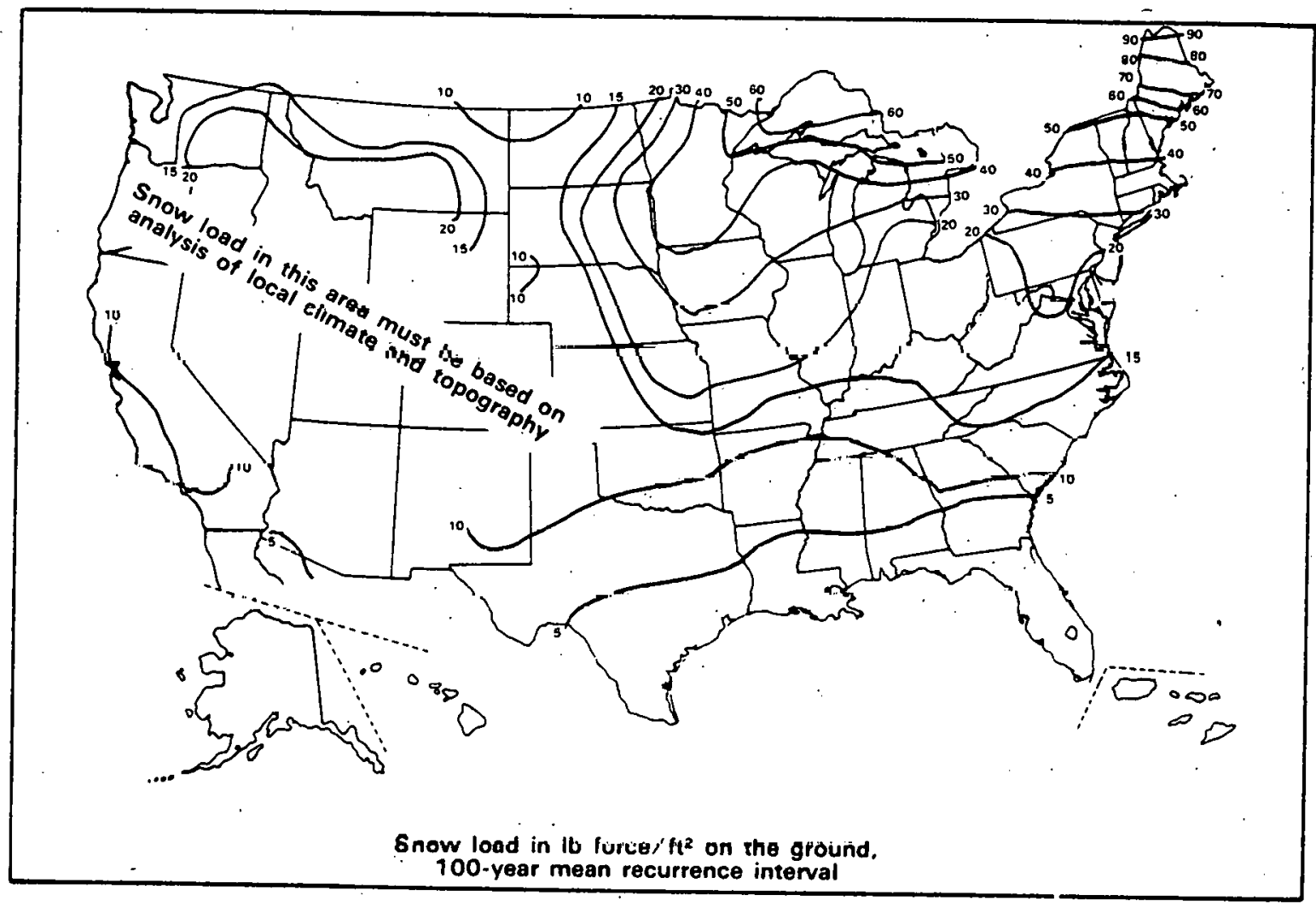

Exhibit 3.8 BOCA-Snow Loads.

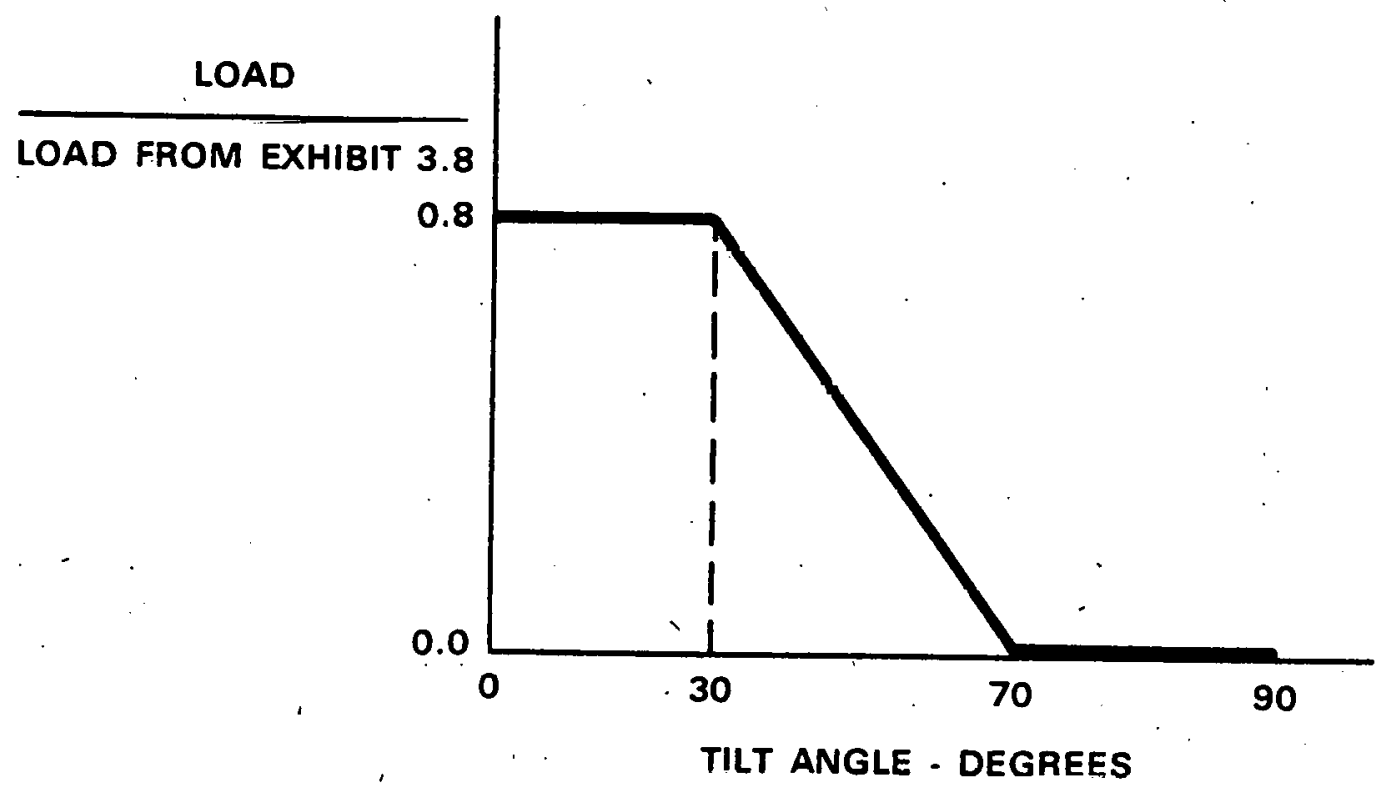

Exhibit 3.9 Snow Loads-Tilt Effect. 


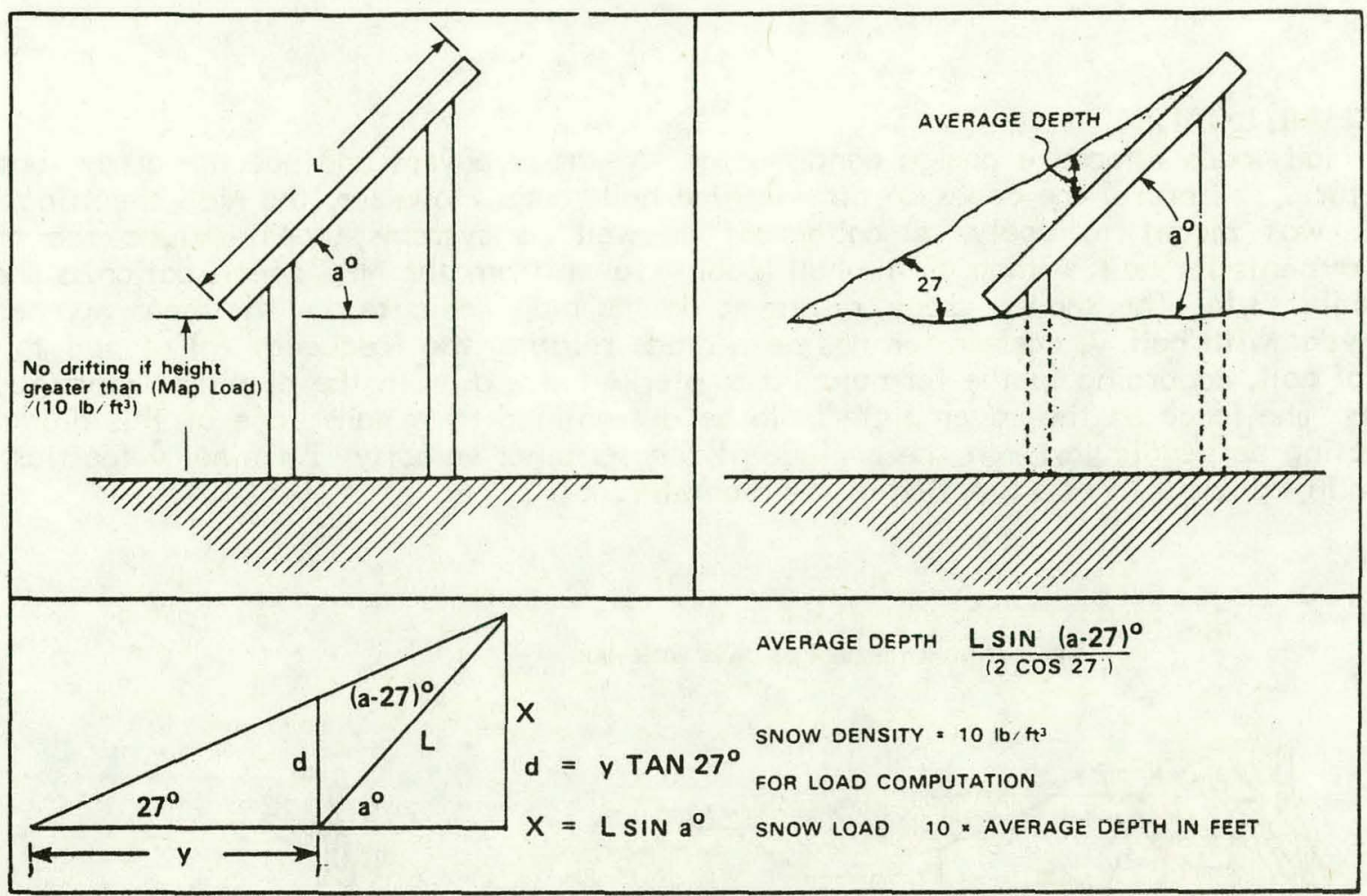

Exhibit 3.10 Snow Loads-Drift Effect.

Despite Previous Computations

\begin{tabular}{|c|c|}
\hline TILT & MINIMUM LOAD \\
\hline $0^{\circ}$ to $8^{\circ}$ & $20 \mathrm{lb} / \mathrm{ft}^{2}$ \\
$18^{\circ}$ to $45^{\circ}$ & $16 \mathrm{lb} / \mathrm{ft}^{2}$ \\
$45^{\circ}$ to $90^{\circ}$ & $12 \mathrm{lb} / \mathrm{ft}^{2}$ \\
\hline
\end{tabular}

Exhibit 3.11 BOCA-Minimum Loads.

Assumed less stringent than wind or snow, since structure unoccupied.

Exhibit 3.12 BOCA-Seismic Loads.

$\begin{array}{cc}\text { ANSI calls for lateral load on signs }=0.3 * Z^{*} W t \\ \text { ZONE } & \boldsymbol{Z} \\ 1 & 0.25 \\ 2 & 0.50 \\ 3 & 1.00\end{array}$

Exhibit 3.13 Seismic Load-Some Complications. 


\subsubsection{HAIL LOADS}

Hail loads impose a design condition on the array covers and not the array support structure. Therefore, the codes do not mention hail loads. However, the NBS specification, which was meant to apply to collectors as well as systems, does include the HUD requirements for hail. A map of the hail loading taken from the NBS specification is shown in exhibit 3.14. The shaded areas, displayed on the map, indicate $d-$ the mean number of days/year with hail. A correlation has been made relating the frequency (d) of hail to the size of hail, according to the formula: diameter $=0.3 \times \mathrm{d}$, with the diameter measured in inches. The force on the cover plate is to be determined by a hail stone of this diameter impacting perpendicularly on the surface at the terminal velocity. Terminal velocities are shown in exhibit 3.15 as a function of the diameter.

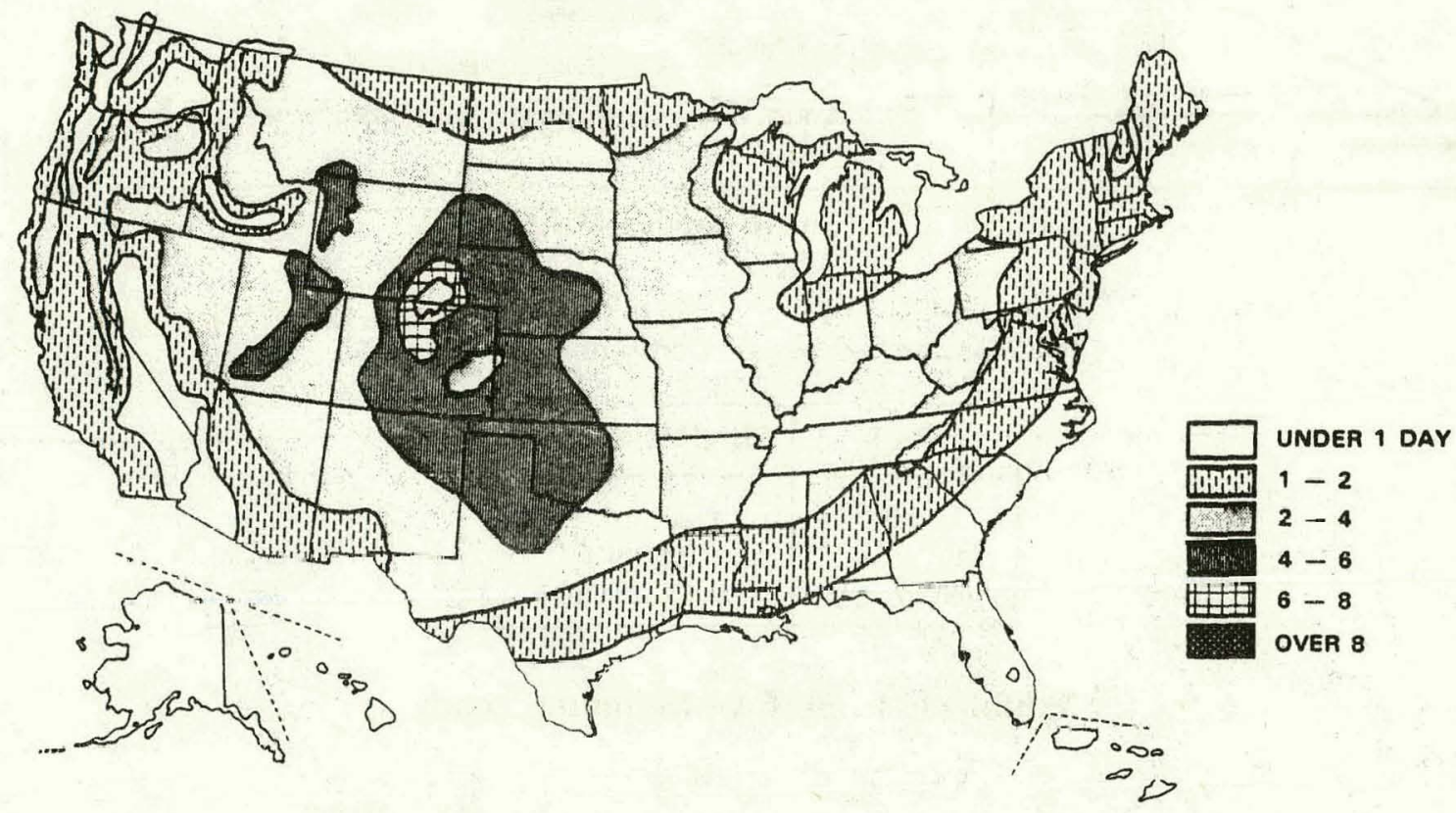

Exhibit 3.14 Mean Annual Number of Days with Hail.

\subsubsection{ICE LOADS}

None of the codes addresses the problems of ice loads (e.g., the icing of wires during freezing rains). The NBS specification uses the map shown in exhibit 3.16 for the mean number of days/year with freezing rain. The table in exhibit 3.17 indicates the corresponding thicknesses of the ice layers on wires exposed in each of these map areas. 


\begin{tabular}{|c|c|c|c|}
\hline DIAMETER & \multicolumn{2}{|c|}{ WEIGHT } & $\begin{array}{c}\text { TERMINAL } \\
\text { VELOCITY }\end{array}$ \\
\hline VIN. & GM & FT / SEC \\
\hline & 0.98 & 0.002 & 51 \\
$1 / 2$ & 3.30 & 0.007 & 62 \\
$3 / 4$ & 7.85 & 0.017 & 73 \\
\hline 1 & 15.33 & 0.034 & 82 \\
$11 / 4$ & 26.50 & 0.058 & 90 \\
$1 \frac{1}{1} / 2$ & 42.08 & 0.093 & 97 \\
$13 / 4$ & 62.81 & 0.138 & 105 \\
\hline 2 & 89.43 & 0.197 & 111 \\
$21 / 4$ & 122.67 & 0.270 & 117 \\
$21 / 2$ & 163.28 & 0.360 & 124 \\
$23 / 4$ & 211.98 & 0.467 & 130 \\
\hline 3 & & & \\
\hline & & & \\
\hline
\end{tabular}

Exhibit 3.15 Values of Weight and Terminal Velocity, Computed for Smooth Ice Spheres.

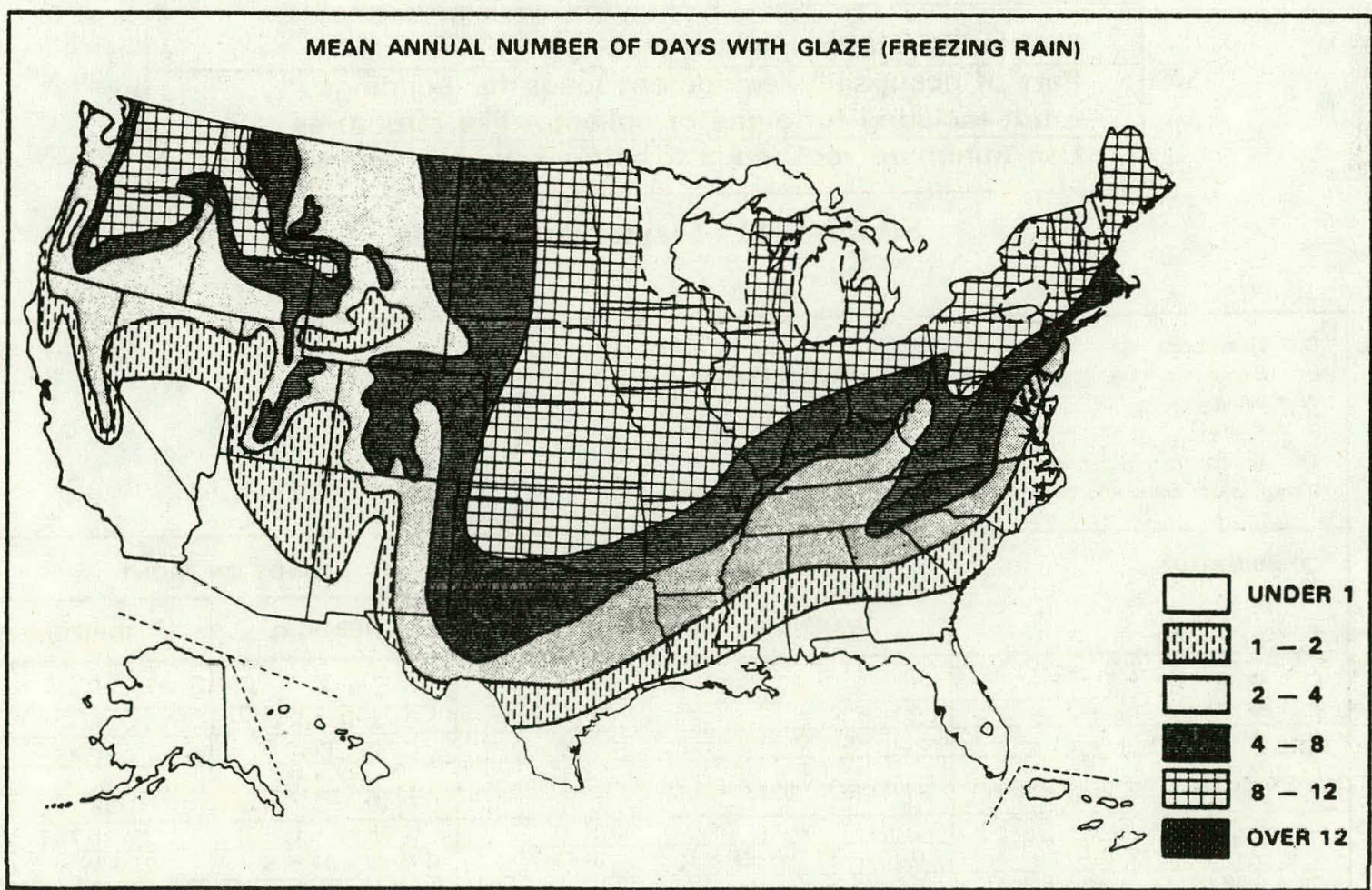

Exhibit 3.16 Mean Annual Number of Days with Glaze (Freezing Rain). 


\subsubsection{MAINTENANCE LOADS}

Maintenance, occupancy, and equipment loads are included in the codes for structures that house people. None of the codes includes such loads on signs or collector-like structures. The designer must use his judgement.

Small collectors would have almost no maintenance loads (exhibit 3.18). Large collectors may have maintenance people climbing on walks or on the panels themselves. Unless details are available for estimating the maintenance load, the table of minimum loads shown in exhibit 3.3 can be used.

\begin{tabular}{|c|c|}
\hline $\begin{array}{c}\text { MEAN NUMBER } \\
\text { OF DAYS WITH } \\
\text { FREEZING RAIN }\end{array}$ & $\begin{array}{c}\text { THICKNESS OF } \\
\text { ICE-IN. }\end{array}$ \\
\hline 1 & 0.00 \\
\hline 1 to 4 & 0.50 \\
4 to 8 & 0.75 \\
\hline 8 & 1.00 \\
\hline
\end{tabular}

Exhibit 3.17 NBS: Ice on Wires.

\subsubsection{COMBINATIONS OF LOADS}

All of the foregoing loads do not act simultaneuusly; those likal do, do not necessarlly have a high probability of simultaneous occurrence. Exhibit 3.19 shows the design loads, $\mathrm{C}_{\mathrm{i}}$, as computed for various combinations of loads. The coefficients of 0.75 and 0.66 account for the reduced probability of occurrence. Each of the combinations, $C_{1}$ through $C_{8}$, must be computed for wind loads from each direction to determine which is the critical load that determines the design.

Live loads due to maintenance

Part of occupancy/equipment loads for buildings not included for signs or collector-like structures

Use minimum roof loads if better estimate unavailable

Exhibit 3.18 Maintenance Loads.

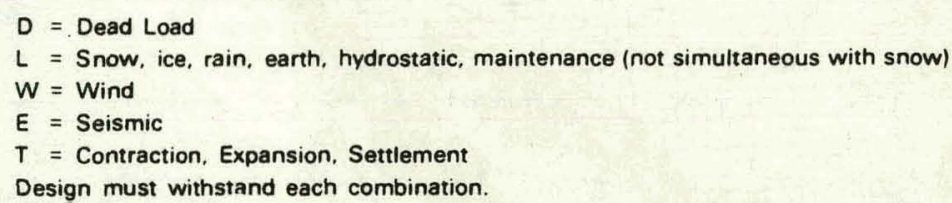

\begin{tabular}{|c|c|c|c|c|}
\hline \multirow[t]{2}{*}{ CUMBINATIOUN } & \multicolumn{2}{|c|}{ WINDS ON BACK } & \multicolumn{2}{|c|}{ WINDS ON FRONT } \\
\hline & VERTICAL & HORIZONTAL & VERTICAL & HORIZONTAL \\
\hline$C_{1}=D$ & - & - & - & - \\
\hline$C_{2}=D+L$ & - & - & $D+L$ & 0 \\
\hline$C_{3}-D+W$ or $E$ & $-0.1 D+W$ & WV & $D+W$ or $E$ & Wor $\mathrm{G}$ \\
\hline$C_{4}=D+T$ & $T$ & 0 & $D+T$ & $T$ \\
\hline$C_{5}=0.75(D+L+W$ or $E+T)$ & - & - & $0.75(D+L+W$ or $E)$ & 0.75 (W or $E)$ \\
\hline$C_{6}=0.75(D+L+T)$ & - & - & $0.75(D+L+T)$ & $0.75 \mathrm{~T}$ \\
\hline$C_{7}=0.75(D+W$ or $E+T)$ & $0.75(-0.1 D+W+T)$ & $0.75 \mathrm{~W}$ & $0.75(D+W$ or $E+T)$ & $0.75(W$ or $E+T)$ \\
\hline$C_{8}=0.66(D+L+W$ or $E+T)$ & - & - & $0.66\left(\mathrm{D}+\mathrm{L}+\mathrm{W}\right.$ or $\mathrm{E}+\mathrm{L}^{\top}$ & $0.66(\mathrm{~W}$ or $\mathrm{E}+\mathrm{T})$ \\
\hline
\end{tabular}

Exhibit 3.19 Combination Loads. 


\subsection{EXAMPLE OF THE USE OF THE BOCA CODE}

Exhibit 3.20 illustrates an example of the use of the BOCA code. The collector (array) is assumed to be 25 feet long and 8 feet wide. This is approximately the largest collector that has been found cost effective in 1978. The collector is assumed to be installed in Albany, New York, at a tilt of $50^{\circ}$ to the horizontal. The weight of the collector is assumed to be $5 \mathrm{lb} / \mathrm{ft}^{2}$. The area equals $25 \times 8=200 \mathrm{ft}^{2}$.

\begin{tabular}{|c|c|}
\hline $\begin{array}{ll}\text { Data: } & \\
\text { Collector: } & 25^{\prime} \text { long } \times 8^{\prime} \text { high } \\
\text { Location: } & \text { Albany, NY } \\
\text { Tilt: } & 50^{\circ} \\
\text { Weight: } & 5 \mathrm{lb} / \mathrm{ft}\end{array}$ & \\
\hline Loads: & Weight \\
\hline $\begin{array}{l}D=\text { Dead Load }=5 \mathrm{lb} / \mathrm{ft}^{2} \times 25^{\prime} \times 8^{\prime} \\
L=\text { Maintenance: } 12 \mathrm{lb} / \mathrm{ft}^{2} \times 25^{\prime} \times 8^{\prime}\end{array}$ & $\begin{array}{l}=1,000 \mathrm{lb} \\
=2,400 \mathrm{lb}\end{array}$ \\
\hline$L=$ Snow: No drift: $25 \mathrm{lb} / \mathrm{ft}^{2} \times \frac{50-30}{50}$ slope $\times 25^{\prime} \times 8^{\prime} \times 50^{\circ}$ & $=1,286 \mathrm{bb}$ \\
\hline Drift: $19 \mathrm{lb} / \mathrm{ft}^{3} \times \frac{8 \sin (50-27)}{2} \times 27^{\circ} \times 8^{\prime}$ & $=3,508 \mathrm{lb}$ \\
\hline $\begin{array}{l}W=\text { Wind: } 15 \mathrm{lb} / \mathrm{ft} \times 25^{\prime} \times 8^{\prime} \\
\mathrm{E}=\text { Seismic, hail and ice }\end{array}$ & $\begin{array}{l}=3,000 \mathrm{lb} \\
=0\end{array}$ \\
\hline$T=$ Deflection: check after design & \\
\hline
\end{tabular}

Exhibit 3.20 Example-by BOCA Code.

The dead load is the weight of the collector, 1,000 pounds. The maintenance load is computed from the minimum lood of $12 \mathrm{lb} / \mathrm{ft}^{2}$ and the area, yielding 2,400 pounds. The snow load without drift, but on the tilted surface, is: $25 \mathrm{lb} / \mathrm{ft}^{2}$ multiplied by the tilt factor, the area, and the cosine of the tilt angle, or 1,286 pounds (exhibit 3.9). When drifting is included, the snow load can be as high as 3,508 pounds. In keeping with the BOCA code for signs, the wind load is computed from a wind force of $15 \mathrm{lb} / \mathrm{ft}^{2}$ and the area, yielding a total load of 3,000 pounds. Seismic loads, hail, ice, and deflection-induced loads are assumed negligible. By mounting the collector so the bottom is clear of the ground, with ic 2.5-foot gap below the collector, the etfect of dritting can be avoided.

Exhibit 3.21 shows the individual loads combined according to the scheme of exhibit 3.19. The most severe loads are circled. Wind from the front and wind from the back are considered separately. The wind from the back is frequently the most stringent, because it causes uplift and overturning. With the wind on the front, two conditions must be checked separately: $C_{3}$ gives the highest horizontal component; $C_{5}$ gives the highest vertical component and the highest resultant combination of the horizontal and vertical. The structure must withstand both $\mathrm{C}_{3}$ and $\mathrm{C}_{5}$. For the example, the resultant load is less than 20 $\mathrm{lb} / \mathrm{ft}^{2}$ vertically and $12 \mathrm{lb} / \mathrm{ft} 2$ horizontally. As will be seen in the following section, most commercially available structures have capabilities for in excess of these loads. 


\subsection{AVAILABLE STRUCTURES}

Small structures, typical of most applications, will use pre-engineered structures. The cost of support structures will be approximately $\$ 40$ per square meter of array. For a system requiring an average power of one kilowatt, the array size will be approximately $60 \mathrm{~m}^{2}$, so the total structure cost will be $\$ 2,400$. The cost of the structure will not only be a small part of the total system cost of nearly $\$ 200,000$, but will also be small compared to the engineering cost that would be required to reduce the structure cost significantly. Only in systems above 10 kilowatts will structural optimization be worthwhile. Because the designs being considered in this seminar are all under $2 \mathrm{kWe}$, the pre-engineered structures are the only consideration.

Exhibits 3.22 through 3.25 illustrate some structural designs available from various manufacturers. The capabilities and typical dimensions are listed in exhibit 3.26. Many structures are copable of hondling wind loods over $150 \mathrm{mph}$, well above any that can be expected. The force resulting from the $150 \mathrm{mph}$ wind is approximately $56 \mathrm{lb} / \mathrm{ft}^{2}$ which is also well above any snow load that might be expected, but the structural members nre lypically only 2 or. 3 inches in diameter.

Perhaps the most important consideration in the selection of the structure is the selection of the material. Hot-dip-galvanized steel, aluminum and pressure-treated lumber are preferred to minimize maintenance, with the last two being most desirable because 20year lives are common. Galvanized steel coatings are frequently chipped or worn off near fasteners, so the corrosion protection is lost.

Exhibit 3.26 also shows the foundations required for the various structures. Concrete footings that extend below the frost line will suffice, although some manufacturers claim that their structures are rigid enough to withstand several inches of frost hegve, so they ran be placed on slabs-on-grade or shallow footings. As is always the case, the foundations must be evaluated carefully in terms of the ground characteristics of the site. A soils engineer should normally be consulted.

\begin{tabular}{|c|c|c|c|c|}
\hline \multirow[t]{2}{*}{ COMBINATION } & \multicolumn{2}{|c|}{ WINDS ON BACK } & \multicolumn{2}{|c|}{ WINDS ON FRONT } \\
\hline & VEATICAL & HORIZONTAL & VEATICAL & HORIZONTAL \\
\hline$C_{1}$ & - & - & - & - \\
\hline $\mathrm{C}_{2}$ & - & $\overline{-}$ & $\begin{array}{c}1.080+3.686 \cos 50 \\
3.369 \mathrm{lb}\end{array}$ & \\
\hline$\overline{C_{3}}$ & $3.000 \cos 50-0.1$ & $3,000 \sin 50$ & $\begin{array}{c}1.000+3.000 \cos 50 \\
2.928 \mathrm{lb}\end{array}$ & $\begin{array}{c}3.000 \sin 50 \\
(2,298) \mathrm{lb}\end{array}$ \\
\hline $\mathrm{C}_{4}$ & $\begin{array}{l}\sigma \\
0\end{array}$ & 0 & $\begin{array}{c}1,000+0 \\
1.000\end{array}$ & r \\
\hline$\overline{C_{3}}$ & - & $\cdots$ & $0.7513 .36 \frac{9}{3.973} 3.000$ 50 & $0.75 \times 2.298$ \\
\hline $\mathrm{C}_{6}$ & - & - & $\begin{array}{c}0.75(3,369+0) \\
2,527\end{array}$ & $0.75 \times 0$ \\
\hline$C_{7}$ & $\begin{array}{c}0.75(1,828+0) \\
1.371\end{array}$ & $\begin{array}{c}0.75 \times 2,298 \\
1.724\end{array}$ & $\begin{array}{c}0.75(2.928+0) \\
2.196\end{array}$ & $\begin{array}{c}0.75(2.298+0) \\
1.729\end{array}$ \\
\hline$\overline{\mathrm{C}_{\mathrm{a}}}$ & - & $=$ & $\begin{array}{c}0.66(3,973,0.76+0) \\
3.496\end{array}$ & $\begin{array}{c}0.66(1.724 / 0.75 \div 0) \\
1.517\end{array}$ \\
\hline
\end{tabular}

Exhibit 3.21 Combination of Loads. 


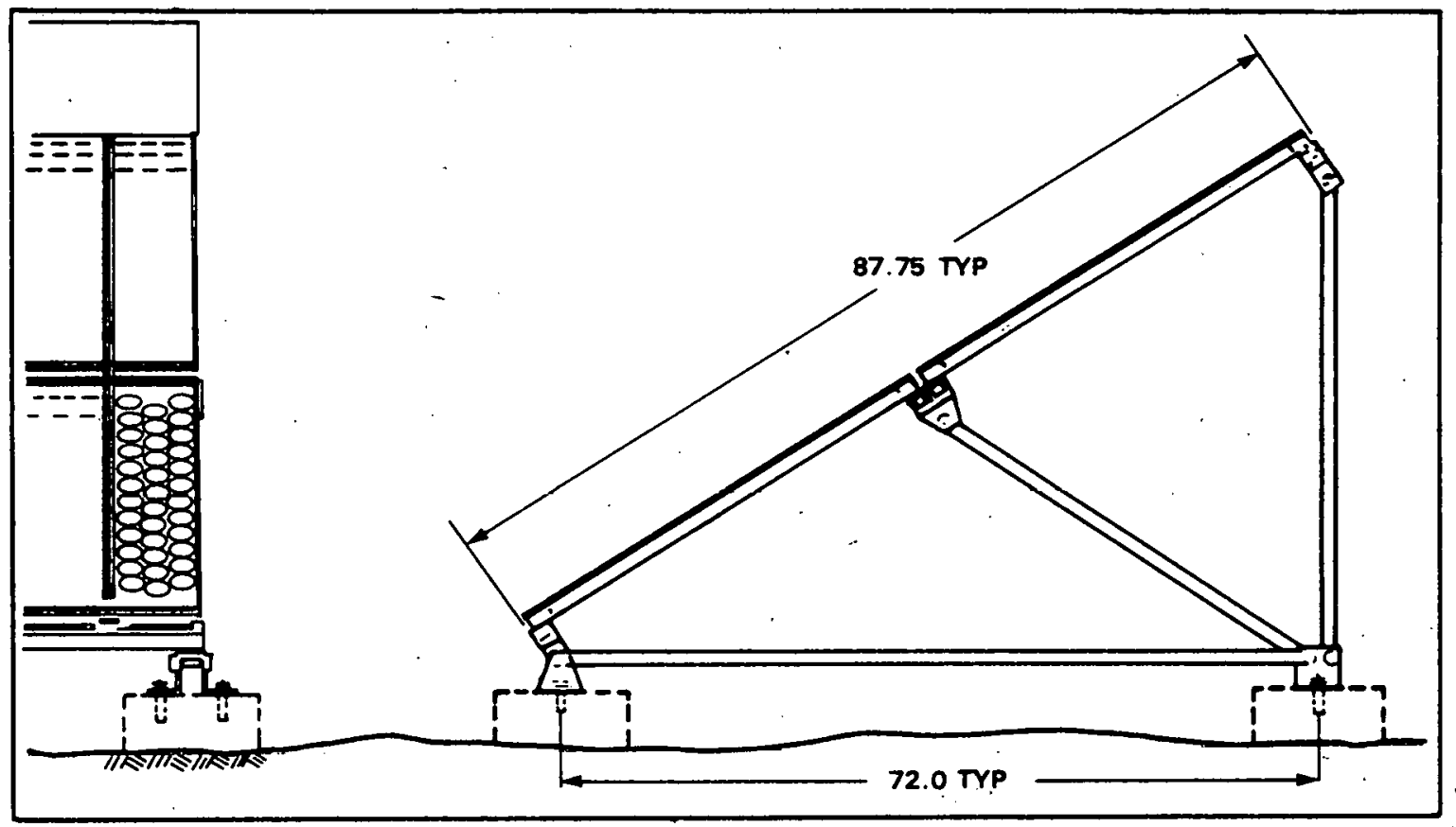

Exhibit 3.22 Truss Structure.

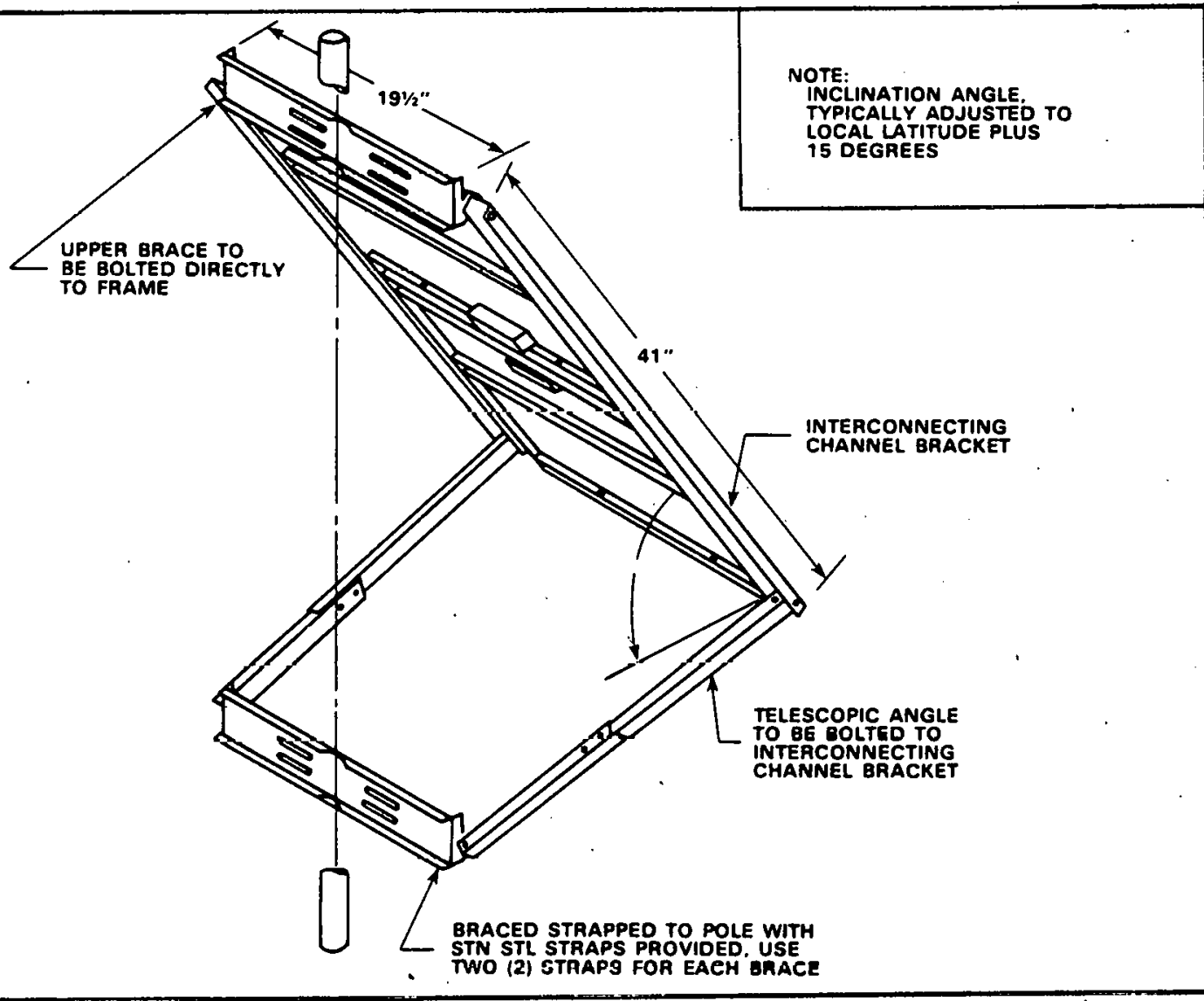

Exhibit 3.23 Truss Structure for Polc Mounting. 


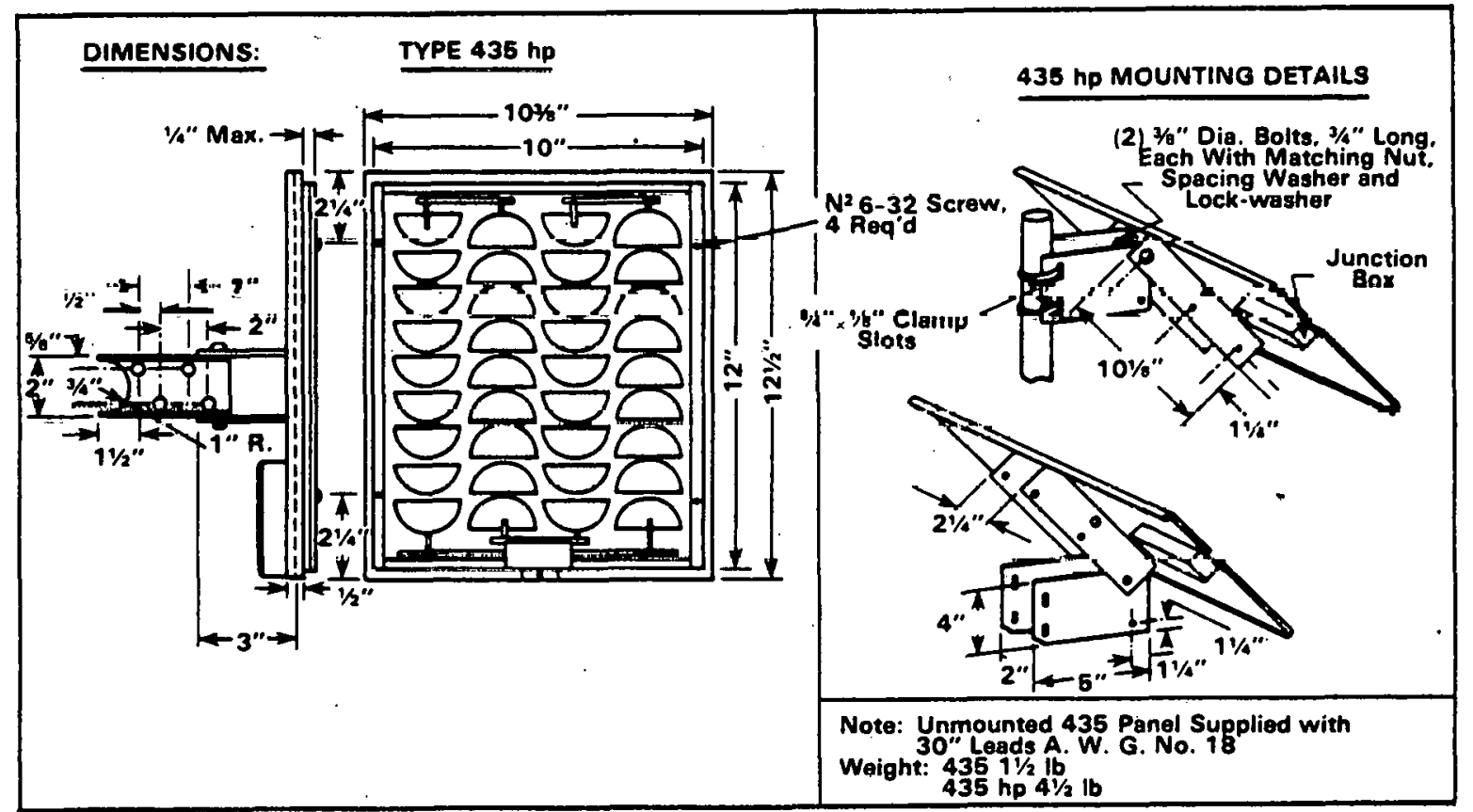

Exhibit 3.24 Structures for Pole Mounting.

\begin{tabular}{|c|c|c|c|c|c|c|c|c|}
\hline & ALLOWABLE & FOUN & DATION & APPROX. & MAT'L. & FACE & LONGEST & ELEMENT \\
\hline & & SLAB & FTO. & & & & & \\
\hline Arco & 100 & $x$ & $12^{\prime \prime} \times 12^{\prime \prime}$ & 30 & $A L$ & $2.2 \times 0.22$ & 2.3 & $2 " \times 2 " \times 1 / 8 "$ \\
\hline $\begin{array}{l}\text { Solar Power } \\
\text { Solarex-Pruss } \\
\text { Solarex-Pole } \\
\text { Solarex-Pole }\end{array}$ & $\begin{array}{l}75 \\
75.100 \\
100 \\
100\end{array}$ & $\bar{x}$ & $\bar{X}$ & $\begin{array}{l}45^{2} \\
602^{2}\end{array}$ & $\begin{array}{l}A L \\
A L\end{array}$ & $\begin{array}{l}1.3 \times 1.8 \\
0.3 \times 0.3\end{array}$ & $\begin{array}{l}1.8 \\
\text { Pole }\end{array}$ & $\begin{array}{c}21 / 2 "{ }^{\prime 1} \times 2^{\prime \prime} \times 1 / 8^{\prime \prime} \\
2^{\prime \prime} \text { Typical }\end{array}$ \\
\hline
\end{tabular}

Exhibit 3.26 Typical Structures and Their Capabilities. 


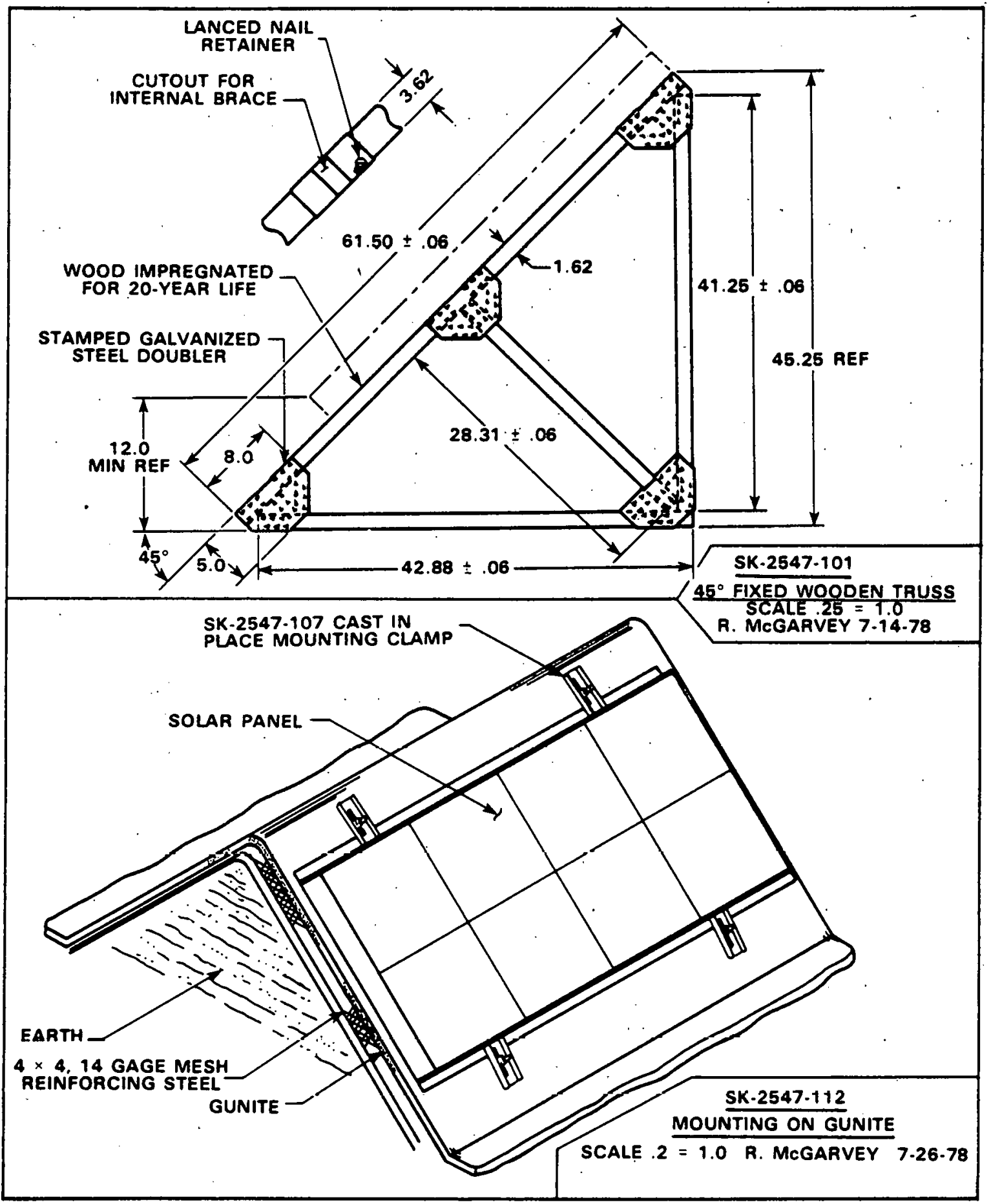

Exhibit 3.25 Proposed Structural Concepts. 


\subsection{THERMAL DESIGN}

Designers have frequently proposed the solar thermal-solar cell total energy system. Solar cells are mounted integrally with thermal collectors, so the solar energy that is not converted to electricity might be used to generate heat useful for heoting and cooling the building on which the solar cells are mounted. The properties of solar cells are such that they usually absorb less heat and emit more heat than the coatings often used in thermal collectors. As a consequence, the solar cells, when mounted on a thermal collector, can degrade the performance of the thermal collector. For the purposes of this seminar, only solar cells will be considered as being mounted on the array. Thermal collectors for total energy systems are being studied in the DOE/NASA/SERI solar thermal power systems program. Generally, total energy systems involve the cogeneration of electricity and heating/cooling or process heat. DOE/NASA/SERI are studying the solar photovoltaic total energy system also as well as the solar thermal total energy system.

Solar cells are cooled by natural convection or by wind. The power required to fan cool the cells far outweighs the added power obtained from operating the cells at the lower temperature available with fan cooling.

The temperature of the cell is computed from an equation that balances the energy input to the cell with the energy output. The computations can be done for a typical square foot of cell. The thermal output is equal to the solar input (insolation) multiplied by $(1-n)$, where $n$ is the electrical efficiency of the cell. This term is illustrated on the third line of exhibit 3.27. The thermal output from the cell is a combination of losses from the rear plus losses from the front. The panels are usually so thin that the temperature inside the panel is nearly equal to the temperatures at the surface of the cell. The maximum temperature of the cell occurs when the insolation is greatest, or approximately $100 \mathrm{~W} / \mathrm{ft}^{2}$. The convective heat transfer coefficients, $h$, depend on wind velocity as indicated by the equation obtained from the American Society of Heating, Refrigerating and Air Conditioning Engineers (ASHRAE) handbook. With still air, the coefficient is $1 \mathrm{Btu} / \mathrm{hr} / \mathrm{sq} \mathrm{ft} / \mathrm{deg} F$. At $15 \mathrm{mph}$, the coefficient is 6 .

With no wind but with a ground-mounted array, both the back and front of the panel are effective in removing the heat. Therefore, the total heat-loss coefficient is the sum of two for the front and two for the rear, for a total of $4 \mathrm{Btu} / \mathrm{hr} / \mathrm{sq} \mathrm{ft} / \mathrm{F}^{\circ}$. If this coefficient is divided into the energy that is not converted to electricity, the temperature is $74^{\circ} \mathrm{F}$ above the ambient temperature, or $174^{\circ} \mathrm{F}$ on a $100^{\circ} \mathrm{F}$ day. To be conservative, it is assumed that the glass surface has zero reflectivity. This assumption will be increasingly more accurate as the technology for antireflection coatings is improved. If a glass reflectance of 10 percent were used, the temperature of the cell would be $167^{\circ} \mathrm{F}$, which is not significantly less, using the temperature differential of $74^{\circ} \mathrm{F}$.

If the panel is root mounted, the back is not tree to lose heat to the surroundings. Instead, the heat radiates to the roof and convects to the space between the roof and the panel. The panel should be mounted either flashed into the roof to prevent leakage, or 3 inches from the roof to permit air to circulate under the panel, thereby removing both heat and moisture. The heat transfer coefficient on the front is given by the same formula as for the freestanding collection. The heat-transfer coefficient for the rear is approximately 1.5 . For the same no-wind case as analyzed above, the temperature of the roof-mounted panel would be $85^{\circ} \mathrm{F}$ above ambient, or $185^{\circ} \mathrm{F}\left(85^{\circ} \mathrm{C}\right)$ on a $100^{\circ} \mathrm{F}$ day. 


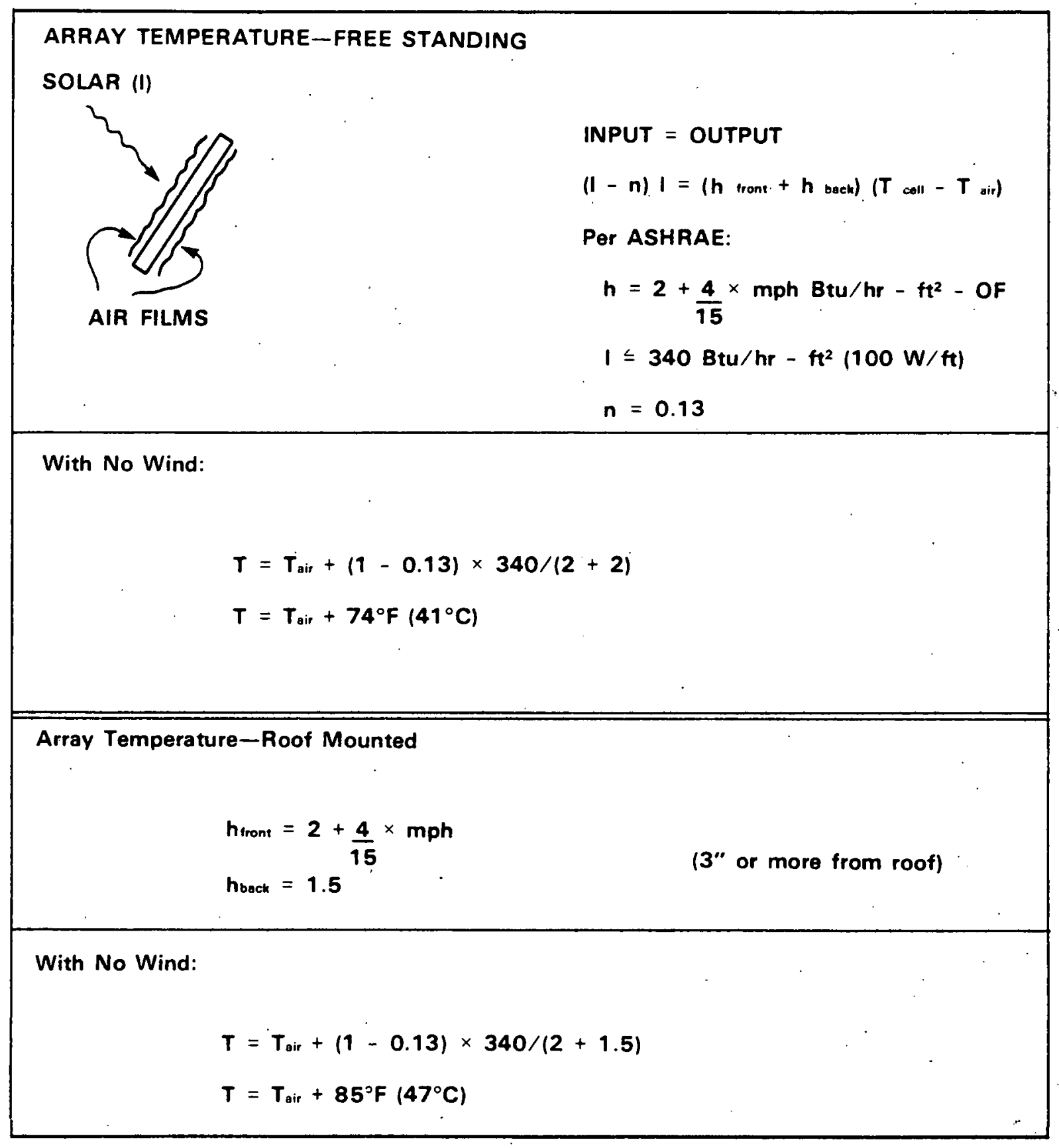

Exhibit 3.27 Typical Solar Array Temperatures. 
In a $15 \mathrm{mph}$ wind, the freestanding collector would be at a temperature of $125^{\circ} \mathrm{F}$ on a $100^{\circ} \mathrm{F}$ day, whereas the roof-mounted array would be at $146^{\circ} \mathrm{F}$. The gverage roof-mounted cell temperature over the life of the cell would probably be close to $15^{\circ} \mathrm{F}$ above the groundmounted temperature. The temperature of the cell is proportional to the insolation, so the above temperature differences can be modified to insolation values other than $100 \mathrm{~W} / \mathrm{ft}^{2}$ by a simple ratio.

Since the panel temperatures are well within the allowable temperature limits for most materials, they are of little concern. However, care must be exercised on still days to prevent personal injury from hot cells. In addition, the high cell temperatures will affect the cell life and, more importantly, the cell efficiency. The temperatures shown in exhibit 3.27 will result in a panel efficiency of 8 percent, as compared to the rated efficiency at $25^{\circ} \mathrm{C}$ of 12 percent.

\subsection{FROST, SNOW, AND ICE}

Because the panels face skyward, their temperatures will frequently be lower than the ambient dew point. As a consequence, frost.will form on the panels, thereby obscuring some of the sunlight. Exhibit 3.28 shows the frost thickness that would not normally last past 10 a.m. on clear and average days. The sun will be bright enough so that, despite the high reflectance of the ice, the warmth of the sunlight will be sufficient to melt the frost. The same curve applies to ice and snow.

Under more severe conditions, the frost, ice, or snow thickness will be greater than could be melted by the sun. Electrical melting by a resistance heater in the panel could be used to de-ice the panel. Exhibit 3.29 shows the tradeoff between the energy required for de-icing and the energy produced from the de-iced cell. It is evident that de-icing will not be profitable under most circumstances, since the power required for de-icing (curves $A$ and C) will not be regained by the array before the next snow fall (curve $D$ ).

Other possibilities exist for de-icing the panels. One promising method calls for heating the snow or ice at the surface of the panel, then allowing the upper layers of ice or snow to slough off (curve $B$ in exhibit 3.29). This method is not effective if the panel has a lip at the lower edge, as many panels do. This lip serves as a snow retainer. More work is required to determine how effective surface melting would be.

Another area requiring further study is the partial melting of the ice/snow covering on the panel. If a strip of ice or snow is melted electrically, a strip of the panel will be exposed to direct solar radiation. As a consequence, the panel will be solar heated without the intermediate step of conversion to electriclty. The strip will widein as the panel is heatcd, until the entire array is freed from snow and ice.

No matter what is done, on cold windy days, there is little hope of removing the ice cover. The experience of homeowners with icing of their southern windows will provide some indication of the severity of the ice problem and the persistence of the ice layer. At present, electrical heating of the panels does not appear worthwhile.

\subsection{CONCLUSION}

As a result of this chapter, agency representatives should be able to estimate the structural load that the collector must withstand, and be able to compare this with the given data on typical solar-panel structures, or with data supplied by the same or other manufacturers for more suitable structures. If the ambient temperatures and the local precipitation indicate ice and snow may cause a serious shortfall of solar-cell output, the ability of a manufacturer's collectors to retain ice and snow should be considered. 


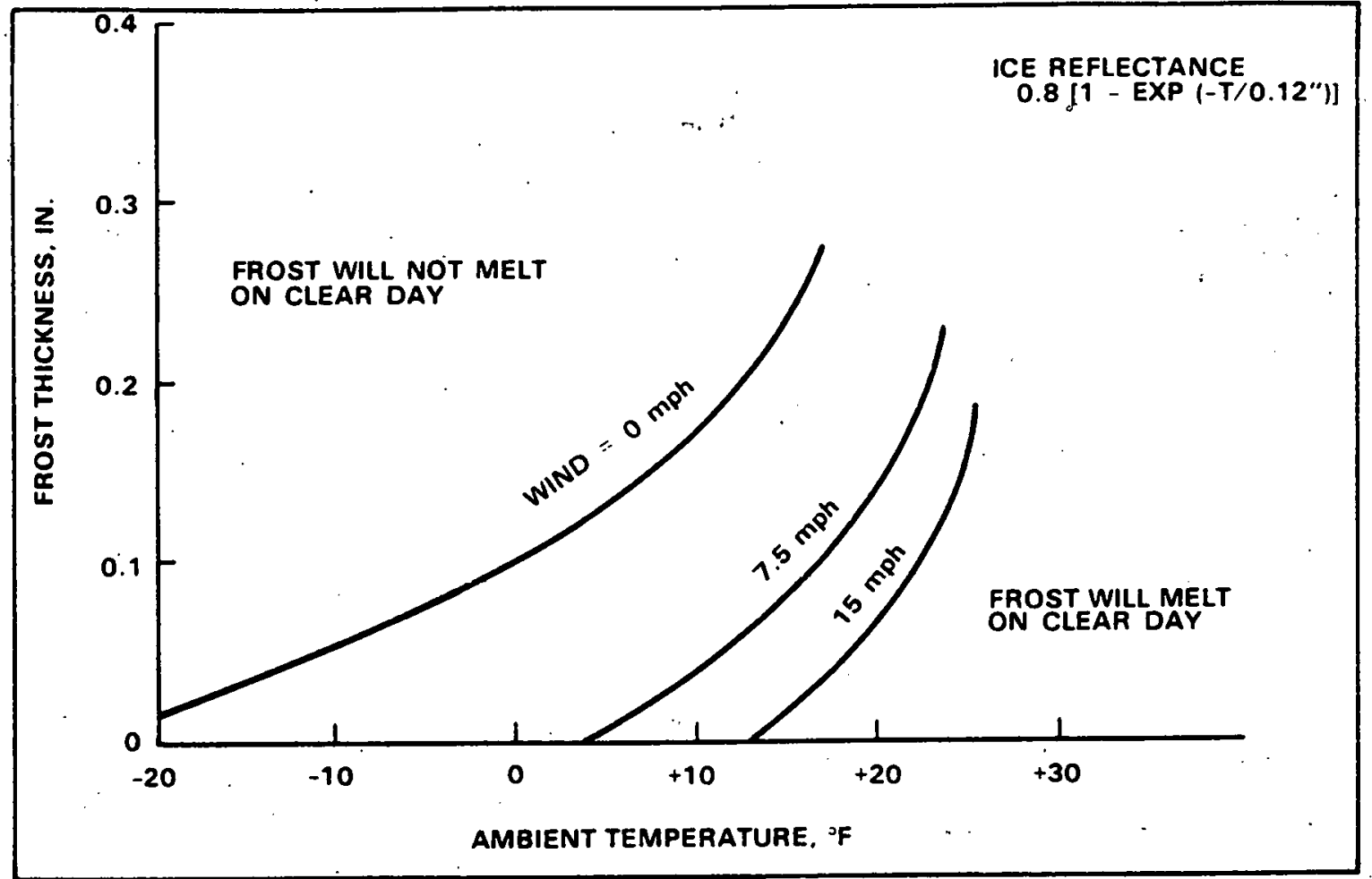

Exhibit 3.28 Frost Removal.

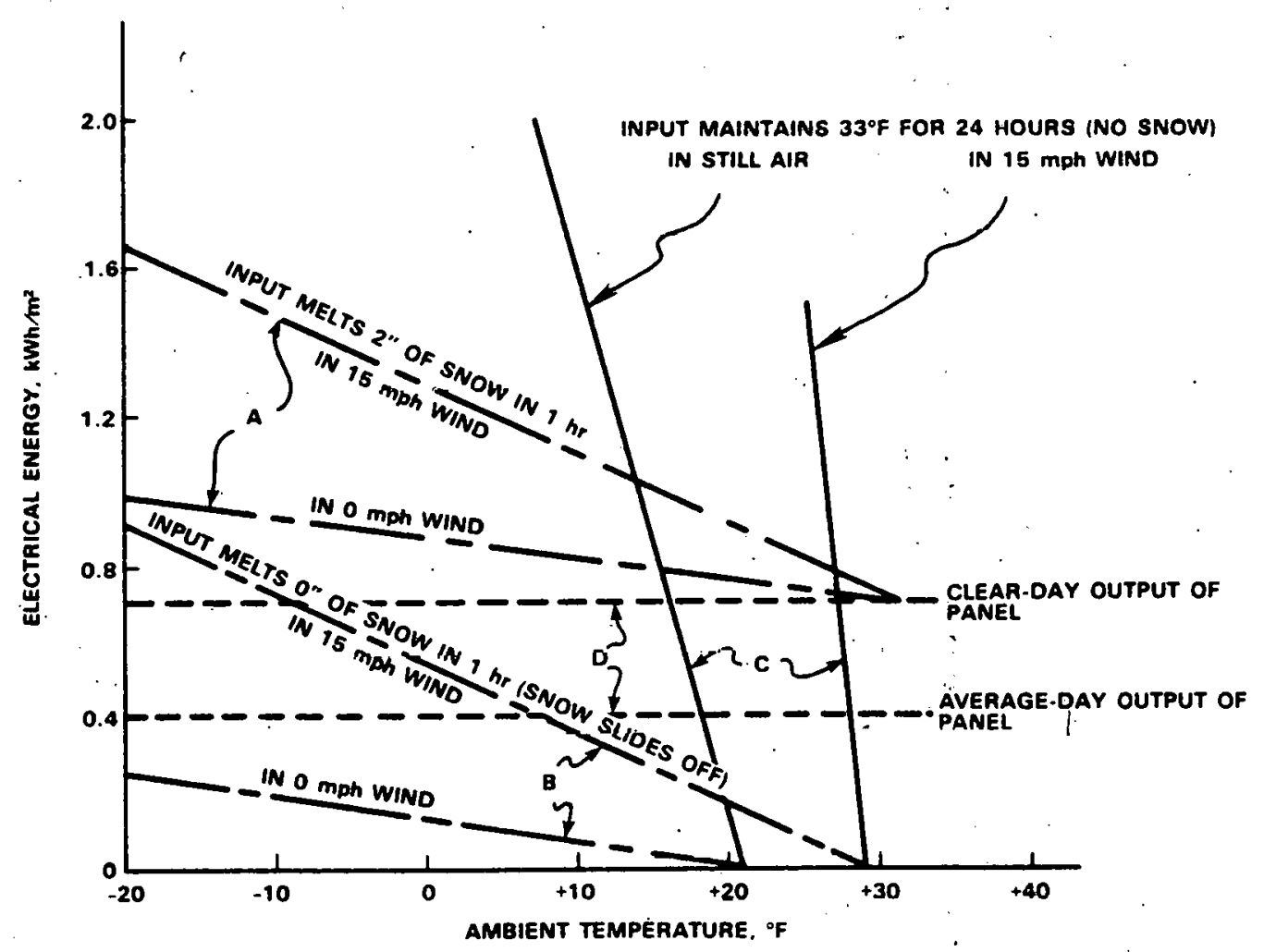

Exhibit 3.29 Thermal Snow Removal. 


\section{THIS PAGE \\ WAS INTENTIONALLY \\ LEFT BLANK}




\section{SECTION 4}

BATTERIES

\subsection{ENERGY STORAGE}

Due to the variations in insolation, and the possible utilization of energy under no-sun conditions, some form of electrical storage normally must be used in conjunction with the PV system.

Of the many possible energy storage systems available, most are not practical due to their high initial costs and energy storage capability mismatch (many can store much more energy than is required). Pumped hydroelectric, compressed air, thermal or hydrogen storage, super conducting magnets, and flywheel storage fall into this category. Chemical storage of electrical energy via batteries proves to be the most cost effective method of storing eniergy in connection with the PV system.

Batteries are storage devices that use chemical reactions to convert chemical energy into electrical energy. The quantity of available electric energy is a function of the inherent potential voltage and efficiency of electrochemical reactions, as well as the amount of active material in the battery. Many combinations of chemicals have been used as energy storage systems, each type possessing advantages and disadvantages with regard to physical and electrical battery characteristics. Energy density, expressed in either $\mathrm{Wh} / \mathrm{lb}$ or $\mathrm{Wh} / \mathrm{in}^{3}$, is one important characteristic to consider. In many applications, energy cost, expressed in dollars per watt-hour, will be the overriding consideration.

\subsection{TERMINOLOGY}

Batteries are normally classified as either primary or secondary. Primary batteries are designed to be utilized orly once because the active chemicals they contain are depleted during the chemical reaction which produces the output electrical energy. They are discarded when they are completely discharged. Secondary batteries are designed to be used repeatedly. Electrical energy, which can be applied to the output terminals, is electrochemically converted, stored as chemical potential energy, and, subsequently, reconverted to electrical energy.

Primary batteries are used in systems that require low current discharge rates and low first cost. The secondary battery, due to the reversibility of its chemical reaction, can be used repeatedly in high-power applications with a recharge included in the duty cycle. Types of batteries include: zinc-carbon, alkaline-manganese, mercury, and lithium for primary cells; and, lead-acid, nickel-cadmium, lithium-sulfur, sodium-sulfur, and sodium-chlorine for secondary application. Lead-acid and nickel-cadmium secondary batteries are the two types most suitable for use with PV systems due to their cost, operation, and availability (shown in exhibit 4.1).

In the following material, detailed discussions of types and operation of batteries are presented. It is expected that this material will allow the PV designer to choose the proper battery for the application at a cost suitable to the projects. 


\begin{tabular}{|c|c|c|c|c|}
\hline ACTIVE MATERIAL & TYPE & V/CELL & Wh/lb & FEATURES \\
\hline Zinc-Carbon & Primary & 1.5 & 35 & Low-cost. wide variety of small sizes. \\
\hline Alkaline-Manganese & - & 1.5 & 42 . & $\begin{array}{l}\text { Good low temperature operation. high efficiency under } \\
\text { high-drain duty, more costly than zine-carbon. }\end{array}$ \\
\hline Mercury & $*$ & $1.3 \cdot 1.4$ & 56 & $\begin{array}{l}\text { Excellent high temperature performance. } \\
\text { Relatively flat discharge characteristics. }\end{array}$ \\
\hline Lithium & - & 2.95 & 150 & $\begin{array}{l}\text { Highest energy densitv, temperature range and shelf } \\
\text { life of primary cells, connains no water. }\end{array}$ \\
\hline Lead-Acid & Secondary & 2.0 & 12 & $\begin{array}{l}\text { Least expensive and most resdily available of } \\
\text { secondary cells. }\end{array}$ \\
\hline Nickel-Cadmium : & - & $\begin{array}{l}1.2 . \\
.\end{array}$ & 16 & $\begin{array}{l}\text { Excellent low temperature operation, low weight. low } \\
\text { maintenance. higher initial cost than lead. }\end{array}$ \\
\hline Lithium-Sulfur & " & $\approx 1.5$ & $=60$ & $\begin{array}{l}\text { Onarata at high temperature, } 100^{\circ} \mathrm{C} \text {, not oureently } \\
\text { commercially available. majerted sosis } \$ 2.5 / \mathrm{kWh}\end{array}$ \\
\hline Sođium/Sulfur & - & 2.2 & $=95$ & $\begin{array}{l}\text { Operate at high temperature. }=300^{\circ} \mathrm{C} \text {. very low self. } \\
\text { discharge. projectod copts are } \$ 20 / \mathrm{kWh} \text {. should bo } \\
\text { rnmmerrially arailahis in mid } 80^{\circ} \circ \text {. }\end{array}$ \\
\hline Sodium/Chlorine & - & 2.12 & $\approx 70$ & $\begin{array}{l}\text { Not commercially availatle, projected costs } \$ 20 \mathrm{kWh} \\
\text { with efficiencies of greater than } 90 \% \text {. operates at } 200^{\circ} \mathrm{C} \text {. }\end{array}$ \\
\hline
\end{tabular}

Exhibit 4.1 Types of Batteries.

Because electrochemical reactions usually produce potentials of no more than a few volts, several cells are usually connected in series to attain a more useful battery voltage. Cells can also be connected in parallel to obtain larger battery current capacity.

Cells are composed of three basic components: (1) the negative electrode that supplies electrons to the external circuit as it is oxidized during the discharge reaction; (2) the positive electrode which accepts electrons from the external circuit when it is reduced during discharge; and, (3) the electrolyte that completes the circuit by furnishing the ions (i.e., electrically charged atoms) needed for conductance between the two electrodes.

The capability of a battery in Ah is expressed as the quantity of discharge current available during a specified length of time and at a given temperature, since charge equals current $x$ time. The output voltage decreases during discharge until the battery reaches a certain state where any further current draw will rapidly decrease the output voltage and possibly damage the battery. This state defines the "cut-off" voltage as shown in exhibit 4.2. During a cycle of use, the battery charge is dissipated and the battery voltage decreases. The energy capacity is the time integral (summation) of the product of the discharge current and voltage from full charge to cut-off voltage and is expressed in watt-hours, since energy equals power $x$ time and power equals voltage $x$ current. A charge or discharge rate is usually referred to as the energy capacity, where one watt-hour equals 360 joules of energy. For example, a battery which has a 150 Ah capacity, $c$, and is supplying 15 . A continuously, will be completely discharged in $150 \mathrm{Ah} / 15 \mathrm{~A}$, or 10 hours. This current draw equals c/10 for a 10-hour discharge rate. The percent of a battery's capacity that has been discharged is termed the depth of discharge. A complete discharge and subsequent recharge is referred to as a cycle. The number of such cycles a battery can undergo before degrading is termed the cycle life and is a function of both the battery type and the depth of discharge the battery is subjected to during the cycle. The deeper the discharge, the shorter the life, as a more complete utilization of the active materials in the battery will result in larger internal stresses. The interdependence of life cycles and depth of discharge is shown in exhibit 4.3. 


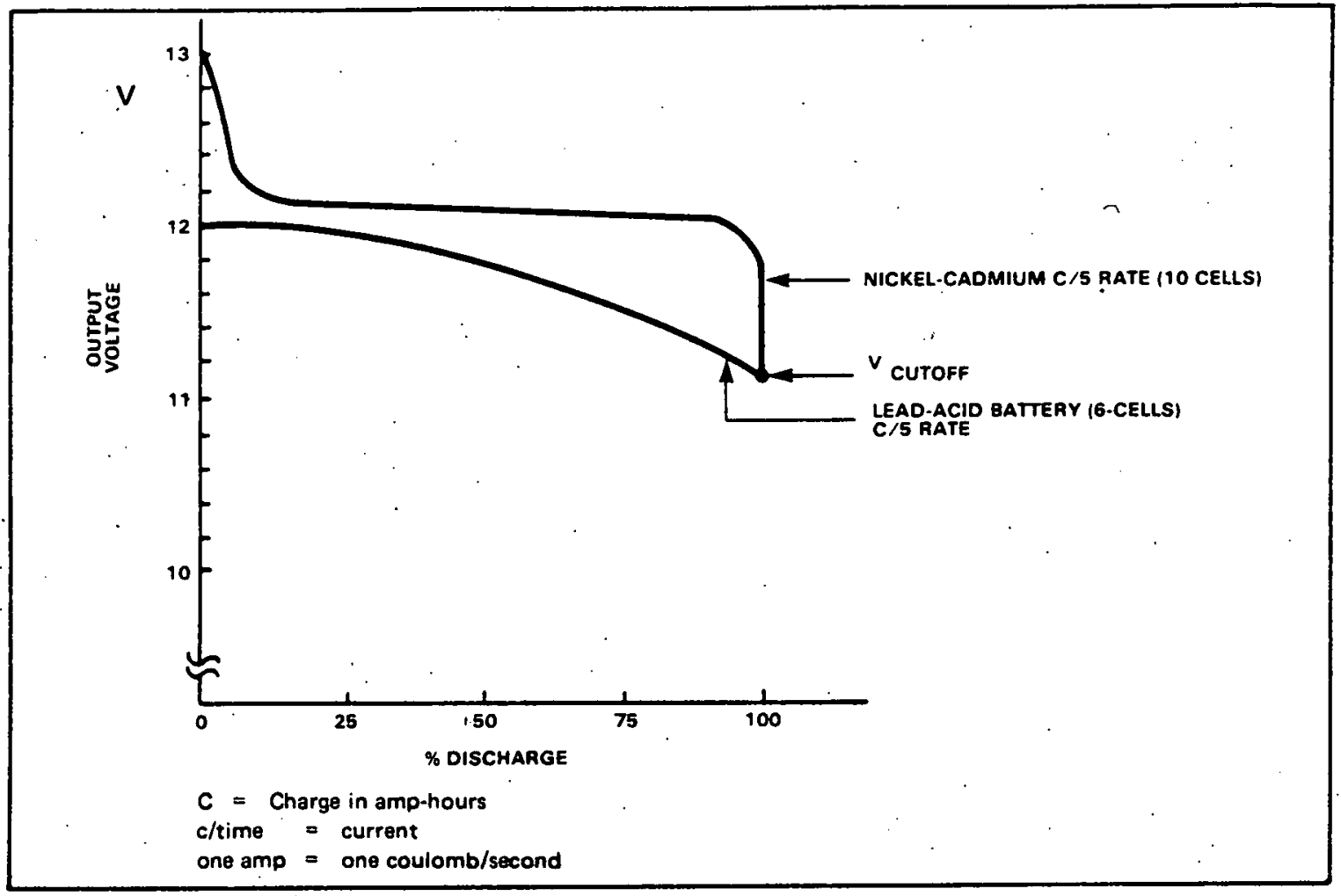

Exhibit 4.2 Comparison of Battery Output Voltages During Constant Discharge.

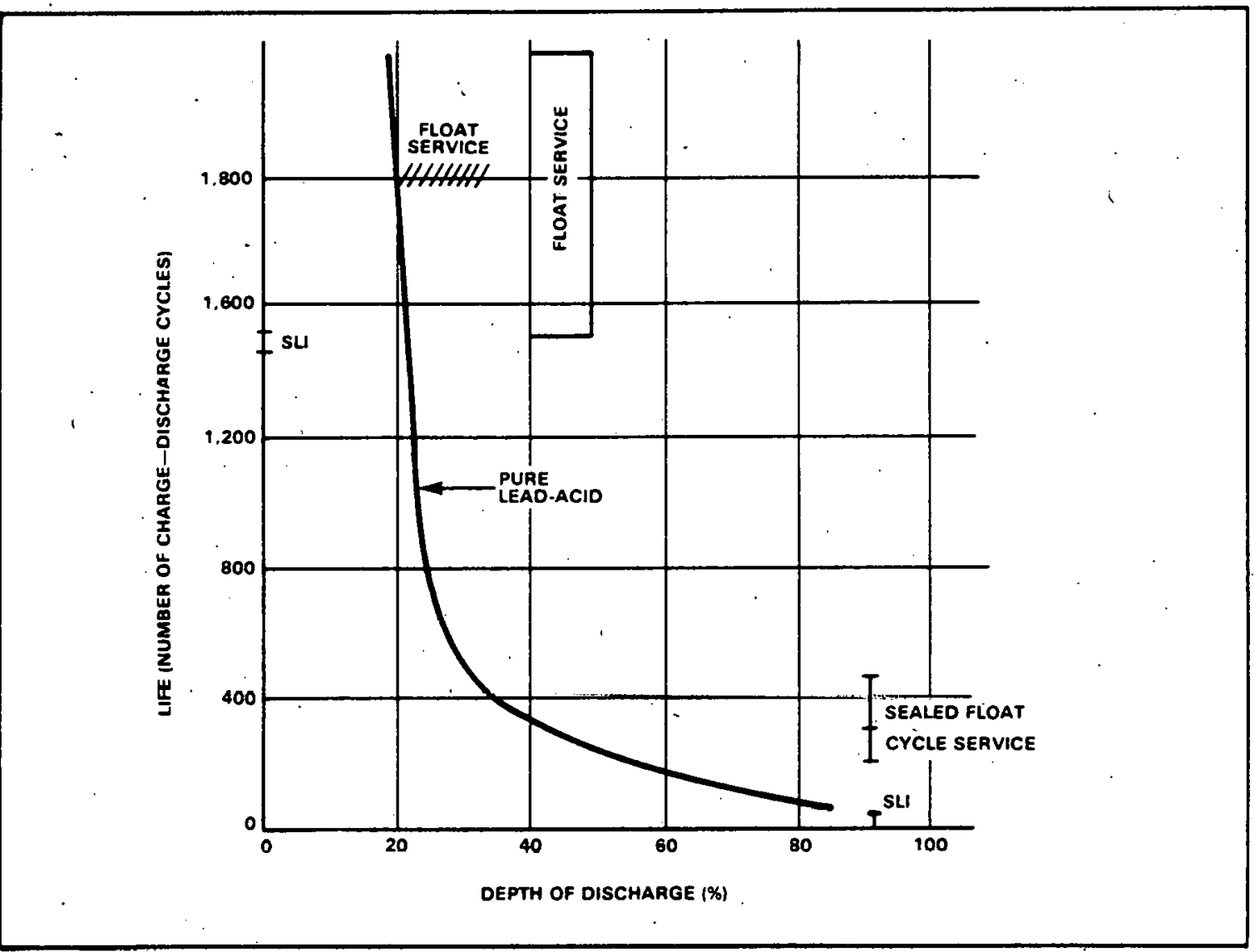

Exhibit 4.3 Battery Cycle Life vs. Depth of Discharge. 
- The coulomb is the common unit of electrical charge where one ampere equals one coulomb per second. In physical terms, the charge of an electron equals $1.6 \times 10^{-19}$ coulombs. The actual number of free electrons in a battery cycle can be calculated by knowing capacity, and vice versa, for battery material choice and chemcial-electrical energy conversion sizing.

\subsection{LEAD-ACID BATTERY}

Lead-acid cells are comprised of lead negative electrodes and lead dioxide positive electrodes. The electrolyte used is an ionic conducting solution of sulfuric acid in water. During discharge, the active material contained in the electrodes is converted to lead sulfate. The equation for this reversible reaction is:

$$
\mathrm{Pb}+\mathrm{PbO}_{2}+2 \mathrm{H}_{2} \mathrm{SO}_{4}=\mathrm{PbSO}_{4}+2 \mathrm{H}_{2} \mathrm{O}
$$

The ion is defined as an electrically charged atom or group of atoms, the electrical charge of which results when a neutral atom or group of atoms lose or gain one or more electrons during chemical reactions; the loss of electrons results in a positively charged ion and the gain of electrons in a negatively charged ion.

The electrolyte concentration of sulfuric acid decreases as the battery is discharged. Upon complete discharge, either the electrolyte or the active material in the electrodes is exhausted. Side reactions also occur, especially during overchorging. One such reaction is responsible for the production of hydrogen and oxygen via a method similar to electrolysis. Alloying materials or impurities in the electrodes can cause the evolution of toxic gases such as stibine $\left(\mathrm{SbH}_{3}\right)$ or arsine $\left(\mathrm{AsH}_{3}\right)$. In designing a lead-acid battery storage system, provisions must be made for venting these gases and for supplying additional water to the batteries. A catalytic converter can be incorporated in the battery cap, reconverting the hydrogen and oxygen into water.

An electrolyte is any substance which in solution or liquid form is capable of conducting an electric current by the movement of its dissnrintert positive and negative ione to the electiviles.

When recharged properly, lead-acid cells typically have coulombic efficiencies of 90 to 95 percent. For example, $111 \mathrm{Ah}$ are required to recharge a 100 Ah battery with a 91 percent coulombic efficiency $(: 00 / 0.91=111)$. Since recharge valtages are higher than discharge voltages, the energy efficiency is 70 to 80 percent. The coulomb is the common unit of electrical charge, and equals one amp-sec.

\subsection{TYPES OF LEAD-ACID BATTERIES}

At present, there are four types of lead-acid batteries, each of which possesses certain features critical to the type of service for which it is designed (see exhibit 4.4). One type of lead-acid battery is used in automobiles for starting, lighting, and ignition (SLI). These batteries are capable of delivering large amounts of energy in a short period of time, resulting in a low depth of discharge. For example, a typical $12 \mathrm{~V}$ car battery has an energy capacity of approximately $0.78 \mathrm{kWh}$. It typically delivers $300 \mathrm{~A}$ for about $20 \mathrm{sec} / \mathrm{start}$, or $0.02 \mathrm{kWh}(12 \mathrm{~V} \times 300 \mathrm{~A} \times 20 / 3600) \mathrm{kWh}$. This corresponds to a depth of discharge of 2.56 percent of rated capacity $(0.78 \mathrm{kWh})$. When utilized in this fashion, an SLI battery will last 4 to 5 years. If the battery undergoes only three to five deep discharges ( 80 to 100 percent of capacity), failure may result. These batteries have thin plates that contain a high antimony content. Tin and arsenic have also been added to give strength and castability to the grids so 
they can withstand the large stresses caused by rapid discharge and recharge cycles, as well as mechanical stresses due to their environment in a moving vehicle. Automotive SLI batteries range in cost from $\$ 35$ to $\$ 54 /$ rated $\mathrm{kWh}$ in sizes from 0.95 to $1.2 \mathrm{kWh}$, respectively. Diesel SLI batteries cost approximately $\$ 50 /$ rated $k W h$, ranging in sizes from 1 to $2.5 \mathrm{kWh}$. The high self-discharge rate and relatively short life of SLI batteries, especially when utilized to a deep discharge state, make this type of lead-acid cell unsuitable for many PV applications.

\begin{tabular}{|c|c|c|c|c|}
\hline \multirow{2}{*}{\multicolumn{2}{|c|}{$\begin{array}{l}\text { BATTERY } \\
\text { TYPE }\end{array}$}} & ENERGY COST & ENERGY DENSITY & \multirow{2}{*}{ CHARACTERISTICS } \\
\hline & & ratod $s / \mathrm{kWh}$ & ratod Wh/lb & \\
\hline \multirow{2}{*}{ SLI } & AUTO & $\$ 35.54$ & $15 \cdot 21$ & \multirow{2}{*}{$\begin{array}{l}\text { High self-discharge. shallow discharge cycle. } \\
\text { shorn life under deep discharge conditions. }\end{array}$} \\
\hline & DIESEL & 550 & $16 \cdot 18$ & \\
\hline \multirow{2}{*}{$\begin{array}{l}\text { MOTIVE } \\
\text { POWER }\end{array}$} & GOLF CART TYPE & $545-60$ & 30-35 & 250 cycle life, high self-discharge. deep cycle. \\
\hline & LONG LIFE & $\$ 160.220$ & 7.11 & $\begin{array}{l}1.000 \cdot 2.000 \text { crcte life, high self-discharge. } \\
\text { deep cycle. }\end{array}$ \\
\hline \multirow{2}{*}{$\begin{array}{l}\text { FLOAT } \\
\text { SERVICE }\end{array}$} & PURE LEAD GRIO & 580.130 & 14.18 & Low self-discharge, low maintenance. \\
\hline & LEAD-CALCIUM & $\$ 180 \cdot 240$ & $7 \cdot 10$ & $\begin{array}{l}1.500 \text { cycle life. low sell-discharge } \\
\text { deep cycle capabilities. }\end{array}$ \\
\hline SEALED & $\begin{array}{l}\text { FLOAT } \\
\text { SERVICE }\end{array}$ & $\$ 140$ & $14 \cdot 18$ & Low maintenance. low self-discharge. \\
\hline
\end{tabular}

\section{Exhibit 4.4 Types of Lead-Acid Batteries.}

\subsubsection{CYCLE SERVICE BATTERIES}

Another battery type may be categorized as providing "cycle" service. Motive power batteries, such as those used to power forklifts and golfcarts, allow greater dep'th of discharge. These batteries may survive one or more years (200 to 300 cycles) of daily deep discharge duty (90 percent of design capasity during an average work day). Plates for use in cycle batteries are made several times thicker than those used in SLI batteries and the number and size of these plates determine the battery capacity and weight. A massive positive electrode current collector (40 percent of the electrode) permits long life regardless of the use of high (5 percent) antimony grid alloys. As with the SLI batteries, most motive power-type batteries suffer from self-discharge problems and may lose up to 30 percent of rated capacity within several days, even if no powar is drawn from them. Prices in the range of $\$ 45$ to $\$ 60 /$ rated $\mathrm{kWh}$ for a single motive battery are typical, with sizes ranging upward from a $2 \mathrm{kWh}$ size. Longer life motive power batteries $(1,000$ to 2,000 cycles) are priced in the $\$ 145$ to $\$ 220 /$ rated $k W h$ range and typically weigh three to five times as much as the less expensive motive batteries for the same $k$ Wh capacity.

\subsubsection{FLOAT SERVICE BATTERIES}

In float-type service, the battery is maintained in a state of. low depth of discharge and only undergoes an occasional deep discharge. Batteries designed for such use have a very low self-discharge rate due to the use of pure lead or calcium alloyed grids, and a low rate of gas evolution (they require about one-tenth the makeup water needed in motive-power batteries). The positive grids are more corrosion resistant in the charge mode as they use no antimony. Therefore, the lifetime of these botteries typically exceeds 5 years' use. For maximum life, a discharge level of less than 20 to 30 percent of full capacity is recommended. These attributes make them ideally suited for use with PV power systems. Prices generally range from $\$ 80$ to $\$ 130 /$ rated $k W h$. (higher for batteries smaller than 1 $\mathrm{kWh}$ ), in sizes up to about $6 \mathrm{kWh}$. 
Deep discharge, float-type batteries are also available at higher costs, with 0.4 to 0.8 percent calcium alloyed with the lead for added strength. This allows the battery to minimize self-discharge problems, thereby retaining a near full capacity for anywhere from 6 months to 1 year while permitting deep discharge cycles. For maximum life expectancy, a discharge level of less than 40 to 50 percent of capacity is recommended. Costs for batteries of this type (which also contain extra electrolyte to prevent damage at full discharge) range from $\$ 200$ to $\$ 240 /$ rated $\mathrm{kWh}$ per single battery, in sizes from $1 \mathrm{kWh}$ and larger. Life expectancy is in the 1,500- to 2,000-cycle range.

\subsubsection{SEALED FLOAT SERVICE BATTERIES}

Sealed float service-type batteries are also now available in sizes up to approximately one-half $\mathrm{kWh}$. These cells use grids composed of calcium and other proprietary alloys. Due to the calcium grids, there is a low rate of gas evolution, allowing the cell to be. sealed, making it virtually maintenance free. Pressure relief valves are provided and will vent hydrogen if the battery is improperly charged. The life expectancy of the cells, as with most calcium grid designs, is 200 to 500 deep discharge cycles. Prices are in the $\$ 140 / \mathrm{kWh}$ range for $0.4 \mathrm{kWh}$ batteries, with higher prices per $\mathrm{kWh}$ for the lower power ratings.

\subsection{FAILURE MECHANISMS OF LEAD-ACID BATTERIES}

A diagram of a lead-acid cell is shown in exhibit 4.5. The chemical conversion of metallic lead and lead dioxide to lead sulfate and vice versa causes internal volume changes

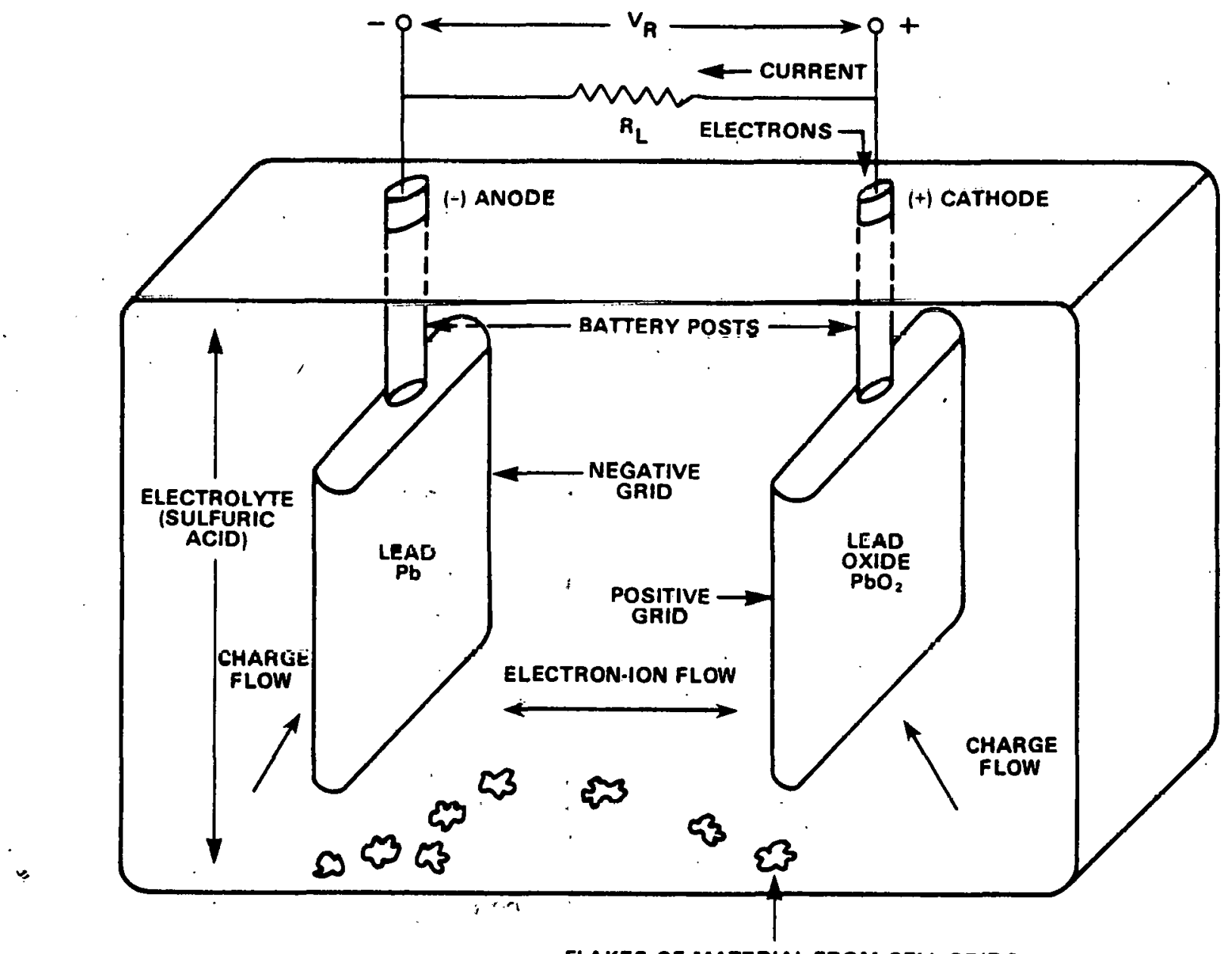

FLAKES OF MATERIAL FROM CELL GRIDS

Exhibit 4.5 Lead-Acid Cell. 
in the electrodes. These changes create stresses on thë electrodes which may cause the active material to flake or shed from the collector grid, and eventually shorting the cell. This process causes the battery to lose capacity and increases the internal resistance. This degradation increases with depth of discharge and results in decreased energy density and current carrying capability. This is why motive batteries, which supply large power loads, have antimony and other alloys to strengthen the electrode grids.

Lead-acid cells will also fail if the quantity of electrolyte is allowed to fall to an excessively low level. This can be prevented by adding additional distilled water, as needed, to the cells and by not overcharging the cells, thereby avoiding outgassing.

Although a battery's capacity increases with temperature, high temperatures actually reduce a battery's life (see exhibit 4:6). While there is considerable disagreement on the exact correlation of life and temperature, a good estimate is that a battery's life is halved if the average temperature is raised from $75^{\circ} \mathrm{F}$ to $110^{\circ} \mathrm{F}$. Energy. conversion efficiency also decreases with increasing temperature due to internal loss processes, such as electrolyte resistance, which shows an exponential increase with temperature.

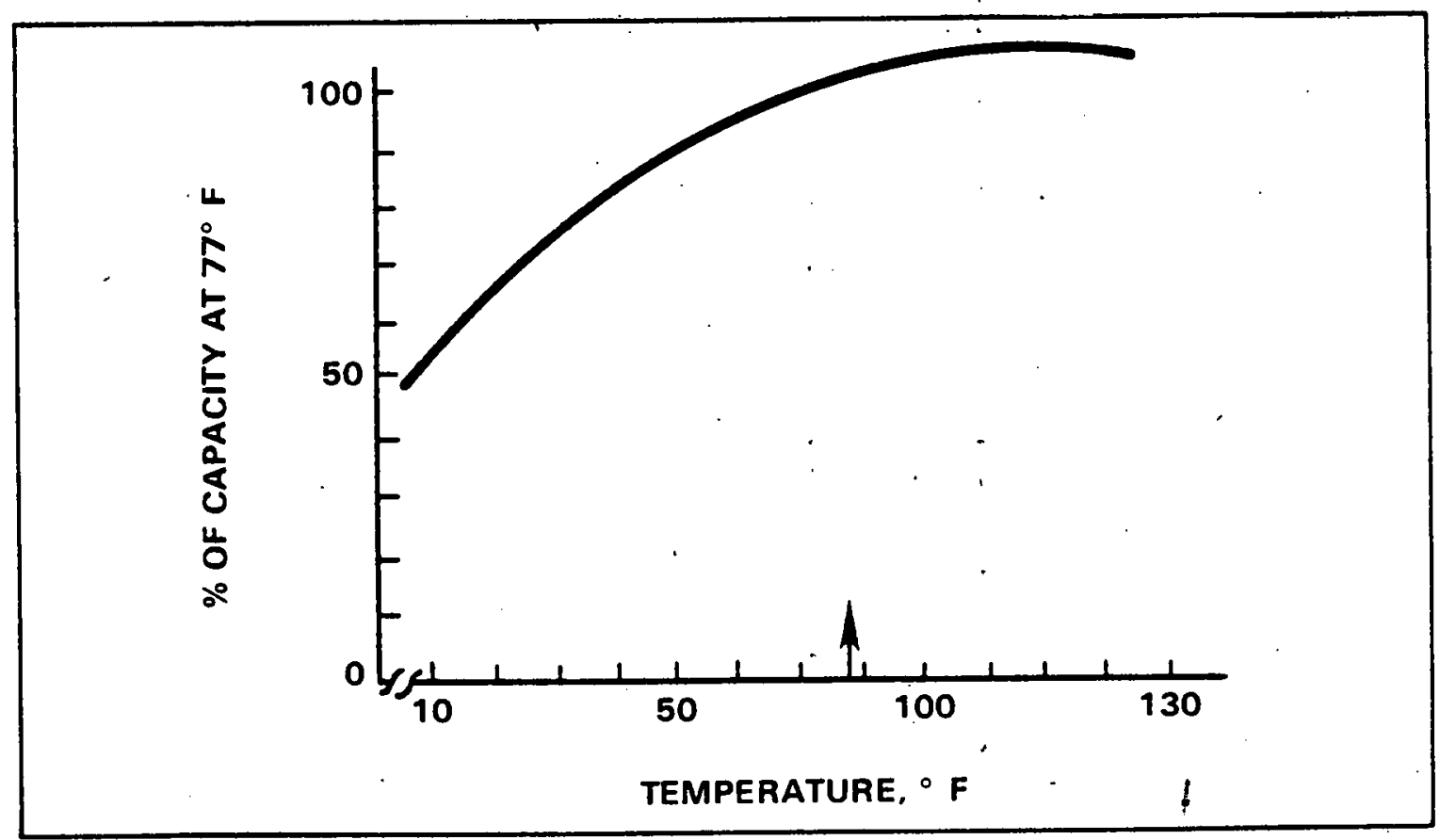

Exhibit 4.6 Relative Capacity of Lead-Acid Batteries as a Function of Temperature.

Care must also be taken to ensure that the batteries do not freeze in cold weather applications, as shown in exhibit 4.7; a fully-charged battery, will freeze at approximately $-60^{\circ} \mathrm{F}$, depending on the concentration of the electrolyte. As the battery discharges, the electrolyte is consumed by the chemical reactions and the concentration decreases. A fully discharged cell will freeze at a temperature somewhat below $32^{\circ} \mathrm{F}$. A high state of charge will prevent the batteries from freezing. 


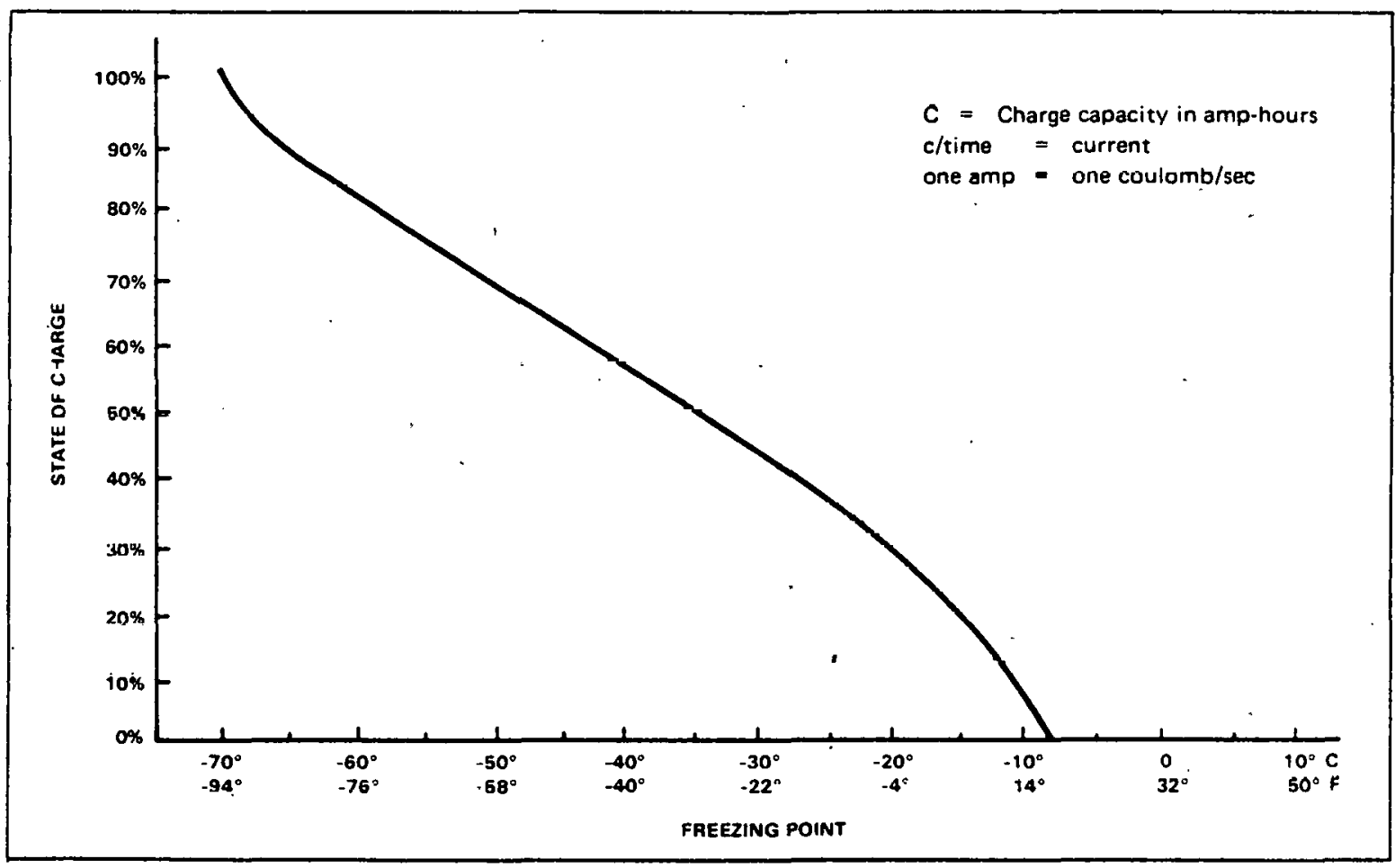

\section{Exhibit 4.7 Freezing Point of Lead-Acid Battery as a Function of State of Charge. Specific Gravity of Electrolyte $=1.310$ at $25^{\circ} \mathrm{C}$ at Full Charge.}

\subsection{DISCHARGE AND RECHARGE CHARACTERISTICS}

\subsection{LEAD-ACID BATTERIES}

The voltage characteristics of typical lead-acid cells during constant current discharge are shown in exhibit 4.8. The higher the rate of discharge, the larger the cell voltage decrease. This is the result of connector and grid structure resistance and electrolyte concentration polarization. Use of different materials and manufacturing techniques will result in actual cells varying from these curves. To assure maximum battery life, discharge past a terminal voltage of approximately $1.8 \mathrm{~V} /$ cell should be avoided.

The same effects which lower the cell's output during discharge contribute toward increasing the required terminal voltage during recharge, as shown in exhibit 4.9. During recharge, efficiency and battery life will be lowered by excessive outgassing and high temperatures. 


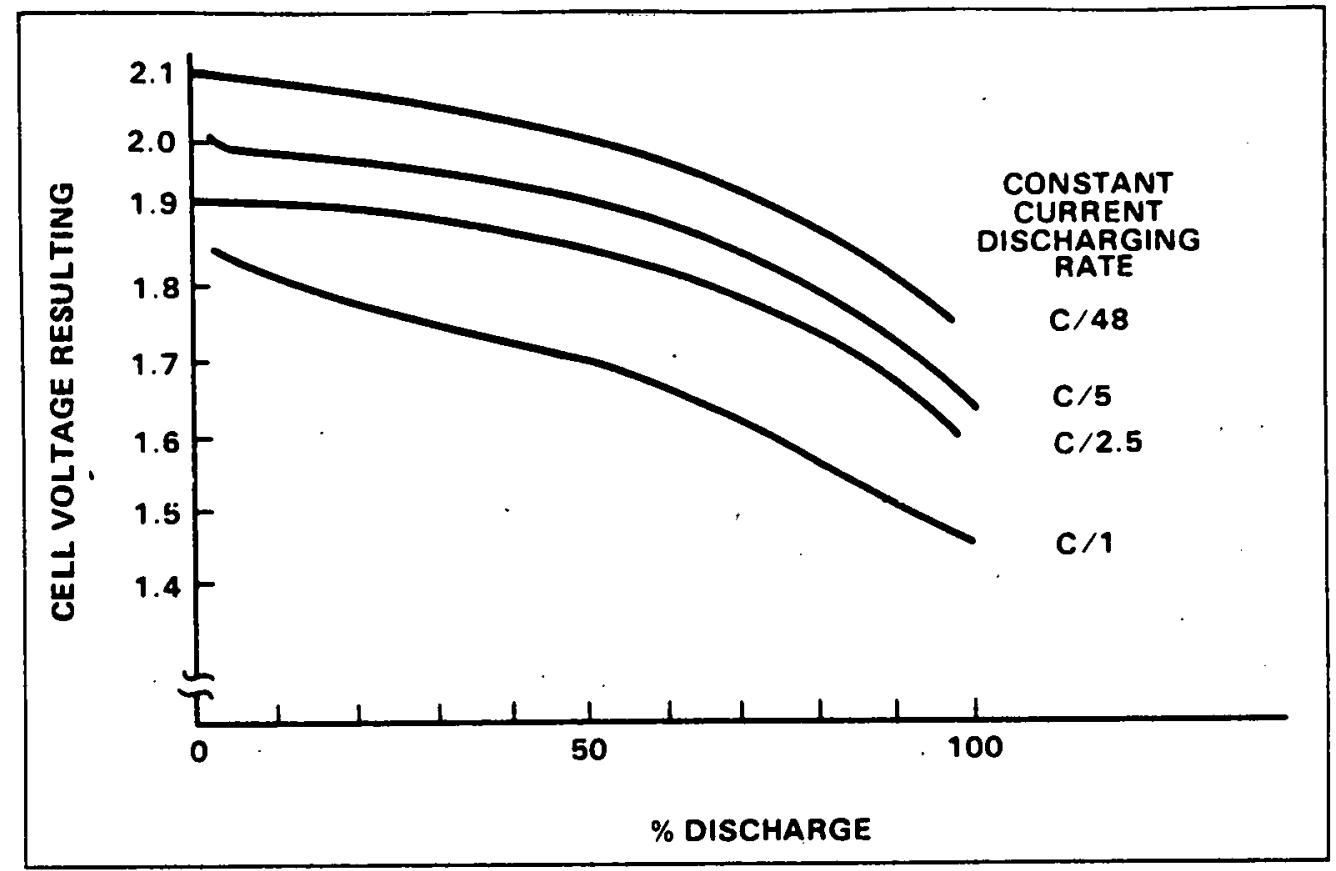

Exhibit 4.8 Typical Discharge Voltage Characteristics.

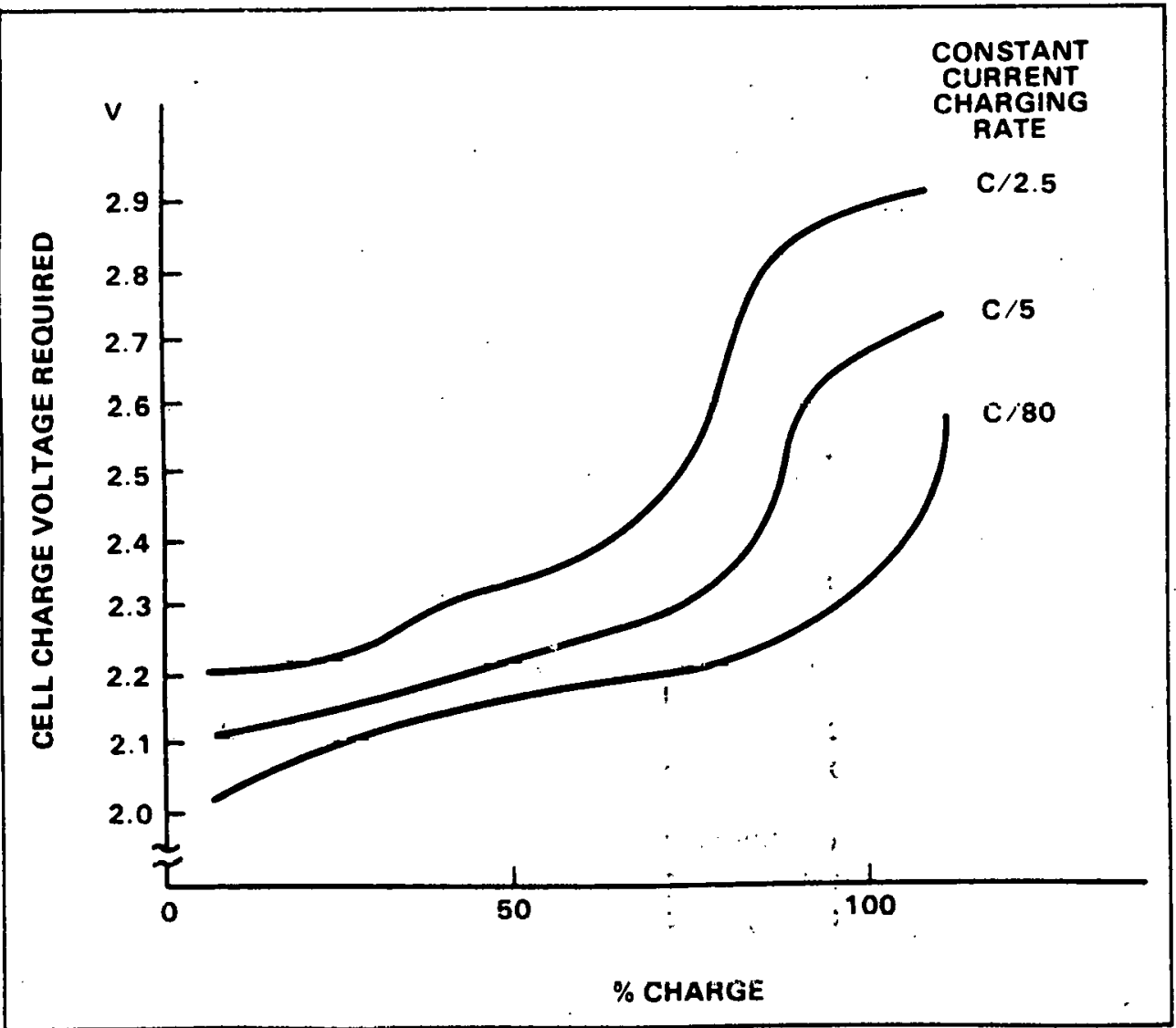

Exhibit 4.9 Typical Charge Voltage Characteristics. 
For standby or float-type service batteries, the charge voltage should be held at $2.25 \mathrm{~V} /$ cell, or $13.5 \mathrm{~V} / 12 \mathrm{~V}$ battery. This will limit the effects of overcharging and significantly increase battery life. This method is commonly referred to as "float voltage" charging at constant voltage, whereas in trickle charging the charging current is held constant and may result in overcharging the battery.

For cyclic use, where the maximum number of recharge cycles is of paramount importance, the battery on-charge voltage should be maintained below $2.4 \mathrm{~V} /$ cell or 14.4 $\mathrm{V} / 12 \mathrm{~V}$ battery. In applications where the batteries are used in both float and cyclic service, a charging voltage of between 2.30 and $2.45 \mathrm{~V}$ should be employed.

One of the simplest recharging circuits for small capacity batteries, a shunt-connected (parallel) zener diode, is shown in exhibit 4.10. This sircuit uses an inexpensive, low wul tuye zener diode (sized to meet the needed recharge voltage) in conjunction with a current limiting resistor.

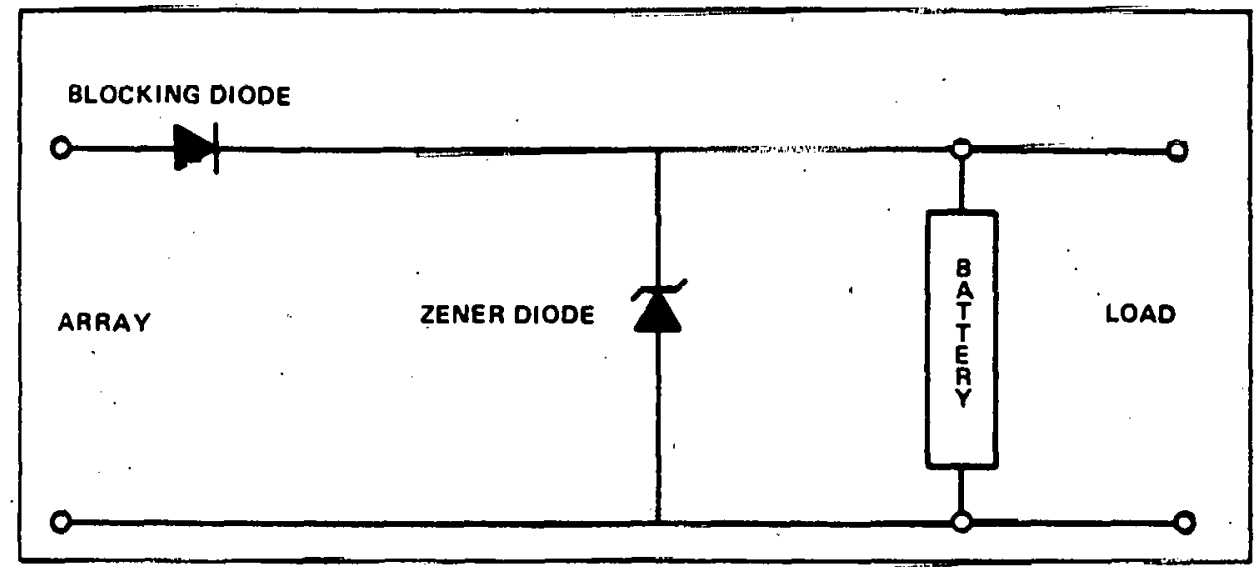

\section{Exhibit 4.10 Battery Charging Regulator with Zener Dissipating Excess Energy.}

Another popular circuit is the shunt (parallel connected) transistor regulator (see exhibit 4.11). This circuit allows a small zener diode to be used ior all battery sizes, since only the transistor base current flows through the zener diode.

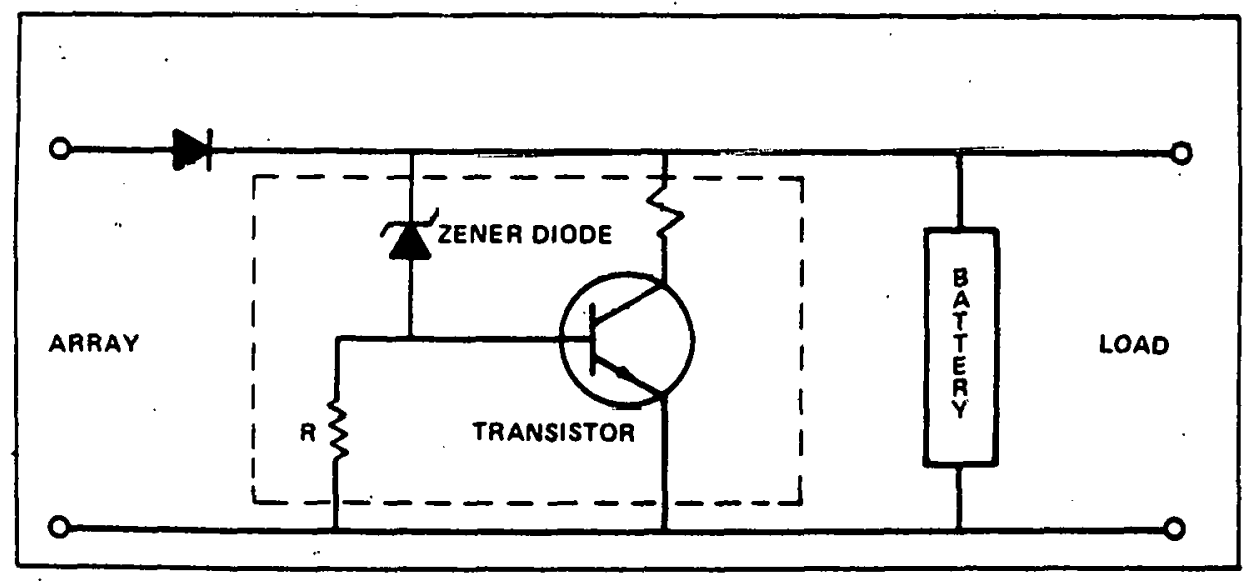

Exhibit 4.11 Battery Charging Regulator with Resistor Dissipating Excess Energy. 


\subsubsection{NICKEL-CADMIUM BATTERIES}

Nickel-cadmium batteries are designed for use in lightweight, portable applications which require long operating lifetimes and little or no maintenance. The electrodes (see exhibit 4.12) in nickel-cadmium cells undergo changes in oxidation state without any changes in physical state. The active materials involved, insoluble in the alkaline electrolyte, remain as solids during oxidation state changes. This results in two favorable properties of nickelcadmium cells: (1) because no chemical mechanism exists which would result in the loss of active material, the electrodes are long lived and (2) the cell potential is essentially constant throughout most of the discharge cycle. Oxidation is defined as the process of increasing the positive valence of or decreasing the negative valence of an element or ion. Electrons are removed from atoms or ions.

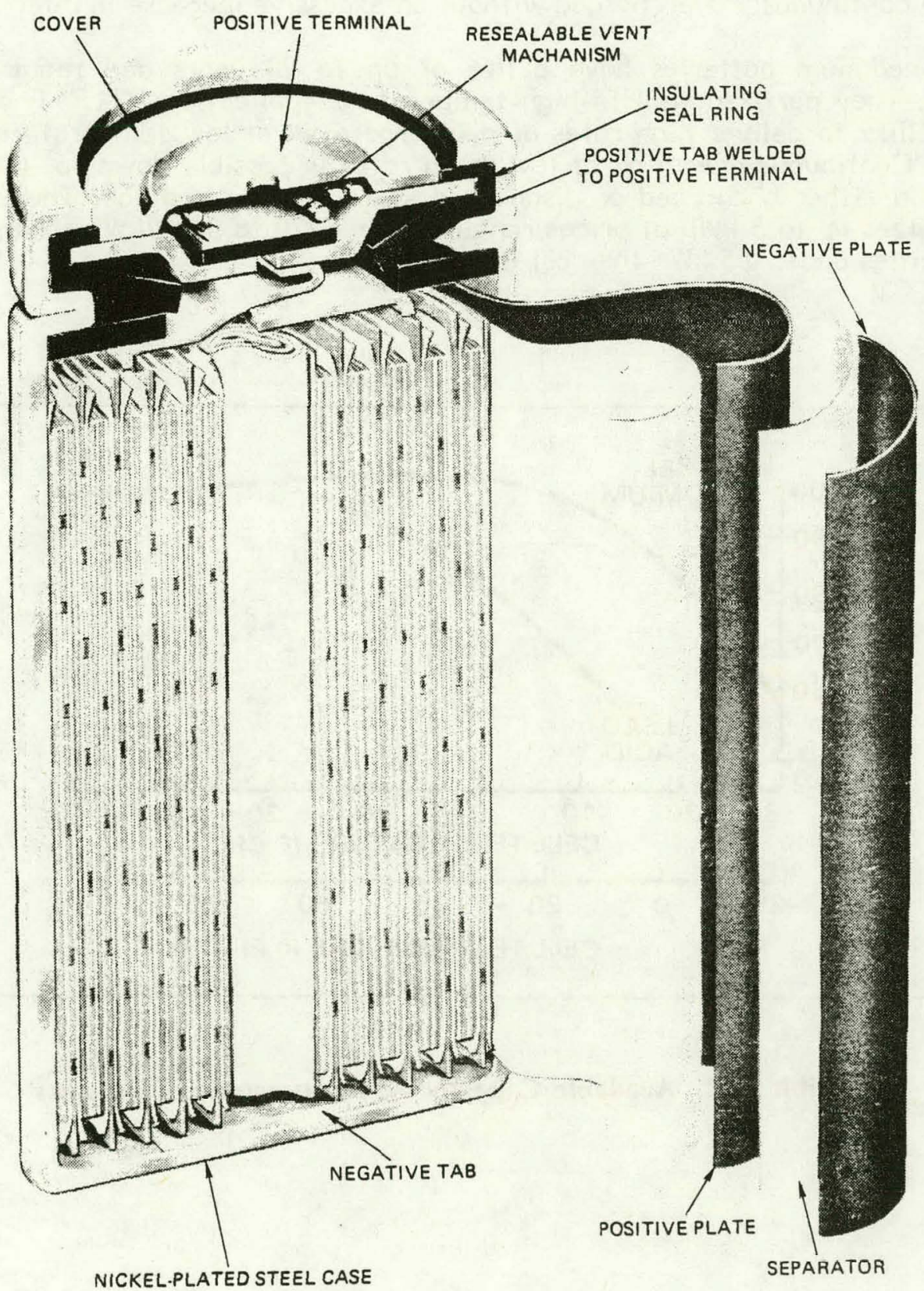

Exhibit 4.12 Cylindrical Sealed Cell Construction. 
Nickel hydroxide $(\mathrm{NiO}(\mathrm{OH}))$ is the active material in the positive plate. During discharge, the charged $\mathrm{NiO}(\mathrm{OH})$ goes into a lower valence state, $\mathrm{Ni}(\mathrm{OH})_{2}$, by accepting electrons from the external circuit. The negative plate, which is composed of cadmium, is oxidized to cadmium hydroxide and releases electrons to the external circuit. During charging, the reactions are reversed, as shown:

$$
\mathrm{Cd}+2 \mathrm{H}_{2} \mathrm{O}+2 \mathrm{NiOOH} \neq 2 \mathrm{Ni}(\mathrm{OH})_{2}+\mathrm{Cd}(\mathrm{OH})_{2}
$$

These batteries do not vent hydrogen because the cells are constructed so the oxygen generated at the positive plate migrates to the negative plate, recombining with the hydrogen. The oxygen acts as a chemical short circuit within the cell, enabling the sealed battery to be continuously overcharged without an excessive increase in internal pressure.

Nickel-cadmium batteries have a life of up to 20 years and require virtually no maintenance. They perform well in high-temperature applications (115\% $\mathrm{F}$ contiruous) and retain the ability to deliver high rates of discharge even at low temperatures, as shown in exhibit 4.13. Continuous operation at low drain rates is possible down to $-40^{\circ} \mathrm{C}$. They also may be left in either a charged or discharged state without dumuye. These batteries are available in sizes up to $5 \mathrm{kWh}$ at prices ranging from $\$ 270$ to $\$ 320 / \mathrm{kWh}$ for over $1 \mathrm{kWh}$. For energy capacities under $0.5 \mathrm{kWh}$ the cost is $\$ 450 / \mathrm{kWh}$.

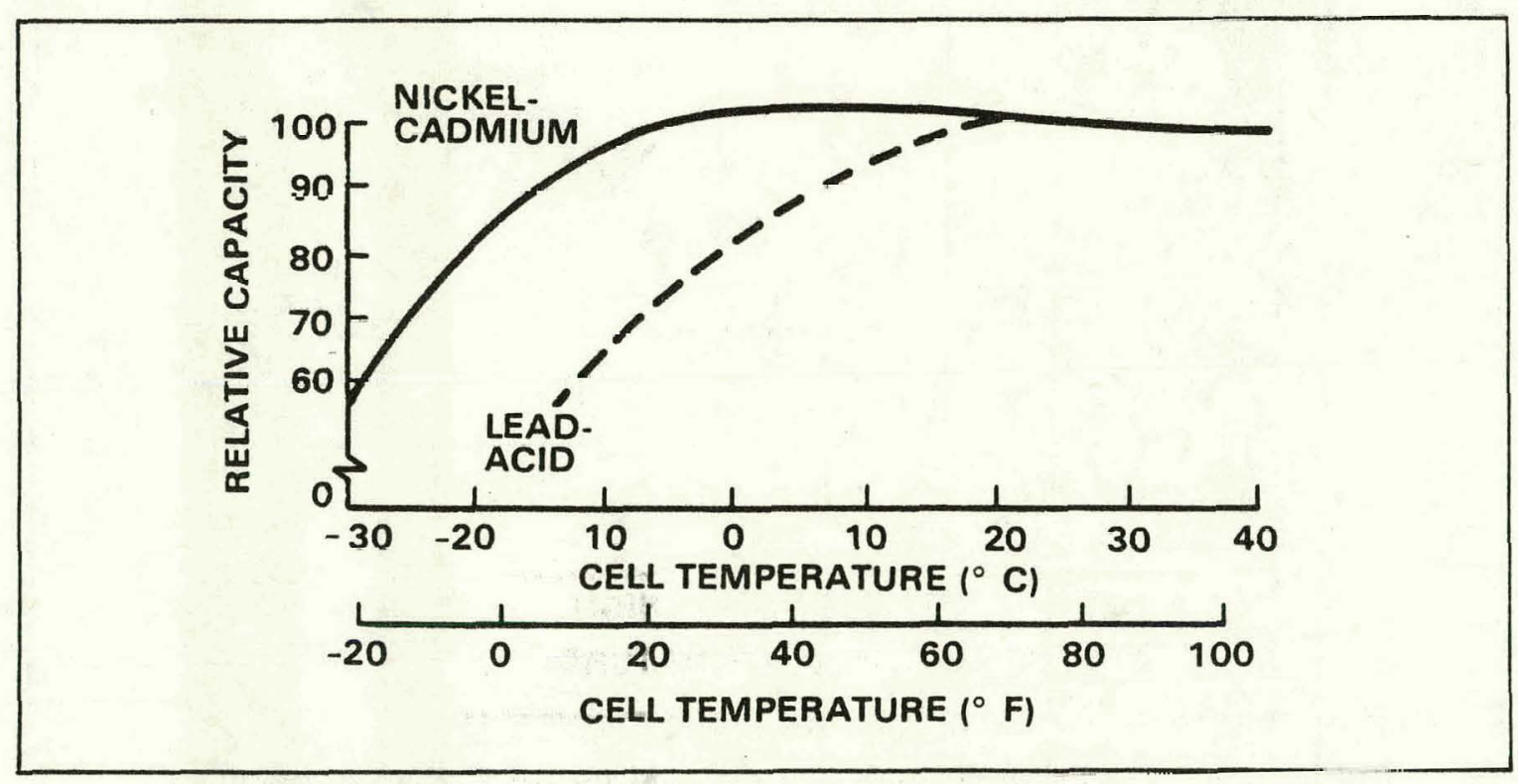

Exhibit 4.13 Available Capacity vs. Temperature of Batteries 


\subsection{FAILURE MECHANISMS}

Failures generally result from either an internal short circuit or an excessive loss of electrolyte. Short circuits are generally created when two plates of opposite polarity, or their attached hardware, make physical contact. This results in the charging current passing through the partial short, forcing the cell voltage to zero almost immediately. The performance of nickel-cadmium batteries is not affected by small losses of electrolyte. However, moderate electrolyte loss will cause slight capacity degradation, which increases to almost complete loss of capacity when large amounts of electrolyte are lost.

Both internal shorts and excessive electrolyte loss are related primarily to time of usage and battery temperature during this period of time. Battery temperature is the most significant parameter in the life expectancy of the battery as high temperatures accelerate performance degradation by increasing self-discharge and reducing charge acceptance (input-output efficiency) as shown in exhibit 4.14. Prolonged exposure to high temperatures will also increase the decomposition of the separator material between the positive and negative electrodes, causing internal shorts. The performance of nickel-cadmium batteries at temperature extremes is superior to that of lead-acid batteries, making them the logical choice for high-temperature applications.

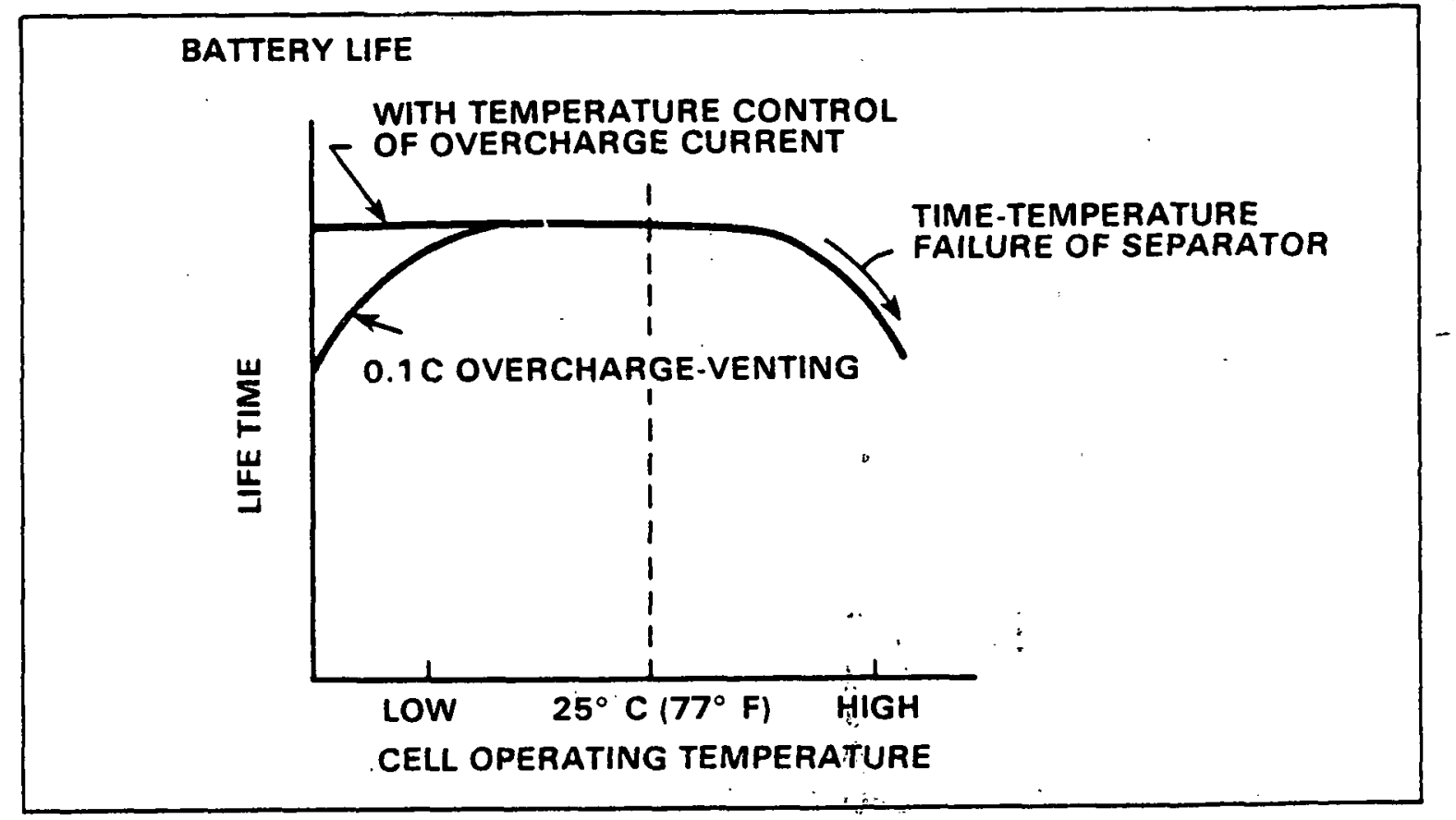

\section{Exhibit 4.14 Life Expectance Relationship òf Nickel-Cadmium Batteries vs. Operating Temperature.}

Nickel-cadmium botteries which are subjected to a repetitive shallow depth of discharge tend to exhibit an apparent loss of capacity, commonly referred to as "memory loss." This problem can be mitigated by deep discharging of the battery and then recharging. The "memory" is thus erased and the original battery capacity restored. This memory problem is exhibited in sintered cell nickel-cadmium batteries but not in cells employing pocket plate construction. 
Nickel-cadmium batteries can tolerate from 0.05 to $0.10 \mathrm{C}$ overcharge for extended periods of time without damage or performance degradation (where $C$ is the battery capacity in Ah). For example, a 220 Ah battery could be recharged with a constant current circuit (see exhibit 4.15) with a maximum charge current of 11 to $22 \mathrm{~A}$. This slow charging rate has high reliability and low cost due to its simplicity, and will tend to minimize cell temperature rise and internal pressure during the recharge.

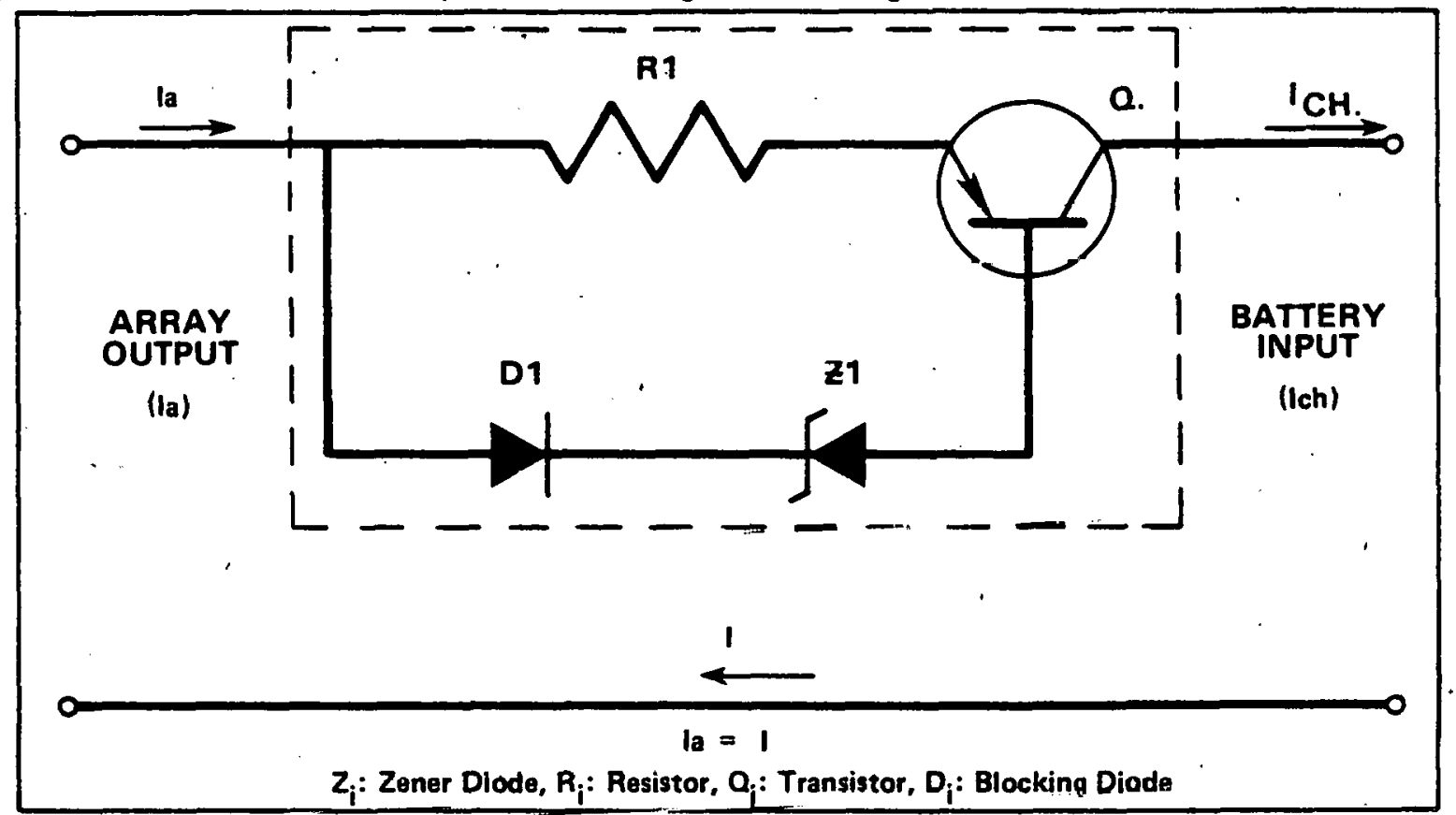

\section{Exhibit 4.15 Constant Current Charging Circuit.}

\subsection{RECHARGING MULTIPLE BATTERY STRINGS}

When recharging series-connectert batterics to obluin higher system voltages, the possibilily of slight differences in capacity between the individual cells exists. When a single voltage suurce is connected across the entire battery string, the same current will flow through all the cells. Due to differing characteristics of batteries, some may overcharge while others remain slightly undercharged. This results in both underutilizing the capocity of the batteries and prematurely aging them. To minimize these adverse effects, installations near hot spots (as temperature affects both cell voltage and capacity) and charging the batteries' string in more than $24 \mathrm{~V}$ battery groups (see exhibit 4.16) should be avoided.

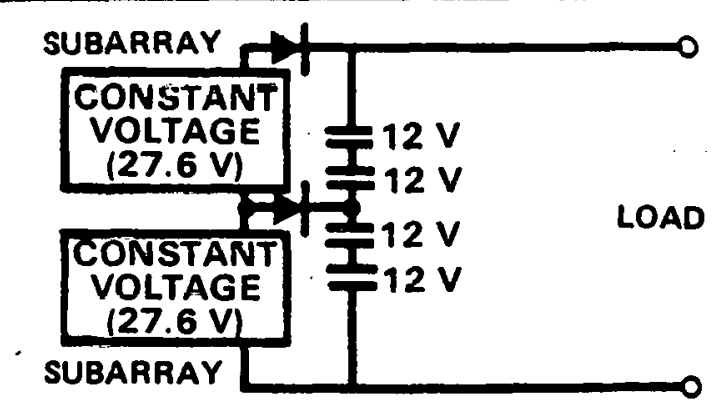

Exhibit 4.16 Charging High Voltage Battery System. 
When connected in parallel, the current from the array will tend to divide equally between the batteries. If batteries of unequal capacity are being recharged, the current tends to divide between the batteries in the ratio of their capacities (due to internal resistance differences).

\subsection{SAFETY PROBLEMS}

Because batteries are hazardous devices and cannot be switched off, the potential hazard of DC electrical shock exists. A few of the considerations are as follows.

Shorting the output terminals by dropping a wrench across them can cause currents high enough to melt metal conductors and result in severe burns.

Lead-acid batteries also pose a hazard"due to the acid electrolyte. Plastic squeezebottle eye-wash devices, sodium carbonate or similar neutralizing agents, and protective clothing (e.g., rubber gloves and aprons) should be kept on hand when servicing lead-acid batteries. Similarly, nickel-cadmium batteries contain a caustic base electrolyte. A mild acidic solution such as boric acid or vinegar should be kept handy as a neutralizing agent.

Battery storage systems generate hydrogen (which may explode) and other potentially dangerous gases such as stibine $\left(\mathrm{SbH}_{3}\right)$ or arsine $\left(\mathrm{AH}_{3}\right)$. A ventilation system and catalytic battery caps should be included in the housing design. Catalytic caps are currently available from various distributors for approximately $\$ 3.75$ per cap.

There are existing standards which are more compulsory that ensure safety.

\subsection{SUMMARY}

Exhibits 4.17 and 4.18. provide a summary for the battery types that have been presented. Since system costs are directly related to the load requirements, current loss due to battery inefficiency and self-discharge can be very expensive. For this reason, the SLI lead-acid or motive-type batteries used in forklifts and golfcarts are generally unacceptable from the standpoint of cost and reliability.

Float-service, lead-acid batteries featuring lead-acid with calcium grid or pure lead grids are the most suited for general PV use. This is due to their very low self-discharge (typically 10 to 15 percent per year) and ti.eir high efficiency (80 percent electricai to chemical energy conversion). In extremely cold conditions, nickel-cadmium cells can offer advantages in increased capacity which may outweigh their higher costs. During electrical to chemical energy conversion, the batteries rêceive charge; and during chemical to electrical energy conversion, the batteries dissipate $e_{i}^{*}$ charge.
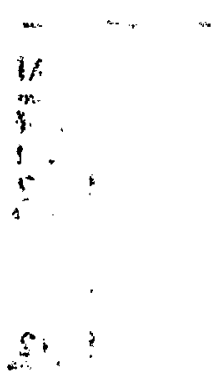


\begin{tabular}{|c|c|c|c|c|}
\hline \multicolumn{2}{|c|}{ TYPE } & \multirow{2}{*}{ 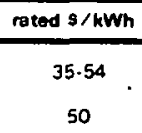 } & \multirow{2}{*}{$\begin{array}{c}\text { rated Wh/lb } \\
15.21 \\
16.18\end{array}$} & \multirow{2}{*}{$\begin{array}{l}\text { CHARACTERISTICS } \\
\text { self-discharge, short tife under deep discharge } \\
\text { ions. }\end{array}$} \\
\hline su & $\begin{array}{l}\text { AUTOMOTIVE } \\
\text { OIESEL }\end{array}$ & & & \\
\hline \multirow{2}{*}{$\begin{array}{l}\text { MOTIVE } \\
\text { POWER }\end{array}$} & $\begin{array}{l}\text { GOLF CART } \\
\text { FORK LIFT }\end{array}$ & 45.60 & 30.35 & 250 cycle life. high self-discharge. \\
\hline & $\begin{array}{l}\text { FOAK LIFT } \\
\text { LONG LIFE }\end{array}$ & $160-220$ & $7: 11$ & $1, \infty 00-2,000$ eycle life. high self-discharge. \\
\hline \multirow{2}{*}{$\begin{array}{l}\text { FLOAT } \\
\text { SEAVICE }\end{array}$} & $\begin{array}{l}\text { LONG LIFE } \\
\text { CALCIUM-GRID }\end{array}$ & $200 \cdot 240$ & 7.10 & $1,500 \cdot 2,000$ cycle life. low self-discharge. \\
\hline & $\begin{array}{l}\text { PURE } \\
\text { LEAD } \\
\text { GRID }\end{array}$ & $80 \cdot 130$ & $14-18$ & Low self-discharge, low maintenance. \\
\hline \multicolumn{2}{|c|}{ SEALED FLOAT SERVICE } & 140 & $14 \cdot 18$ & Very low maintenance. low self-discharge. \\
\hline \multicolumn{2}{|c|}{ NICKEL-CAOMIUM } & מט 270 & $10-15$ & $\begin{array}{l}\text { Very low discharge. good low temperature performance. } \\
\text { long life. "memory" problem. }\end{array}$ \\
\hline
\end{tabular}

Exhibit 4.17 Summary of Lead-Acid and Nickel-Cadmium Batteries.

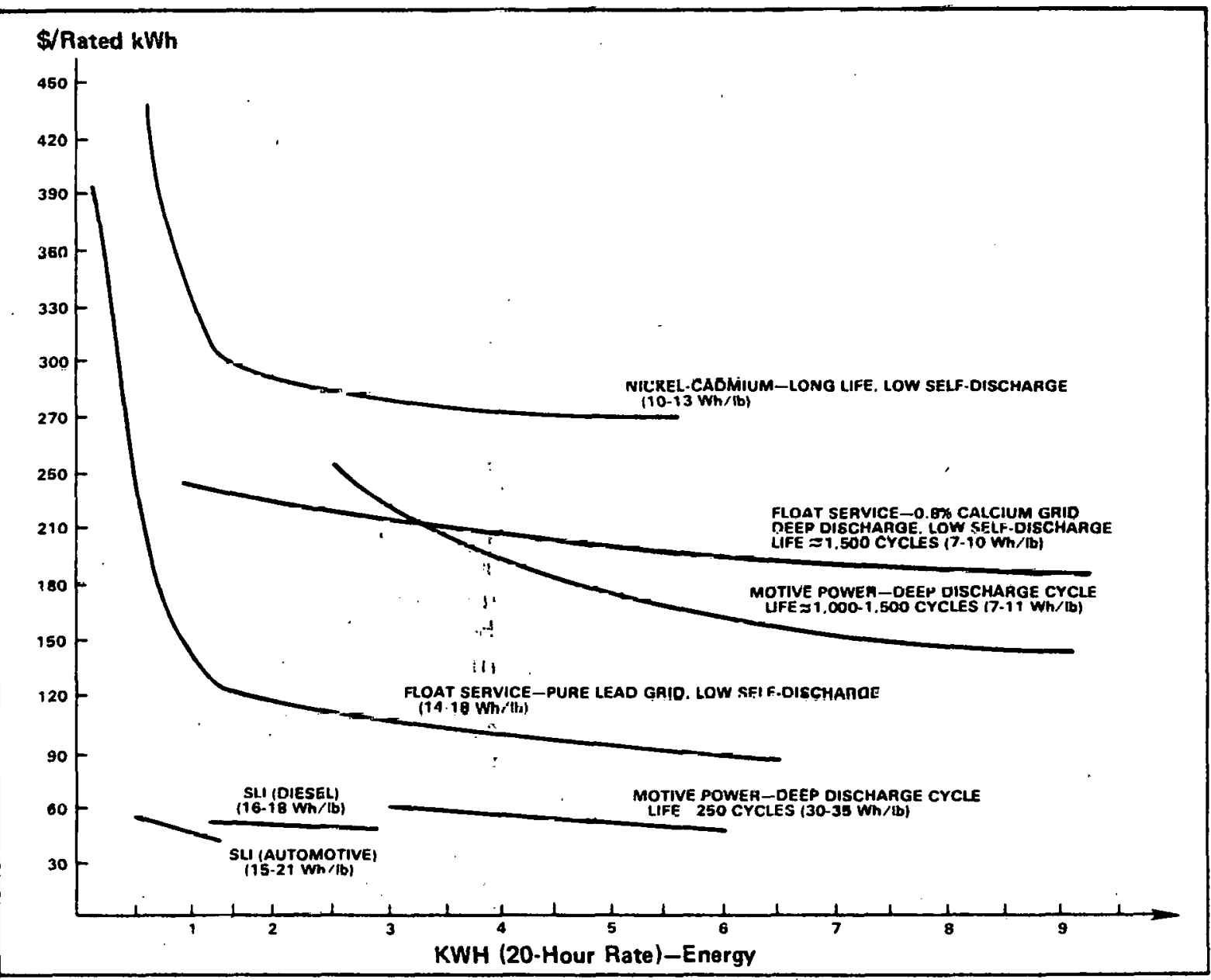

Exhibit 4.18 Comparative Battery Cost. 


\section{SECTION 5 \\ POWER CONDITIONING}

\subsection{POWER CONDITIONING}

The output from the solar array must match the requirements of both the load and the batteries. The power conditioning subsystem matches the load, batteries, array, and possible back-up energy by regulating the DC voltage and current input to the battery subsystem or converting the DC output of the array into AC at a specified voltage, if needed, or both. This section explains the basic operation of each type of power conditioning unit and its function within the electrical power system.

\subsection{SELF-REGULATED PV POWER SYSTEMS}

For the smaller, cost-effective power applications (normally under $2 \mathrm{~kW}$ capacity), the direct connection (through a blocking diode) of the orray to the storage system and then to the load may prove to be the most cost-effective and require the least maintenance (refer to exhibit 5.1). Direct electrical connection of the array to the battery system without regulation is advisable only when the peak output current of the array is less than 5 percent of the charge capacity of the batteries (amp-hour rating) in the system (charge equals current $x$ time).

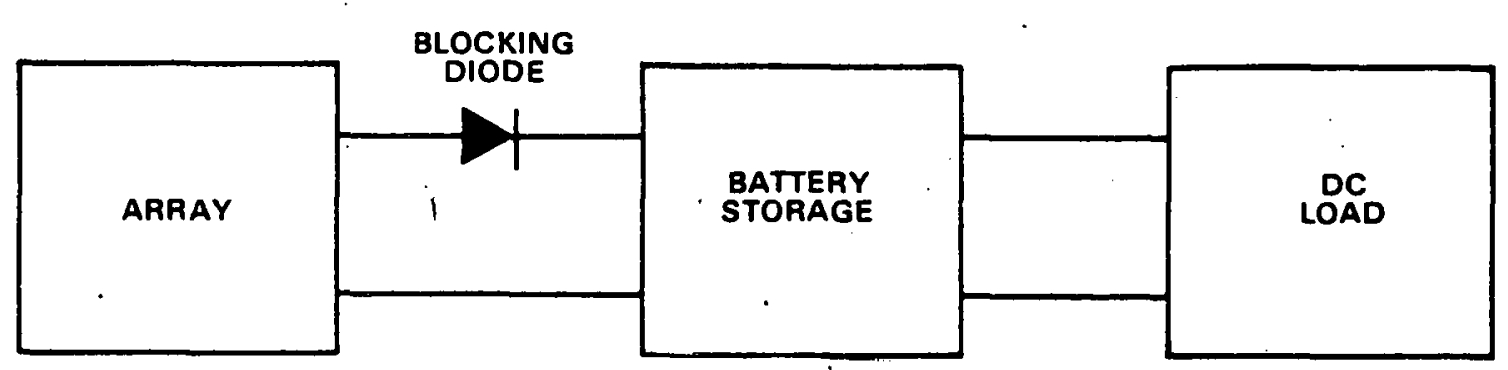

Exhibit 5.1 Self-Regulated PV. System.

The operation of the system shown in exhibit 5.1 is as follows.

1. During periods of insolation, the batteries are charged by the power produced by the PV array at a voltage that causes current to flow from the array into the battery storage system while also supplying the load. When the voltage of the battery storage system equals that of the array (less the initial voltage drop across the blocking diode), current flow into the storage system would stop, with the batteries being at a full state of charge. (NOTE: A difference of potential is required for current to flow.) Voltage equals potential in an electrical energy system and the voltage drop (loss) across the blocking diode is basically current $x$ diode resisturice.

2. A battery storage system continually supplies load requirements. It should be recognized that this system design (see exhibit 5.1) places specific constraints on the selection of the PV array current and voltage operating conditions, resulting in the array operating at other than the maximum power point. These constraints 
are centered around the charging voltage requirements of the battery storage system. The voltage range for a $12 \mathrm{~V}$ lead-acid battery under charge varies from $12.8 \mathrm{~V}$ (at 60 percent discharge) to $14.4 \mathrm{~V}$ (at full charge). To transfer the maximum power from the PV array to the storage system, the voltage-operating point for the array should be approximately $14.40 \mathrm{~V}$ plus $0.75 \mathrm{~V}$ (to account for the voltage drop across the diode) thus $15.15 \mathrm{~V}$. The manufacturer's rated temperature of $27^{\circ} \mathrm{C}$ would cause a different curve-displaced along the voltage axis, as shown in Exhibit 5.2. As shown in exhibit 5.2, for a slight increase in cell voltage above the nominal array operating voltage, cell current will decrease rapidly limiting the charging current. The I-V curve used corresponds to the array's character istics at its nominal operating temperature.

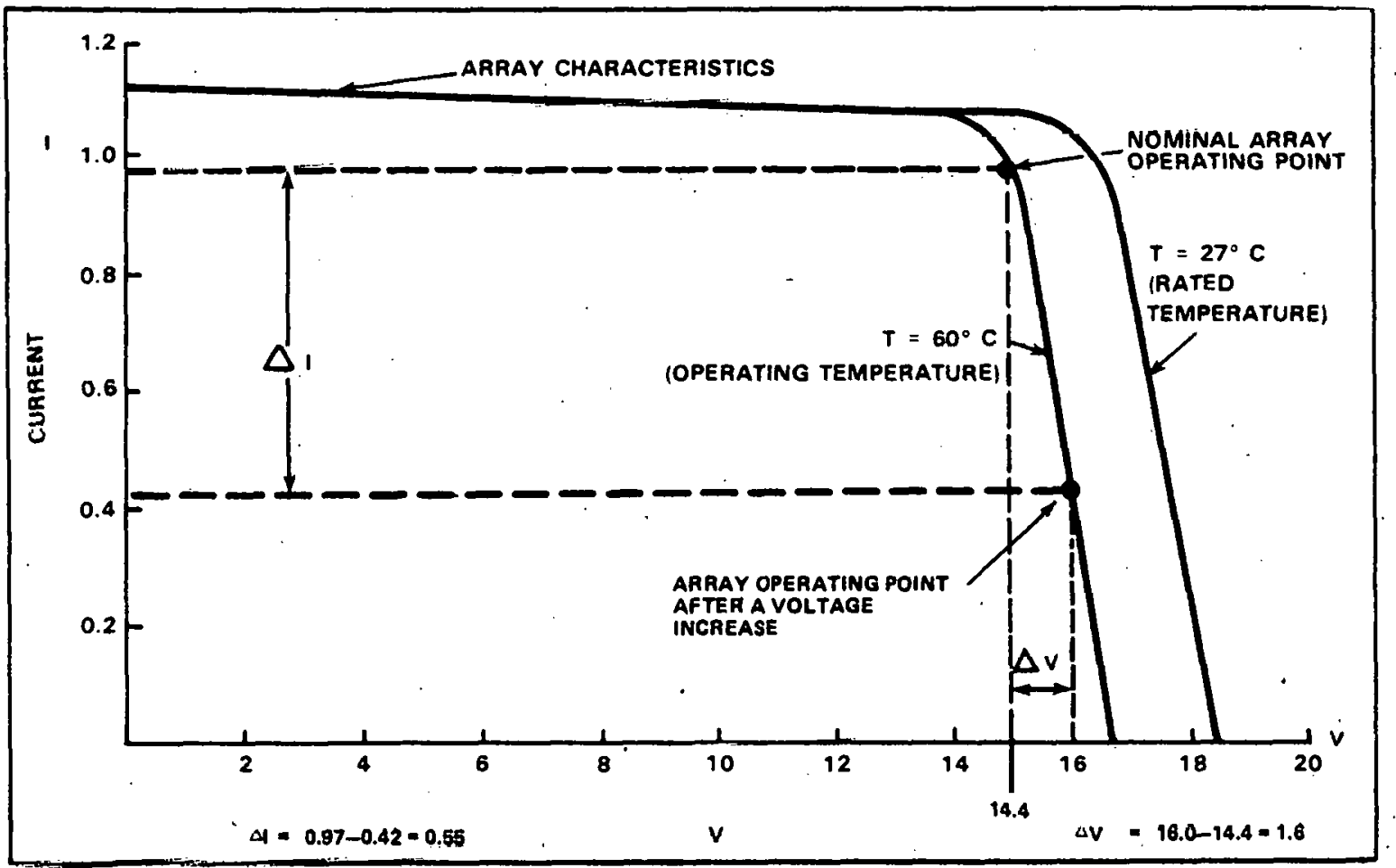

Exhibit 5.2 I-V Curve of PV Module Fxhibiting Self-Regulation.

The possibility of battery gas production (and losses in electrolyte) are higher for a self-limiting system than for a system utilizing an active voltage regulator-battery charger. This is due to the variations in insolation causing instantaneous changes in array voltage and current, resulting in the possibility of overcharging the battery storuge system. Setting the array operating polnt less than that required for maximum charging capability results in a larger and more costly battery storage system to meet the same load.

For the application of directly charging a battery storage system of $12 \mathrm{~V}$, PV modules must be connected to produce approximately $15.15 \mathrm{~V}$ at the designed operating insolation and temperature. A self-regulated system usually operates away from the maximum power point, thereby wasting energy and array capability. Larger systems would use an active device to maintain maximum power utilization. 


\subsection{VOLTAGE REGULATED PV POWER SYSTEMS}

Most PV systems utilize a voltage regulator, either in parallel or in series with the PV array, the storage system, and laad (see exhibit 5.3). PV power systems are normally designed to recover quickly from worst-case load and weather conditions during their total useable life span. Under average weather conditions, this results in the arrays having excess power capability under normal load conditions, especially at the beginning of their lives. To regulate the voltage within required limits to prevent battery overcharge and outgassing, a voltage regulator must dissipate the excess available power.

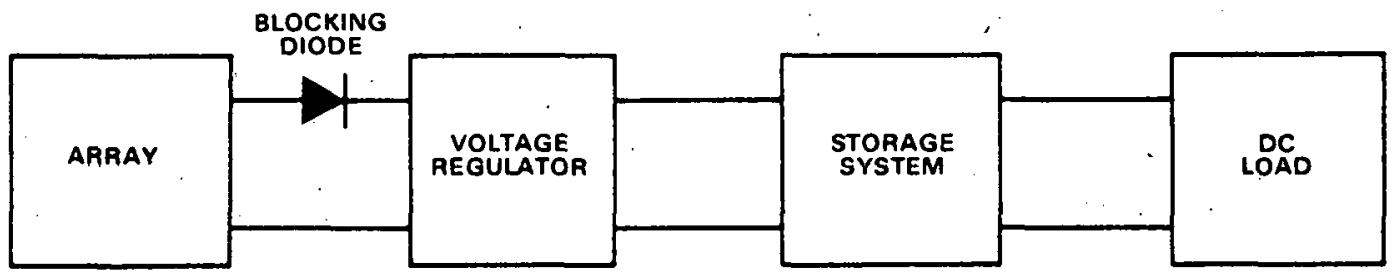

\section{Exhibit 5.3 PV System Utilizing Voltage Regulator.}

There are two types of voltage regulators available as off-the-shelf products. Seriestype voltage regulators utilize a series transistor that electrically blocks the array when the battery reaches a full charge voltage and conducts when battery charging is required. A shunt-type regulator uses a transistor to shunt the excess current to the ground. Series regulators consume power at all times. Shunt regulators only dissipate that power not required by the storage system/load combinations. Exhibits 5.4 and 5.5 show simplified designs of series and shunt (parallel connected) regulators.

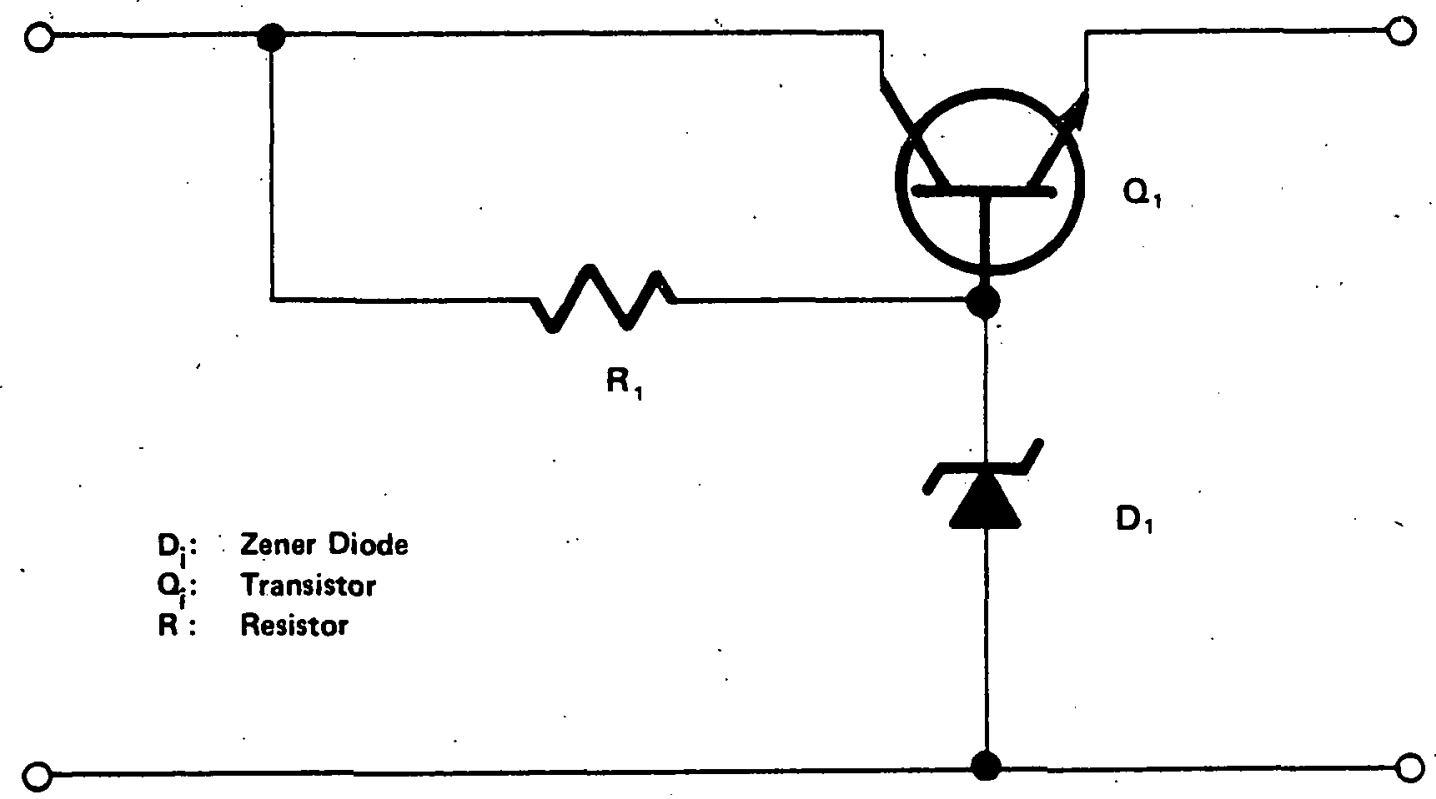

Exhibit 5.4 Series Regulator-Basic Design 


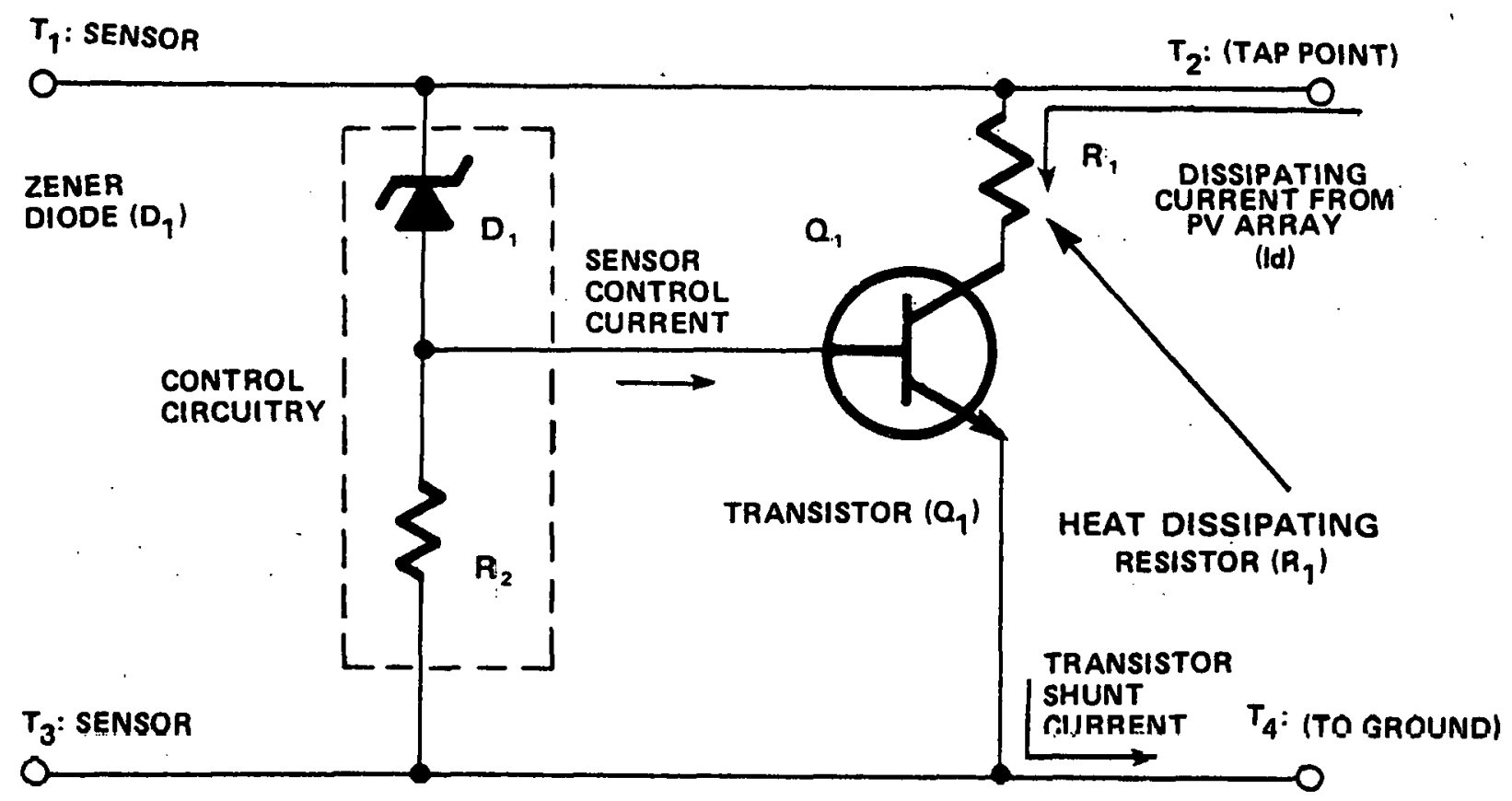

Exhibit 5.5 Shunt Regulation-Simple Design.

Series regulators operate by applying a set voltage to the transistor base, keeping the transistor output at a constant voltage over a large variation in current. All the load current passes through the regulator transistor in a series regulator design. This results in approximately a $1.2 \mathrm{~V}$ drop across the regulator transistor output, and a consequent loss in power (e.g., with a $10 \mathrm{~A}$ current, $\mathrm{P}$ loss $=10 \mathrm{~A} \times 1.2 \mathrm{~V}$ drop $=12 \mathrm{~W}$ loss), where power equals $\mathrm{I} \times \mathrm{V}$.

Exhibit 5.5 shows a simplified design of a shunt regulator. This type of regulator dissipates the excess power to ground. When the load utilizes all of the PV produced power, the shunt regulator consumes no power. Based on the output voltage, the regulator "shunts" current through a regulating transistor to keep the output voltage constant.

An alternative to using a full-shunt regulator is the partial-shunt regulator. Smaller heat dissipating elements and heat sinks are required, so the weight, heat management, and cost problems associated with these dissipating elements and accompanying power transistors are minimized. The partial-shunt regulator, which is connected ocross part of the array, shunts part of the array output at no load conditions; and is fully turned off at fullload, maximum operating temperature, and at the end of the designated life of the system. This type of shunt regulator dissipates less than one-half of the power dissipated in a fullshunt regulator (see exhibit 5.6).

For larger power systems (over several hundred watts), several linear-partial-shunt regulators can be employed, each of which regulates a separate portion of the array (see exhibit 5.7). In this system, the regulators would be operated linearly between saturation and cutoff, one at a time in a voltage-dependent sequence. At full load all the shunt regulators. are off. At no load all regulators are in saturation, except one which is either in saturation or in its linear operating region, depending upon the array temperature. Typical costs for regulators are in the range of $\$ \mathrm{I} / \mathrm{W}$. Linear operation refers to the output voltage function, cutoff refers to zero current, and saturation refers to maximum current. 


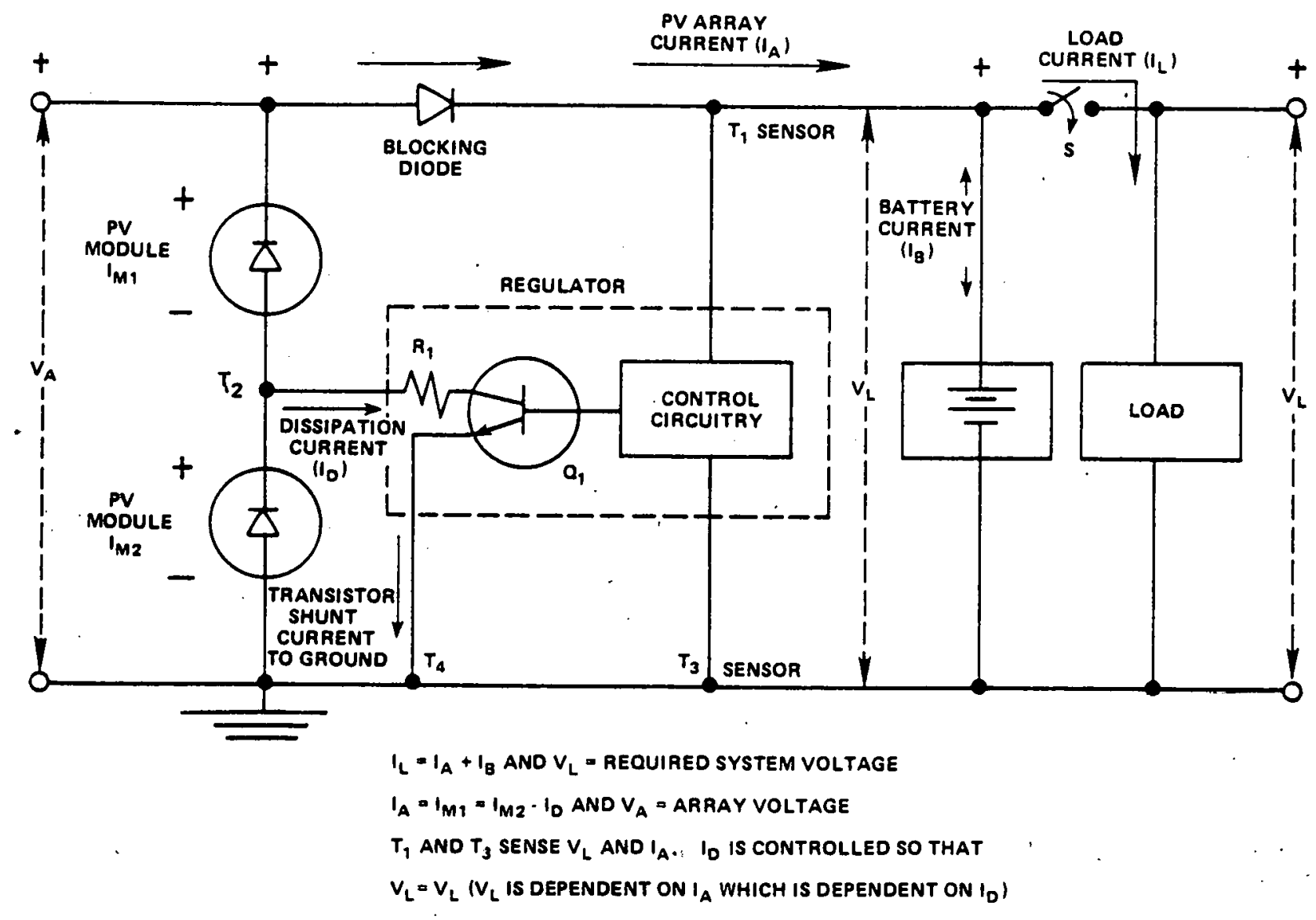

Exhibit 5.6 Partial Shunt Regulation $\left(T_{2}\right.$ on Im )

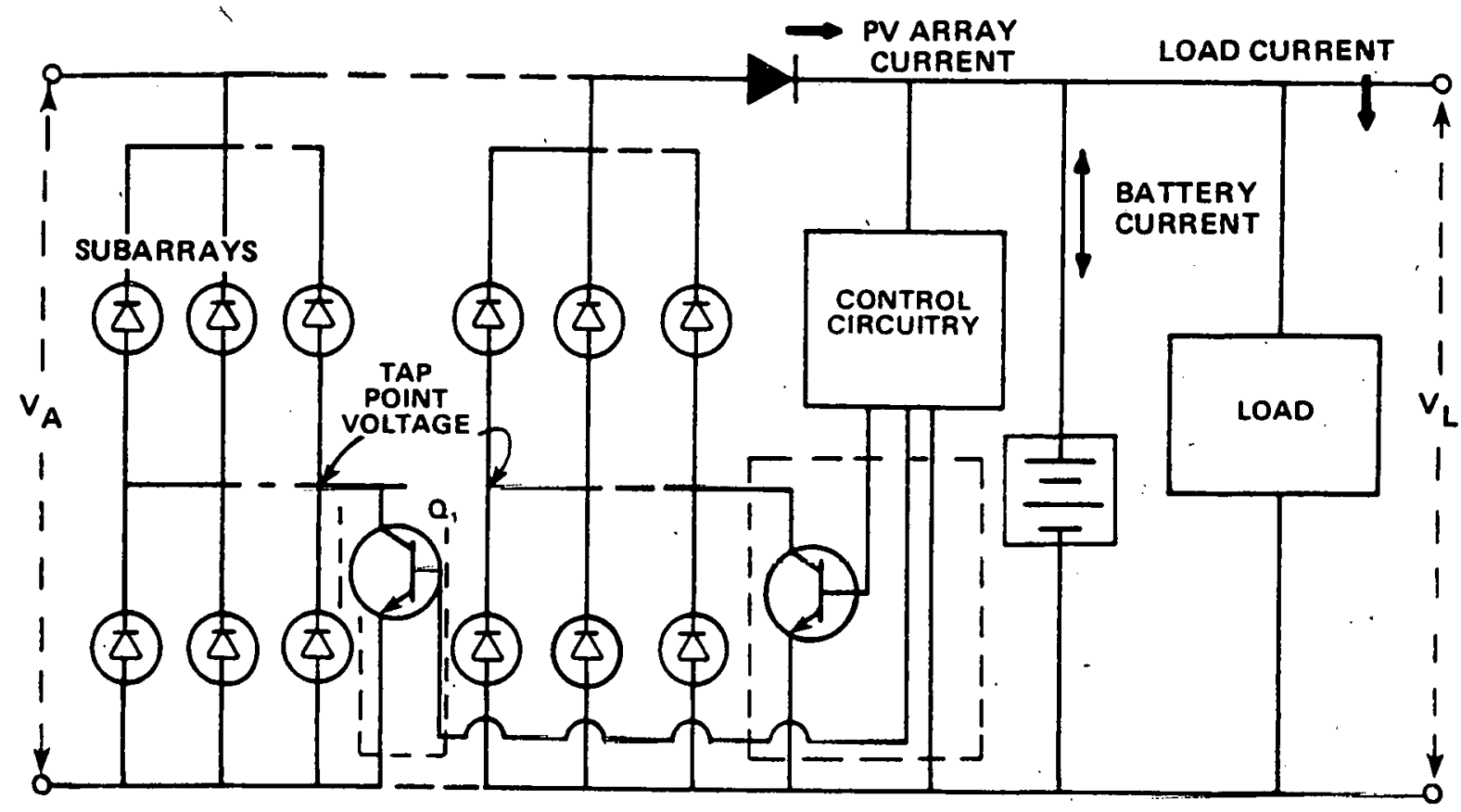

Exhibit 5.7 Partial Shunt Regulators for Multiple Subarrays. 


\subsection{SWITCHING REGULATORS}

Switching regulators are complex, classical electronic devices (see exhibit 5.8) and have a distinct advantage over series or shunt regulators: low power dissipation in the main control device (especially at high power levels). The amount of current dissipated from the array is accomplished by electronic switching, feedback, and control. The transistor is the main element. Diodes, capocitors, inductors, and resistors are also employed, and seriesparallel connections are used. The series part is either saturated or off, and has a low forward voltage drop to neutral when conducting load current and only leakage current flowing when it is not conducting. Power dissipation is small, resulting in high throughput efficiencies. To avoid stability problems, switching regulators must be employed in conjunction with a peak power tracker. They are not used in low power applications. Also, they are not currently available as off-the-shelf items. Series-parallel and delta-star connections are used.

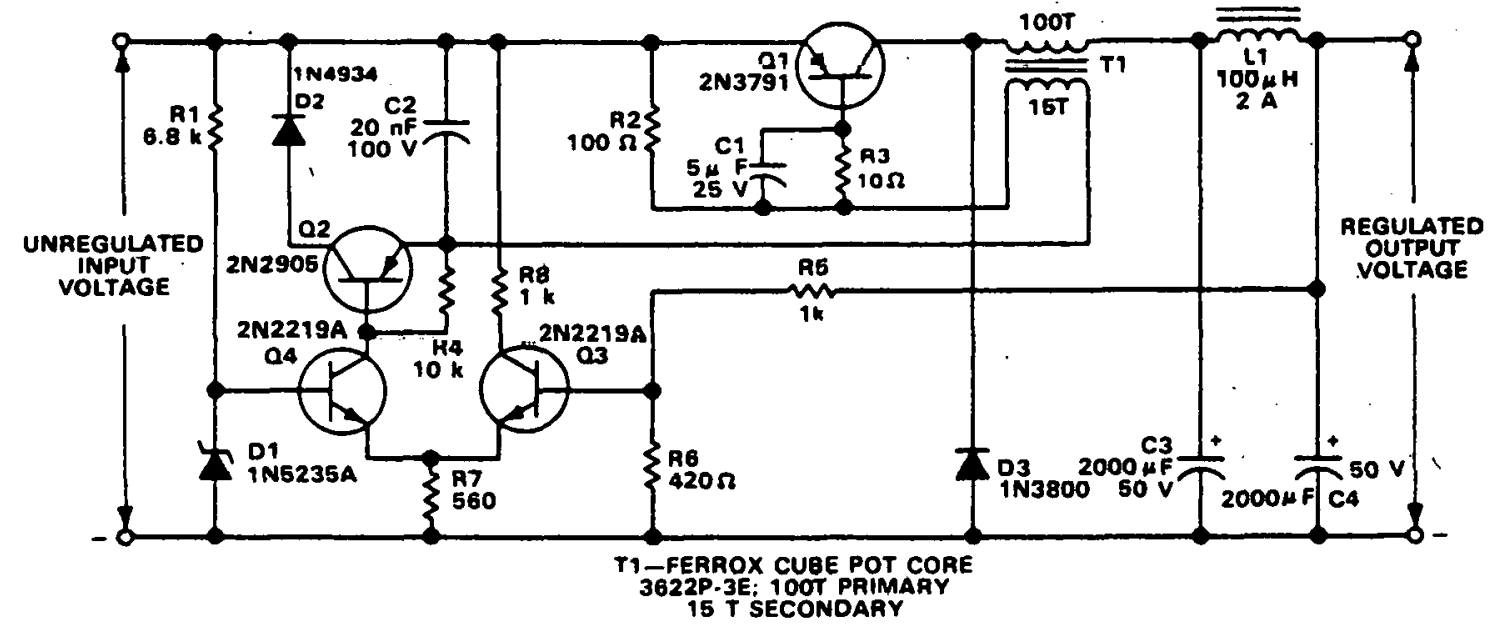

Exhibit 5.8 Electronic Switching Regulator

\subsection{INVERTERS}

An inverter changes $D C$ voltage into $A C$ voltage (see exhibit 5.9). Motor-generator sets, as well as other rotating equipment, have beeti used as inverters. Duc to the maintenance problems associated with mechanical devices, only static, solid-state (electronic) inverters are considered here.

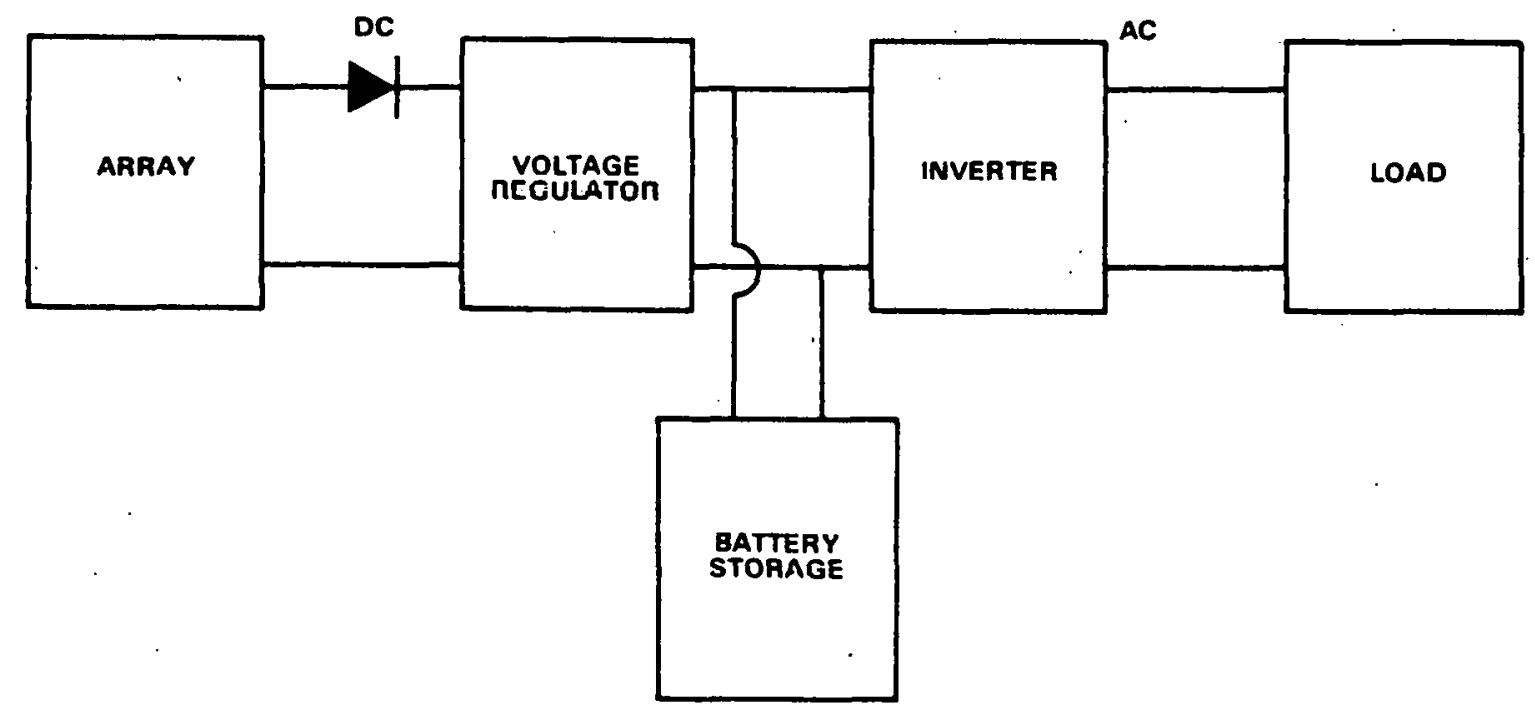

Exhibit 5.9 Regulated System Providing AC Output. 


\subsubsection{SOLID STATE INVERTERS}

Power handling capability and efficiency make electronic switching-type inverters the most commonly used in power conditioning. The main circuit elements are alternately switched electronically between their on and off states. A simplified, single-phase bridge configuration is shown in exhibit 5.10. Electronic switching causes an alternating current, which is roughly equivalent to the pure sine wave of an al ternator.

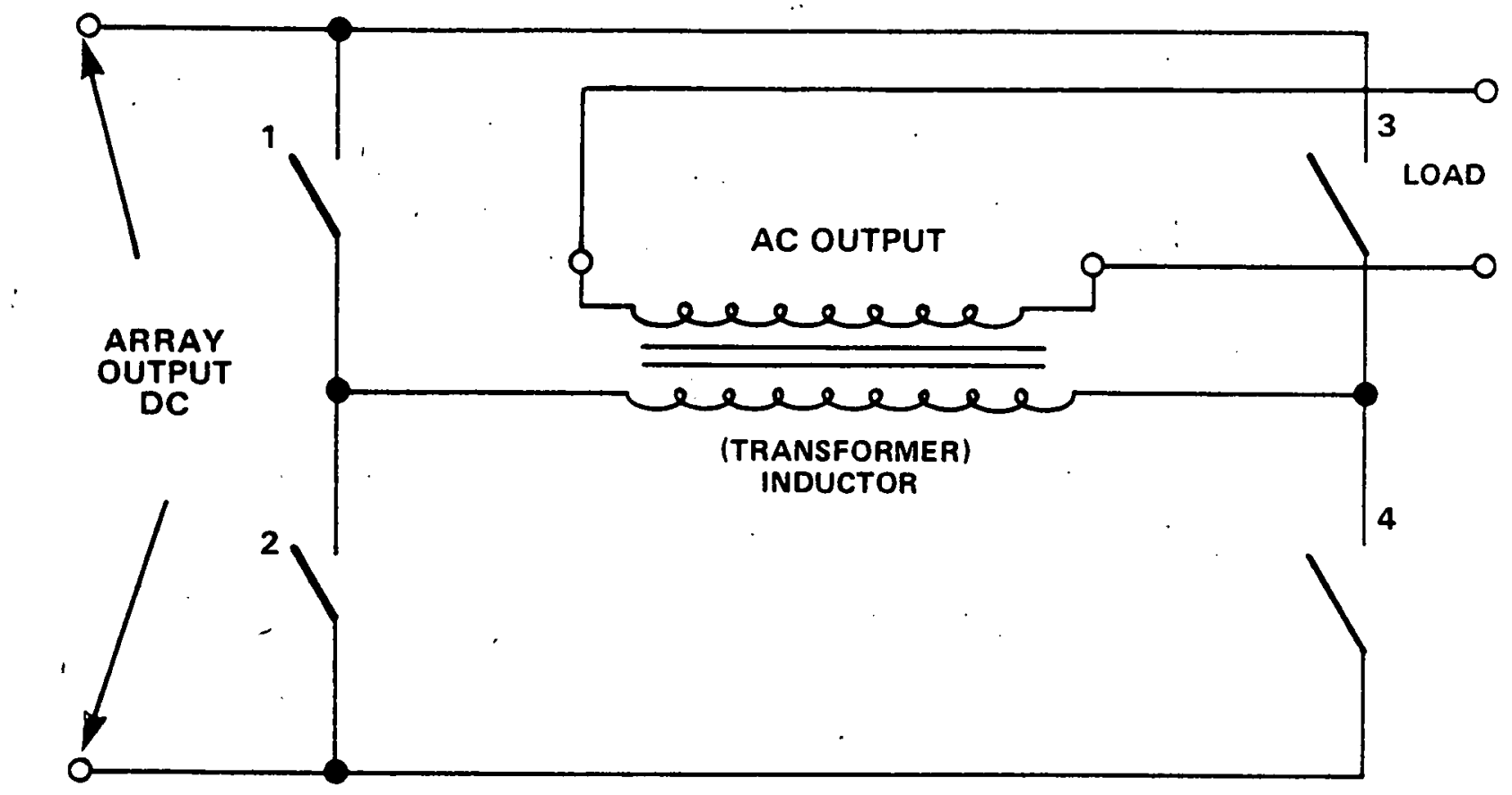

\section{Exhibit 5.10 Conceptual Inverter Design.}

Conceptually, switches 1 and 4 are initially closed while switches 2 and 3 are open. This causes a direct current to flow through the transformer winding. After 8.3 miliseconds (for $60 \mathrm{~Hz} \mathrm{AC}$ operation), switches 1 and 4 are opened and 2 and 3 are closed, causing current to reverse direction in the transformer winding. This cycle is then repeated ot the desired frequency to produce the AC output. In actual inverters, the switches are replaced by transistors or static controlled rectifiers. Rectifiers convert $A C$ vol tage to $D C$ vol tage.

Most low power inverters (less than $1 \mathrm{~kW}$ ) utilize transistors as active electronic switching elements (see exhibit 5.11). Transistors are quiet, reliable, exhibit a low forward voltage drop to the base, and negligible leakage currents. Unfortunately, they require a relatively high control power. This effect can be mitigated by utilizing Darlington transistor configurations, although these devices are generally rated for lower power applications and are also more expensive. Typical efficiencies of commercially available DC to $A C$ inverters range from 65 to 75 percent for $50 \mathrm{~W}$ units, costing $\$ 80$ to $\$ 140$, with less than 3 percent output harmonic distortion. A harmonic is an $A C$ voltage whose frequency is some integral multiple of a fundamental frequency. 


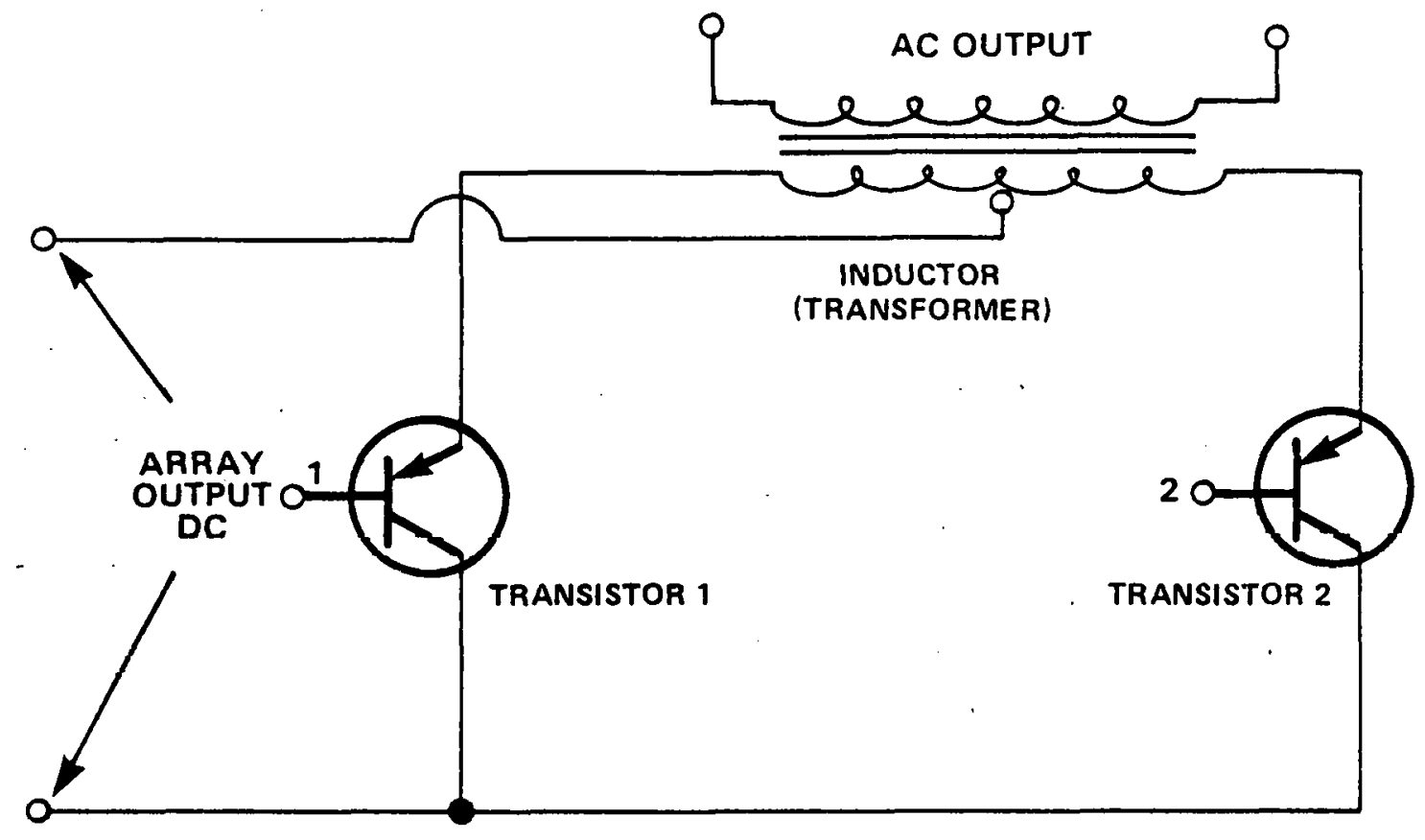

Exhibit 5.11 Actual Transistor Switching Inverter

Higher power inverters (greater than $1 \mathrm{~kW}$ ) generally use static controlled rectifiers (SCR's) as the electronic switching elements. Very little control current, as compared with transistor circuits, is needed to switch the SCR into its "on" state. Unlike transistors, the current through the SCR must go to zero before the device can be turned off. The resulting $A C$ voltage is not exactly equivalent to the transistor circuit $A C$ voltage.

Inverter circuits can be divided into the two broad categories of line or seltcommutated. (Commutation is the switching of current flow from one branch of a circuit to another.) Line-commutated inverters rely on control voltage from a fixed $A C$ voltage bus to provide the electricity, (e.g., to turn off SCR's). This type of circuit is usually used in conjunction with utility line applications. Self-commutated inverters, however, rely on an internal control circuit to electronically switch the active elements on and off. Because transistors can be biased off by an external voltage, transistorized inverters are inherently self-commutating. SCR inverters can be designed for self-commutated service by the addition of internal circuits across the SCR bridge legs. Since output power is reduced, these circuits lead to higher costs, and lower efficiency. A summary of the utility of SCR and transistor inverter characteristics is tabled in exhibit 5.12. 
SCR

- usually line-COMMUTATED

- HIGH POWER, $1 \mathrm{~kW}$

- HIGHER EFFICIENCY

\section{TRANSISTOR}

- SELF-COMMUTATED

- LOW POWER, $1 \mathrm{~kW}$

\section{Exhibit 5.12 The Utility of SCR and Transistor Inverters}

Because high efficiency inverters using electronic switching techniques are constant power devices, they exhibit a negative slope for input impedance. The equation $V \times I=P$ is - a hyperbola for constant $P$, thus the negative slope. PV devices have a fairly sharp knee where the transition between being a voltage source and current source occurs. The peak power point from the source is found somewhere on this knee, and an optimized system should locate and track this peak point. This can be done either by analog tracking or digital tracking, or in combination. Analog refers to the manipulation of voltages and mechanical quantities by the slide-rule concept, whereas digital refers to the use of arithmetic.

Because PV systems cannot store energy and switching inverters (choppers) draw their current in short pulses, there must be a smoothing filter between the inverter and source. By, using as data the modulation of the input current due to chopping, both the current and: change in current can be measured. The voltage on the array multiplied by these factors * determines both actual power and rate of change of power. By a basic arithmetic circuit, the operating power point can be found and tracked. Modulation is a variation in the amplitude, frequency, or phase of a wave in accordance with some signal.

If a hall effect generator were placed in the gap of a simple choke input filter, the hall generator's output would be proportional to current. The chopping or switching frequency itself would serve to modulate the current sufficiently to detect the operating point (see exhibit 5.13).

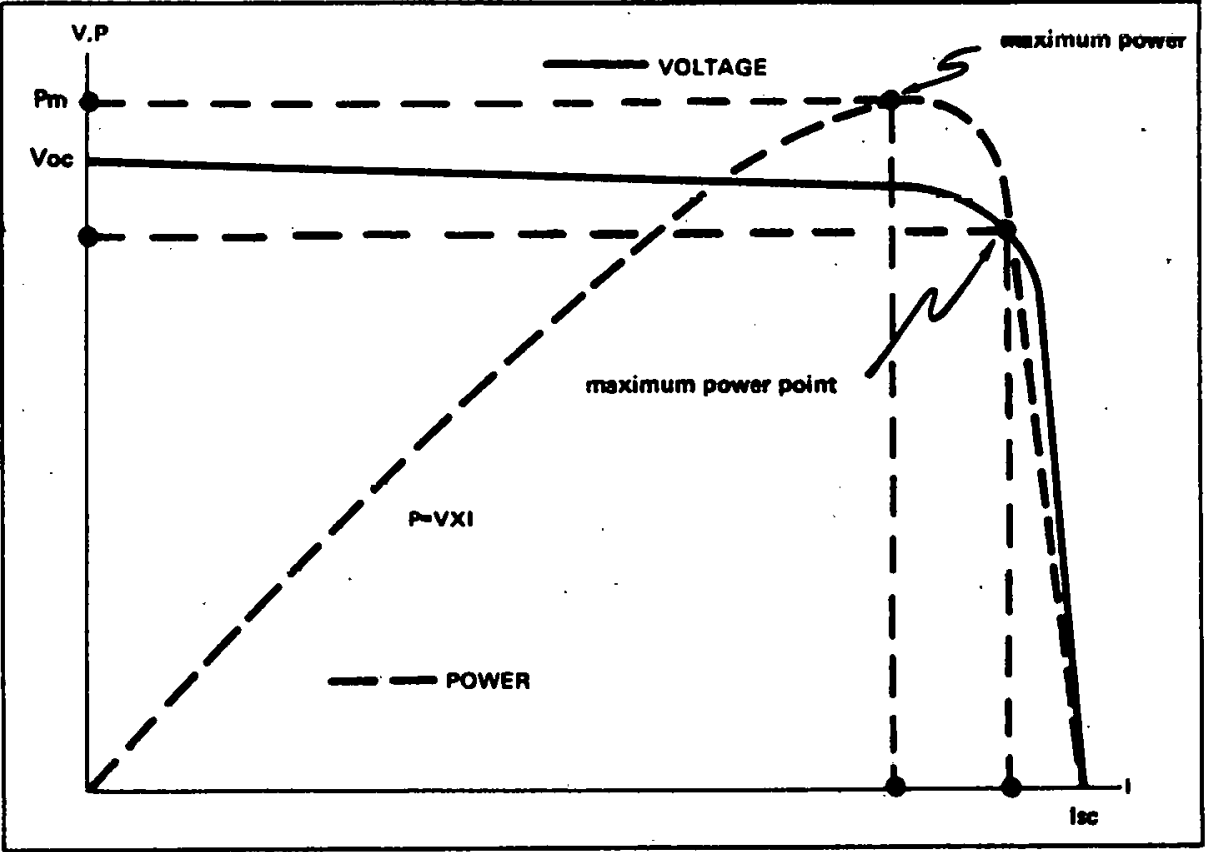

Exhibit 5.13 Peak Power Tracking. 
Comparing the sign of the current modulation to the power determines at which side of the peak point the system is operating. If the current and power are increasing, operation is behind the peak. If current is increosing and power decreasing, it is operating beyond the peak. This information is fed back to the inverter to control the pulse width to either increase or decrease the current demand on the array. The pulse width is signal duration.

\subsubsection{NVERTER TECHNOLOGY}

In initial discussions of inverter technology, system constraints will not be considered; only methods will be considered. Inverter technology is extremely wide ranging and varied. The various techniques can be grouped, by implementation, into some basic classifications.

The simplest inverter technique, a power amplifier, is not widespread because of its low efficiency. Power amplifiers employ a hi-fi amplifier controlled by an oscillator, with voltage regulating feedback. Oscillators produce $A C$ voltage of various frequencies. Hi-fi refers to an almost exact reproduction achieved by low distortion, and a wide range of reproduced frequencies.

Switching inverters, however, generally fall into the two basic classes of current generators and voltage generators. Each of these can be further divided into sub-classes by the techniques used.

Current generators usually feed pulses of current into a resonant electronic tank, which then oscillates electronically at its self-resonant frequency in a decaying sine wave. Control is achieved by varying the amount of electricity fed into the electronic tank so that, when the desired output. voltage is present, the electric energy input is equal to the losses plus the electric load taken from the tank. When a power factor is included in the load, the electronic tank is detuned, changing the frequency of electrical oscillation. However, if the current pulses are synchronized to an external electronic clock, the tank is driven off electrical resonance, and the output electrical frequency is determined by the clock rather than the tuned circuit. Resonance in an electrical circuit occurs at an $A C$ voltage input frequency which causes zero reactance. Reactance is the inductive-capacitive component of impedance. Power factor indicates the percent of resistive component.

Current can be fed into the tank at either a constant value and varying time (duty cycle), or at a constant duty cycle, with the magnitude of current varied. Usually, both duty. cycle and current magnitude are varied in practical circuits, but they have a predictable interdependence. The effective value of an $A C$ voltage is a function of amplitude and duty cycle.

Voltage generators, however, supply a voltage which is related to the source. In these devices, it is usual to have several voltages generated at differing time relationships to obtain the desired output. If two voltages are in phase and have amplitude of 1 , the sum is 2 . If they are $180^{\circ}$ out of phase, their sum is zero; all intermediate conditions can be found by vector addition. The phase angle indicates the electrical waveform time displacement for starting time. Classical $A C$ voltage analysis and synthesis is involved.

Synthesizing a waveform involves the sum of the outputs of many low-powered inverters. By implementation, certain fixed-phase relationships can be achieved that reduce the content of lower harmonics to levels requiring little or no filtering. Summation can be done magnetically with transformers or electrically from separate transformers. Transformers increase amplitude in this case by electromagnetic induction due to an $\mathrm{AC}$ voltage. 
There are also techniques that require little or no output with a transformer operating at the output frequency. These generally can be considered as "carrier" systems. They are sometimes lumped into the synthesized waveform class, but they are different. A carrier is generated at a higher frequency ( 10 to 20 or more times the desired output frequency), and then modulated at the desired output frequency. Either $F M$ or $A M$ is used. With $A M$, there is a fundamental problem in that, theoretically, only 50 percent is in the sidebands. By several simple techniques, the $A M$ can be converted to single sideband, suppressed carrier, so that all the power is in the sideband. Cyclo-converters fall within this carrier technology along with other circuits that obtain the same results by other techniques. FM refers to frequency modulation and $A M$ refers to amplitude modulation.

The Ferroresonant inverter is the most popular lower priced system used today for sinewave outputs. These inverters should be considered as special cases of either voltage or current generators, with a different filter than the usual lumped, constant types.

Filtering of waveforms serves the purpose of separating the fundamental from the switched woveform harmonics in a nonloss manner. In concept, they are quite simple, but in practice they add considerable difficulty insofar as phase shift, dynamic response, and control stability are concerned.

\subsection{INVERTER OUTPUT FILTERS}

In most inverters, a square-wave voltage is produced at some point in the circuit because of the sudden switching action that occurs during the inversion process. Electricity travels as a wave at the speed of light. Changes often appear to occur instantaneously. For simple transistor or SCR-switch inverters, a square-wave voltage is outputed to the load. Square waves contain considerable harmonic content that will cause excessive heating in $A C$ motor loads, resulting in increased motor losses without improving the torque output. Most inverters contain internal filters or resonant circuits that enable them to produce an output voltage that closely approaches a sine wave. Typically, total sinewave output harmonic distortion is less than 3 percent, which is comparable to utility standards. 
THIS PAGE

\section{WAS INTENTIONALLY \\ LEFT BLANK}




\section{SECTIONG \\ . ELECTRIC POWER LOAD ANALYSIS AND SYNTHESIS}

\section{I INTRODUCTION}

To prevent oversizing the solar PV power system (which is a stand-alone system) and unnecessarily increasing the cost, energy demand must be estimated as accurately as possible. As indicated in exhibit 6.1 , the purpose of this section is to enable participants to estimate loads correctly, to reduce unnecessary loads, to determine whether the loads ore best met by $A C$ or $D C$ voltage, and to anticipate potential problems that may occur when converitional loads are combined with the unconventional characteristics of the PV system. The lood analysis also influences the selection and sizing of back-up systems in the areas of quantity and quality, including reliability and the need to maintain frequency stability for $A C$ loads and possible utility grid-connected applications. All loads, generation, transmission, and distribution are analyzed and synthesized.

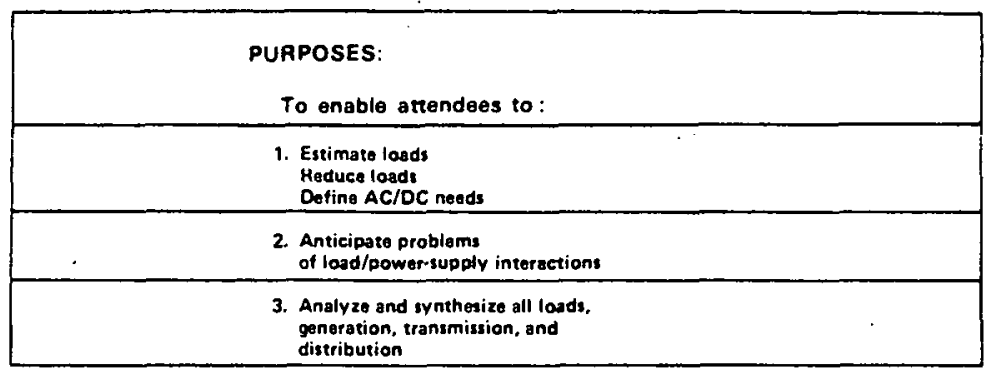

Exhibit 6.1 Load Analysis.

\subsection{ESTIMATING THE LOAD DEMAND}

The first aspect of the load analysis is defining individual energy requirements. These are used to determine the total electric load demand profile. Exhibit 6.2 lists typical electric equipment for remote applications (e.g., fire water towers). The power requirement represents the maximum demand at any one time. Because some of the equipment is operated on a cyclic basis (including the refrigerator), $\div$ e average demand (the energy demand) is considerably less than would be obtained by multiplying these rated demands by $24 \mathrm{hr} /$ day, since energy equals average power $x$ time. Exhibit 6.3 illustrates typical electrical energy requirements, based on the indicated duty cycle--the minutes per day the apparatus is drawing.current. The $1.607 \mathrm{kWh}$ per day energy requirement is only four percent of the $40 \mathrm{kWh}$ per day that would be estimated if the peak demands were assumed to prevail for 24 hours, continuously. The size of the PV array and the cost would be approximately 4 percent of what might have otherwise been estimated. Energy per day ( $\mathrm{kWh} /$ day) can be translated into average power ( $\mathrm{kW} /$ day) by dividing by the duty cycle hours.

\begin{tabular}{|cc|}
\hline EQUIPMENT (12V DC) & $\begin{array}{c}\text { MAXIMUM POWER } \\
\text { REQUIREMENT } \\
\text { (W) }\end{array}$ \\
\hline A) Radio Communication & $\begin{array}{c}\text { (Transmit) } \\
\text { (Standby) }\end{array}$ \\
\hline B) Lighting (Fluorescent) & 25 \\
\hline C) Water Pumping (5 gal/min. 50 gal/day) & 60 \\
\hline D) Refrigerator (25\% Duty Cyele) & 12 \\
\hline E) Mirrnwave Oven & 60 \\
\hline F) $\quad$ N & 1.450 \\
\hline
\end{tabular}

Exhibit 6.2 Estimating the Load. 


\begin{tabular}{|c|c|c|c|c|}
\hline \multirow{2}{*}{ ITEM } & \multirow{2}{*}{$\frac{\text { MAX. POWER }}{\text { LOAD (W) }}$} & \multicolumn{2}{|c|}{ DUTY CYCLE } & \multirow{2}{*}{$\begin{array}{l}\text { ENERGY/DAY } \\
\text { Wh/day }\end{array}$} \\
\hline & & $\mathrm{min} / \mathrm{hr}$ & hr/day & \\
\hline 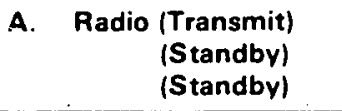 & $\begin{array}{r}25.00 \\
1.08 \\
1.08\end{array}$ & $\begin{array}{l}10 \\
50 \\
60\end{array}$ & $\begin{array}{r}8 \\
8 \\
.16\end{array}$ & $\begin{array}{r}33.33 \\
7.20 \\
17.28\end{array}$ \\
\hline B. Lighting & 60.00 & 60 & 8 & 480.00 \\
\hline C. Water Pump & 12.00 & 10 & 1 & 2.00 \\
\hline D. Refrigerator & 60.00 & 15 & 24 & 360.00 \\
\hline Microwave & $1,450.00$ & 7 & 3 & 570.50 \\
\hline F. TV & 50.00 & 60 & 4 & 200.00 \\
\hline TOTAL & 1659.16 & $\mathbf{M}$ & $H$ & 1607.31 Wh/day \\
\hline Total Demand C & $K w H=$ & & & $1.607 \mathrm{kWh} /$ day \\
\hline
\end{tabular}

Watt-hours per day $=W \times M \times H \times 1 \mathrm{hr} / 60 \mathrm{~min}$

$1 \mathrm{kWh}=1000 \mathrm{WH}$

Exhibit 6.3 Allowance for Duty Cycle.

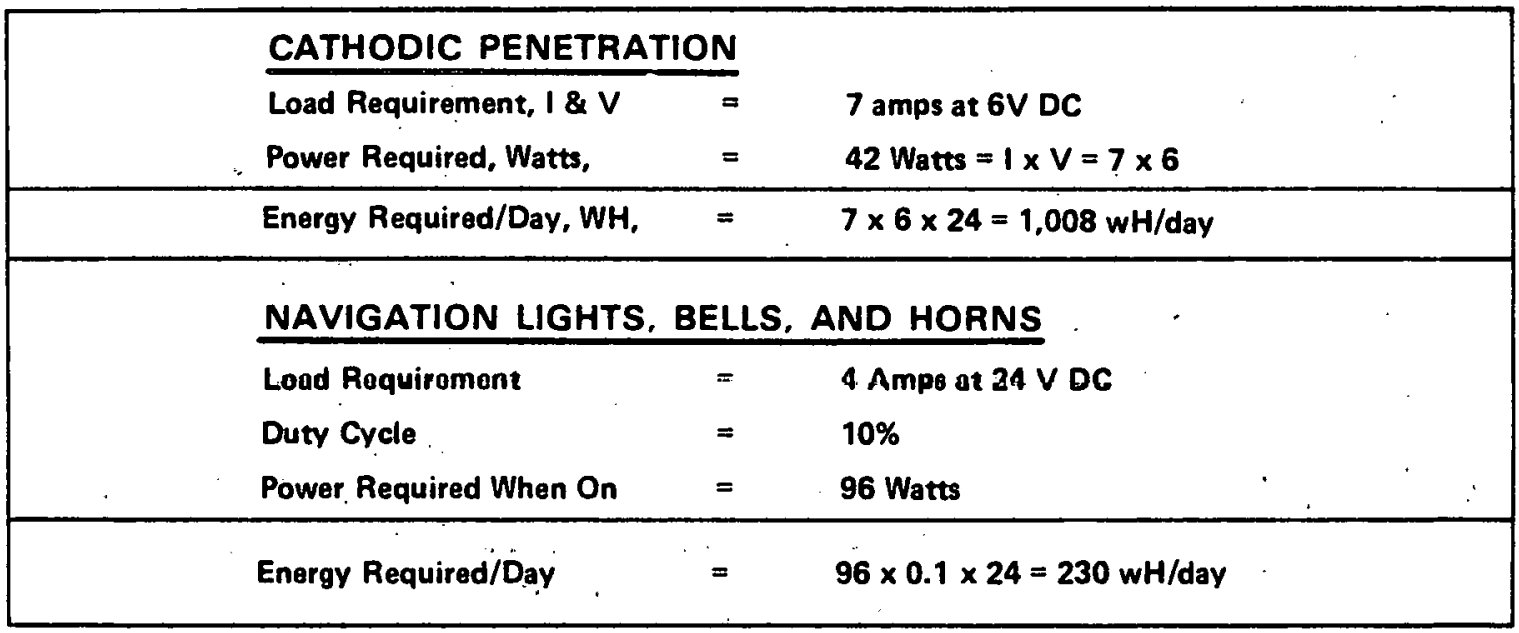

\section{Exhibit 6.4 Other Remote Applications.}

Exhibit 6.4 lists the energy requirements associated with cathodic protection systems and navigational aids. Exhibit 6.5 shows typical energy requirements for pumping applications, including toilet flushers and domestic water supplies with two different flow/lift combinations. Battery storage sizing is heavily dependent on entergy demand and duty cycle as well as peak power demand., 
TOILET FLUSHER

\begin{tabular}{|l|c|l|}
\hline CATEGORY & UNIT & \multicolumn{1}{|c|}{ VALUE } \\
\hline $\begin{array}{l}\text { 1. Load Cycle } \\
\text { 2. Load (I and V) }\end{array}$ & $\begin{array}{c}\text { Amp. } \\
\text { Volt }\end{array}$ & $\begin{array}{l}800 \text { flushes/day, 55 sec/flush } \\
7 A, 24 \mathrm{~V} \text { DC pump motors }\end{array}$ \\
\hline 3. Power Required & W & $7 \times 24=168 \mathrm{~W}(\mathrm{I} \times \mathrm{V})$ \\
\hline 4. Energy Required/Flush & WH & $168 \times 55 \times \frac{1 \mathrm{hr}}{3600}=2.567 \mathrm{Wh}$ \\
\hline 5. Energy Required/Day & WH & $800 \times 2.567=2053 \mathrm{Wh} /$ day \\
\hline
\end{tabular}

WATER PUMPING

\begin{tabular}{|l|l|l|l|}
\hline \multicolumn{1}{|c|}{ CATEGORY } & UNIT & \multicolumn{1}{|c|}{ PUMP 1 } & PUMP 2 \\
\hline $\begin{array}{l}\text { 1. Flow Rate } \\
\text { 2. Head }\end{array}$ & $\begin{array}{c}\text { Gal/ } \\
\text { dav } \\
\text { Ft. }\end{array}$ & $\begin{array}{l}2,300 \\
130\end{array}$ & $\begin{array}{l}200 \\
1,500\end{array}$ \\
\hline $\begin{array}{l}\text { 3. Pump Model No. } \\
\text { 4. Manufacturer }\end{array}$ & & $\begin{array}{l}\text { ALTA-X 600 } \\
\text { Pompes Guinard }\end{array}$ & $\begin{array}{l}50 \mathrm{~W} \\
\text { Jenson }\end{array}$ \\
\hline $\begin{array}{l}\text { 5. Power Required (24 V) } \\
\text { 6. Duty cycle }\end{array}$ & $\begin{array}{c}\text { HP } \\
\text { Hr/day }\end{array}$ & 5 & $3 / 4$ \\
\hline 7. Efficiency & $\%$ & & 5 \\
\hline $\begin{array}{l}\text { 8. Load requirement (a) } \\
\text { (Line 5 } \div \text { Line 7) }\end{array}$ & W & 600 & 75 \\
$\begin{array}{l}\text { 9. Energy Requirement } \\
\text { (Line 6 x Line 8) (b) }\end{array}$ & WH & 3,000 Wh & 746 \\
\hline
\end{tabular}

a. Conversion Factor: $1 \mathrm{HP}=0.746$ watts at $100 \%$ efficiency:

b. One watt-hour equals 3.3 Btu at $100 \%$ efficiency.

Exhibit 6.5 Pumping Applications 
These computations for typical devices, as depicted in exhibits 6.2 to 6.5 , can also be used as a guide in computing the energy demands for other applications. The peak demand must be known and the duty cycle estimated. Dato obtained in the field indicate that some of the nameplate ratings of components can be substantially overstated. Although the overstatement is not important for conventional energy sources, the impact on the cost is enormous for PV systems sized to meet the nameplate ratings. The energy consumption of some equipment is only 40 percent of what the nameplate would imply, even at peak power. It is possible that the PV system will be seriously oversized, to the point of being noncosteffective, if nameplate readings are used directly. For marginal applications, measurement of the power input is advisable.

\subsection{LOAD-REDUCTION STRATEGIES}

When the energy demand of a potential PV system application is analyzed, methods for reducing system requirements are trequently discovered. Exhibit 6.6 lists the most frequent methods of reduction. First, components of the load profile can be operated cyclically. When the transmitter is operating at peak demand, the refrigerator can be shut off. This reduces the system peok power demand and, consequently, the sizes of the equipment and lines. The smaller sizes will result in higher system efficiency during off-peak operation and lower energy consumption. The cyclic operation of the components can be either manual or automatic, although the automatic system will be more costly and will introduce another power-consuming component into the system. The automatic systems will be cost-effective only if the existing peak power under simultaneous component operation is significantly greater than peak power under cyclic operation. At a ratio of approximately $3: 1$ (simultaneous to cyclic), the cyclic operation should be examined. If more than $1 \mathrm{~kW}$ is drawn, the cyclic operation should be examined, and the automatic system should be contemplated. (NOTE: The 3:1 and I kW figures are today's best estimates based upon anticipated pastl load efficiencies. Both figures will decrease as the price of the automatic system is reduced.)

\begin{tabular}{|c|c|}
\hline \multicolumn{2}{|c|}{ CYCLIC OPER̈ATION OF COMPONENTS } \\
\hline $\begin{array}{l}\text { Diversity } \\
\text { Load Shedding }\end{array}$ & $\begin{array}{l}\text { Manual } \\
\text { Automatic }\end{array}$ \\
\hline \multicolumn{2}{|c|}{ Eliminate DC-to-AC Inversion } \\
\hline
\end{tabular}

\section{Exhibit 6.6 Load Reduction Strategies.}

Some of the losses that result from operating power-conversion equipment at less than maximum power can be circumvented if a separate power-conversion device is used for ecch load. For the most part, whenever the load is demanding power, the power converter will pass its rated capacity. In addition, if $A C$ is needed for part of the load and DC for another part, the individual converters would be designed for the respective duties. This scheme's realized energy savings must be compared to the added cost of using individual power converters instead of one central converter. Because their power requirement is small, the individual converters can be simply made; thus, they may be no more expensive than the central unit. 
Cyclic operation of a large number of components permits the undersizing of equipment on the basis of load diversity. When several components drow power from the system, it is unlikely all will draw current simultaneously. The power utilities make constant use of the low odds associated with their enormous systems. For example, suppose there are four components on the line drawing powers of $10,20,30$, and $50 \mathrm{~kW}$ peak randomly with duty cycles of 50, 40, 30 and 20 percent, respectively. The probability that all four loads will operate simultaneously is 1.2 percent, as shown in exhibit 6.7. The system should be designed for the peak demand rather than cyclic operation under these circumstances, because the probability of overload is too great. (The 1.2 percent figure can be translated into 0.012 times 365 days, or four days/yr.) The total electric load demand profile is stochastic.

\begin{tabular}{|c|c|}
\hline LOAO & ON-TIME \\
\hline $10 \mathrm{~W}$ & $50 \%$ \\
$20 \mathrm{~W}$ & $40 \%$ \\
\hline $30 \mathrm{~W}$ & $30 \%$ \\
$40 \mathrm{~W}$ & $20 \%$ \\
\hline Probability of simultaneous operation $=$ \\
$0.5 \times 0.4 \times 0.3 \times 0.2=0.012=1.2 \%$ \\
\hline
\end{tabular}

\begin{tabular}{|c|c|c|}
\hline \multicolumn{3}{|c|}{ PROBABILITY OF OTHER COMBINATIONS } \\
\hline $\mathbf{w}$ & Probability Calculation & Value \\
\hline 10 & $0.5 \times(1-0.4) \times(1-0.3) \times(1-0.2)$ & $=0.168$ \\
\hline 20 & $0.4 \times(1-0.6) \times(1-0.3) \times(1-0.2)$ & $=0.112$ \\
\hline 30 & $0.3 \times(1-0.5) \times(1-0.4) \times(1-0.2)+0.5 \times 0.4 \times(1-0.3) \times(1-0.2)$ & $=0.184$ \\
\hline 40 & $0.5 \times 0.3 \times(1-0.4) \times(1-0.2)+0.2 \times(1.05) \times(1-0.4) \times(1-0.3)$ & $=0.114$ \\
\hline 50 & $0.3 \times 0.4 \times(1-0.5) \times(1-0.2)+0.5 \times 0.2 \times(1-0.4) \times(1-0.3)$ & -0.09 \\
\hline 60 & $0.5 \times 0.4 \times 0.3 \times(1-0.2)+0.2 \times 0.4 \times(1-0.5) \times(1-0.3)$ & $=0.076$ \\
\hline 70 & $0.2 \times 0.3 \times(1-0.5) \times(1-0.4)+0.2 \times 0.4 \times 0.5 \times(1-0.3)$ & $=0.046$ \\
\hline 80 & $0.2 \times 0.3 \times 0.5 \times(1-0.4)$ & $=0.018$ \\
\hline 90 & $0.2 \times 0.3 \times 0.4 \times(1-0.5)$ & $=0.012$ \\
\hline
\end{tabular}

\section{Exhibit 6.7 Load Diversity.}

The probability of having loads ranging from 10 to $90 \mathrm{~W}$ is also indicated in exhibit 6.7. The probability of any one combination of loads can be computed in keeping with the following example: the probability that the load would be $20 \mathrm{~W}$ is equal to the product of the probability that the $20 \mathrm{~W}$ load is on and the probabilities that the three other loads are of $f$, or 11.2 percent, according to the data in the exhibit. 
The foregoing discussion brings us to the logical concept of load shedding. If the probability of simultaneous operation is low, or if the same functions are not critical, the peak demand can be limited by a controller that senses the demand and supplies power to the low-priority components only when the demand on the power system is low. Load shedding involves the automatic tripping of load center circuit breakers, usually in distribution switchboards, by use of relays triggered by sensors. Load shed tripping can also be initiated manually by remote control. The first loads shed are non-vital, then semil-vital, and finally the generators are tripped from the system. Closing is accomplished manudlly.

Reducing the peak load has such an indirect effect on the reduction in energy demand that it is difficult to estimate the energy impact without a computer that tracks system performance on a minute-by-minute basis. Even the simplest analysis can incorporate the energy-saving concept of eliminating DC-to-AC inversion. If the loads would conventionally require $A C$, there is always the option of replocing the $A C$ components with a $D C$ counterpart. Examples are: $A C$ motors can be replaced by DC motors; other components that are normally supplied by $A C$ but actually use $D C$, such as radio transmitters and receivers, can use the solar-produced DC dirertly. If DC is supplied directly, the losses associated with the DC-to-AC inversion (from the array/battery system to the load) can be eliminated, as well as the losses associated with the AC-to-DC conversion in the equipment itself. The overall reduction in energy demand can be as much as 40 percent. However, the tradeoffs between $A C$ and DC must be examined in greater detail for each application, as is discussed in the next section. Modifying or manufacturing DC equipment that normally rectifies or transforms $A C$ electricity prior to equipment use for DC electricity can be accomplished easily on a technological basis.

\subsection{VERSUS AC POWER}

An analysis was undertaken by a DOE contractor to assess the merits of directly supplying certain loads with the DC power available from the solar array/battery power sources rather than inverting the power to $A C$ with losses resulting from inverter inefficiency. In making the assessment, the question of regulation was also considered. Although the inversion of $D C$ to $A C$ carries with it a nominal penalty of 12 percent inefficiency, relatively good output $A C$ regulation $r n$ he nchieved with the inverter within nominal limits of \pm 5 percent. Regulating DC from an unregulated DC source (of which the array/battery combination is typical with a voltage range of 30 percent) also involves an inefficiency penalty of about 12 percent. Thus, power economy benefits would result only when unregulated DC is used. In section 6.4.1, typical loads are assessed as to their general operability with DC. Exhibit 6.8 presents the disndvantages of both $A C$ and DC power. Iransformers, whose purpose is to reduce or increase voltage for an isolated system or load, or just isolate a load or system, and motors rely on induction by electromagnetic fields. This induction can take place only with alternating current (AC) or modified direct current (DC). 


\begin{tabular}{|l|l|l|}
\hline \multirow{2}{*}{ WAVEFORM } & \multicolumn{2}{|c|}{ BUS } \\
\cline { 2 - 3 } & \multicolumn{1}{|c|}{ DC } & \multicolumn{1}{|c|}{ AC } \\
\hline Motor Drive & Brushes wear & \\
\hline Universal/linduction & More expensive & \\
\hline Lights & $\begin{array}{l}\text { Fluorescents less efficient } \\
\text { Loss of incandescent and } \\
\text { fluorescent reliability }\end{array}$ & Requires regulation/rectification \\
\hline Electronics & Requires regulation & Requires inverter \\
\hline PV Output & & Requires rectification \\
\hline Battery & Contact wear & Requires rectification \\
\hline Controls & Not easily accommodated & \\
\hline Multiple Voltages & & \\
\hline
\end{tabular}

Exhibit 6.8 Disadvantages of DC and AC.

\subsection{CLASSIFICATION OF LOADS}

Electric loads can be classified by their type of electrical energy transformation. The four categories useful for PV system considerations are resistance elements, universal motors, induction motors, and induction-coupled loods (transformers).

The resistance elements include simple heat producers such as electric range elements and incandescent elements such as light bulbs. The universal motor is found in a great number of the portable appliances where the compact size and low cost are of primary concern. The universal motor can operate on DC or AC power. Where the low brush life or variable speed of the universal motor is objectionable, the induction motor is used. In longlife, low-maintenance applications, such as refrigerators and washers, the simple squirrel cage wound motor is used. Where occurate speed is needed, such as in timekeeping and sound reproduction, a synchronous induction motor is used where synchronous refers to the matching of electromagnetic fields and speed. The fourth class energy transformation utilizing induction coupling (transformers) is used in such items as fluorescent lamps, some electronics power supplies, and microwave oven supplies.

All electrical loads have impedance, which consists of resistance and reactance. Reactance consists of inductance and capacitance. The power factor indicates the percentage of resistance. The magnitude of impedance equals the square root of the quantity (resistance ${ }^{2}+$ reactance $^{2}$ ). AC voltage equals current $x$ impedance. The common unit of impedance, resistance, and reactance is the ohm, where one ohm equals one volt per ampere.

\subsubsection{CLASSIFICATION OF CONTROLS}

Loads are frequently controlled by two types of control elements. The simple contact pair is the most prevalent and is found in both dry and mercury wetted forms. The second type of control element is the thyristor. Both unipolar (SCR) and bipolar (Triacs) devices are used. The SCR is a static-controlled-rectifier (solid state-transistors). A triac is an alternating-current switch. Thyristors are semicunductor rectifiers. 
The simple contact pair, found in nearly every appliance or circuit, guarantees complete line isolation when the appliance circuit is turned off. An extension of this control is the simple bimetal temperature control found in appliances such as irons and skillets. These switches are required to cycle on the order of four or five times per minute in most applications. A further extension of this technique is found in some universal motor speed controls where a miniature flyball governor is used to open and close contacts, thereby controlling the motor speed. Appliances and controls utilizing relays also rely on the contact pair. Many heating systems use relays for control and interlock functions. A governor is a mechanical device for automatically controlling the speed of an engine or motor by regulating intake power.

The thyristor controls are found in lamp dimmers and motor speed controls where phase control techniques control the effective voltage delivered to the appliance or lighting load. A new application of these devices will he in appliance temperature controls where the thyristor, used in the $A C$ wave zero crossing mode, can eliminate unwanted conducted and radiated electrical noise inherent in contact opening and closing.

Relays are electromagnetic devices activated by a variation in conditions in one electric circuit and control o larger current or activate other devices in the same or another circuit.

\subsubsection{APPLIANCE LISTING}

The most common electric appliances are listed in exhibits 6.9 through 6.16 . They pertain to climate control, food preparation, food preservation, home care, home entertainment, laundry, lighting, and personal care. Included in the listing are overage rated wattage, type of electrical energy transformation employed, types of common controls, and average yearly energy usage. Electrical energy transformation involves resistance, reactance or a combinntion. The average rated wattage and average yeurly energy usage figures were computed from the Niagara Mohawk Power Corporation, NPC 1970 estimate and CEQ 1973 estimate.

The major types of energy transformation and control types have been marked on the tables with an $X$. The minor types have been marked with an $M$. For example, the first entry in the Climate Control list (exhibit 6.9) is the Air Cleaner. It has $X$ marks on the induction motor and transformer-coupled because there is usually an induction motor fan drive and, in the case of electrostatic air cleaners, a high voltage transformer. These units usually have simple on-off switches. However, room air conditioners are available with the three control types indicated. Most radiant heaters do not have air circulation fans, some do use a small induction motor to cool the metallic surfaces of the unit and provide some convection heating. Therefore, there is an $M$ in the Induction Motor column. Induction involves the transfer of electric power by use of electromagnetic fields, and can serve to isolate circuits while reducing or increasing voltage, if necessary, as in transformers. 


\begin{tabular}{|c|c|c|c|c|c|c|c|c|c|c|c|c|}
\hline \multirow[b]{2}{*}{ Appliance , } & \multirow[b]{2}{*}{$\begin{array}{l}\text { Aversge } \\
\text { Rated } \\
\text { Load (W) }\end{array}$} & \multicolumn{4}{|c|}{ ENEAGY TRANSFOAMATION } & \multicolumn{6}{|c|}{ CONTROL TYPE } & \multirow[b]{2}{*}{$\begin{array}{l}\text { Average } \\
\text { Yearly } \\
\text { Energy } \\
\text { Usage } \\
\text { (kWh }\end{array}$} \\
\hline & & 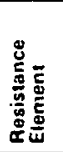 & 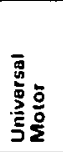 & 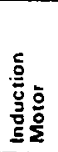 & 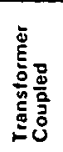 & 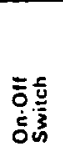 & 紊总 & 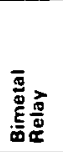 & 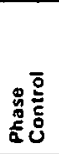 & 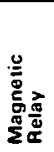 & 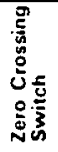 & \\
\hline Air Cleanar & 50 & & & $x$ & $x$ & $x$ & & & & & & 216 \\
\hline Room Air Conditioner & 860 & & & $x$ & & $x$ & $x$ & $x$ & & & & 860 \\
\hline Bod Covering & 177 & $x$ & & & & $x$ & $\bar{x}$ & $x^{\circ}$ & & & & 147 \\
\hline Dehumidifier & 257 & & & $x$ & & $x$ & & $x$ & & & & 377 \\
\hline Fan-Altic & 370 & & & $x$ & & $x$ & & & & & & 291 \\
\hline Fan-Bathroom & 105 & & & $x$ & & $x$ & & & & & & \\
\hline Fan-Circulating & 86 & & & $\mathbf{x}$ & & $x$ & $x$ & & & & & 43 \\
\hline Fan-Window & 200 & & & $x$ & & $x$ & $x$ & & & & & \\
\hline Furnace Fan & 294 & & & $x$ & & $x$ & & $x$ & & $x$ & & \\
\hline Heater-Radiant & 1.250 & $x$ & & $M$ & & $x$ & $x$ & $x$ & & & & 176 \\
\hline Hest Pump & 12.500 & $x$ & & $x$ & & $x$ & & $x$ & & & & \\
\hline Heoring Pad & 65 & $x$ & & & & $x$ & $x$ & & & & & 10 \\
\hline Humidifier & 17 & & & $x$ & & $x$ & $x$ & $x$ & & & & 163 \\
\hline Oil Burner & 263 & & & $x$ & $x$ & $x$ & & $x$ & & $x$ & & \\
\hline
\end{tabular}

Exhibit 6.9 Climate Control.

\begin{tabular}{|c|c|c|c|c|c|c|c|c|c|c|c|c|}
\hline \multirow[b]{2}{*}{ Appliance } & \multirow[b]{2}{*}{$\begin{array}{c}\text { Average } \\
\text { Rated } \\
\text { Load (W) }\end{array}$} & \multicolumn{4}{|c|}{ ENERGY TRANSFORMATION } & \multicolumn{6}{|c|}{ CONTROL TYPE } & \multirow[b]{2}{*}{$\begin{array}{l}\text { Average } \\
\text { Yearly } \\
\text { Energy } \\
\text { Usage } \\
\text { ikwhi }\end{array}$} \\
\hline & & 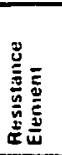 & 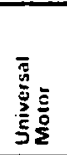 & 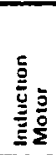 & 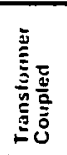 & 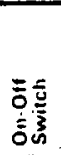 & 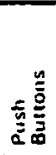 & 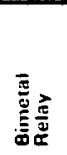 & 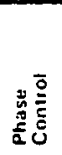 & 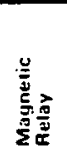 & 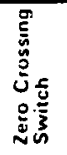 & \\
\hline Blender & 386 & & $x$ & & & $x$ & $x$ & & $\bar{x}$ & & & 15 \\
\hline Broiler & $\begin{array}{l}6700 \\
1400\end{array}$ & $x$ & & $\mathbf{M}$ & & $x$ & $x$ & $x$ & & & & 100 \\
\hline Can Opener & $100^{\circ}$ & & $\bar{x}$ & $\bar{x}$ & & $\bar{x}$ & & & & & & 3 \\
\hline Caring Knife line & 92 & & $\bar{x}$ & & & $\bar{x}$ & & & & & & 8 \\
\hline Carving Knife-banery & 92 & & & & $\bar{x}$ & & & & & & & 8 \\
\hline Colfee Maker & 894 & $x$ & & & & $\bar{x}$ & & $\bar{x}$ & & & & 106 \\
\hline Deep Fryer & $\begin{array}{l}1667 \\
1448\end{array}$ & $x$ & & & & $x$ & & $x$ & & & & 83 \\
\hline Dishwasher & $\begin{array}{l}1250 \\
1202\end{array}$ & $x$ & & $x$ & & $x$ & $x$ & $x$ & & & & 363 \\
\hline Egg Cooker & 500 & $x$ & & & & $x$ & & $x$ & & & & \\
\hline Frying Pan & $\begin{array}{l}1250 \\
1106\end{array}$ & $x$ & & & & $x$ & & $x$ & & & $M$ & 186 \\
\hline Sandwich Grill & $\begin{array}{l}1250 \\
1161\end{array}$ & $x$ & & & & $x$ & & $x$ & & & & 33 \\
\hline Hot Plate & 1257 & $\bar{x}$ & & & & $\bar{x}$ & & $x$ & & & & 90 \\
\hline Microwave Oven & 1450 & & & $M$ & $x$ & $x$ & . & $x$ & & & & 190 \\
\hline Range w Oven & 12.200 & $x$ & & $M$ & $M$ & $x$ & $x$ & $x$ & & $x$ & & 1.175 \\
\hline Range Self-Clean & 12.200 & $\bar{x}$ & & $M$ & $M$ & $\bar{x}$ & $\bar{x}$ & $\bar{x}$ & & $x$ & & 1.205 \\
\hline Poaster & 1.333 & $x$ & $M$ & $N$ & & $x$ & $\bar{x}$ & $x$ & & & & 205 \\
\hline Toaster & $\begin{array}{l}1.250 \\
1.146\end{array}$ & $x$ & . & & & $x$ & & $x$ & & & & 39 \\
\hline Trash Compacior & 400 & & $\bar{x}$ & $\bar{x}$ & & $\bar{x}$ & & & & & & 50 \\
\hline Waffle Iron & 1.116 & $x$ & & & & $\bar{x}$ & & $\bar{x}$ & & & & 22 \\
\hline Waste Dispusal & 445 & & $\bar{x}$ & $x$ & & $x$ & & & & & & 30 \\
\hline
\end{tabular}

Exhibit 6.10 Food Preparation. 


\begin{tabular}{|c|c|c|c|c|c|c|c|c|c|c|c|c|}
\hline \multirow[b]{2}{*}{ Appliance } & \multirow[b]{2}{*}{$\begin{array}{l}\text { Average } \\
\text { Rated } \\
\text { Load (W) }\end{array}$} & \multicolumn{4}{|c|}{ ENERGY TRANSFORMATION } & \multicolumn{6}{|c|}{ CONTROL TYPE } & \multirow[b]{2}{*}{$\begin{array}{l}\text { Average } \\
\text { Yeorly } \\
\text { Energy } \\
\text { Usage } \\
\text { (kWh) }\end{array}$} \\
\hline & & 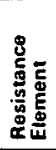 & 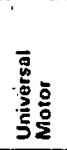 & 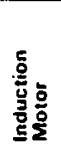 & 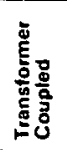 & 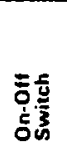 & 常产 & 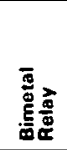 & 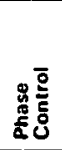 & 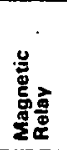 & 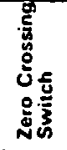 & \\
\hline Freezer $15 \mathrm{cu} \mathrm{ft}$ & 341 & $M$ & & $x$ & & $\bar{x}$ & & & & & & 1.195 \\
\hline Freazer (frostless) $15 \mathrm{cu} \mathrm{it}$ & 440 & $M$ & & $\bar{x}$ & & $x$ & & $\bar{x}$ & & & & 1.761 \\
\hline Retrigerator $12 \mathrm{cu}$ fi & $\begin{array}{l}238 \\
241\end{array}$ & $\mathbf{M}$ & & $x$ & & $x$. & & $x$ & & & & 728 \\
\hline $\begin{array}{l}\text { Refrigerator (frostless) } \\
12 \mathrm{cu} \text { it }\end{array}$ & $\begin{array}{l}333 \\
321\end{array}$ & M & & $x$ & & $x$ & & $x$ & & & & 1.217 . \\
\hline Retrigerator/Freezer $14 \mathrm{cu} \mathrm{Cl}$ & 326 & $M$ & & $x$ & & $x$ & & $x$ & & & & 1.137 \\
\hline $\begin{array}{l}\begin{array}{l}\text { Refrigerator, Freazer } 14 \mathrm{cu} \\
\text { (frostless) }\end{array} \\
\end{array}$ & 615 & $\mathbf{M}$ & 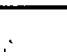 & $x$ & & $x$ & & $x$ & & & & 1,829 \\
\hline
\end{tabular}

Exhibit 6.11 Food Preservation.

\begin{tabular}{|c|c|c|c|c|c|c|c|c|c|c|c|c|}
\hline \multirow[b]{2}{*}{ Appliance } & \multirow[b]{2}{*}{$\begin{array}{l}\text { Average } \\
\text { Ratted } \\
\text { Load iWM }\end{array}$} & \multicolumn{4}{|c|}{ ENERGY TRANSFORMATION } & \multicolumn{6}{|c|}{ SONTROL TYFE } & \multirow[b]{2}{*}{$\begin{array}{l}\text { Average } \\
\text { Yearty } \\
\text { Enargy } \\
\text { Usago } \\
\text { (kWh) }\end{array}$} \\
\hline & & 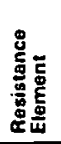 & 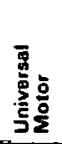 & 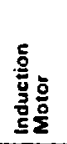 & 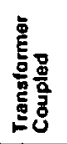 & 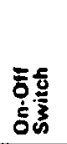 & 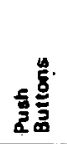 & 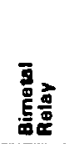 & 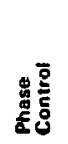 & 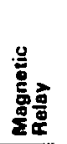 & 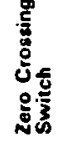 & \\
\hline Clock & 2 & & & $\bar{x}$ & & & & & & & & 17 \\
\hline Floor Polisher & $\begin{array}{l}312 \\
350\end{array}$ & & $x$ & & & $x$ & & & & & & 15 \\
\hline Germicidal Lamp & 20 & $x$ & & & $x$ & & & & & & & 170 \\
\hline Sewing Machine & 75 & & $x$ & & & $\bar{x}$ & & & $x$ & & & 11 \\
\hline Vacuum Cleaner & 630 & & $x$ & & & $x$ & $x$ & & & & & 46 \\
\hline Water Pump & 454 & & & $\bar{x}$ & & $\bar{x}$ & & $x$ & & $\bar{x}$ & & \\
\hline Circular Hand Saw & 800 & & $x$ & & & $x$ & & & & & & \\
\hline Table Șaw & Rno & & $x$ & & & $x$ & & & & & & \\
\hline Drill-Hand & 250 & & $x$ & & & $\bar{x}$ & & & $\bar{x}$ & & & \\
\hline Soldering Iron & 125 & $x$ & & & $\bar{x}$ & $x$ & & & & & & \\
\hline Garage Onnr Oponer & & & $x$ & $x$ & & $x$ & & & & $\bar{x}$ & & \\
\hline
\end{tabular}

Exhibit 6.12 Home Care.

\begin{tabular}{|c|c|c|c|c|c|c|c|c|c|c|c|c|}
\hline \multirow[b]{2}{*}{ Applianse } & \multirow[b]{2}{*}{$\begin{array}{l}\text { Averag口 } \\
\text { Rotred } \\
\text { Losd (W) }\end{array}$} & \multicolumn{4}{|c|}{ enenor Thaivstummalsun } & \multicolumn{6}{|c|}{ CONTROL TYPE } & \multirow[b]{2}{*}{$\begin{array}{l}\text { Average } \\
\text { Yearty } \\
\text { Energy } \\
\text { Usage } \\
\text { itWil }\end{array}$} \\
\hline & & 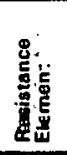 & 覀 & 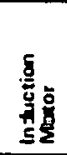 & 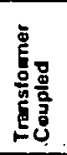 & 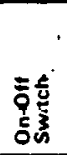 & 它 & $\begin{array}{l}\overline{\mathbf{g}} \\
\bar{q} \\
\overline{\mathbf{m}} \\
\overline{8}\end{array}$ & 㙂 & 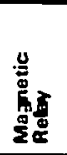 & 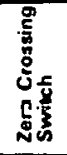 & \\
\hline Redio & 71 & & & & $\bar{x}$ & $\bar{x}$ & & & & & & 86 \\
\hline Radio/Recond Player & 109 & & & & $x$ & $x$ & & & & & & 109 \\
\hline Telovision-8\&W & $\begin{array}{r}160 \\
56 \\
\end{array}$ & & & $:$ & $x$ & $\mathbf{x}$ & . & & & & & $\begin{array}{l}350 \\
120\end{array}$ \\
\hline Tetovitiün-culur & $\begin{array}{l}300 \\
200\end{array}$ & - & & & $x$ & $x$ & & & & & & $\begin{array}{l}660 \\
410\end{array}$ \\
\hline Slide Projector & 300 & $x$ & & $x$ & & $x$ & $x$ & & & & & \\
\hline Movia Projector & 600 & $x$ & & $\bar{x}$ & & $x$ & $x$ & & & & & \\
\hline
\end{tabular}

Exhibit 6.13 Home Entertainment. 


\begin{tabular}{|c|c|c|c|c|c|c|c|c|c|c|c|c|}
\hline \multirow[b]{2}{*}{ Appliance } & \multirow[b]{2}{*}{$\begin{array}{l}\text { Avorage } \\
\text { Ratad } \\
\text { Load (W) }\end{array}$} & \multicolumn{4}{|c|}{ ENERGY TRANSFORMATION } & \multicolumn{6}{|c|}{ CONTROL TYPE } & \multirow[b]{2}{*}{$\begin{array}{l}\text { Average } \\
\text { Yearly } \\
\text { Energy } \\
\text { Usage } \\
\text { (kWh) }\end{array}$} \\
\hline & & 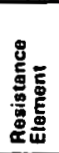 & $\begin{array}{l}5 \\
\frac{3}{9} \\
\frac{3}{5} \\
5\end{array}$ & 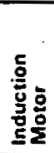 & 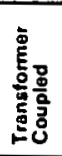 & 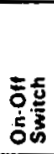 & 둑롤 & . & 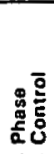 & 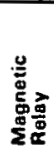 & 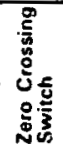 & \\
\hline Clothes Drver & 4.856 & $\bar{x}$ & & $\bar{x}$ & & $\bar{x}$ & & $x$ & & & & 993 \\
\hline Hand Iron & 1.000 & $x$ & . & & $\because$ & $\bar{x}$ & & $\bar{x}$ & & & & 144 \\
\hline Washing Machine-automatic & $\begin{array}{l}500 \\
512\end{array}$ & & & $\mathbf{x}$ & & $x$ & & & & & & 103 \\
\hline Washing Machine-manual & 286 & & & $\bar{x}$ & & $x$ & & & & & & 76 \\
\hline Water Heater & 2.500 & $x$ & & & & & & $\bar{x}$ & & & & 4.219 \\
\hline Quick Recovery & 5.000 & $\bar{x}$ & & & & & & $x$ & & & & 4.811 \\
\hline
\end{tabular}

Exhibit 6.14 Laundry.

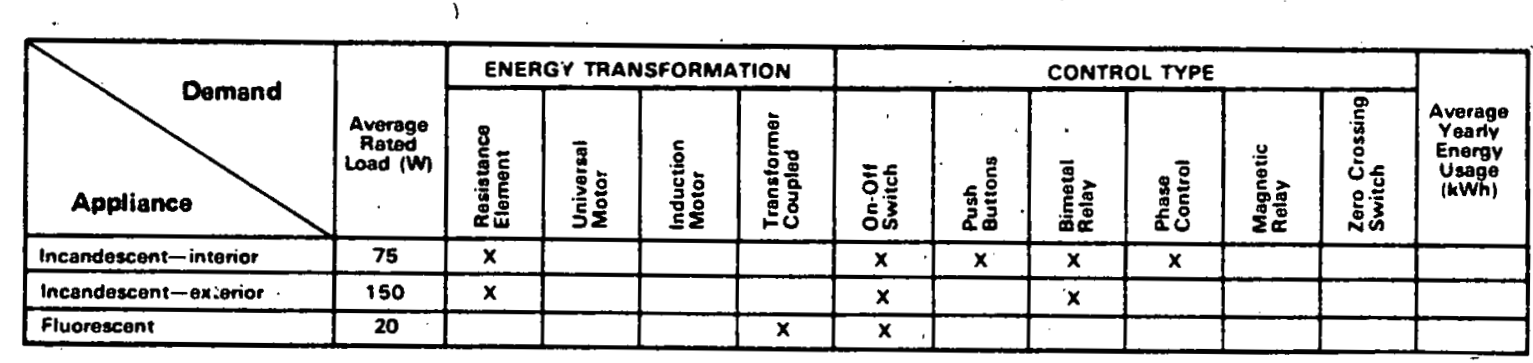

Exhibit 6.15 Lighting.

\begin{tabular}{|c|c|c|c|c|c|c|c|c|c|c|c|c|}
\hline \multirow[b]{2}{*}{ Appliance } & \multirow[b]{2}{*}{$\begin{array}{l}\text { Avoreso } \\
\text { Roted } \\
\text { Lotd im }\end{array}$} & \multicolumn{4}{|c|}{ ENEROY TRANSFORMATION } & \multicolumn{6}{|c|}{ CONTROL TYPE } & \multirow[b]{2}{*}{$\begin{array}{l}\text { Average } \\
\text { Yeariv } \\
\text { Energy } \\
\text { Usago } \\
\text { (kWh) }\end{array}$} \\
\hline & & 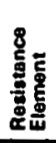 & 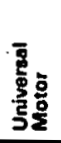 & 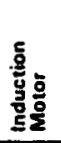 & 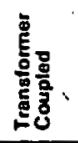 & 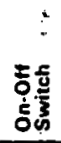 & 卢产 & 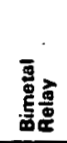 & 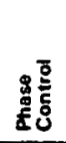 & 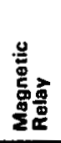 & 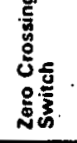 & \\
\hline Hair Dryer & 380 & $x$ & $x$ & $M$ & & $x$ & $x$ & 1 & & & & 14 low \\
\hline Hoat Lamp & 250 & $\mathbf{x}$ & & & & $x$ & $x$ & & & & & 13 \\
\hline Curling Iron & & $x$ & & & & $x$ & & $x$ & & & & \\
\hline Heated Curlers & & $x$ & & & & $x$ & & $x$ & & & & \\
\hline Shover & 14 & & $x$ & & $\bar{x}$ & $x$ & & & & & & 1.8 \\
\hline Sun Lamp & 279 & $x$ & & & & $x$ & & 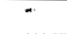 & & & & 16 \\
\hline Toothbrush & & & $x_{1}$ & & $x$ & $x$ & & & & & & .5 \\
\hline Vibrator & & & $\mathbf{x}$ & & & $x$ & $\mathbf{x}$ & $\therefore$ & M & & & 2 \\
\hline Water Pic & & & $x$ & & & $x$ & & & & & & \\
\hline
\end{tabular}

Exhibit 6.16 Personal Care. 


\subsubsection{OPERATION ON DC VOLTAGE}

The operation of the appliances will be discussed with respect to the suitability of the basic electrical energy transformation means to utilization of DC voltage and the suitability of the various controls to operation on DC voltage.

The loads with resistance element energy transformation can operate on DC voltages, as can the universal motor. The induction motors and induction-coupled appliances (transformers) rely on periodic voltage reversals to function (alternating current). These appliances cannot operate on DC voltage.

Of the resistance element loads, those most suitable for $D C$ are the simple heat producers. These units follow a square law relationship between voltage and electric power transformed into heat. The heat flow equals, $I^{2} \times R=V^{2} / R$ which results from $P=V \times \mid$ and $V=I \times R$ (ohm's law). Many of these appliances have thermostatic controls such that the DC voltage might vary moderately ( +10 to 20 percent), while the control would compensate for the input power variation. For example, an electric frying pan set for $350^{\circ} \mathrm{F}$ may only have power supplied to the resistance heater for the first 15 seconds out of a minute during which time the temperature of the load is raised to some upper limit, say $375^{\circ} \mathrm{F}$. During the following 45 seconds, the temperature will decrease to some lower limit, say $325^{\circ} \mathrm{F}$, at which time the bimetal controller applies voltage to the resistance element to start the cycle again. With a low input voltage, the cycle might be 25 second on, 35 second off, while the high input case would result in 10 seconds on and 50 seconds off. Voltage eavals energy per charge and current equals charge per time ( $1 \mathrm{amp}=1$ coulomb per sec). Power is the heat rate in this case (one watt equals one joule per second which equals one volt $x$ one amp).

Due to the square law power conversion of these elements, the most significant variable in performance of these controlled resistance elements would be a low maximum output at low input voltages. Simple uncontrolled heaters, such as hot plates and radiant units, also suffer from this poor regulation. A 10 percent reduction in voltage from the normal operating voltage would result in a 20 percent reduction in heat flow, since $p=V^{2} / R$, and change in power (heat flow) equals $2 \times V / R \times$ change in voltage.

Incandescent lamps (lightbulbs) can operate on DC voltages; however, two phenomena must be considered. First energy output is proportional to the second power of the operating voltage. As the energy output decreases so does the temperature; therefore, the amount of energy emitted in the visible spectrum--the light output--decreases more rapidly. For example, the overall effect of a 10 percent reduction in voltage is a 40 percent reduction in the light output.

The second phenomenon is metallurgical in nature. The incandescent tungsten filament in a lamp undergoes a grain boundary modification on DC operatiun. This results in a reduced life from some lamps when operated on DC voltage. This grain boundary modification is quite temperature sensitive. Lamps operated above $2,800^{\circ} \mathrm{K}$ show little of this phenomenon during life. Lamps operated in this temperature range are used for general household illumination. Long-life lamps and very low wattage types, such as night lights, operate at reduced filament temperatures (below $2,700^{\circ} \mathrm{K}$ ) which make them susceptible to this effect. However, the majority of the lamps used in residences will be unaffected by the $\mathrm{DC}$ operation. $\mathrm{K}=\mathrm{C}+273$, and $\mathrm{F}=1.8 \mathrm{c}+32.2800^{\circ} \mathrm{K}=2527^{\circ} \mathrm{C}=4548.6^{\circ} \mathrm{F}$. 
Fluorescent lomps suffer from an approximate 20-percent loss in efficiency when operated on DC voltage. In addition, the direction of the DC voltage must be reversed approximately once per day to avoid the degradation and premature failure of the electrodes due to ion bombardment. The output of a fluorescent lamp is considerably less sensitive to voltage variations than the incandescent lamp.

Universal motors do not rely on the periodic voltage reversal (AC) to operate. Even though these units can operate from either $A C$ or $D C$ voltoge, most motors incorporated in present appliances have been optimized for AC operation because DC is not readily available. When the motors are operated on DC, they exhibit accelerated negative-brush erosion, resulting in a 50 percent decrease in life. Brushes, commutators, and slip rings, and other methods are used to transfer electricity from a moving conductor in which voltage has been induced.

Some AC-equiped systems, such as refrigerators, are hermetically sealed. Because the brushes of a DC motor must be accessible for repair, hermetic sealing is not feasible when these systems are DC equiped. Therefore, there will be a loss in system reliability when DC is used.

The speed of these motors is also dependent on the input voltage. A 10 percent decrease in voltage would result in a 10-percent decrease in speed. In most blower applications, this would result in a 10-percent reduction in flow rate and a 20 -percent reduction in output pressure.

The induction motor and transformer-coupled devices rely on the periodic sinusoidal voltage reversals to operate. If these units are connected to a DC bus, a heavy current will be drawn with resultant appliance failure, since the reactance component of AC impedance becomes negligible under DC voltage. Power factor indicates the percentage of resistance.

If universal motors are excluded due to life considerations, the controlled resistance elements are the only residential loads suitable for operation with DC voltage.

The contact pair, as used in the typical residence, has been optimized with respect to cost and function. To redu e the cost of contact material and mechanical actuators, switches have been designeu for $A C$ operation only. The arc (which is established 75 the contact pair opens) is extinguished during the periodic current reversal. At the instant of zero current, the arc can be extinguished quite eusily with inexpensive contact materials and small gap spacings. A DC switch must be designed to extinguish an established arc with both voltage and current available to sustain it. Therefore, more massive erosion-resistant contacts are needed with an increased spacing for DC operation.

Appropriate controls are required for the operation of any appliance on DC. Even the simple on-off switches of most appliances would need modifications to operate safely. Arcarresting capacitors should be considered for all DC switches.

Electronic equipment, such as radios and environmental sensors, operate on DC, internally rectifying the $A C$ usually available from the utility. Many devices also operate directly on a battery power supply. Therefore, these devices are ideally suited to operation with a regulated DC output from a PV system. A solar array, if not regulated, may produce operating voltages ranging from $17 \mathrm{~V}$ when new and fully illuminated to $0 \mathrm{~V}$ when dark. Early in the morning, when the sun is just above the horizon, the operating output voltage may be 
only $10 \mathrm{~V}$. Therefore, the unregulated input to the electronic equipment will range from 10 to $17 \mathrm{~V}$. The same range may prevail if a battery provides the only means of regulation, because it operates from nearly $17 \mathrm{~V}$ when fully charged to approximately $10 \mathrm{~V}$ when fully discharged. Many electronic devices will not operate properly over this voltage range and, therefore, will require a system voltage regulator for proper operation.

\subsubsection{CONCLUSIONS}

Voltage regulation will be required under most circumstances. DC voltage control is usually established by designing the solar array voltage higher than the load requirements. The higher voltage is reduced to match the load and system through losses controlled by and incurred in the voltage regulator. $A C$ and $D C$ regulation can be accomplished by a motor/generator set. The motor/generator set converts array electric power to electric power for the load and has built in voltage regulators, power regulators, and frequency control. It is often used to supply syslems of different frequency than the main system, including DC/AC inversion and AC/DC sonversion. AC regulution cán glso he nscomplished by thyristor rontrol (rectifiers). The methods of regulation must be evaluated for each application to determine which is the most economical. This is especially important because the cost of some $A C$ equipment, such as induction motors, is much less than for the corresponding DC equipment. It does appear that electronic equipment is best driven by DC voltage from the array/battery system.

\subsection{LOAD/POWER SYSTEM INTERACTIONS}

Many problems which may be encountered when common components are connected to $A C$ and $D C$ power lines remain to be identified. Exhibit 6.17 lists the various problem areas.

\begin{tabular}{|c|c|}
\hline LOAD & POTENTIAL PROBLEM \\
\hline AC Motors & $\begin{array}{l}\text { Insufficient starting ourrent (turque) } \\
\text { Varying frequanoy } \\
\text { Overvoltage } \\
\text { Nonsinusoidal wave form }\end{array}$ \\
\hline DC Motors & $\begin{array}{l}\text { Inoutficieint starling current (torque) } \\
\text { Overvoltage }\end{array}$ \\
\hline Incandescent Lights & Sensitivity to voltage \\
\hline Fluorescent Lights & $\begin{array}{l}\text { Sensitivity to voltage } \\
\text { Lower efficiency }\end{array}$ \\
\hline Electronics & $\begin{array}{l}\text { Discontinuous voltage changes } \\
\text { from voltage regulator } \\
\text { Sensitivity to voltage }\end{array}$ \\
\hline Battery (Charging) & $\begin{array}{l}\text { Overvoltage } \\
\text { Overcharging }\end{array}$ \\
\hline
\end{tabular}

Exhibit 6.17 Load/PV Interactions. 


\subsubsection{AC MOTORS}

$A C$ motors would be driven from the array/battery combination through a DC-to-AC inverter. If a battery were used, the power system would probably have sufficient current capacity to start the motor. If no battery were used, the array would drive the motor directly and the output current of the array would be limited by the insolation. The problem is illustrated in exhibit 6.18 . When the motor is started, there is no back EinF generated, so the input impedance is very low. EMF is the electromotive force in volts. As a consequence, the load line is as indicated for high current and low voltage. When the motor is running at full speed, the load line is as indicated, corresponding to the higher load impedance due to reactance (back EMF). The zero-speed current is much higher than the running current (the starting current is very brief and looks like a spike function). As a consequence, the starting torque will be nearly equal to the running torque for some types of motors, such as synchronous and squirrel-cage. For universal motors, the zero-speed output torque could be too low to start the motor, especially if the motor were started under load. The load would be picked up as operating speed is approached.

Because permanent-magnet motors and series-wound motors have starting torques somewhat higher than running torques, they are the preferred motors to use with PV systems. The series-wound have a tendency to overspeed under light loads because the field strength is too weak at low currents.

AC motors are designed for a specific frequency, such as 60 or $400 \mathrm{cycles} / \mathrm{sec}$. If the frequency deviates from the design value, the efficiency of the motor will decrease. Frequency deviations of 10 percent would result in an increase in heat generation of 10 to 20 percent.

Waveform is another source of potential difficulties with $A C$ motors. If the inverter does not produce a perfect sine wave, the motor will be less efficient and may overheat. Exhibit 6.19 illustrates this problem. If the motor uses only the square wave equivalent of the sine wave, most of the square wave will not be useful in producing work output. Therefore, the difference in the electric power input from the sine wave and the power input from the square wave must be dissipated as heat. As indicated in exhibit 6.19 , the heat dissipation with a sine wave is 15 percent of the output mechanical power (for an 85 percent efficient motor); but the heat dissipation with a square wave is 115 percent of the output power, as an approximation. 'or a $1.0 \mathrm{hp}$ load, the motor must be rated for this extra input. As shown in exhibit 6.19 , the input electric energy must be 2.15 times the mechanical output for a square wave, so the dissipation is 1.15 times the output. Compensation for this much heat dissipation requires a motor size 7.67 (1.15/0.15) times what would be required with a sine wave, or $7.67 \mathrm{hp}$ for the illustrated case. There is a possibility that a capacitor could be built into the motor to decrease this effect; in fact, the inductive aspect of the motor impedance will provide some filtering, so the dissipation will be somewhat less than shown in exhibit 6.19. Capacitance decreases the reactance component of impedance.

Finally, some difficulties can be expected if the input voltage is allowed to vary significantly. Motors normally can tolerate $a \pm 10$ percent change; however, for the PV system without a battery, the voltoge swing will be from $0 \mathrm{~V}$ to possibly $17 \mathrm{~V}$. The synchronous and squirrel-cage motors will stall at low voltages because the load torque will exceed the pull-out torque. The universal motor will rotate at a speed proportional to the input voltage. If the motor is designed for $12 \mathrm{~V}$ and the solar. intensity is high enough to produce $17 \mathrm{~V}$, the high motor voltage could cause overheating, overspeed, and motor failure. Under most circumstances a voltage regulator is required. 


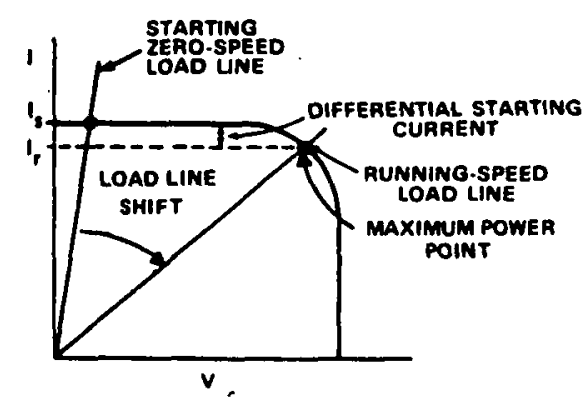

CONVENTIONAL STARTING CURAENT $=6$ - AUNNING CURRENT

MOTOR TORQUE: Proportional to Current

Synchronowe

Squirrel Cege

Sartea Wound

Proportional to Current and Voltage

Strumt Wourid

Compound Wound

$I_{g}=6 \times I_{r}$, thus $I_{g} \cdot I_{r}=5 I_{r}$.

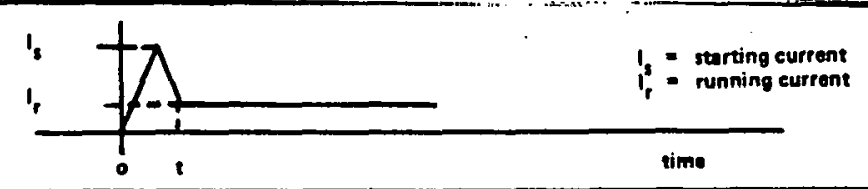

Exhibit 6.18 AC Motor Starting.

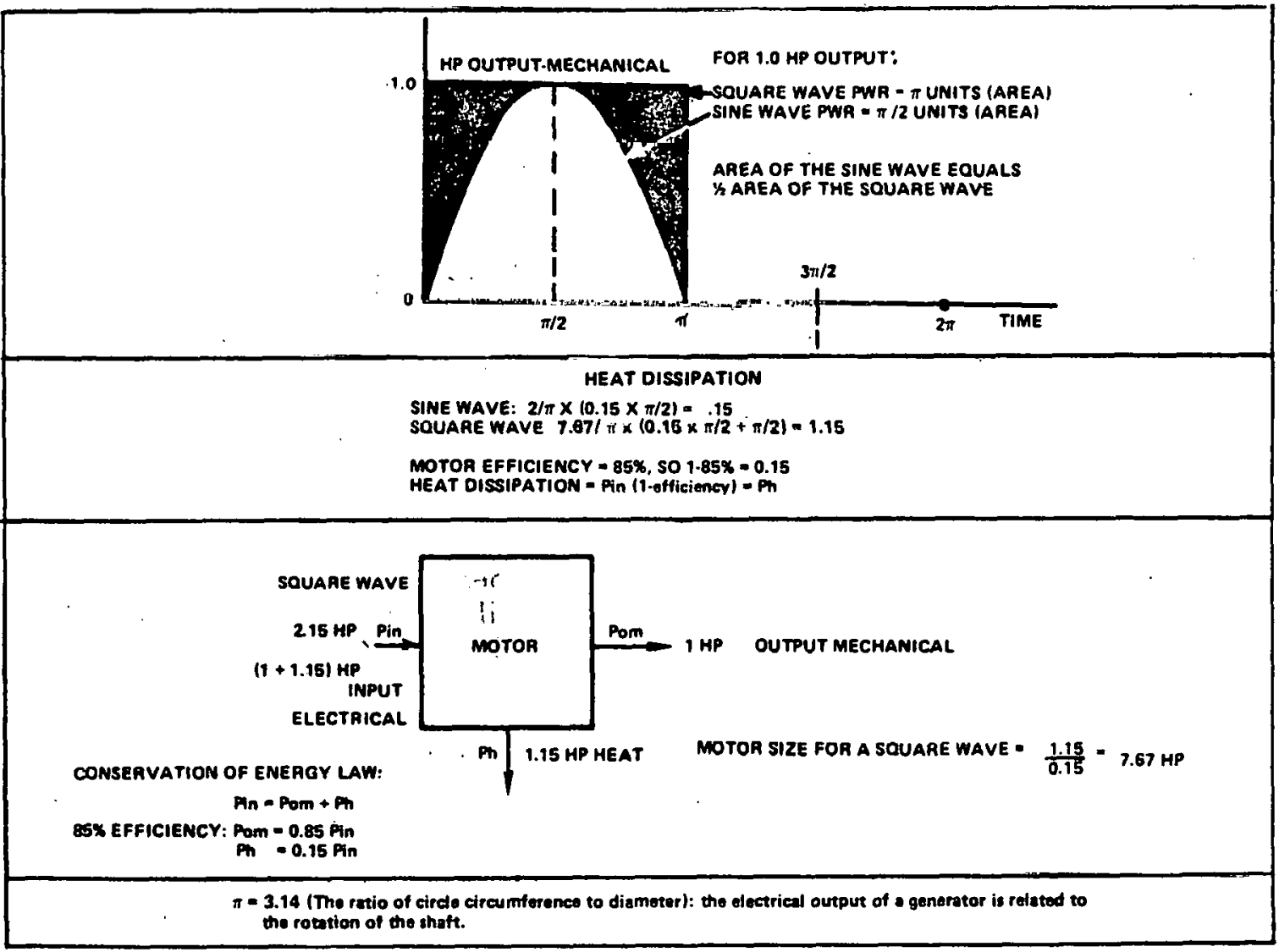

Exhibit 6.19 Estimated Derating for AC Operation Utilizing a Square Wave. 


\subsubsection{MOTORS}

DC motors encounter the same startup problems as AC motors. In addition, the lack of voltage control will cause similar problems. Wave form problems pertain only to AC motors.

\subsubsection{INCANDESCENT LIGHTS}

The output of lights is highly sensitive to the input voltage, so an unregulated DC source--even a battery operating from 11 to $14 \mathrm{~V}$--will cause a significant variation in light output. Over-voltage will seriously shorten the light life; a drop from $14 \mathrm{~V}$ to $1 \mathrm{I} \mathrm{V}$ will result in a drop in intensity to approximately 15 percent of the $14 \mathrm{~V}$ value. As with the motors, a voltage regulator is a good investment. In most designs the voltage regulator is placed upstream of the battery, so the same 11 to $14 \mathrm{~V}$ range will still occur. A separate voltage regulator for the lights might prove necessary.

\subsubsection{FLUORESCENT LIGHTS}

A fluorescent light is a glass tube or bulb coated on the inside with a fluorescent substance that gives off light when mercury vapor in the tube is acted upon by a stream of electrons from the cathode (negative terminal electrode).

Fluorescent lights need a high voltage to start, but then can run with low voltage. (A 12 W DC fluorescent is available.) A starting coil, similar to an automobile ignition coil, is needed to develop the initial high voltage to sturt the. lamp. The coil is usually triggered by a thermal switch. Under DC operation, the efficiency of the fluorescent is approximately 20 percent lower than that attainable with 60-cycle $A C$. In addition, if the ions migrate in one direction only, as they do under DC, the life of the electrodes is seriously degraded. Ions are positive charge atoms or molecules missing an electron. They do not necessarily move physically but rather transfer electrons. The effect is positive charge motion opposite the transfer of electrons. Reversing the direction of the DC on a daily bosis is recommended; ho'vever, if the thermal switch is an electronic switch, DC reversal is not possible. The 11 to $14 \mathrm{~V}$ range may also prove troublesome for maintaining light output.

\subsubsection{ELECTRONICS}

Because the PV system produces DC and electronic equipment normally use DC, powering of electronics is idedly suited to PV systems. Iviost electronics require voltage regulation within 1 or 2 percent, so the variation of orray output voltage can be mitigated only with a regulator. If the regulator is of the electronic switching type, there may be some radio interference from the array itself or from the noise introduced through the power lines to the electronics, because the switching produces square voltage pulses superimposed on the input voltage.

\subsubsection{BATTERIES}

Batteries represent a load during the charge period. If the array is sized to yield $17 \mathrm{~V}$, the voltage will be too high when the battery is fully charged. Overcharging, overheating, electrolyte boil-ofi, and battery failure can result. If the array voltage is designed for a lower voltage, the charge rate may be too small to permit recovery the next day from overnight discharge when the solar intensity is low. In most cases, the battery capacity will span many days, so the recovery rate need not be high; however, there is a definite tradeoff between battery size and array voltage. If the array voltage is designed to be as high as 17 $V$, a voltage regulator will be required to prevent the overcharging. 
The problem associated with charging many batteries in series was discussed in section 4. The combination of needs: charging batteries in banks of low voltage, availability of DC components at low voltages only, and safety, may dictate that the PV system is best designed for low-voltage DC operation. DC-to-AC inverters would then be used for those isolated components requiring $A C$ operation.

\subsubsection{INVERTERS}

DC-to-AC inverters can cause instabilities in the power system that could, when coupled with the output characteristics of the PV array, lead to loss of power. The stability criterion is that the power output of the power system must increase with voltage at a slower rate than the power consumption of the load. This instability can be exhibited by a PV system without a battery when operated at a voltage below the peak-power voltage. For this reason, nnd to permit operutiun of each power-conditioning component at its peak efficiency, there is o possibility that the mnst cost-effcctive systell deslgh will incorporate u DC-to-AC inverter at each $A C$-operated component.

\subsubsection{CONCLUSION}

Exhibit 6.20 provides a convenient checklist of potential problems that must be solved during the design phase of the project. The tradeoffs among the potential solutions will depend on the particular characteristics of each application.

\section{MODULE CHARACTERISTICS}

$\begin{array}{llrl}\text { Sensor Technology: } & 36 \text { cells } & 19.8 \mathrm{Voc} & 0.25 \text { Isc } \\ \text { Solarex }(2 \text { modules) } & 12 \text { cells } & 6.6 \mathrm{Voc} & 1.2 \text { Isc } \\ \text { ARCO } & 36 \text { calls } & 19.3 \mathrm{Voc} & 1.2 \text { lsc }\end{array}$

QUESTIONS TO BE ANSWERED IN THE LABORATORY:

1. How can the I.V curvo be determined for a module?

2. How can the I.V curve be used to judge the quality of the module?

3. How do Voc and lsc vary with insolation?

4. How do Voc arid lac vary when the module is shaded?

9. How aan you judie the current capacity of the module when charging the battery?

6. How can you test the diode that protects the module?

7. How does the output of a $\mathrm{CB}$ radio vary with modute shading?

B. How does the current dernund of a $C_{B}$ 'radio change with transmit/atandby operation?

9. How does the current demand of a permanent-magnet motor chinge with speed?

10. How does the output of a fluorescent lamp van with voltage?

11. How can you test a thodule for open-circuit or short-circuit cells?

12. What is the I-V characteristic of similar modules when connocted properly in parallel? When connected improperty?

13. Whet is the I-V characteriotic of similar modules when connected properly in soriest When connected improperly?

14. What is the I-V cheracteristic of an array consisting of grossly mismatched modules when connected in sories and parallel. properly and improperly? (Sensor Technology and one Solarex)

15. Describe the hot-spot problem. Can it be demonatrated with the four modules provided with the laboratory?

Exhibit 6:20 Checklist and Laboratory Analysis of Arrays and Systems. 


\subsection{LOAD ANALYSIS LAB}

The primary purpose of the laboratory portion of the seminar is to demonstrate PV hardware and subsystems, its construction, operating peculiarities, and potential problems in applications. Solutions to applications problems will be suggested. These solutions are not expected to be the final definitive answers, since PV technology is evolving ropidly. Exhibit 6.20 summarizes the questions the reader should be able to answer via the laboratory.

A series of demonstrations and experiments are planned to highlight various ospects of PV applications. After these demonstrations, there will be an opportunity for the participants to explore problems that have not yet been addressed by the seminar. Three kits of modular components and detailed instructions will be available for tests or experiments. Other seminar 'resources will be available. Participants should feel free to inspect the equipment and subsystems more closely, run their own tests with the demonstration kits, or ask questions during this question and answer session.

Exhibit 6.21 outlines the planned demonstrations and experiments. First, PV arrays have been set up outside to power several pieces of equipment. Lighting, pumping, and communications equipment are represented because the participants may be expected to deal with these applications.

Following the inspection of these PV powered systems, the effects of over-and undervoltage on both incandescent and fluorescent lighting will be demonstrated. Exhibit 6.22 shows the circuit arrangement that has been set up to explore the sensitivity of lighting to voltage. While it is unlikely the lights will be powered directly from a PV orray; the batteries used in the system can be expected to depend on their state-of-charge, exhibiting voltage swings similar to those of the PV array.

The same circuit, as shown in exhibit 6.22 , will be used for showing the effects of voltage variations on a television receiver. It should be noted that, for convenience, the arrays have been set up here to produce a nominal $12 \mathrm{~V}$ output because a variety of $12 \mathrm{~V}, \mathrm{DC}$ equipment is made for the boating and recreational vehicle markets.

Next, the effects of over- and under-voltage and the performance of DC motors driving different types of loads will be explored (exhibit 6.23). In porticular, a centrifugal water pump is used to typify a load characterized by zero torque ot zero speed. A diaphragm-type positive displacement pump typifies a full-torque-at-starting load. Because PV arrays are inherently current-limited power sources, the concern is that some types of DC motors may not be able to turn the full-torque-ot-starting loads. It may be desirable to specify series-wound permanent magnet motors, where available, or to use centrifugal pumps where the application permits. 


\section{EQUIPMENT}

Sensor Technology. Solarex and ARCO modules

Ammeter (5 A DC). Voltmeter (25 V DC), Rheostat (100 ahms)

Baneries $(2-6 \vee$ gel-cells $)$

CB radio. fan/permanent-magnet motor, fluorescent lamp

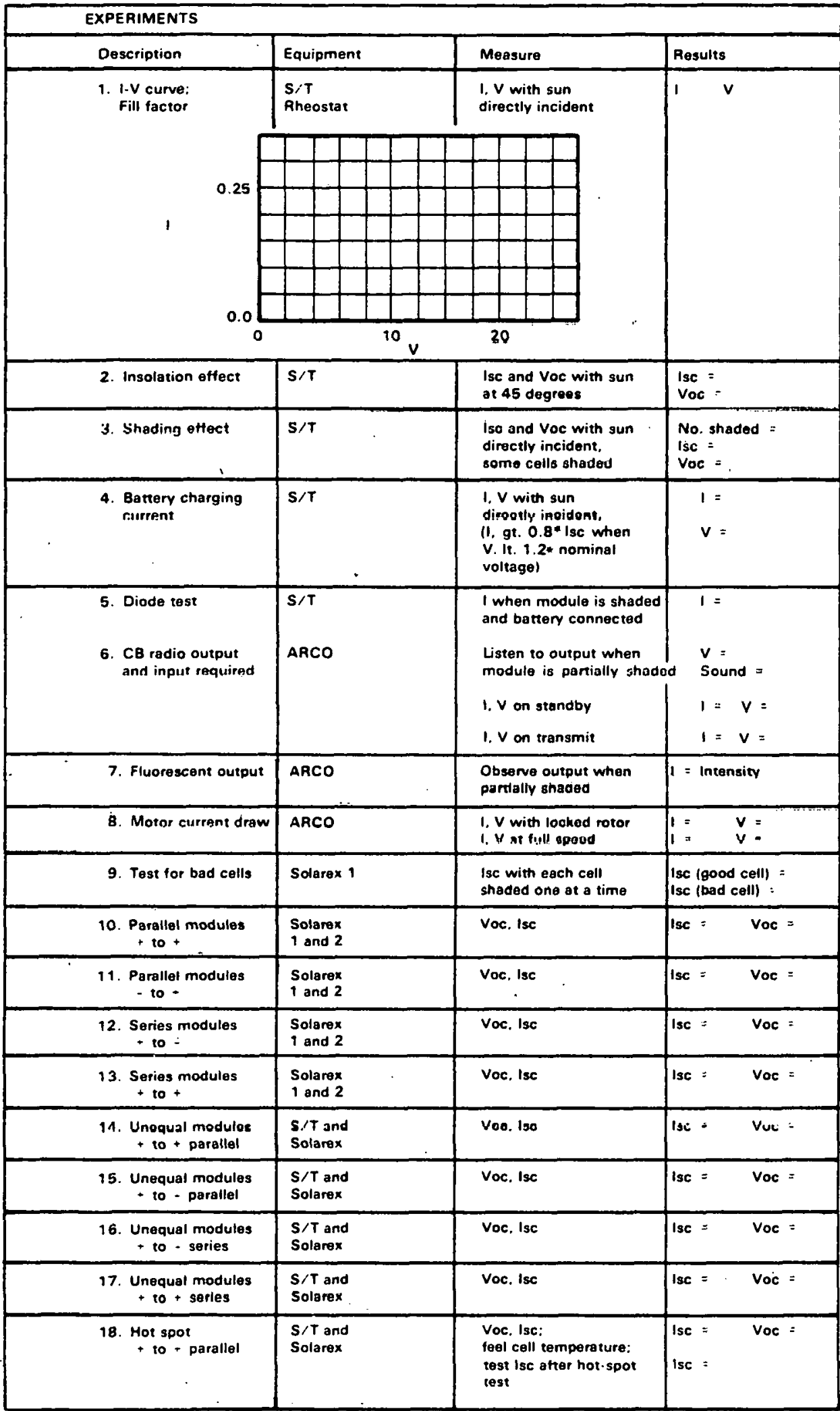

Exhibit 6.21 Laboratory Experiments. 


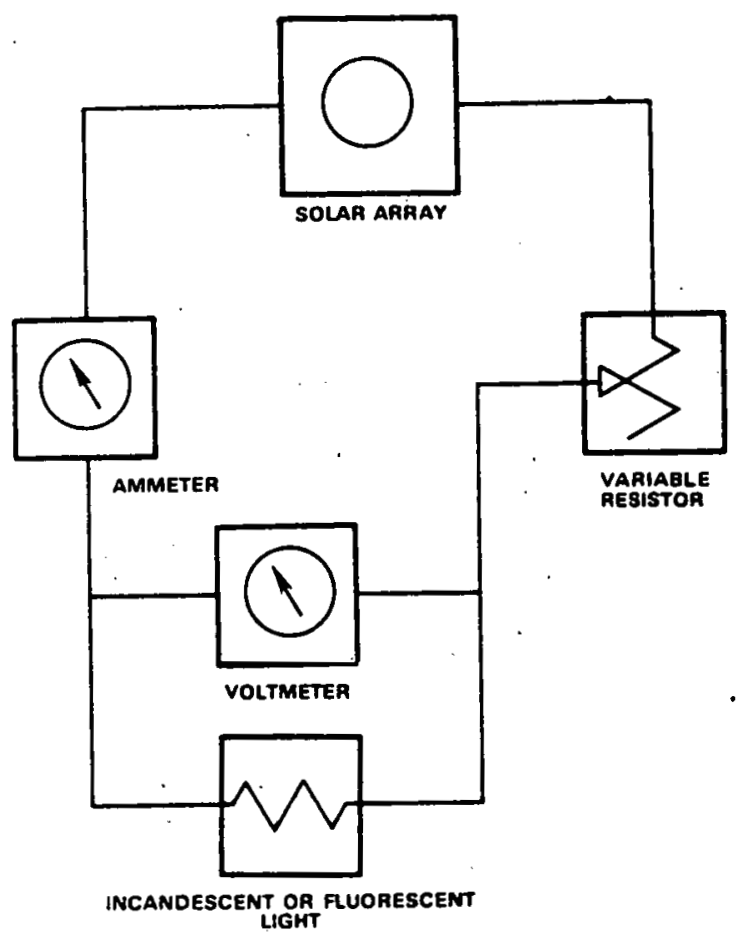

Exhibit 6.22 Effects of Variable Voltage on Lights.

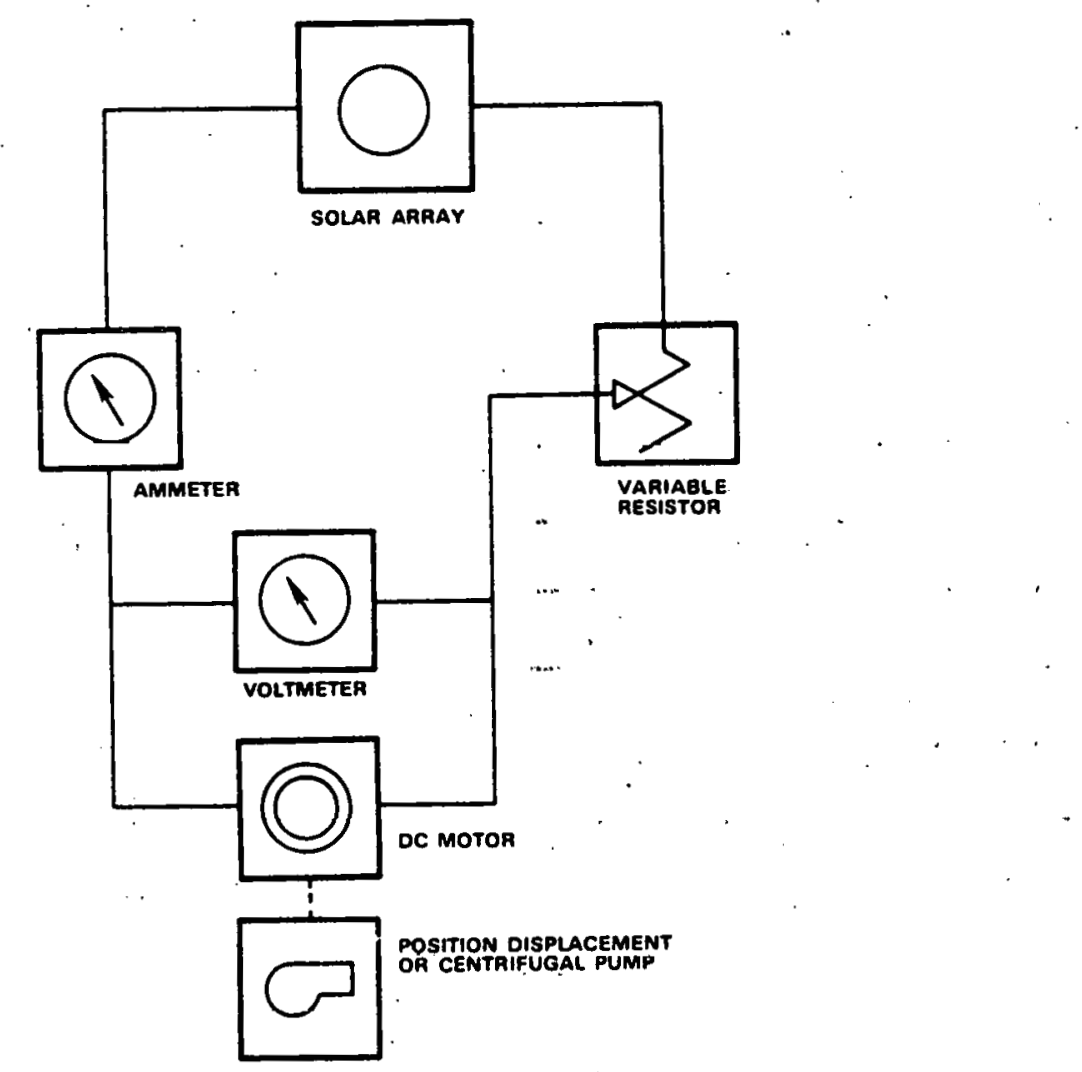

Exhibit 6.23 Effects of Variable Voltáge on DC Motors. 
Lastly, the effects of over-voltage and over-charging on batteries will be explored, because most applications will require batteries for energy storage. Batteries also hove the capability to provide some degree of voltage regulation to the output of the PV array and to provide higher starting currents than the array alone could provide to motors and lights.

\subsection{ELECTRICAL POWER FACTOR CORRECTION (SAME, AUGUST 1976)}

Emphasis on the need for conserving energy in AC systems makes the electrical power factor a subject of continuing interest. When providing the consumer with electrical service, the utility supplier is actually furnishing two types of AC electrical energy. The major type is known as the active or real kilowatt component upon which the cost of service is based. This is the component that is usable and does the work. The other kind of energy is the reactive or magnetizing kilovar component and is needed to magnetize any electrical equipment that requires a magnetic flux from the power system to permit operations. This type of energy does not cause the disk of the watt-hour meter to rotate, although it is drown from the power lines and furnished to the system. Every piece of electrical equipment or appliance whose operation is dependent upon a magnetic circuit requires a supply of exciting or magnetizing current. As an example, a transformer. or a common induction motor receives magnetizing current through the alternating current distribution systems. Accordingly, it can be seen that all inductive equipment such as transformers, motors, and fluorescent fixtures need two kinds of current:

- Working Current -- also known as usable, active and power-producing current which is converted into useful work by electrical equipment, and,

- Magnetizing Current -- also known as wattless, reactive, and nonusable current which cannot be converted into useful work by inductive electrical equipment but which is needed for operation.

Power factor may be defined as the ratio of the working current to the total of the working current plus the magnetizing current. Low power factor means only that an excessive amount of magnetizing current is being drawn from the incoming power lines. During the past years when electrical power could be considered relatively cheap and in plentiful supply, the taking of magnetizing current from the A.C. lines was not considered abjectionable if the effect of the extra current on voltage regulation was not too serious. However, as the tctal electrical load approaches the capasity of the utility supplier's generators, a low power factor will cause the generators to become overloaded and additional expensive generation equipment must be provided. Additionally, a low power factor results in the overheating of conductors and transformers and low voltage throughout the distribution system. This results in inefficient operation of electrical equipment and overheating of induction motors. 
Clarification of the power factor principle can be demonstrated by the following calculations. The power flowing in a direct current (DC) circuit is the product of the volts times the omperes and is expressed in watts. A wattmeter will accurately register this value. If one considers an $A C$ circuit; and the load is pure resistance, then again $E$ (volts) $\times 1$ (amperes) = watts. However, if the $A C$ circuit contains an induction motor, the wattmeter reading is less than the product of $E \times 1$. A fraction of the total amperes is consumed in magnetizing the motor and the balance is used to perform work. Consider the following case: a 240-volt motor draws 10 amperes as measured by an ammeter. The product of volts times amperes would equal 2,400 watts; however, when the power is measured with 'a wattmeter, it is found to register only 1,920 watts. It is apparent the current is doing other than providing useful work. The current necessary to provide the useful work is:

$$
\frac{1,920 \text { watts }}{240 \text { volts }}=8 \text { amperes }
$$

Accordingly, the total current is 10 amperes, and the power factor, previously defined as the ratio of the working current to the total current is:

$$
\begin{aligned}
& \frac{8}{10}=.8 \text { or } 80 \text { percent or as usually_stated: } \\
& \frac{\text { watts }}{\text { volts } \times \text { amperes }}=\frac{1,920}{2,400}=.8 \text { or } 80 \text { percent }
\end{aligned}
$$

In the preceding case, if it was found that the wattmeter had read 2,400 watts in lieu of 1,920 watts, then all of the current would have been performing useful work and the power factor would be 100 percent. This will not be found to be true, however, as the induction motor is a principal source of low power factor. All induction motors operate at considerably less than 100 percent power factor due to their magnetizing current requirements. Unfortunately, the magnetizing current needed is not proportional to the motor load and is very nearly the same at no load as at full load. Therefore, the power factor is dependent upon the load the motor is carrying and becomes lower as the load is reduced. The importance of selecting a motor according to the work it must perform is evident.

Most electrical systems contain quantities of working current and magnetizing current, and one type is just as effective in overloading the utility supplier's system as the other. The utility supplier must provide both, and the magnetizing current constitutes an addtional load without producing revenue. Many utilities compensate for this loss by including a charge for low power factor in their rate schedules. This is a penalty charge and is paid for by requiring the utility to provide magnetizing current as an extra service. A low power factor is not a desirable feature on an electrical distribution system and action should be taken to provide improvement or correction. This means neutralizing the effect of the magnetizing current by the addition of either:

- Synchronous motors, which do work while improving low power factor;

- Static condensors (capacitors), which are employed solely for power factor correction; or

- Solid-state devices, such as silicon-controlled rectifiers and thyristor switches, which are required where frequent and rapid variations in power factor correction are needed. 
Of these, the capacitor is generally the most economical method for power factor improvement and usually pays back capital investment spent for installation in six months to three years. Capacitors may be considered as generators that supply the needed magnetizing current to inductive loads. A bank of serveral capacitors is usually needed and these are normally cluster or rack-mounted on a power pole as near the load as possible.

Power factor correction should be initiated to secure the following benefits:

- Reduction of losses in generators, transformers, and distribution lines to conserve energy;

- Reduction in the cost of electrical bills, particularly when a power factor penalty clouse exists in the rate schedule;

- Increase in system capacity by elimination of excessive line current;

- Increase in voltage levels allowing efficient operation of all equipment; and,

- Increase in illumination from incandescent lamps.

The best solution to obtaining power factor correction is to have a qualified engineer survey the electrical system and select the proper location for the most efficient and economical equipment needed. Power factor calculations are slightly more complicated than indicated. Power factor is the cosine of the angle by which the current lags (or leads) the voltage in an AC circuit, complete with vector diagrams, plus the formula

$$
w=\sqrt{3} \times E \times 1 \times \operatorname{Cos} \theta
$$

where $w=$ three phase $A C$ watts, $E=$ voltage difference between phases (volts), $1=$ current (amps), $\cos \theta=$ power factor, and $\sqrt{3} \times E=3 \times V$ where $V=$ the voltage difference between neutral and phase. Three phase power equals $3 \times$ single phase power (phase to neutral). 


\section{SECTION 7 \\ BACKUP SYSTEMS}

\section{I NTRODUCTION}

If the system requires continuous power, a backup system would be required. The purpose of this chapter is to enable the attendee to list possible backup systems and to evaluate each possibility for his application (see exhibit 7.1).

\section{What Happens if the PV System Fails?}

PURPOSE: To enable attendee to:

\section{List possible backup systems.}

2. Evaluate pros and cons of each in context of the application.

\section{Exhibit 7.1 Backup Systems.}

As shown in exhibit 7.2, the key question to be answered in analyzing the application is: is the occasional loss of power critical? If the loss is not critical, there is no need for the backup system with its attendant complexities. The duration of the possible outage is also part of the criticality. If the system is frequently inspected and spare parts and maintenance crew are nearby, there may be little need for a backup system. Even if the power need is not critical, a backup system may be desirable if the repair cannot be accomplished in a short time. A reliability analysis may be required by legal codes and regulations, and often involves standard protection requirements.

Is loss of power critical?

How long will loss last?

\section{Exhibit 7.2 Need for a Backup System.}

\subsection{CAUSES OF POWER LOSS}

Exhibit 7.3 lists the primary causes of power losses. At this time, there are insufficient data to estimate the frequency of the various interruptions for solar PV power systems. Available information does indicate that total system failures are rare; the major causes of power failures are nature, design deficiencies, and hardware failures.

One known outage due to lightning occurred at Meade, Nebraska. A $25 \mathrm{~kW}$ irrigation facility was struck during its first 15 months of operation. The exact failure mechanism is still unknown, even though lightning protection is a fairly routine analysis. Long periods of little or no sun are another cause of power losses. In the design process, as will be seen in section 8, an allowance is made for the maximum number of low-insolation days. The design will not be based on the worst possible condition, but the worst experienced over the past 10 to 20 years. There is always a possibility that there will be less sim than considered in the design. Unless a backup system is provided, insufficient energy will be possible at a high probability. 
NATURAL

Lightning

Consecutive suinless days

Cold-weather effect on batteries

\title{
DESIGN
}

Less insolation than expected

More load than expected

Maintenance shutdown

\section{HARDWARE FAILURES}

\author{
Array short, opeil=circuit, full or partlal fallure \\ Optical degradation \\ Electronics-power conditioning \\ Batteries
}

\section{Exhibit 7.3 Causes of Power Loss.}

Cold weather, even if it does not cause battery failure, will cause a loss in battery capacity. This loss in capacity can result in deeper discharge of the batteries, with an attendant shortening of the battery life or a loss in the capability to store and later supply the needed energy. Cold weather is considered in the design, but nature may provide colder weather than anticipated.

Power may also be lost due to an inadequnte deșign. In some cases, the enginccring may be at fault. In other cases, basic data may not be sufficiently accurate to permit the development of an adequate design; for example, the insolation in many parts of the country can be estimated only to within 30 percent. Designing with a 30 perrent margin may make the system noncost-effective; the designer must use the best available information, and then qualifies the variance. Similar design deficiencies may present themselves if either the user or the designer underestimates the energy needs of the load or if the load is added to the system subsequent to completion of the system design.

Finally, the user must expect occasional interruptions during scheduled maintenance periods, if only to protect the maintenance worker. In some cases, the power need moy remain throughout the maintenance period and a backup system must be provided or already to go inlinie instantaneously.

The various causes of hardware failure, either partial or total, are listed in exhibit 7.3 under the last category. Optical degradntion of the outer surface is particularly important. In some circumstances, arrays covered with silicon rubber have experienced a 30-percent loss of power in 15 months. Cleaning restores much of this loss, although some ultraviolet degradation persists. Although an allowance is made in the design, loss of transmission through the optical coating could cause significant power losses and ultimately loss of the power supply to the load. Ultraviolet refers to sunlight of a particular energy level, and thus the optical effect on photoconductivity due to material propagation. 


\subsection{POTENTIAL BACKUP SYSTEMS}

Exhibit 7.4 lists several backup systems that might be suitable for the applications envisioned for remote sites. In many cases, the loss of power will not be critical, so backup will not be necessary. In judging the criticality, one must recognize the time that will elapse before the power can be restored. Maintaining some inventory of spares will help keep the elapsed time to a minimum. The modularity of the array will keep the cost of spares, if required, low. .

1. None (e.g., visit daily, carry spares)

2. Manual (e.g., pumping, pedaling)

3. Engine/Generator

4. Primary Battery

5. Emergency Solar-Rechargeable Battery

6. Emergéncy Fossil-Rechargeable Battery

\section{Exhibit 7.4 Potential Backup Systems.}

Manual backups are a viable, low-cost alternative. Hand pumping can be used for village water on an emergency basis, although provision must be made in the initial design for hand pumping. Centrifugal pumps cannot be manually operated, but positive-displacement pumps can. For small radio systems, pedal-powered generators can be used in emergencies.

For larger pumping operations or large-power operations, an engine can be justified for the backup system. If an engine is rarely used, it should be started regularly to prevent problems. The maintenance costs associated with the weekly operation must be factored into the analysis.

Battery backups can be the most desirable. Primary batteries, such as zinc-air batteries, can be used in some circumstances. Once discharged, the zinc-air batteries must be manually replaced, so the maintenance costs could be high. If standby rechargeable batteries are used, battery replacement can be avoided. For example, lead-acid batteries could be used that have their charge maintained by the solar cells, but are not connected to the main battery-storage system, which is used for nonemergency. energy storage. The advantage of the solar-recharged battery is that it can recover from an emergency condition without separate servicing by a mechanic. The batteries might also be recharged by a portable generator carried by the mechanic. The portable generator would be especially useful as a backup in the rare circumstance of extremely low insolation for many days. 
The various advantages and disadvantages just described are summarized in exhibit 7.5. Most of the systems would cost opproximately $\$ 150$ for $3 \mathrm{kWh} /$ day applications, if servicing can be accomplished within I day. An engine-generator is the only device that could be considerably more expensive, because the sets do not come in small sizes (less than $500 \mathrm{~W}$ ). Storage and safety of fuels for engine-generators and maintainability of the sets are some of the primary considerations in using portable generators.

A motor-generator set using electricity from a small electric utility consisting of a small transmission and distribution network is a secondary possibility. Natural gas utilities, and energy technologies such as the solar thermal-solar cell (PV) total energy system, and radioisotope powered photovoltaic cells are other secondary possibilities.

\begin{tabular}{|c|c|c|c|c|}
\hline BACK.UP SYSTEM & $\frac{\text { TYPICAL COET }}{--}$ & $\frac{\text { LOAD-kWW }}{3 k W h / D_{Q Y}}$ & $\frac{\text { ADYANTAOES }}{\text { Simole }}$ & $\begin{array}{l}\text { DISADVANTAGES } \\
\text { Nonsrititial } \\
\text { applicationg only }\end{array}$ \\
\hline 2. Manual & $\$ 150$ & ". & Simple. reliable & Require antendance \\
\hline 3. Engine & 2.000 & " & High power & $\begin{array}{l}\text { Frooventiy won't start: } \\
\text { high moiviterlarice }\end{array}$ \\
\hline 4. Primary Batrery & 150 & " & $\begin{array}{l}\text { Can be remote } \\
\text { pellable }\end{array}$ & One-shot only \\
\hline $\begin{array}{l}\text { 5. PV Recharged } \\
\text { Ballery }\end{array}$ & 150 & ". & $\begin{array}{l}\text { Can be remote } \\
\text { Aoliúlule }\end{array}$ & $\begin{array}{l}\text { Cannot recover } \\
\text { voithiuut sun }\end{array}$ \\
\hline $\begin{array}{l}\text { 6. Fossil.Fueled } \\
\text { Recharged Battery }\end{array}$ & 150 & . & $\begin{array}{l}\text { Can be remote } \\
\text { Retiatile }\end{array}$ & Fuel, engine transpon \\
\hline
\end{tabular}

Exhibit 7.5 Advantages and Disadvantages of Potential Backup Systems.

\subsection{APPROPRIATENESS OF VARIOUS BACKUP SYSTEMS}

The appropriateness of various backup systems depends on the application. The primary considerations are listed in exhibit 7.6. As shown, the first consideration is the remoteness of application. The second consideration is whether the loss of power is critical.

\begin{tabular}{|l|l|l|l|l|}
\hline \multirow{2}{*}{ APPLICATION } & \multicolumn{2}{|c|}{ UNATTENDED } & \multicolumn{2}{c|}{ INHABITED } \\
\cline { 2 - 5 } & CRITICAL & NONCRITICAL & \multicolumn{1}{|c|}{ CRITICAL } & NONCRITICAL \\
\hline Radio & Battery, alarm & Nono & Battery, pedal & Noilo \\
\hline Lighting & Battery, alarm & None & Engine & None \\
\hline Pump & Battery, alarm & None, alarm & Engine & None \\
\hline Refrigerator & Battery, alarm & None, alarm & Engine & None, alarm \\
\hline TV & Battery, alarm & None & Battery, pedal & - None \\
\hline Cathodic Protection & Battery, alarm & None, alarm & Battery, alarm & None, alarm \\
\hline Field Instruments & Battery, alarm & None, alarm & Engine, battery, & None, alarm \\
\hline
\end{tabular}

Exhibit 7.6 Appropriateness of Various Backup Systems. 
In many circumstances, the loss of power will be obvious because the equipment will not be working--the light will not come on, the radio will not receive or transmit. In other applications, the power loss will not be detectable. For example, because the refrigerator compressor operates cyclically, an observer will not know if the compressor is in an off period or is not being supplied by power. For these cases an alorm is needed to warn either that power has been lost or that the system is operating on emergency (backup) power.

Systems operating in a heavily traveled area and requiring large power consumption can use a gasoline or diesel engine for backup, because the engine operators and servicemen will be nearby. Under automatic startup, operation of the engine will be apparent; therefore; no alarm is needed in these coses.

\subsection{INTEGRATION OF THE BACKUP INTO THE SYSTEM}

Once the type of backup system has been selected, it must be integrated with the PV array. Means of integration are listed in exhibit 7.7. In monitoring or controlling an automatic actuator, a manual transfer switch is clean, direct, and involves no energy loss. The manual system requires inspection and onsite correction.

\section{MANUAL TRANSFER SWITCH}

\section{Simple}

No energy loss

Requires detection, onsite correction

\section{REMOTE MANUAL TRANSFER SWITCH}

No energy loss

Requires two-way telemetry

Requires actuator

\section{AUTOMATIC TRANSFER SWITCH}

\section{Continuous energy loss-possible only when on backup}

\section{Exhibit 7.7 Considerations for the Integration of the Backup into the PV System.}

For remote sites, especially those with a telemetry system that transmits when the system is not performing properly, a second communication link can be supplied by which a person at a central station can radio to the site for the backup system to come on. A remote actuator will be required for this type of backup, although the actuator can be an electrical relay or solid-state electronic switch. A telemeter is any device which measures physical phenomena (temperature, radiation, etc.) at some remote point and transmits the values obtained to a distant indicator, recorder, or observer. Transmission is by radio or wire. 
Automatic switchover is also possible, although the sensing and controlling function will then result in an additional power drain through the controller. It would be possible to have the relay or solid-state switch arranged so that power drain occurs only during those infrequent periods when the backup system is on, so the total energy loss would be insignificant. The relay would be the holding-coil type; the solid-state switch, a triac.

\subsection{CONCLUSION}

Except for a costly engine that might be required for a large-power backup supply, the backup systems add significantly to the reliability of the electric power supply system at minimal cost. Where required, the backup will add to the suitability of the solar PV system in remote applications. The use of back-up systems for supplement depends primarily on insolation, the electric load demand profile, and system capacity. Implementation involves the use of distribution switchboards or minor switching, and manual or automatic control. There ore some applications which ore not isolated but becouse of the importance of application and the duty cycle, require high reliability back-up regardless of remoteness. 


\section{SECTION 8 \\ PV SYSTEM DESIGN}

\subsection{NTRODUCTION}

This section presents an introductory detailed engineering method for the design of a solar PV system. This type of design is frequently used by system designers, however, it is too conceptual to be used as a design manual.

An overview of electric power system design: was given in the Introduction to this seminar (see section 1). Since then, the discussion has been centered on each subsystem and component in the system and how they interact with the load. This section takes what was discussed in the previous chapters and integrates it into a system schematic and an analysis and synthesis of system performance, which involves basic, equivalent networks and block diagrams, load analyses, insolation computation, battery energy storage, and system capacity.

In designing an electric power. system, one follows the sequence shown in exhibit 8.1. The load is analyzed according to the procedures discussed in section 6.

\section{DESIGN SEQUENCE}

1. Load Analysis

- Chaprar 6

2. Quick Sizing of Components-Monthly Analysis

3. Computer Analysis of Performance

\section{Exhibit 8.1 PV System Design.}

Exhibit 8.2 illustrates typical system schematics for PV power supplies. The top exhibit shows a system supplying DC; the bottom, AC. To illustrate the method for sizing a system and computing its performance, an example of a DC system will be provided. Power is sent from the solar array, through a protecting diode, either to a battery or directly to the load, or both. If the voltage being supplied by the array is too large (for example, over (4.4 V), the shunt regulator will be activated so the excess power is dissipated in the regulator and the output voltage is maintained at approximately the battery voltage (for example, 14.4 V). If insufficient power is being supplied by the array, the battery will supply the deficit. 

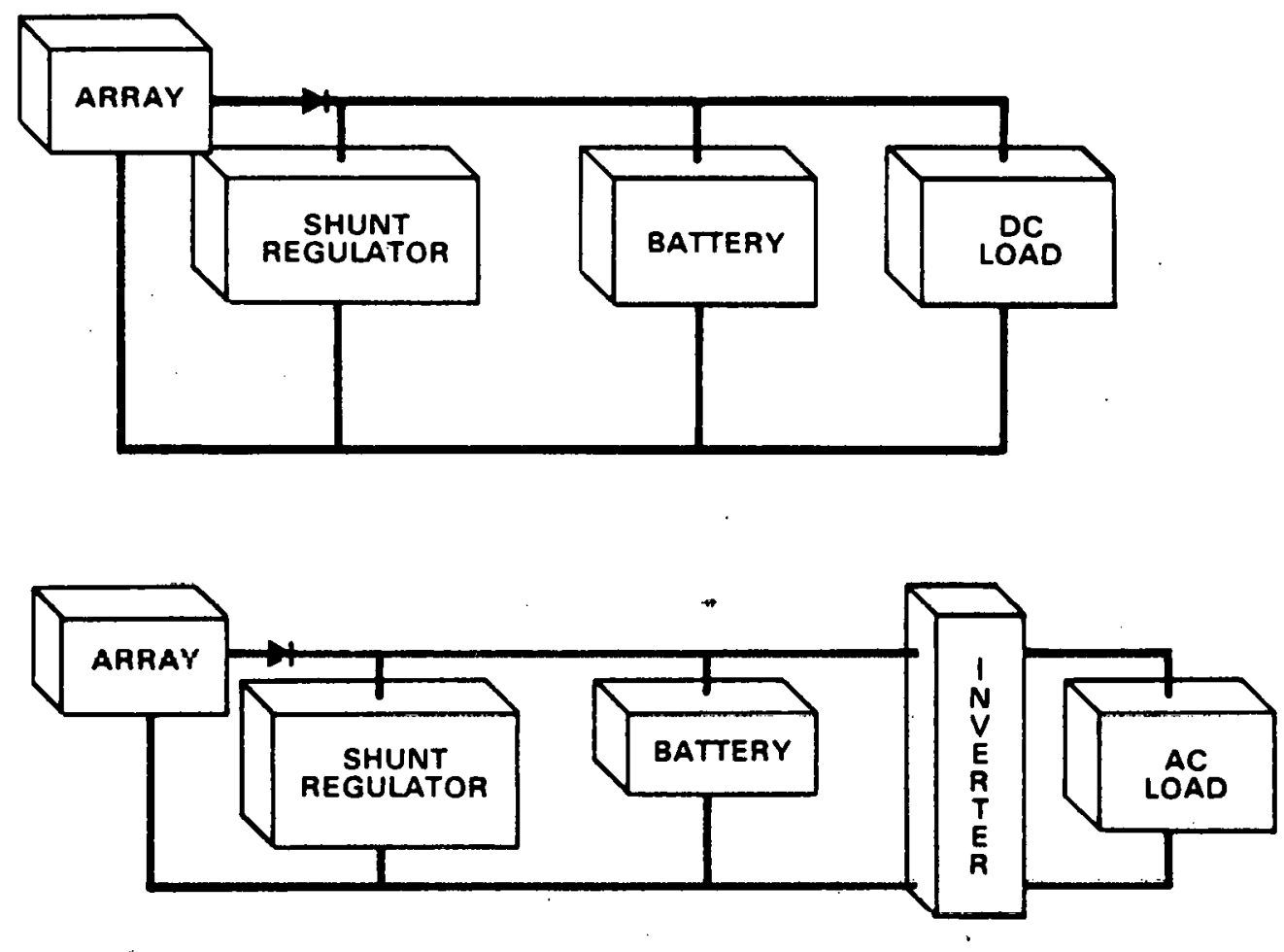

4

Exhibit 8.2 Representative PV System Schematic for DC and AC Loads

With the schematic now defined for the typical PV application, the sizing of the system and its subsystems and components can be addressed. An analysis and synthesis of the system performance will follow.

\subsection{QUICK SIZING OF COMPONENTS AND SUBSYSTEMS}

Exhibit 8.3 outlines the steps to be taken in estimating the sizes of the components. These sizes will be used in the detailed analysis of "the system performance. Component sizing is an iterative process aimed at selecting the combination of sizes that minimizes the system life cycle cost. The collector tilt, collector area, and battery size are the key factors governing the minimization process. The collector tilt is a compromise among the seasonal demands and available insolation during each of the seasons. The tilt that maximizes the energy collected in January may provide too little energy in July, or may require such a large collector area that a combination of less tilt, less area, and larger batteries may be less costly. One-axis and two-axis tracking collectors are being studied in the solar thermal power systems program, solar heating/cooling, and solar total energy system programs. Insolation is the rate of solar radiation per unit of surface area. 


\section{SEQUENCE}
A. Select array size, tilt
B. Compute insolation on average day for each month
C. Compute output of array

STORAGE REQUIRED

D. Compute costs of array, battery

E. Repeat for other array sizes, tilts until minimum cost is found.

\section{Exhibit 8.3 Quick Sizing of Components.}

The sequence illustrated in exhibit 8.3 calls for the estimation of the collector tilt and size. The computational procedure will yield the required battery size. The system cost can be computed on the basis of the array and battery sizes. As shown in step $E$, the process is repeated with other tilts and array areas until the minimum cost is determined.

The method for selecting array size is outlined in exhibit 8.4. First, the tilt angle must be estimated. If the tilt angle is equal to the latitude of the installation, the noon insolation will be the same in winter as in summer. However, the array will produce a righ energy output during the summer, due to the longer summer days, and a low output during the winter. By increasing the design tilt angle to be $5^{\circ}$ to $20^{\circ}$ greater than the latitude angle, significantly more energy can be obtained ot winter noon, partially offsetting the shorter day. The tilt angle should be selected as latitude plus $15^{\circ}$. For illustrative purposes, the first tilt angle to be evaluated is $45^{\circ}$, as indicated in exhibit 8.4 . The insolation for this tilt angle is obtainable from available maps of insolation on tilted surfaces, such as shown in exhibits 8.5 to 8.8 . For the first iteration, the collector size should be selected on the basis of the minimum insolation over a 4-month period. Data from the maps can be used to estimate this minimum, as will be illustrated in a subsequent section of this section, where the monthly insolation values are tabulated (see exhibit 8.19). The average of the three lowest months is shown in exhibit $8.4\left(4.0 \mathrm{kWh} / \mathrm{m}^{2}\right.$ per day). Insolation is given as energy per unit orea per day.

The electrical energy available to the load is equal to the incident solar energy times the electrical efficiency of the PV power system. The efficiency of the power system must be estimated taking the load demand into consideration. An initial estimate of the needed array size can then be mode.

Exhibit 8.2 illustrates a system whose components display the following throughput efficiencies: PV cells - 7.5 percent; array interconnections and leads -95 percent; and, battery storage -80 percent. 
1. Select tilt of $45^{\circ}$ for convenience, since data is readily available

2. Use the load computed according to the methods of Chapter $6,3.6 \mathrm{kWh} /$ day for this example

3. Compute the minimum 3-month insolation lor take it from charts such as exhibit 8.6: Insolation $=4.0 \mathrm{kWh} / \mathrm{day}-\mathrm{m}^{2}$

4. Compute the average system efficiency

For power directly from array to load

0.075 collector efficiency $\times 0.95$ distribution efficiency $=0.07125$

For power from array to battery to load 0.075 cullectoi efficiancy" 0.95 dist,ibutiun efficiency $\times 0.8$ battery officiency $=0.057$

Weighted average based on $8 \mathrm{hr}$ direct and $16 \mathrm{hr}$ via the battery: $(8 \times 0.07125+16 \times 0.057) / 24 \mathrm{hr}=0.06175$

5. Compute array area

Array area $=10 a d /$ (insolation $\times$ system efficiency)

Array area $=3.6 \mathrm{kWh} /$ day load $/\left(4.0 \mathrm{kWh} / \mathrm{day}-\mathrm{m}^{2}\right.$ insolation $\times 0.06175$ system efficiency) $=14.67 \mathrm{~m}^{2}$

6. Compute peak output from the array

Peak output $=1.0 \mathrm{~kW} / \mathrm{m}^{2}$ peak insolation $\times 0.075$

array efficiency $\times 14.67 \mathrm{~m}^{2}$ array area $=1.1 \mathrm{kWp}$

\section{Exhibit 8.4 Estimating the Array Size and Tilt.}

Because the battery is bypassed during the daytime, a weighted average of the day and nighttime efficiencies must be used as an estimate of the system efficiency.

Daytime efficiency $=(0.075 \times 0.95) \times 100 \%=7.125 \%$.

Nighttime efficiency $=(0.075 \times 0.95 \times 0.80) \times 100 \%=5.7 \%$

If the load is constant and there is an average of 8 hours of daylight per day at this site, the overall efficiency of the system is:

$$
\text { System efficiency }=\frac{7.125 \times 8+5.1 \times 16}{24}=6.115 \%
$$

The initial estimate of the needed array size can now be calculated.

$$
\text { Array size }\left(\mathrm{M}^{2}\right)=\frac{(\text { Load demand/day }(\mathrm{kWh} / \text { day })) .}{\begin{array}{c}
\text { Efficiency } x \text { average insolation of the } \\
4 \text { lowest months }\left(\mathrm{kWh} / \text { day }-\mathrm{m}^{2}\right)
\end{array}}
$$$$
=\text {. }
$$$$
3.6
$$

$$
0.06175 \times 4.0
$$




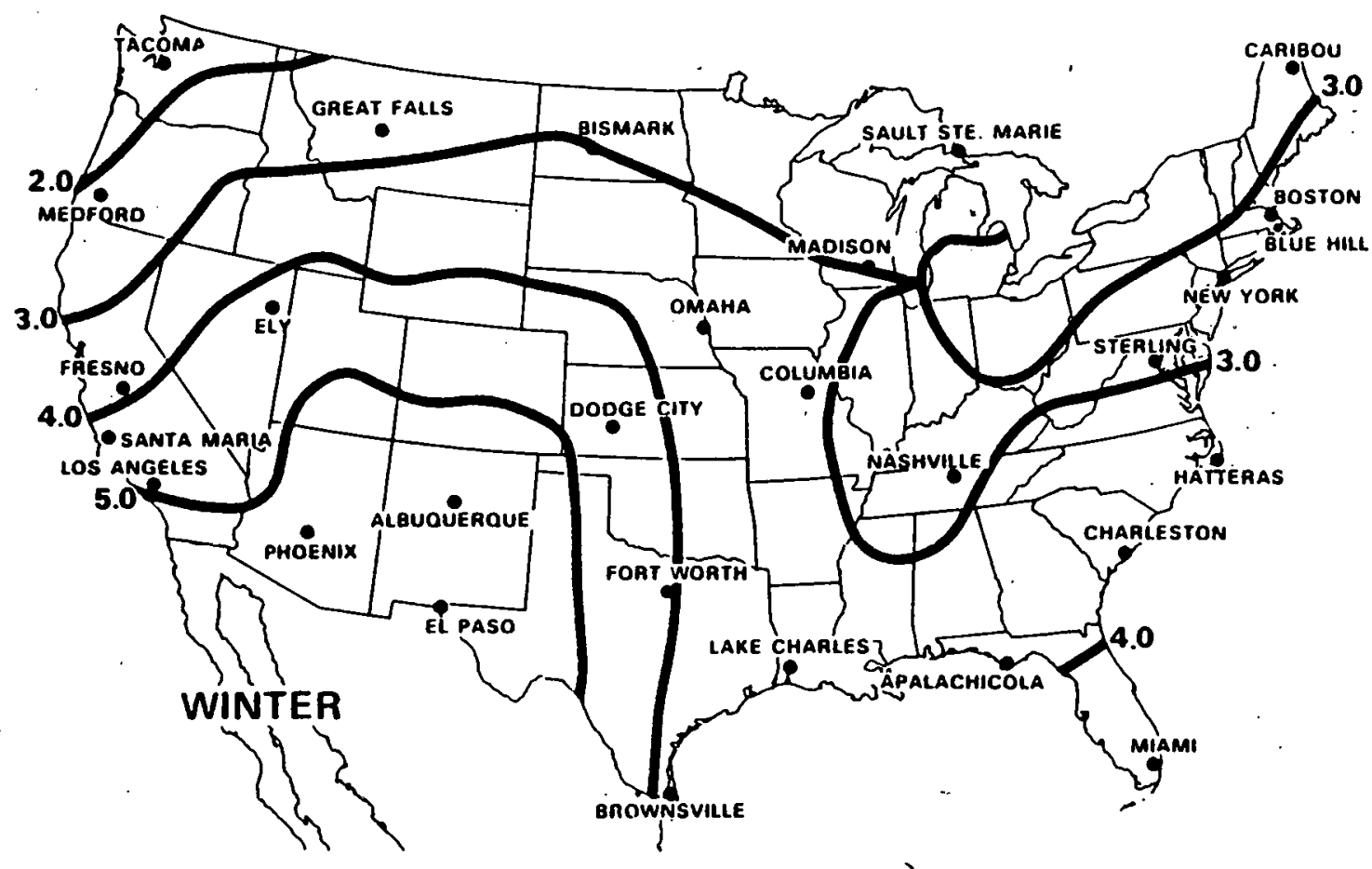

Exhibit 8.5 Total Insolation on a Collector Tilted $45^{\circ}$ above the Horizontal: Winter.

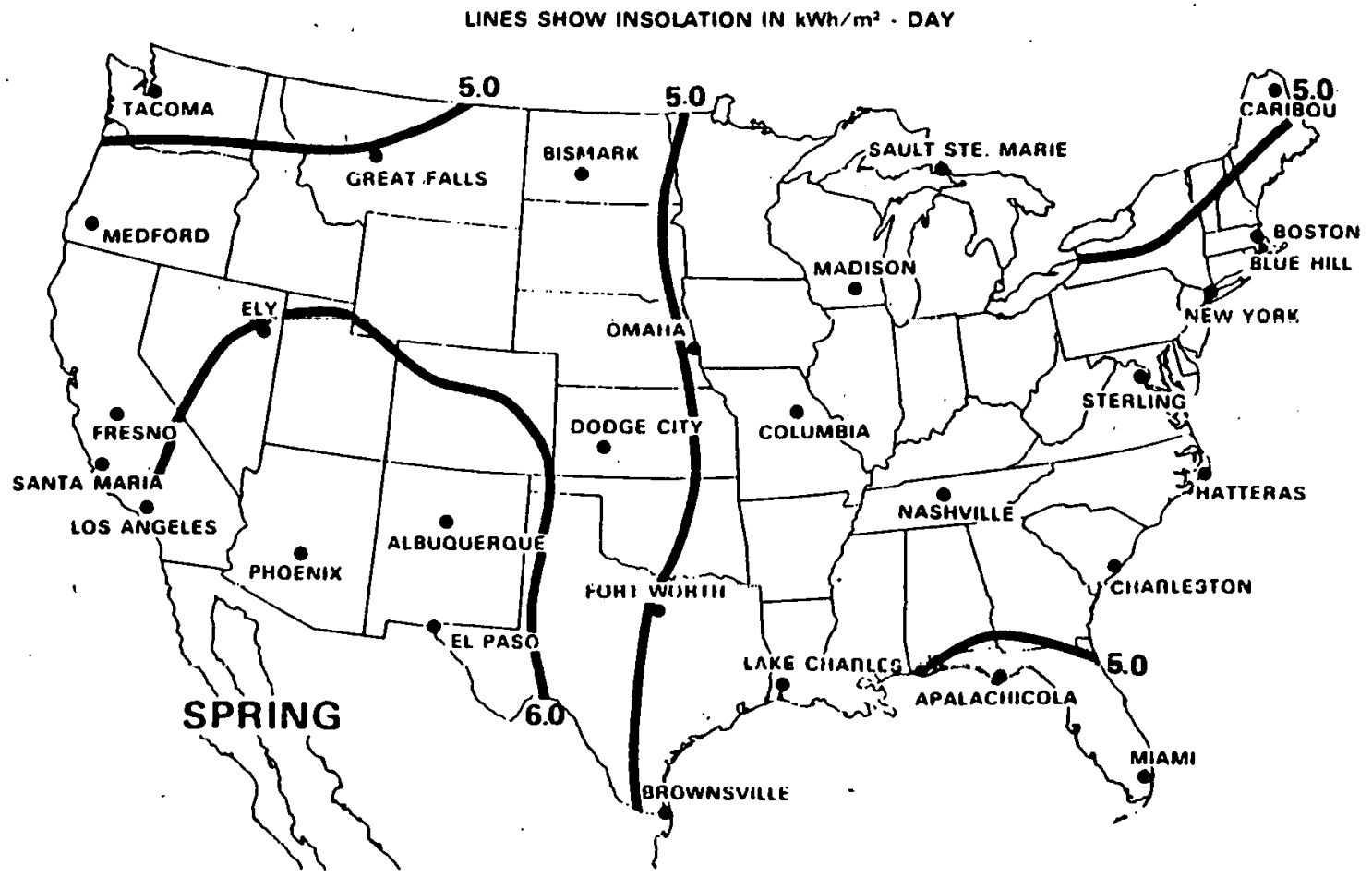

Exhibit 8.6 Total Insolation on a Collector Tilted $45^{\circ}$ above the Horizontal: Spring. 


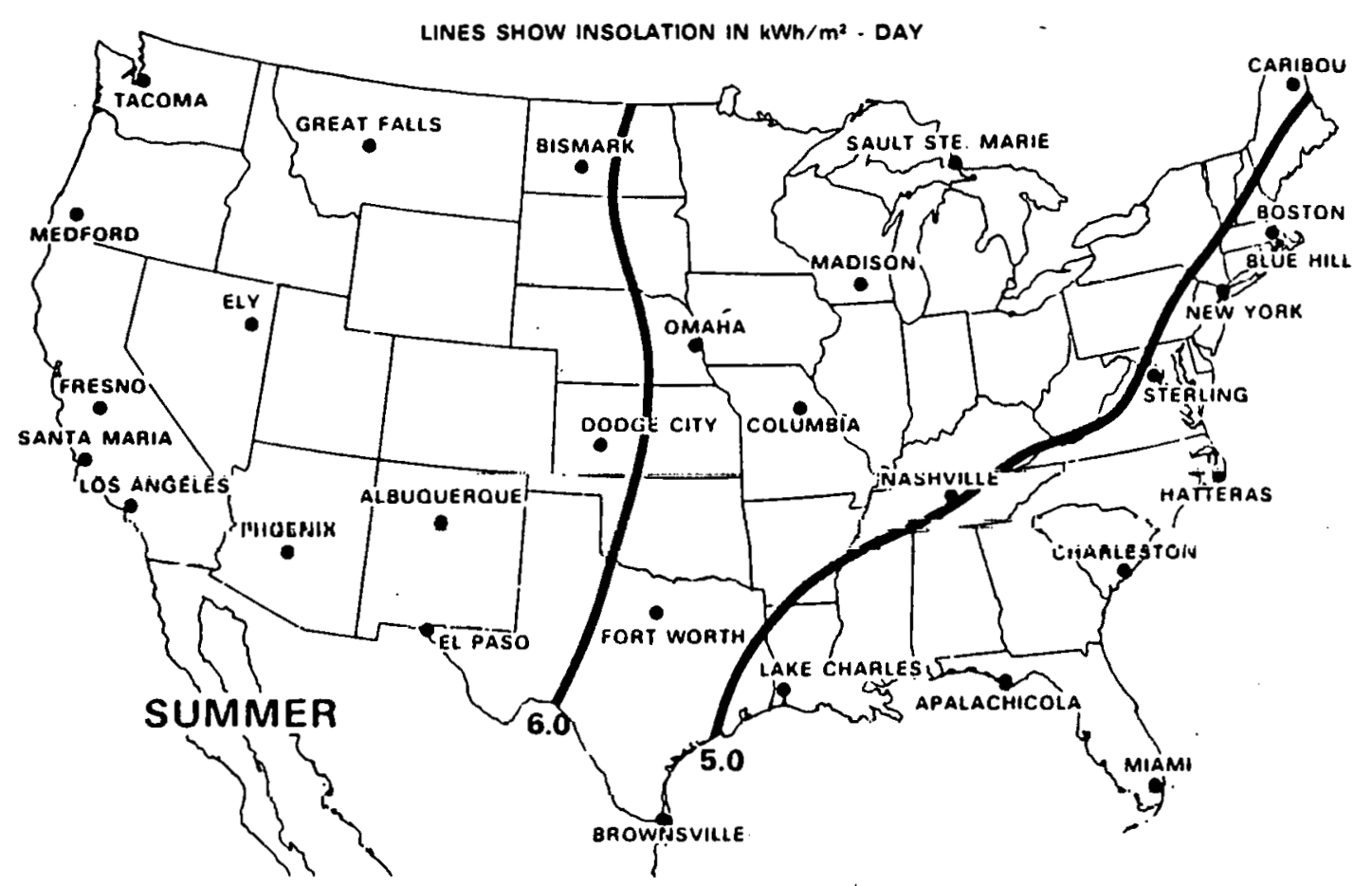

Exhibit 8.7 Total Insolation on a Collector Tilted $45^{\circ}$ above the Horizontal: Summer.

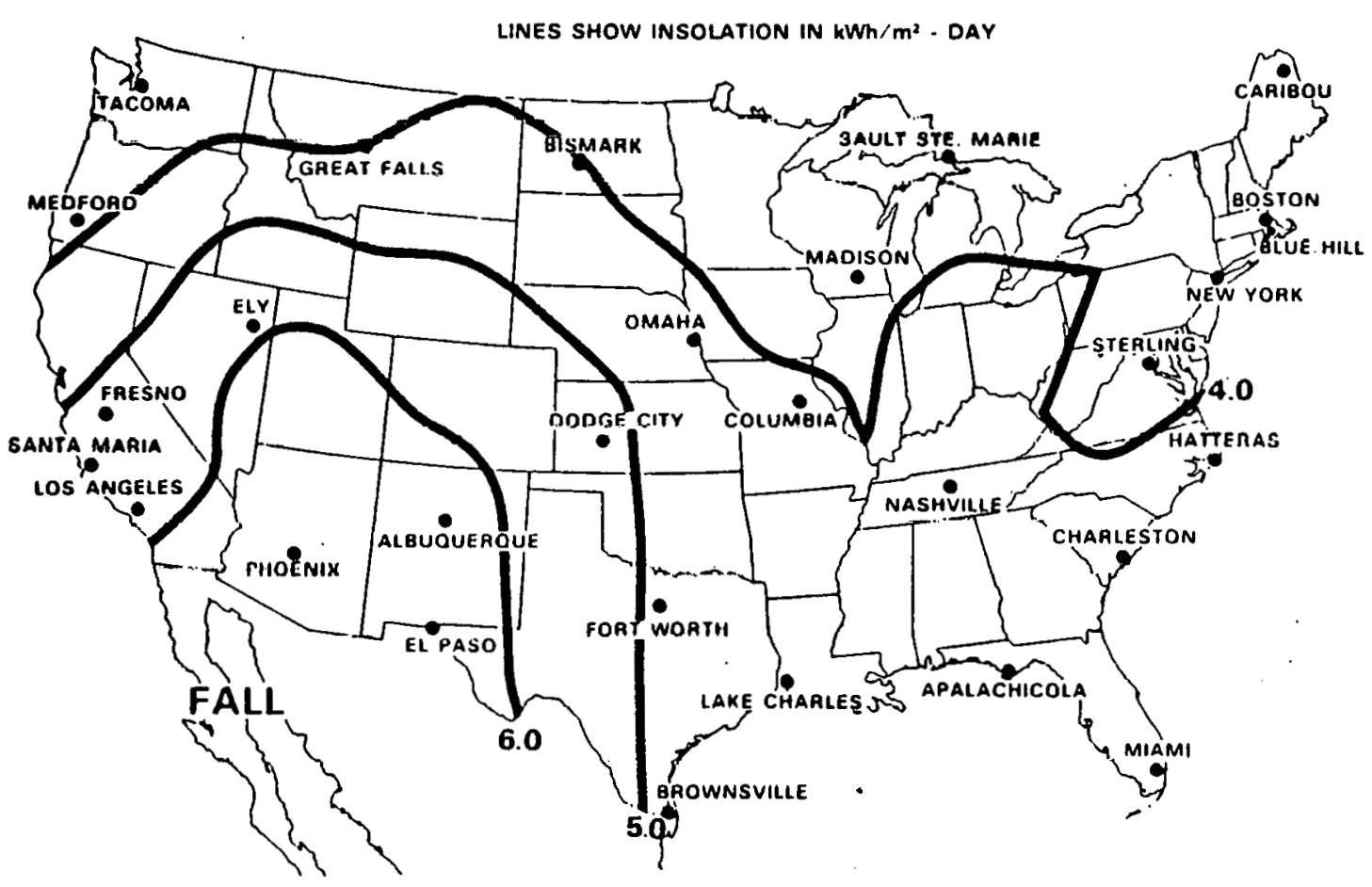

Exhibit 8.8 Total Insolation of a Collector Tilted $45^{\circ}$ above the Horizontal: Fall. 
The array size can also be expressed in terms of the peak electric power output when the insolation is $1.0 \mathrm{~kW} / \mathrm{m}^{2}$, a common rating point for arrays. In terms of output,

$$
\begin{aligned}
& \text { Array size }(\mathrm{KWP})=\text { area } \times \text { array efficiency } \times 1.0 \mathrm{~kW} / \mathrm{m}^{2} \\
& =14.67 \times 0.075 \times 1.0 \\
& =1.10 \mathrm{kWp} .
\end{aligned}
$$

This is the size and the tilt of the array that will be used in the first more-detailed computation of system performance.

The first step in estimating the system performance more accurately is to determine the insolation on the tilted array. This step was indicated as step B in exhibit 8.3. The computation of the insolation is perhaps the most complicated computation required to estimate the system performance in detail. The following section deals with on approximation for conceptual design, and incorporates resource assessment of the solar thermal power systems program, the solar heating/cooling and solar total energy systems programs.

\subsection{INSOLATION COMPUTATION}

\subsection{INTRODUCTION}

The output of the solar cells depends on the amount of sunshine striking the solar cells. One of the first steps in computing the system performance involves the computation of the solar input, or insolation (see exhibit 8.9). The procedure that will be followed here uses the method devised by Liv and Jordan and published by ASHRAE and is applicable to systems anolysis and synthesis. The method was applied by Duffie and Beckman in the development of the insolation tables. After reading this section, the reader should be able to use these tables and compute the insolation on solar cells tilted at any angle, facing south (north in the southern hemisphere), at any location in the world. The data are most accurate in the United States, where some of the empirical functions were determined. For the present purposes, the method will be accurate enough anywhere.

\section{OBJECTIVES:}

To enable the reader to:

1. Use the tables of insolation.

2. Compute the insolation

On tilted eollectors

Anvwhere in the world.

\section{Exhibit 8.9 Computation of Insolation.}


The data for Fairbanks, Alaska are in total disagreement with the other data, apparently due to instrument error. The correlation between cloud cover, $C C$, and. $\bar{K}_{T}$ is shown in exhibit 8.13. The scatter is considerably greater. If $C C$ were 0.5 , the $\bar{K}$ could be somewhere between 0.50 and 0.65 , a 27 -percent range. If $F$ were $0.65, \bar{K}_{T}$ would be between 0.51 and 0.61 , a range of 18 percent. In estimating insolation, pyranometer data should be sought first, fraction-sunshine data second, and, as a last resort, cloud cover data.

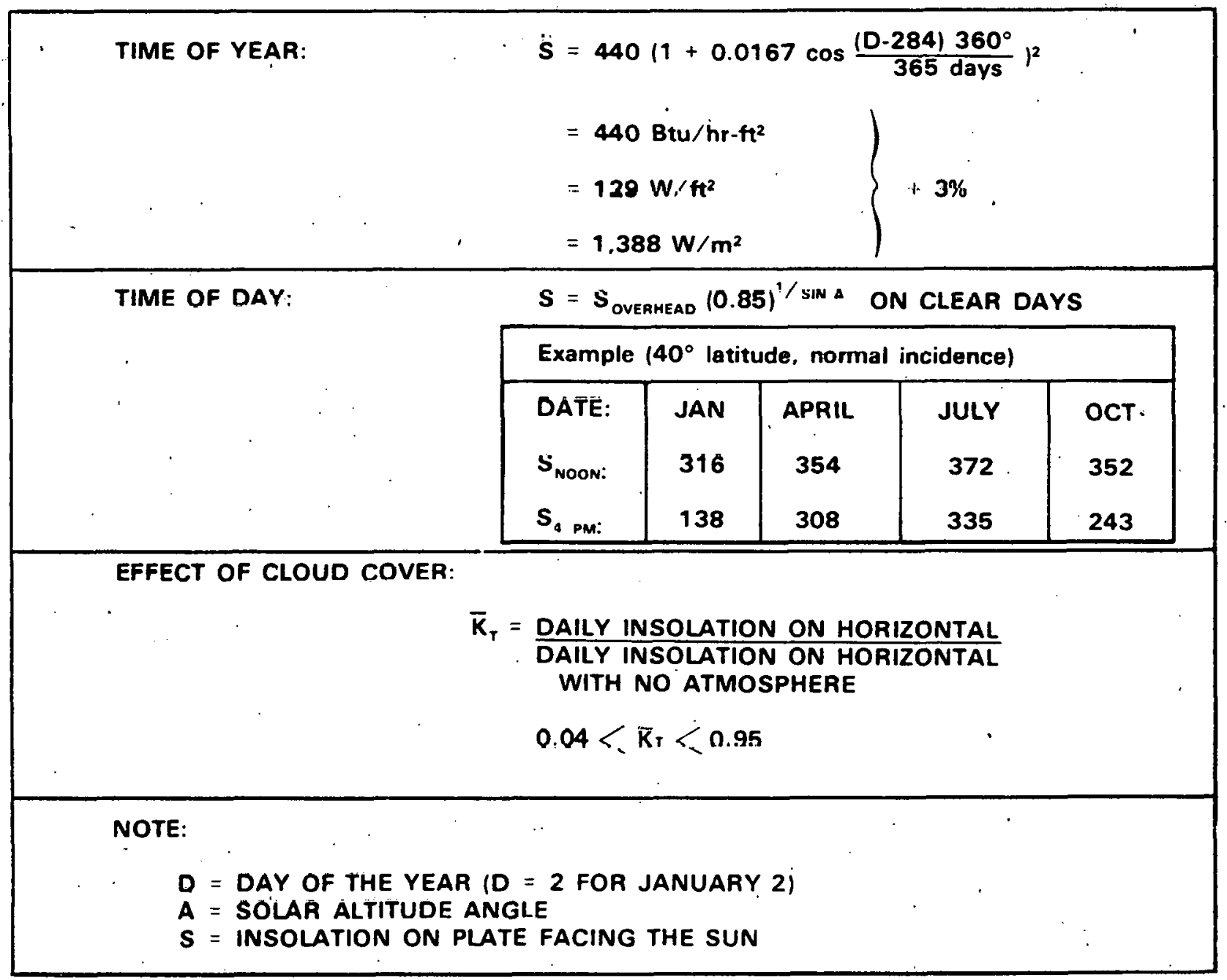

\section{Exhihit 8.10 Varrability of Insolation.}

Possibly the best procedure for estimating insolation at a site with no pyranometer data is to find the closest station having pyranometer data. Then the correlations for fraction sunshine or cloud cover can be used to adjust the pyranometer data to the location of interest. 


\subsubsection{VARIABILITY OF INSOLATION}

The computation of insolation must take into account the variation of insolation throughout the year, throughout the day, and throughout clear and cloudy days. Exhibit 8.10 shows the three effects and gives numerical evaluations of each. Because the distance between earth and sun varies throughout the year, the insolation varies plus or minus 3 percent over the year. Outside the atmosphere, on a plate held perpendicular to the solar rays, the solar flux is $129 \mathrm{~W} / \mathrm{ft}^{2}$ or $1,388 \mathrm{~W} / \mathrm{m}^{2}$, on the average over the year, where flux equals power per unit area.

Of more importance than the variation throughout the year is the variation throughout the day. At noon, the solar rays must penetrate the least amount of atmosphere, so the attenuation is least. On a clear day, the insolation at noon is approximately 85 percent of the insolation that would impinge on a plate outside the atmosphere. At times other than noon, the solar rays must penetrate a greater depth of atmosphere. The effect of the penetration distance is given by the simple power low shown in exhibit 8.10. If $A$ is $30^{\circ}$, sin $A$ is 0.5 and the reciprocal of $\sin A$ is 2 , then the atmospheric transmission is $0.85^{2}$, or 0.5625 (approximately 56 percent). The table in exhibit 8.10 provides additional examples for a site at $40^{\circ} \mathrm{N}$ latitude. It is evident that at $4 \mathrm{p} . \mathrm{m}$. in January, the transmission is quite low, whereas at $4 \mathrm{p} . \mathrm{m}$. in July, the transmission is still high. (The plate is assumed to be held perpendicular to the sun's rays and the numbers given are for the direct sunlight only. The total sunlight would be somewhat higher due to reflection from the same atmospheric particles that cause the loss in direct transmission.)

\section{DEFINITION OF $\overline{\mathrm{K}}_{\mathrm{T}}$}

Cloud cover has the greatest single influence on insolation. If a ratio, $\bar{K}_{\mathrm{T}}$, is defined as the ratio of total flux on a horizontal surface over the entire day to the fotal flux that would be received if there were no atmosphere (called the extraterrestrial flux), then $\bar{K}_{T}$ would be found to range from 0.05 to 0.95 , normal values being approximately 0.60 . Thus, the clouds can cause the insolation to be only 5 percent of what might otherwise reach the surface of the earth.

\subsubsection{MEASUREMENTTS OF INSOLATION}

The most accurate measurement of local insolation is obtained from a heat-flux meter called a pyranometer. The instrument measures the temperature reached by a blackened cavity when exposed to sunlight and protected from the ambient air b; a glass dome. The instrument is calibrated by illuminating it with a beam of known intensity. Instead of a black cavity, some pyranometers measure the temperature difference between black and white strips and infer the insolation from this temperature difference. These black-and-white pyranometers are more difficult to calibrate and degrade more rapidly than the more precise black-cavity pyranometer. Intensity is defined as energy per unit time per unit area.

Few weather stations are equipped with pyranometers. As indicated in exhibit 8.11, some have Campbell-Stokes sunshine meters while others report the weatherman's estimate of the fraction of the sky that is covered by clouds. The Campbell-Stokes instrument focuses the sunlight onto a strip of paper, which is burned when the sunlight is bright enough. The length of the burn indicates the hours of sunshine. Actually, the instrument indicates the hours the sunshine was bright enough to burn the paper, so the hours are the hours of direct sunlight. Newer sun-actuated switches provide similar data. Exhibit 8.12 compares a correlation between (hours of sunshine as a fraction of possible sunshine $F)$ to $\left(\bar{K}_{T}\right)$. Good correlation is obtained with $K_{T}=0.85 \times F$. 


$$
\begin{aligned}
& \text { Pyranometer - energy measurement } \\
& \text { Fraction of Possible Sunshine - burning of paper } \\
& \text { Cloud Cover - human visual observation, judgment }
\end{aligned}
$$

\section{Exhibit 8.11 Measurement of Insolation.}

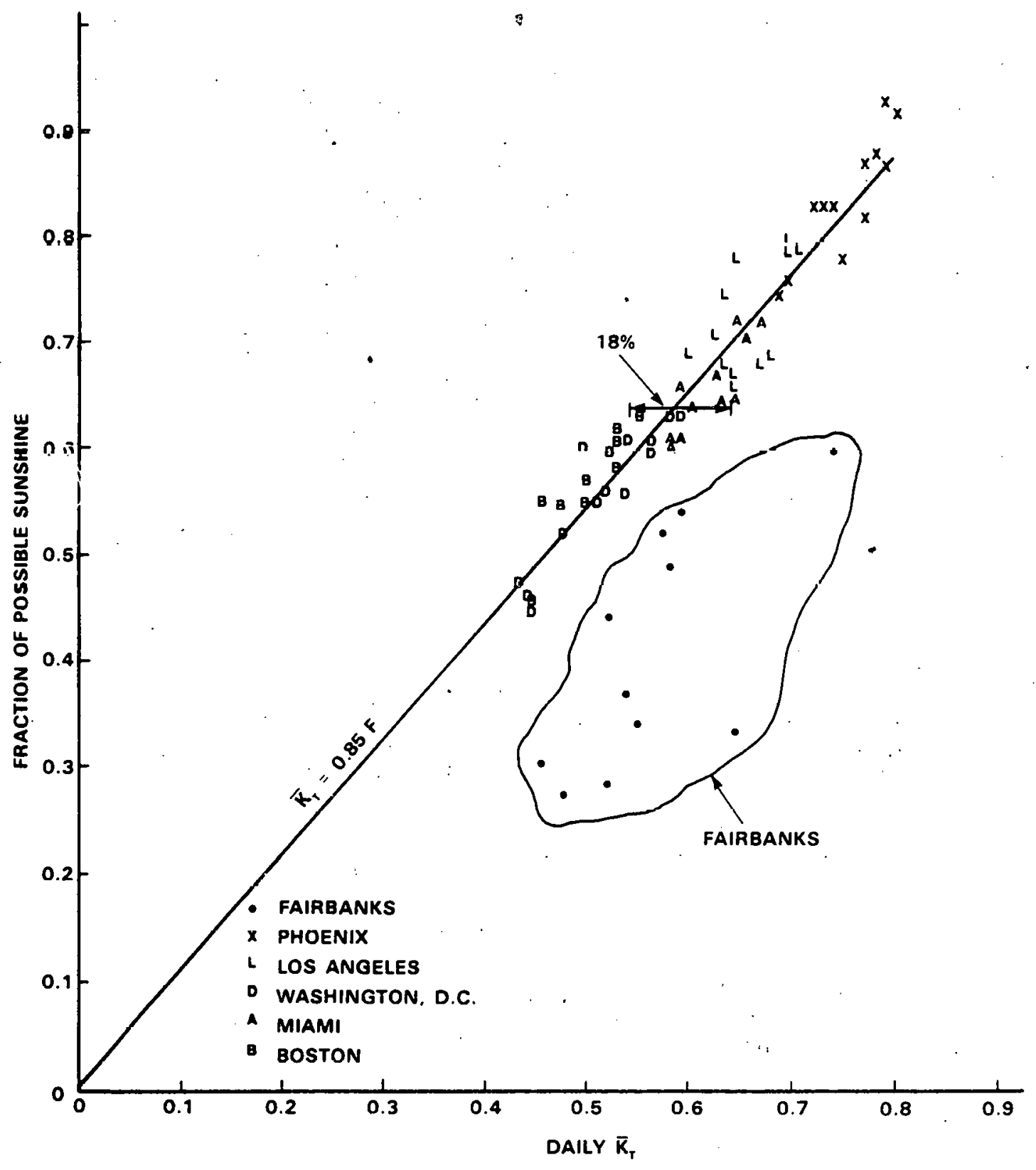

Exhibit 8.12 Correlation between $\bar{K}_{T}$ and Fraction of Sunshine. 


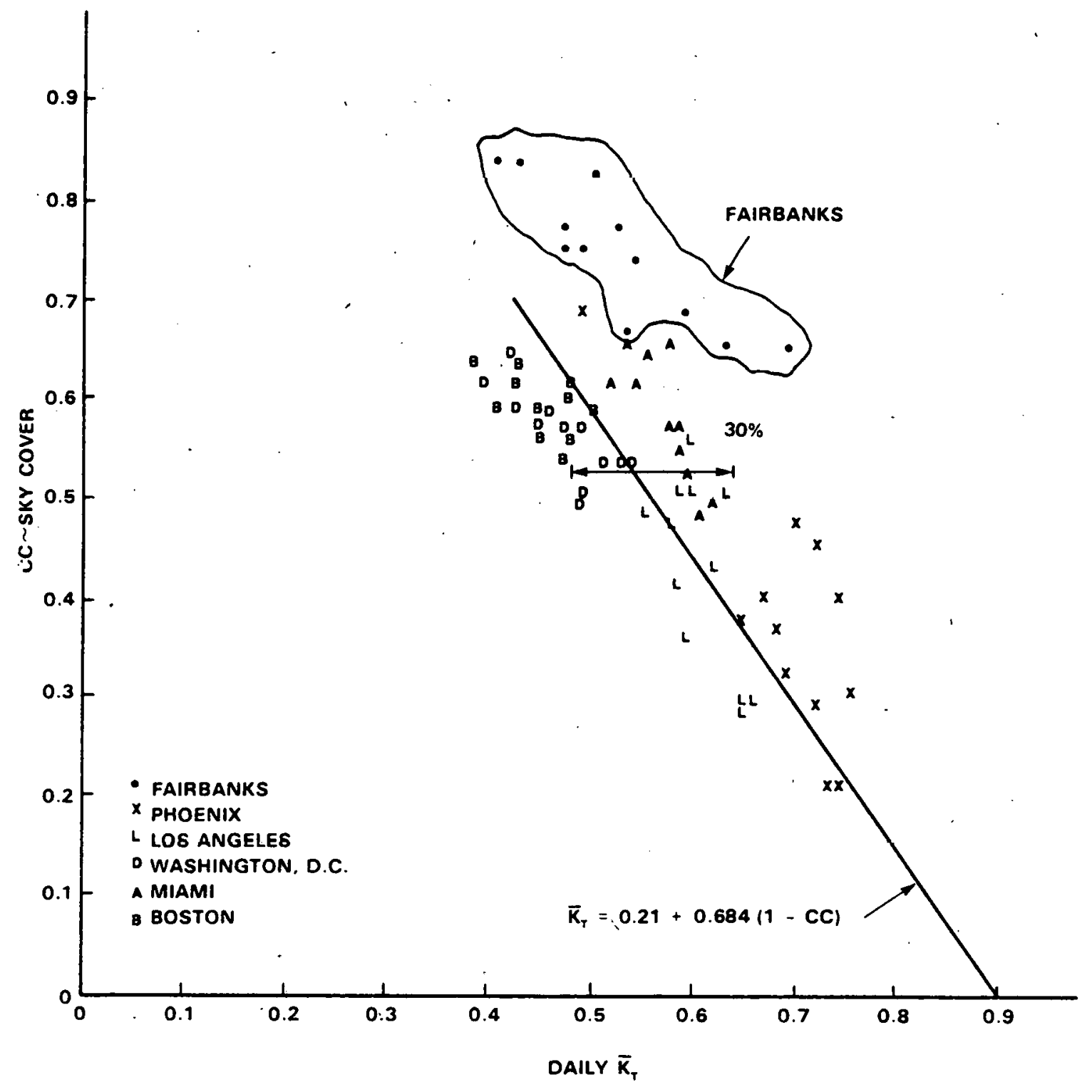

Exhibit 8.13 Correlation between $\bar{K}_{r}$ and Cloud Cover. 
For example, suppose one needed $K_{T}$ for location $A$ and the closest pyranometer data were for location B, 100 miles away. Suppose both locations had data on fraction sunshine, as indicated in exhibit 8.14; 0.60 for $A$ and 0.65 for $B$, with $\bar{K}_{T}$ at 0.52 for $B$. From exhibit $8.12, F=0.65$ and $\bar{K}_{T}=0.52$ are not consistent with the correlation curve. To adjust to location $\mathrm{A}$, one can use the coefficient 0.85 of the correlation equation to write:

$$
\bar{K}_{T}=0.52-0.85 \times(0.65-0.60)=0.48
$$

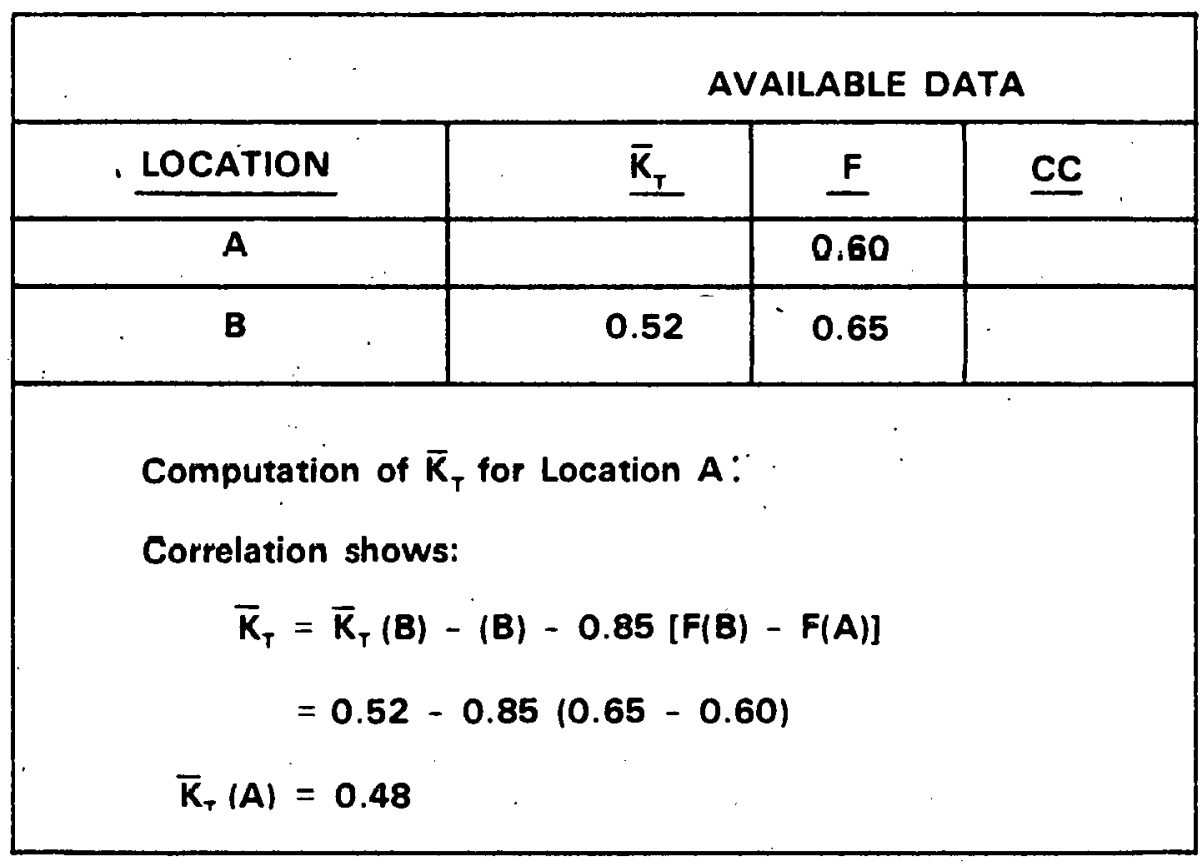

\section{Exhibit 8.14 Sample Computation Procedure.}

\subsubsection{VALUES OF $\bar{K}_{T}$}

Values of $R_{T}$ were derived from measurements of the National Weather Service and extrapolated by Duffie and Beckman in a manner similar to that just described to produce the tables for the United States (exhibit 8.15). These tables show not only the flux ratio, but also the horizontal flux; the average outdoor-air temperature for the month, and the number of heating degree-days accumulated that month, on the average. For world insolation see World Distribution of Solar Radiation, Report No. 21 by George O. O. Lof, John A. Duffie, and Clayton 0. Smith, Solar Energy Laboratory, The University of Wisconsin, July, 1966. The insolation can be converted to $\bar{K}_{\mathrm{T}}$ values by dividing by the extraterrestrial flux, as tabulated in exhibit 8.25 for each latitude and month. On exhibit 8.15 , SI units have been used, so the degree-days, for example, are in C degrees. One MJ (megajoule) is equal to one million $W$-seconds of energy, where $W$ represents watts of power. 


\begin{tabular}{|c|c|c|c|c|c|c|c|c|c|c|c|c|}
\hline Comb & JaA & $\mathbf{n s}$ & mas & APR & $\mathrm{mr}$ & Jose & JuLY & AUG & SEP & OCt & nov & DEC \\
\hline 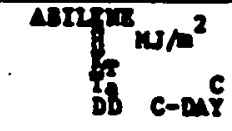 & $\begin{aligned} & 2 x(40 \\
& 11.47 \\
& .98 \\
& 66.0 \\
& 369 .\end{aligned}$ & $\begin{array}{r}600 \\
266 .\end{array}$ & $\begin{array}{r}18.42 \\
1 i^{61} \\
197^{\circ}\end{array}$ & $\begin{aligned} 22.11 \\
18.01 \\
58.0\end{aligned}$ & $\begin{array}{r}26.84 \\
23.61 \\
6.0\end{array}$ & $\begin{array}{r}27.05 \\
2 i^{66} \\
0 .\end{array}$ & $\begin{array}{l}25.41 \\
29.63 \\
0 .\end{array}$ & $\begin{array}{r}23.74 \\
29.64 \\
0 .\end{array}$ & $\begin{array}{r}20.43 \\
24.03 \\
0 .\end{array}$ & $\begin{array}{l}16.24 \\
19.6 \\
49 .\end{array}$ & $\begin{array}{l}12.31 \\
139 \\
18 i .\end{array}$ & $\begin{aligned} 10.51 \\
53 \\
830 \\
32 i\end{aligned}$ \\
\hline 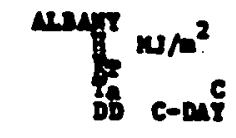 & $\begin{array}{r}x_{3}(14 \\
3.19 \\
-3.0 \\
i 69 .\end{array}$ & $\frac{60.0}{666 .}$ & $\begin{array}{r}14.36 \\
: 35 \\
54.0\end{array}$ & $\begin{array}{r}15.22 \\
563 \\
302 .\end{array}$ & $\begin{array}{r}18.64 \\
14.0 \\
18 i .\end{array}$ & $\begin{aligned} 23.22 \\
19.61 \\
22 .\end{aligned}$ & $\begin{array}{r}22.37 \\
23.56 \\
5 .\end{array}$ & $\begin{array}{r}18.63 \\
292 \\
21.0 \\
12 .\end{array}$ & $\begin{array}{r}13.05 \\
1 i^{450} \\
7 j^{\circ}\end{array}$ & $\begin{array}{r}10.75 \\
10.0 \\
234 .\end{array}$ & $\begin{array}{r}9.20 \\
4.62 \\
4.0 \\
423 .\end{array}$ & $\begin{array}{l}5.86 \\
-8.80 \\
673 .\end{array}$ \\
\hline 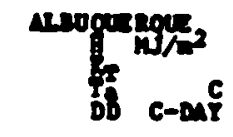 & 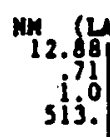 & की.0. & $\begin{aligned} 21.41 \\
; i 3 \\
33 i .0\end{aligned}$ & $\begin{array}{r}26.35 \\
134 \\
157 .\end{array}$ & $\begin{array}{r}28.77 \\
173 \\
172.0\end{array}$ & $\begin{array}{r}30.90 \\
23 ; 0 \\
0 .\end{array}$ & $\begin{array}{r}28.86 \\
25_{j}^{80}\end{array}$ & $\begin{array}{r}26.60 \\
237 \\
0 .\end{array}$ & $\begin{array}{r}23.67 \\
20.8 \\
4 .\end{array}$ & $\begin{array}{l}18.69 \\
13.0 \\
12 \mathrm{i} .\end{array}$ & $\mid \begin{array}{r}14.13 \\
764 \\
362.0 \\
342 .\end{array}$ & $\begin{array}{r}11.75 \\
71.0 \\
496 .\end{array}$ \\
\hline 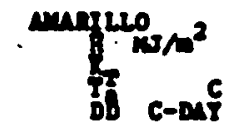 & 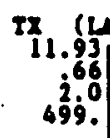 & $\begin{array}{r}35 . \\
i 3.26 \\
566 \\
393 .\end{array}$ & $\begin{array}{r}19.17 \\
863 \\
334 . \\
334 .\end{array}$ & $\begin{array}{r}23.61 \\
16 \% \\
153 .\end{array}$ & $\begin{array}{r}23.83 \\
19.65 \\
43 .\end{array}$ & $\begin{array}{r}27.38 \\
24.67 \\
6 .\end{array}$ & $\begin{array}{r}26.75 \\
26.66 \\
0 .\end{array}$ & $\begin{array}{r}25.12 \\
25 \% 0 \\
0 .\end{array}$ & $\begin{array}{r}21.23 \\
21.0 \\
11 .\end{array}$ & $\begin{array}{r}16.83 \\
15^{68} \\
114 .\end{array}$ & $\begin{array}{r}12.98 \\
688 \\
312.0\end{array}$ & $\begin{array}{r}10.72 \\
65 \\
455^{6}\end{array}$ \\
\hline 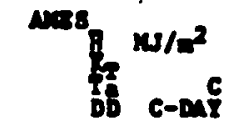 & $\begin{array}{l}\text { IA, } 14 \\
1.28 \\
i^{53} \\
79.0\end{array}$ & $\begin{array}{r}1042 . \\
5.5 \\
-45 \\
639 .\end{array}$ & $\begin{array}{r}13.67 \\
.32 \\
339.0 \\
39 .\end{array}$ & $\begin{array}{r}16.89 \\
990 \\
260.0\end{array}$ & $\begin{array}{r}20.07 \\
151 \\
106.0 \\
106 .\end{array}$ & $\begin{array}{r}22.62 \\
20.55 \\
18 .\end{array}$ & $\begin{aligned} 22.61 \\
236 \\
23.0 \\
0 .\end{aligned}$ & $\begin{array}{r}19.24 \\
23.5 \\
8 .\end{array}$ & $\begin{array}{r}15.35 \\
173.0 \\
38 .\end{array}$ & $\begin{array}{l}11.46 \\
11.54 \\
206 .\end{array}$ & $\begin{aligned} 7.82 \\
.852 \\
250 \\
463 .\end{aligned}$ & $\begin{array}{l}3.98 \\
-4.48 \\
-49.9 \\
69 .\end{array}$ \\
\hline 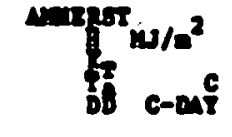 & 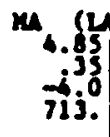 & $\begin{array}{r}7.40 \\
-390 \\
.61 i^{30}\end{array}$ & $\begin{array}{r}12.55 \\
12.5 \\
i^{4} 5^{2} \\
\end{array}$ & $\begin{aligned} 16.51 \\
300.0 \\
30.0\end{aligned}$ & $\begin{array}{r}18.02 \\
14.6 \\
139 .\end{array}$ & $\begin{array}{r}21.49 \\
192 \\
23.0 \\
23 .\end{array}$ & $\begin{array}{r}21.58 \\
21.0 \\
: 4 .\end{array}$ & $\begin{array}{r}18.40 \\
28.0 \\
12.0\end{array}$ & $\begin{array}{r}13.80 \\
16.8 \\
68 .\end{array}$ & $\begin{array}{r}10.45 \\
1 i^{49} \\
22 i .\end{array}$ & $\begin{array}{r}6.40 \\
4.3 \\
4.0 \\
403 .\end{array}$ & $\begin{array}{l}3.19 \\
-34.0 \\
644 .\end{array}$ \\
\hline
\end{tabular}

\begin{tabular}{|c|c|c|c|c|c|c|c|c|c|c|c|c|}
\hline 7 & SAB & $\mathrm{ms}$ & $m$ & APR & $\operatorname{maY}$ & sure & ת几ाY & AUC & SEP & $\infty \subset$ & nov & DEC \\
\hline 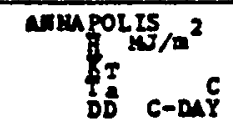 & RD & 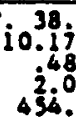 & $\begin{array}{r}14.24 \\
531 \\
376^{\circ}\end{array}$ & $\begin{array}{r}17.54 \\
12^{51} \\
183 .\end{array}$ & $\begin{array}{r}20.43 \\
1532 \\
158.0 \\
58 .\end{array}$ & $\begin{array}{r}23.32 \\
2536 \\
0.0\end{array}$ & $\begin{array}{r}22.69 \\
24.56 \\
0.0 \\
0 .\end{array}$ & $\begin{array}{r}19.64 \\
23.04 \\
0 .\end{array}$ & $\begin{array}{r}16.03 \\
833 \\
20.0 \\
16 .\end{array}$ & $\begin{array}{r}12.31 \\
153 \\
13 j^{\circ} .\end{array}$ & $\begin{array}{r}7.91 \\
8.66 \\
293.0\end{array}$ & $\begin{array}{r}6.49 \\
3.45 \\
484 .\end{array}$ \\
\hline 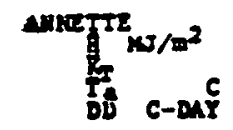 & $\begin{array}{rl}A x & .64 \\
2.64 \\
.43 \\
327^{\circ}\end{array}$ & $4 i^{2}$ & $\begin{array}{r}9.88 \\
391 \\
j .0 \\
468 .\end{array}$ & $\begin{aligned} 15.24 \\
532 \\
350 \\
301 .\end{aligned}$ & $\begin{array}{r}18.30 \\
999 \\
281 .\end{array}$ & $\begin{array}{r}18.34 \\
4 i^{45} \\
18 i^{0}\end{array}$ & $\begin{array}{r}18.34 \\
15.0 \\
143 .\end{array}$ & $\begin{array}{l}14.28 \\
18.4 \\
124 .\end{array}$ & $\begin{array}{l}10.80 \\
12.0 \\
196 .\end{array}$ & $\begin{array}{c}5.11 \\
37 \\
830 \\
315 .\end{array}$ & $\begin{array}{r}2.47 \\
4.34 \\
4.00\end{array}$ & $\begin{array}{r}1.72 \\
336 \\
2.00 \\
499 .\end{array}$ \\
\hline 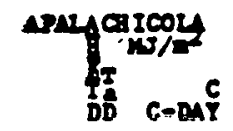 & $\begin{array}{r}12 . \\
12.25 \\
125 \\
1250 \\
193 .\end{array}$ & $\begin{array}{l}13.0 \\
144 .\end{array}$ & $\begin{array}{l}18.44 \\
15^{59} \\
100^{\circ}\end{array}$ & $\begin{array}{r}23.00 \\
193 \\
18.0 \\
18 .\end{array}$ & $\begin{array}{r}25.47 \\
23.04 \\
0.0\end{array}$ & $\begin{array}{r}24.71 \\
26.01 \\
0 .\end{array}$ & $\begin{array}{r}22.62 \\
27.97 \\
0 .\end{array}$ & $\begin{array}{r}21.20 \\
27.56 \\
0 .\end{array}$ & $\begin{array}{r}19.24 \\
26.08 \\
0 .\end{array}$ & $\begin{array}{r}17.48 \\
21.63 \\
9 .\end{array}$ & $\begin{array}{r}13.93 \\
16.62 \\
85.0\end{array}$ & $\mid \begin{aligned} 11.04 \\
135 \\
1390 \\
173^{3}\end{aligned}$ \\
\hline 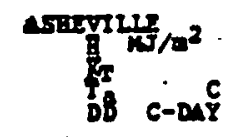 & $\begin{array}{r}\text { Ic } \\
9 . \\
4 \\
46\end{array}$ & 4.0 & $\begin{array}{r}16.93 \\
.95 \\
5290 \\
329 .\end{array}$ & $\begin{aligned} 20.51 \\
138 \\
135.0\end{aligned}$ & $\begin{array}{r}23.28 \\
189 \\
36.0\end{array}$ & $\begin{array}{r}23.78 \\
2 i^{88} \\
8 .\end{array}$ & $\mid \begin{array}{r}23.26 \\
23.0 \\
0 .\end{array}$ & $\begin{aligned} 21: 56 \\
23.8 \\
23.0 \\
0 .\end{aligned}$ & $\begin{array}{r}18.25 \\
19.8 \\
28 .\end{array}$ & $\begin{array}{r}14.86 \\
16.00 \\
149 .\end{array}$ & $\begin{aligned} 10.47 \\
95 \\
890 \\
312 .\end{aligned}$ & $\begin{array}{l}8.46 \\
51.0 \\
453.0 \\
453 .\end{array}$ \\
\hline 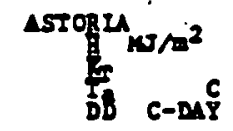 & $\begin{aligned} & \text { or }(2) \\
& 3.85 \\
& 34.0 \\
& 35.0 \\
& 420 .\end{aligned}$ & $\begin{array}{r}6.52 \\
639 \\
333.0\end{array}$ & $\begin{array}{r}1.17 \\
.460 \\
3950 .\end{array}$ & $\begin{array}{r}15.31 \\
848 \\
28 \% .\end{array}$ & $\begin{array}{r}20.49 \\
11^{3} 3 \\
219 .\end{array}$ & $\begin{array}{r}20.16 \\
13.0 \\
142 .\end{array}$ & $\begin{array}{r}22.37 \\
15^{56} \\
9 \mathrm{i} .\end{array}$ & $\begin{array}{r}19.09 \\
195 \\
19.0 \\
84 .\end{array}$ & $\begin{array}{l}13.01 \\
153 \\
112.0 \\
112 .\end{array}$ & $\begin{array}{l}8.82 \\
11^{47} \\
210 .\end{array}$ & $\begin{array}{r}4.77 \\
8.38 \\
308 .\end{array}$ & $\begin{array}{c}3.26 \\
3.33 \\
3.0 \\
382 .\end{array}$ \\
\hline 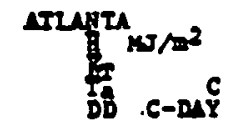 & $\begin{array}{r}e .5 \\
9.53 \\
850 \\
6.0 \\
389 .\end{array}$ & $\begin{array}{r}580 \\
319 \\
319\end{array}$ & $\begin{array}{l}15.77 \\
193 \\
246.0\end{array}$ & $\begin{array}{r}20.28 \\
16.56 \\
80.0 \\
80 .\end{array}$ & $\begin{array}{r}22.37 \\
200^{96} \\
13 .\end{array}$ & $\begin{array}{r}23.17 \\
24.60 \\
0 .\end{array}$ & $\begin{array}{r}22.50 \\
25.96 \\
0 .\end{array}$ & $\begin{array}{r}20.99 \\
23.6 \\
0.0\end{array}$ & $\begin{array}{r}17.23 \\
23.0 \\
4 .\end{array}$ & $\begin{array}{r}14.64 \\
175 \\
76.0\end{array}$ & $\begin{array}{r}11.08 \\
195 \\
22 i^{\circ}\end{array}$ & $\begin{array}{r}8.41 \\
.48 \\
371.0 \\
371 .\end{array}$ \\
\hline
\end{tabular}

Exhibit 8.15 Values of $\vec{K}_{\mathrm{T}}$ and Insolation for the United States. 


\begin{tabular}{|c|c|c|c|c|c|c|c|c|c|c|c|c|}
\hline Location Month & SNS & T23 & we & $\Delta P Q$ & $M Y Y$ & JNE & JoLY & AUC & SEP & $O C T$ & Hov & DEC \\
\hline 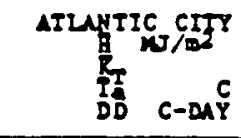 & $\begin{array}{l}\text { MJ } \\
\dot{j} \\
\text { s2 }\end{array}$ & $i^{51}$ & $\begin{array}{r}16.12 \\
359 \\
507\end{array}$ & $\begin{array}{r}18.09 \\
10.92 \\
24 i^{0}\end{array}$ & $\begin{array}{r}20.64 \\
15.5 \\
150.0 \\
100 .\end{array}$ & $\begin{array}{r}23.99 \\
25.58 \\
8.0\end{array}$ & $\begin{array}{r}23.78 \\
2399 \\
0 .\end{array}$ & $\begin{array}{r}19.97 \\
235 \\
23.0 \\
0 .\end{array}$ & $\begin{array}{r}16.41 \\
20.55 \\
13 . \\
13.0\end{array}$ & $\begin{array}{r}12.64 \\
15.56 \\
11.0\end{array}$ & $\begin{array}{r}8.83 \\
553 \\
99.0 \\
27 \%\end{array}$ & $\begin{array}{r}6.66 \\
437 \\
430 \\
470 .\end{array}$ \\
\hline 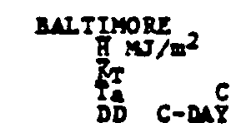 & MD & $\begin{array}{r}390 \\
470 . \\
40 .\end{array}$ & $\begin{array}{r}14.24 \\
552 \\
38.0 \\
382 .\end{array}$ & $\begin{array}{r}17.34 \\
12.51 \\
189 .\end{array}$ & $\begin{array}{r}20.43 \\
10^{35} \\
6 i^{3}\end{array}$ & $\begin{array}{r}23.32 \\
23.50 \\
0 .\end{array}$ & $\begin{array}{r}22.69 \\
2560 \\
0.0\end{array}$ & $\begin{array}{r}19.64 \\
26.54 \\
0.0 \\
0 .\end{array}$ & $\begin{array}{r}16.03 \\
20.93 \\
15.0\end{array}$ & $\begin{array}{r}12.31 \\
1954 \\
139.0\end{array}$ & $\begin{array}{r}7.91 \\
\dot{8} 4 \\
315\end{array}$ & $\begin{aligned} 6.49 \\
i^{4} .6 \\
\text { sit. }\end{aligned}$ \\
\hline 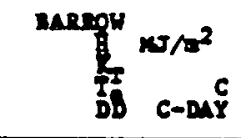 & $\begin{array}{r}\Delta x .1 \frac{1}{13} \\
-200 \\
-2590 \\
1398 .\end{array}$ & $\begin{array}{r}-289 \\
-2296.0 \\
1296 .\end{array}$ & $\begin{array}{r}7.99 \\
-26.1 \\
137 i \\
139\end{array}$ & $\begin{array}{r}16.85 \\
-18.76 \\
1080.0\end{array}$ & $\begin{array}{r}20.95 \\
-8.60 \\
803 .\end{array}$ & $\begin{array}{r}22.87 \\
.94 \\
547 .\end{array}$ & $\begin{array}{r}17.73 \\
346 \\
36.0 \\
46 .\end{array}$ & $\begin{array}{r}10.62 \\
339 \\
367.0 \\
467 .\end{array}$ & $\begin{array}{r}4.81 \\
.34 \\
575^{0}\end{array}$ & $\begin{array}{l}1.72 \\
-92 . \\
833 .\end{array}$ & $\begin{array}{r}.29 \\
-1.00 \\
1095.0\end{array}$ & 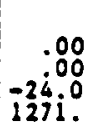 \\
\hline 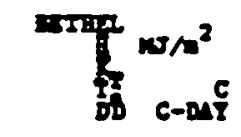 & $\begin{array}{r}x_{1.5} \\
-1.4 \\
1057\end{array}$ & -1 & $\begin{array}{r}11.79 \\
-11.0 \\
919 .\end{array}$ & $\begin{array}{r}18.57 \\
56.8 \\
532 .\end{array}$ & $\begin{array}{r}19.19 \\
45 \\
46.8\end{array}$ & $\begin{array}{l}18.78 \\
184.8 \\
223 .\end{array}$ & $\begin{array}{l}15.47 \\
1348 \\
177 .\end{array}$ & $\begin{array}{l}10.62 \\
11.8 \\
219 .\end{array}$ & $\begin{array}{r}8.32 \\
4.4 \\
340 .\end{array}$ & $\begin{array}{l}4.89 \\
379.6\end{array}$ & $\begin{array}{l}1.88 \\
-848 \\
797 .\end{array}$ & $\begin{array}{r}.96 \\
-1367 \\
1037 .\end{array}$ \\
\hline 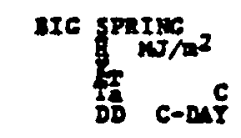 & 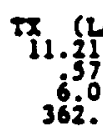 & $\begin{array}{r}32 . \\
i 4.39 \\
58.0 \\
260.0\end{array}$ & $\begin{array}{r}19.49 \\
196 \\
139.0 \\
179 .\end{array}$ & $\begin{array}{r}24.38 \\
168.0 \\
50 .\end{array}$ & $\begin{array}{r}23.92 \\
23.60 \\
0 .\end{array}$ & $\begin{array}{r}24.80 \\
26.01 \\
0 .\end{array}$ & $\begin{array}{r}23.08 \\
28.57 \\
0 .\end{array}$ & $\begin{array}{r}19.53 \\
28.52 \\
0 .\end{array}$ & $\begin{array}{r}21.87 \\
24.67 \\
0.0\end{array}$ & $\begin{array}{r}16.14 \\
18.61 \\
68 .\end{array}$ & $\begin{array}{l}12.17 \\
128 \\
212 .\end{array}$ & $\begin{array}{r}10.87 \\
; 99 \\
329 .\end{array}$ \\
\hline 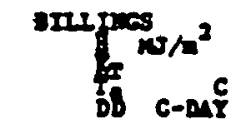 & $\begin{array}{l}6.8 \mathrm{l} \\
6.62 \\
-36 \\
74.0 \\
742 .\end{array}$ & 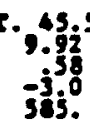 & $\begin{array}{r}15.03 \\
.61 \\
350^{\circ}\end{array}$ & $\begin{array}{r}19.09 \\
58 . \\
360^{3}\end{array}$ & $\begin{array}{r}22.65 \\
13.58 \\
10.5 .\end{array}$ & $\begin{array}{r}25.63 \\
17 \% \\
73.0\end{array}$ & $\begin{array}{r}26.63 \\
23.5 \\
6 .\end{array}$ & $\begin{array}{r}23.12 \\
2 i^{67} \\
8 .\end{array}$ & $\begin{array}{r}17.75 \\
15.65 \\
12 j .\end{array}$ & $\begin{array}{r}10.68 \\
195 \\
27 i^{5 .}\end{array}$ & $\begin{array}{r}7.24 \\
356 \\
280 \\
488 .\end{array}$ & $\begin{array}{l}3.97 \\
-354 \\
658 .\end{array}$ \\
\hline
\end{tabular}

\begin{tabular}{|c|c|c|c|c|c|c|c|c|c|c|c|c|}
\hline ation Month & SAM & $\mathrm{rat}$ & $\mathrm{mu}$ & $\boldsymbol{N m}$ & $\mathrm{mr}$ & Jun & Jor & A.DC & SEP & OCT & mov & DEC \\
\hline 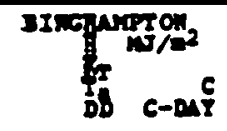 & $\begin{array}{l}m_{3} . \\
j i\end{array}$ & $\begin{array}{l}-5^{4} \\
6535\end{array}$ & $\begin{array}{r}12.48 \\
.48 \\
58 i^{\circ}\end{array}$ & $\begin{array}{r}15.99 \\
547 \\
338 .\end{array}$ & $\begin{array}{r}20.39 \\
13^{35} \\
178 .\end{array}$ & $\begin{array}{r}23.32 \\
186 \\
42 .\end{array}$ & $\begin{array}{r}22.90 \\
21.0 \\
12 .\end{array}$ & $\begin{array}{r}19.72 \\
20.0 \\
22 .\end{array}$ & $\begin{array}{r}15.24 \\
193 \\
16.0 \\
96 .\end{array}$ & $\begin{array}{l}10.80 \\
19.91 \\
253 .\end{array}$ & $\begin{array}{r}6.03 \\
340 \\
447 .\end{array}$ & $\begin{array}{l}6.77 \\
-39 \\
-48.0 \\
682 .\end{array}$ \\
\hline 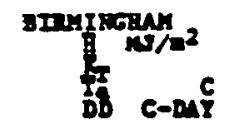 & 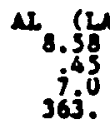 & 28 & $\begin{array}{l}15.32 \\
12^{51} \\
216 .\end{array}$ & $\begin{array}{r}20.56 \\
1957 \\
19.0\end{array}$ & $\begin{array}{r}23.40 \\
23^{39} \\
11 .\end{array}$ & $\begin{array}{r}23.57 \\
25 \% \\
5 \%\end{array}$ & $\begin{array}{r}22.94 \\
27.0 \\
0 .\end{array}$ & $\begin{array}{l}21.189 \\
26.00 \\
0 .\end{array}$ & $\begin{array}{r}18.09 \\
23.06 \\
3 .\end{array}$ & $\begin{aligned} 15.03 \\
198 \\
190.0 \\
76 .\end{aligned}$ & $\begin{aligned} 10.38 \\
191 \\
21 \%\end{aligned}$ & $\begin{array}{r}8.12 \\
560 \\
34 i^{60}\end{array}$ \\
\hline 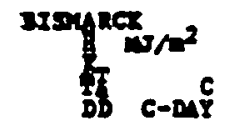 & $\begin{array}{r}100.61 \\
6.61 \\
-1390 \\
978 .\end{array}$ & $-\frac{1}{80} i^{\circ}$ & $\begin{array}{l}14.68 \\
-361 \\
-387.0\end{array}$ & $\begin{array}{r}18.78 \\
58 \\
665^{6} \\
367 .\end{array}$ & $\begin{array}{r}23.04 \\
13.00 \\
188 .\end{array}$ & $\begin{array}{r}24.53 \\
179 \\
68 .\end{array}$ & $\begin{array}{r}25.59 \\
21.64 \\
10 .\end{array}$ & $\begin{array}{c}21.66 \\
20.62 \\
19 .\end{array}$ & $\begin{array}{r}15.98 \\
149 \\
140.0\end{array}$ & $\begin{aligned} 11.42 \\
j_{1}^{61} \\
313 .\end{aligned}$ & $\begin{array}{l}6.73 \\
-154 \\
602.0\end{array}$ & $\begin{array}{l}3.19 \\
-54 \\
851.0\end{array}$ \\
\hline 10 & $:$ & $\mathbf{j i j}^{\mathbf{4}} \mathbf{0}$ & $\begin{array}{r}12.71 \\
5 \\
320 \\
320 .\end{array}$ & $\begin{array}{r}15.89 \\
597 \\
322 .\end{array}$ & $\begin{array}{r}19.70 \\
130^{50} \\
148^{0}\end{array}$ & $\begin{array}{r}21.62 \\
1.32 \\
1890 \\
38 !\end{array}$ & $\begin{aligned} 20.91 \\
2 \mathrm{i}^{32} \\
0 .\end{aligned}$ & $\begin{array}{r}28.15 \\
291 \\
29 \\
92\end{array}$ & 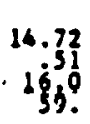 & $\begin{array}{r}10.41 \\
1349 \\
114 .\end{array}$ & $\begin{array}{l}6.62 \\
544 \\
383 ? .\end{array}$ & $\begin{array}{l}5.39 \\
-j_{34}^{4}\end{array}$ \\
\hline 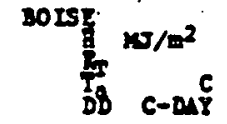 & -1 & $\begin{array}{r}43 \\
9.7 \\
13 \\
474\end{array}$ & $\begin{array}{r}14.18 \\
535 \\
30.0 \\
40 i .\end{array}$ & $\begin{array}{r}20.32 \\
96.1 \\
243 .\end{array}$ & $\begin{array}{r}24.55 \\
163 \\
136.0 \\
136 .\end{array}$ & $\begin{array}{r}26.72 \\
18.63 \\
45 .\end{array}$ & $\begin{array}{r}27.98 \\
23,0 \\
0 .\end{array}$ & $\begin{array}{r}23.80 \\
23.67 \\
0 .\end{array}$ & $\begin{array}{l}19.07 \\
17.69 \\
73 .\end{array}$ & $\begin{array}{l}13.13 \\
1.64 \\
231.0\end{array}$ & $\begin{array}{r}7.57 \\
853 \\
4.0 \\
440 .\end{array}$ & $\begin{array}{l}3.14 \\
.44 \\
565 .\end{array}$ \\
\hline${ }^{\text {NOSTOM }} \mathrm{m} / \mathrm{m}^{2}$ & $\begin{array}{l}m \text { s. } \\
s .81 \\
-i .02 \\
604 .\end{array}$ & $\begin{array}{r}42 . \\
8.28 \\
.43 \\
340 .\end{array}$ & $\begin{array}{r}12.25 \\
.47 \\
470 .\end{array}$ & $\begin{array}{r}25.22 \\
565 \\
285^{6 .}\end{array}$ & $\begin{array}{r}19.78 \\
19.90 \\
116 .\end{array}$ & $\begin{array}{r}20.87 \\
20.0 \\
20 .\end{array}$ & $\begin{array}{r}20.74 \\
23^{5} \\
0 .\end{array}$ & $\begin{array}{r}17.77 \\
2 i_{30}^{0} \\
3 .\end{array}$ & $\begin{array}{r}16.26 \\
18.69 \\
33^{\circ}\end{array}$ & $\begin{array}{l}9.95 \\
1340 \\
176 .\end{array}$ & $\begin{array}{r}6.06 \\
j^{41} \\
335^{0}\end{array}$ & $\begin{array}{c}4.98 \\
4.41 \\
i^{4} .0 \\
546 .\end{array}$ \\
\hline
\end{tabular}




\begin{tabular}{|c|c|c|c|c|c|c|c|c|c|c|c|c|}
\hline Month & SAN & RB & mar & APR & $\operatorname{MuY}$ & JURE & JoLY & $A U C$ & SEP & $\alpha \boldsymbol{T}$ & HOV & DEC \\
\hline 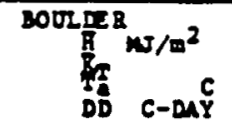 & 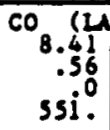 & $\begin{array}{l}\text { i.j } \\
659 .\end{array}$ & $\begin{array}{r}16.79 \\
562 \\
3.0 \\
449 .\end{array}$ & $\begin{array}{r}19.26 \\
i^{35} \\
268 .\end{array}$ & $\begin{array}{r}19.26 \\
14.40 \\
13 i^{\circ} .\end{array}$ & $\begin{aligned} 21.98 \\
193 \\
49 .\end{aligned}$ & $\begin{array}{r}21.77 \\
23.54 \\
3 .\end{array}$ & $\begin{array}{r}18.38 \\
22.0 \\
0 .\end{array}$ & $\begin{array}{r}17.25 \\
1798 \\
77 .\end{array}$ & $\begin{array}{r}12.98 \\
158 \\
208 .\end{array}$ & $\begin{array}{r}9.29 \\
357 \\
383 .\end{array}$ & $\begin{array}{l}7.62 \\
.56 \\
\text { soj. } \\
\text { s. }\end{array}$ \\
\hline 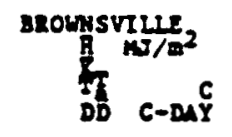 & $\begin{array}{r}2 x \\
12.0 \\
1 j^{5} \\
125\end{array}$ & $\begin{array}{r}190 \\
19^{50}\end{array}$ & $\begin{array}{r}16.81 \\
20.51 \\
49 .\end{array}$ & $\begin{array}{r}19.15 \\
33.5 \\
3 . \mathrm{c} \\
0 .\end{array}$ & $\begin{array}{r}23.25 \\
26.0 \\
0 .\end{array}$ & $\begin{array}{r}25.26 \\
28.63 \\
0 .\end{array}$ & $\begin{array}{r}25.89 \\
28.65 \\
0 .\end{array}$ & $\begin{array}{r}23.21 \\
28.6 ! \\
0 .\end{array}$ & $\begin{array}{r}19.45 \\
275 \\
59 \\
0 .\end{array}$ & $\begin{array}{r}16.98 \\
24.58 \\
j .\end{array}$ & $\begin{array}{r}11.88 \\
19.48 \\
19 .\end{array}$ & $\begin{array}{r}10.58 \\
16.47 \\
81.0\end{array}$ \\
\hline 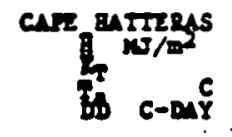 & $\begin{aligned} & \pi c(\mathrm{IA} \\
& 10.20 \\
& 57 \\
& 390 \\
& 339 .\end{aligned}$ & $8^{38} .0$ & $\begin{array}{r}18.07 \\
10.02 \\
253 .\end{array}$ & $\begin{array}{r}23.88 \\
16.67 \\
10.0\end{array}$ & $\begin{array}{r}26.56 \\
1967 \\
26 .\end{array}$ & $\begin{array}{r}26.97 \\
23.66 \\
0 .\end{array}$ & $\begin{array}{r}26.30 \\
25.05 \\
0.0\end{array}$ & $\begin{array}{r}23.29 \\
23.63 \\
0 .\end{array}$ & $\begin{array}{r}19.74 \\
2363 \\
0.0\end{array}$ & $\begin{array}{l}13.10 \\
18.6 \\
42 .\end{array}$ & $\begin{array}{r}11.88 \\
13.62 \\
154 .\end{array}$ & $\begin{array}{r}9.03 \\
.94 \\
99.0 \\
298 .\end{array}$ \\
\hline 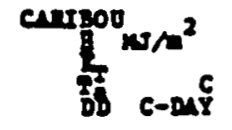 & $\begin{array}{l}0 . \\
-19 \\
93\end{array}$ & $\begin{array}{r}-10 \\
81\end{array}$ & $\begin{array}{r}15.33 \\
-6.64 \\
727 .\end{array}$ & $\begin{array}{r}16.73 \\
332 \\
350 \\
679\end{array}$ & $\begin{array}{r}19.82 \\
10^{\circ 1} \\
260 .\end{array}$ & $\begin{array}{r}20.07 \\
15.09 \\
102 .\end{array}$ & $\begin{array}{r}21.29 \\
183 \\
43 . \\
83 .\end{array}$ & $\begin{array}{r}18.82 \\
17^{34} \\
64 .\end{array}$ & $\begin{array}{r}13.93 \\
152 \\
18.0 \\
187 .\end{array}$ & $\begin{array}{r}8.78 \\
797 \\
379 .\end{array}$ & $\begin{array}{l}4.60 \\
.37 \\
580.0\end{array}$ & $\begin{array}{l}4.43 \\
-8.66 \\
833.0\end{array}$ \\
\hline 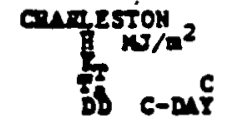 & 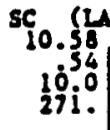 & $\begin{array}{l}2.67 \\
10.0 \\
216 .\end{array}$ & $\begin{array}{r}16.39 \\
14.04 \\
162 .\end{array}$ & $\begin{array}{r}21.58 \\
19.0 \\
30.0\end{array}$ & $\begin{array}{r}23.00 \\
23_{3}^{5} .0 \\
0 .\end{array}$ & $\begin{array}{r}23.62 \\
2350 \\
6 .\end{array}$ & $\begin{array}{r}21.87 \\
27.00 \\
0 .\end{array}$ & $\begin{array}{r}20.74 \\
26.56 \\
0 .\end{array}$ & $\begin{array}{r}17.06 \\
29.0 \\
0.0 \\
0 .\end{array}$ & $\begin{array}{rl}16.34 & 35 \\
1990 \\
33 .\end{array}$ & $\begin{array}{r}11.92 \\
588 \\
1550 \\
159 .\end{array}$ & $\begin{array}{l}9.03 \\
500 \\
1000 \\
262 .\end{array}$ \\
\hline 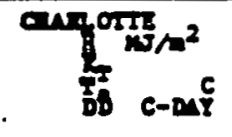 & $\begin{array}{l}\text { Ic } 14 \\
9.29 \\
652 \\
690 \\
396 .\end{array}$ & $\begin{array}{r}i 2.35 \\
590 \\
327 .\end{array}$ & $\begin{array}{r}16.20 \\
1055 \\
1560 \\
256 .\end{array}$ & $\begin{array}{r}21.39 \\
16.00 \\
8 i .\end{array}$ & $\begin{array}{r}23.07 \\
20.5 \\
19 .\end{array}$ & $\begin{array}{r}24.455 \\
24.50 \\
0 .\end{array}$ & $\begin{array}{r}23.65 \\
26.59 \\
0 .\end{array}$ & $\begin{array}{r}21.69 \\
2590 \\
0 .\end{array}$ & $\begin{array}{r}18.21 \\
23.0 \\
6 .\end{array}$ & $\begin{array}{r}14.86 \\
160 \\
16.0 \\
84 .\end{array}$ & $\begin{array}{r}10.31 \\
135 \\
11^{3} \\
233 .\end{array}$ & $\begin{array}{r}8.54 \\
5.51 \\
38.0 \\
388 .\end{array}$ \\
\hline
\end{tabular}

\begin{tabular}{|c|c|c|c|c|c|c|c|c|c|c|c|c|}
\hline Month & SM & rs & $\mathrm{mex}$ & APR & $\operatorname{mar}$ & JUSR & SILY & AUG & SEP & OCT & MOV & DEC \\
\hline 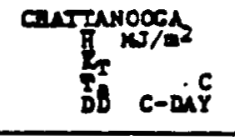 & $\begin{array}{r}8 . \\
3 \\
42 \\
\end{array}$ & $60^{\circ}$ & $\begin{array}{r}14.49 \\
10.49 \\
268 .\end{array}$ & $\begin{array}{r}19.51 \\
16.5 \\
92 .\end{array}$ & $\begin{array}{r}22.57 \\
20.57 \\
20 .\end{array}$ & $\begin{array}{r}23.32 \\
24.5 \\
0 .\end{array}$ & $\begin{array}{r}22.90 \\
265 \\
36.0 \\
0 .\end{array}$ & $\begin{array}{r}21.02 \\
2635 \\
0.0\end{array}$ & $\begin{array}{r}17.84 \\
23.5 \\
3 .\end{array}$ & $\begin{array}{r}13.94 \\
196 \\
10.0 \\
10 \mathrm{i} .\end{array}$ & $\begin{array}{r}9.46 \\
.49 \\
9.0 \\
268 .\end{array}$ & $\begin{array}{r}7.58 \\
345 \\
410 .\end{array}$ \\
\hline 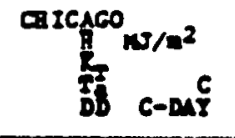 & $\begin{array}{l}\pi, j \\
i \frac{1}{5} \\
70 \mathrm{i}\end{array}$ & 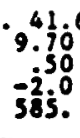 & $\begin{array}{r}13.63 \\
: 52 \\
3.0 \\
486 .\end{array}$ & $\begin{array}{l}16.31 \\
10.8 \\
252.0 \\
252 .\end{array}$ & $\begin{array}{r}20.78 \\
163 \\
16.0 \\
116 .\end{array}$ & $\begin{array}{r}23.13 \\
2 i^{36} \\
14.0\end{array}$ & $\begin{aligned} 22.04 \\
24.55 \\
0.0\end{aligned}$ & $\begin{array}{r}20.32 \\
235 \\
23.0 \\
4 .\end{array}$ & $\begin{array}{r}16.06 \\
195 \\
1950 \\
32 .\end{array}$ & $\begin{array}{r}11.08 \\
13.02 \\
136 .\end{array}$ & $\begin{array}{r}6.57 \\
4.3 \\
410 .\end{array}$ & $\begin{array}{r}5.48 \\
-i^{4} .0 \\
629 .\end{array}$ \\
\hline 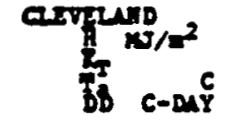 & $\begin{array}{l}\mathrm{OR}_{5} . \\
-2 \\
69\end{array}$ & $-i$ & $\begin{array}{r}13.05 \\
349 \\
498.0\end{array}$ & $\begin{array}{r}15.77 \\
j 96 \\
278.0\end{array}$ & $\begin{array}{r}21.87 \\
15.6 \\
136 .\end{array}$ & $\begin{array}{r}23.38 \\
20.97 \\
22.0\end{array}$ & $\begin{array}{r}23.04 \\
2 \dot{2}^{3} \\
\dot{3} .0\end{array}$ & $\mid \begin{array}{r}20.62 \\
21.57 \\
9.0 \\
9 .\end{array}$ & $\begin{array}{r}13.72 \\
194.0 \\
53 .\end{array}$ & $\begin{aligned} 11.00 \\
1251 \\
1950 \\
19 \%\end{aligned}$ & $\begin{array}{r}3.90 \\
3.38 \\
390.0\end{array}$ & $\begin{array}{r}4.81 \\
.37 \\
598.0\end{array}$ \\
\hline 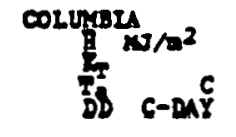 & $\begin{array}{r}\text { no } \\
7.5 \\
39.5 \\
390\end{array}$ & 4 & $\begin{array}{r}16.39 \\
.52 \\
69.0 \\
398 .\end{array}$ & $\begin{array}{rl}18.11 & .12 \\
12.0 \\
180 .\end{array}$ & $\begin{array}{r}22.21 \\
186 \\
67.0\end{array}$ & $\begin{array}{r}23.88 \\
23.58 \\
7.0\end{array}$ & $\begin{array}{r}24.00 \\
25.00 \\
0 .\end{array}$ & $\begin{array}{r}22.00 \\
24.60 \\
0 .\end{array}$ & $\begin{array}{r}18.73 \\
20.62 \\
30.0\end{array}$ & $\begin{array}{r}13.55 \\
159 \\
1390 \\
139\end{array}$ & $\begin{array}{r}9.28 \\
354 \\
6.0 \\
363 .\end{array}$ & $\begin{array}{r}7.07 \\
.49 \\
537^{\circ}\end{array}$ \\
\hline $\begin{array}{l}\text { COLOnBos } \\
\text { Db } C-\operatorname{DAY}\end{array}$ & $\begin{array}{c}045.3 \\
: 3 \\
-i .3 \\
604\end{array}$ & $\begin{array}{c}.41 \\
52 i^{0}\end{array}$ & $\begin{array}{r}12.38 \\
446 \\
440 \\
449 .\end{array}$ & $\left|\begin{array}{r}16.43 \\
10^{48} \\
235^{0}\end{array}\right|$ & $\begin{array}{r}20.41 \\
16.52 \\
95 .\end{array}$ & $\begin{array}{r}23.50 \\
2 i^{57} \\
15 .\end{array}$ & $\begin{array}{r}22.67 \\
23.06 \\
0 .\end{array}$ & $\mid \begin{array}{r}19.95 \\
22_{3}^{5} \\
3 .\end{array}$ & $\begin{array}{r}17.65 \\
189 \\
47.0 \\
49\end{array}$ & $\begin{array}{r}11.96 \\
134 \\
193 .\end{array}$ & $\begin{array}{r}7.46 \\
546 \\
397.0\end{array}$ & $\begin{array}{l}5.52 \\
.41 \\
57 i^{\circ}\end{array}$ \\
\hline 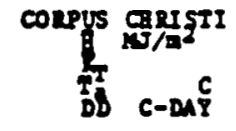 & $\begin{array}{r}7 x .(4) \\
10.97 \\
1 j .9 \\
169 .\end{array}$ & 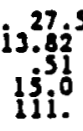 & $\begin{array}{r}17.29 \\
1890 \\
67 .\end{array}$ & $\begin{array}{r}19.85 \\
2 i_{0}^{54} \\
0 .\end{array} \mid$ & $\begin{array}{r}23.499 \\
33.09 \\
0 .\end{array}$ & $\begin{array}{r}25.29 \\
27.63 \\
0 .\end{array}$ & $\mid \begin{array}{l}26.33 \\
2966 \\
0.0\end{array}$ & $\mid \begin{array}{r}23.36 \\
29.62 \\
0 .\end{array}$ & $\begin{aligned} 19.68 \\
2758 \\
0 .\end{aligned}$ & $\begin{array}{r}17.08 \\
290 \\
2.0 \\
4 .\end{array}$ & $\begin{array}{r}11.93 \\
1.51 \\
45^{4}\end{array}$ & $\begin{array}{l}10.23 \\
15^{4} \\
122\end{array}$ \\
\hline
\end{tabular}




\begin{tabular}{|c|c|c|c|c|c|c|c|c|c|c|c|c|}
\hline Month & SAS & res & WNS & APR & MAY & JURE & JURY & 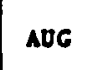 & SEP & OCT & nOV & DEC \\
\hline 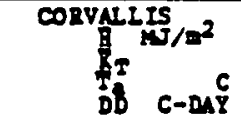 & $\begin{array}{r}02 \\
4 \\
4 \\
4\end{array}$ & $\begin{array}{l}3^{33} \\
341 . \\
341 .\end{array}$ & $\begin{array}{r}11.75 \\
5.47 \\
336 .\end{array}$ & $\begin{array}{r}16.89 \\
10.01 \\
248 .\end{array}$ & $\begin{array}{l}21.24 \\
133 \\
163 . \\
163 .\end{array}$ & $\begin{array}{r}24.30 \\
169 \\
160 .\end{array}$ & $\begin{array}{r}28.02 \\
1.70 \\
34.0 \\
34 .\end{array}$ & $\begin{array}{r}22.87 \\
18.65 \\
31 .\end{array}$ & $\begin{array}{r}16.69 \\
16.60 \\
67 .\end{array}$ & $\begin{array}{l}9.83 \\
24.9 \\
203.0\end{array}$ & $\begin{array}{r}5.86 \\
j^{7} .03 \\
328 .\end{array}$ & $\begin{array}{r}3.39 \\
531 \\
513.0\end{array}$ \\
\hline 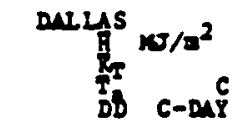 & 33 & $\begin{array}{l}10^{33} .0 \\
243 .\end{array}$ & $\begin{array}{r}16.49 \\
13.0 \\
174 .\end{array}$ & \begin{tabular}{r|r|}
19.01 \\
193 \\
1990 \\
39.
\end{tabular} & $\begin{array}{r}21.82 \\
235.0 \\
0 .\end{array}$ & $\begin{aligned} 24.91 \\
28.61 \\
0 .\end{aligned}$ & $\begin{array}{r}24.62 \\
30.61 \\
0 .\end{array}$ & $\begin{array}{r}22.52 \\
30.00 \\
0 .\end{array}$ & $\begin{array}{r}19.17 \\
26.09 \\
0 .\end{array}$ & $\begin{array}{r}15.20 \\
2098 \\
30^{\circ} .\end{array}$ & $\begin{array}{l}10.93^{\circ} \\
13^{3} 3^{\circ} \\
158^{\circ}\end{array}$ & $\begin{array}{r}9.25 \\
951 \\
2890\end{array}$ \\
\hline 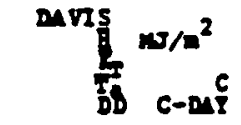 & $\begin{array}{l}a_{6} \\
3\end{array}$ & $\begin{array}{r}5^{50} \\
230^{\circ}\end{array}$ & $\begin{array}{r}16.81 \\
1 \mathrm{i} .0 \\
184 .\end{array}$ & $\begin{array}{r}22.08 \\
16.64 \\
99.0\end{array}$ & $\begin{array}{r}26.60 \\
197 \\
40.0\end{array}$ & $\begin{array}{r}29.36 \\
21.0 \\
0 .\end{array}$ & $\begin{array}{r}28.86 \\
23.0 \\
0.0\end{array}$ & $\begin{array}{r}25.55 \\
23.00 \\
0 .\end{array}$ & $\begin{array}{r}20.83 \\
21.69 \\
0 .\end{array}$ & $\begin{array}{r}14.55 \\
196.0 \\
3 i^{3} .\end{array}$ & $\begin{array}{l}9.03 \\
132 \\
158.0 \\
178 .\end{array}$ & $\begin{array}{r}6.19 \\
7.62 \\
303 .\end{array}$ \\
\hline 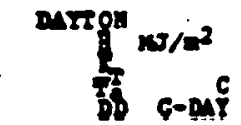 & 0 & $-i^{2}$ & 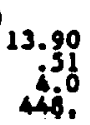 & $\begin{array}{r}17.46 \\
11 \\
229 \\
229\end{array}$ & $\begin{array}{r}21.69 \\
169 \\
92.0\end{array}$ & $\begin{array}{r}24.07 \\
23.98 \\
9 .\end{array}$ & $\begin{array}{r}23.61 \\
24.95 \\
0 .\end{array}$ & $\begin{array}{r}21.52 \\
23.09 \\
4 .\end{array}$ & $\begin{array}{r}17.79 \\
199 \\
19.0 \\
33 .\end{array}$ & $\begin{array}{r}12.94 \\
133^{3} \\
17 i .\end{array}$ & $\begin{array}{r}7.83 \\
549 \\
389 .\end{array}$ & $\begin{array}{l}6.07 \\
-i^{44} \\
38 i^{\circ}\end{array}$ \\
\hline 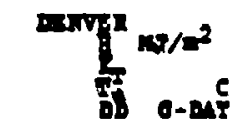 & $-i$ & 301. & $\begin{array}{r}18.25 \\
3.67 \\
+02 .\end{array}$ & $\begin{array}{r}21.73 \\
693 \\
292 .\end{array}$ & $\begin{array}{r}24.37 \\
18.62 \\
161 .\end{array}$ & $\begin{array}{r}27.38 \\
19.68 \\
46 .\end{array}$ & $\begin{array}{r}26.50 \\
23.66 \\
0.0\end{array}$ & $\begin{array}{r}24.79 \\
23.8 \\
8 .\end{array}$ & $\begin{array}{r}20.68 \\
1799 \\
67 .\end{array}$ & $\begin{array}{r}15.49 \\
11.69 \\
227 .\end{array}$ & $\begin{array}{r}10.97 \\
: 66 \\
+27 .\end{array}$ & $\begin{array}{r}9.13 \\
.653 \\
998 .\end{array}$ \\
\hline Des pres & $\begin{array}{l}14.8 \\
7.8\end{array}$ & 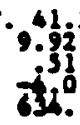 & $\begin{array}{l}13.48 \\
\text { j3i } \\
536 .\end{array}$ & $\begin{array}{r}17.79 \\
1932 \\
238 .\end{array}$ & $\begin{array}{r}21.52 \\
155 \\
1039\end{array}$ & $\begin{array}{r}23.78 \\
2 i^{57} \\
14 .\end{array}$ & $\begin{array}{r}23.78 \\
24.9 \\
0 .\end{array}$ & $\begin{array}{c}20.93 \\
13 \\
130 \\
i .\end{array}$ & $\begin{array}{r}16.96 \\
198 \\
192 \\
32 .\end{array}$ & $\begin{array}{r}12.60 \\
12^{38} \\
196 .\end{array}$ & $\begin{array}{r}7.91 \\
\text { 351 } \\
453 .\end{array}$ & $\begin{array}{l}3.78 \\
-45 \\
689 \\
689 .\end{array}$ \\
\hline
\end{tabular}

\begin{tabular}{|c|c|c|c|c|c|c|c|c|c|c|c|c|}
\hline cortion Month & SAN & rzs & me & APR & $M Y$ & Jणne & $\operatorname{suL} x$ & AUC & SE? & OCI & wov & DEC \\
\hline 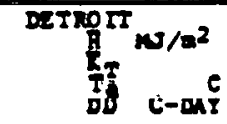 & $\begin{array}{r}-40 \\
-498 .\end{array}$ & & $\begin{array}{r}12.52 \\
548 \\
512 .\end{array}$ & $\begin{array}{r}16.20 \\
59.0 \\
288 .\end{array}$ & $\begin{array}{r}20.89 \\
1853 \\
136 .\end{array}$ & $\begin{array}{r}23.24 \\
2.56 \\
20 .\end{array}$ & $\begin{array}{r}23.36 \\
238 \\
32\end{array}$ & $\begin{array}{r}20.26 \\
23_{3}^{3} \\
.9\end{array}$ & $\begin{array}{r}16.03 \\
1.96 \\
139 .\end{array}$ & $\begin{array}{r}11.39 \\
154 \\
1090 \\
209 .\end{array}$ & $\begin{array}{r}6.20 \\
441 \\
415 .\end{array}$ & $\begin{array}{r}4.81 \\
-39 \\
629 .\end{array}$ \\
\hline 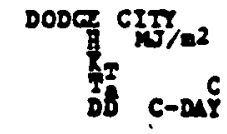 & $\begin{array}{c}u_{10} \\
-i \\
36\end{array}$ & & $\begin{array}{r}18.07 \\
5.04 \\
410 .\end{array}$ & $\begin{array}{r}22.58 \\
12^{6} \\
19 i .\end{array}$ & $\begin{array}{r}23.54 \\
17.0 \\
64 .\end{array}$ & $\begin{array}{r}27.56 \\
23.0 \\
12 .\end{array}$ & $\begin{array}{r}27.18 \\
26.0 \\
0 .\end{array}$ & $\begin{array}{r}24.38 \\
25.07 \\
0 .\end{array}$ & $\begin{array}{r}20.62 \\
20.67 \\
23 .\end{array}$ & $\begin{array}{r}15.89 \\
13.69 \\
137^{60}\end{array}$ & $\begin{array}{r}11.71 \\
3.66 \\
370 .\end{array}$ & $\begin{array}{r}9.70 \\
.64 \\
544 .\end{array}$ \\
\hline 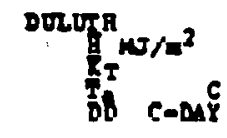 & -1 & -1 & $\begin{array}{l}13.54 \\
-56 \\
735.0\end{array}$ & $\begin{array}{r}16.70 \\
35 \\
3.0 \\
40.0\end{array}$ & $\begin{array}{r}20.72 \\
132 \\
860.0 \\
869 .\end{array}$ & $\begin{array}{r}23.11 \\
196 \\
100 . \\
100\end{array}$ & $\begin{array}{r}23.19 \\
198 \\
39 .\end{array}$ & $\begin{array}{r}19.55 \\
1968 \\
190^{\circ} .\end{array}$ & $\begin{array}{l}13.94 \\
132 \\
19 \% .0\end{array}$ & $\begin{array}{l}9.71 \\
5 \frac{71}{32} \\
9390\end{array}$ & $\begin{array}{l}3.32 \\
-34.0 \\
610 .\end{array}$ & $\begin{array}{r}4.35 \\
-1.45 \\
-872 .\end{array}$ \\
\hline 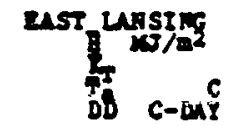 & 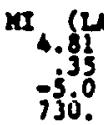 & - & $\begin{array}{r}12.29 \\
.675 \\
533 .\end{array}$ & $\begin{array}{r}14.18 \\
842 \\
38 \mathrm{~s} .\end{array}$ & $\begin{array}{r}19.65 \\
530 \\
136^{\circ}\end{array}$ & $\begin{array}{r}21.70 \\
132 \\
13^{0}\end{array}$ & $\begin{array}{r}21.37 \\
2 i_{3}^{33}\end{array}$ & $\begin{array}{r}18.44 \\
.52 \\
20^{0.0} \\
13 .\end{array}$ & $\begin{array}{r}14.76 \\
19.9 \\
16.0 \\
94 .\end{array}$ & $\begin{array}{r}10.12 \\
10^{48} \\
234 .\end{array}$ & $\begin{array}{r}3.39 \\
390 \\
460\end{array}$ & $\begin{array}{l}4.35 \\
-i 35 \\
653 .\end{array}$ \\
\hline 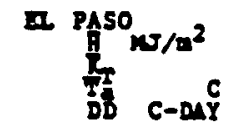 & $\begin{array}{r}5 x .84 \\
13.84 \\
j^{69} \\
38 i .\end{array}$ & $\begin{array}{l}319 \\
i 8.07 \\
972 \\
247 .\end{array}$ & $\begin{array}{r}22.96 \\
1935 \\
175 .\end{array}$ & $\begin{array}{r}27.39 \\
17.6 \\
38.0\end{array}$ & $\begin{array}{r}29.90 \\
29.75 \\
0.0\end{array}$ & $\begin{array}{r}30.53 \\
2 i, 0 \\
0 .\end{array}$ & $\begin{array}{r}28.02 \\
27.0 \\
0 .\end{array}$ & $\begin{array}{r}26.72 \\
26.00 \\
0 .\end{array}$ & $\begin{array}{r}24.05 \\
23.04 \\
0 .\end{array}$ & $\begin{array}{r}19.32 \\
183 \\
47^{7} \\
4 .\end{array}$ & $\begin{array}{l}15.35 \\
172 \\
230.0\end{array}$ & $\begin{array}{r}13.09 \\
790 \\
360^{\circ}\end{array}$ \\
\hline 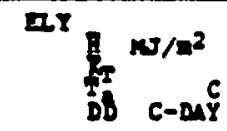 & $\begin{array}{l}80.94 \\
9.95 \\
664 \\
72 i .0\end{array}$ & $\begin{aligned} i 3.9 j \\
-367 \\
397 .\end{aligned}$ & $\begin{array}{r}19.40 \\
.70 \\
543 .\end{array}$ & $\begin{array}{r}23.59 \\
356 \\
373 .\end{array}$ & $\begin{array}{r}26.10 \\
186.0 \\
253 .\end{array}$ & $\begin{array}{l}29.61 \\
14.0 \\
125 .\end{array}$ & $\begin{array}{r}27.10^{\circ} \\
19.6 \\
16 .\end{array}$ & $\begin{array}{r}25.43 \\
18.0 \\
24 .\end{array}$ & $\begin{array}{r}21.70 \\
13.0 \\
130 .\end{array}$ & $\begin{array}{r}16.43 \\
77 \\
730 \\
329 .\end{array}$ & $\begin{array}{r}12.00 \\
i .0 \\
i .0 \\
522 .\end{array}$ & $\begin{array}{r}9.20 \\
-3.65 \\
658 .\end{array}$ \\
\hline
\end{tabular}




\begin{tabular}{|c|c|c|c|c|c|c|c|c|c|c|c|c|}
\hline Month & د. & $\sqrt{153}$ & Me & APR & $M \gamma$ & תRा & JoLX & AUG & SEP & OCT & nov & DEC \\
\hline 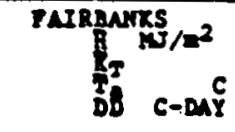 & 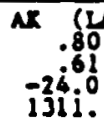 & $\begin{array}{l}\text { T. } 3.18 .18 \\
3.193 \\
-1958 \\
3056 .\end{array}$ & $\begin{array}{r}9.74 \\
-13.0 \\
-136 . \\
966 .\end{array}$ & $\begin{array}{r}16.10 \\
-6.64 \\
593 .\end{array}$ & $\begin{array}{r}19.95 \\
856 \\
308.0\end{array}$ & 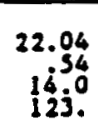 & $\begin{array}{r}18.57 \\
1550 \\
95 .\end{array}$ & $\begin{array}{r}15.18 \\
152 \\
184 . \\
184 .\end{array}$ & $\begin{array}{l}7.69 \\
843 \\
3550\end{array}$ & $\begin{array}{l}3.60 \\
-34.5 \\
-3.0 \\
668 .\end{array}$ & $\begin{array}{r}1.13 \\
-151 \\
-16.0 \\
1018 .\end{array}$ & $\begin{array}{r}.25 \\
249 \\
-22^{4} \\
1252 .\end{array}$ \\
\hline 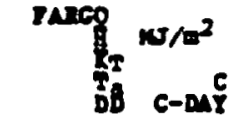 & $\begin{array}{l}\text { Do. } \\
\text { - } 10.6 \\
101\end{array}$ & $-\frac{1}{3}$ & 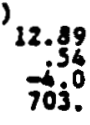 & $\begin{array}{r}17.54 \\
54 \\
638.0 \\
378 .\end{array}$ & $\begin{array}{rl}21.91 & 0 \\
13 & 5 \\
186 . & 0\end{array}$ & $\begin{array}{r}22.02 \\
193.0 \\
54.0\end{array}$ & $\begin{array}{r}23.19 \\
2 i^{58} \\
.\end{array}$ & $\begin{array}{r}19.89 \\
21.0 \\
18 .\end{array}$ & $\begin{array}{r}14.57 \\
196 \\
130.0 \\
130 .\end{array}$ & $\begin{array}{r}10.13 \\
584 \\
310.0 \\
310 .\end{array}$ & $\begin{array}{l}5.48 \\
-24.4 \\
607 .\end{array}$ & $\begin{array}{r}4.94 \\
-151 \\
-19.0 \\
896 .\end{array}$ \\
\hline 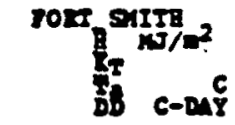 & ${ }^{4}$ & $\begin{array}{r}4.49 \\
33^{6} .0\end{array}$ & $\begin{array}{r}15.11 \\
552 \\
1050 \\
262 .\end{array}$ & $\begin{array}{r}18.80 \\
190 \\
193 \\
73\end{array}$ & $\begin{array}{r}22.02 \\
21.96 \\
9 .\end{array}$ & $\begin{array}{r}23.61 \\
285 \\
0.0 \\
0 .\end{array}$ & $\begin{array}{r}22.86 \\
28.57 \\
0.0\end{array}$ & $\begin{array}{r}21.86 \\
25.90 \\
0 .\end{array}$ & $\begin{array}{r}18.13 \\
2358 \\
0 .\end{array}$ & $\begin{array}{r}14.19 \\
195 \\
1550 \\
75.0\end{array}$ & $\begin{array}{l}9.80 \\
1.31 \\
240 \\
243 .\end{array}$ & $\begin{array}{r}7.83 \\
53 \\
50.0 \\
605 .\end{array}$ \\
\hline 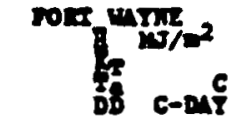 & 68 & $\begin{array}{l}-4109 \\
9.08 \\
-4260 \\
38.8 .\end{array}$ & 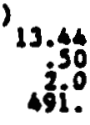 & $\begin{array}{r}17.08 \\
10.9 \\
262.0\end{array}$ & $\begin{array}{r}22.06 \\
15.0 \\
120.0\end{array}$ & $\begin{array}{r}26.45 \\
2 i^{39} \\
13 .\end{array}$ & $\begin{array}{r}23.82 \\
233^{59} \\
0 .\end{array}$ & $\begin{array}{r}21.10 \\
2390 \\
i .\end{array}$ & $\begin{array}{r}16.54 \\
1960 \\
50.0\end{array}$ & $\begin{array}{r}12.35 \\
135 \\
102 .\end{array}$ & $\begin{array}{r}7.07 \\
54.0 \\
413 .\end{array}$ & $\begin{array}{l}5.65 \\
-i^{4} \\
62 i^{0}\end{array}$ \\
\hline 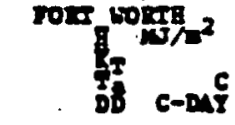 & in. & $\begin{array}{r}\text { is.32: } \\
535 \\
249 .\end{array}$ & $\begin{array}{r}17.77 \\
139 \\
139 .\end{array}$ & $\begin{array}{r}12.25 \\
18.34 \\
53.0\end{array}$ & $\begin{array}{r}23.56 \\
22_{0}^{5} .0 \\
0 .\end{array}$ & $\begin{array}{r}26.85 \\
23^{66} \\
0 .\end{array}$ & $\begin{array}{r}25.59 \\
29.60 \\
0 .\end{array}$ & $\begin{array}{r}26.39 \\
29.0 . \\
0 .\end{array}$ & $\begin{array}{r}20.91 \\
25.00 \\
0 .\end{array}$ & $\begin{array}{r}16.48 \\
19.63 \\
36 .\end{array}$ & $\begin{array}{l}12.46 \\
13.00 \\
180 .\end{array}$ & $\begin{array}{r}10.30 \\
56 \\
896 \\
298.8\end{array}$ \\
\hline 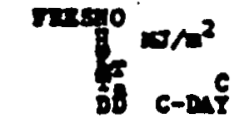 & 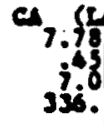 & 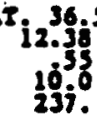 & $\begin{array}{r}18.32 \\
18.60 \\
186 .\end{array}$ & $\begin{array}{r}22.78 \\
16.0 \\
90.9\end{array}$ & $\begin{array}{r}26.66 \\
1967 \\
34.0\end{array}$ & $\begin{array}{r}29.15 \\
2 j_{j}^{15} \\
j^{2} .\end{array}$ & $\begin{array}{r}27.93 \\
27.0 \\
0 .\end{array}$ & $\begin{array}{r}23.34 \\
26.8 \\
0 .\end{array}$ & $\begin{array}{r}21.03 \\
23.08 \\
0 .\end{array}$ & $\begin{array}{r}13.68 \\
18.6 \\
45 .\end{array}$ & {$\left[\begin{array}{r}10.08 \\
35 \\
19 j \\
19 j .\end{array}\right.$} & $\begin{array}{r}6.69 \\
542 \\
32 i^{\circ}\end{array}$ \\
\hline
\end{tabular}

\begin{tabular}{|c|c|c|c|c|c|c|c|c|c|c|c|c|}
\hline ocation Month & $\mathrm{JNM}$ & $\mathbf{n u}$ & $m$ & APR & MAY & STIE & soly & $A D C$ & SE2 & OCT & Nov & DEC \\
\hline 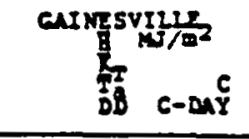 & $\begin{array}{l}71.5 \\
11.63 \\
134 \\
13.0 \\
164 .\end{array}$ & $\begin{array}{l}159 \\
133^{5} \\
\end{array}$ & $\begin{array}{r}18.61 \\
1 i^{59} \\
73 .\end{array}$ & $\begin{array}{r}22.54 \\
2 i^{6} \\
119\end{array}$ & $\begin{array}{r}24.51 \\
24.62 \\
0 .\end{array}$ & $\begin{array}{r}22.73 \\
26.50 \\
0 .\end{array}$ & $\begin{array}{r}21.73 \\
27.0 \\
0 .\end{array}$ & $\begin{array}{r}21.24 \\
256 \\
0.0 \\
0 .\end{array}$ & $\begin{array}{r}18.57 \\
26.56 \\
0 .\end{array}$ & $\begin{array}{r}15.39 \\
256 \\
23.0\end{array}$ & $\begin{array}{r}13.30 \\
17.59 \\
69 .\end{array}$ & $\begin{array}{r}10.62 \\
.53 \\
14.0 \\
143 .\end{array}$ \\
\hline 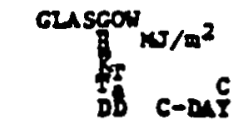 & 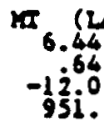 & $\begin{array}{l}-80.0 \\
799.0\end{array}$ & $\begin{array}{r}15.77 \\
-35 \\
659 \\
659\end{array}$ & $\begin{array}{r}19.03 \\
660 \\
680 .\end{array}$ & $\begin{array}{r}23.50 \\
126.0 \\
186 .\end{array}$ & $\begin{array}{r}23.63 \\
17.62 \\
83 .\end{array}$ & $\begin{array}{r}26.76 \\
21.67 \\
17.0\end{array}$ & $\begin{array}{r}22.33 \\
2 i^{65} \\
26.0\end{array}$ & $\begin{array}{r}17.15 \\
165 \\
150 .\end{array}$ & $\begin{array}{r}11.21 \\
\dot{8}^{6.3} \\
338.0\end{array}$ & $\begin{array}{l}6.48 \\
-57 \\
-1.0 \\
613 .\end{array}$ & $\begin{array}{l}4.94 \\
-i^{5} 7 \\
814\end{array}$ \\
\hline 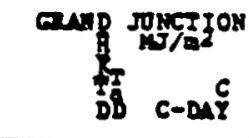 & $\begin{array}{l}\infty \\
9 . \\
-3 \\
67\end{array}$ & $\begin{array}{l}.65 \\
\text { soi. }\end{array}$ & $\begin{array}{r}17.98 \\
369 \\
405 .\end{array}$ & $\begin{array}{r}22.29 \\
1 i^{6} \\
215.9\end{array}$ & $\begin{array}{r}25.30 \\
16.64 \\
81 .\end{array}$ & $\begin{array}{r}29.61 \\
22.0 \\
12 .\end{array}$ & $\begin{array}{r}28.06 \\
25.00 \\
0 .\end{array}$ & $\begin{array}{r}24.30 \\
24.67 \\
0.0\end{array}$ & $\begin{array}{r}20.95 \\
1990 \\
19 .\end{array}$ & $\begin{array}{r}15.81 \\
13.6 \\
174.0\end{array}$ & $\begin{array}{r}11.00 \\
863 \\
435 .\end{array}$ & $\begin{array}{l}9.03 \\
-64 \\
617.0\end{array}$ \\
\hline 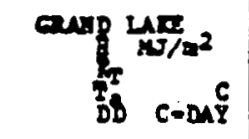 & $\begin{array}{l}{ }^{c 0} 8 . \\
-9 \\
86\end{array}$ & 7iz. & $\begin{array}{r}17.71 \\
-4.65 \\
720.0\end{array}$ & $\begin{array}{r}21.44 \\
.65 \\
523^{\circ}\end{array}$ & $\begin{array}{r}23.11 \\
39 \\
38.0 \\
38 .\end{array}$ & $\begin{array}{r}26.46 \\
10.64 \\
250 .\end{array}$ & $\begin{array}{r}25.12 \\
13.2 \\
153 .\end{array}$ & $\begin{array}{r}21.14 \\
138 \\
19.0 \\
174 .\end{array}$ & $\begin{array}{r}19.93 \\
667 \\
280.0 \\
280 .\end{array}$ & $\begin{array}{r}15.11 \\
568 \\
46.0\end{array}$ & $\begin{array}{l}9.80 \\
-6.61 \\
-63.0 \\
653 .\end{array}$ & $\begin{array}{r}7.70 \\
-85 \\
-820 \\
820 .\end{array}$ \\
\hline 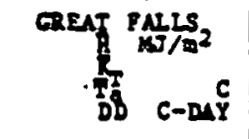 & $\begin{array}{l}5.72 \\
5.77 \\
-5.04 \\
749 .\end{array}$ & $\begin{array}{l}9.58 \\
-359 \\
-24.0 \\
641 .\end{array}$ & $\begin{array}{l}15.14 \\
.64 \\
59 i^{\circ}\end{array}$ & $\begin{array}{r}17.94 \\
564 \\
359 \\
359\end{array}$ & $\begin{array}{r}21.91 \\
135 \\
213 .\end{array}$ & $\begin{array}{r}24.78 \\
16.0 \\
103 .\end{array}$ & $\begin{array}{r}26.36 \\
25.7 \\
16 .\end{array}$ & $\begin{array}{r}22.12 \\
20.64 \\
29 .\end{array}$ & $\begin{array}{r}16.89 \\
.653 \\
14.0 \\
143 .\end{array}$ & $\begin{array}{r}10.96 \\
960 \\
902 .\end{array}$ & $\begin{array}{l}6.44 \\
\text { is. } \\
\text { sit. }\end{array}$ & $\begin{array}{l}4.68 \\
-351 \\
-3.00 \\
649 .\end{array}$ \\
\hline 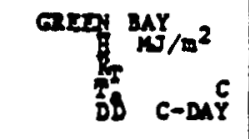 & $\begin{array}{r}\text { UI. } 5 \\
5.74 \\
-9.96 \\
854.0\end{array}$ & $\begin{array}{r}4.79 \\
8.99 \\
73^{3} \\
9 .\end{array}$ & $\begin{array}{r}13.10 \\
-352 \\
62 j^{\circ} .\end{array}$ & $\begin{array}{r}16.08 \\
548 \\
353 .\end{array}$ & $\begin{array}{r}20.47 \\
12.33 \\
18.0 \\
18.9\end{array}$ & $\begin{array}{r}22.69 \\
185 \\
18.0 \\
5 i .\end{array}$ & $\begin{array}{r}22.552 \\
2 i^{36} \\
12 .\end{array}$ & $\begin{array}{r}19.34 \\
250.5 \\
30 .\end{array}$ & $\begin{array}{r}14.78 \\
1553 \\
106.0 \\
100 .\end{array}$ & $\begin{array}{l}10.05 \\
1900 \\
272.0\end{array}$ & $\begin{array}{r}5.82 \\
542 \\
513.0\end{array}$ & $\begin{array}{r}4.60 \\
4.42 \\
7590 .\end{array}$ \\
\hline
\end{tabular}




\begin{tabular}{|c|c|c|c|c|c|c|c|c|c|c|c|c|}
\hline Month & JAH & TEB & MuR & $A P R$ & $M Y$ & JUNE & JULY & $\triangle D G$ & SEP & OCT & Nov & DEC \\
\hline 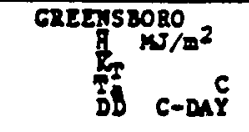 & $\begin{array}{r}86.51 \\
8.57 \\
349 \\
453 .\end{array}$ & $\begin{array}{r}50 \\
3790 \\
390 .\end{array}$ & $\begin{array}{r}14.80 \\
.931 \\
302.0\end{array}$ & $\begin{array}{r}19.61 \\
196 \\
113^{\circ} \\
11 .\end{array}$ & $\begin{array}{r}22.29 \\
1960 \\
33 .\end{array}$ & $\begin{array}{r}23.50 \\
23.0 \\
0 .\end{array}$ & $\begin{array}{r}22.62 \\
2356 \\
0.0\end{array}$ & $\begin{array}{r}19.86 \\
24.54 \\
0.0 \\
0 .\end{array}$ & $\begin{array}{r}17.23 \\
295 \\
i j . \\
13 .\end{array}$ & $\begin{array}{r}13.76 \\
14.06 \\
116 .\end{array}$ & $\begin{array}{r}10.16 \\
855 \\
89.0 \\
278 .\end{array}$ & $\begin{array}{r}7.78 \\
448 \\
437 .\end{array}$ \\
\hline 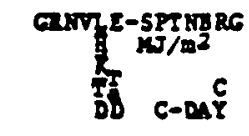 & $\begin{array}{r}\text { Me. } 3 \frac{4}{8} \\
: 51 \\
6.0 \\
391 .\end{array}$ & $32 \mathrm{i}$. & $\begin{array}{r}16.29 \\
1055 \\
250 .\end{array}$ & $\begin{array}{r}21.39 \\
16.60 \\
80.0\end{array}$ & $\begin{array}{r}23.15 \\
21.8 \\
16 .\end{array}$ & $\begin{array}{r}23.32 \\
24.07 \\
0.0\end{array}$ & $\begin{array}{r}23.19 \\
26.0 \\
0 .\end{array}$ & $\begin{array}{r}21.48 \\
25^{88} \\
0.0\end{array}$ & $\begin{array}{r}17.75 \\
23.56 \\
5 .\end{array}$ & $\begin{array}{r}14.99 \\
16.60 \\
81 .\end{array}$ & $\begin{array}{r}10.76 \\
155 \\
2330 .\end{array}$ & $\begin{array}{l}8.58 \\
5.51 \\
38.0 \\
381 .\end{array}$ \\
\hline 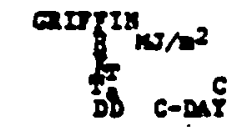 & 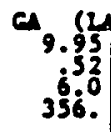 & 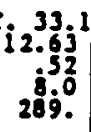 & $\begin{aligned} 16.23 \\
1 i^{54} \\
228 .\end{aligned}$ & $\begin{array}{r}21.70 \\
16.0 \\
61 .\end{array}$ & $\begin{array}{r}24.13 \\
21.61 \\
11 .\end{array}$ & $\begin{array}{r}24.25 \\
24.59 \\
0.0\end{array}$ & $\begin{array}{r}23.38 \\
25.58 \\
0 .\end{array}$ & $\begin{array}{r}21.87 \\
259 \\
0 .\end{array}$ & $\begin{array}{r}18.27 \\
23.0 \\
11 .\end{array}$ & $\begin{array}{r}15.56 \\
17.60 \\
6 i .\end{array}$ & $\begin{array}{l}12.04 \\
1 i^{39} \\
200.0\end{array}$ & $\begin{array}{c}8.78 \\
; .9 \\
339 .\end{array}$ \\
\hline 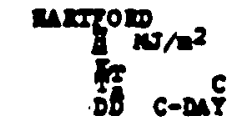 & 69.0. & 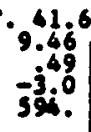 & $\begin{aligned} 13.73 \\
352 \\
2.0 \\
306 .\end{aligned}$ & $\begin{array}{r}16.12 \\
\dot{9.8} \\
268.0\end{array}$ & $\begin{array}{r}19.85 \\
15.5 \\
126 .\end{array}$ & $\begin{array}{r}22.36 \\
20.54 \\
13 .\end{array}$ & $\begin{array}{r}22.15 \\
235 \\
0 .\end{array}$ & $\begin{array}{r}19.22 \\
21.54 \\
7 .\end{array}$ & $\begin{array}{r}15.22 \\
192 \\
19.0 \\
59 .\end{array}$ & $\begin{aligned} 11.05 \\
10^{51} \\
21 j \\
21 .\end{aligned}$ & $\begin{array}{r}6.91 \\
35 \\
395 \\
39 .\end{array}$ & $\begin{array}{l}7.91 \\
-3.62 \\
634 .\end{array}$ \\
\hline 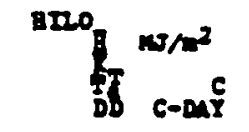 & $2 i^{2}$ & 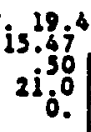 & $\begin{array}{r}18.73 \\
2 i^{34} .0 \\
0 .\end{array}$ & $\begin{array}{r}18.02 \\
22.08 \\
0.0\end{array}$ & $\begin{array}{r}18.73 \\
23.08 \\
0 .\end{array}$ & $\begin{aligned} 24.00 \\
23.6 ! \\
0 .\end{aligned}$ & $\begin{array}{r}22.33 \\
235_{0}^{\circ} \\
0 .\end{array}$ & $\begin{array}{r}20.49 \\
24.04 \\
0 .\end{array}$ & $\begin{array}{r}18.90 \\
24.53 \\
24.0 \\
0 .\end{array}$ & $\begin{array}{r}14.18 \\
23.00 \\
0 .\end{array}$ & $\begin{array}{r}12.63 \\
2 i^{45} \\
0 .\end{array}$ & $\begin{array}{r}11.00 \\
2 i^{4.3} \\
0 .\end{array}$ \\
\hline 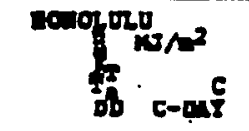 & 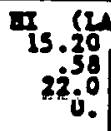 & $\begin{array}{r}27^{21} \cdot 5^{2} \\
23_{30}^{3}\end{array}$ & $\begin{array}{r}21.60 \\
22^{63} \\
\quad 0^{6}\end{array}$ & $\begin{array}{r}23.40 \\
23.62 \\
6 .\end{array}$ & $\begin{array}{r}25.83 \\
24 \mathrm{~g}^{6}\end{array}$ & $\begin{array}{r}25.75 \\
25 \% \\
0 .\end{array}$ & $\begin{array}{r}25.73 \\
2660 \\
0 .\end{array}$ & $\begin{array}{r}25.62 \\
26.8 \\
8 .\end{array}$ & $\begin{array}{r}23.99 \\
268 \\
26.0 \\
0.0\end{array}$ & $\begin{array}{r}21.23 \\
1.68 \\
0 .\end{array}$ & $\begin{array}{r}37.86 \\
26^{66} \\
0 .\end{array}$ & $\begin{array}{r}15.53 \\
23.03 \\
0 .\end{array}$ \\
\hline
\end{tabular}

\begin{tabular}{|c|c|c|c|c|c|c|c|c|c|c|c|c|}
\hline Month & SAX & TES & wn & APR & $\operatorname{mar}$ & Jone & JULY & ANG & SEP & OCT & Hov & DEC \\
\hline 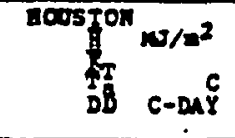 & $\begin{array}{r}\pi x .54 \\
10.05 \\
1 i^{47} \\
23 i^{0}\end{array}$ & $\begin{array}{l}2.64 \\
1390 \\
163.0\end{array}$ & $\begin{array}{r}16.37 \\
162 \\
165 \\
105\end{array}$ & $\begin{array}{r}18.97 \\
213 \\
13 .\end{array}$ & $\begin{array}{rl}23.11 & .38 \\
24 & 0.0 \\
0 .\end{array}$ & $\begin{array}{r}23.08 \\
26^{66} \\
0 .\end{array}$ & $\begin{array}{r}24.93 \\
28.0 \\
0 .\end{array}$ & $\begin{array}{r}21.68 \\
29.00 \\
0 .\end{array}$ & $\begin{array}{r}19.09 \\
26.08 \\
0 .\end{array}$ & $\begin{array}{r}16.58 \\
260 \\
23.0 \\
13 .\end{array}$ & $\begin{array}{c}11.59 \\
16.32 \\
86 .\end{array}$ & $\begin{array}{l}9.5 n \\
13.8 \\
13.0 \\
183 .\end{array}$ \\
\hline 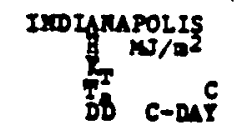 & $\begin{array}{l}\text { IN } 6.1 \\
-i \\
639\end{array}$ & $\begin{array}{r}39 . \\
8.95 \\
.43 \\
533 .\end{array}$ & $\begin{array}{r}13.05 \\
48 . \\
436.0 \\
436\end{array}$ & $\begin{array}{r}16.4 \\
14.8 \\
215 .\end{array}$ & $\begin{array}{r}20.53 \\
17.52 \\
88 .\end{array}$ & $\begin{array}{r}22.87 \\
22.0 \\
6 .\end{array}$ & $\begin{array}{r}22.67 \\
24.56 \\
0 .\end{array}$ & $\begin{array}{r}20.32 \\
2350 \\
3 .\end{array}$ & $\begin{array}{r}16.94 \\
195 \\
35.0\end{array}$ & $\begin{array}{r}12.25 \\
1350 \\
168 .\end{array}$ & $\begin{array}{c}7.36 \\
5.44 \\
388 .\end{array}$ & $\begin{array}{l}5.44 \\
.39 \\
587^{\circ}\end{array}$ \\
\hline 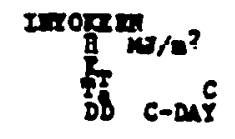 & $\begin{array}{r}c a .04 \\
19.05 \\
3^{7} 50 \\
34 i .\end{array}$ & $\begin{array}{r}7.75 \\
21.0 \\
218 .\end{array}$ & $\begin{array}{r}24.27 \\
18.03 \\
148 .\end{array}$ & $\begin{array}{r}29.32 \\
18.83 \\
7 i .\end{array}$ & $\begin{array}{r}32.49 \\
23.0 \\
6 .\end{array}$ & $\begin{array}{r}34.96 \\
285 \\
27.0 \\
0 .\end{array}$ & $\begin{array}{r}32.79 \\
328 \\
0.0\end{array}$ & $\begin{array}{r}30.86 \\
3 i .0 \\
0 .\end{array}$ & $\begin{array}{r}27.10 \\
27.06 \\
0 .\end{array}$ & $\begin{array}{r}20.24 \\
20.02 \\
24 .\end{array}$ & $\begin{array}{l}13 . \$ 1 \\
130 \\
176.0\end{array}$ & $\begin{array}{r}12.34 \\
8.75 \\
334 .\end{array}$ \\
\hline 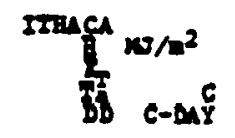 & $\begin{array}{r}\pi_{3} .1 \\
-j_{3}^{3} \\
723\end{array}$ & $\begin{array}{l}.42 . \\
8.49 \\
4.45 \\
646 .\end{array}$ & $\begin{array}{r}11.79 \\
.465 \\
562 .\end{array}$ & $\begin{array}{r}14.35 \\
59.5 \\
332 .\end{array}$ & $\begin{array}{l}19.61 \\
130 \\
135.0\end{array}$ & $\begin{array}{r}22.54 \\
1980 \\
39.0\end{array}$ & $\begin{array}{r}22.37 \\
296 \\
11.0 \\
1 .\end{array}$ & $\begin{array}{r}19.24 \\
19.94 \\
29 .\end{array}$ & $\begin{array}{r}16.89 \\
159 \\
87.0 \\
87 .\end{array}$ & $\begin{array}{r}10.37 \\
10^{49} \\
243 .\end{array}$ & $\begin{array}{r}3.23 \\
353 \\
4.00 \\
423 .\end{array}$ & $\begin{array}{l}4.14 \\
-34 \\
-3.94 \\
654 .\end{array}$ \\
\hline 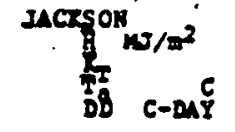 & 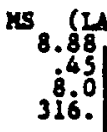 & $\begin{array}{r}11.73 .9 \\
10^{48} \\
246.0\end{array}$ & $\begin{array}{r}15.83 \\
15.82 \\
13.0 \\
194 .\end{array}$ & $\begin{array}{r}20.18 \\
196 \\
49 .\end{array}$ & $\begin{array}{r}22.94 \\
23.58 \\
3 .\end{array}$ & $\begin{array}{r}23.53 \\
265 \\
26.0\end{array}$ & $\begin{array}{r}22.78 \\
28.57 \\
0 .\end{array}$ & $\begin{array}{r}21.39 \\
25.07 \\
0 .\end{array}$ & $\begin{array}{rl}17.96 \\
24 & 55 \\
24 & 0 \\
0 .\end{array}$ & $\begin{array}{l}19 . \frac{1}{58} \\
19^{58} \\
51 .\end{array}$ & $\begin{array}{l}10.42 \\
50 \\
13.0 \\
167 .\end{array}$ & $\begin{array}{r}8.46 \\
9.46 \\
280 .\end{array}$ \\
\hline 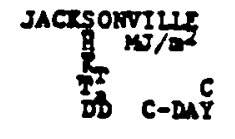 & $\begin{array}{r}\pi,(2 A \\
11.18 \\
13 \\
12.0 \\
193 .\end{array}$ & $\begin{array}{l}30.2 \\
14.49^{2} \\
13^{35} \\
157^{\circ}\end{array}$ & $\begin{array}{r}17.71 \\
185 \\
169 \\
98 .\end{array}$ & $\begin{array}{r}21.55 \\
20.09 \\
13.0\end{array}$ & $\begin{array}{r}23.28 \\
23.0 \\
23.0\end{array}$ & $\begin{array}{r}21.98 \\
26.54 \\
0.0\end{array}$ & $\begin{array}{r}21.86 \\
27.05 \\
0 .\end{array}$ & $\begin{array}{r}19.93 \\
27.03 \\
0 .\end{array}$ & $\begin{array}{r}16.03 \\
25.0 \\
25 .\end{array}$ & $\begin{aligned} 13.86 \\
2 i_{1}^{5} \\
1 i^{\circ}\end{aligned}$ & $\begin{array}{c}11.45 \\
162 \\
89.0 \\
89 .\end{array}$ & $\begin{array}{l}9.63 \\
12^{49} \\
176 .\end{array}$ \\
\hline
\end{tabular}




\begin{tabular}{|c|c|c|c|c|c|c|c|c|c|c|c|c|}
\hline ocation Month & JAH & FEs & $\mathrm{MAR}$ & $\triangle P R$ & $m Y$ & JUXE & $\operatorname{sul} Y$ & AUG & SEP & $\alpha \boldsymbol{C T}$ & Nov & DEC \\
\hline 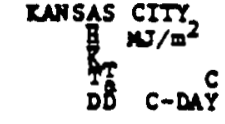 & $\begin{array}{r}00.62 \\
7.62 \\
-2.49 \\
64 i .\end{array}$ & & $\begin{array}{r}14.28 \\
552 \\
510 \\
414 .\end{array}$ & $\begin{array}{rl}18.46 & 5 \\
13 & 5.0 \\
174 & 0\end{array}$ & $\begin{array}{r}21.81 \\
1.55 \\
18.0 \\
62 .\end{array}$ & $\begin{array}{r}26.70 \\
23.00 \\
7 .\end{array}$ & $\begin{array}{r}24.24 \\
26.0 \\
0 .\end{array}$ & $\begin{array}{r}22.02 \\
25.01 \\
0.0\end{array}$ & $\begin{array}{r}17.79 \\
20.99 \\
23 .\end{array}$ & $\begin{array}{r}13.40 \\
159 \\
135 . \\
131 .\end{array}$ & $\begin{array}{r}9.00 \\
54 \\
55.0 \\
357 .\end{array}$ & $\begin{array}{r}6.87 \\
.49 \\
363 .\end{array}$ \\
\hline 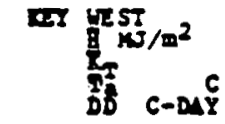 & $\begin{array}{r}77.67 \\
13.69 \\
2150 \\
9.0\end{array}$ & $2 i_{0}^{\circ 0}$ & $\begin{array}{r}20.51 \\
23.52 \\
3 .\end{array}$ & $\begin{aligned} 23.95 \\
23.64 \\
0 .\end{aligned}$ & $\begin{array}{r}24.24 \\
2 i^{62} \\
0.0\end{array}$ & $\begin{array}{r}22.73 \\
28.57 \\
0 .\end{array}$ & $\begin{array}{r}22.36 \\
29.0 \\
0 .\end{array}$ & $\begin{array}{r}20.97 \\
295 \\
0.0\end{array}$ & $\begin{array}{r}18.63 \\
28.54 \\
0.0\end{array}$ & $\begin{array}{r}16.49 \\
265 \\
0.0 \\
0 .\end{array}$ & $\begin{array}{r}13.90 \\
23.95 \\
0.0\end{array}$ & $\begin{array}{r}12.23 \\
21.53 \\
10 .\end{array}$ \\
\hline 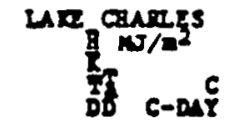 & $\begin{array}{r}40.80 \\
10.40 \\
1 i^{4}: 0 \\
212 .\end{array}$ & $13^{50}$ & $\begin{array}{r}16.56 \\
153 \\
150 . \\
108 .\end{array}$ & $\begin{array}{r}20.20 \\
20.53 \\
22.0\end{array}$ & $\begin{array}{r}23.17 \\
23.58 \\
0 .\end{array}$ & $\begin{array}{r}24.34 \\
27.60 \\
0 .\end{array}$ & $\begin{array}{r}21.79 \\
28.94 \\
0 .\end{array}$ & $\begin{array}{r}21.08 \\
25 \% \\
56.0 \\
0 .\end{array}$ & $\begin{array}{r}18.73 \\
2557 \\
0 .\end{array}$ & $\begin{array}{r}16.81 \\
2 i^{62} \\
11 .\end{array}$ & $\begin{array}{r}12.38 \\
156 \\
15.0 \\
117 .\end{array}$ & $\begin{array}{l}9.70 \\
1390 \\
189 .\end{array}$ \\
\hline 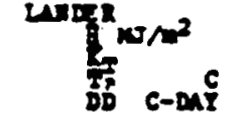 & - & $-\mathbf{j}$ & $\begin{array}{r}18.86 \\
.72 \\
565.0\end{array}$ & $\begin{array}{r}23.13 \\
869 \\
363 . \\
363 .\end{array}$ & $\begin{array}{r}24.66 \\
12^{63} \\
212 .\end{array}$ & $\begin{array}{r}28.23 \\
168 \\
85.0\end{array}$ & $\begin{array}{r}27.109 \\
21.69 \\
3 .\end{array}$ & $\begin{array}{r}24.25 \\
21.88 \\
11 .\end{array}$ & $\begin{array}{r}19.40 \\
130 \\
113 .\end{array}$ & $\begin{array}{r}14.89 \\
89.0 \\
908 .\end{array}$ & $\begin{array}{r}9.91 \\
.67 \\
567^{\circ}\end{array}$ & $\begin{array}{l}8.26 \\
-88 \\
-8.0 \\
722 .\end{array}$ \\
\hline 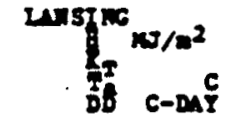 & $\begin{array}{l}\text { II } 3.65 \\
3.65 \\
-340 \\
730 .\end{array}$ & $\begin{array}{l}42 . \\
8.92 \\
6.7 \\
630 .\end{array}$ & 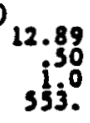 & $\begin{array}{r}14.99 \\
84.5 \\
308.0\end{array}$ & $\begin{array}{r}20.89 \\
1.83 \\
156^{\circ} .\end{array}$ & $\begin{array}{r}23.13 \\
1960 \\
27 .\end{array}$ & $\begin{array}{r}22.78 \\
22^{57} \\
5 .\end{array}$ & $\begin{array}{r}20.14 \\
21.5 \\
15.0\end{array}$ & $\begin{array}{r}15.78 \\
165 \\
16.0 \\
74 .\end{array}$ & $\begin{array}{r}10.80 \\
151 \\
\frac{15}{236.0} \\
23 .\end{array}$ & $\begin{array}{r}3.86 \\
4.40 \\
4.0 \\
43 .\end{array}$ & $\begin{array}{r}4.69 \\
-399 \\
-353.0\end{array}$ \\
\hline 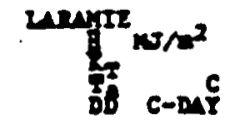 & 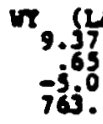 & $\frac{i}{63}$ & $\begin{array}{r}17.73 \\
-65 \\
653.0\end{array}$ & $\begin{array}{r}20.83 \\
63 . \\
653 . \\
453 .\end{array}$ & $\begin{array}{r}22.92 \\
998 \\
296 .\end{array}$ & $\begin{array}{r}26.22 \\
14.63 \\
143^{6}\end{array}$ & $\begin{array}{r}26.80 \\
182 \\
39.0 \\
39 .\end{array}$ & $\begin{array}{r}22.00 \\
1901 \\
56.0\end{array}$ & $\begin{array}{r}17.36 \\
132^{60} \\
192 .\end{array}$ & $\begin{array}{r}13.36 \\
662 \\
376 .\end{array}$ & $\begin{array}{r}9.49 \\
.670 \\
\text { s7i. }\end{array}$ & $\begin{array}{r}7.65 \\
399 \\
71.00 \\
715 .\end{array}$ \\
\hline
\end{tabular}

\begin{tabular}{|c|c|c|c|c|c|c|c|c|c|c|c|c|}
\hline Month & JAN & ras & $m$ & $\Delta P \mathbb{R}$ & $\mathbf{M r}$ & JUNE & JURY & AUE & SEP & oct & nov & DEC \\
\hline 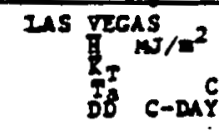 & $\begin{array}{r}7.67 \\
11.67 \\
667 \\
382 .\end{array}$ & $27 i^{\mathbf{9}}$ & $\begin{array}{l}21 . \frac{12}{72} \\
12^{3} \\
186.0\end{array}$ & $\begin{array}{r}26.0 .9 \\
17.9 \\
62.9\end{array}$ & $\begin{array}{r}29.36 \\
233^{6} \\
j .\end{array}$ & $\begin{array}{r}31.20 \\
28.76 \\
0.0\end{array}$ & $\begin{array}{r}28.27 \\
31.0 \\
0.0\end{array}$ & $\begin{array}{r}26.22 \\
30.0 \\
0 .\end{array}$ & $\begin{array}{r}23.29 \\
26.5 \\
0.0\end{array}$ & $\begin{array}{r}17.94 \\
19.0 \\
43 .\end{array}$ & $\begin{array}{r}13.30 \\
1 i^{11} \\
215^{\circ} .\end{array}$ & $\begin{array}{r}10.87 \\
58 \\
343 .\end{array}$ \\
\hline 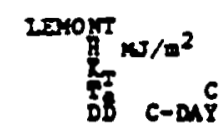 & $\begin{array}{l}\text { II, } \\
-j \\
i 0\end{array}$ & $\begin{array}{l}-30 \\
-i_{8}^{3} \\
58.0\end{array}$ & $\begin{array}{r}13.63 \\
3.3 \\
3.0 \\
486 .\end{array}$ & $\begin{array}{r}16.3 . \\
10.9 \\
253 .\end{array}$ & $\begin{array}{r}20.78 \\
163 \\
116.0\end{array}$ & $\begin{array}{r}23.13 \\
2.56 \\
2 i^{5.0} \\
14 .\end{array}$ & $\begin{array}{r}22.04 \\
24.55 \\
. \quad 0.0\end{array}$ & $\begin{array}{r}20.32 \\
235 \\
4 . \\
4 .\end{array}$ & $\begin{array}{r}16.95 \\
1955 \\
32 .\end{array}$ & $\begin{array}{l}11.08 \\
13.0 \\
176 .\end{array}$ & $\begin{array}{r}6.57 \\
5.43 \\
410.0\end{array}$ & $\begin{array}{r}5.48 \\
-i^{4} .0 \\
629 .\end{array}$ \\
\hline 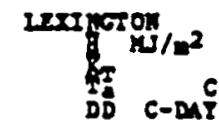 & $\begin{array}{r}12 x .15 \\
7.15 \\
.44 \\
553^{\circ}\end{array}$ & $\begin{array}{r}50 \\
\dot{z}_{0}\end{array}$ & $\begin{array}{r}13.22 \\
554 \\
674.0 \\
374 .\end{array}$ & $\begin{array}{r}19.91 \\
13.5 \\
168.9\end{array}$ & $\begin{array}{r}24.09 \\
16.01 \\
59.0\end{array}$ & $\begin{array}{r}25.97 \\
23.3 \\
4.0\end{array}$ & $\begin{array}{r}25.76 \\
2564 \\
25.0 \\
0.0\end{array}$ & $\begin{array}{r}23.34 \\
24.64 \\
0 .\end{array}$ & $\begin{array}{r}20.53 \\
20^{6.5} \\
22 .\end{array}$ & $\begin{array}{r}15.10 \\
16.5 \\
137 .\end{array}$ & $\begin{array}{r}9.83 \\
i 56 \\
340.0\end{array}$ & $\begin{array}{r}7.13 \\
4.48 \\
308 .\end{array}$ \\
\hline 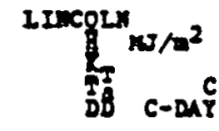 & $\begin{array}{r}\pi \times 9.95 \\
7.95 \\
-940 \\
687.0\end{array}$ & $\begin{array}{l}-i^{53} \\
568 .\end{array}$ & $\begin{array}{r}14.51 \\
354 \\
3.0 \\
63 .\end{array}$ & $\begin{array}{r}17.75 \\
15 \\
2230 \\
239\end{array}$ & $\begin{array}{r}20.75 \\
16.5 \\
95.0\end{array}$ & $\begin{array}{r}22.79 \\
23^{35} \\
17 .\end{array}$ & $\begin{array}{r}22.46 \\
25.5 \\
25.0 \\
\quad 0 .\end{array}$ & $\begin{array}{r}21.54 \\
24.0 \\
3 .\end{array}$ & $\begin{array}{r}17.23 \\
198 \\
42.0 \\
42 .\end{array}$ & $\begin{array}{r}13.39 \\
13^{62} \\
167 .\end{array}$ & $\begin{array}{r}8.66 \\
i^{4} .0 \\
405 .\end{array}$ & $\begin{array}{l}7.19 \\
354 \\
592.0\end{array}$ \\
\hline 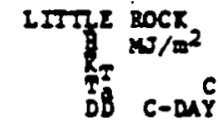 & $\begin{aligned} & A 2 \text { (L } \\
& 8.28 \\
& 4.5 \\
& 4.0 \\
& 420 .\end{aligned}$ & $\begin{array}{l}6^{47} \\
32 \mathrm{i} \\
32 .\end{array}$ & $\begin{array}{l}14.97 \\
130.0 \\
26.9 .\end{array}$ & $\begin{array}{r}19.03 \\
165 \\
16.90\end{array}$ & $\begin{array}{r}22.08 \\
2 i .96 \\
5 .\end{array}$ & $\begin{array}{r}23.50 \\
2657 \\
0 .\end{array}$ & $\begin{array}{r}r 3.34 \\
23.8 \\
27 \%\end{array}$ & $\begin{array}{r}21.58 \\
27.08 \\
0 .\end{array}$ & $\begin{array}{r}18.48 \\
23.0 \\
5 .\end{array}$ & $\begin{array}{r}14.47 \\
1975 \\
7 i^{\circ}\end{array}$ & $\begin{array}{r}10.20 \\
10^{52} \\
298 .\end{array}$ & $\begin{array}{r}7.82 \\
346 \\
398 .\end{array}$ \\
\hline 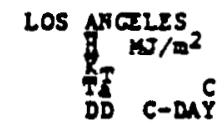 & $\begin{array}{l}94.12 \\
10.75 \\
1350 \\
184 .\end{array}$ & $\begin{array}{r}33 . \\
i_{4}^{3.39} \\
13.0 \\
150 .\end{array}$ & $\begin{array}{r}19.19 \\
13.0 \\
148 .\end{array}$ & $\begin{array}{r}21.68 \\
13.6 .9 \\
108 .\end{array}$ & $\begin{array}{r}24.09 \\
16.01 \\
63 .\end{array}$ & $\begin{array}{r}25.80 \\
186 \\
39 .\end{array}$ & $\begin{array}{r}27.14 \\
20.7 \\
11 .\end{array}$ & $\begin{array}{r}24.63 \\
20.66 \\
8 .\end{array}$ & $\begin{array}{r}21.08 \\
206 \\
13 .\end{array}$ & $\begin{array}{r}19.39 \\
18.0 \\
43 .\end{array}$ & $\begin{array}{r}12.25 \\
15.61 \\
88 .\end{array}$ & $\begin{array}{r}10.29 \\
1350 \\
155 .\end{array}$ \\
\hline
\end{tabular}




\begin{tabular}{|c|c|c|c|c|c|c|c|c|c|c|c|c|}
\hline Month & JAN & rEs & we & $\Delta \mathbf{P R}$ & $m y$ & Jone & Jor & $\triangle D C$ & SEP & 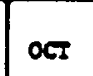 & BOP & Dec \\
\hline 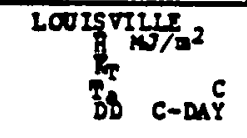 & $\begin{array}{r}\pi_{6} \\
54\end{array}$ & $\begin{array}{l}24.3 \\
454 .\end{array}$ & $\begin{array}{r}13.61 \\
649 \\
665\end{array}$ & $\begin{aligned} 17.58 \\
515 \\
1 j^{5} \\
159 \\
9.0\end{aligned}$ & $\begin{array}{r}21.56 \\
1.55 \\
198.0 \\
58 .\end{array}$ & $\begin{array}{r}23.455 \\
22.05 \\
0 .\end{array}$ & $\begin{array}{r}23.03 \\
24.00 \\
0 .\end{array}$ & $\begin{array}{r}20.85 \\
24.00 \\
0 .\end{array}$ & $\begin{array}{r}17.08 \\
20.96 \\
19.0\end{array}$ & $\mid \begin{array}{r}12.69 \\
154 \\
134.0 \\
134 .\end{array}$ & $\begin{array}{r}7.95 \\
j 46 \\
333.0\end{array}$ & $\begin{array}{r}6.28 \\
i^{4} .0 \\
506 .\end{array}$ \\
\hline 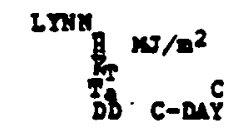 & 60 & $\begin{array}{r}.42 \\
8.7 \\
540\end{array}$ & 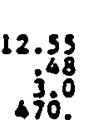 & $\begin{array}{r}16.48 \\
\dot{9} 9 \\
28 j^{0}\end{array}$ & $\begin{array}{r}18.99 \\
154.0 \\
116 .\end{array}$ & $\begin{array}{r}22.30 \\
20.54 \\
20.0\end{array}$ & $\begin{array}{r}22.54 \\
236 \\
0.0 \\
0 .\end{array}$ & $\mid \begin{array}{r}17.65 \\
23.0 \\
3.0\end{array}$ & $\begin{array}{r}14.22 \\
1849 \\
18.0 \\
3 j .\end{array}$ & $\begin{array}{l}9.66 \\
1346 \\
136 .\end{array}$ & $\begin{array}{r}5.56 \\
7^{3} 37 \\
333^{\circ}\end{array}$ & $\begin{array}{l}4.28 \\
3.34 \\
34.0 \\
546 .\end{array}$ \\
\hline 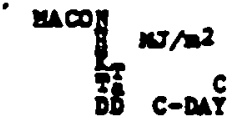 & $\begin{array}{rl}4 & .34 \\
10.38 \\
533 \\
302.0 \\
30.0\end{array}$ & $\begin{array}{l}100^{3} \\
235^{3} \\
\end{array}$ & $\begin{array}{l}16.41 \\
14.34 \\
166.0\end{array}$ & $\begin{array}{r}21.39 \\
1939 \\
37.0\end{array}$ & $\begin{array}{r}23.70 \\
2300 \\
3 .\end{array}$ & $\begin{array}{r}23.995 \\
26.0 \\
0 .\end{array}$ & $\mid \begin{array}{r}23.07 \\
27 \\
27.0 \\
0 .\end{array}$ & $\begin{array}{r}21.81 \\
2 j .8 \\
0 .\end{array}$ & $\mid \begin{array}{r}18.13 \\
246 \\
24.0 \\
0 .\end{array}$ & $\begin{array}{r}15.81 \\
199 . \\
46 .\end{array}$ & $\begin{array}{l}1.14 \\
1354 \\
169.0\end{array}$ & $\begin{array}{r}9.08 \\
9.50 \\
288 .\end{array}$ \\
\hline${ }^{m 0230 x}{ }^{w 0 / m^{2}}$ & $i$ & $\begin{array}{l}-43 . \\
9.23 \\
-90 \\
69.0 \\
696 .\end{array}$ & $\begin{array}{r}13.99 \\
.96 \\
390^{\circ}\end{array}$ & $\begin{array}{r}16.53 \\
549 \\
32 i^{0}\end{array}$ & $\begin{array}{r}19.82 \\
13^{5} \\
163^{0}\end{array}$ & $\begin{array}{l}24.07 \\
196 \\
60.0\end{array}$ & $\mid \begin{array}{r}23,73 \\
21.00 \\
4 .\end{array}$ & $\begin{array}{r}19,236 \\
20.06 \\
2 i ?\end{array}$ & $\begin{array}{r}16.49 \\
13.0 \\
96 .\end{array}$ & $\begin{array}{l}11.39 \\
100^{30} \\
263 .\end{array}$ & $\begin{array}{l}6.31 \\
\text { i.t. } \\
\text { sos. }\end{array}$ & $\begin{array}{l}3.63 \\
38 \\
343.0 \\
743 .\end{array}$ \\
\hline 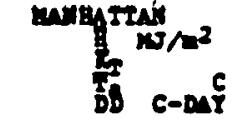 & 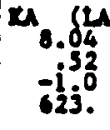 & 49 & $\begin{array}{l}14.44 \\
: 32 \\
401 .\end{array}$ & $\begin{array}{r}18.13 \\
135 \\
133^{5} \\
18 .\end{array}$ & $\begin{array}{r}22.06 \\
10^{36} \\
69 .\end{array}$ & $\begin{array}{r}23.07 \\
236 \\
7.0 \\
7 .\end{array}$ & $\mid \begin{array}{r}22.23 \\
26.35 \\
0.0 \\
0 .\end{array}$ & $\begin{array}{r}22.02 \\
25.01 \\
0 .\end{array}$ & $\begin{array}{r}17.17 \\
20.97 \\
32.0\end{array}$ & $\begin{array}{l}12.23 \\
19.0 \\
190 .\end{array}$ & $\begin{array}{r}9.50 \\
657 \\
673.0\end{array}$ & $\begin{array}{l}6.53 \\
.46 \\
344 .\end{array}$ \\
\hline 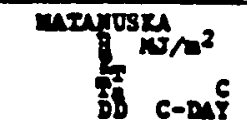 & 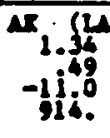 & $\begin{array}{l}0 . \\
3.7 \\
71\end{array}$ & $\begin{array}{l}10.13 \\
-3.04 \\
689 .\end{array}$ & 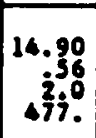 & $\begin{array}{r}18.29 \\
3^{35} \\
\text { 310. }\end{array}$ & $\begin{array}{r}19.34 \\
12^{48} \\
168 .\end{array}$ & $\mid \begin{array}{r}17.12 \\
14.03 \\
129 .\end{array}$ & $\mid \begin{array}{l}13.15 \\
12.63 \\
169 .\end{array}$ & $\begin{array}{r}8.29 \\
.42 \\
28.0 \\
28 .\end{array}$ & $\begin{array}{r}4.18 \\
502 \\
326.0 \\
326 .\end{array}$ & $\begin{array}{r}1.59 \\
842 \\
738 .\end{array}$ & $\begin{array}{r}.63 \\
-37 \\
-10.0 \\
906 .\end{array}$ \\
\hline
\end{tabular}

\begin{tabular}{|c|c|c|c|c|c|c|c|c|c|c|c|c|}
\hline Mnnth & INA & $\mathbf{E 3}$ & wax & APR & $\operatorname{my}$ & Jore & soLY & AUC & SEP & OCT & 10V & DEC \\
\hline $\begin{array}{l}\text { REDFog } w / a^{2} \\
\text { Df } c-m x^{c}\end{array}$ & $\begin{array}{r}02 .(\mathrm{rA} \\
4.94 \\
36 \\
33.0 \\
489 .\end{array}$ & $\begin{array}{r}8.87 \\
5.46 \\
3.0 \\
369 .\end{array}$ & 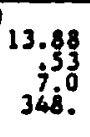 & $\begin{array}{r}20.24 \\
10.00 \\
245^{\circ}\end{array}$ & $\begin{array}{r}24.63 \\
163 \\
199 .\end{array}$ & $\begin{array}{r}27.31 \\
1866 \\
52 .\end{array}$ & $\begin{array}{r}29.23 \\
2 i^{2} .0 \\
6 .\end{array}$ & $\begin{array}{r}25.17 \\
21.0 \\
13 .\end{array}$ & $\begin{array}{r}18.78 \\
18.65 \\
49 .\end{array}$ & $\begin{array}{r}11.63 \\
5350 \\
1380 .\end{array}$ & $\begin{array}{r}6.36 \\
843 \\
6.00 \\
358 .\end{array}$ & $\begin{array}{r}3.85 \\
31 \\
3.0 \\
470 .\end{array}$ \\
\hline 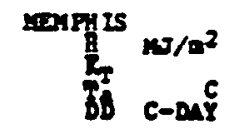 & $\begin{array}{r}\pi n .8 \mathrm{LA} \\
8.04 \\
340 \\
422.0\end{array}$ & $\begin{array}{r}35.0 \\
1.10^{\circ} \\
j^{4.8} \\
330^{\circ}\end{array}$ & $\begin{array}{r}15.03 \\
51 \\
11^{5} .0 \\
254 .\end{array}$ & $\begin{array}{r}19.68 \\
1750 \\
73 .\end{array}$ & $\begin{array}{r}23.19 \\
23.09 \\
12 .\end{array}$ & $\begin{array}{r}24.66 \\
26.60 \\
0 .\end{array}$ & $\begin{array}{r}24.41 \\
28.61 \\
0 .\end{array}$ & $\begin{array}{r}22.40 \\
27.6 ! \\
0 .\end{array}$ & $\begin{array}{r}18.50 \\
239.0 \\
4 .\end{array}$ & $\begin{array}{r}14.82 \\
17.60 \\
79 .\end{array}$ & $\begin{array}{l}9.96 \\
10^{52} \\
233^{\circ} .\end{array}$ & $\begin{array}{r}7.70 \\
846 \\
384.0 \\
384 .\end{array}$ \\
\hline 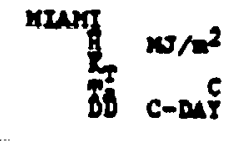 & $\begin{array}{r}\pi, 34 \\
14.34 \\
190.0 \\
41^{0}\end{array}$ & $\begin{array}{l}25.5 \\
7.40^{5} \\
19^{62} \\
3 i^{\circ} .\end{array}$ & $\begin{array}{r}20.53 \\
2 i^{63} \\
11 .\end{array}$ & $\begin{array}{r}22.75 \\
236.0 \\
0 .\end{array} \mid$ & $\begin{array}{r}23.08 \\
235 \% \\
8 .\end{array}$ & $\begin{array}{r}22.21 \\
2 i^{5} \\
8 .\end{array}$ & $\begin{array}{r}22.46 \\
2 i^{3} \\
8 .\end{array}$ & $\begin{array}{r}21.24 \\
28.56 \\
8 .\end{array}$ & $\begin{array}{r}18.69 \\
2750 \\
8 .\end{array}$ & $\begin{array}{r}16.27 \\
25.00 \\
0 .\end{array}$ & $\mid \begin{array}{r}14.80 \\
23.60 \\
0 .\end{array}$ & $\begin{array}{r}13.36 \\
20.00 \\
36 .\end{array}$ \\
\hline 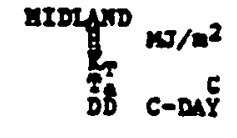 & $\begin{array}{r}\pi .94 \\
11.75 \\
0.58 \\
362.0 \\
36.0\end{array}$ & $\begin{array}{r}31.6 \\
3.05^{6} \\
360 \\
260^{6 .}\end{array}$ & $\begin{array}{r}19.95 \\
12.8 \\
179 .\end{array}$ & $\begin{array}{r}23.04 \\
19.8 \\
50^{\circ}\end{array}$ & $\begin{array}{r}25.80 \\
28.8 \\
0 .\end{array}$ & $\begin{array}{r}25.72 \\
26.8 \\
0 .\end{array}$ & $\begin{array}{r}25.68 \\
29.0 \\
0 .\end{array}$ & $\begin{array}{r}24.55 \\
29.8 \\
0 .\end{array}$ & $\begin{array}{r}21.37 \\
23.60 \\
0 .\end{array}$ & $\begin{array}{r}16.69 \\
18.63 \\
42 .\end{array}$ & $\begin{array}{r}13.47 \\
11^{6}, 0 \\
212 .\end{array}$ & $\begin{array}{l}11.37 \\
j .51 \\
329 .\end{array}$ \\
\hline 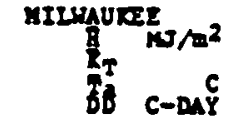 & $\begin{array}{l}\text { WI }(24 \\
6.26 \\
-7.66 \\
786 .\end{array}$ & $\begin{array}{c}4.2 .6 \\
8.79 \\
-3.7 \\
661 . \\
66 .\end{array}$ & $\begin{array}{r}13.10 \\
930 \\
379 .\end{array}$ & $\begin{array}{r}16.83 \\
590 \\
338 .\end{array}$ & $\begin{array}{r}21.27 \\
1254 \\
193 .\end{array}$ & $\begin{array}{r}23.65 \\
185 \\
50.0 \\
50 .\end{array}$ & $\begin{array}{r}23.57 \\
2 i^{59} .0 \\
8 .\end{array}$ & $\begin{array}{r}20.31 \\
21.57 \\
20.0\end{array}$ & $\begin{array}{r}16.415 \\
16.0 \\
76 .\end{array}$ & $\begin{array}{r}11.18 \\
11^{53} \\
24.0 \\
24 .\end{array}$ & $\begin{array}{r}6.74 \\
\dot{3} 46 \\
475 .\end{array}$ & $\begin{array}{l}3.02 \\
-8.2 \\
703.0 \\
703 .\end{array}$ \\
\hline 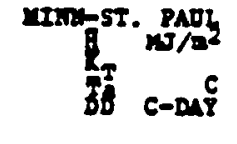 & $\begin{array}{r}3.99 \\
-1.49 \\
-11.0 \\
909 .\end{array}$ & $\begin{array}{l}9.4 i^{3} \\
9.32 \\
-9350 \\
734 .\end{array}$ & $\begin{array}{l}12.821 \\
-2^{51} \\
632^{\circ}\end{array}$ & $\begin{array}{r}16.54 \\
590 \\
332 . \\
332 .\end{array}$ & $\begin{array}{r}20.18 \\
52 \\
15 i^{2} \\
15 .\end{array}$ & $\begin{array}{r}22.44 \\
19.58 \\
36 .\end{array}$ & $\begin{array}{r}22.82 \\
22^{57} \\
6 .\end{array}$ & $\begin{array}{r}19.31 \\
215 \\
21.0 \\
12 .\end{array}$ & $\begin{array}{r}14.99 \\
16.54 \\
16.0 \\
96 .\end{array}$ & $\begin{array}{c}10.80 \\
10.0 \\
262 .\end{array}$ & $\begin{array}{r}6.03 \\
.440 \\
543^{\circ}\end{array}$ & $\begin{array}{r}4.69 \\
-i^{63} \\
799 .\end{array}$ \\
\hline
\end{tabular}




\begin{tabular}{|c|c|c|c|c|c|c|c|c|c|c|c|c|}
\hline ention Month & SWI & 728 & max & $\mathbf{P R}$ & $\mathbf{m r}$ & $\operatorname{sen}$ & $\operatorname{sel}$ & $\triangle \nabla G$ & $\sin$ & OCI & mor & DEC \\
\hline 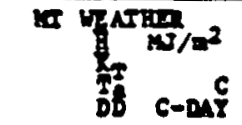 & 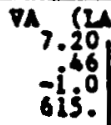 & & $\begin{array}{r}14.15 \\
351 \\
353 \\
453\end{array}$ & $\begin{array}{r}17.39 \\
1050 \\
251 .\end{array}$ & $\begin{array}{r}21.273 \\
1954 \\
102 .\end{array}$ & $\begin{array}{r}21.98 \\
20.93 \\
13 .\end{array}$ & $\begin{array}{r}21.35 \\
23.53 \\
0 .\end{array}$ & $\begin{aligned} 18.00 \\
210 \\
20 \\
j .\end{aligned}$ & $\begin{array}{r}15.70 \\
183 \\
44.0\end{array}$ & $\begin{array}{r}11.96 \\
132 \\
189.0\end{array}$ & $\begin{array}{r}8.46 \\
590 \\
370 .\end{array}$ & $\begin{array}{r}7.03 \\
.040 \\
373^{\circ} .\end{array}$ \\
\hline 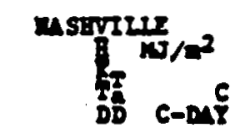 & 5x. & $\begin{array}{c}53 \\
373 .\end{array}$ & $\begin{array}{r}13.76 \\
4.80 \\
291 .\end{array}$ & $\begin{array}{r}18.82 \\
1353 \\
98.0\end{array}$ & $\begin{array}{r}23.62 \\
200^{55} \\
23 .\end{array}$ & $\begin{array}{r}23.71 \\
23.58 \\
0.0\end{array}$ & $\begin{array}{r}23.13 \\
26.5 \\
0 .\end{array}$ & $\begin{array}{r}20.66 \\
25.56 \\
0 .\end{array}$ & $\begin{array}{r}17.90 \\
23.0 \\
6 .\end{array}$ & $\begin{array}{r}13.67 \\
16.0 \\
100 .\end{array}$ & $\begin{array}{r}9.07 \\
\dot{69} \\
277^{\circ}\end{array}$ & $\begin{array}{r}6.79 \\
562 \\
426 .\end{array}$ \\
\hline 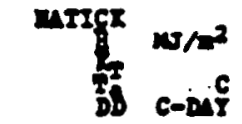 & $m$ & ;i & $\begin{array}{r}13.63 \\
53 \\
53 \\
487 .\end{array}$ & $\begin{array}{r}16.39 \\
289^{40} \\
28 .\end{array}$ & $\begin{array}{r}20.91 \\
153 \\
1530 \\
123 .\end{array}$ & $\begin{aligned} 17.40 \\
20.1 \\
10^{40}\end{aligned}$ & $\begin{array}{r}21.45 \\
23.03 \\
0 .\end{array}$ & $\begin{aligned} 19.5 \frac{1}{53} \\
22.0 \\
6 .\end{aligned}$ & $\begin{array}{r}15.01 \\
152 \\
190 \\
36 .\end{array}$ & $\begin{array}{l}10.91 \\
12^{32} \\
203 .\end{array}$ & $\begin{array}{l}6.15 \\
6.1 \\
375.0 \\
375 .\end{array}$ & $\begin{array}{r}5.60 \\
.66 \\
600^{\circ}\end{array}$ \\
\hline 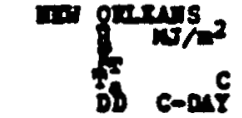 & 202 & $133^{42}$ & $\begin{array}{r}14.03 \\
13.5 \\
107 .\end{array}$ & $\begin{array}{r}17.25 \\
20.07 \\
22 .\end{array}$ & $\begin{array}{r}18.80 \\
23.70 \\
0 .\end{array}$ & $\begin{array}{r}18.55 \\
26.6 \\
0.0\end{array}$ & $\begin{array}{r}17.46 \\
23 \% 0\end{array}$ & $\begin{aligned} 17.42 \\
28.60 \\
0.0\end{aligned}$ & $\begin{array}{r}16.03 \\
23.04 \\
0 .\end{array}$ & $\begin{aligned} 26.95 \\
20.94 \\
20.0 \\
11 .\end{aligned}$ & $\begin{array}{l}11.64 \\
1530 \\
107 .\end{array}$ & $\begin{array}{l}8.29 \\
13.02 \\
179 .\end{array}$ \\
\hline 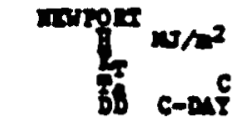 & $\begin{array}{l}2 I .84 \\
6.46 \\
.450 \\
367^{\circ}\end{array}$ & $\begin{array}{r}.49 \\
53 i^{0}\end{array}$ & $\begin{array}{r}13.80 \\
52 \\
350 \\
687 .\end{array}$ & $\begin{array}{r}16.52 \\
549 \\
360 .\end{array}$ & $\begin{array}{r}20.45 \\
1352 \\
19 i^{0}\end{array}$ & $\begin{array}{r}22.50 \\
1754 \\
55 .\end{array}$ & $\begin{array}{r}21.62 \\
21.54 \\
0.0\end{array}$ & $\begin{array}{r}18.78 \\
21.0 \\
9 .\end{array}$ & $\begin{array}{r}15.89 \\
18.0 \\
43 .\end{array}$ & $\begin{aligned} 11.42 \\
133 \\
13 i^{\circ}\end{aligned}$ & $\begin{array}{l}7.32 \\
30^{47} \\
330 .\end{array}$ & $\begin{array}{r}5.90 \\
366 \\
301 .\end{array}$ \\
\hline 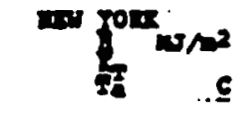 &. .06 & i! & $\begin{array}{r}12.26 \\
349\end{array}$ & $\begin{array}{r}13.45 \\
\frac{14}{440} \\
230 .\end{array}$ & $\begin{array}{r}18.09 \\
176.0 \\
69 .\end{array}$ & $\begin{array}{r}19.68 \\
22_{0}^{68} \\
j .\end{array}$ & $\begin{array}{r}19.22 \\
23.0 \\
0.0\end{array}$ & $\begin{array}{r}16.29 \\
26,05 \\
0 .\end{array}$ & $\begin{array}{r}13.86 \\
20.70 \\
10^{6}\end{array}$ & $\begin{array}{r}10.13 \\
13.6 \\
134 .\end{array}$ & $\begin{array}{r}6.25 \\
930 \\
293.0\end{array}$ & $\begin{array}{r}4.95 \\
360 \\
4930 \\
4930\end{array}$ \\
\hline
\end{tabular}

\begin{tabular}{|c|c|c|c|c|c|c|c|c|c|c|c|c|}
\hline ition Momin & SNI & ns & $\min$ & ard & $m$ & SUNE & 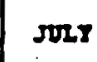 & ADE & sEs & $\infty \mathrm{CT}$ & not & Dece \\
\hline 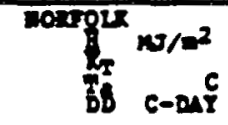 & 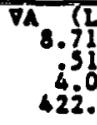 & 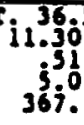 & $\begin{aligned} 15.57 \\
594 \\
296.0 \\
296 .\end{aligned}$ & $\begin{array}{r}19.97 \\
193 \\
126.0 \\
126 .\end{array}$ & $\begin{array}{r}22.61 \\
1957 \\
29.0\end{array}$ & $\begin{array}{r}23.95 \\
2350.0 \\
0.0\end{array}$ & $\begin{array}{l}23.03 \\
25^{57} \\
0.0\end{array}$ & $\begin{array}{r}20.14 \\
245 \\
24.0 \\
0 .\end{array}$ & $\begin{array}{r}16.66 \\
2 i^{54} \\
5 .\end{array}$ & $\begin{array}{r}12.98 \\
196 \\
198 .\end{array}$ & $\begin{array}{l}9.34 \\
1051 \\
223 .\end{array}$ & $\begin{array}{r}7.70 \\
\dot{3} \times 9 \\
39: .\end{array}$ \\
\hline 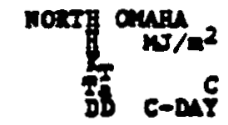 & 政. & $\begin{array}{r}-539 \\
626 .\end{array}$ & $\begin{array}{l}16.90 \\
536 \\
320 \\
322 .\end{array}$ & $\begin{array}{r}19.38 \\
1057 \\
258.0\end{array}$ & $\begin{array}{r}21.48 \\
195 \\
116.0\end{array}$ & $\begin{array}{r}23.57 \\
237 \\
23.0 \\
23 .\end{array}$ & $\begin{array}{r}23.78 \\
259 \\
30.0\end{array}$ & $\begin{array}{r}21.81 \\
23.6 \\
7 .\end{array}$ & $\begin{array}{r}16.58 \\
1957 \\
98.0\end{array}$ & $\begin{array}{r}12.31 \\
135 \\
198 .\end{array}$ & $\begin{array}{r}6.29 \\
\dot{933} \\
460 .\end{array}$ & $\begin{array}{l}6.95 \\
-2.98 \\
-353.0\end{array}$ \\
\hline 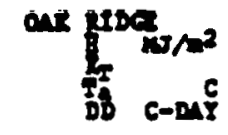 & 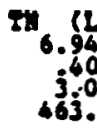 & $\begin{array}{r}9.93 \\
5.40 \\
383 .\end{array}$ & $\begin{array}{r}13.63 \\
.47 \\
30 \%\end{array}$ & $\begin{array}{r}18.69 \\
15.5 \\
122.0\end{array}$ & $\begin{array}{r}21.54 \\
1954 \\
63.0\end{array}$ & $\begin{array}{r}22.79 \\
2353 \\
0.0\end{array}$ & $\begin{aligned} 21.79 \\
25 \\
25.0 \\
0.0\end{aligned}$ & $\begin{array}{r}19.91 \\
24.6 \\
0.0\end{array}$ & $\begin{array}{r}17.44 \\
2 i^{36} \\
11 .\end{array}$ & $\begin{array}{r}13.34 \\
1535 \\
120 .\end{array}$ & $\begin{array}{r}8.82 \\
4.47 \\
298 .\end{array}$ & $\begin{array}{r}6.73 \\
642 \\
6.0 \\
46 .\end{array}$ \\
\hline 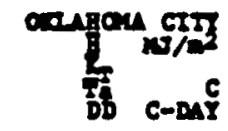 & $\begin{aligned} 02.32 \\
10.66 \\
339 \\
486.0\end{aligned}$ & $\begin{array}{r}135 . \\
13.25 \\
i 38 \\
369.0\end{array}$ & $\begin{aligned} 17.92 \\
938 \\
296.0\end{aligned}$ & $\begin{array}{r}20.83 \\
159.0 \\
100 .\end{array}$ & $\begin{array}{r}22.58 \\
2057 \\
20.0\end{array}$ & $\begin{array}{r}26.39 \\
23.6 \\
0 .\end{array}$ & $\begin{array}{l}25.31 \\
27.35 \\
0.0 \\
0.0\end{array}$ & $\begin{array}{r}24.59 \\
27.67 \\
0.0\end{array}$ & $\begin{array}{r}20.24 \\
23.0 \\
i .\end{array}$ & $\begin{array}{r}15.83 \\
16.60 \\
82 .\end{array}$ & $\begin{array}{r}11.88 \\
\dot{962} \\
26 j^{0}\end{array}$ & $\begin{array}{r}9.91 \\
600 \\
631 .\end{array}$ \\
\hline 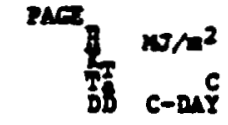 & $\begin{aligned} 42.58 \\
12.56 \\
.73 \\
59 i^{\circ} .\end{aligned}$ & 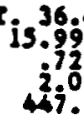 & $\begin{array}{r}22.02 \\
59.0 \\
396.0\end{array}$ & $\begin{array}{r}23.87 \\
10.9 \\
240 .\end{array}$ & $\begin{array}{r}29.10 \\
194 \\
107 .\end{array}$ & $\begin{array}{r}29.60 \\
20.72 \\
20.0\end{array}$ & $\begin{array}{r}28.47 \\
24.0 \\
0.0\end{array}$ & $\begin{array}{r}24.95 \\
23.6 \\
6.0 \\
6 .\end{array}$ & $\begin{array}{r}21.60 \\
19.0 \\
41 .\end{array}$ & $\begin{array}{r}16.83 \\
190 \\
189.0\end{array}$ & $\begin{array}{r}12.98 \\
370 \\
390 .\end{array}$ & $\begin{array}{r}10.17 \\
.64 \\
362^{0}\end{array}$ \\
\hline 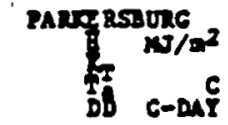 & 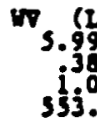 & $\begin{array}{l}399 \\
8.46 \\
4.40 \\
47 i .\end{array}$ & $\begin{array}{r}12.64 \\
12.66 \\
301 . \\
30.0\end{array}$ & $\begin{array}{r}13.87 \\
136 \\
170^{6}\end{array}$ & $\begin{array}{r}20.39 \\
183 \\
67 .\end{array}$ & $\begin{aligned} 22.65 \\
22.55 \\
4.0\end{aligned}$ & $\begin{array}{r}22.02 \\
24.53 \\
0.0\end{array}$ & $\begin{array}{c}20.26 \\
23^{56} \\
0 .\end{array}$ & $\begin{array}{r}16.50 \\
2555 \\
20.0 \\
26 .\end{array}$ & $\begin{array}{r}11.89 \\
53 \\
16.0 \\
169 .\end{array}$ & $\begin{array}{r}6.99 \\
42 \\
333^{0}\end{array}$ & $\begin{array}{r}3.33 \\
399 \\
3150\end{array}$ \\
\hline
\end{tabular}




\begin{tabular}{|c|c|c|c|c|c|c|c|c|c|c|c|c|}
\hline Month & JAI & rES & MR & APR & $M Y$ & Jais & 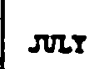 & AUC & SEP & oCI & Hov & DEC \\
\hline 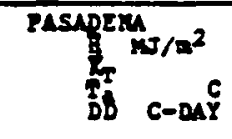 & 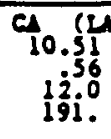 & $\begin{array}{l}\text { ijso } \\
\text { isi. }\end{array}$ & $\begin{array}{r}18.38 \\
1362 \\
134 \\
141 .\end{array}$ & $\begin{array}{r}21.31 \\
150 \\
94.0\end{array}$ & $\begin{array}{r}23.82 \\
17.0 \\
47 .\end{array}$ & $\begin{array}{r}24.28 \\
199 \\
26.0\end{array}$ & $\begin{array}{r}26.34 \\
23.66 \\
0 .\end{array}$ & $\begin{array}{r}25.08 \\
23.08 \\
0 .\end{array}$ & $\begin{array}{r}20.18 \\
22^{63} .0 \\
6 .\end{array}$ & $\begin{array}{r}13.32 \\
19.60 \\
29 .\end{array}$ & $\begin{array}{r}11.35 \\
155 \\
15 i .0 \\
91 .\end{array}$ & $\begin{array}{l}9.88 \\
135 \\
136.0 \\
166 .\end{array}$ \\
\hline 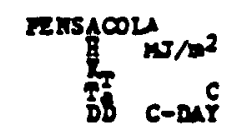 & $\begin{array}{l}n \\
10 . \\
23\end{array}$ & $\begin{array}{l}133 \\
1790 .\end{array}$ & $\begin{array}{l}16.96 \\
1534 \\
115 .\end{array}$ & $\begin{array}{r}21.31 \\
20^{59} \\
2 \mathrm{i} .\end{array}$ & $\begin{array}{r}23.53 \\
2390 \\
0 .\end{array}$ & $\begin{array}{r}23.78 \\
26.58 \\
0 .\end{array}$ & $\begin{array}{r}22.48 \\
27^{36} \\
0 .\end{array}$ & $\begin{array}{r}21.31 \\
2750 \\
0 .\end{array}$ & $\begin{aligned} 18.00 \\
2555 \\
25.0 \\
0 .\end{aligned}$ & $\begin{array}{r}16.49 \\
21^{6 .} \\
18 .\end{array}$ & $\mid \begin{array}{l}11.64 \\
153 \\
1550 \\
105 .\end{array}$ & $\begin{array}{l}9.38 \\
1348 \\
199 .\end{array}$ \\
\hline 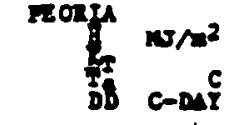 & 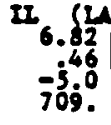 & -380 & $\begin{array}{r}13.48 \\
30 \\
333^{\circ}\end{array}$ & $\begin{array}{r}17.67 \\
1 i^{32} \\
231.0\end{array}$ & $\begin{array}{r}21.31 \\
154 \\
160.0 \\
100 .\end{array}$ & $\begin{array}{r}23.99 \\
23.98 \\
9 .\end{array}$ & $\begin{array}{r}23.57 \\
259 \\
24.0 \\
0 .\end{array}$ & $\begin{array}{r}21.02 \\
23.08 \\
4.0\end{array}$ & $\begin{array}{r}17.04 \\
198 \\
19.00 \\
.99 .\end{array}$ & $\begin{array}{r}12.32 \\
137 \\
182.0\end{array}$ & $\begin{array}{r}7.75 \\
: 48 \\
418.0\end{array}$ & $\begin{array}{l}3.82 \\
-2.43 \\
637^{4}\end{array}$ \\
\hline${ }^{2}{ }^{2}{ }^{2}$ & $\begin{array}{r}42 .(44 \\
12.43 \\
10^{65} \\
250 .\end{array}$ & $\begin{array}{l}33 . \\
7.06 \\
13.0\end{array}$ & $\begin{array}{r}21.79 \\
1530 \\
105^{\circ}\end{array}$ & $\left|\begin{array}{r}26.898 \\
1990 \\
3 j .\end{array}\right|$ & $\begin{array}{r}30.28 \\
2 i_{0}^{60} \\
0 .\end{array}$ & $\begin{array}{r}30.95 \\
29.05 \\
0 .\end{array}$ & $\begin{array}{r}27.27 \\
33_{0}^{68} \\
0 .\end{array}$ & $\begin{array}{r}25.99 \\
31,69 \\
0 .\end{array}$ & $\begin{array}{r}23.75 \\
28.0 \\
i .0\end{array}$ & $\begin{array}{r}18.90 \\
2 i_{3}^{3} \\
j .\end{array}$ & $\mid \begin{array}{r}16.18 \\
25.0 \\
10 i .\end{array}$ & $\begin{array}{l}11.71 \\
1.66 \\
11.0 \\
116 .\end{array}$ \\
\hline 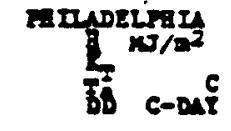 & $\begin{array}{l}34 .: 54 \\
7.43 \\
.460 \\
363 .\end{array}$ & io. & $\begin{array}{r}16.53 \\
533 \\
398.0 \\
398 .\end{array}$ & $\left|\begin{array}{r}17.79 \\
152 \\
190 \\
20.0\end{array}\right|$ & $\begin{array}{l}20.64 \\
.192 \\
.68 .0\end{array}$ & $\begin{array}{r}23.19 \\
22.0 \\
0 .\end{array}$ & $\begin{array}{r}22.52 \\
26.56 \\
0 .\end{array}$ & $\mid \begin{array}{r}19.47 \\
23.64 \\
0 .\end{array}$ & $\begin{array}{r}16.24 \\
20.94 \\
21.0\end{array}$ & $\begin{array}{r}12.27 \\
14.54 \\
138 .\end{array}$ & $\begin{array}{r}8.00 \\
68.0 \\
313.0\end{array}$ & $\begin{array}{l}6.36 \\
346 \\
313.0\end{array}$ \\
\hline 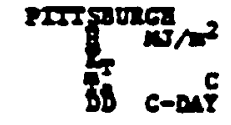 & $\begin{array}{l}24.640 \\
6.62 \\
592^{0} \\
592 .\end{array}$ & $\begin{array}{l}.409 \\
: 9 i^{4} \\
31 j^{0}\end{array}$ & $\begin{array}{r}23.48 \\
350 \\
32.0 \\
42 .\end{array}$ & $\begin{array}{r}16.75 \\
11.9 \\
212 .\end{array}$ & $\begin{array}{r}20.39 \\
352 \\
390 \\
69.0\end{array}$ & $\begin{array}{r}23.400 \\
237.0 \\
6.0\end{array}$ & $\begin{array}{r}22.90 \\
24.37 \\
0.0\end{array}$ & $\begin{array}{c}20.18 \\
23.5 \\
23.0 \\
j .\end{array}$ & $\begin{array}{r}17.000 \\
1970 \\
19.0 \\
32 .\end{array}$ & $\begin{array}{l}12.31 \\
135 \\
166.0 \\
166 .\end{array}$ & $\begin{array}{r}7.70 \\
348 \\
348 \\
348\end{array}$ & $\begin{array}{l}3.94 \\
34.60 \\
368 .\end{array}$ \\
\hline
\end{tabular}

\begin{tabular}{|c|c|c|c|c|c|c|c|c|c|c|c|c|}
\hline cation Month & MAS & nz & we & $\Delta P 2$ & $\max$ & JUNR & SOLY & AUC & S8P & $\alpha C$ & nov & DEC \\
\hline 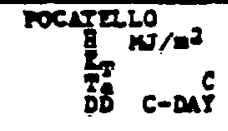 & 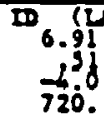 & 554. & $\begin{array}{r}15.28 \\
350 \\
310 .\end{array}$ & $\begin{array}{r}21.97 \\
39.8 \\
328 .\end{array}$ & $\begin{array}{r}24.28 \\
136.8 \\
18 \%\end{array}$ & $\begin{array}{r}27.42 \\
178 \\
77 .\end{array}$ & $\begin{array}{r}28.26 \\
23.0 \\
0 .\end{array}$ & $\begin{array}{r}24.70 \\
2 i^{69} \\
11 .\end{array}$ & $\begin{array}{r}19.89 \\
15.69 \\
107 .\end{array}$ & $\begin{array}{r}13.82 \\
966 \\
286 .\end{array}$ & $\begin{array}{r}8.58 \\
598 \\
53.0 \\
488 .\end{array}$ & $\begin{array}{l}6.49 \\
-5.94 \\
-390 \\
636 .\end{array}$ \\
\hline 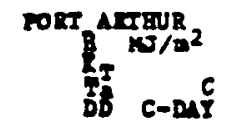 & $\begin{array}{l}5 x \\
9 . \\
\frac{1}{23}\end{array}$ & $\begin{array}{l}13.48 \\
168 .\end{array}$ & $\begin{array}{l}16.08 \\
16.0 \\
112 .\end{array}$ & $\begin{array}{r}18.21 \\
20^{\circ} \\
18.0\end{array}$ & $\begin{array}{r}22.54 \\
25.0 \\
0.0\end{array}$ & $\begin{array}{r}23.86 \\
2399 \\
0.0\end{array}$ & $\begin{array}{r}22 . \frac{11}{55} \\
28.0 \\
0 .\end{array}$ & $\begin{array}{r}20.47 \\
28.94 \\
0.0\end{array}$ & $\begin{array}{r}18.09 \\
2653 \\
0.0 \\
0 .\end{array}$ & $\begin{array}{r}15.99 \\
2 i^{38} \\
19 .\end{array}$ & $\begin{array}{l}10.36 \\
16.0 \\
102 .\end{array}$ & $\begin{array}{l}8.92 \\
13.0 \\
190 .\end{array}$ \\
\hline 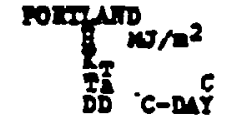 & $=$ & $-i$ & $\begin{array}{r}13.01 \\
.99 \\
379 .\end{array}$ & 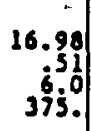 & $\begin{array}{r}21.45 \\
11^{5} \\
207 .\end{array}$ & $\begin{array}{r}22.62 \\
17^{5} \\
62.0\end{array}$ & $\begin{array}{r}23.46 \\
20.8 \\
30 \\
i .\end{array}$ & $\begin{array}{r}20.16 \\
19.93 \\
29 .\end{array}$ & $\begin{array}{r}16.02 \\
13.85 \\
108 .\end{array}$ & $\begin{array}{l}11.42 \\
\$ 9.8 \\
282 .\end{array}$ & $\begin{array}{r}6.57 \\
4.8 \\
448 .\end{array}$ & $\begin{array}{c}3.770 \\
-350 \\
-364 .\end{array}$ \\
\hline 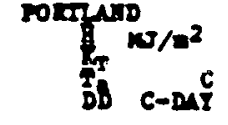 & $\begin{array}{r}3 \\
46\end{array}$ & $\begin{array}{l}38 \\
3.0 \\
346.0\end{array}$ & $\begin{array}{r}10.03 \\
48.0 \\
332 .\end{array}$ & $\begin{array}{r}16.78 \\
10.0 \\
240 .\end{array}$ & $\begin{array}{r}17.71 \\
14.6 \\
149 .\end{array}$ & $\begin{aligned} 19.80 \\
19.8 \\
7 i .\end{aligned}$ & $\begin{array}{r}23.15 \\
19.8 \\
27 .\end{array}$ & $\begin{array}{r}18.59 \\
1953 \\
31.0\end{array}$ & $\begin{array}{r}16.36 \\
1752 \\
66 .\end{array}$ & $\begin{array}{l}8.41 \\
13.0 \\
193 .\end{array}$ & $\begin{array}{l}4.86 \\
37 \\
328 .\end{array}$ & $\begin{array}{l}3 . \$ 3 \\
530 \\
418 .\end{array}$ \\
\hline 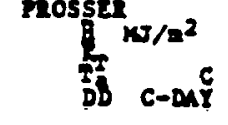 & $\begin{array}{l}n .92 \\
4.90 \\
-i 4.0 \\
624 .\end{array}$ & $\begin{array}{r}96.2 \\
9.29 \\
535 \\
6.0^{2} \\
428 .\end{array}$ & $\begin{array}{r}14.70 \\
6.1 \\
376.0 \\
378 .\end{array}$ & $\begin{array}{r}21.81 \\
10.6 \\
240 .\end{array}$ & $\begin{array}{r}25.79 \\
\cdot 16.0 \\
127 .\end{array}$ & $\begin{array}{r}28.47 \\
18.6 \\
69 .\end{array}$ & $\begin{array}{r}29.60 \\
21.0 \\
i .\end{array}$ & $\begin{array}{r}25.29 \\
20.0 \\
16 .\end{array}$ & $\begin{array}{r}19.18 \\
16.0 \\
66.0 \\
66 .\end{array}$ & $\begin{array}{l}11.47 \\
10.6 \\
231 .\end{array}$ & $\begin{array}{r}5.69 \\
445 \\
4.0 \\
413 .\end{array}$ & $\begin{array}{l}4.19 \\
.42 \\
543 .\end{array}$ \\
\hline 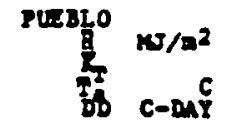 & $\begin{array}{r}c 0 .(2) \\
11.39 \\
-170 \\
601 .\end{array}$ & $\begin{array}{r}38.2 \\
i 4.76^{2} \\
69 \\
470^{\circ}\end{array}$ & $\begin{array}{r}18.67 \\
: 67 \\
43 i^{0} \\
4\end{array}$ & $\begin{array}{r}22.78 \\
1196 \\
225 .\end{array}$ & $\begin{array}{r}25.20 \\
16.04 \\
82 .\end{array}$ & $\begin{array}{r}28.05 \\
29.6 \\
16 .\end{array}$ & $\begin{array}{r}27.13 \\
25.67 \\
0 .\end{array}$ & $\begin{array}{r}25.08 \\
26.09 \\
0.0\end{array}$ & $\begin{array}{r}21.10 \\
196 \\
31 .\end{array}$ & $\begin{array}{r}16.50 \\
12.0 \\
186 .\end{array}$ & $\begin{array}{r}12.31 \\
\dot{5}^{71} \\
40 j^{1}\end{array}$ & $\begin{array}{r}10.01 \\
i^{68} \\
35 i^{0}\end{array}$ \\
\hline
\end{tabular}




\begin{tabular}{|c|c|c|c|c|c|c|c|c|c|c|c|c|}
\hline Month & SAS & TES & MAR & APR & $m \boldsymbol{x}$ & JURE & $\sin x$ & $\triangle D G$ & SEP & $\alpha C T$ & sov & DEC \\
\hline 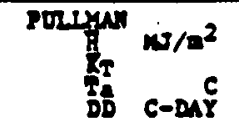 & 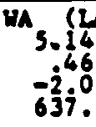 & & $\begin{array}{r}12.42 \\
3.0 \\
460 .\end{array}$ & $\begin{array}{r}19.07 \\
3^{39} \\
317^{0}\end{array}$ & $\begin{array}{r}22.67 \\
1190 \\
206 .\end{array}$ & $\begin{array}{r}28.69 \\
15.69 \\
105 .\end{array}$ & $\begin{array}{r}29.32 \\
19.9 \\
26 .\end{array}$ & $\begin{array}{r}23.08 \\
18.60 \\
42 .\end{array}$ & $\begin{array}{r}17.90 \\
15.06 \\
114 .\end{array}$ & $\begin{array}{r}10.71 \\
357 \\
271 .\end{array}$ & $\mid \begin{array}{r}6.15 \\
350 \\
3.0 \\
652 .\end{array}$ & $\begin{array}{r}4.01 \\
.460 \\
572 .\end{array}$ \\
\hline PUT- & $\begin{array}{l}\text { OR } 3.81 \\
3.03 \\
-i 3 \\
-i .0 \\
661 .\end{array}$ & $\begin{array}{l}-i{ }^{4} \\
576 .\end{array}$ & $\begin{array}{r}12.21 \\
346 \\
301 .\end{array}$ & $\begin{array}{r}15.43 \\
946 \\
283.0\end{array}$ & $\begin{array}{r}20.66 \\
1353 \\
123 .\end{array}$ & $\begin{array}{r}22.67 \\
2155 \\
13.0\end{array}$ & $\begin{array}{r}23.71 \\
26.59 \\
0 .\end{array}$ & $\begin{array}{r}21: 58 \\
23.00 \\
0 .\end{array}$ & $\begin{array}{r}16.69 \\
197 \\
22.0\end{array}$ & $\begin{array}{r}12.34 \\
16.0 \\
134 .\end{array}$ & $\begin{array}{l}6.57 \\
643 \\
367.0\end{array}$ & $\begin{array}{l}4.64 \\
.36 \\
589.0\end{array}$ \\
\hline 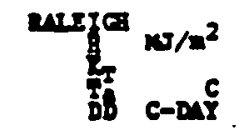 & $\begin{array}{l}\text { ac } 9.5 \\
9.95 \\
556 \\
53.0 \\
422 .\end{array}$ & 3 & $\begin{array}{r}16.81 \\
158 \\
299.0\end{array}$ & $\begin{array}{r}19.78 \\
156 \\
100 .\end{array}$ & $\begin{array}{r}20.87 \\
20.03 \\
2 j .\end{array}$ & $\begin{array}{r}23.84 \\
26.58 \\
0 .\end{array}$ & $\begin{array}{r}22.71 \\
25.56 \\
0 .\end{array}$ & $\begin{array}{r}20.20 \\
2550 \\
0 .\end{array}$ & $\begin{array}{r}16.18 \\
232 \\
22.0 \\
7 .\end{array}$ & $\begin{array}{r}13.09 \\
193 \\
10.0 \\
103 .\end{array}$ & $\mid \begin{array}{r}10.000 \\
1053 \\
250 .\end{array}$ & $\begin{array}{r}8.49 \\
532 \\
610.0 \\
610 .\end{array}$ \\
\hline 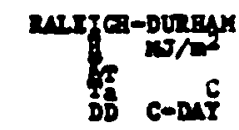 & 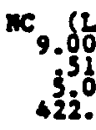 & 354. & $\begin{array}{r}15.66 \\
590^{\circ} \\
239 .\end{array}$ & $\begin{array}{r}20.22 \\
1357 \\
100 .\end{array}$ & $\begin{array}{r}21.645 \\
20.05 \\
27 .\end{array}$ & $\begin{array}{r}22.464 \\
2 i_{0}^{3.6} \\
0 .\end{array}$ & $\begin{array}{r}23.15 \\
235 \% \\
8 .\end{array}$ & $\mid \begin{array}{r}20.145 \\
25 \% \\
25 \\
8 .\end{array}$ & $\begin{array}{r}16.62 \\
25^{53} \\
3 .\end{array}$ & $\begin{array}{r}13.23 \\
154 \\
1650 \\
103 .\end{array}$ & $\mid \begin{array}{r}10.05 \\
1053 \\
250 .\end{array}$ & $\begin{array}{r}8.16 \\
530 \\
410 .\end{array}$ \\
\hline 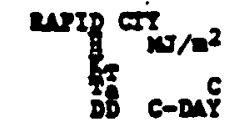 & $\begin{array}{l}50.72 \\
7.76 \\
-562 \\
74 i .0\end{array}$ & $-\frac{5}{63}$ & $\begin{array}{r}16.69 \\
.66 \\
38.0\end{array}$ & $\begin{array}{r}20.28 \\
5 i \\
342 . \\
34 .\end{array}$ & $\begin{array}{r}22.45 \\
13^{58} \\
18 i .\end{array}$ & $\begin{array}{r}24.76 \\
18.80 \\
70^{\circ} .\end{array}$ & $\begin{array}{r}24.88 \\
23^{62} \\
12.0\end{array}$ & $\begin{array}{r}22.62 \\
23^{6.0} \\
i .\end{array}$ & $\begin{array}{r}17.98 \\
16.0 \\
92.0\end{array}$ & $\begin{array}{r}13.13 \\
106 \% \\
26 \%\end{array}$ & $\begin{array}{r}8.57 \\
362 \\
498 .\end{array}$ & $\begin{array}{l}6.61 \\
-39 \\
-251.0\end{array}$ \\
\hline 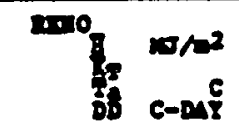 & 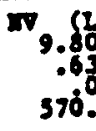 & $\begin{array}{r}3.56 \\
3^{6} \\
4^{6} \dot{3}^{\circ}\end{array}$ & $\left\{\begin{array}{r}18.80 \\
68 \\
426\end{array}\right.$ & $\begin{array}{r}24.79 \\
8 \% \\
303 .\end{array}$ & $\begin{array}{r}27.80 \\
17 \\
182.0\end{array}$ & $\begin{array}{r}29.89 \\
16.0 \\
16.0\end{array}$ & $\begin{array}{r}29.60 \\
20,0 \\
9.0\end{array}$ & $\begin{array}{r}27.05 \\
19.8 \\
28 .\end{array}$ & $\begin{array}{r}22.27 \\
1340 \\
93 .\end{array}$ & $\begin{array}{r}16.54 \\
193 \\
253^{0 .}\end{array}$ & $\begin{array}{r}11.60 \\
6 \% \\
615 .\end{array}$ & $\begin{array}{r}8.75 \\
.62 \\
35 i^{0}\end{array}$ \\
\hline
\end{tabular}

\begin{tabular}{|c|c|c|c|c|c|c|c|c|c|c|c|c|}
\hline cotion Month & SAII & $\sqrt{10}$ & $\mathrm{man}$ & As & $\operatorname{mr}$ & Jons & $\pi \Omega x$ & $\Delta 6$ & 827 & OCT & mov & DEC \\
\hline 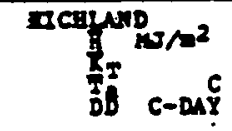 & su & 395. & $\begin{array}{r}13.93 \\
585 \\
3250 \\
325\end{array}$ & $\begin{array}{r}19.53 \\
13.60 \\
183 .\end{array}$ & $\begin{array}{r}21.58 \\
196 \\
72.0\end{array}$ & $\begin{array}{r}27.06 \\
26.05 \\
17.0\end{array}$ & $\begin{array}{r}24.09 \\
24.00 \\
\quad .7 .\end{array}$ & $\begin{array}{r}23.13 \\
23.0 \\
16 .\end{array}$ & $\begin{array}{r}16.23 \\
1860 \\
43 .\end{array}$ & $\begin{array}{l}9.58 \\
151 \\
2020 \\
202 .\end{array}$ & $\begin{array}{r}3.19 \\
54 . \\
3830\end{array}$ & $\begin{aligned} 4.14 \\
.42 \\
303 \\
303 .\end{aligned}$ \\
\hline 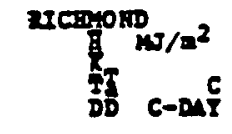 & ${ }^{\mathrm{A}}$, & 49 & $\begin{array}{r}13.07 \\
53 \\
830 \\
316 .\end{array}$ & $\begin{array}{r}19.36 \\
15 \\
12.5 \\
126 .\end{array}$ & $\begin{array}{r}21.86 \\
295 \\
36.0\end{array}$ & $\begin{array}{r}23.49 \\
2357 \\
0 .\end{array}$ & $\begin{array}{r}23.40 \\
2358 \\
0 .\end{array}$ & $\begin{array}{r}20.14 \\
23.0 \\
0 .\end{array}$ & $\begin{array}{r}16.54 \\
25.0 \\
12 .\end{array}$ & $\begin{array}{r}12.64 \\
15.54 \\
113.0\end{array}$ & $\begin{array}{r}8.67 \\
599 \\
267 .\end{array}$ & $\begin{array}{r}7.12 \\
449 \\
448 .\end{array}$ \\
\hline 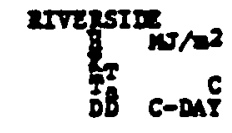 & $\begin{array}{c}01 \\
11 \\
1\end{array}$ & $\begin{array}{l}13^{64} \\
17 j^{\circ}\end{array}$ & $\begin{array}{r}20.01 \\
13.07 \\
157 .\end{array}$ & $\begin{array}{r}22.65 \\
135.0 \\
93 .\end{array}$ & $\begin{array}{r}26.08 \\
18.06 \\
4 i .\end{array}$ & $\begin{array}{r}28.47 \\
20.6 \\
22 .\end{array}$ & $\begin{array}{r}28.18 \\
24.0 \\
0 .\end{array}$ & $\begin{array}{r}25.87 \\
24.0 \\
0 .\end{array}$ & $\begin{array}{r}22.40 \\
22.0 \\
3 .\end{array}$ & $\begin{array}{r}17.04 \\
18.67 \\
96 .\end{array}$ & $\begin{array}{r}13.36 \\
167 \\
118.9 \\
118 .\end{array}$ & $\begin{array}{r}11.30 \\
1.64 \\
10.0 \\
208 .\end{array}$ \\
\hline 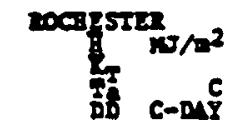 & $x$ & $\frac{-6}{626}$ & $\begin{array}{r}12.64 \\
i 49 \\
351.0\end{array}$ & $\begin{array}{r}16.56 \\
58^{50} \\
315 .\end{array}$ & $\begin{array}{r}21.55 \\
155 \\
150 .\end{array}$ & $\begin{array}{r}23.95 \\
1980 \\
26.0\end{array}$ & $\begin{array}{r}24.03 \\
22.00 \\
5 .\end{array}$ & $\begin{array}{r}20.51 \\
2 i^{5} .0 \\
16 .\end{array}$ & $\begin{array}{r}15.53 \\
1535 \\
70 .\end{array}$ & $\begin{aligned} 10.55 \\
151 \\
15 i .0 \\
22 i .\end{aligned}$ & $\begin{array}{r}6.03 \\
54.2 \\
508 .\end{array}$ & $\begin{array}{l}4.77 \\
-3.40 \\
632 .\end{array}$ \\
\hline 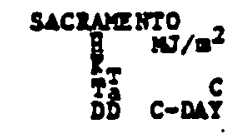 & $\begin{array}{r}c_{0.82}(12 \\
.82 \\
i .0 \\
343 .\end{array}$ & $\begin{array}{l}38 . \\
i 1.10 \\
1052 \\
237^{\circ}\end{array}$ & $\begin{array}{r}16.62 \\
1260 \\
207 .\end{array}$ & $\begin{array}{r}22.23 \\
139.4 \\
126.0\end{array}$ & $\begin{array}{r}28.39 \\
18.0 \\
67 .\end{array}$ & $\begin{array}{r}23.68 \\
2 i^{60} \\
11 .\end{array}$ & $\begin{array}{r}28.47 \\
24.0 \\
0 .\end{array}$ & $\begin{array}{r}24.37 \\
23.0 \\
0 .\end{array}$ & $\begin{array}{r}20.43 \\
22.06 \\
3 .\end{array}$ & $\begin{array}{r}15.28 \\
196.0 \\
56 .\end{array}$ & $\begin{array}{l}9.42 \\
15.54 \\
200.0\end{array}$ & $\begin{array}{r}6.36 \\
543 \\
33 i^{4} \\
33 .\end{array}$ \\
\hline 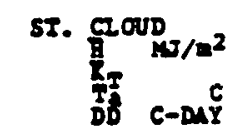 & $\begin{array}{r}7.1 \\
-12 . \\
966\end{array}$ & $\begin{array}{r}10.50 \\
-6.60 \\
-30.0\end{array}$ & $\begin{array}{r}15.31 \\
-62 \\
673.0 \\
673 .\end{array}$ & $\begin{array}{r}17.69 \\
394 \\
368.0 \\
368 .\end{array}$ & $\begin{array}{r}20.83 \\
13.0 \\
180 .\end{array}$ & $\begin{array}{r}22.62 \\
1855 \\
49 .\end{array}$ & $\begin{array}{r}23.21 \\
2 i^{58} \\
10.0\end{array}$ & $\begin{array}{r}20.53 \\
2099 \\
21 .\end{array}$ & $\begin{array}{r}13.09 \\
135 \\
123 . \\
127 .\end{array}$ & $\begin{aligned} 10.08 \\
592 \\
99.0 \\
299 .\end{aligned}$ & $\begin{array}{r}6.11 \\
.47 \\
583 .\end{array}$ & $\begin{array}{r}5.14 \\
-950 \\
-84 \% \\
849\end{array}$ \\
\hline
\end{tabular}




\begin{tabular}{|c|c|c|c|c|c|c|c|c|c|c|c|c|}
\hline Month & $J \Delta B$ & rE & MAR & $\triangle P Q$ & mat & SOIIE & Jolर & ADG & SEP & OCT & MOV & DEC \\
\hline 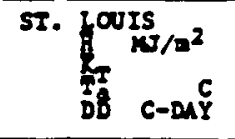 & $\begin{array}{r}\text { no } 7.37 \\
.46 \\
38 i^{\circ}\end{array}$ & $\begin{array}{r}5.49 \\
465.0\end{array}$ & $\begin{array}{r}14.49 \\
532 \\
69.0 \\
379 .\end{array}$ & $\begin{array}{r}18.00 \\
352 \\
14.0 \\
151 .\end{array}$ & $\begin{array}{r}22.15 \\
1960 \\
59 .\end{array}$ & $\begin{array}{r}23.95 \\
24.0 \\
6 .\end{array}$ & $\begin{array}{r}33.78 \\
26.0 \\
0 .\end{array}$ & $\begin{array}{r}20.93 \\
2537 \\
5.0\end{array}$ & $\begin{array}{r}17.90 \\
5.98 \\
21.00 \\
19 .\end{array}$ & $\begin{array}{r}12.89 \\
1956 \\
124 .\end{array}$ & $\begin{array}{l}8.71 \\
551 \\
330 \\
333 .\end{array}$ & $\begin{array}{l}6.45 \\
54 \\
523 \\
52.0\end{array}$ \\
\hline 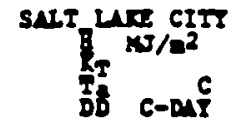 & $\begin{array}{l}{ }_{0} \\
6 . \\
-i \\
63\end{array}$ & 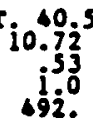 & $\begin{array}{r}14.82 \\
555 \\
4370\end{array}$ & $\begin{array}{r}20.06 \\
10.99 \\
263 .\end{array}$ & $\begin{array}{r}23.87 \\
15.0 \\
132 .\end{array}$ & $\begin{array}{r}26.00 \\
19.03 \\
99 .\end{array}$ & $\begin{array}{r}25.96 \\
25_{0}^{64} \\
0 .\end{array}$ & $\begin{array}{r}23.07 \\
26.04 \\
3 .\end{array}$ & $\begin{array}{r}18.67 \\
18.63 \\
58 .\end{array}$ & $\begin{array}{r}13.23 \\
150 \\
223 .\end{array}$ & $\begin{array}{r}8.54 \\
543 \\
4.00 \\
432 .\end{array}$ & $\begin{array}{r}6.11 \\
.46 \\
598 .\end{array}$ \\
\hline 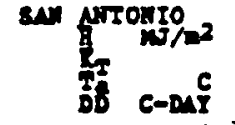 & 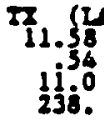 & $\begin{array}{l}i_{4.53}^{29.3} \\
1456 \\
1590 \\
159 .\end{array}$ & $\begin{array}{l}17.52 \\
1656 \\
108 .\end{array}$ & $\begin{array}{r}18.82 \\
20^{51} \\
22^{3}\end{array}$ & $\begin{array}{r}22.54 \\
24.57 \\
0 .\end{array}$ & $\begin{array}{r}25.26 \\
27.02 \\
0 .\end{array}$ & $\begin{array}{r}26.14 \\
285 \\
0.0 \\
0 .\end{array}$ & $\begin{array}{r}24.34 \\
28.05 \\
0 .\end{array}$ & $\begin{array}{r}20.49 \\
26.02 \\
0 .\end{array}$ & $\begin{array}{r}16.52 \\
2 i^{60} \\
1 i^{\circ}\end{array}$ & $\mid \begin{array}{r}12.17 \\
15.5 \\
113 . \\
11 .\end{array}$ & $\mid \begin{array}{l}10.54 \\
132 \\
1202 . \\
202 .\end{array}$ \\
\hline 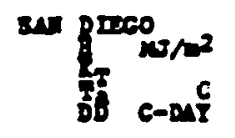 & ca. & 13 & $\begin{array}{l}17.92 \\
14^{39} \\
122.0\end{array}$ & $\begin{array}{r}19.63 \\
15.54 \\
80^{5} .\end{array}$ & $\begin{array}{r}20.64 \\
135.0 \\
4.5\end{array}$ & $\begin{array}{r}21.95 \\
1852 \\
29 . \\
29 .\end{array}$ & $\begin{array}{r}22.90 \\
20.07 \\
3 .\end{array}$ & $\begin{array}{r}20.89 \\
2 i_{0.0}^{96} \\
0 .\end{array}$ & $\begin{array}{r}18.67 \\
21.98 \\
9 .\end{array}$ & $\begin{array}{r}15.51 \\
18.0 \\
26.0\end{array}$ & $\begin{array}{r}11.89 \\
1590 \\
70 .\end{array}$ & $\mid \begin{array}{r}10.26 \\
56 \\
13.0 \\
14 j .\end{array}$ \\
\hline 3w Ructsed & 24 & 18i. & $\begin{array}{r}17.08 \\
13^{60} \\
184 .\end{array}$ & $\begin{array}{r}21.64 \\
13.61 \\
162 .\end{array}$ & $\begin{array}{r}26.20 \\
16.0 \\
163 .\end{array}$ & $\begin{array}{r}24.99 \\
15.0 \\
108 .\end{array}$ & $\begin{array}{l}22.61 \\
19.50 \\
112.0\end{array}$ & $\begin{array}{r}20.01 \\
15.55 \\
98 .\end{array}$ & $\begin{array}{r}17.75 \\
19.0 \\
57.0\end{array}$ & $\begin{array}{r}13.90 \\
1659 \\
7 i^{\circ}\end{array}$ & $\begin{array}{l}9.63 \\
9.94 \\
14.0 \\
129 .\end{array}$ & $\begin{array}{l}7.33 \\
1.48 \\
224.0\end{array}$ \\
\hline 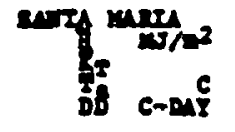 & $\begin{aligned} 41.84 \\
10^{60} \\
235 .\end{aligned}$ & $\begin{array}{l}36.5 \\
46.64 \\
16.5 \\
106 .\end{array}$ & $\begin{array}{r}20.32 \\
1 i^{69} \\
202\end{array}$ & $\begin{array}{r}23.42 \\
13^{66} \\
15 \%\end{array}$ & $\begin{array}{r}26.64 \\
13.67 \\
129 .\end{array}$ & $\begin{array}{r}29.11 \\
13.1 \\
92 .\end{array}$ & $\begin{array}{r}28.46 \\
16.0 \\
55.0\end{array}$ & $\begin{array}{r}25.59 \\
16.69 \\
52 .\end{array}$ & $\begin{array}{r}21.91 \\
179.60 \\
53 .\end{array}$ & $\begin{array}{r}17.48 \\
19.0 \\
81 .\end{array}$ & $\begin{array}{r}13.01 \\
19.6 \\
150 .\end{array}$ & $\begin{array}{l}10.58 \\
10.2 \\
217 .\end{array}$ \\
\hline
\end{tabular}

\begin{tabular}{|c|c|c|c|c|c|c|c|c|c|c|c|c|}
\hline ation Month & SN & TEB & Whe & APR & $M Y$ & SUNE & SULY & AUG & SEP & גד & Nov & DEC \\
\hline 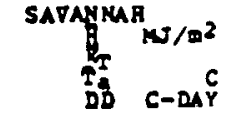 & $\begin{array}{r}G 0.30 \\
10.30 \\
1052 \\
10.0 \\
268 .\end{array}$ & 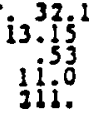 & $\begin{array}{r}16.87 \\
145 \\
14.0 \\
142 .\end{array}$ & $\begin{array}{r}21.64 \\
19.80 \\
39 .\end{array}$ & $\begin{array}{r}23.57 \\
23.0 \\
0 .\end{array}$ & $\begin{array}{r}23.19 \\
26.57 \\
0 .\end{array}$ & $\begin{array}{r}22.44 \\
25^{56} \\
0 .\end{array}$ & $\begin{array}{r}20.97 \\
2 i^{56} \\
0 . \\
0 .\end{array}$ & $\begin{array}{r}16.87 \\
355 \\
35.0 \\
0 .\end{array}$ & $\begin{array}{r}14.57 \\
30.55 \\
33 .\end{array}$ & $\begin{aligned} 11.10 \\
53 \\
14.00 \\
141^{\circ}\end{aligned}$ & $\begin{array}{l}8.96 \\
10.9 \\
254 .\end{array}$ \\
\hline 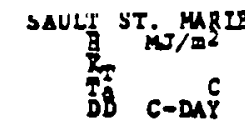 & 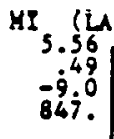 & $\begin{array}{r}46.3 \\
9.45^{3} \\
-90^{57} \\
-969 \\
69\end{array}$ & $\begin{array}{l}14.89 \\
-i^{6 .} .0 \\
709 .\end{array}$ & $\begin{array}{r}77.52 \\
3.4 \\
350.0 \\
450 .\end{array}$ & $\begin{array}{r}22.00 \\
59 \\
9.0 \\
265 .\end{array}$ & $\begin{array}{l}23.00 \\
196 \\
11.0 \\
112 .\end{array}$ & $\begin{array}{r}23.96 \\
1960 \\
53.0\end{array}$ & $\begin{array}{r}19.95 \\
17.57 \\
58 .\end{array}$ & $\begin{array}{r}13.47 \\
130 \\
135.0\end{array}$ & $\begin{array}{r}9.03 \\
7.88 \\
322 .\end{array}$ & $\begin{array}{r}4.39 \\
.35 \\
528.0\end{array}$ & $\begin{array}{r}3.97 \\
-6.41 \\
759 .\end{array}$ \\
\hline 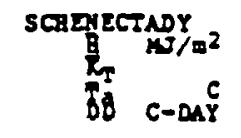 & $\begin{array}{l}\text { Ix } 3.64 \\
3.40 \\
-5.0 \\
744 .\end{array}$ & $\begin{array}{l}42.5 \\
8.4 i^{5} \\
-4.40 \\
64 i^{\circ}\end{array}$ & $\begin{array}{r}11.46 \\
i^{4.4} \\
363 .\end{array}$ & \begin{tabular}{r|r|}
14.22 \\
$8^{4} .2$ \\
$13^{3}$ & 0
\end{tabular} & $\begin{array}{r}17.31 \\
15.44 \\
136.0\end{array}$ & $\begin{array}{r}18.78 \\
20.0 \\
80 .\end{array}$ & $\begin{array}{r}18.57 \\
23.6 \\
4 .\end{array}$ & $\begin{aligned} 16.69 \\
21.47 \\
11 .\end{aligned}$ & $\begin{array}{r}12.55 \\
194.0 \\
16 .\end{array}$ & $\begin{array}{l}9.16 \\
13 j^{64}\end{array}$ & $\begin{array}{l}3.39 \\
4.36 \\
4.0 . \\
420 .\end{array}$ & $\begin{array}{l}4.35 \\
-3.36 \\
653 .\end{array}$ \\
\hline 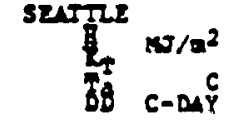 & $\begin{array}{l}H \Delta .(24 \\
3.260 \\
35.0 \\
310 . \\
410 .\end{array}$ & 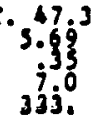 & $\begin{array}{r}1.04 \\
5.7 \\
32 i .\end{array}$ & $\begin{array}{r}16.56 \\
10.92 \\
220.0\end{array}$ & $\begin{array}{r}20.95 \\
135 \\
134 .\end{array}$ & $\begin{array}{r}21.79 \\
163 \\
65 .\end{array}$ & $\begin{array}{r}23.71 \\
189 \\
28.0\end{array}$ & $\begin{array}{r}19.86 \\
1.57 \\
18.0 \\
26 .\end{array}$ & $\begin{array}{r}13.72 \\
20_{1}^{3} \\
72^{0} .\end{array}$ & $\begin{array}{l}7.90 \\
1243 \\
183 .\end{array}$ & $\begin{array}{l}4.43 \\
3.37 \\
802.0 \\
302 .\end{array}$ & $\begin{array}{r}2.68 \\
29 \\
6.5 \\
365 .\end{array}$ \\
\hline 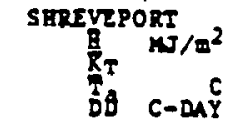 & $\begin{array}{r}24.45 \\
9.48 \\
88.0 \\
307 .\end{array}$ & $\begin{array}{l}32.2 \\
1.67^{2} \\
10^{48} \\
237^{\circ}\end{array}$ & $\begin{array}{r}15.81 \\
192 \\
14.0 \\
169 .\end{array}$ & $\begin{array}{r}19.53 \\
18.5 \\
45.0\end{array}$ & $\begin{array}{r}22.92 \\
22_{0} .0 \\
0 .\end{array}$ & $\begin{array}{r}22.75 \\
26.56 \\
0 .\end{array}$ & $\begin{array}{r}23.50 \\
238 \\
28.0 \\
0 .\end{array}$ & $\begin{aligned} 21.75 \\
28.58 \\
0 .\end{aligned}$ & $\begin{array}{r}17.36 \\
25.54 \\
0 .\end{array}$ & $\begin{array}{r}14.47 \\
195 \\
26.0\end{array}$ & $\begin{array}{l}10.16 \\
13.09 \\
165 .\end{array}$ & $\begin{array}{r}8.28 \\
945 \\
965 .\end{array}$ \\
\hline $\begin{array}{rr}\text { SILVER RIIL } & \text { RU/m2 } \\
T_{T} & c \\
\text { DS } & C-\text { LI }\end{array}$ & $\begin{array}{l}\text { m } 7 \mathrm{L1} \\
7.6 \frac{1}{48} \\
30.0 \\
306 .\end{array}$ & $\begin{array}{r}10.28 \\
.20^{\circ} \\
3^{48} \\
43 i^{\circ}\end{array}$ & $\begin{array}{r}14.22 \\
51 \\
5.0 \\
343 .\end{array}$ & $\begin{array}{rl}18.32 & 2 \\
13 & 3 \\
147^{\circ} & 0\end{array}$ & $\begin{array}{r}21.45 \\
18.54 \\
40.0\end{array}$ & $\begin{array}{r}23.21 \\
23.56 \\
0 .\end{array}$ & $\begin{array}{r}21.58 \\
254 \\
50 \\
0 .\end{array}$ & $\begin{array}{r}19.19 \\
24.6 \\
0 .\end{array}$ & $\begin{array}{r}16.60 \\
2 \mathrm{i} 5 \\
8.0 \\
8 .\end{array}$ & $\begin{array}{r}12.34 \\
153 \\
106 .\end{array}$ & $\begin{array}{r}8.45 \\
599 \\
283 .\end{array}$ & $\begin{array}{r}6.82 \\
47 \\
3.0 \\
476 .\end{array}$ \\
\hline
\end{tabular}




\begin{tabular}{|c|c|c|c|c|c|c|c|c|c|c|c|c|}
\hline Month & JAN & $\pi 3$ & MAR & $\Delta P R$ & KuY & SONE & SULY & ADG & SEP. & OCr & Nov & DEC \\
\hline 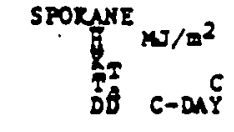 & $\begin{array}{r}4.94 \\
4.94 \\
-3.0 \\
682 .\end{array}$ & $310^{\circ}$ & $\begin{array}{r}13.26 \\
356 \\
3.0 \\
474 .\end{array}$ & $\begin{array}{r}19.53 \\
86 . \\
315 .\end{array}$ & $\begin{array}{r}23.25 \\
13.00 \\
182 .\end{array}$ & $\begin{array}{r}25.30 \\
16.61 \\
80 .\end{array}$ & $\begin{array}{r}27.73 \\
21.0 \\
12 .\end{array}$ & $\begin{array}{r}23.17 \\
20.67 \\
26 .\end{array}$ & $\begin{array}{r}17.02 \\
15.04 \\
109.0\end{array}$ & $\begin{array}{r}8: 62 \\
; 4.0 \\
296 .\end{array}$ & $\begin{array}{l}5.48 \\
i^{4.6} \\
492 .\end{array}$ & $\begin{array}{r}3.18 \\
-35 \\
-1.00 \\
620 .\end{array}$ \\
\hline 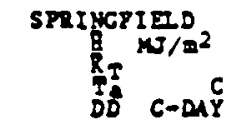 & 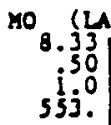 & 光. & $\begin{array}{r}15.41 \\
5.54 \\
367.0\end{array}$ & $\begin{array}{r}18.59 \\
153 \\
1530 \\
153 .\end{array}$ & $\begin{array}{r}22.11 \\
186 \\
52.0\end{array}$ & $\begin{array}{r}23.91 \\
23.50 \\
6 .\end{array}$ & $\begin{array}{r}23.61 \\
259 \\
0 .\end{array}$ & $\begin{array}{r}22.15 \\
25.60 \\
j .\end{array}$ & $\begin{array}{r}19.01 \\
2 i^{62} \\
19.0\end{array}$ & $\begin{array}{r}13.94 \\
19.99 \\
126 .\end{array}$ & $\begin{array}{l}99.22 \\
5 ; 52 \\
325.0 \\
325 .\end{array}$ & $\begin{array}{r}7.54 \\
349 \\
490 \\
49 .\end{array}$ \\
\hline 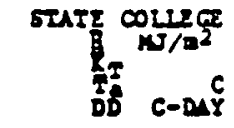 & & -2.0 & $\begin{array}{r}12.42 \\
.46 \\
489 .\end{array}$ & $\begin{array}{r}13.60 \\
946 \\
267^{\circ}\end{array}$ & $\begin{array}{r}19.53 \\
150 \\
115.0\end{array}$ & $\begin{array}{r}22.75 \\
2095 \\
13 .\end{array}$ & $\begin{array}{r}22.08 \\
235 \\
j .0\end{array}$ & $\begin{array}{r}18.999 \\
21.0 \\
8 .\end{array}$ & $\begin{array}{r}15.10 \\
1950 \\
61 .\end{array}$ & $\begin{aligned} 11.90 \\
11^{32} \\
214 .\end{aligned}$ & $\begin{array}{r}6.48 \\
3.4 \\
400 \\
40.0\end{array}$ & $\begin{array}{l}5.02 \\
-138 \\
-1.00 \\
609 .\end{array}$ \\
\hline 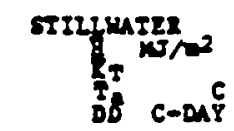 & 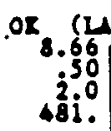 & $\begin{array}{l}5^{3} .3 \\
338.0\end{array}$ & $\begin{array}{r}16.23 \\
: 56 \\
289 .\end{array}$ & $\begin{array}{r}19.07 \\
195 \\
169 \\
97 .\end{array}$ & $\begin{array}{r}20.99 \\
200^{3} \\
21 .\end{array}$ & $\begin{array}{r}24.80 \\
25.60 \\
0 .\end{array}$ & $\begin{array}{r}24.80 \\
26^{62} \\
0 .\end{array}$ & $\begin{array}{r}22.67 \\
27.0 \\
0 .\end{array}$ & $\begin{array}{r}19.03 \\
23.01 \\
6 .\end{array}$ & $\begin{array}{r}14.72 \\
19.0 \\
81 .\end{array}$ & $\begin{array}{r}10.75 \\
958 \\
9.0 \\
258 .\end{array}$ & $\begin{array}{r}8.53 \\
553 \\
54.0 \\
429 .\end{array}$ \\
\hline 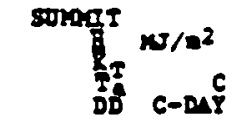 & $\begin{array}{l}\pi_{3} \\
-8 \\
83\end{array}$ & $\begin{array}{l}-544 \\
6991.0\end{array}$ & 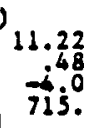 & $\begin{array}{r}17.33 \\
55 \\
518 . \\
\text { sis. }\end{array}$ & $\begin{array}{r}19.34 \\
530 \\
397^{\circ}\end{array}$ & $\begin{array}{r}20.64 \\
10.90 \\
253 .\end{array}$ & $\begin{array}{r}23.45 \\
159 \\
14.0 \\
143 .\end{array}$ & $\begin{array}{r}21.35 \\
13.62 \\
17 i^{1 .}\end{array}$ & $\begin{aligned} 14.82 \\
\dot{8} 56 \\
80.0 \\
30 \dot{2}\end{aligned}$ & $\begin{array}{r}9.04 \\
.51 \\
459 .\end{array}$ & $\begin{array}{l}4.27 \\
-2.38 \\
649 .\end{array}$ & $\begin{array}{l}3.18 \\
395 \\
79 ;\end{array}$ \\
\hline 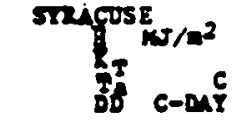 & $\begin{array}{l}\pi \text { r. } \\
3.40 \\
-540 \\
713.0\end{array}$ & 628 & 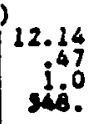 & $\begin{array}{r}15.66 \\
847 \\
308 .\end{array}$ & $\begin{array}{r}20.28 \\
152 \\
15 i^{\circ} \\
15\end{array}$ & $\begin{array}{r}23.1 \frac{1}{156} \\
19.0 \\
26 .\end{array}$ & $\begin{array}{r}23.36 \\
238 \\
23.0\end{array}$ & $\begin{array}{r}19.72 \\
215 \\
10 .\end{array}$ & $\begin{array}{r}14.99 \\
1753 \\
67 .\end{array}$ & $\begin{array}{r}10.09 \\
11^{49} \\
218 .\end{array}$ & $\begin{array}{r}5.19 \\
36 \\
300 \\
400 .\end{array}$ & $\begin{array}{r}4.31 \\
-35 \\
-32.0 \\
436 .\end{array}$ \\
\hline
\end{tabular}

\begin{tabular}{|c|c|c|c|c|c|c|c|c|c|c|c|c|}
\hline Month & JNA & res & mar & APZ & $M Y Y$ & JUNE & JULY & AUE & SEP & OCT & NOV & DEC \\
\hline 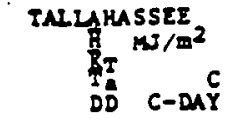 & 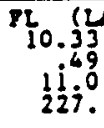 & $\begin{array}{l}1 \dot{3}^{51} \\
1390 \\
139 .\end{array}$ & $\begin{array}{l}17.69 \\
157 \\
106.0\end{array}$ & $\begin{array}{r}20.20 \\
195 \\
19.0 \\
19 .\end{array}$ & $\begin{array}{r}22.92 \\
23.0 \\
0 .\end{array}$ & $\begin{array}{r}19.91 \\
26.0 \\
0.0\end{array}$ & $\begin{array}{r}22.75 \\
2757 \\
0 .\end{array}$ & $\begin{array}{r}22.46 \\
27.00 \\
0 .\end{array}$ & $\begin{array}{r}17.73 \\
255 \\
25.0\end{array}$ & $\begin{array}{r}14.76 \\
20.96 \\
17 .\end{array}$ & $\begin{array}{l}15.22 \\
15.0 \\
113^{60}\end{array}$ & $\begin{array}{r}13.01 \\
13.67 \\
209 .\end{array}$ \\
\hline 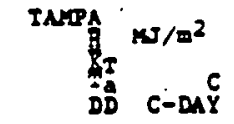 & $\begin{array}{r}72.64 \\
13.67 \\
16.0 \\
113 .\end{array}$ & $\begin{array}{r}16.1 \\
98 .\end{array}$ & $\begin{array}{r}19.95 \\
19.62 \\
50 .\end{array}$ & $\begin{array}{r}22.79 \\
21.02 \\
5.0\end{array}$ & $\begin{array}{r}24.88 \\
26.63 \\
24.0\end{array}$ & $\begin{array}{r}23.96 \\
26.59 \\
0 .\end{array}$ & $\begin{array}{r}22.29 \\
27.56 \\
0 .\end{array}$ & $\begin{array}{r}20.70 \\
27.55 \\
0 .\end{array}$ & $\begin{array}{r}18.99 \\
26.0 \\
0 .\end{array}$ & $\begin{array}{r}16.94 \\
23.00 \\
0 .\end{array}$ & $\begin{array}{r}14.93 \\
196.0 \\
39 .\end{array}$ & $\begin{array}{r}12.63 \\
16.60 \\
94 .\end{array}$ \\
\hline 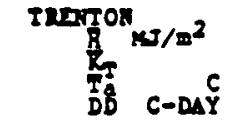 & $\begin{array}{l}\text { RJ } 7.24 \\
7.24 \\
.48 \\
56 i^{\circ} .\end{array}$ & & $\begin{array}{r}14.36 \\
5.53 \\
5.00 \\
410 .\end{array}$ & $\begin{array}{rl}17.75 \\
52 \\
11^{2} \\
21 & 0 \\
0\end{array}$ & $\begin{array}{r}20.56 \\
152 \\
16.0 \\
15 .\end{array}$ & $\begin{array}{r}22.86 \\
215 \\
21.0 \\
0.0\end{array}$ & $\begin{array}{r}22.61 \\
24.56 \\
24.0\end{array}$ & $\begin{array}{r}19.64 \\
23.54 \\
0 .\end{array}$ & $\begin{array}{r}16.29 \\
195 \\
192.0 \\
22 .\end{array}$ & $\begin{array}{r}12.35 \\
135 \\
13.0 \\
140 .\end{array}$ & $\begin{array}{r}8.160 \\
750 \\
310^{\circ}\end{array}$ & $\begin{array}{r}6.49 \\
148 \\
518.0\end{array}$ \\
\hline 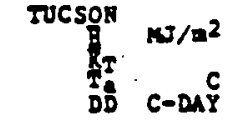 & $\begin{array}{r}12.61 \\
13.09 \\
1060 \\
262.0\end{array}$ & $11^{6.0}$ & $\begin{array}{r}22.83 \\
145 \\
134.0\end{array}$ & $\begin{array}{r}27.85 \\
18.0 \\
42 .\end{array}$ & $\begin{array}{r}30.98 \\
23.8 \\
\text { j. }\end{array}$ & $\begin{array}{r}29.65 \\
27.0 \\
0 .\end{array}$ & $\begin{array}{r}26.26 \\
30^{65} \\
0 .\end{array}$ & $\begin{array}{r}24.67 \\
28.66 \\
0 .\end{array}$ & $\begin{array}{r}26.30 \\
26.5 \\
0 .\end{array}$ & $\begin{array}{r}18.69 \\
209 ! \\
14 .\end{array}$ & $\begin{array}{l}24.85 \\
141 \\
128.0 \\
128 .\end{array}$ & $\begin{array}{l}12.42 \\
10.0 \\
226 .\end{array}$ \\
\hline 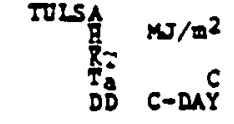 & $\begin{array}{r}0 R_{8} . \\
j \\
48\end{array}$ & $\begin{array}{r}36 . \\
11.43 \\
5.51 \\
370.0\end{array}$ & $\begin{aligned} 15.45 \\
594 \\
993 .\end{aligned}$ & $\begin{array}{r}18.30 \\
162 \\
98.0 \\
98 .\end{array}$ & $\begin{array}{r}23.56 \\
20.55 \\
16.0 \\
16 .\end{array}$ & $\begin{array}{r}24.51 \\
25.59 \\
0 .\end{array}$ & $\begin{array}{r}23.86 \\
289 \\
28.0 \\
0 .\end{array}$ & $\begin{array}{r}22.23 \\
27.60 \\
0 .\end{array}$ & $\begin{array}{r}18.34 \\
239 . \\
6 .\end{array}$ & $\begin{array}{r}13.77 \\
197 \\
79 .\end{array}$ & $\begin{array}{l}9.80 \\
2053 \\
10.0 \\
260 .\end{array}$ & $\begin{array}{r}8.16 \\
551 \\
43.0 \\
43 .\end{array}$ \\
\hline 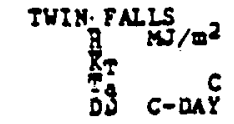 & $\begin{aligned} & \text { ID } \text { (L } \\
& 6.82 \\
&-i^{46} \\
& 84.0 \\
& 84 .\end{aligned}$ & $\begin{array}{r}i 0.09 \\
50 \\
50.0 \\
490 .\end{array}$ & $\begin{array}{r}14.86 \\
555 \\
450 \\
454 .\end{array}$ & $\begin{array}{r}19.34 \\
9.57 \\
290.0 \\
290 .\end{array}$ & $\begin{array}{r}23.11 \\
139 \\
161\end{array}$ & $\begin{array}{r}24.79 \\
1790 \\
73 .\end{array}$ & $\begin{array}{r}25.20 \\
22^{63} \\
0.0 \\
0 .\end{array}$ & $\mid \begin{array}{c}22.61 \\
21.63 \\
12.0\end{array}$ & $\begin{array}{r}18.09 \\
16.0 \\
99 .\end{array}$ & $\begin{array}{r}11.97 \\
10.54 \\
260 .\end{array}$ & $\begin{array}{r}7.37 \\
446 \\
46.0 \\
46 .\end{array}$ & $\begin{array}{r}3.49 \\
.41 \\
589 .\end{array}$ \\
\hline
\end{tabular}




\begin{tabular}{|c|c|c|c|c|c|c|c|c|c|c|c|c|}
\hline Month & JN & r28 & MN & APR & MMY & תRת & sताy & AUG & SEP & OCI & MOV & DEC \\
\hline 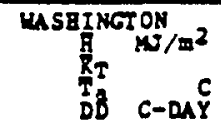 & $\begin{aligned} D C .62 \\
6.65 \\
42.2 \\
484 . \\
484 .\end{aligned}$ & $\begin{array}{l}938 \\
9.62 \\
445 \\
43.0 \\
423 .\end{array}$ & $\begin{array}{r}13.38 \\
7.88 \\
348 .\end{array}$ & $\left|\begin{array}{r}16.85 \\
14.9 \\
130.0 \\
160 .\end{array}\right|$ & $\begin{array}{r}18.69 \\
18.47 \\
4 i .\end{array}$ & $\begin{array}{r}23.34 \\
235 \\
0.0\end{array}$ & $\begin{array}{r}22 . \frac{12}{55} \\
25.00 \\
0 .\end{array}$ & $\begin{array}{r}19.32 \\
243 \\
24.0 \\
0 .\end{array}$ & $\begin{array}{r}13.35 \\
2.51 \\
21.0 \\
18 .\end{array}$ & $\begin{aligned} 11.75 \\
151 \\
121.0\end{aligned}$ & $\begin{array}{r}8.82 \\
991 \\
288.0\end{array}$ & $\begin{array}{r}6.15 \\
3.42 \\
463 .\end{array}$ \\
\hline 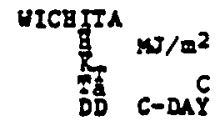 & $\begin{aligned} & \text { RS } \text {.L } \\
& 0.29 \\
& .56 \\
& 581.0 \\
& 58 .\end{aligned}$ & $\begin{array}{r}379 \\
i 1.97 \\
955 \\
4470\end{array}$ & $\begin{array}{r}15.99 \\
566 \\
365.0 \\
373 .\end{array}$ & $\begin{array}{r}19.76 \\
135 \\
1350 \\
153 .\end{array}$ & $\begin{array}{r}22.78 \\
198 \\
90.0 \\
90 .\end{array}$ & $\begin{array}{r}23.20 \\
26.5 \\
4 .\end{array}$ & $\begin{array}{r}24.41 \\
27.61 \\
0 .\end{array}$ & $\begin{array}{r}22.57 \\
26.62 \\
0 .\end{array}$ & $\begin{array}{r}28.71 \\
2.61 \\
28.0\end{array}$ & $\begin{aligned} 24.40 \\
15.61 \\
115^{6}\end{aligned}$ & $\begin{array}{r}10.26 \\
738 \\
337 .\end{array}$ & $\begin{array}{r}8.29 \\
54 \\
528 \\
526 .\end{array}$ \\
\hline 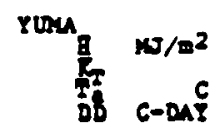 & $\begin{array}{r}42 \\
12 . \\
13 \\
17\end{array}$ & $\begin{array}{r}132.79 \\
169 \\
15.0 \\
10 \%\end{array}$ & $\begin{array}{r}21.64 \\
17.0 \\
17.0 \\
54 .\end{array}$ & $\begin{array}{r}26.50 \\
21.4 \\
13.0\end{array}$ & $\begin{array}{r}29.43 \\
25.9 \\
0.0 \\
0 .\end{array}$ & $\begin{array}{r}29.32 \\
29.0 \\
0 .\end{array}$ & $\begin{array}{r}27.30 \\
36.68 \\
0 .\end{array}$ & $\begin{array}{r}24.58 \\
33.66 \\
0.0\end{array}$ & $\begin{array}{r}22.19 \\
30.69 \\
0 .\end{array}$ & $\begin{array}{r}18.30 \\
24.0 \\
34.0\end{array}$ & $\begin{array}{r}13.82 \\
17.67 \\
60 .\end{array}$ & $\begin{array}{r}11.35 \\
13.02 \\
153 .\end{array}$ \\
\hline 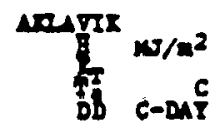 & 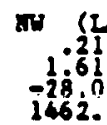 & $\begin{array}{r}2.099 \\
-2756 \\
1298 .\end{array}$ & $\begin{array}{r}8.45 \\
-232.0 \\
2268 .\end{array}$ & $\begin{array}{r}16.23 \\
-12.69 \\
-930 .\end{array}$ & $\begin{array}{r}21.54 \\
.62 \\
59 i^{0}\end{array}$ & $\begin{array}{r}22.08 \\
934 \\
96.0 \\
268 .\end{array}$ & $\begin{array}{r}18.65 \\
13.9 \\
152 .\end{array}$ & $\begin{array}{r}12.42 \\
1044 \\
255\end{array}$ & $\begin{array}{r}6.52 \\
341 \\
3.0 \\
48 .\end{array}$ & $\begin{array}{r}2.59 \\
-7.04 \\
78.0 .\end{array}$ & $\begin{array}{r}.46 \\
-1968 \\
1947 .\end{array}$ & $\begin{array}{r}.04 \\
-260 \\
1406.0\end{array}$ \\
\hline 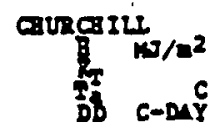 & $\begin{array}{r}\mathrm{MA} .7 \mathrm{~L} \\
2.75 \\
-27^{65} \\
142 i .\end{array}$ & $\begin{aligned} 6.38 \\
6.25 \\
-267 \\
-26.0 \\
+265 .\end{aligned}$ & $\begin{array}{l}12.75 \\
-19.73 \\
1183 .\end{array}$ & $\begin{array}{r}18.61 \\
-610 \\
-10.0 \\
872 .\end{array}$ & $\begin{array}{r}21.33 \\
-3.38 \\
64 i .\end{array}$ & $\begin{array}{r}22.16 \\
54 \\
573.0 \\
373 .\end{array}$ & $\begin{array}{r}21.12 \\
155 \\
200 . \\
200 .\end{array}$ & $\begin{array}{r}15.89 \\
191 \\
\frac{1}{20.0} \\
208 .\end{array}$ & $\begin{array}{r}9.41 \\
34.4 \\
390 .\end{array}$ & $\begin{array}{l}4.81 \\
-i^{401} \\
601 .\end{array}$ & $\begin{array}{r}2.51 \\
-1.67 \\
-680 .\end{array}$ & $\begin{array}{r}1.46 \\
-21^{49} \\
1269 .\end{array}$ \\
\hline 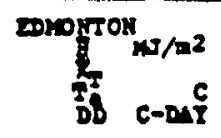 & $\begin{array}{r}49.52 \\
3.72 \\
-163 \\
1006.0\end{array}$ & 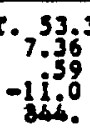 & $\begin{array}{r}13.05 \\
-65.04 \\
739.0\end{array}$ & $\begin{array}{r}17.27 \\
358 \\
425 .\end{array}$ & $\begin{array}{r}21.29 \\
11^{57} \\
222.0 \\
252 .\end{array}$ & $\begin{array}{r}21.43 \\
14.0 \\
123.0\end{array}$ & $\begin{array}{r}22.00 \\
16.96 \\
4 i .\end{array}$ & $\begin{array}{r}17.10 \\
152 \\
1000 \\
100 .\end{array}$ & $\begin{array}{r}12.46 \\
10.52 \\
230^{\circ} .\end{array}$ & $\begin{array}{r}7.86 \\
533 \\
5.00 \\
410 .\end{array}$ & $\begin{array}{r}4.64 \\
3.56 \\
673^{4}\end{array}$ & $\begin{array}{r}2.76 \\
-1.48 \\
-690^{\circ} .\end{array}$ \\
\hline
\end{tabular}

\begin{tabular}{|c|c|c|c|c|c|c|c|c|c|c|c|c|}
\hline corion Month & JNA & 728 & ms & APR & $\mathbf{m r}$ & JONE & $\pi r y$ & AUG & SEP & $\infty C T$ & nov & PEC \\
\hline 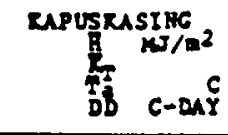 & $\begin{array}{l}\sigma_{4} . \\
-18 \\
113\end{array}$ & -16 & $\begin{array}{r}12.96 \\
-9.97 \\
868 .\end{array}$ & $\begin{array}{r}13.4 \\
.49 \\
343\end{array}$ & $\begin{array}{r}17.15 \\
54.0 \\
328 .\end{array}$ & $\begin{array}{r}20.07 \\
14.9 \\
1.3 .\end{array}$ & $\begin{array}{r}20.07 \\
16.51 \\
4 i .\end{array}$ & $\begin{array}{r}16.93 \\
15.9 \\
95 .\end{array}$ & $\begin{array}{r}11.29 \\
10^{4} .0 \\
223^{\circ}\end{array}$ & $\begin{array}{r}6.69 \\
39 \\
4.0 \\
420 .\end{array}$ & $\begin{array}{r}3.35 \\
-31 \\
-4.0 \\
693 .\end{array}$ & $\begin{array}{r}3.35 \\
-1.42 \\
1004 .\end{array}$ \\
\hline 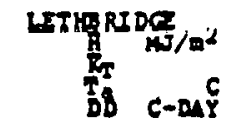 & $\begin{array}{l}\Delta \tau_{3} \\
-8 \dot{8}\end{array}$ & $7^{599} i^{50}$ & $\begin{array}{l}14.22 \\
-2.63 \\
644.0\end{array}$ & $\begin{array}{r}17.55 \\
39 \\
387\end{array}$ & $\begin{array}{r}21.75 \\
15 \\
224 \\
22 .\end{array}$ & $\begin{array}{r}24.25 \\
149 \\
118 .\end{array}$ & $\begin{array}{r}25.51 \\
17^{64} \\
3 i .\end{array}$ & $\begin{array}{r}21.75 \\
16.64 \\
62.0\end{array}$ & $\begin{array}{l}15.47 \\
12.60 \\
177^{\circ}\end{array}$ & $\begin{array}{r}10.04 \\
59 \\
5390 \\
339 .\end{array}$ & $\begin{array}{r}3.86 \\
.53 \\
962.0\end{array}$ & $\begin{array}{r}3.76 \\
-447 \\
-409.0\end{array}$ \\
\hline 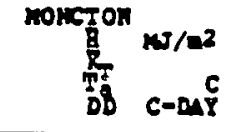 & $\begin{array}{l}-6^{3} b^{3} \\
823 .\end{array}$ & $\begin{array}{l}-8.35 \\
742 .\end{array}$ & $\begin{array}{r}12.13 \\
-350 \\
663 .\end{array}$ & $\begin{array}{r}15.85 \\
34 \\
438 \\
438\end{array}$ & $\begin{array}{r}18.40 \\
590 \\
260.0\end{array}$ & $\begin{array}{r}18.82 \\
13^{46} \\
95.0\end{array}$ & $\begin{array}{r}19.65 \\
17.09 \\
34 .\end{array}$ & $\begin{array}{r}17.15 \\
164 \\
16.0 \\
58 .\end{array}$ & $\begin{array}{r}12.96 \\
13.48 \\
153 .\end{array}$ & $\begin{array}{r}8.78 \\
376 \\
339 .\end{array}$ & $\begin{array}{r}.60 \\
336 \\
i .0 \\
495 .\end{array}$ & $\begin{array}{l}3,76 \\
-3,8.0 \\
746 .\end{array}$ \\
\hline${ }_{D B}^{C-D A Y}$ & 8 & $\begin{array}{l}-4^{4} \cdot 8 \\
767 .\end{array}$ & $\begin{array}{r}13.38 \\
-55 \\
-550 \\
653\end{array}$ & $\begin{array}{r}16.75 \\
35.9 \\
380\end{array}$ & $\begin{array}{r}19.65 \\
135 \\
176 .\end{array}$ & $\begin{array}{r}20.49 \\
1390 \\
38.0\end{array}$ & $\begin{array}{r}21.33 \\
188_{j}^{33} \\
5 .\end{array}$ & $\begin{array}{r}18.40 \\
193 \\
24.0 \\
24 .\end{array}$ & $\begin{array}{r}12.96 \\
13.9 \\
92 .\end{array}$ & $\begin{array}{r}8.36 \\
843 \\
289^{\circ}\end{array}$ & $\begin{array}{r}4.18 \\
i^{3} .0 \\
490^{\circ}\end{array}$ & $\begin{array}{r}3.35 \\
-3.0 \\
-613.0\end{array}$ \\
\hline 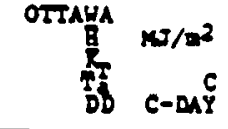 & $\begin{array}{l}0 \% \text {. } \\
6.0 \frac{1}{2} \\
-10.1 \\
-10.0 \\
902 .\end{array}$ & $\begin{array}{r}-10^{5} \\
80 \mathrm{i}^{\circ}\end{array}$ & $\begin{aligned} 14.01 \\
-5.5 \\
-3.0 \\
684 .\end{aligned}$ & $\begin{array}{r}16.85 \\
359 \\
393\end{array}$ & $\begin{array}{r}20.78 \\
13.9 \\
189 .\end{array}$ & $\begin{array}{r}23.34 \\
196 \\
90.0 \\
50 .\end{array}$ & $\begin{array}{r}22.87 \\
179 \\
14.0\end{array}$ & $\begin{array}{r}19.61 \\
16.5 \\
49 .\end{array}$ & $\begin{array}{r}14.85 \\
145 \\
123 .\end{array}$ & $\begin{array}{r}9.24 \\
\because 48 \\
8.00 \\
315 .\end{array}$ & $\begin{array}{r}5.14 \\
.39 \\
320 .\end{array}$ & $\begin{array}{r}4.56 \\
-84 \\
816.0\end{array}$ \\
\hline 3I. JQRNS & T3. & $\begin{array}{l}-47 \\
6.25 \\
-39 \\
-630 \\
630 .\end{array}$ & $\begin{array}{r}10.04 \\
-4.42 \\
-359 \\
639 .\end{array}$ & $\begin{array}{r}13.38 \\
54 \\
515\end{array}$ & $\begin{array}{r}16.73 \\
34.0 \\
394 .\end{array}$ & $\begin{array}{r}17.98 \\
10.4 \\
240.0 \\
440 .\end{array}$ & $\begin{array}{r}18.40 \\
15.46 \\
103 .\end{array}$ & $\begin{array}{r}14.22 \\
15.0 \\
100 .\end{array}$ & $\begin{array}{r}11.71 \\
11.04 \\
190.0\end{array}$ & $\begin{array}{r}7,15 \\
6.39 \\
362 .\end{array}$ & $\begin{array}{r}3.35 \\
328 \\
462.0\end{array}$ & $\begin{array}{l}2.93 \\
-1.0 \\
618 .\end{array}$ \\
\hline
\end{tabular}


For greatest accuracy, a month-by-month computation. must be made of system performance; exhibit 8.15 shows the monthly values. Exhibit 8.16 is an example of the use of exhibit 8.15. Albany, N.Y., in January has a $\bar{K}_{T}$ of 0.38 (see exhibit 8.16). The overage ambient temperature is $-5.0^{\circ} \mathrm{C}\left(22^{\circ} \mathrm{F}\right)$. The insolation is $11.47 \mathrm{~mJ} / \mathrm{m}^{2}$ on a horizontal surface, and $367^{\circ} \mathrm{C}$ days are normally accumulated.

Albany, N.Y. in January:

$$
\begin{aligned}
\bar{K}_{T} & =0.38 \\
\bar{T}_{A} & =5.0^{\circ} \mathrm{C} \\
\bar{H} & =11.47 \mathrm{MJ} / \mathrm{m}^{2} \\
D D & =367 / \text { month }
\end{aligned}
$$

\section{Exhibit 8.16 Example of Use of Exhibit 8.15}

For most PV systems, one must design not only for average power, but for days in which there is no sunshine. One must know how much energy storage is needed to carry through to the next day or so without sunshine. The insolation on one day is poorly correlated with the insolation on the next. If today is cleor, tomorrow has an equal chance of being clear or cloudy. The lack of correlation eases the problem of estimating the number of sequential days the insolation will fall below any specified value. Exhibits 8.17 and 8.18 present the correlation devised by Liu and Jordan that shows the frequency of occurrence of each value of $\bar{K}_{T}$, given the average value, $\bar{K}_{T}$. The curves show the cumulative probability. For example (exhibit 8.19 ), if $\bar{K}_{T}$ were 0.5 , the probability that $\bar{K}_{T}$ would be equal to or less than 0.40 would be 0.323 (from exhibit 8.18 ). The probability that $\bar{K}_{T}$ would be less than 0.4 for $n$ days in a row would be given by exhibit 8.20 . Cumulative probability gives the probability that a value or those less than the value will occur. The frequency distribution gives the probability.

$$
\begin{gathered}
P_{n}=p^{n} \\
\text { or, for two days in a row, } \\
P_{2}=(0.323)^{2}=0.104
\end{gathered}
$$

The probability that $\bar{K}_{T}$ would be less than 0.4 for four days in a row would be $(0.323)^{4}$, or 0.01 , once out of every 100 days. 


\begin{tabular}{|l|l|l|l|l|l|}
\hline \multirow{2}{*}{$K_{T}$} & \multicolumn{5}{|c|}{ VALUE OF f FOR $\bar{K}_{T}=$} \\
\cline { 2 - 6 } & .3 & .4 & .5 & .6 & .7 \\
\hline .04 & .073 & .015 & .001 & .000 & .000 \\
.08 & .162 & .070 & .023 & .008 & .000 \\
\hline .12 & .245 & .129 & .045 & .021 & .007 \\
.16 & .299 & .190 & .082 & .039 & .007 \\
\hline .20 & .395 & .249 & .121 & .053 & .007 \\
.24 & .496 & .298 & .160 & .076 & .007 \\
\hline .28 & .513 & .346 & .194 & .101 & .013 \\
.32 & .579 & .379 & .234 & .126 & .013 \\
\hline .36 & .628 & .438 & .277 & .152 & .027 \\
.40 & .687 & .493 & .323 & .191 & .034 \\
\hline .44 & .748 & .545 & .358 & .235 & .047 \\
.48 & .793 & .601 & .400 & .269 & .054 \\
\hline .52 & .821 & .654 & .460 & .370 & .081 \\
.56 & .861 & .719 & .509 & .360 & .128 \\
\hline .60 & .904 & .760 & .614 & .410 & .161 \\
.64 & .936 & .827 & .703 & .467 & .228 \\
\hline .68 & .953 & .888 & .792 & .538 & .295 \\
.72 & .967 & .931 & .873 & .648 & .517 \\
\hline .76 & .979 & .967 & .945 & .758 & .678 \\
.80 & .986 & .981 & .980 & .884 & .859 \\
\hline .84 & .993 & .997 & .993 & .945 & .940 \\
.88 & .995 & .999 & 1.000 & .985 & .980 \\
\hline .92 & .998 & .999 & & .996 & 1.000 \\
.96 & .998 & 1.000 & & .999 & \\
\hline 1.00 & 1.000 & & & 1.000 & \\
\hline & & & & & \\
\hline
\end{tabular}

Exhibit 8.17 Generalized $K_{\mathrm{T}}$ Distribution Curves.

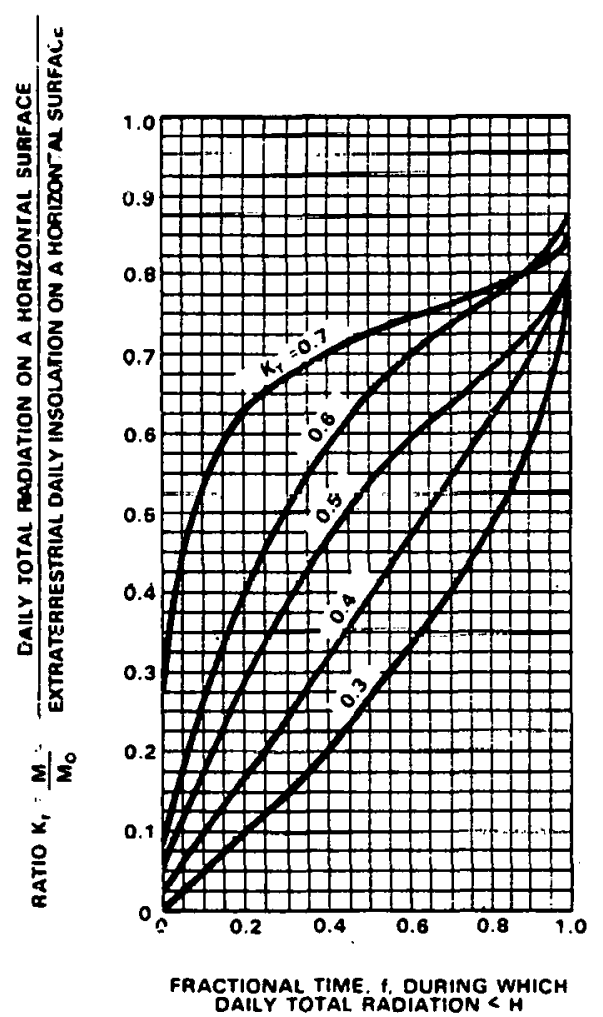

Exhibit 8.18 The Generalized K Tistribution Curves. 


\begin{tabular}{l} 
Given: $K_{\mathrm{T}}=0.5$ \\
What is probability $K_{\mathrm{T}} \leq 0.40 ?$ \\
$\begin{array}{l}\text { a. Enter exhibit } 8.13 \text { or exhibit } 8.14 \text { at } K_{\mathrm{T}}=0.5 \\
\text { b. Read to } K_{\mathrm{T}}=0.40 \\
\text { c. Read probäbility }=0.323\end{array}$ \\
\hline
\end{tabular}

\section{Exhibit 8.19 Use of Frequency Charts.}

\begin{tabular}{|c|c|c|c|}
\hline \multicolumn{4}{|l|}{ Probability $=\mathbf{P}^{M}$} \\
\hline м: & 1 & 2 & 4 \\
\hline Probability: & 0.323 & 0.104 & 0.010 \\
\hline
\end{tabular}

\section{Exhibit 8.20 Consecutive Days of $K_{T}<0.4$.}

\subsubsection{INSOLATION ON TILTED SURFACES}

The insolation computed and the probabilities of experiencing this insolation; pertain to insolation on a flat, horiznntal surface. Most solar cells will be tilted toward the sun to take advantage of the higher incident flux. The method of Liu and Jordan can also be used to devise a way of computing the flux on the tilted surface.

First, the flux must be broken into the direct and diffuse components. The diffuse component is due to the scattering of sunlight in the atmosphere by dust, clouds, water vapor, etc. One assumes that the diffuse flux is isotropic, coming uniformly from every sector of the sky (isotropic refers to the same value regardless of direction of measurement). If the diffuse flux is known for the horizontal surface, which "sees" the entire sky, the diffuse flux for the tilted surface can be computed by correcting for the amount of sky "seen" by the tilted surface. The ratio of views is given by the formula shown in exhibit 8.21 , where $A$ is the tilt of the surface ( $A$ is zero for a horizontal suface; $90^{\circ}$ for a vertical surface). If the direct sunlight onto the horizontal surface is known, the flux can be divided by the cosine of the angle of incidence (solar-altitude angle) to determine the intensity of the beam flux. This intensity can be multiplied by the cosine of the angle of incidence (with respect to the tilted surface) to determine the direct flux on the tilted surface. Summing the direct and diffuse fluxes gives the total insolation on the tilted surface, except for the flux reflected from the surroundings. A typical value for the ground reflectance is 0.1 ; for snow, it is 0.46 to 0.86 . The flux received by the tilted surface due to ground reflectance is given by the last formula in exhibit 8.21 , where $S_{H}$ is the insolation on the horizontal surface. Exhibit 8.22 gives the relationship between $\mathcal{K}_{T}$ and the diffuse flux, as obtained by 
Liu and Jordan. The computation of the flux on a tilted surface is complicated. Wlost of the computation effort lies in determining. the angle of incidence to the horizontal and to the tilted area. Fortunately, Duffie and Beckman have performed the computations and have tabulated the results (see exhibit 8.23). The following computational procedure is based on the use of their tables.

Direct Sunlight: By cosine of angle with solar rays

Diffuse Sunlight: By view of sky, $F=(1+\cos A) / 2$

Ground-Reflected Sunlight: By view of earth, $(1-F) R_{c} S_{11}$

$$
\text { Ro } \simeq 10 \%
$$

\section{Exhibit 8.21 Conversion tu Flux on Tilted Surfaces}

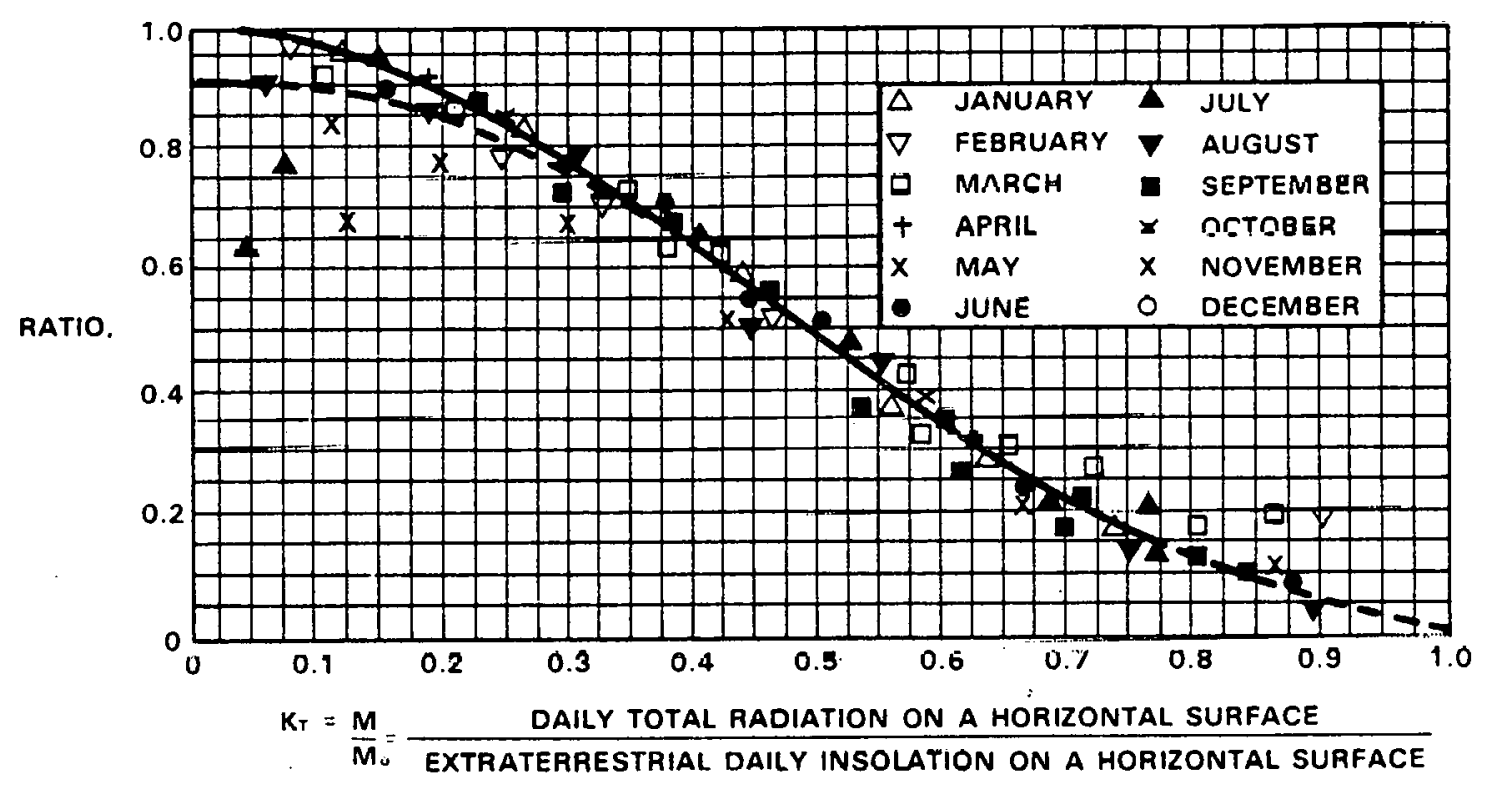

Exhibit 8.22 Ratio of Daily Diffuse Radiation on a Horizontal Surface to the Daily Total Radiation on a Horizontal Surface as a Function of the Cloudiness Index $K_{\mathrm{T}}$. 


\begin{tabular}{|c|c|c|c|c|c|c|c|c|c|c|c|c|}
\hline Letroes & $\boldsymbol{J \Delta I}$ & 78 & an & APE & nat & sur & JvL & $400^{\circ}$ & 28 & oer & nov & ose \\
\hline $\begin{array}{l}25 \\
90 \\
35 \\
60 \\
49 \\
90 \\
93\end{array}$ & $\begin{array}{l}1.05 \\
1.13 \\
1.83 \\
1.34 \\
1.31 \\
1.77 \\
8.26\end{array}$ & $\begin{array}{l}1.06 \\
1.10 \\
1.15 \\
1.22 \\
1.31 \\
1.46 \\
1.19\end{array}$ & $\begin{array}{l}1.03 \\
1.09 \\
1.01 \\
1.11 \\
1.15 \\
1.21 \\
1.29\end{array}$ & $\begin{array}{l}1.00 \\
1.01 \\
1.01 \\
1.02 \\
1.03 \\
1.05 \\
1.08\end{array}$ & 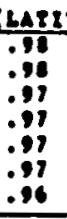 & $\begin{array}{l}08-31 \\
.19 \\
.17 \\
.96 \\
.95 \\
.96 \\
.93 \\
.93\end{array}$ & 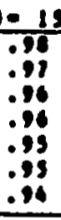 & $\begin{array}{l}.99 \\
.99 \\
1.00 \\
1.00 \\
1.00 \\
1.01 \\
1.02\end{array}$ & $\begin{array}{l}1.02 \\
1.03 \\
1.09 \\
1.08 \\
1.10 \\
1.14 \\
1.11\end{array}$ & $\begin{array}{l}1.05 \\
1.08 \\
1.18 \\
1.10 \\
1.25 \\
1.39 \\
1.30\end{array}$ & $\begin{array}{l}1.08 \\
1.13 \\
1.80 \\
1.30 \\
1.43 \\
1.67 \\
8.04\end{array}$ & $\begin{array}{l}1.05 \\
1.16 \\
1.25 \\
1.38 \\
1.38 \\
1.91 \\
2.33\end{array}$ \\
\hline $\begin{array}{l}25 \\
30 \\
35 \\
10 \\
15 \\
30 \\
35\end{array}$ & $\begin{array}{l}1.87 \\
1.24 \\
1.33 \\
1.64 \\
1.65 \\
1.96 \\
2.31\end{array}$ & $\begin{array}{l}0.11 \\
1.13 \\
1.20 \\
1.27 \\
1.37 \\
1.32 \\
1.15\end{array}$ & $\begin{array}{l}1.06 \\
1.05 \\
1.08 \\
1.11 \\
1.113 \\
1.21 \\
1.29\end{array}$ & $\begin{array}{l}.91 \\
.97 \\
.81 \\
.98 \\
.19 \\
1.00 \\
1.01\end{array}$ & $\begin{array}{l}4421 \\
.93 \\
.98 \\
.91 \\
.19 \\
.90 \\
.89 \\
.05\end{array}$ & $\begin{array}{r}28-1 \\
.81 \\
.10 \\
.89 \\
.87 \\
.84 \\
.85 \\
.84\end{array}$ & $\begin{array}{l}-10 \\
18 \\
.11 \\
.89 \\
.89 \\
.81 \\
.87 \\
.86\end{array}$ & $\begin{array}{l}.95 \\
.95 \\
.95 \\
.14 \\
.94 \\
.95 \\
.95\end{array}$ & $\begin{array}{l}1.01 \\
1.02 \\
1.03 \\
1.05 \\
1.08 \\
1.11 \\
1.16\end{array}$ & $\begin{array}{l}1.00 \\
1.11 \\
1.16 \\
1.21 \\
1.28 \\
1.10 \\
1.54\end{array}$ & $\begin{array}{l}1.16 \\
1.21 \\
1.28 \\
1.41 \\
1.51 \\
1.82 \\
2.25\end{array}$ & $\begin{array}{l}1.19 \\
1.11 \\
1.38 \\
1.53 \\
1.76 \\
2.14 \\
2.00\end{array}$ \\
\hline $\begin{array}{l}25 \\
30 \\
35 \\
40 \\
13 \\
30 \\
39\end{array}$ & $\begin{array}{l}T .71 \\
1.38 \\
1.37 \\
1.31 \\
1.71 \\
2.08 \\
2.63\end{array}$ & $\begin{array}{l}1.11 \\
1.15 \\
1.20 \\
1.27 \\
1.37 \\
1.32 \\
1.85\end{array}$ & $\begin{array}{l}1.00 \\
1.01 \\
1.03 \\
1.06 \\
1.20 \\
1.13 \\
1.23\end{array}$ & $\begin{array}{l}911 \\
.90 \\
980 \\
.90 \\
90 \\
918 \\
.91\end{array}$ & $\begin{array}{l}\text { (LAS1 } \\
.11 \\
.83 \\
.81 \\
.81 \\
.00 \\
.79 \\
.18\end{array}$ & $\begin{array}{c}08-71 \\
.81 \\
.80 \\
.79 \\
.77 \\
.18 \\
.14 \\
.13\end{array}$ & $\begin{array}{l}0-19 \\
781 \\
.81 \\
.80 \\
.78 \\
.87 \\
.76 \\
.75\end{array}$ & $\begin{array}{l}.16 \\
.87 \\
.86 \\
.16 \\
.85 \\
.85 \\
.83\end{array}$ & $\begin{array}{l}.86 \\
.87 \\
.81 \\
.99 \\
1.01 \\
1.06 \\
1.00\end{array}$ & $\begin{array}{l}1.07 \\
1.10 \\
1.14 \\
1.11 \\
1.28 \\
1.38 \\
1.54\end{array}$ & $\begin{array}{l}1.24 \\
1.38 \\
1.44 \\
1.31 \\
1.89 \\
1.39\end{array}$ & $\begin{array}{l}1.25 \\
1.32 \\
1.43 \\
1.00 \\
1.84 \\
2.86 \\
3.09\end{array}$ \\
\hline $\begin{array}{l}25 \\
90 \\
39 \\
40 \\
43 \\
90 \\
93\end{array}$ & $\begin{array}{l}.96 \\
1.04 \\
1.18 \\
1.33 \\
1.38 \\
1.193 \\
2.35\end{array}$ & $\begin{array}{l}.11 \\
.13 \\
.86 \\
1.04 \\
1.18 \\
1.36 \\
1.42\end{array}$ & $\begin{array}{l}.68 \\
.67 \\
.72 \\
.78 \\
.96 \\
.95 \\
1.06\end{array}$ & $\begin{array}{l}.75 \\
.92 \\
.35 \\
.35 \\
.64 \\
.61 \\
.14\end{array}$ & $\begin{array}{l}.65 \\
.48 \\
.47 \\
.30 \\
.93 \\
.30 \\
.60\end{array}$ & $\begin{array}{l}\text { erica } \\
.46 \\
.42 \\
.44 \\
.47 \\
.49 \\
.32 \\
.35\end{array}$ & $\begin{array}{l}.61 \\
.43 \\
.45 \\
.48 \\
.31 \\
.36 \\
.38\end{array}$ & $\begin{array}{l}.65 \\
.48 \\
.51 \\
.53 \\
.51 \\
.63 \\
.67\end{array}$ & $\begin{array}{l}.35 \\
.69 \\
.65 \\
.10 \\
.76 \\
.82 \\
.81\end{array}$ & $\begin{array}{l}.73 \\
.79 \\
.18 \\
.85 \\
1.08 \\
1.20 \\
1.00\end{array}$ & $\begin{array}{l}.70 \\
.91 \\
1.10 \\
1.23 \\
1.45 \\
1.75 \\
2.84\end{array}$ & $\begin{array}{l}.17 \\
1.10 \\
1.84 \\
1.46 \\
1.78 \\
2.17 \\
3.00\end{array}$ \\
\hline
\end{tabular}

$\bar{K}_{T}=0.30$

Exhibit 8.23 Values of $\bar{R}$ for $\bar{K}_{r}$ 
VALUES OF $\overline{\mathbf{R}}$ FOR $\bar{K}_{\mathbf{T}}=\mathbf{0 . 4 0}$

\begin{tabular}{|c|c|c|c|c|c|c|c|c|c|c|c|c|}
\hline LATITUDE & jaI & 188 & mar & $\Delta P E$ & Mat & Jua & 302 & $\triangle U G$ & set & oct & nor & Dec \\
\hline $\begin{array}{l}35 \\
30 \\
39 \\
40 \\
43 \\
30 \\
35\end{array}$ & $\begin{array}{l}1.11 \\
1.20 \\
1.31 \\
1.46 \\
1.69 \\
2.06 \\
2.68\end{array}$ & $\begin{array}{l}1.04 \\
1.13 \\
1.20 \\
1.30 \\
1.63 \\
1.61 \\
1.09\end{array}$ & $\begin{array}{l}1.04 \\
1.08 \\
1.11 \\
1.19 \\
1.21 \\
1.30 \\
1.41\end{array}$ & $\begin{array}{l}1.01 \\
1.01 \\
1.03 \\
1.04 \\
1.06 \\
1.09 \\
1.12\end{array}$ & 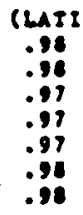 & $\begin{array}{c}02-811 \\
.91 \\
.96 \\
.95 \\
.94 \\
.94 \\
.96 \\
.93\end{array}$ & $\begin{array}{l}013 \\
.98 \\
.97 \\
.96 \\
.96 \\
.93 \\
.93 \\
.93\end{array}$ & $\begin{array}{l}0 \\
1.00 \\
1.00 \\
1.00 \\
1.01 \\
1.02 \\
1.00 \\
1.00\end{array}$ & $\begin{array}{l}1.03 \\
1.05 \\
1.07 \\
1.20 \\
1.15 \\
1.20 \\
1.28\end{array}$ & $\begin{array}{l}1.07 \\
1.11 \\
1.17 \\
1.25 \\
1.15 \\
1.69 \\
1.30\end{array}$ & $\begin{array}{l}1.10 \\
1.10 \\
1.20 \\
1.41 \\
1.61 \\
1.90 \\
2.61\end{array}$ & $\begin{array}{l}1.23 \\
1.22 \\
1.36 \\
1.32 \\
1.79 \\
2.22 \\
3.06\end{array}$ \\
\hline $\begin{array}{l}25 \\
30 \\
35 \\
40 \\
45 \\
90 \\
35\end{array}$ & $\begin{array}{l}1.24 \\
1.34 \\
1.46 \\
1.46 \\
1.90 \\
2.32 \\
3.03\end{array}$ & $\begin{array}{l}1.15 \\
1.21 \\
1.29 \\
1.39 \\
1.39 \\
1.36 \\
2.04\end{array}$ & $\begin{array}{l}1.06 \\
1.08 \\
1.13 \\
1.19 \\
1.23 \\
1.32 \\
1.43\end{array}$ & $\begin{array}{l}.98 \\
.96 \\
.99 \\
1.00 \\
1.02 \\
1.00 \\
1.09\end{array}$ & $\begin{array}{l}\text { L472 } \\
.92 \\
.91 \\
.91 \\
.90 \\
.90 \\
.90 \\
.90\end{array}$ & $\begin{array}{l}05-711 \\
.90 \\
.80 \\
.87 \\
.86 \\
.86 \\
.85 \\
.84\end{array}$ & $\begin{array}{l}0 \\
.91 \\
.90 \\
.89 \\
.81 \\
.81 \\
.87 \\
.87\end{array}$ & 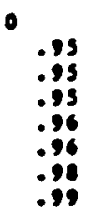 & $\begin{array}{l}1.03 \\
1.06 \\
1.07 \\
1.20 \\
1.16 \\
1.19 \\
1.27\end{array}$ & $\begin{array}{l}1.12 \\
1.17 \\
1.23 \\
1.31 \\
1.62 \\
1.98 \\
1.80\end{array}$ & $\begin{array}{r}1.22 \\
1.30 \\
1.61 \\
1.37 \\
1.39 \\
2.13 \\
2.71 .\end{array}$ & $\begin{array}{l}1.27 \\
1.38 \\
1.32 \\
1.73 \\
2.06 \\
2.36 \\
3.54\end{array}$ \\
\hline $\begin{array}{l}25 \\
30 \\
39 \\
40 \\
45 \\
90 \\
35\end{array}$ & $\begin{array}{l}1.31 \\
1.41 \\
1.36 \\
1.73 \\
2.01 \\
2.65 \\
3.26\end{array}$ & $\begin{array}{l}1.87 \\
1.25 \\
1.31 \\
1.61 \\
1.96 \\
1.77 \\
2.09\end{array}$ & $\begin{array}{l}1.02 \\
1.06 \\
1.09 \\
1.13 \\
1.19 \\
1.27 \\
1.39\end{array}$ & $\begin{array}{l}.11 \\
.91 \\
91 \\
912 \\
93 \\
99 \\
918\end{array}$ & $\begin{array}{l}\text { Ch81 } \\
.92 \\
.81 \\
.80 \\
.80 \\
.79 \\
.79 \\
.79\end{array}$ & $\begin{array}{l}08-781 \\
.78 \\
.77 \\
.76 \\
.79 \\
.76 \\
.73 \\
.72\end{array}$ & $\begin{array}{l}10-15 \\
.10 \\
.78 \\
.78 \\
.78 \\
.76 \\
.18\end{array}$ & $\begin{array}{l}.189 \\
.186 \\
.86 \\
.86 \\
.87 \\
.88 \\
.89\end{array}$ & $\begin{array}{l}190 \\
.98 \\
1.01 \\
1.06 \\
1.08 \\
1.13 \\
1.19\end{array}$ & $\begin{array}{l}1.12 \\
1.17 \\
1.23 \\
1.31 \\
1.42 \\
1.30 \\
1.81\end{array}$ & $\begin{array}{l}1.27 \\
1.36 \\
1.69 \\
1.64 \\
1.87 \\
2.24 \\
2.83\end{array}$ & $\begin{array}{l}1.35 \\
1.46 \\
1: 62 \\
1: 84 \\
2.10 \\
2.70 \\
3.80\end{array}$ \\
\hline $\begin{array}{l}23 \\
30 \\
39 \\
40 \\
35 \\
30 \\
35\end{array}$ & $\begin{array}{l}1.05 \\
1.18 \\
1.35 \\
1.31 \\
1.88 \\
2.36 \\
3.18\end{array}$ & $\begin{array}{l}.86 \\
.96 \\
1.05 \\
1.18 \\
1.36 \\
1.60 \\
1.99\end{array}$ & $\begin{array}{l}.63 \\
.69 \\
.78 \\
.86 \\
.96 \\
1.06 \\
1.21\end{array}$ & $\begin{array}{l}.46 \\
.49 \\
.96 \\
.98 \\
.69 \\
.18\end{array}$ & $\begin{array}{l}.36 \\
.39 \\
.49 \\
.47 \\
.38 \\
.35 \\
.60\end{array}$ & $\begin{array}{l}28 \pi c a 1 \\
.34 \\
.36 \\
.39 \\
.62 \\
.66 \\
.30 \\
.34\end{array}$ & $\begin{array}{l}.35 \\
.37 \\
.11 \\
.46 \\
.18 \\
.38 \\
.36\end{array}$ & $\begin{array}{l}.40 \\
046 \\
09 \\
.53 \\
.98 \\
.63 \\
.69\end{array}$ & $\begin{array}{r}.34 \\
.60 \\
.86 \\
.83 \\
.81 \\
.80 \\
1.00\end{array}$ & $\begin{array}{l}.77 \\
.85 \\
.98 \\
1.06 \\
1.21 \\
1.40 \\
1.66\end{array}$ & $\begin{array}{l}.99 \\
1.11 \\
1.16 \\
1.46 \\
1.73 \\
2.12 \\
8.76\end{array}$ & $\begin{array}{l}2.12 \\
1.26 \\
1.49 \\
1.71 \\
2.08 \\
2.68 \\
3.70\end{array}$ \\
\hline
\end{tabular}

VALUES OF $\overline{\mathbf{R}}$ FOR $\overline{\mathbf{K}}_{\mathbf{T}}=\mathbf{0 . 5 0}$

\begin{tabular}{|c|c|c|c|c|c|c|c|c|c|c|c|c|}
\hline LATISUOS & $\boldsymbol{J \Delta U}$ & 18: & nat & Ara & mat & J4: & 206 & AUE & ET & ams & nop & ase \\
\hline $\begin{array}{l}23 \\
30 \\
33 \\
60 \\
43 \\
30 \\
35\end{array}$ & $\begin{array}{l}1.16 \\
1.25 \\
1.37 \\
1.35 \\
1.82 \\
2.26 \\
2.98\end{array}$ & $\begin{array}{l}1.91 \\
1.16 \\
1.26 \\
1.36 \\
1.51 \\
1.73 \\
3.06\end{array}$ & $\begin{array}{l}1.05 \\
1.08 \\
1.13 \\
1.19 \\
1.26 \\
1.36 \\
1.30\end{array}$ & $\begin{array}{l}1.01 \\
1.02 \\
1.03 \\
1.05 \\
1.08 \\
1.12 \\
1.16\end{array}$ & $\begin{array}{c}\text { (2458 } \\
198 \\
.97 \\
.97 \\
.97 \\
988 \\
1998\end{array}$ & $\begin{array}{l}08=7 \\
.17 \\
.96 \\
.93 \\
.94 \\
.94 \\
.94 \\
.94\end{array}$ & $\begin{array}{l}101 \\
.17 \\
.96 \\
.96 \\
.96 \\
.96 \\
.96\end{array}$ & $\begin{array}{l}0 \\
1.00 \\
1.00 \\
1.01 \\
1.02 \\
1.03 \\
1.06 \\
1.08\end{array}$ & $\begin{array}{l}1.05 \\
1.06 \\
1.09 \\
1.13 \\
1.18 \\
1.25 \\
1.34\end{array}$ & $\begin{array}{l}1.04 \\
1.13 \\
1.20 \\
1.30 \\
1.42 \\
1.39 \\
1.83\end{array}$ & $\begin{array}{l}1.18 \\
1.81 \\
1.33 \\
1.49 \\
1.92 \\
2.08 \\
8.67\end{array}$ & $\begin{array}{l}1.13 \\
1.20 \\
1.41 \\
1.13 \\
1.93 \\
2.43 \\
3.44\end{array}$ \\
\hline $\begin{array}{l}25 \\
30 \\
35 \\
40 \\
45 \\
30 \\
35\end{array}$ & $\begin{array}{l}1.29 \\
1.40 \\
2.36 \\
1.79 \\
2.08 \\
2.57 \\
3.04\end{array}$ & $\begin{array}{l}1.19 \\
1.26 \\
1.35 \\
1.48 \\
1.45 \\
1.89 \\
2.26\end{array}$ & $\begin{array}{l}1.08 \\
1.11 \\
1.16 \\
1.22 \\
1.30 \\
1.10 \\
1.34\end{array}$ & $\begin{array}{l}.98 \\
.99 \\
1.08 \\
1.02 \\
1.04 \\
1.08 \\
1.12\end{array}$ & $\begin{array}{l}\text { L478 } \\
.91 \\
.91 \\
.90 \\
.90 \\
.90 \\
.91 \\
.92\end{array}$ & $\begin{array}{l}08-71 \\
.88 \\
.87 \\
.86 \\
.86 \\
.85 \\
.85 \\
.85\end{array}$ & $\begin{array}{l}.90 \\
.89 \\
.88 \\
.88 \\
.87 \\
.87 \\
.88\end{array}$ & $\begin{array}{l}.75 \\
.15 \\
.78 \\
.97 \\
.98 \\
1.90 \\
1.02\end{array}$ & $\begin{array}{l}1.06 \\
1.06 \\
1.09 \\
1.13 \\
1.14 \\
1.25 \\
1.34\end{array}$ & $\begin{array}{l}1.13 \\
1.21 \\
1.28 \\
1.38 \\
1.32 \\
1.70 \\
1.92\end{array}$ & $\begin{array}{l}1.26 \\
1.36 \\
1.90 \\
1.68 \\
1.95 \\
3.36 \\
3.04\end{array}$ & $\begin{array}{l}1.37 \\
1.65 \\
1.13 \\
1.67 \\
2.25 \\
2.65 \\
6.02\end{array}$ \\
\hline $\begin{array}{l}23 \\
30 \\
35 \\
40 \\
45 \\
90 \\
39\end{array}$ & $\begin{array}{l}1.38 \\
1.30 \\
1.68 \\
1.81 \\
2.82 \\
2.73 \\
3.68\end{array}$ & $\begin{array}{l}1.22 \\
1.29 \\
1.39 \\
1.32 \\
1.35 \\
1.94 \\
2.32\end{array}$ & $\begin{array}{l}1.05 \\
1.98 \\
1.13 \\
1.19 \\
1.20 \\
1.30 \\
1.30\end{array}$ & $\begin{array}{r}.91 \\
.91 \\
.92 \\
.94 \\
.96 \\
.98 \\
1.98\end{array}$ & $\begin{array}{c}645 \\
.81 \\
.80 \\
.80 \\
.79 \\
.79 \\
.79 \\
.00\end{array}$ & $\begin{array}{l}.817 \\
.77 \\
.76 \\
.75 \\
.74 \\
.73 \\
.73 \\
.78\end{array}$ & $\begin{array}{l}10-18 \\
.79 \\
.74 \\
.77 \\
.76 \\
.76 \\
.76 \\
.79\end{array}$ & $\begin{array}{l}.86 \\
.86 \\
.86 \\
.81 \\
.88 \\
.81\end{array}$ & $\begin{array}{r}.99 \\
1.01 \\
1.04 \\
1.08 \\
1.12 \\
1.19 \\
1.87\end{array}$ & $\begin{array}{l}1.10 \\
1.22 \\
1.30 \\
1.60 \\
1.33 \\
1.78 \\
1.99\end{array}$ & $\begin{array}{l}1.33 \\
1.44 \\
1.58 \\
1.78 \\
2.06 \\
2.48 \\
3.28\end{array}$ & $\begin{array}{l}1.43 \\
1.57 \\
1.73 \\
2.08 \\
2.43 \\
3.09 \\
4.34\end{array}$ \\
\hline $\begin{array}{l}25 \\
30 \\
35 \\
40\end{array}$ & $\begin{array}{l}1.13 \\
1.29 \\
1.68 \\
1.74\end{array}$ & $\begin{array}{l}.09 \\
1.00 \\
1.13 \\
1.29\end{array}$ & $\begin{array}{l}.63 \\
.71 \\
.79 \\
\div 89\end{array}$ & .53 & $\begin{array}{l}.32 \\
.35 \\
.48 \\
.46\end{array}$ & $\begin{array}{l}18 x e d \\
.39 \\
.38 \\
.35 \\
.39\end{array}$ & $\begin{array}{r}.30 \\
.33 \\
.37 \\
.48\end{array}$ & $\begin{array}{l}.37 \\
.41 \\
.47 \\
.32\end{array}$ & $\begin{array}{l}.53 \\
.68 \\
.67 \\
.79\end{array}$ & $\begin{array}{r}.89 \\
.89 \\
1.01 \\
1.16\end{array}$ & $\begin{array}{l}1.00 \\
1.20 \\
1.30 \\
1.61\end{array}$ & $\begin{array}{l}1.21 \\
1.38 \\
1.50 \\
1.91\end{array}$ \\
\hline
\end{tabular}


VALUES OF $\overline{\mathrm{R}}$ FOR $\overline{\mathrm{K}}_{\mathrm{T}}=\mathbf{0 . 6 0}$

\begin{tabular}{|c|c|c|c|c|c|c|c|c|c|c|c|c|}
\hline 10.817008 & SAC & Tes & man & Ape & mar & sun & JOL & AUE & sep & oCs & nor & DeE \\
\hline $\begin{array}{l}25 \\
30 \\
35 \\
40 \\
43 \\
50 \\
35\end{array}$ & $\begin{array}{l}1.15 \\
1.27 \\
1.61 \\
1.62 \\
1.92 \\
2.60 \\
3.24\end{array}$ & $\begin{array}{l}1.11 \\
1.18 \\
1.28 \\
1.11 \\
1.38 \\
1.83 \\
2.20\end{array}$ & $\begin{array}{l}1.06 \\
1.10 \\
1.15 \\
1.21 \\
1.30 \\
1.11 \\
1.51\end{array}$ & $\begin{array}{l}1.01 \\
1.02 \\
1.06 \\
1.07 \\
1.10 \\
1.16 \\
1.19\end{array}$ & 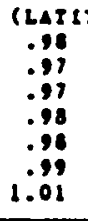 & $\begin{array}{l}98-\pi 11 \\
.96 \\
99 \\
94 \\
94 \\
94 \\
94 \\
94\end{array}$ & $\begin{array}{l}1015 \\
.99 \\
96 \\
96 \\
99 \\
.96 \\
.96\end{array}$ & $\begin{array}{l}1.00 \\
1.00 \\
1.01 \\
1.08 \\
1.04 \\
1.07 \\
1.10\end{array}$ & $\begin{array}{l}1.04 \\
1.01 \\
1.10 \\
1.15 \\
1.21 \\
1.29 \\
1.39\end{array}$ & $\begin{array}{l}1.09 \\
1.19 \\
1.23 \\
1.34 \\
1.60 \\
1.67 \\
1.95\end{array}$ & $\begin{array}{l}1.14 \\
1.24 \\
1.38 \\
1.36 \\
1.22 \\
2.22 \\
2.89\end{array}$ & $\begin{array}{l}1.18 \\
1.29 \\
1.46 \\
1.70 \\
2.03 \\
2.64 \\
3.13\end{array}$ \\
\hline $\begin{array}{l}25 \\
30 \\
35 \\
40 \\
43 \\
30 \\
35\end{array}$ & $\begin{array}{l}1.33 \\
1.46 \\
1.63 \\
1.88 \\
2.23 \\
2.18 \\
3.16\end{array}$ & $\begin{array}{l}1.21 \\
1.21 \\
1.30 \\
1.40 \\
1.35 \\
1.84 \\
2.02 \\
2.43\end{array}$ & $\begin{array}{l}1.09 \\
1.13 \\
1.19 \\
1.26 \\
1.35 \\
1.48 \\
1.63\end{array}$ & $\begin{array}{l}.98 \\
.99 \\
1.01 \\
1.03 \\
1.06 \\
1.010 \\
1.15\end{array}$ & $\begin{array}{l}\text { (LAT12 } \\
.91 \\
.90 \\
90 \\
990 \\
911 \\
.92 \\
.91\end{array}$ & $\begin{array}{l}98-714 \\
.89 \\
.86 \\
.85 \\
.85 \\
.89 \\
.89 \\
.89\end{array}$ & $\begin{array}{l}1 . \\
.89 \\
.88 \\
.81 \\
.81 \\
.81 \\
.86\end{array}$ & $\begin{array}{c}.95 \\
.95 \\
.86 \\
.97 \\
.98 \\
1.02 \\
1.09\end{array}$ & $\begin{array}{l}1.05 \\
1.08 \\
1.11 \\
1.16 \\
1.22 \\
1.30 \\
1.41\end{array}$ & $\begin{array}{l}1.17 \\
1.26 \\
1.33 \\
1.04 \\
1.39 \\
1.81 \\
2.12\end{array}$ & $\begin{array}{l}1.30 \\
1.42 \\
1.31 \\
1.78 \\
2.08 \\
2.34 \\
3.31\end{array}$ & $\begin{array}{l}1.31 \\
1.31 \\
1.11 \\
1.99 \\
2.41 \\
3.10 \\
4.41\end{array}$ \\
\hline $\begin{array}{l}25 \\
30 \\
35 \\
40 \\
45 \\
30 \\
35\end{array}$ & $\begin{array}{l}1.43 \\
1.97 \\
1.76 \\
2.02 \\
2.40 \\
2.99 \\
4.04\end{array}$ & $\begin{array}{l}1.26 \\
1.34 \\
1.49 \\
1.00 \\
1.80 \\
2.09 \\
2.32\end{array}$ & $\begin{array}{l}1.01 \\
1.11 \\
1.16 \\
1.23 \\
1.32 \\
1.46 \\
1.35\end{array}$ & $\begin{array}{l}.91 \\
.92 \\
.93 \\
.93 \\
.98 \\
1.01 \\
1.05\end{array}$ & $\begin{array}{l}\text { (LAII } \\
.80 \\
.78 \\
.79 \\
.79 \\
.78 \\
.80 \\
.81\end{array}$ & $\begin{array}{l}08-712 \\
.73 \\
.14 \\
.73 \\
.73 \\
.72 \\
.72 \\
.72\end{array}$ & $\begin{array}{l}10-13 \\
.17 \\
.76 \\
.76 \\
.75 \\
.75 \\
.75 \\
.76\end{array}$ & $\begin{array}{l}.86 \\
.86 \\
.86 \\
.89 \\
.98 \\
.93\end{array}$ & $\begin{array}{l}1.00 \\
1.03 \\
1.06 \\
1.11 \\
1.16 \\
1.24 \\
1.34\end{array}$ & $\begin{array}{l}1.19 \\
1.28 \\
1.35 \\
1.49 \\
1.62 \\
1.14 \\
8.15\end{array}$ & $\begin{array}{l}1.39 \\
1.31 \\
1.67 \\
1.90 \\
2.22 \\
2.71 \\
3.32\end{array}$ & $\begin{array}{l}1.49 \\
1.69 \\
1.86 \\
2.19 \\
2.63 \\
3.37 \\
4.98\end{array}$ \\
\hline $\begin{array}{l}25 \\
30 \\
35 \\
40 \\
45 \\
30 \\
35\end{array}$ & $\begin{array}{l}1.20 \\
1.37 \\
1.39 \\
1.81 \\
2.30 \\
2.93 \\
4.01\end{array}$ & $\begin{array}{l}.92 \\
1.04 \\
1.19 \\
1.37 \\
1.64 \\
1.93 \\
2.39\end{array}$ & $\begin{array}{l}.63 \\
.78 \\
: 11 \\
.82 \\
1.05 \\
1.21 \\
1.61\end{array}$ & $\begin{array}{l}.39 \\
.46 \\
.52 \\
.39 \\
.96 \\
.84\end{array}$ & $\begin{array}{l}.28 \\
.32 \\
.37 \\
.42 \\
.48 \\
.36 \\
.60\end{array}$ & $\begin{array}{l}83 \mathrm{CAL} \\
.25 \\
.28 \\
.32 \\
.37 \\
.62 \\
.197 \\
.32\end{array}$ & $\begin{array}{l}.26 \\
.30 \\
.36 \\
.39 \\
.66 \\
.30 \\
.35\end{array}$ & $\begin{array}{l}.34 \\
.39 \\
.69 \\
.31 \\
.38 \\
.69 \\
.32\end{array}$ & $\begin{array}{r}.33 \\
.60 \\
.68 \\
.77 \\
.87 \\
.99 \\
1.13\end{array}$ & $\begin{array}{l}.12 \\
.93 \\
1.06 \\
1.21 \\
1.40 \\
1.65 \\
2.00\end{array}$ & $\begin{array}{l}1.12 \\
1.28 \\
1.48 \\
1.73 \\
2.08 \\
2.61 \\
3.46\end{array}$ & $\begin{array}{l}1.28 \\
1.48 \\
1.73 \\
2.07 \\
8.56 \\
3.34 \\
4.80\end{array}$ \\
\hline
\end{tabular}

VALUES OF $\bar{R}$ FOR $\bar{K}_{T}=0.70$

\begin{tabular}{|c|c|c|c|c|c|c|c|c|c|c|c|c|}
\hline GATITUDS & 3Ax & ers & An & ARE & Mr & jun & sol & $\triangle 06$ & $\mathbf{s e p}$ & oct & $\operatorname{nov}$ & Des \\
\hline $\begin{array}{l}25 \\
30 \\
35 \\
60 \\
45 \\
50 \\
53\end{array}$ & $\begin{array}{l}1.17 \\
1.30 \\
1.46 \\
1.69 \\
2.03 \\
2.36 \\
3.50\end{array}$ & $\begin{array}{l}1.12 \\
1.20 \\
1.31 \\
1.45 \\
1.65 \\
1.93 \\
2.34\end{array}$ & $\begin{array}{l}1.06 \\
1.11 \\
1.17 \\
1.24 \\
1.34 \\
1.47 \\
1.64\end{array}$ & $\begin{array}{l}1.01 \\
1.03 \\
1.05 \\
1.04 \\
1.12 \\
1.16 \\
1.22\end{array}$ & $\begin{array}{c}\text { PLI } \\
.99 \\
.97 \\
.99 \\
.98 \\
.99 \\
1.00 \\
1.02\end{array}$ & $\begin{array}{l}.8-71 \\
.96 \\
.95 \\
.94 \\
.94 \\
.94 \\
.94 \\
.94\end{array}$ & $\begin{array}{l}19 \\
.97 \\
.96 \\
.95 \\
.95 \\
.96 \\
.97 \\
.98\end{array}$ & $\begin{array}{l}1.00 \\
1.00 \\
1.01 \\
1.03 \\
1.06 \\
1.09 \\
1.13\end{array}$ & $\begin{array}{l}1.04 \\
1.07 \\
1.12 \\
1.17 \\
1.26 \\
1.33 \\
1.45\end{array}$ & $\begin{array}{l}1.10 \\
1.18 \\
1.26 \\
1.38 \\
1.33 \\
1.75 \\
2.06\end{array}$ & $\begin{array}{l}1.16 \\
1.27 \\
1.42 \\
1.62 \\
1.192 \\
2.36 \\
3.11\end{array}$ & $\begin{array}{l}1.19 \\
1.33 \\
1.51 \\
1.71 \\
2.18 \\
2.13 \\
1.07\end{array}$ \\
\hline $\begin{array}{l}23 \\
30 \\
35 \\
40 \\
45 \\
50 \\
35\end{array}$ & $\begin{array}{l}1.37 \\
1.92 \\
1.71 \\
1.98 \\
2.38 \\
3.00 \\
6.09\end{array}$ & $\begin{array}{l}1.24 \\
1.34 \\
1.46 \\
1.62 \\
1.84 \\
2.15 \\
2.61\end{array}$ & $\begin{array}{l}1.11 \\
1.16 \\
1.22 \\
1.30 \\
1.40 \\
1.33 \\
1.72\end{array}$ & $\begin{array}{l}.08 \\
1.00 \\
1.02 \\
1.05 \\
1.00 \\
1.13 \\
1.19\end{array}$ & $\begin{array}{l}\text { C612 } \\
.90 \\
.90 \\
.90 \\
.90 \\
.91 \\
.92 \\
.96\end{array}$ & $\begin{array}{l}08-21 \\
=14 \\
.15 \\
.85 \\
.14 \\
.84 \\
.15 \\
.05\end{array}$ & $\begin{array}{l}0 \\
.189 \\
.87 \\
.87 \\
.87 \\
.87 \\
.84 \\
.89\end{array}$ & $\begin{array}{r}.95 \\
.95 \\
.96 \\
.98 \\
1.00 \\
1.03 \\
1.07\end{array}$ & $\begin{array}{l}1.00 \\
1.09 \\
1.13 \\
1.19 \\
1.26 \\
1.35 \\
1.47\end{array}$ & $\begin{array}{l}1.20 \\
1.27 \\
1.37 \\
1.30 \\
1.67 \\
1.91 \\
2.29\end{array}$ & $\begin{array}{l}1.34 \\
1.47 \\
1.64 \\
1.80 \\
2.81 \\
2.73 \\
3.39\end{array}$ & $\begin{array}{l}1.41 \\
1.58 \\
1.80 \\
2.11 \\
8.34 \\
3.39 \\
4.81\end{array}$ \\
\hline $\begin{array}{l}25 \\
30 \\
35 \\
60 \\
45 \\
30 \\
95\end{array}$ & $\begin{array}{l}1.69 \\
1.65 \\
2.06 \\
2.15 \\
2.38 \\
3.24 \\
4.41\end{array}$ & $\begin{array}{l}1.30 \\
1.39 \\
1.32 \\
1.65 \\
1.91 \\
2.23 \\
2.72\end{array}$ & $\begin{array}{l}1.09 \\
1.16 \\
1.20 \\
1.20 \\
1.34 \\
1.32 \\
1.49\end{array}$ & $\begin{array}{r}.91 \\
.82 \\
.94 \\
.96 \\
.99 \\
1.04 \\
1.09\end{array}$ & $\begin{array}{l}\text { (24) } \\
.78 \\
.78 \\
.78 \\
.78 \\
.78 \\
.80 \\
.81\end{array}$ & $\begin{array}{l}2-71 \\
.73 \\
.72 \\
.72 \\
.72 \\
.71 \\
.72 \\
.72\end{array}$ & $\begin{array}{l}0-15 \\
.16 \\
.75 \\
.75 \\
.14 \\
.75 \\
.75 \\
.76\end{array}$ & $\begin{array}{l}.18 \\
.86 \\
.87 \\
.88 \\
.89 \\
.92 \\
.96\end{array}$ & $\begin{array}{l}1.01 \\
1.04 \\
1.09 \\
1.14 \\
1.20 \\
1.29 \\
1.40\end{array}$ & $\begin{array}{l}1.23 \\
1.30 \\
1.31 \\
1.34 \\
1.71 \\
1.96 \\
2.31\end{array}$ & $\begin{array}{l}1.16 \\
1.38 \\
1.86 \\
2.02 \\
2.37 \\
2.92 \\
3.93\end{array}$ & $\begin{array}{l}1.96 \\
1.73 \\
1.98 \\
2.32 \\
2.13 \\
9.65 \\
9.23\end{array}$ \\
\hline $\begin{array}{l}29 \\
30 \\
35 \\
40 \\
45 \\
30 \\
35\end{array}$ & $\begin{array}{l}1.26 \\
1.46 \\
1.90 \\
2.93 \\
2.48 \\
3.19 \\
6.19\end{array}$ & $\begin{array}{l}.06 \\
1.09 \\
1.26 \\
1.96 \\
1.78 \\
8.07 \\
2.59\end{array}$ & $\begin{array}{l}.04 \\
.73 \\
.84 \\
.98 \\
1.10 \\
1.20 \\
1.90\end{array}$ & $\begin{array}{l}.37 \\
.78 \\
.51 \\
.59 \\
.67 \\
.78 \\
.47\end{array}$ & $\begin{array}{l}.35 \\
.29 \\
.35 \\
.10 \\
.47 \\
.53 \\
.60\end{array}$ & $\begin{array}{l}.264 \\
.21 \\
.25 \\
.29 \\
.36 \\
.10 \\
.45 \\
.51\end{array}$ & $\begin{array}{r}.23 \\
.27 \\
.32 \\
.37 \\
.43 \\
.48 \\
.95\end{array}$ & $\begin{array}{l}.31 \\
.37 \\
.43 \\
.30 \\
.37 \\
.45 \\
.74\end{array}$ & $\begin{array}{r}.38 \\
.60 \\
.69 \\
.79 \\
. .90 \\
. .09 \\
1.19\end{array}$ & $\begin{array}{l}.95 \\
.97 \\
1.11 \\
1.28 \\
1.49 \\
1.97 \\
2.15\end{array}$ & $\begin{array}{l}1.16 \\
1.35 \\
1.37 \\
1.86 \\
2.25 \\
8.83 \\
1.70\end{array}$ & $\begin{array}{l}1.34 \\
1.37 \\
1.13 \\
2.23 \\
2.78 \\
3.53 \\
5.27\end{array}$ \\
\hline
\end{tabular}




\subsubsection{COMPUTATIONAL PROCEDURE}

The flux on a tilted surface on any day with clearness $\bar{K}_{T}$ can be computed as follows (see exhibit 8.24).

STEP 1. For month, location, use exhibit 8.16 to obtain $\bar{K}_{T}$ (or choose $K_{T}$ per Exhibit 8.14).

STEP 2. Read $\overline{\mathbf{R}}$ from exhibit 8.24 .

STEP 3. Read E the extraterrestrial flux, from exhibit 8.26.

STEP 4. Compute the flux on the tilted surface from

$$
\mathbf{Q}=K_{\mathbf{T}} \overline{\mathbf{R}} \mathbf{E} \text {. }
$$

Mamphis $50^{\circ}$ tilt

June

1. $\bar{K}_{T}=0.60$

2. $\overline{\mathbf{R}}=0: 73$

3. $E=41.2$

4. $Q=0.60 \times 0.73 \times 41.2=18.05 \mathrm{MJ} / \mathrm{m}^{2}-$ Day

$=18.05 / 3.6=5.01 \mathrm{kWh} / \mathrm{m}^{2}$ Day

\section{Exhibit 8.24 Computational Procedure.}

Step One

For the month and location, determine $R_{T}$ from exhibit 8.15 (using part $A$ for the United States and several cities in Canada and part $B$ for other cities throughout the world) if average conditions are desired $\left(K_{T}=\bar{K}_{T}\right)$. If nonaverage conditions are being anulyzed, refer to exhibit 8.17 or 8.18 . If the world dats on insolation ars used, the flux must be curiverted to $\bar{k}$ by multiplying the tabular values by 0.0481 to convert to $\mathrm{MJ} / \mathrm{m}^{2}$-day and then by dividing by the appropriate extraterrestrial flux as listed in exhibit 8.25.

\begin{tabular}{|c|c|c|c|c|c|c|c|c|c|c|c|c|}
\hline MONTHUDE & JAN & FEB & MAR & APR & MAY & JUN & JUL & AUG & SEP & OCT & NOV & DEC \\
\hline 25 & 23.9 & 28.2 & 33.0 & 37.1 & 39.4 & 40.1 & 39.6 & 37.9 & 34.4 & 29.5 & 24.9 & 22.7 \\
\hline 30 & 21.1 & 25.7 & 31.3 & 36.5 & 39.6 & 40.7 & 40.1 & 37.6 & 33.1 & 27.3 & 22.1 & 19.7 \\
\hline 35 & 18.1 & 23.1 & 29.3 & 35.5 & 39.6 & 41.2 & 40.3 & 37.0 & 31.5 & 24.9 & 19.2 & 16.7 \\
\hline 40 & 15.1 & 20.3 & 27.2 & 34.3 & 39.3 & 41.4 & 40.3 & 36.2 & 29.7 & 22.3 & 16.3 & 13.6 \\
\hline 45 & 12.0 & 17.5 & 24.8 & 32.8 & 38.8 & 41.3 & 40.0 & 35.1 & 27.7 & 19.6 & 13.3 & 10.0 \\
\hline 50 & 9.0 & 14.5 & 22.3 & 31.2 & 38.1 & 41.2 & 39.6 & 33.8 & 25.4 & 16.7 & 10.3 & 7.0 \\
\hline 55 & 6.1 & 11.5 & 19.5 & 29.3 & 37.2 & 40.9 & 39.1 & 32.4 & 23.0 & 13.8 & 7.3 & 4.0 \\
\hline
\end{tabular}

Exhibit 8.25 Monthly Average Daily Extraterrestrial Radiation M]/m²-Day. 
Step Two

Obtain the value of $\bar{R}$, the ratio of flux on the tilted surface to the flux on the horizontal, trom exhibit 8.23.

Step Three

Determine the extraterrestrial flux on a horizontal surface from exhibit 8.25 .

Step Four

Determine the flux on the tilted surface from the equation on exhibit $8.24, Q=K_{T} \overrightarrow{R E}$, where $E$ is the insolation outside the atmosphere (extroterrestrial).

The flux so computed is the daily flux, $M J / \mathrm{m}^{2}$ per day. If $\mathrm{K}_{\mathrm{T}}$ were chosen randomly, then the frequency plot (exhibit 8.17 or 8.18 ) could be used to determine the frequency of the value of $K_{T}$ and thence, the frequency of the value of the corresponding insolation, $Q$. $A$ plot of frequency versus flux will give sufficient statistical dato to compute the average-day performance and the frequency of non-overoge occurrences.

\subsubsection{COMPUTATION OF INSOLATION ON THE AVERAGE DAY}

The use of the computational procedure to obtain the insolation on an array tilted at latitude plus $15^{\circ}$ for an average day in June in Memphis, Tennessee, will be illustrated. From exhibit 8.15 the value of $\vec{K}_{T}$ is 0.60 for June. From exhibit 8.23 , for June, at the latitude of Memphis $\left(35^{\circ}\right)$, with the array. tilted at $50^{\circ}, \bar{R}$ is equal to 0.73 . Latitude is the angular distance, measured in degrees, north or south from the equator. Also for June at this latitude, the extraterrestrial insolation, $E$, is found on exhibit 8.25 to be $41.2 \mathrm{MJ} / \mathrm{m}^{2}-$ day. Therefore the daily insolation on the array is

$$
\begin{aligned}
& Q=\bar{K}_{T} \bar{R} E, \\
= & 0.60 \times 0.73 \times 41.2, \\
= & 18.05 \mathrm{MJ} / \mathrm{m}^{2}-\text { day, }
\end{aligned}
$$

or in more useful units of $\mathrm{kWh} / \mathrm{m}^{2}-$ day,

$$
\begin{aligned}
Q & =18.05 \times 10^{6} / 3,600,000 . \\
& =5.01 \mathrm{kWh} / \mathrm{m}^{2}-\text { day. }
\end{aligned}
$$

\subsubsection{COMPUTATION OF AVERAGE INSOLATION}

Exhibit 8.24 illustrates the use of the insolation-computation procedure when the average insolation is needed, rather than the insolation on the average day, as illustrated above.

The computational procedure will be illustrated for Fresno, California (latitude = $36.5^{\circ}$ ) in December. We wish to determine the frequency distribution for the flux on a solar array tilted at the latitude angle above the horizontal and facing south. 
For $K_{T}=0.1$, the insolation is 97-percent diffuse, as indicated in exhibit 9.22 , so $R$ is equal to $F$.

For convenience, one estimates the values of $R$ by interpolation/extrapolation to be 1.17 at $K_{T}=0.2$ and 1.97 at $K_{T}=0.9$. To improve on this estimate, an hour-by-hour computation would be necessary.

From exhibit 8.25 (step C), the extraterrestrial flux is calculated by interpolation, with the result shown in exhibit ${ }^{2} .2^{2}$.

$$
\begin{aligned}
E & =16.7+\frac{36.5-35}{40-35}(13.6-16.7) \\
& =17.6 \mathrm{MJ} / \mathrm{m}^{2} \text {-doy }
\end{aligned}
$$

\section{Exhibit 8.28 Extraterrestrial Insolation Calculation.}

Step $D$ now can be used to compute the insolation versus frequency, as shown in exhibit $2.2^{n}$.

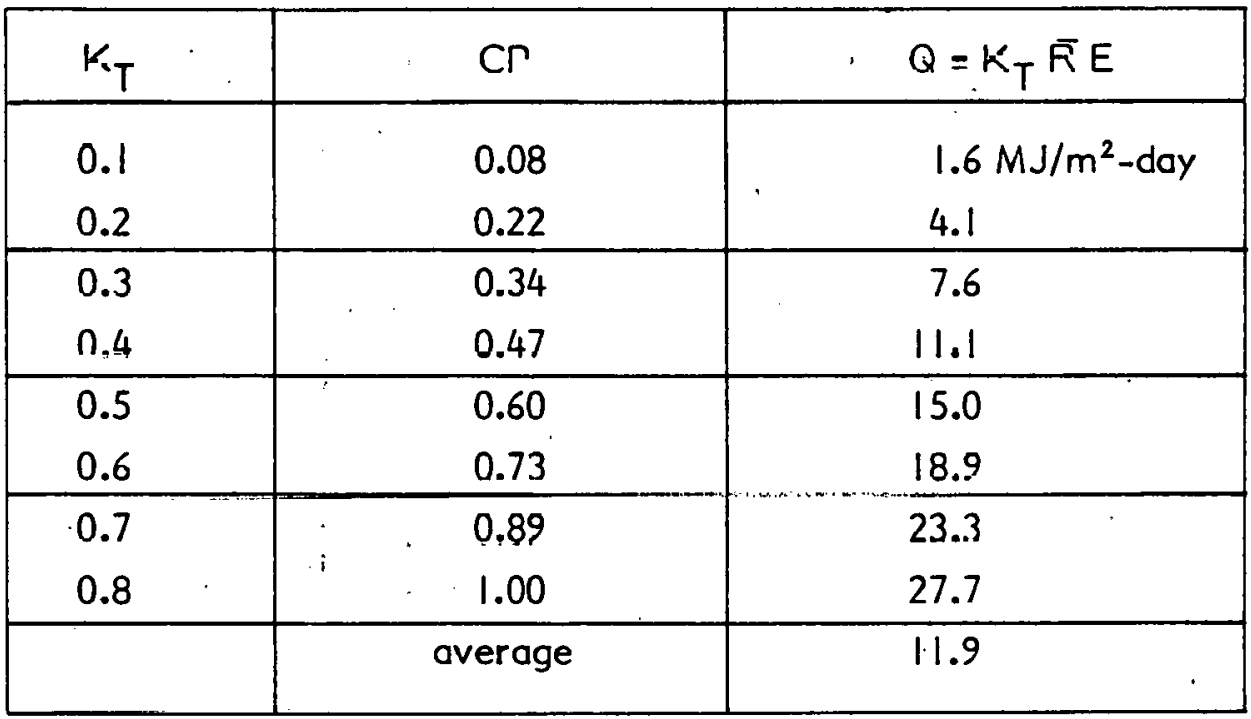

Exhibit 8.29 Flux (Q) on the Tilted Surface.

The median day $(C P=n 5)$ gives $Q=12.0 \mathrm{MJ} / \mathrm{m}^{2}$-day. The average day $\left(\mathbb{K}_{T}=\right.$ $0.42)$ gives $Q=11$. i iJ/m2-day. If the system was designed for the average day $\left(K_{T}=\right.$ 0.42 , $C P=0.46$ ), the probability of falling short of solar power for $m$ days consecutively would be $0.496^{\mathrm{m}}$, or

\begin{tabular}{|c|c|c|c|c|c|c|c|}
\hline $\mathrm{m:}$ & 1 & 2 & 3 & 4 & 5 & 6 & 10 \\
\hline Probability: & 0.496 & 0.246 & 0.122 & 0.060 & 0.030 & 0.015 & 0.001 \\
\hline
\end{tabular}

Exhibit 8.30 Probability of Having $m$ Consecutive Days with $K_{r}$ Less Than 0.42. 
This table does not indicate the number of days of battery storage required, because no indication is given regarding how much above $0.42 \mathrm{~K}$ the. insolation will be on the clearer days. The storage requirements must be computed from an analysis of insolation under extreme conditions. The next section contains the data for designing the storage system.

\subsubsection{CONCLUSION}

The computation of insolation is unavoidable. Although it must be performed only once for each site, it must be performed for three or four tilt angles so the optimum tilt can be determined, as described next. The step-by-step procedure of section 6 above should at least reduce the time required for the computations.

\subsection{MONTHLY PERFORMANCE COMPUTATION}

The apparent digression concerning insolation was necessary so the system input could be computed. The electrical-system performance can be calculated. In accordance with the simplified computation of the section on "Quick Sizing of Components," a first estimate of the required array size was obtained. In the example, $14.67 \mathrm{~m}^{2}$ of arroy was needed to feed the 6.17 percent efficient system. Although the previous section presents only typical insolation computations, it is assumed that the insolation had been computed in detail and the results were as illustrated in exhibit 8.31, column A, (a computation sheet that makes the work of the preliminary system-performance analysis easier). It is also assumed that the load had been computed as tabulated in column $C$.

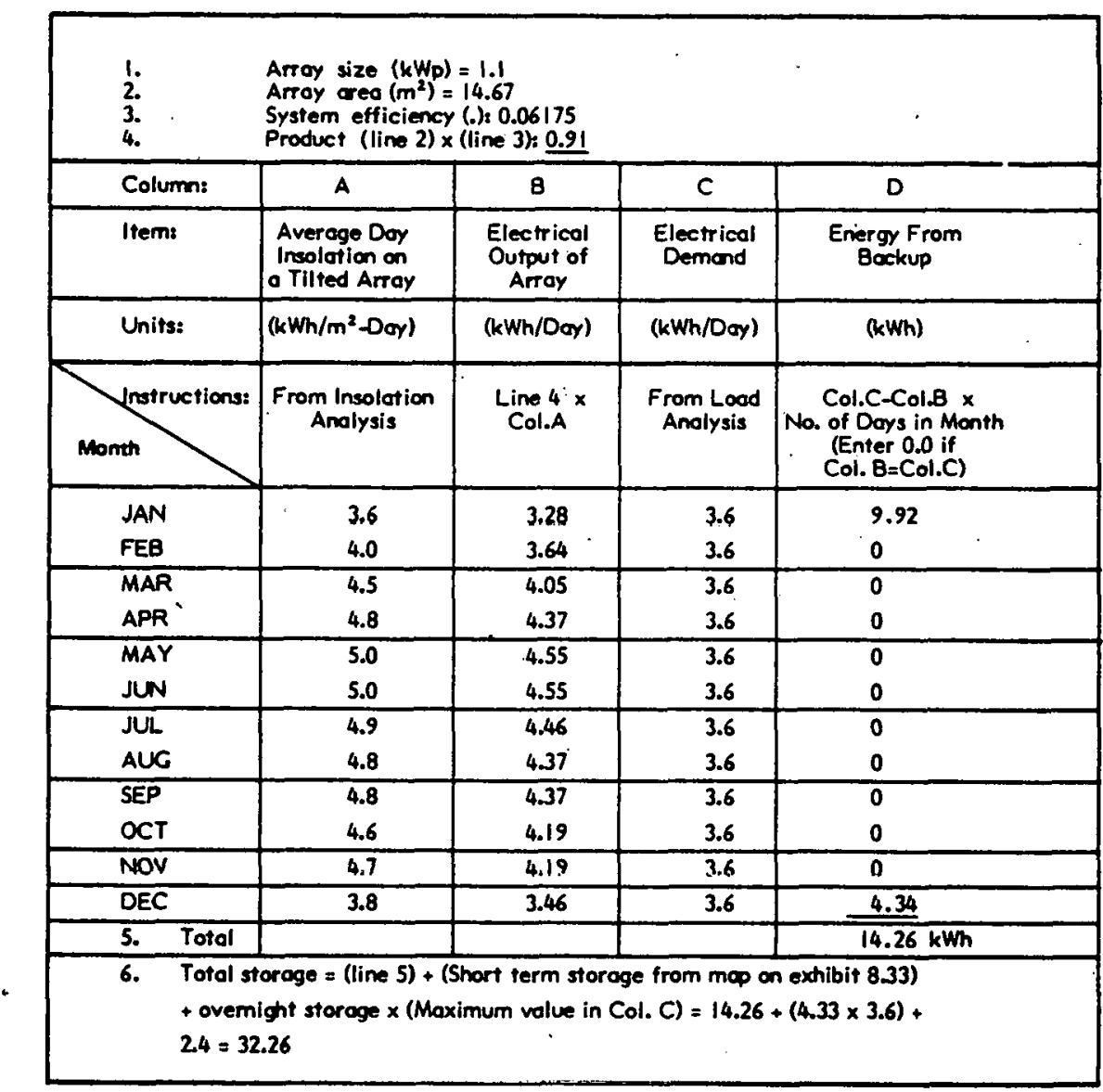

\section{Exhibit 8.31 Computation of System Performance.}


The computation sheet is to be used to determine the performance of a system with given arroy size. The calculated array size is entered on the first and second lines and the estimated system efficiency on the third. The product of system efficiency and collector area is entered on line 4. The monthly insolation on the tilted surface, as computed by the procedures described in the previous section, is entered in column A of the table. The electrical demand, as computed by the procedures in section 6 of this seminar manual, is entered in column C. The output of the PV system is computed in column B by multiplying the insolation by the system efficiency and the collector area (line 4). (The difference between column $B$ and column $C$ represents the amount of energy that must be supplied by the batteries, or by an auxiliary power source.) If column B exceeds column $C$, zero is entered in column D for that month; otherwise, column $C$ minus column $B$ times the number of days in the month is entered in column $D$.

As indicated on line 5 of exhibit 8.31, the amount of long-term storage rcquircd, either in terms of fuel or batteries, is the sum of the values in column D. The long-term stnrnge is required to overcome seosonal differences in insolation and system demands. In addition to the long-term storage, some allowance must be made for the short-term storage requirements to compensate for consecutive days without sunshine. Exhibit ${ }^{n} .32$ has been prepared to indicate the short-term storage needs. The short-term needs have been computed based both on direct weather (insolation) data and on weather simulations. As indicated on line 6, the total storage requirement is the sum of the long-term and short-term needs. The shortterm need will almost always be supplied by the batteries; the long-term, by either botteries or a backup system.

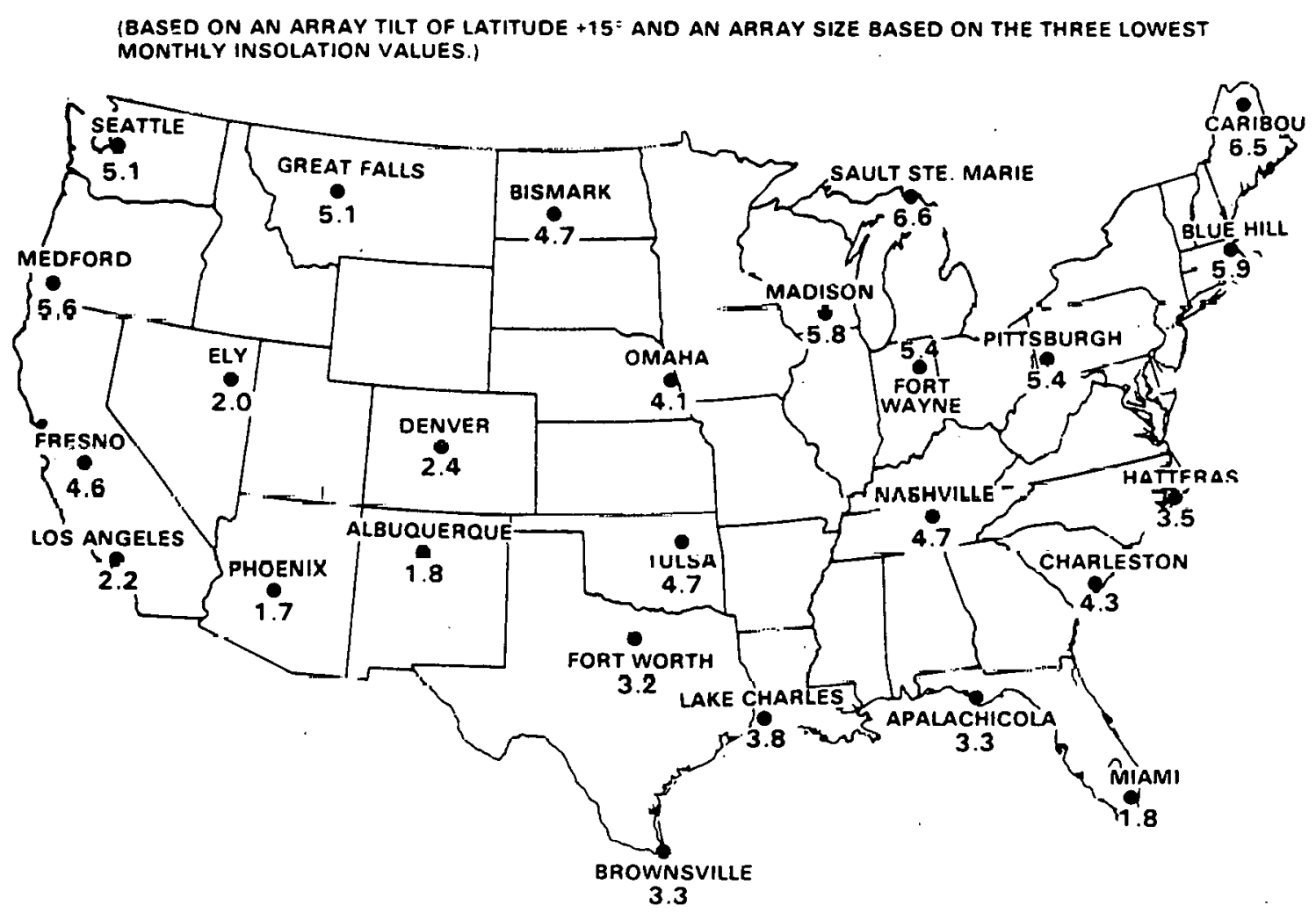

Exhibit 8.32 Short Term Storage Requirements for Selected Cities. 
The sample case, in which a collector area of $14.67 \mathrm{~m}^{2}$ was required, has been continued in the example illustrated on figure 8.31. On line 6 for this collector area and tilt angle, the batteries must be sized to deliver $32.26 \mathrm{kWh}$. Since the collector area and the battery size are both based on useful capacities, some adjustment is required to allow for system degradation, allowable depth of discharge, etc., as described in the next section and tobulated in exhibit 8.33 .

\begin{tabular}{|l|c|c|c|}
\hline \multicolumn{1}{|c|}{ COMPONENT } & Nominal Size & Adjustment Foctor & Size \\
\hline Array: & $1.10 \mathrm{kWp}$ & $\div 0.8$ for degradation & $1.38 \mathrm{kWp}$ \\
\hline Battery: & $32.26 \mathrm{kWh}$ & $\begin{array}{c}\div 0.6 \text { for depth of } \\
\text { discharge }\end{array}$ & $53.77 \mathrm{kWh}$ \\
\hline Regulator: & $12 \mathrm{~V}, 92 \mathrm{~A}$ & $\begin{array}{l}\div 0.8 \text { for array } \\
\text { degradatlon }\end{array}$ & $12 \mathrm{~V}, 115 \mathrm{~A}$ \\
\hline Tronsmission Lines: & $12 \mathrm{~V}, 92 \mathrm{~A}$ & $\begin{array}{c}0.8 \text { for array } \\
\text { degradation }\end{array}$ & $12 \mathrm{~V}, 115 \mathrm{~A}$ \\
\hline
\end{tabular}

\section{Exhibit 8.33 Component Sizes.}

\subsection{COMPONENT SIZES}

Once the operating sizes of the array and storage system have been computed, all the components of the PV system can be sized. The necessary array size has already been calculated, but must be adjusted to allow for degradation with time (see exhibit 8.33). The sizing of the battery system for charge will equal the storage energy required ( $k W h$ ), divided by the system voltage (V), divided by the allowed percent discharge level of the batteries. Energy divided by voltage equals charge, where one coulomb of charge equals one joule of energy divided by one volt. Kwh can be converted to joules and Ah can be converted to coulombs. The allowed discharge of the battery depends on the type of batteries to be used. For example, lead-acid-cadmium grid batteries should be discharged only 40 percent of their rated copacity, so the amount of battery storage required is equal to the calculated requirement divided by 0.4 .

If 20-percent degradation is allowed for the solar array over its lifetime, the orray output initially will be $1 / 0.8=1.25$ times its required output.

The regulator should be sized to handle the maximum power from the array under noon insolation on a clear day. The sea-level insolation under such conditions is approximately $1 \mathrm{~kW} / \mathrm{m}^{2}$, down from the $1.35 \mathrm{~kW} / \mathrm{m}^{2}$ in outer space due to the atmospheric attenuation. Therefore, in the installation year, when the efficiencies are highest, the power transmitted through the regulator could be as high as the product: $1 \mathrm{Kw} / \mathrm{m}^{2} \times 1.25 \times$ array efficiency $\times$ array area with the product having the units of $\mathrm{kW}$. Therefore, the regulator in the example problem should be sized for $1 \times 1.25 \times 0.075 \times 14.67=1.38 \mathrm{~kW}$ of power.

The transmission lines should be sized also to handle the noon load on a clear day $(1.38 \mathrm{~kW} / 12 \mathrm{~V}=115 \mathrm{~A})$, where $P / V=1$, and one $k W$ equals $1000 \times$ one volt $x$ one amp.

A DC/AC inverter, if used, must supply the peak load, so the maximum power through the inverter would be dictated by the peak power demand of the load. If the peak power input to the inverter could be the same as the peak power output of the array, the inverter size would be determined in the same way as the size of the regulator, as discüssed above (II5 A). 


\subsection{DESIGN OPTIMIZATION}

What has been calculated, for a given array tilt angle, are the necessary array size and storage requirements to meet the load demands. An optimization methodology must include. the costs of these components, as well as the other system components, to determine the minimum-cost system.

Exhibit 8.34 presents a form for the computation of life cycle cost. The cost of the various devices must be determined from the vendors. Typical prices and the names of several vendors are provided in section 9 of this manual. The engineering estimates of installation costs must be based on the site conditions. The factors for estimating the maintenance and operating costs are indicated in exhibit 8.34 and represent the best estimates available to date for long-term annual costs. The bottom line (line 20) is the life cycle cost of a PV system with the calculated collector area and collector tilt.

One ean now alter the angle uf lilie uriuy by $\pm 5^{\circ}$ frurn the prevlous tilt angle issed. This will result in different insolation values, a new array size, and a different battery storage requirement. The life cycle cost should be computed for each iteration and compared with the previous results. Ennugh tilt angles and array sizes must he evaluated so that the combination yielding the minimum life cycle cost will be established.

A full consideration of life cycle costing and design optimization is beyond the scope of this seminar and will be considered in subsequent seminars.

\begin{tabular}{|c|c|c|c|}
\hline \multicolumn{4}{|c|}{ Component Sizes } \\
\hline $\begin{array}{l}\text { I. } \\
2 . \\
3 . \\
4 .\end{array}$ & $\begin{array}{l}\text { PV arroy: nominal area } * \text { degradation foctor } \\
\text { Power conditioner from orray: } \\
\text { Battery size: } \\
\text { Power conditioner to loads }\end{array}$ & & $\begin{array}{l}\frac{18.34}{1.38} \mathrm{k} \mathrm{m}^{2} \\
\frac{80.7}{\mathrm{k} W h} \\
=\mathrm{kWp}\end{array}$ \\
\hline \multicolumn{3}{|c|}{ Component Costs } & Spares \\
\hline $\begin{array}{l}5 . \\
6 . \\
7 . \\
8 . \\
9 . \\
10 .\end{array}$ & $\begin{array}{l}\text { PV Ponels } \\
\text { Slructure } \\
\text { Power conditioner for arroy } \\
\text { Butlery } \\
\text { Power conditioner to lood } \\
\text { Monitoring system (telemetry) }\end{array}$ & $\frac{\frac{15,525}{320}}{\frac{800}{8,100}} \frac{-}{-100}$ & $\frac{775}{\frac{-1}{800}} \frac{}{810}$ \\
\hline II. & Total components & 25,245 & 2,386 \\
\hline $\begin{array}{l}12 . \\
13 . \\
14 .\end{array}$ & $\begin{array}{l}\text { Engineering } \\
\text { Installation } \\
\text { Project Management }\end{array}$ & $\frac{2,500}{1,000}$ & 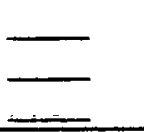 \\
\hline 15. & $\begin{array}{l}\text { Total First Costs } \\
\text { (sum Lines } 110,116,12,13,14,15)\end{array}$ & 31.031 & \\
\hline 16. & \multicolumn{3}{|c|}{$\begin{array}{l}\text { Annug Sosts } \\
\text { Maintenance }=1.78 \times(0.01 \times \text { Line } 5+0.02 \times \text { Line } 7 \times \text { Line } 8+\text { Line } 9\end{array}$} \\
\hline $\begin{array}{l}17 . \\
18 .\end{array}$ & $\begin{array}{l}\text { Replacements (bosed on } 7 \text { percent inflation o } \\
\text { Battery } \\
\text { Power conditioners }\end{array}$ & $\begin{array}{l}\text { cent return } \\
\underline{5,670} \\
\end{array}$ & tment) \\
\hline 19. & Total & 5,670 & \\
\hline 20. & Life cycle cost $=$ Line $15+$ Line $19+8.514 \times$ & 42,324 & \\
\hline
\end{tabular}

Exhibit 8.34 Life Cycle Cost Computation. 


\section{References:}

1. Shellard, H.C., communication to the authors.

2. Dubief, J., "Le Climat Du Sähara," 43, 44 Alger (1959).

3. Lewis, B.M., communication to the authors.

4. Dubief, J., "Le Climat Du Sahara," 55, Alger (1959).

5. WMO Records of Solar Radiation for IGY and IGC.

6. Soares de Azeudeo, M., communication to the authors.

7. Boletim Actinometrico de Portugal, Ano I-V, Lisboa.

8. Morse, R.N., communication with authors.

9. Director of Meterology, Australian Dept. of the Interior, communication with authors.

10. Simmer, R.G., Acting Director, New Zealand Meterological Service, communication to authors.

11. Fritz, S., "Solar Radiation Measurements in the Artic Ocean," Polar Atmosphere Symposium, Oslo, 159 (New York 1958).

12. Reed, W.W., Supplement No. 32, Monthly Weather Review, Washington, 1929.

13. Bonython; C.W.. , communication to the authors.

14. W.B. Rimmer and C.W. Allen, "Solar Radiation Observations on Mount Stromlo 19271939, "Memoirs of the Commonwealth Observatory, Mt. Stromlo, No. II, 1950.

15. Collmann, W., "Diagramme zum Strahlungsklima Europos," Berichte des Deutschen Wetterdienstes, Band $\underline{6}$, No. 42 (1958).

16. Reed, W.W., Monthly Weather Review, Band $\underline{6}$, Supplement No. 31, Washington 1928.

- 17. Teixeira, T.R., communication to the authors.

18. Bahli, F., communication with authors.

19. United States Department of Commerce Weather Bureau, 1950-1961, Climatological Data, National Summary.

20. Duffie and Beckinan, Solar Design by the F-Chart Method, Wiley 1978. 


\section{THIS PAGE \\ WAS INTENTIONALLY \\ LEFT BLANK}

5

$8-42$ 


\subsection{INTRODUCTION}

In this section, the characteristics of typical available components and subsystems are presented in terms of efficiency, control, price, and availability. The data have been taken from manufacturers' or suppliers' literature. The specifications of several designs are compared in each case, so the reader can recognize the correlation between price and performance and determine the reasonableness of various performance requirements.

\subsection{AVAILABLE COMPONENTS}

A brief survey of manufacturers' and standard catalogs reveals the availability and costs of the major components for solar PV power systems. The results of a survey conducted in preparation for this seminar are presented in exhibits 9.1 to 9.8. For typical systems under $1.5 \mathrm{kWp}$ array sizes, there are components ovailable off the shelf (within approximately 16 weeks). The price range is high because many of the components, designed to service laboratory instruments or computers, have more stringent requirements than would normally prevail for solar PV power systems. (A discussion of the requirements for various potential loads can be found in section 6, Lood Analysis.) Therefore, selection of components must be related to the system design. For example, some of the less expensive power conditioners are more efficient becouse they do less power conditioning; however, the higher efficiency is accompanied by a less regulated output, resulting in less efficient operation of the load. The combined efficiency of the power conditioner and the load, therefore, must be considered in performing the subsystem trodeoffs.

An individual discussion will be provided for each of the components. The data are arranged in the order that the components appear in the system, starting with the solar array.

\subsection{SOLAR PANELS}

V-I Dato were obtained from representative solar panel manufacturers listed by GSA. The data are summarized in exhibits 9.1 and 9.2. Exhibit 9.1 illustrates the combinations of currents and voltages available in modules that can be ordered from the GSA list. Exhibit 9.2 summarizes typical specifications. Note from exhibit 9.2 that the costs are all approximately $\$ 16.5 / \mathrm{W}$ of peak power. Panel sizes have not been standardized; however, most manufacturers supply their own structures, so standardization is not important.

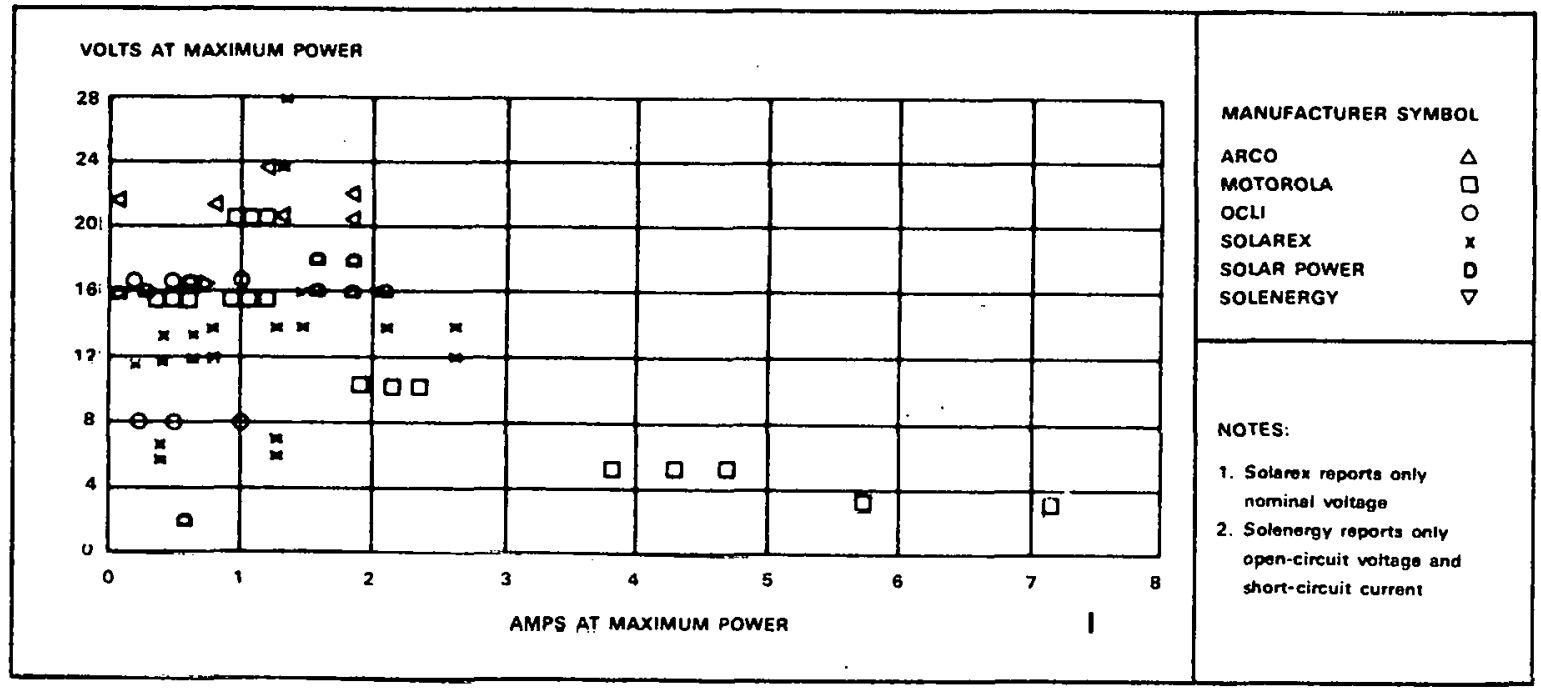

Exhibit 9.1 GSA-Listed PV Modules as of 11/1/78. 


\begin{tabular}{|c|c|c|c|c|c|c|c|}
\hline $\begin{array}{l}\text { WMUUfAC } \\
\text { SOARER }\end{array}$ & MOTOROLA & MOTOROLA & ARCO & SOLAR POWER & SOLAA POWEA & SOLAAEX & SOLAREX \\
\hline Model & MSPO1E30 & MSPÓ'AंA30 & ASI16.1200 & P.1012 & M12-381 & 435 & HB.506 \\
\hline Priea! & 8414.555 & $3414-555$ & $3225 \cdot 268$ & 354.60 & $8390-485$ & $595-179$ & $5494-936$ \\
\hline Delivery & 30 davs & 30 dovs & $30-60$ doys & $30-60$ days & $30-60$ devs & 90 days & 90 days \\
\hline Efficioncy ${ }^{2}$ & $7.4 \%$ & $7.4 \%$ & $7.8 \%$ & $5.3 \%$ & $6.8 \%$ & $7.9 \%$ & $11.2 \%$ \\
\hline $\begin{array}{l}\text { Outpre } \\
W \\
v \\
\text { Protection } \\
T_{\mathrm{c}}-T_{\mathrm{V}} \\
\text { Power Tomp. } \\
\text { Coefficient }\end{array}$ & $\begin{array}{l}25.3 \\
3.52 \\
\text { No } \\
20^{\circ} \mathrm{C} \\
-0.5 \% /{ }^{\circ} \mathrm{C}\end{array}$ & $\begin{array}{l}25.3 \\
21.10 \\
\text { No } \\
20^{\circ} \mathrm{C} \\
-0.5 \% /{ }^{\circ} \mathrm{C}\end{array}$ & $\begin{array}{l}19.4 \\
16.2 \\
\text { No }\end{array}$ & \begin{tabular}{|l|}
1.4 \\
16.5 \\
Diode
\end{tabular} & $\begin{array}{l}33.5 \\
16.5 \\
\text { Diode }\end{array}$ & $\begin{array}{l}6.6 \\
16.0 \\
\text { Optionsl }\end{array}$ & $\begin{array}{l}\cdot \\
35.0 \\
28 \\
\text { Optional }\end{array}$ \\
\hline 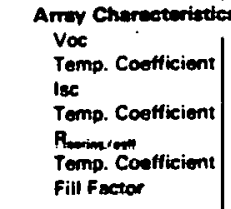 & $\begin{array}{l}4.50 \\
-0.4 \% /^{\circ} \mathrm{C} \\
7.8 \\
+0.08 \% /^{\circ} \mathrm{C} \\
0.047 \mathrm{nhm} \\
0.72\end{array}$ & $\mid \begin{array}{l}27.0 \\
-0.4 \% / 0 \mathrm{c} \\
1.3 \\
+0.08 \% \%^{\circ} \mathrm{c} \\
0.047 \mathrm{ohm} \\
0.72\end{array}$ & $\begin{array}{l}20.9 \\
-0.4 \% \\
1.3 \\
0.0 \% \\
0.064 \text { onnı } \\
0.71\end{array}$ & 1 & $\begin{array}{l}21.0 \\
-0.4 \% \\
2.05 \\
0.032 \mathrm{ohm} \\
0.72\end{array}$ & $\mid \begin{array}{l}20.0 \\
-0.4 \% \\
0.45 \\
+0.1 \% \\
0.115 \mathrm{ohm} \\
0.7 \mathrm{~J}\end{array}$ & $\left\{\begin{array}{l}36 \\
-0.4 \% \\
1.42 \\
+0.4 \% \\
0.096 \mathrm{chm} \\
0.68\end{array}\right.$ \\
\hline \multicolumn{8}{|l|}{ MTRF } \\
\hline $\begin{array}{l}\text { Po. of Colls } \\
\text { Dimensions } \\
\text { Cover } \\
\text { Woight } \\
\text { Ambient Temp. Lim } \\
\text { Insulstion } \\
\text { Mar. Snow Losd } \\
\text { Max. Wind Loed }\end{array}$ & $\begin{array}{l}6 P \times 850 \\
23^{*} \times 23^{\circ} \\
\times 2^{n \prime} . \\
\text { Gloss } \\
12 \mathrm{tb} \\
\text { it }-40^{\circ} \mathrm{C} \text { to } \\
+80^{\circ} \mathrm{C} \\
600 \mathrm{Wdc} \\
60 \mathrm{lb} / \mathrm{H}^{2} \\
100 \mathrm{mph}\end{array}$ & $\begin{array}{c}1 P \times 4850 \\
23^{*} \times 23^{*} \\
\times 22^{*} \\
\text { Glass } \\
12 \mathrm{lb} \\
-40^{\circ} \mathrm{C} \text { to } \\
+80^{\circ} \mathrm{C} \\
600 \mathrm{vdc} \\
60 \mathrm{lb} / \mathrm{h}^{2} \\
100 \mathrm{mph}\end{array}$ & $\begin{array}{l}1 P \times 3650 \\
9^{\prime \prime} \times 43^{\circ} \\
\times 1.4^{\prime \prime} \\
\text { Glass } \\
7.3 \mathrm{lb} \\
-40^{\circ} \mathrm{C} \text { to } \\
+90^{\circ} \mathrm{C}\end{array}$ & $\begin{array}{l}13.6^{\prime \prime} \times 3^{\prime \prime} \\
\times 0.3^{\prime \prime} \\
\text { Polvearbonate } \\
0.616\end{array}$ & $\begin{array}{l}1 P \times 36 S 0 \\
46^{*} \times 15.3^{*} \\
\times 2^{\circ} \\
\text { Siticon } \\
13 \mathrm{tb} . \\
-55^{\circ} \mathrm{C} \text { to } \\
+60^{\circ} \mathrm{C} \\
175 \mathrm{mph}\end{array}$ & $\begin{array}{l}1 \mathrm{P} \times 365 \\
10.4^{\prime \prime} \\
\times 12.5^{\prime \prime} \\
\text { Silicon } \\
-70^{\circ} \mathrm{C} 10 \\
+70^{\circ} \mathrm{C} \\
175 \mathrm{mph}\end{array}$ & $\begin{array}{l}1 P \times 64 S \\
21^{*} \times 24^{*} \\
\text { Glass } \\
12 \mathrm{Ib} \\
-40^{\circ} \mathrm{C} \text { to } \\
+100^{\circ} \mathrm{C} \\
1.500 \mathrm{vdc} \\
260 \mathrm{mph}\end{array}$ \\
\hline Dual Leeds & Yes & Yas & Yos: & Yes & Yes & & Yes \\
\hline \multicolumn{8}{|l|}{ Intermediate Top } \\
\hline GSA Listed & Yes & Yos & Yros & Yos & Yes & 790 & Yes \\
\hline \multicolumn{8}{|l|}{ Pessed DPL Teat } \\
\hline $8 W^{\prime}$ & $376.4-621.9$ & (56.4.321.9 & sti.6-313.7 & $338.6-342.9$ & \$12.6-615.2 & & काय.3.826.7 \\
\hline
\end{tabular}

- Rame on GSA List

2 Based on grose fromal areo

At $\mathrm{t} \mathrm{iW} / \mathrm{m}^{2} .25^{\circ} \mathrm{C}$ ambient

\section{Exhibit 9.2 Comparison of Typical Specifications for PV Modules}

The open-circuit voltage ( $\left.V_{n}\right)$, short-circuit current (lsc), and series resistance (Rseries) are important in combining arrays (see sections 1 and 2 for a discussion on solarpanel theory). For the same reason, the temperature coefficients are important. Arrays matched at one temperature may not be matched at another. As indicated in exhibit 9.2, the reported temperature coefficients are all approximately 0.4 percent per ${ }^{\circ} \mathrm{C}$. The temperature coefficient gives the change in electrir power at a particular temperuture.

Some arrays come with dual leads from each cell, module, panel and subarray. If one should fail, the other will suffice. None of the GSA-listed arrays come with an intermediate tap that would permit the use of a partial shunt regulator. The partial-shunt regulator is described in Section 5, Power Conditioning.

The NASA Jet Propulsiọn Laboratory (JPL), California Institute of Technology has purchased models from many manufacturers and has subjected them to extensive life and performance testing. The specifications in the second part of this section are based on, but are less severe than, the .JPI specifications and test' conditions. Although many manufacturers have submitted panels for JPL testing, few of the production models have been evaluated. In total, there are approximately II manufacturers from whom modules can be bought off-the-shelf. 


\subsection{REGULATORS}

Data were obtained from solar cell manufacturers on the DC regulators offered with the panels. The data that should be specified are listed in exhibit 9.3. Costs will be on the order of $\$ 1 / W$. The use of regulators is shown in Sections 1.2 and 5.

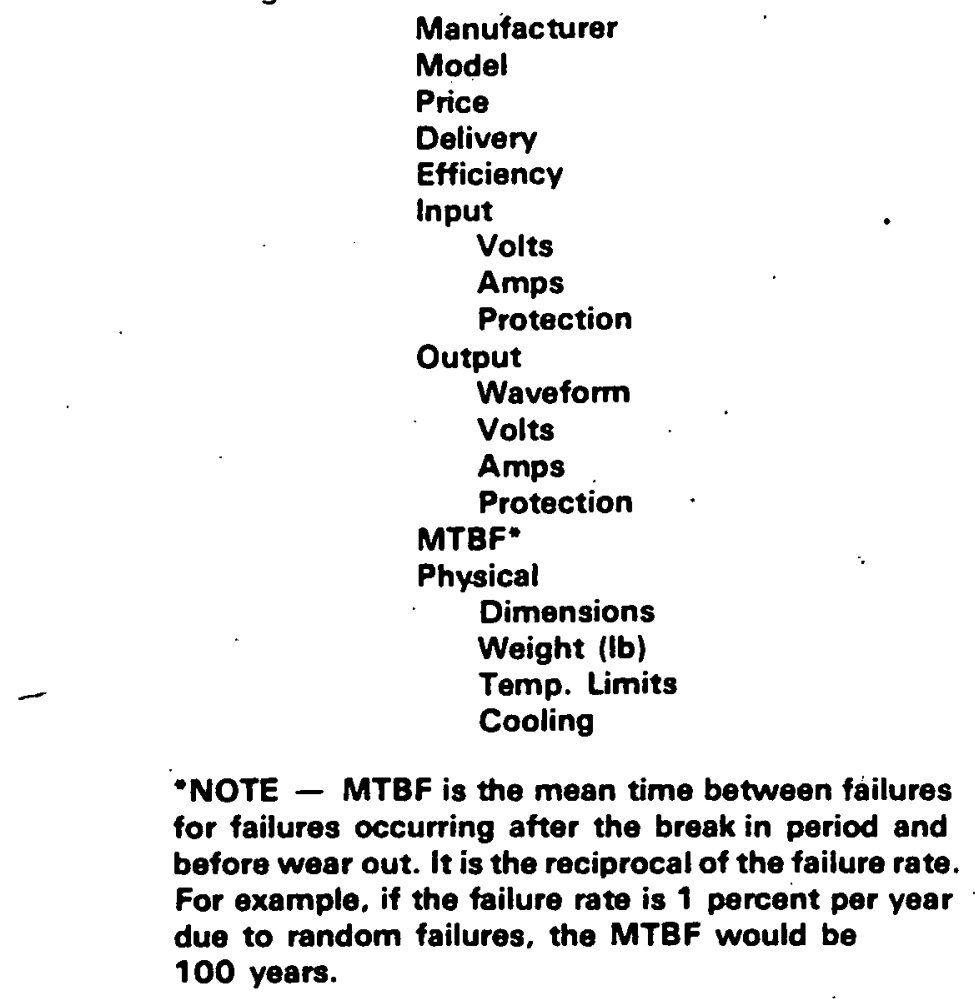

\section{Exhibit 9.3 Specification Items for DC Regulators.}

Normally, DC regulators for PV applications are designed and configured by the array nanufacturer, and are the key components in successful stand-alone systems.

\subsection{BATTERIES}

Both nickel-cadmium and lead-acid battery specifications are tabled in exhibit 9.4. The prime contenders for use with PV systems are the nickel-cadmium, the $C D$, and the DD33 batteries. The cost per $\mathrm{kWh}$ delivered over the life of the battery is lowest for the $C D$; the cost per product of $\mathrm{kWh}$ and the life in years is lowest for nickel-cadmium and DD33. The actual battery size usually is not important, because batteries can be grouped to obtain the desired voltage and current. For very small applications, automotive batteries, sized to prevent more than a 10-percent discharge, might be the most cost effective. Cost per $k W h$ is the energy cost and discharge refers to either $k W h$ or $A H$ (amp-hours), where $k W h e$ is electric energy and AH is charge. The amp-sec is a coulomb of charge, and the $k W e$ is 1000 watts of electric power. The product of $\mathrm{kWhe}$ and the life in years is used for the cost per energy per year.

\subsection{DC/AC INVERTERS}

Exhibits 9.5 and 9.6 list the characteristics of typical DC inverters. If the solar PV system were to supply $A C$ voltage to the load, the inverter would be used. An inverter is approximately 90 percent efficient. Its cost is approximately $\$ 2 / W$ if a sine wave is needed, but only $\$ 0.60 / \mathrm{W}$ if a square wave is acceptable. The use of inverters is shown in Sections 1.2 and 5. 


\begin{tabular}{|c|c|c|c|c|c|c|c|c|}
\hline & \multicolumn{4}{|c|}{ Nickel Cadmium } & \multicolumn{4}{|c|}{ Lead-Acid } \\
\hline Spec & $\mathbf{G}$ & ME. & ME & ME & EL & $C D$ & wI & wI \\
\hline $\begin{array}{l}\text { Model } \\
\text { Price total } \\
\quad \text { S/rated kWh } \\
\text { Delivery } \\
\text { Efficiency }\end{array}$ & $\begin{array}{l}\$ 249 \\
1.383\end{array}$ & $\begin{array}{l}\text { CED } \\
\$ 1.260 \\
273\end{array}$ & $\begin{array}{l}\text { CED. } 79 \\
\$ 360 \\
312\end{array}$ & $\begin{array}{l}\text { CED } .43 \\
\$ 248 \\
448 \\
0715\end{array}$ & $\begin{array}{l}\text { Molive } \\
\text { \$191 } \\
36\end{array}$ & $\begin{array}{l}\text { Calcium } \\
\$ 1.110 \\
192\end{array}$ & $\begin{array}{l}\text { Molive } \\
\$ 934 \\
156\end{array}$ & $\begin{array}{l}\text { Molive } \\
\text { S469 } \\
235\end{array}$ \\
\hline $\begin{array}{l}\text { Inpur (at } 5 \text { hi rate) } \\
\text { a. Charging } \\
\text { Max; volts/cell } \\
\text { Max rate } \\
\text { b. Overcharging } \\
\text { Max volts } \\
\text { Max. current } \\
\end{array}$ & $10 \mathrm{hr}$ & $10 \mathrm{ht}$ & $10 \mathrm{hr}$ & $10 \mathrm{hr}$ & 2.4 & & & \\
\hline $\begin{array}{l}\text { Output lat } 8 \mathrm{hr} \text { rate } \\
\text { kWh } \\
\text { V } \\
\text { Max. current }\end{array}$ & 0.17 & 4.8 & 1.2 & 0.56 & $5.4(20 \mathrm{hr})$ & 54 & $54(6 \mathrm{hr})$ & $1816 \mathrm{nrl}$ \\
\hline $\begin{array}{l}\text { Life } \\
\text { io\% oepth } \\
50 \% \text { depth } \\
\text { 80\% depth } \\
\text { Ehoif }\end{array}$ & $3.00 n$ & $\begin{array}{l}2.900 \\
20, i\end{array}$ & $\begin{array}{l}3,000 \\
20.1 \\
\end{array}$ & \multirow[t]{2}{*}{$\begin{array}{l}2.000 \\
20 \mathrm{yr}\end{array}$} & 250 суй & $1.790^{\circ}$ & $1.250^{\circ}$ & 1.250 \\
\hline \multicolumn{4}{|l|}{ 6olf Disohorgs } & & & & & \\
\hline $\begin{array}{l}\text { Physical } \\
\text { Oimensions } \\
\text { Weight } \\
\text { Temp. Limits } \\
0 \% \text { charge } \\
50 \% \text { charge } \\
100 \% \text { charge }\end{array}$ & $\begin{array}{l}11.5 \mathrm{lb} \\
-40^{\circ} \mathrm{F}\end{array}$ & $\begin{array}{l}22 " .12 " \\
400 \mathrm{lb} \\
.5{ }^{\prime \prime} \\
-40^{\circ} \mathrm{F}\end{array}$ & $\begin{array}{l}15^{\prime \prime} \cdot 10^{\prime \prime} \\
100 \mathrm{lb} \\
-40^{\circ} \mathrm{F}\end{array}$ & $\frac{11 " 9 *}{6313^{\prime \prime}}$ & $21 " \div 15^{\circ}$ & $\begin{array}{l}9^{\prime \prime} \cdot 11^{\prime \prime} \\
\cdot 18^{\prime \prime} \\
70810 \\
-60^{\circ} \mathrm{F}\end{array}$ & $\begin{array}{l}31 \% 8^{*} \\
\cdot 23^{*} \\
546 \text { ib } \\
-60 \mathrm{~F}\end{array}$ & $228 \mathrm{bb}$ \\
\hline siusable kWh & $1.537^{2}$ & $303^{2}$ & $347^{2}$ & 4087 & 44 & 258 & T94 & 293 \\
\hline $\begin{array}{l}\text { Based on } 80 \% \text { disc } \\
\text { Based on } 90 \% \text { dise }\end{array}$ & except as & & & & & . & & \\
\hline
\end{tabular}

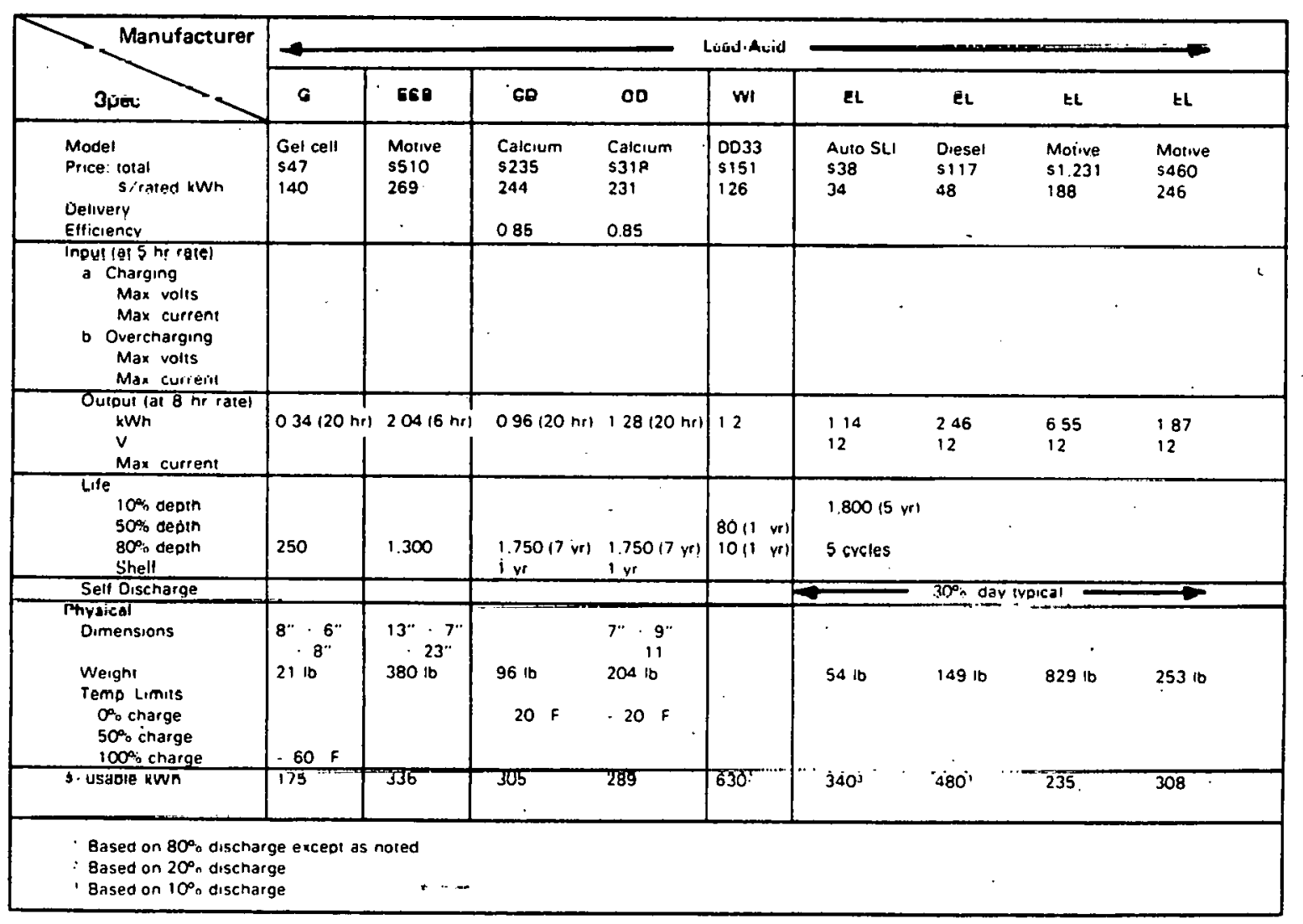

Exhibit 9.4 Specification for Batteries. 


\section{Some manufacturers of self-commutated and line-commutated inverters are (SEIA):}

Abacus Controls. Inc.. Summerville. $\forall . J$. Abacus is finalizing the development of a $10 \mathrm{kw}$. single phase self-commutated inverter under contract to N.AS.A.Lewis. This system was devel. oped for use with photownltaic arrays but is capable of heing used with other sources as well.

The inverter is capable of operating in parallel with a utility line or as a stand-alone svistem. A digitally control. led transistor bridge provides a very" high quality power output with less than $\bar{i} r$ total current harmonic con. tent when interfaced and with less than tre total voltage harmonic distortion when operating in a stand-alone mode. The phase angle of the sutput can be controlled $(=90:)$ to give either a leading or lagging puwer factor. Efficiency is approximately 90 r for nutputs over $25 \%$ with a no-load draw of 250 watts. Input voltage range is 160.240 volts $D r$.

The equipment is presently arailable in small quantitites at a cost of apprnxinately $\$$ lfinolikw capacity includine the photovoltaic interface and an isolattion transformer. Projected crists for a mullerate prodiction level are $5=011 / \mathrm{kw}$

Delta Electronic Control Corp.. Irvine. Calif. Delta produces a line if self-commutated. line-feedinz ar standalnne inverters with rated canacities up to $300 \mathrm{hl}$. Single and three-phase inverters up to : $\mathrm{KV}^{\circ}+$ use transistors for the switching element. Hithor capacity equipment uses a pulse width modulated control concept with silicon controlled rectifiers.

The total voltage harmonic distortion for the transistor model s is less than $3 \%$ when operating in the stand-alone node. The total current harmonic distortion when inter-connected to the utility is also held to less than $3 \%$ Efficieney is typically $90 \%$ for loads in excess of 10\%.

Inverter costs, including an isolation transformer and photovoltaic input filter. range from approximately $\$ 1.000 /$ hit capacity at the $10 \mathrm{KV} d$ level and $\$ 20 n / K i t$ at the $30 n \mathrm{Kl}$ A level.

Exide-Power Systems Division. Rale.itrh. L.C. Exide manufactures a line if Cninterruptable Power Supplies ( I'PS) ranging in capacity from 30 in tho Kl.A. C"nder contract in TEAM. Inc.. and the Department of Energs. Fovide is atlapting two ton $\mathrm{kl}$. commsuiat:ed inverters (which are normally part of a UPS packate for use with it photowoltaic array and battery bank. These systems will he synchonired with the uility line hut will not be able to feed eneres into it.

These systems typically have a total roltage harmonic distortion of $5 \%$ with nn single harmonic in excess of 37 . Fifficiencies range from $70-75$, at 25 , load to $\times(1)-(9) 29$ at full load. Costs. including an tisnlation transformer. range

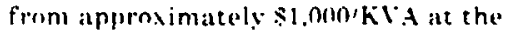

$30 \mathrm{KVA}$ level to approximately $\$ 200 /$ KVA at the $400 \mathrm{KVA}$ level.

Windworks. Inc., Mukwonago. Wisc. Windworks manufactures and distributes line-commutated, line feeding in. certers known as Gemini Synchronous Inverters. Units are available up to 15 $\mathrm{kw}$, single phase and up to $1500 \mathrm{kw}$, three phase. Applications of this equipment include wind systems, photovol taic arrays. small hydroelectric instal lations, solar thermal electric systems. and industrial waste energy recovery.

These systems can only be used in the presence of a lower impedance AC source such as the utility grid or a remote diesel/generator set. Total volt. age harmonic distortion for this type of equipment is a function of the relative impedances of the two sources but is typically less thân $1 \%$ for utility applications. Total current harmonic distortion can be as high as $30 \%$ for a single phase system. Power factor is essentialy proportional to voltage and reaches a maximum of 0.7 for single phase equipment and 0.85 for three phase units. Efficiency of the basic inverter, exclusive of input filters and isolation trans. formers is approximately $90 \%$ at $10 \%$ load and $96-98 \%$ at full load.

Costs for the basic inverter range from $\$ 180 / \mathrm{kw}$ to $\$ 200 / \mathrm{kw}$ for single phase systems and from $\$ 250 / \mathrm{kw}(20 \mathrm{kw}$ (apacity) to $\$ 50 / \mathrm{ku}(1000 \mathrm{kw}$ capacity for three phase equipment.

\begin{tabular}{|c|c|c|c|c|}
\hline Spec & CML-M & CML-M & EICO & POWERMAKER \\
\hline $\begin{array}{l}\text { Model } \\
\text { Price } \\
\text { Deliver } \\
\text { Efficiency }\end{array}$ & $\begin{array}{l}\text { MNS-50 } \\
72 \% \\
\end{array}$ & $\begin{array}{l}\text { DRS-50 } \\
67 \%\end{array}$ & $\begin{array}{r}1080 \\
\$ 86\end{array}$ & $\begin{array}{l}2124 \\
\$ 139 \\
67 \%\end{array}$ \\
\hline $\begin{array}{l}\text { Tnput } \\
\text { V } \\
\text { Wires } \\
\text { Protection }\end{array}$ & $\begin{array}{l}22.30 \\
2 \\
\text { Lead Rov. Fuse }\end{array}$ & $\begin{array}{l}22-30 \\
2 \\
\text { Lead Rev. Fuse }\end{array}$ & 12 & $\begin{array}{l}11-15 \\
\text { Lead Rev. }\end{array}$ \\
\hline $\begin{array}{l}\text { Output } \\
\text { Wave form } \\
\text { V } \\
\text { Temp. Stab. } \\
\text { V.A (W) } \\
\text { Phases } \\
\text { Freq. } \\
\text { Temp. Stab. } \\
\text { Harmonic Dist. } \\
\text { Wires. } \\
\text { Protection }\end{array}$ & $\begin{array}{l}\text { Sine } \\
105 / 115 / 125 \pm 6 \% \\
0.03 \% /^{\circ} \mathrm{C} \\
50 \\
1 \\
60 \pm 0.5 \% \\
0.05 \% /{ }^{\circ} \mathrm{C} \\
3 \% \\
2 \\
\text { Fuse }\end{array}$ & $\begin{array}{l}\text { Sine } \\
115 \pm 0.5 \% \\
0.01 \% / 0 \mathrm{C} \\
50 \\
1 \\
400 \pm 0.5 \% \\
0.05 \% / 0 \mathrm{C} \\
1 \% \\
2 \\
\text { Fuse }\end{array}$ & $\begin{array}{l}117 \\
220 \\
1\end{array}$ & $\begin{array}{l}\text { Square } \\
120 \\
300 \\
60 \\
\\
\text { Yes }\end{array}$ \\
\hline MTBF & & & & \\
\hline $\begin{array}{l}\text { Physical } \\
\text { Dimensions } \\
\text { Weight } \\
\text { Temp. Limits } \\
\text { Cooling }\end{array}$ & $\begin{array}{l}5^{\prime \prime} \times 11^{\prime \prime} \times 11^{\prime \prime} \\
28 \mathrm{lb} \\
=20^{\circ} \mathrm{C} \text { to }+50^{\circ} \mathrm{C} \\
\text { Passive }\end{array}$ & $\begin{array}{l}5^{\prime \prime} \times 11^{\prime \prime} \times 8^{\prime \prime} \\
25 \mathrm{lb} \\
=20^{\circ} \mathrm{C} \text { to }+50^{\circ} \mathrm{C} \\
\text { Passive }\end{array}$ & $\begin{array}{l}4^{\prime \prime} \times 6^{\prime \prime} \times 6^{\prime \prime} \\
7 \mathrm{lb} \\
.\end{array}$ & $\begin{array}{l}6^{\prime \prime} \times 7^{\prime \prime} \times 6^{\prime \prime} \\
13 \mathrm{tb} \\
0^{\circ} \mathrm{C} \text { to } 40^{\circ} \mathrm{C}\end{array}$ \\
\hline
\end{tabular}

Exhibit 9.5 DC/AC Inverters (0.05 kW Output). 


\begin{tabular}{|c|c|c|c|c|}
\hline Spec Manufacturer & CML-M & CML-M & CML-M & BATP' \\
\hline Model & MNS.500 & MRS-500 & DRS-500 & DA.500S \\
\hline Price (aty \#1) & $\$ 1,090$ & & & $\$ 7.645$ \\
\hline Delivery & 16 wks & & & \\
\hline Efficiency & $72 \%$ & $67 \%$ & $67 \%$ & \\
\hline $\begin{array}{l}\text { Input } \\
V \\
\text { Wires } \\
\text { Protection }\end{array}$ & $\begin{array}{l}22 \cdot 30 \\
2 \\
\text { CB overvoltage Lead Rev. }\end{array}$ & $\begin{array}{l}22.30 \\
2 \\
\text { C8 overvoltage Lead Rev. }\end{array}$ & \begin{tabular}{|l|}
22.30 \\
2 \\
CB overvoltage Lead
\end{tabular} & $\begin{array}{l}22 \cdot 30 \\
\text { Rev. }\end{array}$ \\
\hline $\begin{array}{l}\text { Output } \\
\text { Form } \\
\text { V } \\
\text { Temp. Stab. } \\
\text { V.A. } \\
\text { Phases } \\
\text { Hz } \\
\text { Temp. Stab. } \\
\text { Harmonic Dist. } \\
\text { Wires } \\
\text { Protection }\end{array}$ & $\begin{array}{l}\text { Sine } \\
105 / 115 / 125 \pm 6 \% \\
0.3 \% /{ }^{\circ} \mathrm{C} \\
500 \\
1 \\
60 \pm 0.5 \% \\
0.05 \% /{ }^{\circ} \mathrm{C} \\
3 \% \\
2 \\
\mathrm{CB} .\end{array}$ & $\begin{array}{l}\text { Sine } \\
115 \pm 0.5 \% \\
0.01 \% /^{\circ} \mathrm{C} \\
500 \\
1 \\
60 \pm 0.5 \% \\
3 \% \\
2 \\
\mathrm{CB}\end{array}$ & $\begin{array}{l}\text { Sine } \\
115 \pm 0.5 \% \\
0.01 \% /^{\circ} \mathrm{C} \\
500 \\
1 \\
400 \pm 0.5 \% \\
1 \% \\
2 \\
\mathrm{CB}\end{array}$ & $\begin{array}{l}\text { Sine } \\
115 / 230 \pm 1 \% \\
500 \\
1 \\
60 \pm 0.015 \% \\
3 \%\end{array}$ \\
\hline MTEF & & & & \\
\hline $\begin{array}{l}\text { Physical } \\
\text { Dimonoiono } \\
\text { Weight } \\
\text { Temp. Limits } \\
\text { Cooling } \\
\text { Date }\end{array}$ & $\begin{array}{l}0^{\prime \prime \prime} 14^{\prime \prime}: 14^{\prime \prime} \\
80 \mathrm{lb} \\
-20^{\circ} \mathrm{C} \mathrm{to}+50^{\circ} \mathrm{C} \\
\text { Fan } \\
8.74\end{array}$ & $\begin{array}{l}12^{\prime \prime} \times 14^{\prime \prime}, 14^{\circ} \\
150 \mathrm{lb} \\
-20^{\circ} \mathrm{C} \text { to }+50^{\circ} \mathrm{C} \\
\text { Fan } \\
8,74\end{array}$ & $\begin{array}{l}81 " \times 14^{\prime \prime} \times 16^{\prime \prime} \\
70 \text { lb } \\
-20^{\circ} \mathrm{C} \text { to }+50^{\circ} \mathrm{C} \\
\text { Fan } \\
8 / 74\end{array}$ & $\begin{array}{l}1 f^{\prime \prime} \times 15^{\prime \prime} \times 19^{\prime \prime} \\
130 \mathrm{lb} \\
0^{\circ} \mathrm{C} 1050^{\circ} \mathrm{C}\end{array}$ \\
\hline
\end{tabular}

\begin{tabular}{|c|c|c|c|c|}
\hline Spec & $T$ & $\mathbf{T}$ & POWERVERTER & POWERVERTER \\
\hline Model & 5212.26 & $5006 \mathrm{~W}$ & PV-550 & PV-500FC \\
\hline Price (qty\#1) & $\$ 1.416$ & $\$ 1.195$ & $\$ 139$ & $\$ 278$ \\
\hline Delivery & & 3 wks & & \\
\hline Efficiency & $70 \%$ & $70 \%$ & & \\
\hline $\begin{array}{l}\text { Tnpuf } \\
\text { V } \\
\text { Wires } \\
\text { Protection }\end{array}$ & $\begin{array}{l}11-15 / 22-30 \\
\text { C8 Lead Rev. }\end{array}$ & $11-15 / 22-30$ & 12. & 12 \\
\hline $\begin{array}{l}\text { Output } \\
\text { Fuı'II } \\
\text { V } \\
\text { Temp. Stab. } \\
\text { V.A. } \\
\text { Phases } \\
\text { Hz } \\
\text { Temp. Stab. } \\
\text { Harmonic Dist. } \\
\text { Wires } \\
\text { Protection } \\
\end{array}$ & $\begin{array}{l}\sin \theta \\
116 \pm 1 \% \\
500 \\
1 \\
60 \pm 0.5 \%\end{array}$ & $\begin{array}{l}\sin \theta \\
115 \pm 5 \% \\
500 \\
1 \\
60 \pm 0.5 \%\end{array}$ & $\begin{array}{l}11 \% \\
550 \\
1 \\
60\end{array}$ & $\begin{array}{l}117 \\
500 \\
1 \\
60 \pm 2 \%\end{array}$ \\
\hline MTBF & & & & \\
\hline $\begin{array}{l}\text { Physiral } \\
\text { Dimensions } \\
\text { Weight } \\
\text { Tomp. Limits } \\
\text { Cooling } \\
\text { Date }\end{array}$ & $\begin{array}{l}7^{\prime \prime} \times 19^{\prime \prime} \times 13^{\prime \prime} \\
65 \\
-10 \text { to }+55^{\circ} \mathrm{C}\end{array}$ & $8 / 74$ & $5^{\prime \prime} \times 10^{\prime \prime} \times 9 "$ & $\begin{array}{l}5^{\prime \prime} \times 10^{\prime \prime} \times 9^{\prime \prime} \\
19\end{array}$ \\
\hline
\end{tabular}

Exhibit 9.6 DC/AC Inverters (0.50 kW Output). 


\subsection{AC/DC CONVERTERS}

For large $A C$ systems, an $A C / D C$ converter might be used to convert the regulated $A C$ to $D C$ for charging a battery. An additional inverter is used to invert the battery current to $A C$ for the load. The losses associated with such a system are tolerable only if there is a gain due to better matching of load demand and array output. The converters for which data are shown are all approximately 65-85 percent efficient and cost approximately $\$ 1 / W$ (see exhibit 9.7). A converter is useful for $A C$ loads if the demand requirements are drastically reduced when stored energy is used. The use of converters is shown in Section 1.2 for supplying $A C$ with battery storage for low night demands.

\begin{tabular}{|c|c|c|c|c|}
\hline Spec & $\cdot$ & SOLA & SOLA & SOLA \\
\hline $\begin{array}{l}\text { Model } \\
\text { Price } \\
\text { Delivery } \\
\text { Efficiençy }\end{array}$ & $\begin{array}{l}\text { APS-24-2.2 } \\
\$ 70 \\
65 \% \\
\end{array}$ & $\begin{array}{l}83 \cdot 12 \cdot 250-1 \\
\$ 81\end{array}$ & $\begin{array}{l}28 \cdot 28-243 \\
\$ 151\end{array}$ & $\begin{array}{l}281561-2 \\
\$ 306 \\
75 \%\end{array}$ \\
\hline $\begin{array}{l}\text { Tnput } \\
\text { Wave Form } \\
\text { V } \\
\text { A } \\
\text { Frequency } \\
\text { Phases }\end{array}$ & $\begin{array}{l}\text { Sine } \\
115 / 230=10 \% \\
47-63 \\
1\end{array}$ & $\begin{array}{l}108 \text { to } 132 \\
50-400\end{array}$ & $\begin{array}{l}100 \text { to } 300 \\
60\end{array}$ & $\begin{array}{l}100 \text { to } 130 \\
60\end{array}$ \\
\hline $\begin{array}{l}\text { Output } \\
\text { VA } \\
\text { V } \\
\text { Temp. Stab. } \\
\text { Ripple. } \\
\text { Protection }\end{array}$ & $\begin{array}{l}53 \\
24 \pm 0.2 \% \\
5 \mathrm{mV} \\
\text { overload }\end{array}$ & $\begin{array}{l}60 \\
11.5 \text { to } 125 \pm 0.05 \% \\
0.03 \% /^{\circ} \mathrm{C} \\
10 \mathrm{mV}\end{array}$ & $\begin{array}{l}120 \\
28 \pm 1 \% \\
0.03 \% /^{\circ} \mathrm{C} \\
300 \mathrm{mV} \\
\text { isolated current } \\
\text { limiting }\end{array}$ & $\begin{array}{l}480 \\
48 \pm 1 \% \\
1 \% \\
\text { current limiting }\end{array}$ \\
\hline MTBF & & & & \\
\hline $\begin{array}{l}\text { Physical } \\
\text { Dimensions } \\
\text { Weight } \\
\text { Temp.. Limits } \\
\text { Cooling }\end{array}$ & $0^{\circ} \cdot 1065^{\circ} \mathrm{C}$ & $\begin{array}{l}7 \mathrm{lb} \\
0^{\circ} \text { to } 55^{\circ} \mathrm{C} \\
\text { pessive }\end{array}$ & $\begin{array}{l}14 \mathrm{lb} \\
0^{\circ} \text { to } 50^{\circ} \mathrm{C}\end{array}$ & $5^{\prime \prime} \times 2^{\prime \prime} \times 10^{\prime \prime}$ \\
\hline
\end{tabular}

Source: 1979 Allied Electronics Catalog

\section{Exhibit 9.7 AC/DC Converters (Rectifiers, Power Suppliers) $(0.05$ kW)}

\subsection{MONITORING INSTRUMENTATION}

Some transducers that might be useful in monitoring the system performance on:a continuing basis are listed in exhibit 9.8. Transducers can supply measurements to recording equipment and digital and analog computers. The current transducer is inexpensive, and the power transducer has a high price, especially relative to a system that might only cost $\$ 3,000$. If the current transducer is used in conjunction with the battery, however, an approximate value of the power can be obtained, since the battery voltage varies only \pm 15 percent, and power $=$ voltage $\times$ current $(D C$ power $=$ wattage $)$. For $n$ single phasc $A C$ circuit, Wattage $=$ Voltage $\times$ Current $\times$ Power Factor (cosine of phase angle) where power 
factor is the percentage of total power due to resistance. For a three phase $A C$ circuit, the total wattage $(W)=3 \times$ single phase wattage where the single phase voltage $=1 / 3$ (square root 3 ) $x$ voltage between phases. The single phase voltage is the voltage between phase and neutral, thus a three phase AC circuit has "four" phases where the neutral is the "fourth phase". The amount of total power in an AC circuit due to excitation and field flux is termed reactive power (VAR). Total $A C$ power $=$ square root $\left(W^{2}+V A R^{2}\right)$.

\begin{tabular}{|c|c|c|}
\hline Manufact & OSI-PC8 & OSI-CT-L \\
\hline & DC W Transducer & Current Transducer \\
\hline $\begin{array}{l}\text { Price } \\
\text { Inst. Voltage }\end{array}$ & $\begin{array}{l}\$ 450 \\
115 \text { VAC }\end{array}$ & $\$ 97$ \\
\hline $\begin{array}{l}\text { Tnput } \\
\text { V } \\
\text { A } \\
\text { Frequency } \\
\text { Resp. Time } \\
\text { Ohms }\end{array}$ & $\begin{array}{l}25 \text { to } 600 \\
0 \text { to } 650 \\
D C \text { to } 400 \mathrm{~Hz} \\
50 \text { microsec } \\
\text { (into } 0 \text { to } 10,000 \text { ohms) }\end{array}$ & $\begin{array}{l}50 \text { to } 2,000 \\
0 \mathrm{C} \text { to } 5,000 \text { to } \mathrm{Hz} \\
50 \text { microsec } \\
4\end{array}$ \\
\hline $\begin{array}{c}\text { Output } \\
V \\
A \\
\text { Ripule } \\
\text { Accuracy } \\
\text { Temp Stab } \\
\text { ohms }\end{array}$ & $\begin{array}{l}1 \text { ma max. } \\
0.01 \text { ma } \\
\pm 1 \% \\
\pm 1 \%, 0^{\circ} \text { to } 40^{\circ} \mathrm{C}\end{array}$ & $\left\{\begin{array}{l}30 \mathrm{mV} \\
\pm 0.5 \% \text { linearity } \\
-0.15 \% /^{\circ} \mathrm{C},-40 \text { to } 65^{\circ} \mathrm{C} \\
3\end{array}\right.$ \\
\hline
\end{tabular}

\section{Exhibit 9.8 Transducers}

\subsection{COMPONENT MANUFACTURER̄S}

The component manufacturers listed in this section are meant to be representative of what is available. The list is far from exhaustive. For each application, a similar survey will be necessary.

Exhibit 9.9, which is a partial list of manufacturers, follows. 


\begin{tabular}{|c|c|c|}
\hline $\begin{array}{c}\text { Address } \\
\text { Manufacturer }\end{array}$ & Corporate & Wash., D.C. Representative \\
\hline CMLM' & $\begin{array}{l}\text { CML-Macara, Inc. } \\
\text { 166 National Road } \\
\text { Edison, NJ } 08817 \\
\text { (201) } 287-2810\end{array}$ & $\begin{array}{l}\text { Gans-Fryling, Inc. } \\
206214 \text { th Street } \\
\text { Arlington, VA 22201 } \\
\text { (703) } 527-3262\end{array}$ \\
\hline$A C D C$ & $\begin{array}{l}\text { ACDC Elec tronics, Inc. } \\
\text { Oceanside Industrial Ctr. } \\
\text { Oceanside, CA } 92054 \\
\text { (714) } 757-1880\end{array}$ & $\begin{array}{l}\text { Electronics Marketing } \\
\text { Associates, Inc. } \\
11501 \text { Huff Court } \\
\text { Kensington, MD } 20795 \\
\text { (202) } 381-5300\end{array}$ \\
\hline BATP & $\begin{array}{l}\text { Bulova American lime } \\
\text { Products } \\
\text { Bulova Water Co., Inc. } \\
61-20 \text { Woodside Avenue } \\
\text { Woodside, NY } 11377 \\
\text { (212) 335-6000 }\end{array}$ & . \\
\hline$T$ & $\begin{array}{l}\text { Topaz Electronics } \\
3855 \text { Ruffin Road } \\
\text { San Diego, CA } 92123 \\
\text { (714) 279-0111 }\end{array}$ & $\begin{array}{l}\text { Bartlett Associates } \\
4405 \text { East-West Highway } \\
\text { Bethesda, MD } 20014 \\
(301) 656-3061\end{array}$ \\
\hline \multicolumn{3}{|l|}{ EICO } \\
\hline OSI & $\begin{array}{l}\text { Ohio Semitronics, Inc. } \\
\text { 1205 Chesopeoke Avenue } \\
\text { Columbus, OH } 43212 \\
(614) 486-9561\end{array}$ & \\
\hline ME & $\begin{array}{l}\text { McGraw Edison } \\
\text { Edison Battery Division } \\
210 \text { Redstone Hill Road } \\
\text { Bristol, CT } 06010 \\
\text { (203) } 582-6321\end{array}$ & \\
\hline$E L$ & $\begin{array}{l}\text { Electro Lite } \\
\text { 1225 E. 40th Street } \\
\text { Chattanooga, TN } 37407 \\
(615) 867-4750\end{array}$ & \\
\hline$G$ & $\begin{array}{l}\text { Globe-Union Inc. } \\
5757 \text { North Green Bay Ave. } \\
\text { Milwaukee, WI } 53201 \\
\text { (414) 228-2394 }\end{array}$ & $\begin{array}{l}\text { Perrot Engineering Labs } \\
1020 \mathrm{~N} \text {. Fillmore } \\
\text { Arlington, VA } 22201 \\
\text { (703) } 528-5861\end{array}$ \\
\hline
\end{tabular}

Exhibit 9.9 Partial List of Manufacturers. of System Components 


\section{THIS PAGE \\ WAS INTENTIONALLY \\ LEFT BLANK}

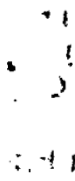

4

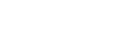




\section{SECTION 10 \\ MODEL SYSTEMS SPECIFICATIONS}

\subsection{INTRODUCTION}

JPL has developed and published specifications for the PV. modules; however, industry work standards have not yet been agreed upon. The Solor Energy Research Institute is the lead agency in this area. Support is provided by the Electric Power Research Institute and the Solar Energy Industry Association.

This section has been prepared to assist those who will be writing specifications for procurement. The following specifications should be used as a model in the development of specifications for any particular installation.

This model specification is designed: (1) to enable the system designer to define the system in sufficient detail for the manufacturers to supply suitable equipment and for the installer to install the system so it will meet the desired functional requirements; (2) to define the quality of the equipment and installation so the maintenance and life of the system will be os anticipated in the design selection and life-cycle cost analyses; and (3) to control the quality of the manufacture and installation of the system. The designer should keep these purposes in mind when applying the model specifications. If the designer deletes an item, he should be willing to accept the corresponding loss of system definition and quality control. If an item is added, it should be done because there is a need for a more complete definition and quality control.

It is expected that, as experience is gained with terrestrial applications of solar PV systems, standards will be developed by the industry. In the absence of widely accepted standards and test procedures, the following model specification represents one effort at documenting requirements for design, manufacture, installation, service life, and the quality control associated with each (See exhibit 10.1). The JPL module specification was the basis for section 10.4, appendices 1 .A and 1 ..B, and section 14.3.

General Photovoltaic Solar System Requirements (see section 10.2)

System Requirements (see section 10.3)

PV Module Specifications (seo section 10.4)

Inverter (see appendix 8.7)

Battery Voltage Regulator (see appendix B.8)

Converter (see appendix B.9)

Battery (see appendix B.10)

Electrical

Structure

Fence

Foundations

Test and Accuptance (see appendix B.11)

Warranty (see appendix B.12)

Appendices

11A. Performance Measurement Procedures

11B. Test Procedures

Exhibit 10.1 Typical Outline for System Specifications. 


\subsection{GENERAL PHOTOVOLTAIC (PV) SYSTEM REQUIREMENTS}

A. General

1. Drawings:

a) The drawings diagrammatically illustrate the arrangements of the principal equipment and shall be followed as closely as possible.

b) The drowings shall be neat and the arrangements well spaced throughout.

c) The drawings and specifications need not include exact equipment dimensions, locations, nor complete accessory items and control wiring devices required for each manufacturer's equipment.

d) The drawings shall provide complete and properly functioning systems that comply with the standards and performance requirements specified herein.

2. Changes in Location:

a) Changes in locations of equipment from the locations indicated on the drawings, to suit the actual conditions of the work, require written approval of the contracting officer.

b) Changes in location after the equipment has been installed, if directed by the contracting officer, shall be made in accordance with applicable provisions of the general conditions.

B. Mate-ials

1. General:

a) Materinls, when not otherwise definitely specified, shall conform to applicable national specifications and standards, such as Underwriters Laboratories, National Electrical Manufacturers Association Code, National Fire Code, National Electrical Code, and the Uniform Building Code.

b) All material shall be new and in perfect condition.

C. Items Requiring Submittal

1. Shop Drawings:

a) Refer to A.I, Drawings.

b) In addition to the above requirements, detailed shop drawings are required for, but not limited to, the following:

(1) solar reill modules

(2) power-conditioning equipment

(3): battery and housing for same. 


\subsection{SYSTEM REQUIREMENTS}

The accompanying engineering drawings, which are part of this specification, describe the system and most of its requirements. The PV system, which is comprised of the PV array, the support structure, the wiring and connectors, the power-conditioning equipment (regulators, inverters, converters, etc.), the batteries, the housings for the equipment, and - buses, substations, switchboards, load centers, transmission, distribution, and control shall supply all the energy required to operate: an irrigation pump, a transmitter, a radio receiver, and a telemetry system (See exhibit 10.2). The power and energy requirements of these devices are shown on exhibit 10.3 of these specifications. The area of the power vs. time'profile equals energy, where energy equals the summation of power $x$ time duration.

What is included in the PV System: Array. Structures, . . .

What the system powers: Pump. Transmitter. . . .

Where the system will be installed: Billings, Montana

Overview of unusual requirements

Insolation: Tabulation of monthly averages, NWS for more data

Load: Graphical representation

Life: 20 years (10 years for battery)

Energy storage required: 10 days without sun

Telemetry/Alarm: 20 miles, signal once per hour besed on battery voltage, adds to load

\section{Exhibit 10.2 General Data Needed to Design a Solar PV System}

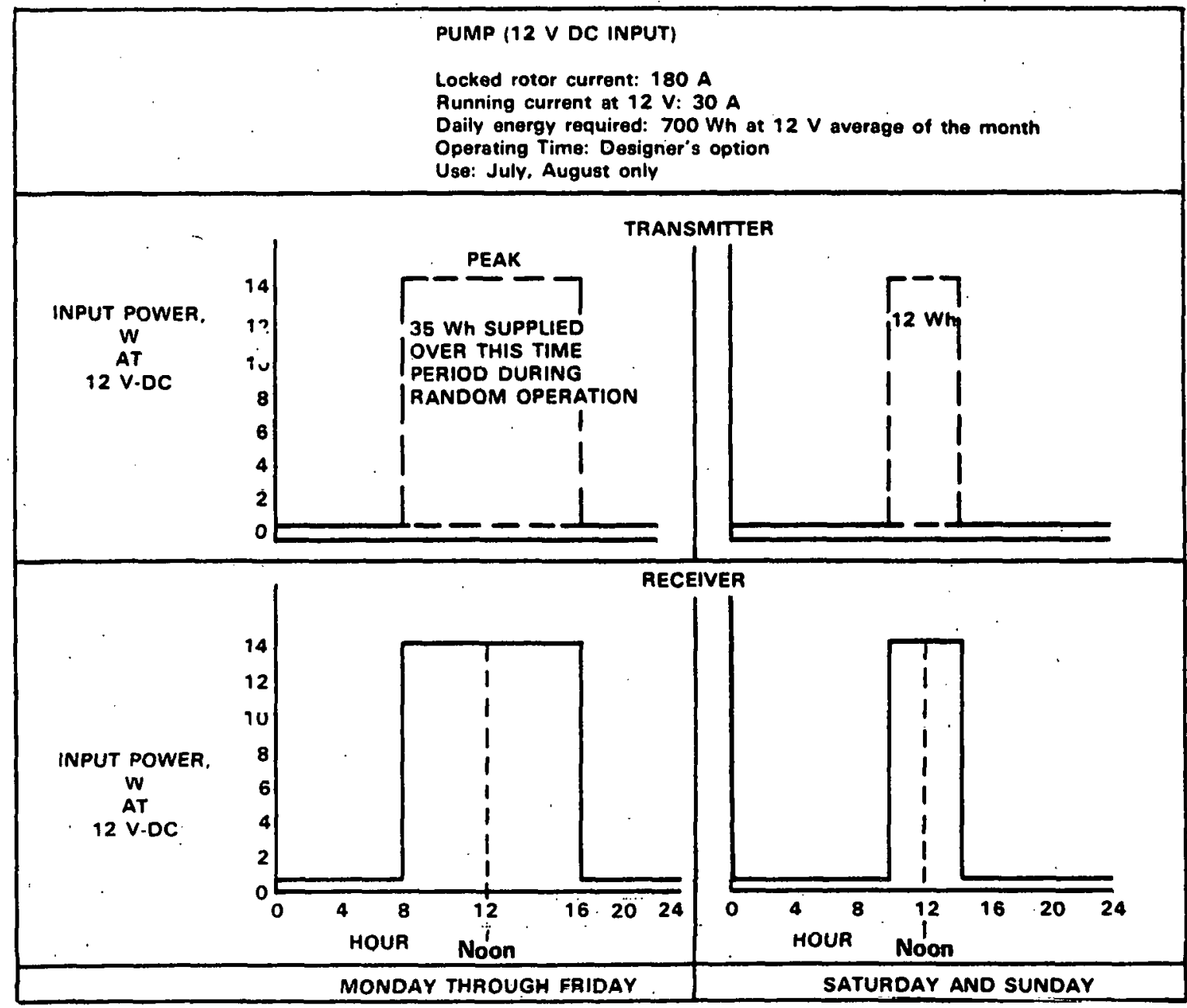

Energy = Summation of power $x$ time duration, which equals the area of the power profile.

Exhibit 10.3 Power and Energy Requirements.

10-3 
The equipment and installation are to be suitable for use over a 20-year period in Billings, Montana (example). The system is to be designed for insolation corresponding to that shown on exhibit 10.4. If more insolation detail is required in the design, the insolation data avalable from the National Wegther Service shall be used. The structure shall be designed according to the local codes pertaining to Billings, Montana. Weather data are also available from the National Weather Service for this location.

If storage is required, the battery storage capacity shall be sufficient to supply the energy needs of the devices described above for a period of 10 days without any energy input from the solar array. The batteries shall be designed for a minimum of 10 years' life. A radio-telemetry alarm system shall be provided to transmit a signal proportional to the battery voltage to a receiver 20 miles from the array installation site.

The contractor is to provide both the alnrm transmitter and receiver. The signal transimitted will require power in addition to that of the load described on exhibit 10.3. However, the power requirements are to be kept as low as possible by only transmitting the signal approximately once per hour. When the battery voltage falls below that specified in section 10.7, an alarm must light to indicate to the attendant at the receiver that the PV. system is not performing within the specifications.

\subsection{PV MODULE SPECIFICATIONS}

Solar cell modules meeting the requirements of this specification shall be mounted or grouped into an array structure compatible with system design constraints for the PV application described in section 10.3. The module, panel, subarray, and array designs shall satisfy the following general design criteria (See exhibit 10.5). 
Exhibit 10.4 Insolation for Billings, Montana ( $45^{\circ}$. Latitude)

MONTH

January

February

March

April

May

June

July

August

September

October

November

December
-ÁVERAGE DÁLY INSOLATION ON A HORIZONTAL SURFACE*(i $\mathrm{J} / \mathrm{m}^{2} /$ day)

6.62

9.92

15.03

19.09

22.65

25.62

26.63

23.32

17.75

10.68

7.24

5.57

*NOTE: 1. Divide by 3.6 to convert $\mathrm{MJ} / \mathrm{m}^{2} /$ day to $\mathrm{kWh} / \mathrm{m}^{2} /$ day.

2. . One MJ equals I million joules of energy.

3. One kWh equals I thousand watt-hours of energy.

4. Une watt equals one joule per second of power.

5. Insolation equals solar flux which equals intensity which equals power density which equals energy per. unit time per unit area.

6. The unit of area used is the square meter, the unit of time used is the day, the unit of power used is the watt, and the unit of energy used is the joule or the kilowatt-hour. 


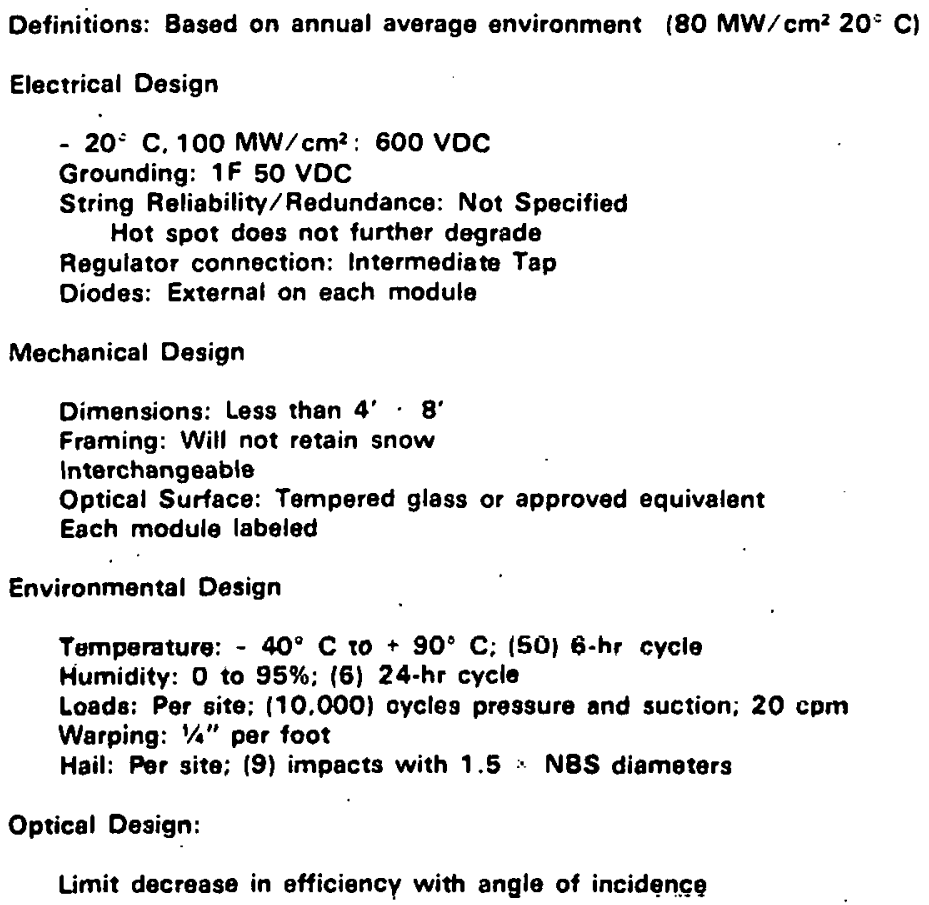

Limit decrease in efficiency with angle of incidencẹt

Exhibit 10.5 PV Module Specifications.

\subsubsection{DESIGN REQUIREMENTS}

\subsubsection{PERFORMANCE MEASUREMENT DEFINITIONS}

The following standard performance-measurement definitions shall be utilized:

(1) Nominal Power Output - The power output of individual modules shall be determined per appendix 1 . A.I as the product of the module Nominal Operating Voltage $\left(V_{L O}\right)$ and the medule current measured at $V_{N O}$ under Standard Operating Conditions (SOC) defined as an irradiance level of $100 \cdot \mathrm{mW} / \mathrm{cm}^{2}$ and cell temperature equal to the Vominal Operating Cell Temperature (NOC.T).

(2) Nominal Operating Voltage - $V_{N O}$ is the reference voltage level at which the modules are designed to provide maximum power output at standard operating conditions $\left(100 \mathrm{~mW} / \mathrm{cm}^{2}, \mathrm{NOCT}\right)$. The $\mathrm{V}_{\mathrm{NO}}$ level shall be selected by the module manufocturer/system designer.

(3) NOCT - NOCT is the module cell temperature under operating conditions in the Nominal Thermal Environment (NTE) which is defined as: Insolation $=80 \mathrm{~mW} / \mathrm{cm}^{2}$. Air Temperature $=20^{\circ} \mathrm{C}$. Wind Average Velocity $=1 \mathrm{~m} / \mathrm{s}$. Mounting = Tilted, Open Bock, Open Circuit.

The NOCT measurement procedure is described in Appendix II.A.2.

* The NTE approximates the annual average environment. 


\subsubsection{ELECTRICAL DESIGN REQUIREMENTS}

The electrical design of the module shall meet the following requirements:

(1) Electrical Voltage Isolation - All module circuitry, including output terminals, shall be insulated from exterial surfaces. The manufacturer/contractor shall establish the voltage isolation requirement on the basis of the maximum open circuit voltage of the complete system at an ambient temperature of $-20^{\circ} \mathrm{C}$, with $100 \mathrm{~mW} / \mathrm{cm}^{2}$ irradiance. The voltage level shall be set at a minimum of 600 VDC.

(2) Electrical Grounding and Safety - To minimize electrical hazard to personnel, all modules shall be provided with an external grounding terminal or stud serving as a common grounding point for all exposed external conductive surfaces not part of the module circuitry. A grounding stud is not required for modules designed for systems with operating voltage levels less than $50 \mathrm{~V}$ or for modules without exposed conductive surfaces, unless removal of covers or mounting hardware will expose such surfaces. Lightning protection must be provided for the array.

(3) Cell String Circuit Reliability/Redundancy - Circuit redundancy features shall be incorporated where cost effective to enhance the reliability of completed modules. Any conflict between minimum reliability and cost shall be in favor of minimum reliability, per se. Design features may include, but are not limited to the following:

(a) Redundant interconnections between solar cells, including redundant cell attachment points,

(b) Series/parallel interconnection of cells within the module, and

(c) By-pass diodes with each module.

The decision to incorporate redundancy features shall be based on minimum reliability and the expected percent improvement in lifetime/yield and replacement cost as contrasted with the percent increase in module cost/watt. Series/parallel circuit arrangements, when used, shall be designed so that "hot spot" cell heating does not lead to further module permanent degradation under worst-case-single-cell failure conditions defined as follows:

(a) The module output is short circuited.

(b) A single representative solar cell is open circuited to represent a single cell failure.

(c) The incident irradiance is $100 \mathrm{~mW} / \mathrm{cm}^{2}$, air-mass equal to 1.5 .

(d) The thermal boundary conditions are adjusted so that the equilibrium solar cell temperature outside the hot-spot region is equal to NOCT + $20^{\circ} \mathrm{C}$.

(4) Connections for a Shunt Voltage Regulator - If a shunt type voltage regulator will be used, an intermediate lead must be provided on each module. The output voltage to ground of this lead shall be between 10 and $11 \mathrm{~V}$, such that the shunt regulator can be connected between the intermediate lead and the high-voltage terminal of the array, since a shunt connection is a parallel connection. 
(5) Diodes - All diodes used to prevent reversed current through the modules must be replaced without damage to the module, when diode performance becomes unacceptable.

\subsubsection{MECHANICAL DESIGN REQUIREMENTS}

The mechanical design of the module shall meet the following requirements:

(1) Module Geometry - To meet the array/system requirements for mounting, each module shall meet the envelope, mechanical, and interface requirements specified by an Interface Control Drawing to be prepared by the manufacturer/contractor, providing as a minimum the following information:

. (a) Niaximum envelope dimensions and tolerances,

(b) Location of output terminals,

(c) Mounting hole or attochment provisions, dimensions, and tolerance,

(d) Illuminated (active) surface dimensions and shadowing or view angle constraints for low level ronnentrators, and

(e) Nominal electrical performance.

To allow for convenient handling, the maximum module dimensions shall not exceed $48 \mathrm{in}$. by $96 \mathrm{in}$. Suggested standard dimensions and hole locations are provided in exhibit 10.6. In this exhibit, the module width may be any dimension up to the 96 in. maximum. The framing for the module shall be designed so snow is not accumulated by the frame such that the cells remain covered.

(2) Interchangeability - All modules shall be physically and functionally interchangeahle. Tolerances on all external nudule dimensions shall be maintained at a level consistent with module interchangeability. Surfaces, mounting holes, and any attachment hardware associated with the attachment interfaces shall be maintained within tolerance specified in the Interface Control Drawing.

(3) Optical Surface Soiling - The illuminated optical surface(s) of the module shall be smooth and generally free of projections that could promote entrapment of dust and other debris. Particular attention shall be given to the selection of materials for the optical surfuce(s) which will minimize the accumulation of nonremovable contaminants, fungus growth, particulate matter and stains, and will promote self-cleaning by natural processes such as wind and rain. A sheet of tempered glass meets these requirements, however, other ilulerials may also prove suitable, but must be approved by the owner. 


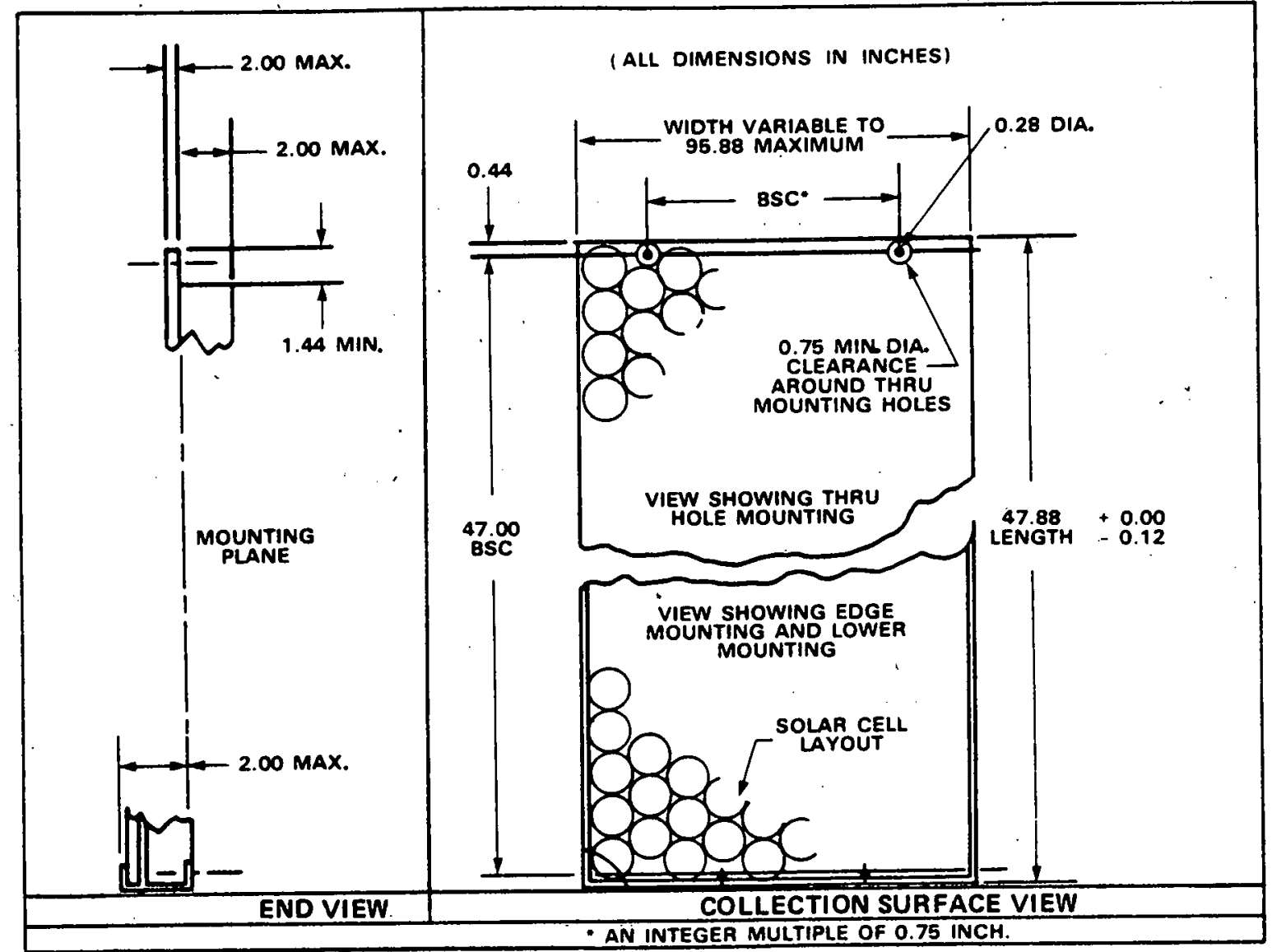

Exhibit 10.6 Suggested Module Standard Dimensions.

(4) Module Labeling and Identification - Each module shall be identified in a permanent and legible manner with suitable labels or markings specifying the manufacturer's module model number (or drawing) and revision, sequential serial number, year and week of manufacture, and maximum system operating voltage for which the module is designed. Additional information may include the $V$ and power of the module. The identification shall be installed at a position that is visible from the front (illuminated) side of each module, when installed in the array. The polarity of each electrical terminal shall be marked in a permanent and legible manner in a position that is visible when accessing the electrical terminals in a completed array. Positive, negative, neutral, "and ground shall be explicitly indicated.

\subsubsection{ENVIFONMENTAL DESIGN REQUIREMENTS}

Environments to be considered in assessing possible degradation of module electrical performance and physical properties include: solor exposure (particularly ultraviolet-UV); thermal conditions, including freezing and thawing; effects of wind, rain, snow, ice, hail, salt mist, and atmospheric oxidants; dust and debris accumulation, especially nonremovable 
stains or contamination; and, dynamic looding effects of wind, snow, and hail. As a minimum, the module design shall be capable of withstanding exposure to the following environmental test environments:

(1) Thermal cycling from $-40^{\circ} \mathrm{C}$ to $+90^{\circ} \mathrm{C}$ per Test Procedure in appendix 11.8 .1 .

(2) Humidity per Test Procedure appendix II.B.2.

(3) Mechanical cyclic loading per procedure in appendix 1/.B.3. The test load level shall be determined by the manufacturer/contractor on the basis of the anticipated application site maximum wind gust velocity. (A test level pressure of \pm 50 $\mathrm{lb} / \mathrm{ft}^{2}$ is tommon practice.)

(4) Warped mounting surface of $y_{4} \mathrm{in} / \mathrm{ft}$ per Test Procedure in appendix 11:B.4.

(5) Hail impact testing per Test Procedure in appendix 11.B.5. The maximum size hailstones that the module can withstand shall be determined by the manufacturer/contractor on the basis of an assessment of the hail risk at the intended application site.

The manufacturer/contractor shall establish additional environmental requirements dictated by special environmental conditions at the intended application site.

\subsubsection{OPTICAL DESIGN REQUIREMENTS}

As the angle of incidence of the insolation to collection area changes, the optical surface coating will result in a decrease in module output for a given insolation. Both reflection and absorption increase with angle of incidence. The tests and ratings resulting from these specifications will be useful in designing the system for year-to-year use. The output of the module shall not be less than that indicated in exhibit 10.7 as the angle of incidence changes, for any azimuth angle of the sun.

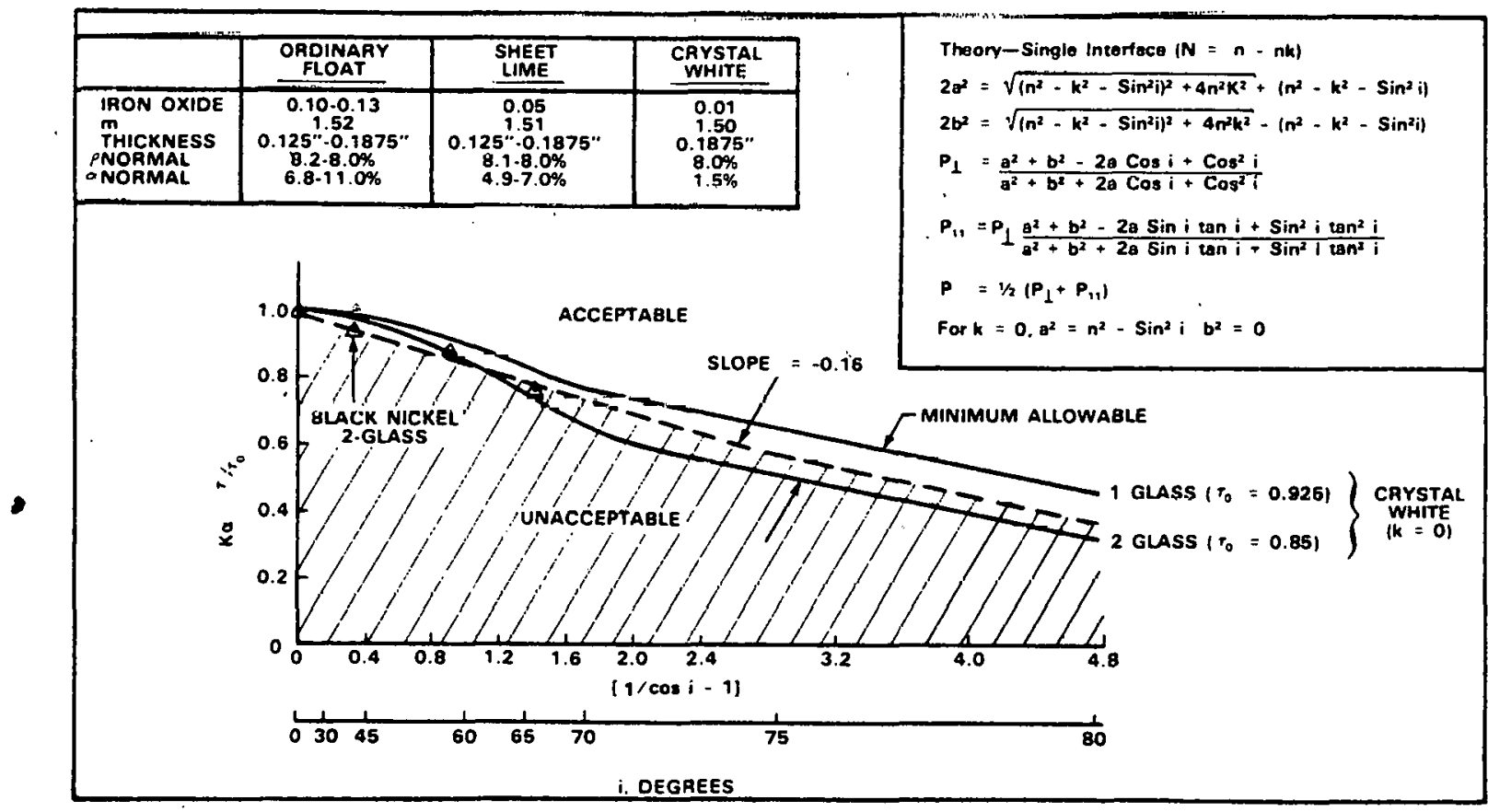

Exhibit 10.7 Transmission Properties of Glass. 


\subsubsection{QUALIFICATION REQUIREMENTS}

\subsubsection{PERFORMANCE CHARACTERIZATION REQUIREMENTS}

The tests included in this section shall be performed to characterize the module performance and to provide a high level of confidence that all modules will function within the specified performance requirements.

(1) Determination of NOCT - For purposes of providing a measurement of module performance that is representative of the anticipated terrestrial application, all module performance measurements are referenced to the NOCT. NOCT is defined as the average cell temperature in the module under operating conditions in the NTE. NTE is characterized by $80 \mathrm{~mW} / \mathrm{cm}^{2}$ insolation, ambient air temperature of $20^{\circ} \mathrm{C}$, average wind velocity of $1.0 \mathrm{~m} / \mathrm{s}$, with the module mounted in an open back condition (i.e., not insulated on back side). Actual cell temperatures shall be taken at conditions approximating NTE to obtain the solar cell NOCT. The NOCT shall be used for all measurements of module performance at SOC. The approved techniques for performing the NOCT characterization test are included in appendix 11.A.2.

(2) Initial Electrical Mieasurement - A minimum of five production module samples shall be used to determine module baseline electrical performance.

Measurements shall be conducted at the NOCT determined per paragraph 10.4.2.1 and the $V$ specified by the manufacturer/contractor for the intended application. In addition to obtaining a baseline $1-V$ characteristic curve for each module, the average output power $(P$ avg $)$ at $V_{N O}$ shall be calculated from measurements of all prototype samples. Any sample producing less than the nominal average power. under SOC shall be replaced by an acceptable module prior to subjecting these modules to the design qualification tests in 10.4.2.2. The Nominal Average Power is defined as the average power of SOC and $V_{1}$ according to the manufacturer's specification, as used in the system design. The output power determined for each module shall be the calculation base for determining extent of performance degradation during environmental qualification testing.

\subsubsection{DESIGN QUALIFICATION TEST REQUIREMENTS}

This section specifies the minimum tests that shall be performed by the contractor/ manufacturer to verify that the modules will satisfy the design requirements of this specification (see exhibit 10.8). Owners may, at their option, in addition to the characterization tests described in 10.4.2.1, perform any or all of these tests on submitted prototype modules prior to approval of the module design. Modules shall be mounted on rigid structural frame simulating the selected mounting interface and configuration for all design qualification testing. As a minimum, the following qualification tests shall be performed in the order listed. 


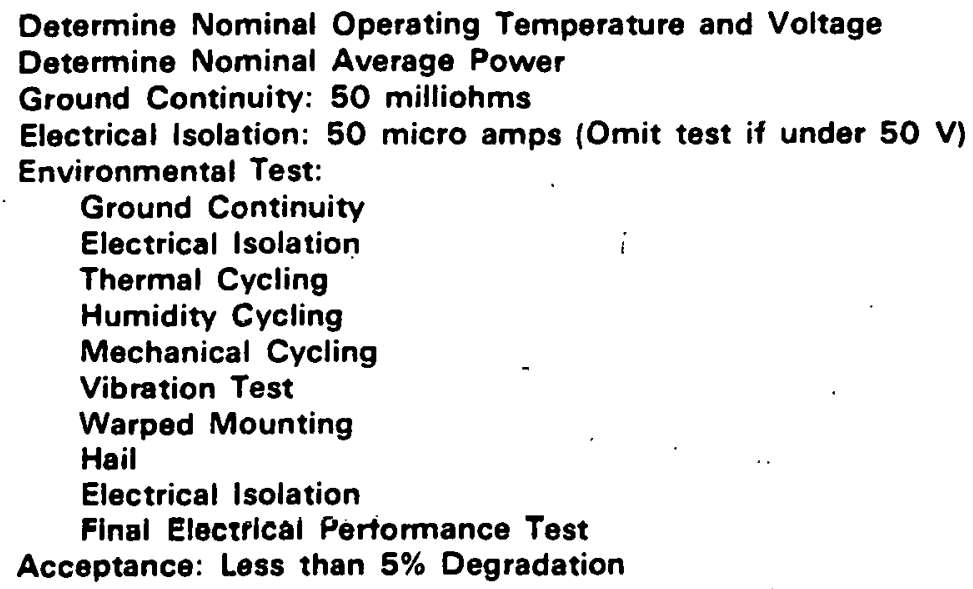

Exhibit 10.8 Qualification Test for PV Module.

\section{(1) Grounding Continuity Test}

Each module having exposed external condirrtive surfaces (i.c., frame or structural members) shall be tested using a suitable continuity tester to verify that electrical continuity exists between all such surfaces and the module grounding point. The maximum resistance to ground shall be 50 milliohms $(50 \times$ $10^{-3} \mathrm{ohms}$ ).

(2) Electrical Isolation Test

Each module shall be subjected to a DC-voltage-withstanding (Hi-Pot) test to assure the capability of the encopsulation system to provide adequate electrical isolation of internal circuitry. This test level shall equal the design level established by the manufacturer per paragraph 10.4.1.2. Leakage current during the test shall not exceed 50 micro $A\left(50 \times 10^{-6} \mathrm{~A}\right)$ and there shall be no evidence of arcs, or flashover indicating insulation foilure. Modules for which the maximum system voltage does not exceed $50 \mathrm{~V} D C$ are exempt from this requirement.

\section{(3) Environmental Testing}

Each module shall be subjected to the following environmental exposures, plus any additional special environmental tests which the manufacturer/contractor may require for the intended application. These tests shall be conducted in the order indicated:

(a) Ground continuity test

(b) Electrical isolation tests per appendix II.B.6

(c) Thermal cycling test per appendix II.B.I

(d) Humidity cycling test per appendix 11.8 .2 
(e) Mechanical cycling test per appendix II.B.3

(f) Vibration test - not port of JPL specification

(g) Warped mounting surface test per appendix II.B.4

(h) Hail impact test per appendix II.B.5

(i) Electrical isolation tests per appendix |I.B.I

(j) Final electrical performance test per appendix II.A.1.

(4) Qualification Pass/Fail Criteria--The output power degradation of each tested module, determined after completion of all qualification tests, shall not exceed 5 percent of the initial electrical performance determined per 10.4.2.1. The module shall pass the electrical isolation test (appendix II.B.6.) when retested at completion of qualification tests. The allowable level of observable cracks or other mechanical degradation (such as delamination of coatings) shall be determined by the manufacturer/contractor. Acceptable performance under the qualification testing requirements is a prerequisite for owner approval of the module design. All test reports showing the results of the qualification tests shall be submitted to the owner.

\subsubsection{MODULE ACCEPTANCE REQUIREMENTS}

(1) Electrical Performance Each module shall be measured to determine its current-voltage characteristics (I-V curve). Measurements shall be made in accordance with appendix 11.A.I. No module shall be accepted for delivery which produces less than the Nominal Average Power under SOC, where $P=V \times I$.

(2) Electrical Isolation

Each module shall be subjected to a DC 'Hi-Pot' test to assure adequate electrical isolation for safety of operating personnel at system operating voltages. The expose $\mathrm{d}$, external, conducting surfaces shall be electrically isolated from any PV voltage. 


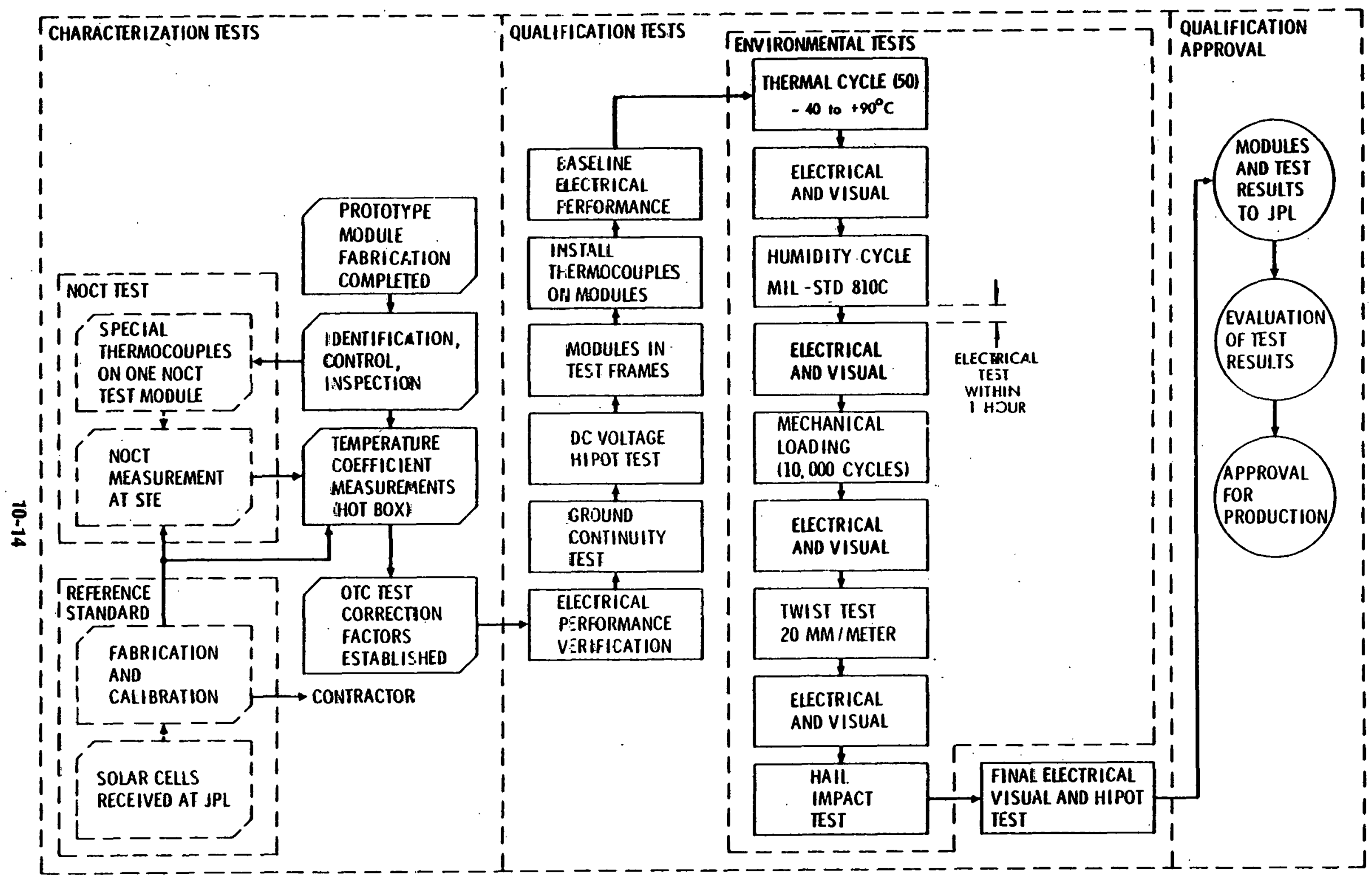

Claracterization and, Qualification Test Flow Plan 


\section{SECTION 11.4 \\ MANUFACTURERS SUPPLEMENT}

\section{SOLAR POWER CORPORATION}

Affiliate of Exxon Enterprises, Inc.

INSTALATION AND MAINTENANCE MANUAL

SERIES "M" ARRAY

SOLAR ELECTRIC GENERATOR 


\section{SECTION II \\ INSTALLATION, OPERATION, AND MAINTENANCE}

\section{II.I INTRODUCTION}

A PV manufacturer has provided an installation and maintenance manual, with troubleshooting and onsite testing procedures. That manual is included in 11.4.

Solar power system performance is usually monitored onsite, however, remote monitoring via telemetry is possible. Because the choice of monitoring techniques can have a significant impact on life cycle cost, a discussion of the economics of the monitoring procedure is included.

\subsection{OPERATION AND MAINTENANCE}

Generally, PV power systems are trouble free, requiring only periodic inspections. The conditions of the array should be noted and meticulously recorded after each inspection, as well as information required by DOE in degradation reports, which should be recorded and forwarded.

Foreign matter coming into contact with the array surface will normally fall away due to the mounting angle or be washed away during rain. Some sources of array soiling are dust, pollen, airborne emission particles, and bird droppings.

A sound maintenance practice is to permanently install monitoring meters between subassemblies and ensure isolation capability. It is also desirable to be able to switch the meters into the circuits, as required (see exhibit 11.1). The cost for the meters and switches and the effort to wire them into the circuits will be well worth the work and extra costs involved when routine monitoring or troubleshooting is required.

The specific gravity of battery electrolyte must be inspected, if possible and applicable, to ascertain the charge of the batteries, where specific gravity equals the ratio of electrolyte density to water density. An immersion thermometer and hydrometer are required. The hydrometer reading should be corrected for temperature using the temperature of the electrolyte solution and not the ambient air temperature. The specific gravity in the batteries should not differ from each other by more than 0.020 . The state-ofcharge of the batteries should be consistent with the season and recent weather conditions. The hydrometer is an instrument for measuring the specific gravity of liquids: it is a graduated, weighted tube that sinks in a liquid up to the point determined by the density of the liquid.

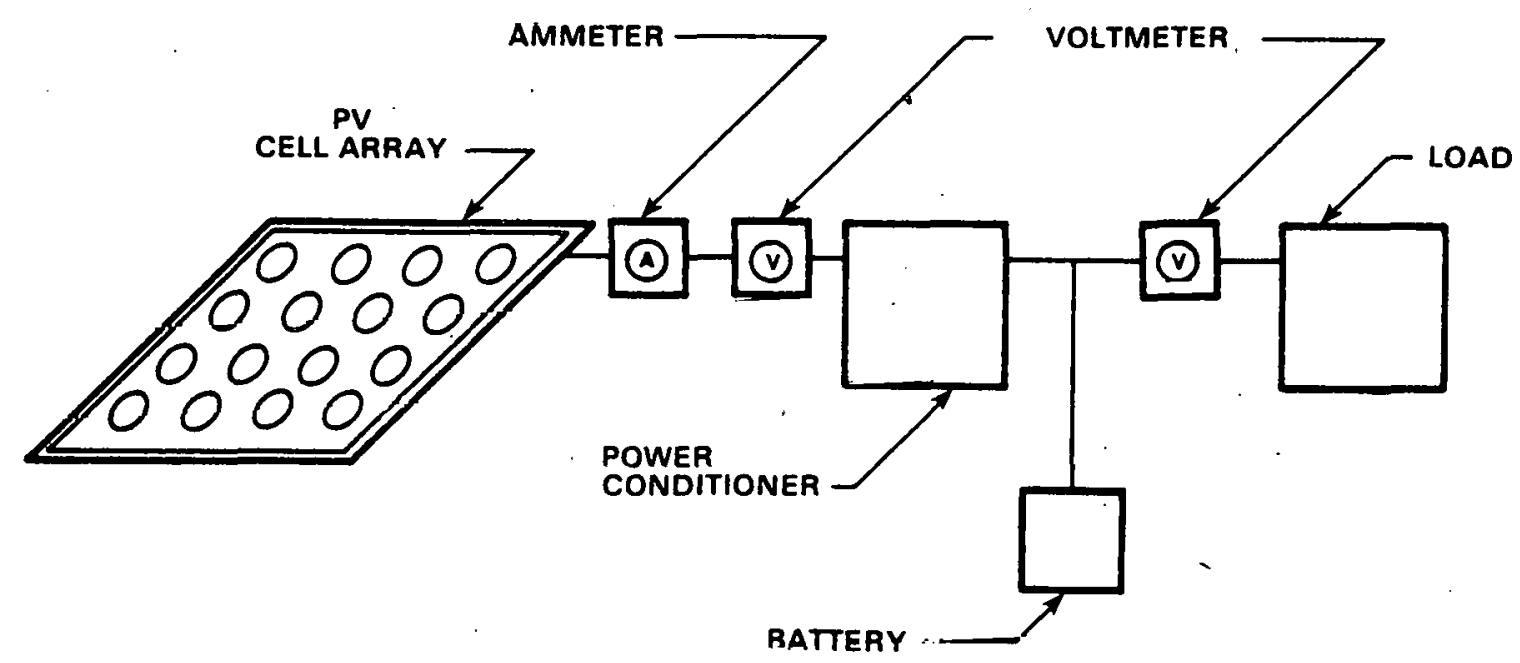

Exhibit 11.1 Proposed Installation for Monitoring/Troubleshooting. 


\subsection{MAINTENANCE AND REMOTE MONITORING WITH TELEMETRY}

Maintenance can be handled through a maintenance contract with the supplier or a local contractor. The cost of such an arrangement is easily determined from quotations. Because high-technology maintenance specialists are not required for most PV systems under most circumstances, the Federal agency will usually handle the maintenance itself.

When the agency performs the maintenance, there is a tradeof $f$ between the frequency of site visits and the use of telemetry systems to radio the condition of the system. For example, if I week of power outage is the maximum tolerable in a remote site, then weekly inspections are necessary. However, if a radio telemetry system is used, inspections may not be needed. Only the repair crew will be required to visit the site and, then, only in the event of a failure. The cost of the inspection labor must be weighed against the cost of telemetry systems. A telemeter is any device for measuring temperature, radiation, etc. at some remote point and transmitting values obtained to a distunt indicator, recorder, or observer.

Telemetry systems can be obtained that cost approximataly $\$ 400$ for trunsmission distances under 0.5 mile, with an additional $\$ 50$ for each mile from the receiver. The telemetry-3ystem sost rins not increase mush with the amount of data transmitted. The simplest system would transmit a constant signal to a munied, ecntral statinn. If the PV system should fail, the signal would be discontinued. The maintenance crew would then be dispatched to the site so the system could be repaired within the allowable downtime. If the telemetry system should fail, the signal would again be'stopped and the maintenance crew would again be sent out for repair. There would be no difficulty in making the signal proportional to the output current or some similar indicator of system performance.

In some applications, such as forest observation stations and American Indian villages, daily inspection is practical because people are near the systems everyday. In other applications, the PV system is powering a transmitter, so the loss of the transmitted signal would indicate failure. Alternatively, the transmitter could be used to signal that the emergency (backup) battery is being used, so the PV system needs attention. In applications such as these, a separate telemetry system is not required. Periodic inspection of the system moy still be desiroble to determine if some element of the system not covered by the telemetry system is not functioning properly. Two visits jer ycar would he recusonable, in light of the past, favorable experience with solar PV systems, If the systems prove sufficiently reliable and durable, the semiannual inspections may alleviate the need of the telemetry system.

In the absence of reliability data, the tradeoff must be made between the cost of a telemetry system and the cost of frequent site visits. The cost of the site visit will be approximately $\$ 20 / \mathrm{hr}$, when wages and overhead are included. The time on site will be approximately 60 minutes. Additional time will be required for travel. Assuming the travel can be accomplished at $40 \mathrm{mph}$, the tradeoff between telemetry and site visits is shown on cxhibit 11.2. Only if the solar-powered system can be out of commission for more than approximately 100 days should personal inspection be used. (Early in the project, weekly inspections may be desirable to learn of any unforeseen problems. However, over the 20year life, the frequent visits have little effect on the life cycle cost tradeoff.) For most unattended, remote systems the telemetry system will be cost effective. 


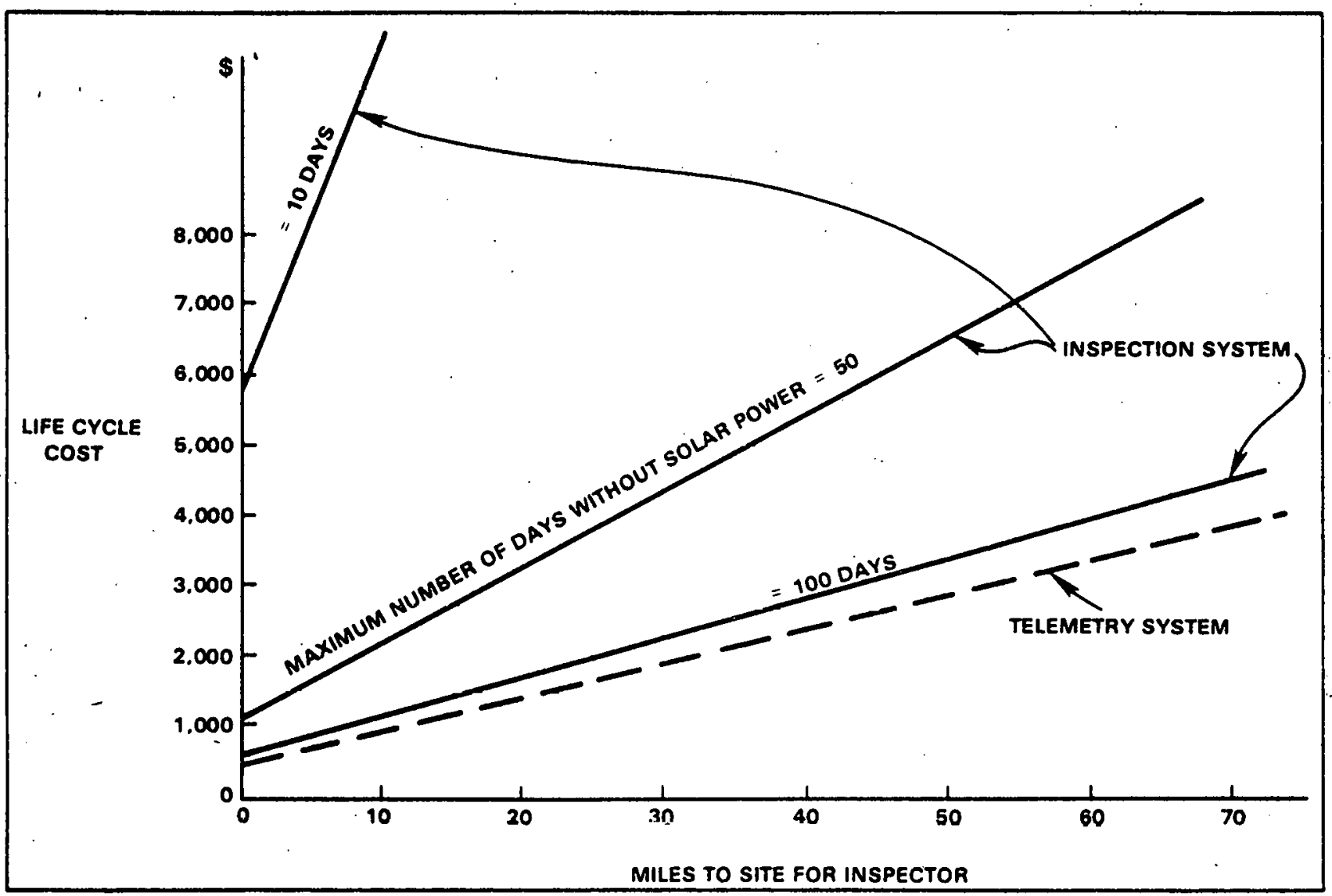

Exhibit 11.2 Comparison of Life Cycle Costs for Telemetry vs. Inspection Systems. 


\subsection{Installation and Maintenance Manual}

\section{TABLE OF CONTENTS}

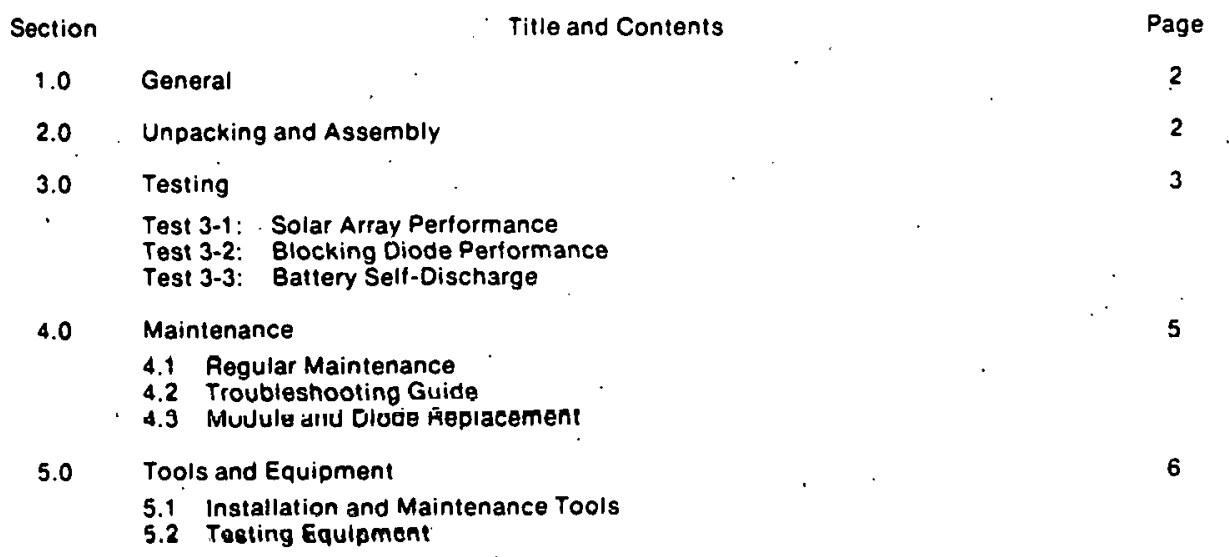

1

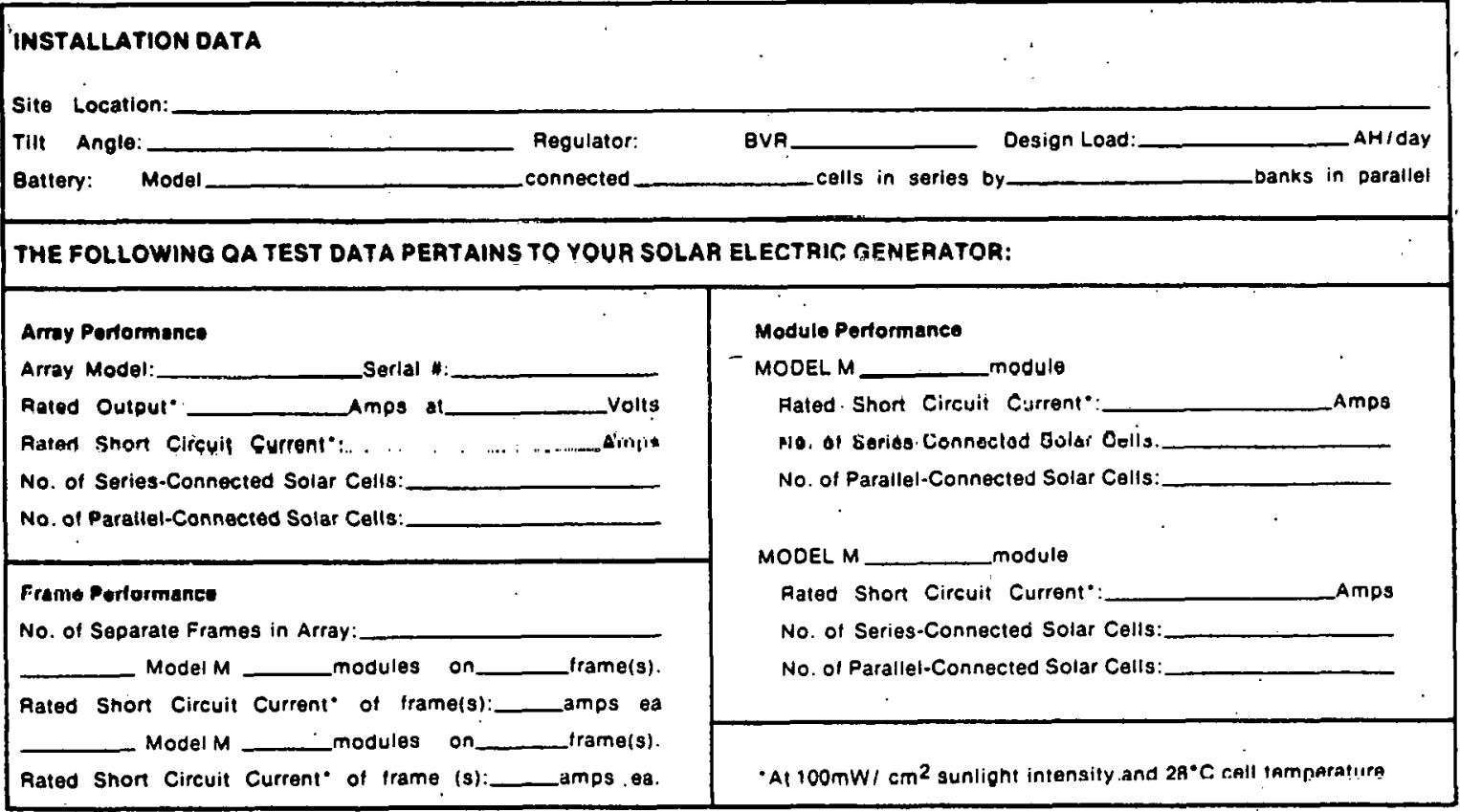


1.0 GENERAL

1.1 This manual includes the unpacking, assembly, and maintenance instructions for the Solar Power Corporation Solar Electric Generator Series M Arrays. For information relating to the installation and maintenance of the storage battery system, refer to Manufacturer's Instructions.

1.2 Properly installed solar electric generator sysiems should only require regular maintenance visits once a year. Maintenance recommendations are given in Section 4.1.

1.3 If any trouble does develop. Sections 3.0 and 4.0 give complete test. troubleshooting, and repair procedures. If additional help is required. contact the Technical Service Department at Solar Power Corporation.

\subsection{UNPACKING AND ASSEMBLYINSTRUCTIONS}

2.0.1 Because the arrays may be anchored to different types of mounting surfaces. the customer is expected to supply mounting hardware.

2.0.2 If the tolal array consists of more than one frame. repeat all instructions for each frame.

\subsection{UNPACKING AND ASSEMBLY.} ARRAYS WITH TELESCOPING LEGS

2.1.1 Open the crate. Remove the layer of packing material and any other hardware or items that are on top of the array.

2.1.2 Lift the array out of the crate and hold it nearly vertical or place it on the ground, front module surface facing uP. DO NOT PUT THE ARRAY ON THE GROUND FACING DOWN
2.1.3 Unbolt the mounting feet from the leg sections.

Retain this hardware and all other hardware removed in the following steps: it will be needed to assemble the legs and mounting feet to the array frame (reference Figure $1 \mathrm{~b}$ ).

2.1.4 Unbolt the two large leg sections (larger cross sectional area) from the bottom mounting brackets. Attach one mounting foot to one end of each of these leg sections as shown in Figure 1. Point C. It may be necessary to loosen the bolt holding the small leg to the top mounting bracket. thus allowing the leg to swing.

2.1.5 Altach the remaining two mounting feet to the bottom mounting brackets (the ones that do not have leg sections attached to them) as shown in Figure 1. Point $D$.

2.1.6 The array is now fully assembled and ready to be moved to its installation location and oriented. If the array must be disassembled or recrated, reverse the above procedure.

\subsection{ORIENTING THE ARRAY .} ARRAYS WITH TELESCOPING LEGS

2.2.1 When selecting a mounting location, make sure that the bottom of the array will be at least 3 feet (or 1 meter) higher than the maximum snow depth level.

2.2.2 IMPORTANT: THE ARRAY MUST BE ALIGNED SUCH THAT THE FRONT (MODULE) SURFACE DIRECTLY FACES DUE SOUTH (DUE NORTH IN THE SOUTHERN HEMISPHERE). WHEN USING A MAGNETIC COMPASS MAKE SURE TO CORRECT FOR THE LOCAL DIFFERENCE BETWEEN MAGNETIC DIRECTION AND TRUE DIRECTION. Anchor the front mounting leet once the array is aligned.

FIGUAE 1

(1) (A)


2.2.3 Anchor the legs' mounting feet to the mounting surface. It is best to make support spacing (B) equal to array dimension ( $A$ ) (reference Figure $2 b$ ). Other positions may be used as necessary depending on the angle required and / or terrain considerations.

2.2.4 To set the tilt angle of the array, remove the long bolts anchoring the leg sections logether (reference Figure 1. Point $B$ ) and adjust the length of the telescoping legs. The tilt angle of the array (the angle the array surface makes with a horizontal surface) should be adjusted to within 2 degrees of the specified angle. An inclinometer (adjustable angle liquid (evel) is most useful in measuring this angle, although, with care, a protractor and an ordinary bubble level may aiso be used. Reinsert the bolts and tighten.

2.2.5 Tighten all nuts and bolts.

FIGUAE 2

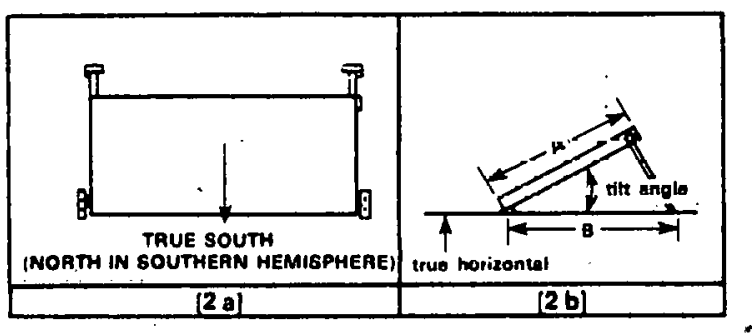

2.3 ATTACHMENT OF CAELES

2.3.1 If a battery voltage regulator is included in the system or if one is added to the system, follow the instructions included with the regulator.

2.3.2 Single Freme Arrays: The output cable can be attached directly to the battery. Observe the correct polarity; black is positive, white is negative (reference Figure 4). If the polarity is accidentally reversed, no damage will result to either the array or the battery (assuming the battery is of the proper voltage for the array). However, if the polarity is left reversed for more than a few hours, the solar electric generator system will not function and the battery may become discharged.

2.3.3 Multiframe Arrays: Each frame is supplied with a separate output cable. Attach the output cable directly to the appropriate battery terminal (observe correct polarity).

2.3.4 After connecting cable(s) to the battery and connecting any required battery interceil connecting wires, protect all battery terminals from corrosion with a layer of grease.

FIGURE 3

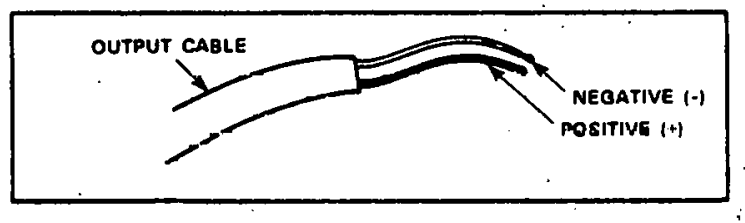

3.0 TESTING

There are several tests that can be conducted to check system performance: Test 3-1 (Solar Array Performance) Test 3-2 (Blocking Diode Performance), Tost 3-3 (Battery Selt-Discharge). These can be performed either independently or in conjunction with the Troubleshooting Guide, Section 4.2.
Upon installation, Test 3-1 (Solar Array Performance) should be conducted. Tests 3-2 and 3-3 should be performed if trouble occurs.

The annual maintenance visit can include the following simple check of the solar electric generator system performance. Measure the specific gravity of the battery electrolyte (for lead-acid batteries) with a standard battery hydrometer. Correct the readings to $77^{\circ} \mathrm{F}\left(25^{\circ} \mathrm{C}\right)$ using Table 3-1. Refer to Table 3-2 and relate the percent of battery capacity remaining to the corrected electrolyte specific gravity. If battery electrolyte specific gravity is low, refer to Section 4.2, Conditions 1 and 2.

\section{TEST 3-1: SOLAR ARRAY PERFORMANCE}

1. This test must be performed during the middle hours of a sunny day. The sun must be clearly visible with no thick haze present.

2. Disconnect array cable(s) or voltage regulator-battery cable from the battery terminals. If the system contains a rogulator(b), disconnect regulator(s) following instructions provided with regulator(s) before proceeding to the nert stan.

3. Connect a suitable ammeter across the two disconnected cable leads (reference Figure 4). The ammeter's resistance should be such that the voltage drop across the ammeter is less than 0.3 volt. Adjust the tilt angle of the array to obtain the maximum current output as indicaled by lite ammeter.

FIGURE 4

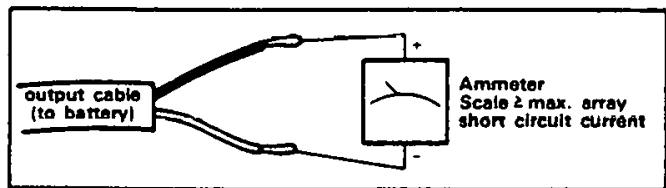

4. The ammeter current reading (short circujt current) should be approximately $70 \%$ of the $100 \mathrm{~mW} / \mathrm{cm}^{2}$ short oircuit current (listed under "Array Performance" on the front cover').

5. Disconnect the ammeter. Connect across the same cable leads (reference Figure 5) a voltmeter having an impedance of at least $1,000 \mathrm{ohms}$ per volt. The volt . mater reading (open cirsuit voltage) should be greater than 0.48 volt times the total number of solar cells in series (listed under "Array Performance" on the front cover).

FIGURE 5

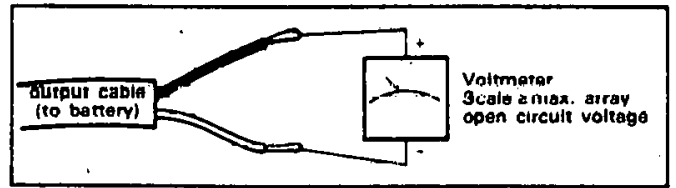


6. Disconnect the voltmeter. Reconnect the negative lead to the battery. Connect an ammeter between the positive cable lead and the positive battery terminal (positive ammeter lead to the positive cable lead). Connect the voltmeter to the iwo cable leads (reference Figure 6 ). If this voltage is less than 2.2 volts times the number of series-connected lead-acid battery cells, the measured current should be at least $80 \%$ of the current measured in Step 4 (assuming sunlight conditions unchanged since Step 3).

FIGURE 6

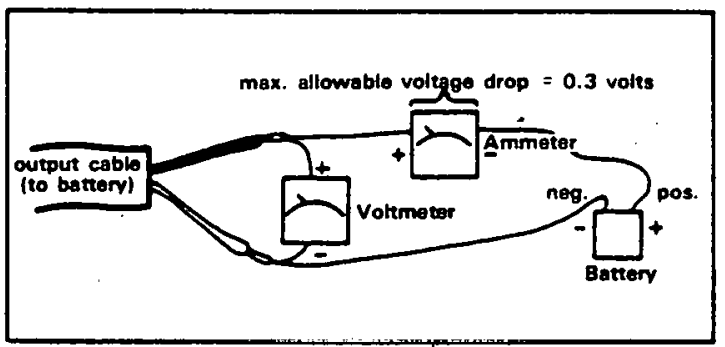

7. For multiframe arrays this test procedure can be repeated for each individual array section by making these tests at each individual array cable termination. For each

individual array section, disconnect the cable leads from the terminal block inside the external junction box and repeat Steps 3 through 6 . The corresponding information for each frame is listed on the front cover of this manual (reference "Frame Performance").

8. If an array does not pass this test, refer to the Troubleshooting Guide, Section 4.2.

TABLE 3-1

HYOROMETER AEADING CORRECTIONS TO $77^{\circ} \mathrm{F}$

\begin{tabular}{|c|c|}
\hline $\begin{array}{c}\text { Electrolyte Temporature } \\
\text { ['F] }\end{array}$ & $\begin{array}{c}\text { Correction } \\
\text { [add to reading] }\end{array}$ \\
\hline 140 & +0.024 \\
130 & +0.020 \\
120 & +0.016 \\
110 & +0.012 \\
100 & +0.008 \\
90 & -0.004 \\
\hline 80 & +0.000 \\
\hline 70 & -0.004 \\
60 & -0.008 \\
50 & -0.012 \\
40 & -0.016 \\
30 & -0.020 \\
20 & -0.024 \\
10 & -0.028 \\
\hline 0 & -0.032 \\
\hline-10 & -0.036 \\
-20 & -0.040 \\
-30 & -0.044 \\
-40 & -0.048 \\
\hline
\end{tabular}

NOTE: The temperature of the electrolyte solution. not the ambient air temperature, should be measured with an immersion type thermometer. Some hydrometers have a thermometer and temperature correction scale built in.
TABLE 3-2

PERCENT OF. 500 HOUR RATE CAPACITY REMAINING

ELECTROLYTE SPECIFIC GRAVITY (CORRECTED TO $77^{\circ} \mathrm{F}$ )

\begin{tabular}{|c|c|c|c|}
\hline \multirow{2}{*}{} & \multicolumn{3}{|c|}{$\begin{array}{c}\text { Initial Electrolyto } \\
\text { Specitic Gravity }\end{array}$} \\
\cline { 2 - 4 }$\%$ Capacity & \multicolumn{3}{|c|}{ Hydrometer Reading } \\
\cline { 2 - 4 } Remaining & 1.210 & 1.250 & 1.300 \\
\hline 100 & 1.210 & 1.250 & 1.300 \\
90 & 1.197 & 1.235 & 1.283 \\
80 & 1.185 & 1.221 & 1.266 \\
70 & 1.172 & 1.206 & 1.249 \\
60 & 1.160 & 1.192 & 1.232 \\
\hline 50 & 1.147 & 1.177 & 1.215 \\
40 & 1.135 & 1.163 & 1.198 \\
30 & 1.122 & 1.148 & 1.181 \\
20 & 1.110 & 1.134 & 1.164 \\
10 & 1.097 & 1.119 & 1.147 \\
0 & 1.085 & 1.105 & 1.130 \\
\hline
\end{tabular}

TEST 3-2: BLOCKING DIODE PERFORMANCE

1. This test must be performed either at night with no. artificial light striking the array or with a black opaque cloth covering the entire array (reference Figure 7).

2. Disconnect the positive lead of the array cable(s) or the voltage regulator-battery cable from the battery terminals. Connect a milliammeter between this disconnected lead and the positive battery terminal (positive milliammeter lead to the positive battery terminal) (reterence Figure 7 ). The current measured should be less than $4 \mathrm{~mA}$ times the number of solar cells connected in parallel (listed under "Array Performance" on the front cover).

3. A current exceeding the above value indicates that the diode(s) has developed excessive reverse leakage current. If the array contains a diode mounted in a junction box, it should be replaced (reference Section 4.3). If the system includes a voltage regulator(s), refer to Regulator Manual for testing procedure. Otherwise, the diode(s) is located inside the module(s) and this test should be repeated. for each module on the affected frame. Access to each module's leads may be obtained by removing the attached junction box cover. Disconnect the leads at the terminal block before starting the test. Remember that no light can strike the module's surface. Diodes located in the terminal box attached to the back of each module are sealed and cannot be replaced in the field. Any module that shows excessive reverse leakage current should be replaced (reference Section 4.3 ).

\section{TEST 3-3: BATTERY SELF-DISCHARGE} [LEAD-ACID BATTERIES]

NOTE: THIS TEST WILL REQUIRE REMOVAL OF THE BATTERY STSTEM PROM THE AARAY SITE.

1. Disconnect all cables from the battery terminals. Charge the battery or battery cell at a current rate not exceeding the battery's capacity in ampere hours divided by 20 hours (e.g., a 100-ampere hour battery would be charged at a current of 5 amperes or less). A standard battery charger should suffice for this purpose. Discontinue charging when the battery's terminal voltage exceeds 2.3 volts per series-connected battery cell.

2. Take a specific gravity reading of the electrolyte in each baltery cell and record the corrected values (use Table 3-1 and an immersion thermometer). 
3. Allow the baltery to stand idie at room temperature for a week At the end of the week take a seccnd sel of specific gravily readings. Compare with readings taken in Slep 2. Correcled readings diflerıng by more than 15 points 10.015 indicate a battery cell with excessively high selt. discriarge.

4.0 MAINTENANCE

4.1 REgULAR MAINTENANCE

(Yearly intervals recommended.)

41.1 Check oattery electrolyte level. Replenish with distilled water. If necessary. When checking or adding to the battery electrolyte. the battery manufacturer's recommendations should be lollowed.

4.1.2 Check the module surface(s) lor dirt buildup. Normal rainfall will usually be sufficient to provide for self-cleaning. It the array is lilted at $15^{\circ}$ or more from the horizontal.

However. il dirt buildup becomes excessive. either plain water or a mild detergent solution followed by a water rinse may be used. DO NOT USE SOLVENTS OR STRONG DETERGENTS.

\subsection{TROUBLESHOOTING GUIDE}

Most problems can be isolated with the aid of the tollowing guide. If it is impossible to locate the problem. please contact the Technical Service Department at Solar Power Corporation for assistance.

\section{SYMPTON \\ Battery electrolyte specific gravity low \\ Other Symptoms \\ Specitic gravities of all battery cells differ no more than 20 points $(0.020)$}

\section{Checks and Repairs}

1. Check all battery electrical connections for corrosion and mechanical soundness. Clean and/or repair.

2. Check to see if there are any obstructions that shadow any portion of the array during any part of the day. If this condition exists, either the obstruction must be removed or the array must be moved to an unobstructed location.

3. Check the orientation of the array. Make sure it is facing directly due south (north in the southern hemisphere) and the tilt angle is correct (reference Section 2.2 or 2.3).

4. Check the load current. Calculate the equivalent number of amp hours per day required by the load. Compare this calculation against the design load listed under "Installation Data" on the front cover. If the measured load exceeds the design load. contact the Technical Service Department at Solar Power Corporation. Each solar electric generator system is designed for a specific load. Deviations trom that load may result in unsatisfactory operation.
5. Check the solar array output by following the instructions in Test 3-1. Refer to Conditions 3, 4, 5, or 6 (Section 4.2) as necessary.

6. Check the blocking diode(s) by following the instructions in Test 3-2.

7. Check tor high battery sell-discharge oy following the instructions in Test 3-3. If the baltery or part of the total baltery system lails'inis test. replace the defective battery cell(s).

\section{SYMPTOM Other Symptoms \\ Same as Specific gravity of \\ Condition 1 only one or a few} battery cells low

\section{Checks and Repairs}

1. Check for excessively high electrolyte level. If so. sheller battery to prevent rain from entering Inrough the vent hole(s).

2. Check the affected cells for high battery self-discharge by following the instructions in Test 3-3. Replace battery cell or battery containing bad celt.

\section{SYMPTOM}

\section{Array open circuit}

voltage equal to

zero [from Test 3-1]

Checks and Repairs

1. Single Frame Arrays:

(a) If the array consists of only one module. that module must be replaced (reference Section 4.3).

(b) If the array consists of more than one module, remove the cover of the junction box mounted to the back of the array. With the output cable disconnected from the battery terminals, test for voltage at the individual module leads. If voltage is present, there are bad contacts. At the terminal block, or the crimp connectors attached to the output cable are not making contact to the wire, or the output cable's conductors are broken. Clean all connections. Test crimp connectors by pulling on wires. Recrimp or attach wire directly to terminal block if necessary. Test the cable with an ohmmeter or continuity tester. Replace output cable if it is an open circuit.

\section{Multiframe Arrays:}

(a) Remove junction box cover. Check for loose connections at the terminal block. Tighten if necessary.

FIGUAE 7

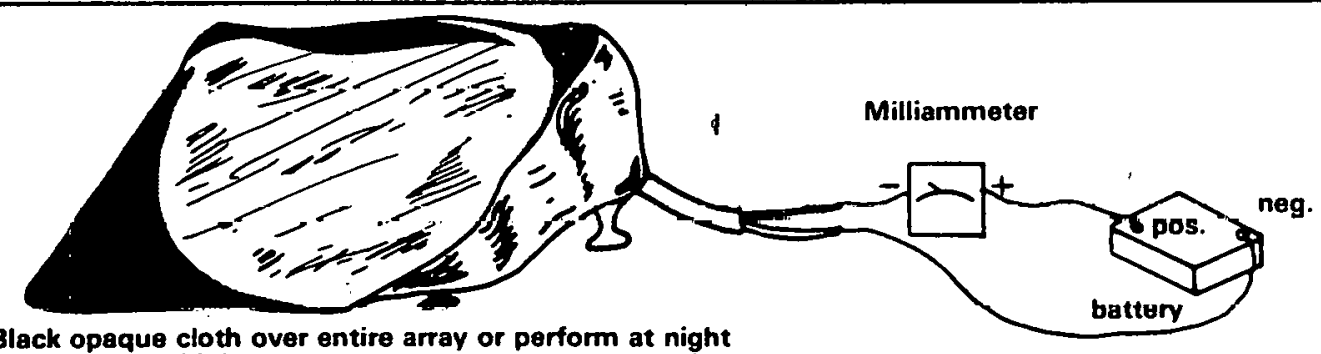

Black opaque cloth over entire array or perform at night with no light shining on arroy. 
(b) Test for voltage at the individual array cable terminations. Il voltage is present there. proceed to Step 2 (c). If no voitage is present at any of the cable terminations, each array section must be checked individually as described in Step $1(b)$.

(c) Make sure that at least one lead of the battery cable is disconnected from the battery terminals. Connect a jumper wire between any positive array cable terminal and the positive battery cable terminal. If voltage is now present at the battery cable leads. and there is a blocking diode within an external junction box. either the blocking diode is defective or one of the wires connecting the diode to the terminal block is broken. Detach the plate on which the terminal block is mounted by removing the four corner screws. The blocking diode is located beneath the plate. inspect for any broken wires and if none are lound. replace the diode (reference Section 4.3).

(d) Check the continuity of the battery cable with an ohmmeter or continuity tester. Replace output cable if it is an open circuit.

\section{SYMPTOM}

Array open circuit

voltage low firom

Test 3-1]

Checks and Repairs

1. Check that the voltmeter's resistance is greater than 1,000 ohms per volt, that the sun is clearty visible, that there is no thick haze blocking the sun, and that the array is aimed lowards the sun.

2. Single Frame Arrays:

(a) If the array consists of only one module, that module should be replaced (reference Section 4.3).

2. (b) If the array consists of more than one module, remove the cover of the junction box mounted in the back of the array. Disconnect the cable leads from each module. Test each module individually for low open circuit voltage. The voltmeter reading (open circuit voltage) should be greater than 0.48 volt times the number of solar cells in series (listed under "Module Performance" on the front cover). Any module that does not pass this test should be replaced (reference Section 4.3).

3: Multiframe Arrays: Disconnect the array cables from the terminal block in the external junction box or from the battery terminals. Check the ope- circuit voltage at each individual cable pair of wires to isolate the affected array section. The voltmeter reading (open circuit voltage) should be greater than 0.48 volt times the number of solar cells in series (listed under "Array Pertormance" on the front cover). To locate the defective module in the array section isolated above, follow the instructions in Step 2(b)

\section{SYMPTOM}

Array short circuit

current low [from

Tesi 3-1]

\section{Chocks and Rapairs}

1. Check for dirt buildup on any module or portion of a module. Clean according to Section 4.1.2.

2. Check for condensation, snow, or ice on module or any portion of a module. Wipe clean.

3. Check for shading of any module or portion of a module. Retest after removing obstruction.

4. Make sure array is aimed directly at the sun. Retest after rnrrerting tilt

5. Single frame arrays or when the problem is isolated to a single array frame (reference Step 6): Remove the attached junction box confer. Test each individual module for short circuit current as described in Test 3-1. Steps 3 and 4. Compare these values to the short circuit current values listed on the front cover under "Moduie Performance". Replace any module (reference Section 4.3) which fails Test 3-1. Make sure all connections in the junction box are tight and clean.

6. Muitiframe Arrays: Perform Test 3-1 for each array section (cable) to determine the faulty section. Check for any loose terminals or broken wires within an external junction box. Also check all connecrors. II driy. lor corrosion and tight mating of the male and female contacts. Clean or replace as necessary.

\section{SYMPTOM}

Excessive dillerence between array short circuit and battery charging current [Irom

charging
Test 3-11]
Checks and Aepairs

1. Check for corrosion at the battery terminals. Clean terminals arid cable leads. Retest.

2. If the array has a junction box(es) (either internal or external), remove the cover(s) and inspect for corrosion on all electrical connections within the box. Clean or replace, damaged components. Retest.

3. Test each module individually as described in Sleps 3.4 and 6 of Test 3-1: Compare these values to the short circuit current values as listed on the front cover under "Module Performance". Replace any module(s) that fail Test 3-1 (reference Section 4.3).

\subsection{MODULE AND DIODE REPLACEMENT}

When it has been determined that a module or a blocking diode needs replacing. proceed as follows:

4.3.1 Replacement of Module

(a) Remove cover of junction box attached to array frame.

(b) Disconnect at the terminal strip the cable leads of the module being replaced.

(c) Loosen the threaded gland of the cable fitting through which the module cable enters the junction box. Pull the end of the cable out of the junction box.

(d) Remove the nuts and bolts holding the module onto the array frame. Lift off the module: save the hardware removed.

(e) Insertion of Replacement Module: Reverse the removal procedure: (a) through (a) above.

4.3.2. Replacement of Blocking Diode Located in Junction Box

(a) Disconnect battery cable at battery terminals.

(b) Remove the junction box cover and the metal plate on which the terminal block is mounted. Loosen or remove cable leads from the terminal block, if necessary.

(c) The diode will usually be mounted on a heat sink Make a sketch showing which lead goes to which terminal and how the hardware is assembled. Unsolder the leads to the diode. Remeve the diode.

(d) Insert the new diode (exact same number as the diode being replaced). Take care to replace the hardware in the same order as was on the removed diode.

(e) Solder the leads to the new diode. Take care that the leads go to the same terminals as on the removed diode. (Array positive lead to anode: battery positive lead to catnode.)

(f) Replace the metal plate and the junction box cover.

(g) Reconnect the battery cable to the battery terminals.

4.3.3. Replacement of Blocking Diode Located in Module

$\wedge$ blocking dinde within the module cannot be replaced in lield. The module should be removed from the array irame (relerence Section 4.3.1,a-d) and relurned to Solar Power corporation tor repair.

\subsection{TOOLS AND EOUIPMENT}

5.1 INSTALLATION \& MAINTENANCE TOOLS

Ratchet Handle (3/8" or $1 / 2$ " drive)

6 " Extension

112 " Socket and Wrench

$9 / 16$ " Socket and Wrench

$7 / 16$ " Socket and Wrench

$3 / 4^{\prime \prime}$ Socket and Wrench

Screw Urivers $(1 / 8$ " (1 $1 / 2 "$ "wide)

Slip Joint Pliers (11/2" diameter grip)

Locking Pliers

Crimping Tool (VACO 22-10 or equivalent) and

assorted crimp terminars

Wire Strippers

Diagonal Cutters (medium)

Compass (magnetic)

inclinometer

5.2 TESTING EOUIPMENT

Simpson 260 VOM (or equivalent)

immerslon Thermometer

Battery Hydrometer 


\section{APPENDIX II.A \\ PERFORMANCE MEASUREMENT PROCEDURES}

\section{A.I ELECTRICAL PERFORMANCE}

Electrical performance measurements shall be referenced to SOC defined as 100 $\mathrm{mW} / \mathrm{cm}^{2}$ irradiance, air-mass 1.5 , NOCT. All procedures, equipment, and standards related to measurements shall conform to the latest revision of date of the contract of NASA TM: 73702, Terrestrial PV Measurement Procedures, dated June 1977. Manufacturer's conformance to this procedure is subject to acceptance by the owner. If a reference cell is used, it shall be traceable to NBS and shall be the only irradiance reference used.

To provide for efficient modile testing, module pcrformance may be Lused on module output at either SOC conditions or at Optional Test Conditions (OTC) definen ns $100 / \mathrm{mW} / \mathrm{cmin}^{2}$ ir made at OTC the power output $(P)$ at NOCT cell temperature shall be determined as follows:

$$
P=V_{\text {NO }}\left(l_{\text {OTC }}+\Delta l\right),
$$

where

$$
\begin{aligned}
& V_{N O}=\text { Module nominal operating voltage at NOCT. } \\
& \mathrm{I}_{\mathrm{OTC}}=\text { Module current measured at OTC and at a voltage } \\
& \text { equal to }\left(V_{N O}+\Delta V\right) \text { volts. } \\
& \Delta \mathrm{I}=\text { Current Tempernture Correction (delta 1). } \\
& =1 @\left(\text { NOCT, } V^{\prime}\right)-1 @\left(\text { OTC, } V^{\prime}=V^{\prime}+\Delta V\right) \text {. } \\
& \Delta V \text { - Vülluge Temperature Correction (delta V). } \\
& =\quad V @\left(O T C, I=I^{\prime}-\Delta I\right)-V @(\text { NOCT, I'). }
\end{aligned}
$$

NOCT

\section{OPEN CIRCUIT}

\section{CLEAN}

$T_{\text {CELL }}=T_{\text {AMB }}+R * S+$ EXHIBIT A-3 CORRECTION

DETERMINE R EXPERIMENTALLY

CORRECT TO NOMINAL $T_{\text {AMB }}$ AND $S$

GEOMETRY: TILTED, 2 ft ABOVE GROUND, WIND NOT EAST OR WEST

Exhibit A.1 Performance Measurement Procedure. 


$$
\begin{aligned}
& V^{\prime}=\text { Voltage }=0.6 V_{o c} @ \text { NOCT. } \\
& \prime^{\prime}=\text { Current }=0.9 \mathrm{I}_{\mathrm{sC}} @ \text { NOCT. }
\end{aligned}
$$

Determination of the temperature correction factors, $\Delta l$ and $\Delta y$, in the above equation shall be based on actual measurements of a minimum of 5 production modules at both OTC and.NOCT $+2^{\circ}$ cell temperature. The current-voltage (I-V) characteristics of each module shall be measured at both conditions. The corresponding $1-V$ curves for the two temperatures may then_be overlayed to determine the correction factors. A simultaneous translation of the curves along both current and voltage axes may be made until an accurate match of the curves is accomplished at two points near the maximum power point. The OTC curve should match the NOCT curve at a point where the NOCT current is approximately 90 percent of $I_{\text {SP }}\left(l^{\prime}\right.$, above), and at a second point where the NOCT voltage is approximately 60 percent of $S \mathcal{S}$ ( $V$ ', above). The current and voltage shift required to produce the curve match shall be determined for the exact cell temperature difference between tests. The change per degree $C$ for each factor is then calculated and multiplied by the difference between NOCT and the temperature used for OTC. The resulting $\Delta \mathrm{I}$ and $\triangle \mathrm{V}$ shall be averaged for the modules tested to establish temperature correction factors to be used when testing modules at other than SOC. Alternate temperature correction procedures such as that provided by a computer controlled, Large Area Pulsed Solar Simulator (Xenon source) may be used if approved by the owner.

\section{A.2 NOCT}

\section{A.2.1 Purpose}

The purpose of this test is to acquire sufficient dato to make an accurate determination of the NOCT's of a terrestrial module's solar cells. By definition, NOCT is the module cell temperature under operating conditions in the nominal thermal environment (NTE), which is defined as:

Insolation $=80 \mathrm{~mW} / \mathrm{cm}^{2}$

Air temperature $=20^{\circ} \mathrm{C}$

Wind average velocity $=1 \mathrm{~m} / \mathrm{s}$

Mounting = tilted, open back, open circuit.

The NOCT test procedure is based on gathering actual measured cell temperature data via thermocouples attached directly to the cells of interest, for a range of environmental conditions similar to the NTE. The data are then presented in a way that allows accurate and repeatable interpolation of the NOCT temperature.

\section{A.2.2 Determination of NOCT}

The temperature of the solar cell $\left(T_{c_{e}}\right)$ is primarily a function of the air temperature $\left(T_{\text {gir }}\right)$, the average wind velocity $(V)$, and the total solar insolation $(L)$ impinging on the acfive side of the solar array module. The approach for determining NOCT is based on the fact that the temperature difference $\left(T_{c e l l}-T_{a i r}\right)$ is largely independent of air temperature and is essentially linearly proportional foll the insolation level. Analyses indicate that the linear assumption is quite good for insolation levels greater than about $40 \mathrm{~mW} / \mathrm{cm}^{2}$. The procedure calls for plotting ( $T_{\text {cef }}-T_{\text {air }}$ ) against the insolation level for a period when wind conditions are favorable. The FUCT is then determined by adding $T$ air $=20^{\circ} \mathrm{C}$ to the value of ( $T_{\text {cell }}-T_{\text {aif }}$ ) interpolated for the NTE insolation level of $80 \mathrm{~mW} / \mathrm{cm}^{2}$, i.e., $N O C T=\left(T_{\text {cell }}\right.$ - Tair gNTE + $20^{\circ}$ C. 
The plot of ( $T_{\text {cell }}-T_{\text {gir }}$ ) versus I shall be determined by conducting a minimum of two field tests in which fhel modure being characterized is tested under terrestrial environmental conditions approximating the NTE in accordance with the testing guidelines which follow. Each test shall consist of acquiring a semicontinuous record of $\left(T_{\text {cell }}{ }^{-} T_{\text {air }}\right.$ ) over a 1- or 2day period, together with other measurements, as required, to characterize the terrestrial environment during the testing period. Acceptable data shall consist of measurements made when the average wind velocity is $1 \mathrm{~m} / \mathrm{s} \pm 0.75 \mathrm{~m} / \mathrm{s}$ and with gusts less than $4 \mathrm{~m} / \mathrm{s}$ for a period of 5 minutes prior to and up to the time of measurement. Local air temperature during the test period shall be $20^{\circ} \mathrm{C} \pm 15^{\circ} \mathrm{C}$. Using only acceptable data as so defined, a plot shall be constructed from a set of measurements made either prior to solar noon or after solar noon which defines the relationship between $\left(T_{\text {cell }}-T_{\text {air }}\right)$ and the insolation level (L) for $L \geq 40 \mathrm{~mW} / \mathrm{cm}^{2}$.*

When ( $T_{\text {cell }}-T_{\text {gir }}$ ) is plotted as a functlon of $L$ for average wind velocities less than $1.75 \mathrm{~m} / \mathrm{s}$, results similar to those shown in exhihit A.2 are obtained. For the dulu sliown, the local air temperature was $15.6^{\circ} \mathrm{C} \pm 4.5^{\circ} \mathrm{C}$ and the wind speed varied from zero to less than $4 \mathrm{~m} / \mathrm{s}$ with an average of $1 \mathrm{~m} / \mathrm{s}$. Using the plot of $\left(T_{\text {cell }}-T_{\text {gir }}\right)$ vs. $L$, the value of $\left(T_{\text {cell }}-\right.$ $T_{\text {gir }}$ ) at NTE is determined by interpolating the average value of ( $T_{\text {ce }}-T$ air ${ }$ for $L=80$ $\mathrm{mW} / \mathrm{cm}^{2}$. Using the data in exhibit A.2 as an example, ( $T_{\text {gell }}-T_{\text {gir }}$ ) af $V$ TE is determined to be $20.2^{\circ} \mathrm{C}$. The preliminary value of NOCT is thus $20.2^{\circ} \mathrm{Eell}+20^{\circ} \mathrm{qu}=40.2^{\circ} \mathrm{C}$.

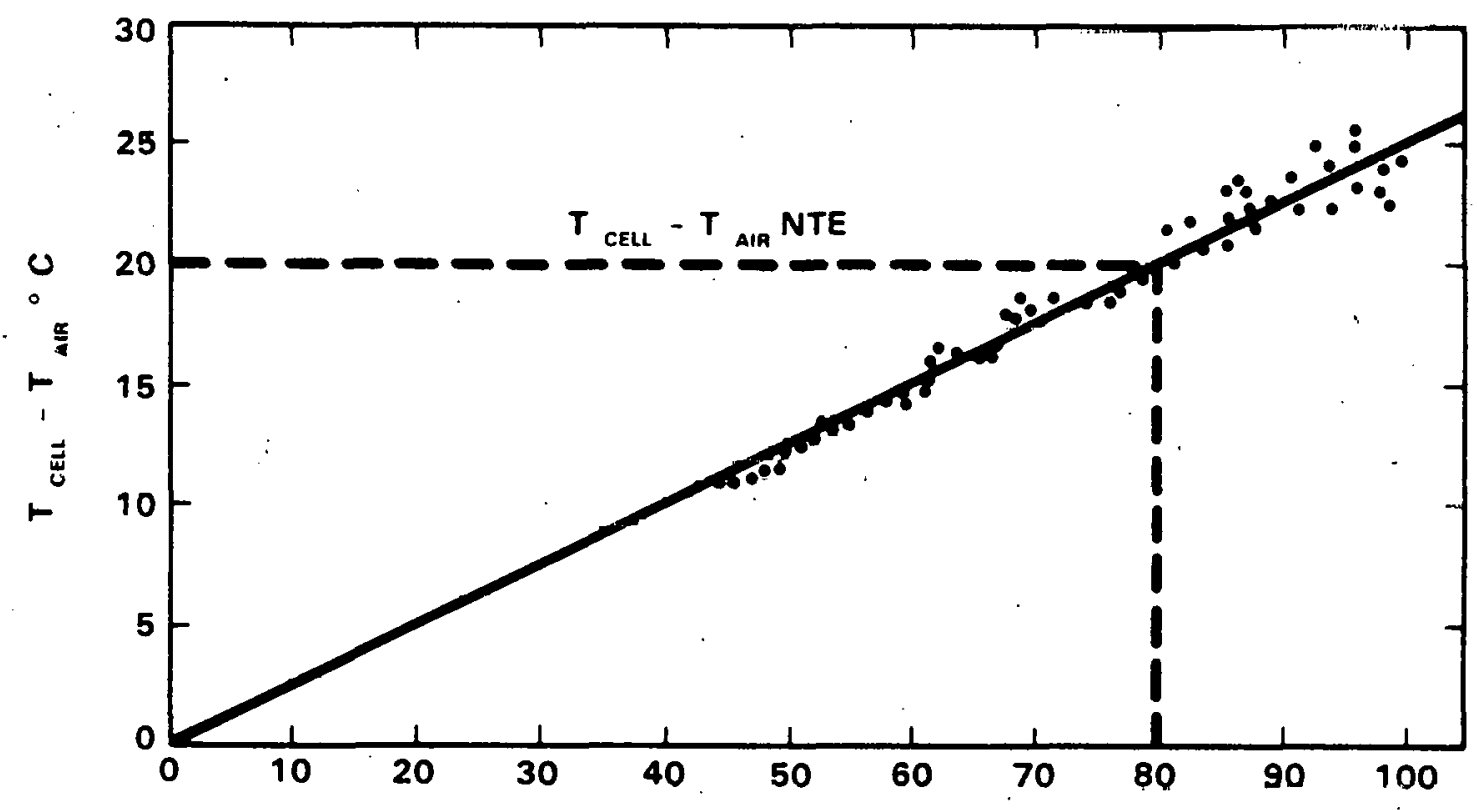

INTENSITY, $\mathrm{mW} / \mathrm{cm}^{2}$

\section{Exhibit A.2 Typical Cell Temperature Data.}

* The two sets of measurements can be combined into a single set provided the overage air temperature of the two sets does not differ by more than approximately $5^{\circ} \mathrm{C}$. If the average air temperature is significantly different, the resulting effect appears as an increase in the scatter of the plotted data. As a result the data will be more difficult to fit and a less accurate result is possible. 


\section{A.2.3 Air Temperature and Wind Correction}

A correction factor to the preliminary NOCT for overage air temperature and wind velocity is determined from exhibit A.3. This value is added to the preliminary NOCT and corrects the data to $20^{\circ} \mathrm{C}$ and $1 \mathrm{~m} / \mathrm{s}$. T air and $\bar{V}$ are the average temperature and wind velocity for the test period.

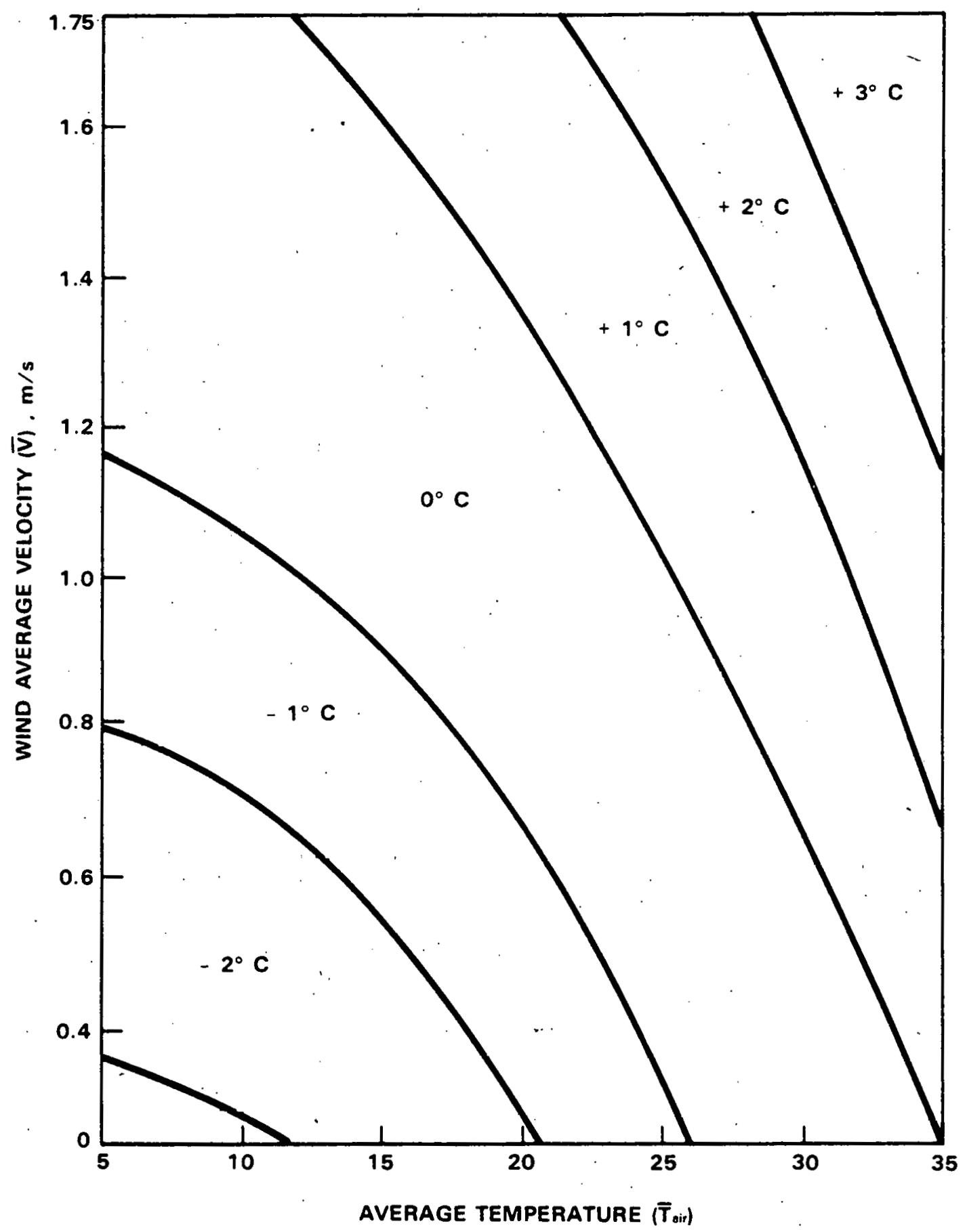

Exhibit A.3 NOCT Correction Factor. 
For the test data shown in exhibit A.2, $\bar{\nabla}$ is $1 \mathrm{~m} / \mathrm{s}$ and $\vec{T}_{\text {in }}$ is $15.6^{\circ} \mathrm{C}$. From exhibit A.3, the correction factor is $0^{\circ} \mathrm{C}$. The NOCT is, therefore, $40.2^{\text {aik. }}$.

\section{A.2.4 Test Geometry}

a. Tilt Angle. The plane of the module shall be positioned so that it is normal to the sun $\left( \pm 5^{\circ}\right)$ at solar noon.

b. Height. The bottom edge of the module shall be 2 feet or more above the local horizontal plane or ground level.

c. Panel Configuration. The module shall be located in the interior of a $1.2 \mathrm{~m} \times 1.2$ $m\left(4^{\prime} \times 4^{\prime}\right)$ panel. Black aluminum panels or other modules of the same design shall be used to fill in any remaining open area of the panel structure. The back of the panel shall be exposed.

d. Surrounding Area. There shall be no obstructions to prevent full irradiance of the module beginning a minimum of 4 hours before solar noon and up to 4 hours after solar noon. The ground surrounding the module shall not have a high snlnr reflestance and shall be flat and/or sloping away from the test fixture. Grass and various types of ground covers, blacktop, and dirt are recommended for the local surrounding area. Buildings having a large solar reflective finish shall not be present in the immediate vicinity. Good. engineering judgment shall be exercised to assure that the module, front and back sides, is receiving a minimum of reflected solar energy from the surrounding area.

e. Wind Direction. The wind shall not be predominantly from due west; flow parallel to the plane of the array is not acceptable and can result in a lower than typical operating cell temperature.

f. Module Electrical Load. Data shall be obtained for a module open-circuit condition corresponding to zero electrical power output.

\section{A.2.5 Test Equipment}

a. Pyranometer. The total solar irradiance on the active side of the module shall be measured by a reference cell traceable to the National Bureau of Standards or by a pyranometer. The device shall be mounted on the plane of the module and within $.3 \mathrm{~m}$ ( 1 foot) of the array. The pyranometer used shall have a traceable annual calibration to a recognized standard instrument and shall be either: (1) a temperature-compensated unit which has less than \pm 1 percent deviation in sensitivity over a range $-20^{\circ}$ to $+40^{\circ} \mathrm{C}$, or (2) a unit which incorporates a temperature sensor and has a sensitivity-temperature correction supplied with its calibration.

b. Wind Measurement. Both the wind direction and wind speed shall be measured of the approximate height of the module and as near to the module as feasible.

c. Air Temperature. The local air temperature shall be measured at the approximate height of the module. The measurement shall be made in the shadow of the module and shall be accurate to $+1^{\circ} \mathrm{C}$. (Note: An average local air temperature is desired. This is obtained satisfactorily by increasing the thermal mass of the thermocouple by imbedding the thermocouple in a solder sphere of approximately 1/4-inch diameter.) The measurement must be appropriately shielded and vented.

d. Cell Temperature. The temperature of at least two represcntative interior solar cells shall be measured to $\pm 1^{\circ}$ C. Thermocouples shall be 36 gauge, and shall be softshouldered directly to the back of the cells.

e. Substrate Surface Temperature. The exterior temperafure of the rear of the solar module shall be measured to $\pm 1^{O} \mathrm{C}$ beneath a representative cell and, when practical beneath a representative space between cells. Thermocouples shall be 26 gauge, and shall be bonded down with 57-C epoxy or the equivolent. 


\section{A.2.6 Data Recording}

All data shall be printed out approximately every 2 minutes. In addition, solar intensity, wind speed, wind direction, and air temperature shall be continuously recorded.

\section{A.2.7 Cleaning}

The active side of the solar cell module and the pyranometer bulb shall be cleaned before the start of each test. Dirt shall not be allowed to build up. Cleaning with a mild soap solution followed by a rinse with distilled water has proven to be effective.

\section{A.2.8 Equipment Calibration}

A calibration check shall be made of all the equipment prior to the start of the test. 


\section{APPENDIX $11 . B$ TEST PROCEDURES}

\section{B.I THERMAL CYCLING TEST PROCEDURE}

The module shall be subjected to the thermal cycling procedure per exhibit B.I, consisting of 50 cycles with the cell temperature varying between $-40^{\circ} \mathrm{C}$ and $+90^{\circ} \mathrm{C}$. The temperature shall vary approximately. linearly with time at a rate not exceeding $100^{\circ} \mathrm{C}$ ger hour and with a period not greater than 6 hours per cycle (from ambient to $-40^{\circ} \mathrm{C}$ to $+90^{\circ} \mathrm{C}$ to ambient). The module circuitry shall be instrumented and monitored throughout the test to verify that no open circuits or short circuits occur during the exposure.

\section{B.2 HUMIDITY TEST PROCEDURE}

The module shall be subjected to the humidity cycling procedure per exhibit B.2. The module shall be tested in the open circuit condition, but with terminals protected from water condensation. Electrical performance test, per appendix A.l, shall be performed within I hour after removal from the humidity chamber, or within another mutually agreed on time period, if the testing is subcontracted.

\section{B.3 MECHANICAL CYCLING TEST PROCEDURE}

The module shall be subjected to a cyclic load test in which the module is supported only at the design support points and a uniform load normal to the module surface is cycled 10,000 times in an alternating negative and positive direction. Cycle rate shall not exceed $20 \mathrm{cycles} / \mathrm{min}$. The module circuitry shall be instrumented to verify that no open circuitry or short circuits occur during the test. JPL Document 5101-19 "Cyclic Pressure-Load Developmental Testing of Solar Panels," February 1977, describes techniques suitable to the performance of this test.

\section{B.4 WARPED MOUNTING SURFACE TEST PROCEDURE}

The module shall be subjected to a twist test oy deflection of the substrate to which it is mounted. The deviation from a true flat surface during the test shall be $+1 / 4$ inch, measured along either mounting surface as shown in exhibit B.3. The module circuitry shall be instrumented to verify that no open circuits or short circuits orcur during the deflection test.

\section{B.5 HAIL IMPACT TEST PROCEDURE}

The module shall be subjected to normal impact loading with the required diameter iceball traveling at terminal velocity for the specified size. Typical hail characteristics are provided in exhibit B.4. At least three different points of impact shall be selerted to include the test specimen's most sensitive exposed point, and each point will be struck at least three times (a minimum of nine impacts). The most sensitive exposed point on a test specimen must be determined experimentally through destructive testing of a sample panel. Iceballs I.5 times the required diameter shall be fired at candidate sensitive points with increasing velocity until the panel is broken. Several different points on the panel should be broken, and the points broken at the lowest velocities should be used for subsequent testing.

The candidate points selected shall include (where applicable) the following:

- Corners and edges of the module;

- Edges of cells, especially ar.ound electrical contacts; 
- Points of support for any superstrate material; and,

- Points of maximum distance from points of support noted above.

Some scatter is expected in hitting a location on a module. Three repeated impacts are required to assure that a sensitive point has been struck. Error of up to $1 / 2$ inch in the location hit is acceptable. Either pneumatic or spring-actuated guns for projecting the iceballs against the modules are acceptable, however iceball velocity at impact must be controlled to within +5 percent of terminal velocity for the required hailstone size. Iceballs shall be generally spherical in shape with a maximum deviation in diameter of $+1 / 8$ inch. The iceballs shall be cooled to $-10^{\circ} \mathrm{C}+2^{\circ}$ as measured in the compartment where they are stored. The module shall be mounted in a manner representative of that used for actual installation of the module in the array. After each impact, the module shall be inspected for evidence of visible domage. Iceballs are the only acceptable hailstone simulation. Dropped steel balls, for example, shall not be used.

\section{B.6 ELECTRICAL ISOLATION TEST PROCEDURE}

The module 'hi-pot' test shall be conducted with the output terminals short-circuited. Test leads from a suitable DC voltage power supply shall be connected with the positive lead on the terminals and the negative lead on the module grounding stud.

In the case of modules not required to provide a grounding stud, the mounting structure shall be used as the second test point. Voltage shall be applied at a rate not to exceed $500 \mathrm{~V} / \mathrm{sec}$ up to the test voltage, and shall then be held at the required test voltage for I minute. The module shall be observed during the test for signs of arcing or flashover. Leakage current shall be monitored during the test to verify that leakage current does not exceed 50 microomps $\left(50 \times 10^{-6} \mathrm{~A}\right)$.

\section{B.7 DC/AC INVERTER .}

The inverter for converting $D C$ to $A C$ voltage shall be designed in conformance'with the general provisions contained in section B.1/ (See exhibit B.5), and the following.

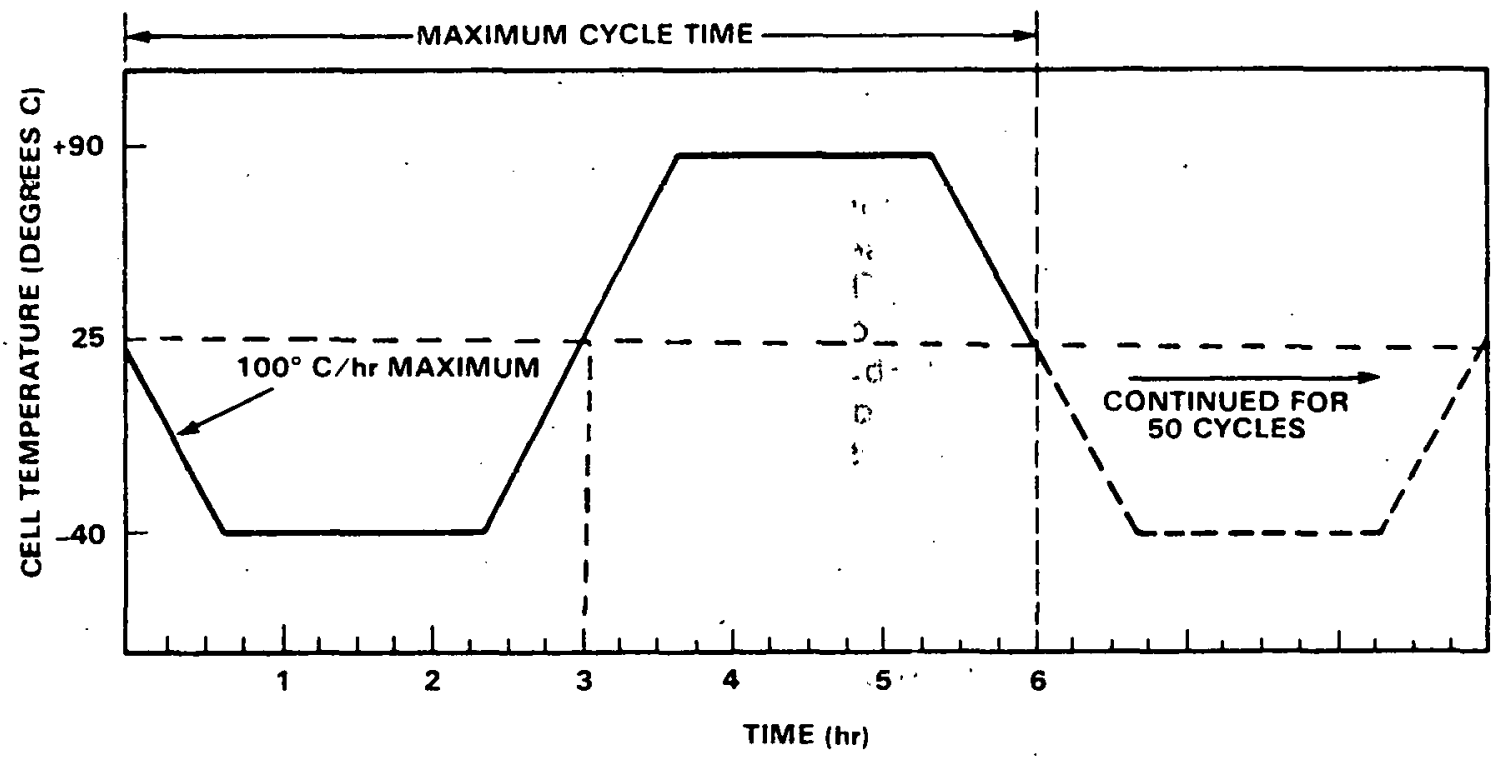

Exhibit B.1 Thermal Cycle Test. 


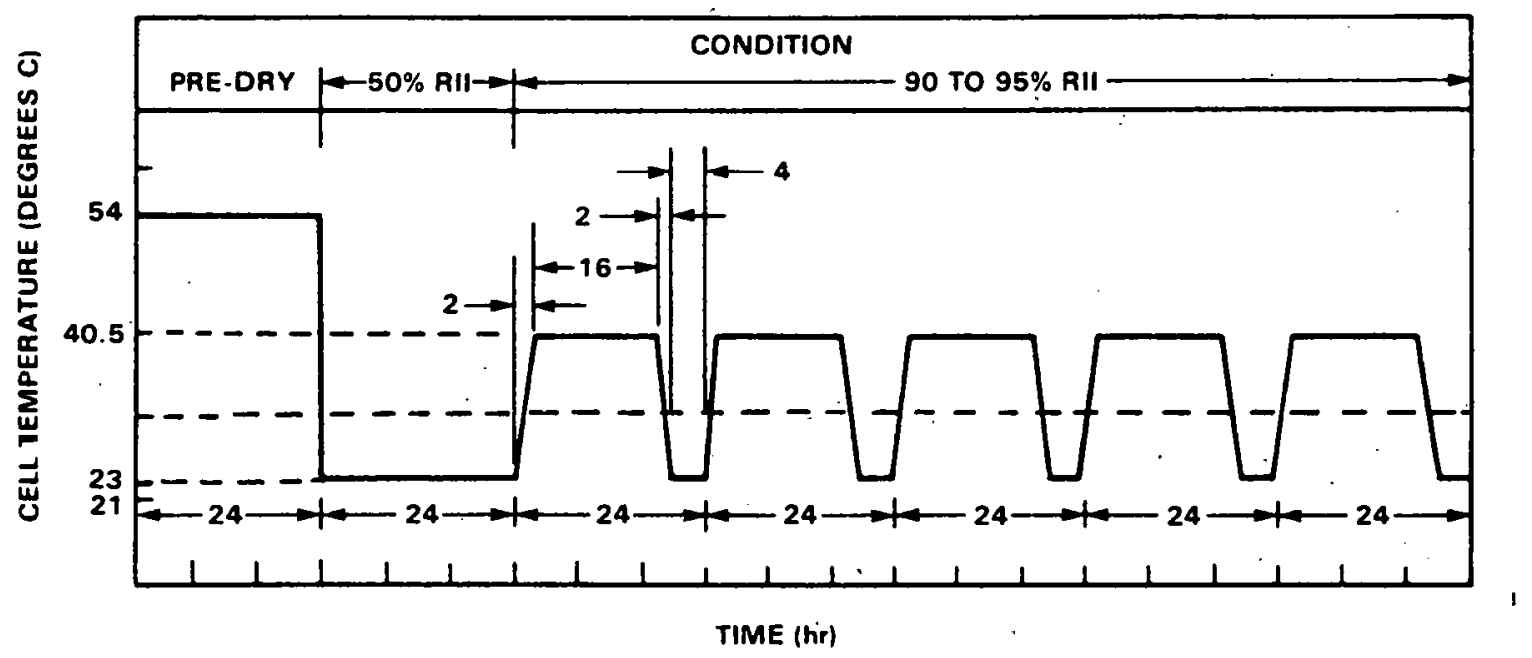

Exhibit B.2 Humidity Cycle Test.

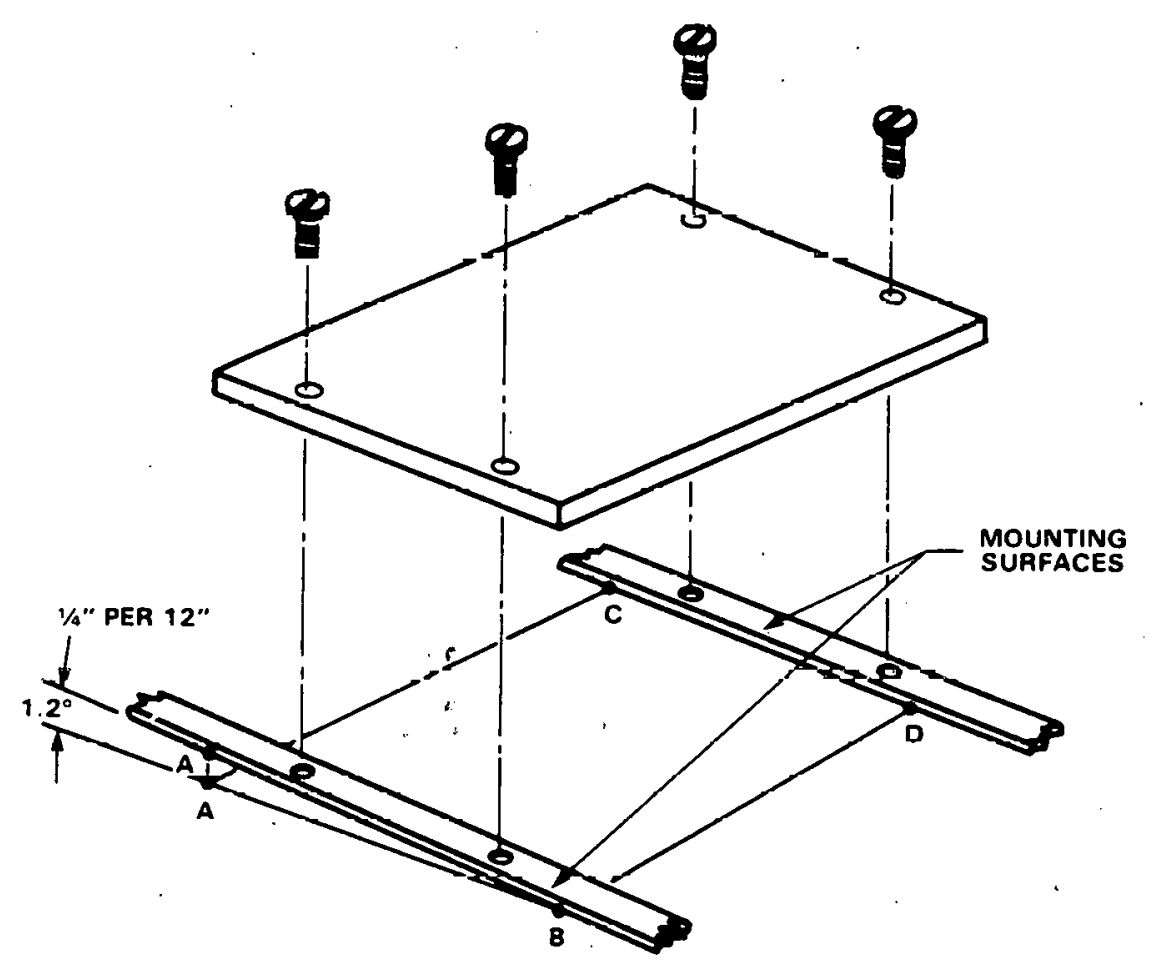

Exhibit B.3 Graphical Representation of "Twisted Mounting Surface".Requirement. 


\section{Design}

The inverter shall be totally enclosed, passively cooled in a weatherproof* metal container, grounded in accordance with the NEC (National Electrical Code). All connectors shall be weatherproof, strain-relieved, and compatible with aluminum/copper wire and cable, with proper polarity indicated by embossing or other permanent marking. All electrical connections shall be three-wire, with one wire serving as ground, and output and input clearly labeled.

* Some applications do not need weather protection (if mounted in a shed) or need only be weather resistant.

\begin{tabular}{|c|c|c|}
\hline \multicolumn{3}{|c|}{$\begin{array}{l}\text { HAIL CHARACTERISTICS } \\
\text { WEIGHT AND TERMINAL VELOCITY }\end{array}$} \\
\hline $\begin{array}{l}\text { SIZE } \\
\text { in. }\end{array}$ & WEIGHT: & $\begin{array}{l}\text { TERMINAL VELOCITY } \\
\text { mph }\end{array}$ \\
\hline $1 / 2$ & 0.0021 & 36 \\
\hline $3 / 4$ & 0.0072 & 45 \\
\hline 1 & 0.017 & 52 \\
\hline $11 / 4$ & 0.033 & 58 \\
\hline $11 / 2$ & 0.057 & 63 \\
\hline 2 & 0.136 & 73 \\
\hline $21 / 2$ & 0.266 & 81 \\
\hline 3 & 0.459 & 89 \\
\hline
\end{tabular}

- DENSITY OF ICE TAKEN AS $0.9 \mathrm{gm} / \mathrm{cm}^{3}$

Exhibit B.4 Typical Hail Characteristics.

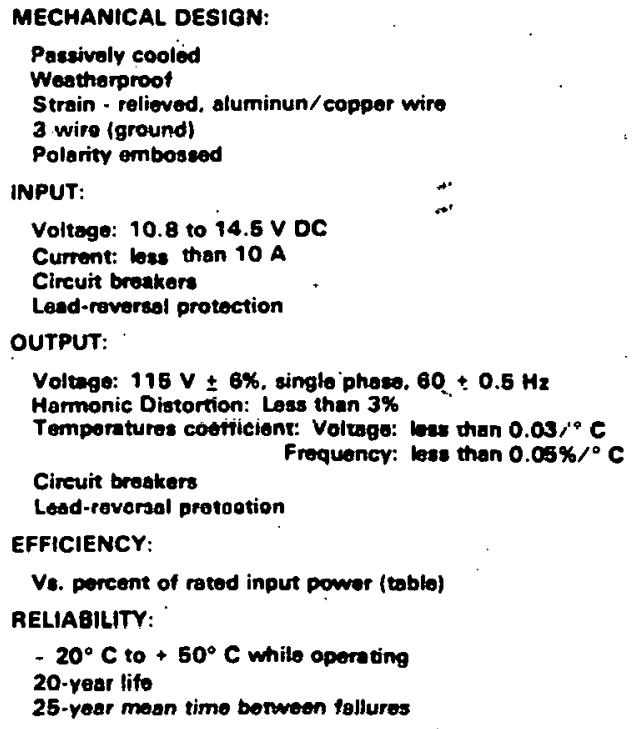

Exhibit B.5 DC/AC Inverter Component Specifications. 


\section{Input}

10.8 to $14.5 \vee D C$ (variable) for a 12 V System

10 A maximum

The inverter shall be self-protected from lead reversal with a keyed connector. Shorting of input leads and over-voltage protection shall be provided with circuit breakers or other devices that require manual resetting, but not replacement of a part. Fuses are not acceptable. Circuit breakers shall not emit smoke, fire, or visible arcs, shall have ambient temperature compensation and manual reset. The internal resistance shall be less than 1 micro-ohm. The circuit breakers shall fail open, but shall have a life exceeding 200 cycles.

\section{Output}

The output from the inverter shall be a sine wave at $11.5 \mathrm{~V}(\mathrm{RMS})+6$ percent, single phase, 60 cycles per second $(60 \mathrm{Hertz})$ frequency \pm 0.5 percent. The härmonic distortion shall be no greater than 3 percent. The output voltage shall change no more than 0.03 percent per degree $C$ change in the ambient air temperature. The output frequency shall change no more than 0.05 percent $/^{\circ} \mathrm{C}$. The inverter shall be protected from lead reversal with a keyed connector and from a direct short of the output leads by a circuit breaker or other resettable device as described in the previous section. Fuses are not acceptable. The RMS value of an $A C$ voltage is the $D C$ equivalent value (the effective value).

\section{Efficiency}

The efficiency of the inverter, defined as the ratio of the product of RMS (root-meansquare) output voltage and current to the product of the input current and voltage, shall be no less than indicated on the following table; where $P=V \times I$ and efficiency equals Pout $/ P$ in.

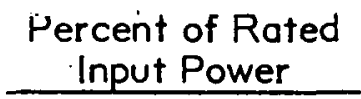

$10 \%$

$20 \%$

$30 \%$

$40 \%$

$50 \%$

$60 \%$

$70 \%$

$80 \%$

$90 \%$

$100 \%$
Ratio

(efficiency)

$40 \%$

$57 \%$

$66 \%$

$72 \%$

$76 \%$

$80 \%$

$82 \%$

$84 \%$

$85 \%$

$87 \%$ 
5. Reliability

The inverter shall be capable of meeting these specifications when operating in ambient-air temperatures ranging from $-20^{\circ} \mathrm{C}$ to $+50^{\circ} \mathrm{C}$. The mean time between failures (MTBF) for the inverter shall be no less than 25 years. Suitable redundancy shall be incorporated in the inverter to achieve this reliability. The life of the inverter shall exceed 20 years.

\section{B.8 BATTERY VOLTAGE REGULATOR}

The voltage regulator that controls the input voltage to the battery during charging operations shall be designed in conformance with the general provisions contained in section B.II, and the following.

\section{Design}

The regulator shall be totally enclosed, passively cooled in a weatherproof* metal container, grounded in accordance with the NEC. All connectors shall be weatherproof, strain-relieved, and compatible with aluminum/copper wire and cable, with proper polarity indicated by embossing or other permanent marking. All electrical connections shall be three-wire, with one wire serving as ground, and shall be clearly labeled for series, parallel, or partial shunt operation, and for output and input.

2. Input

The input to the battery voltage regulotor shall be:

13 to 17 VDC (variable)

10 A maximum

The regulator shall be self-protected from lead reversal, shorting of input leads, and over-voltage by circuit breakers or other devices that require resetting, but not replacement of a part. Fuses are not acceptable. The control voltage into the regulator shall be the battery terminal voltage.

\section{Output}

The output from the regulator. shall be used directly for connection to the battery. The output current shall be $10 \mathrm{~A}$ when the battery voltage is equal to or less than $13.0 \mathrm{~V}$ and shall decrease to $0 \mathrm{~A}$ when the battery voltage is equal to or greater than $14.4 \mathrm{~V}$. The output shall be self-protected from a direct short of the output leads by a circuit breaker or other resettable device. Fuses are not acceptable. The regulator shall also be self-protected from a lead reversal at the output terminals. If the leads to the battery are reversed, no currential is to flow to or from the battery.

\section{Efficiency}

The efficiency of the regulator, defined as the ratio of the product of output voltage and current to the product of the input current and voltage, shall be greater than 90 percent at $13.0 \mathrm{~V}$ and $10 \mathrm{~A}$ input, where $\mathrm{P}=\mathrm{V} \times \mathrm{I}$ and efficiency equals Pout $/ \mathrm{Pin}$.

\section{Reliability}

The regulator shall be capable of meeting the specified requirements while operating in ambient-air temperatures ranging from $-20^{\circ} \mathrm{C}$ to $+50^{\circ} \mathrm{C}$. The mean time between failures (MTBF) for the regulator shall be no less than 25 years. Suitable redundancy shall be incorporated in the regulator to achieve this reliability. The life of the regulator shall exceed 20 years.

*Some applications do not need weather protection (if mounted in a shed) or need only be weather resistant. 


\section{B.9 CONVERTER}

The converter that converts the alternating-current voltage input into a direct-current output shall be designed in conformance with the general provisions contained in section B.Il, and the following.

\section{Design}

The converter shall be totally enclosed, passively cooled in a weatherproof* metal container, grounded in accordance with the NEC. All connectors shall be weatherproof, strain-relieved, and compatible with aluminum/copper wire and cable, with proper polarity indicated by embossing or other permanent marking, with one wire serving as ground, and output and input clearly labeled.

\section{Input}

The input to the converter shall be:

I05 to $140 \mathrm{~V}$ AC (vuriable) slne wave

57 to 63 cycle per second frequency (Hertz), single phase

$10 \mathrm{~A}$ maximum

The convertor shall be self-protected from lead reversal, shorting of input leads and over-voltage by circuit breakers or other devices that require resetting, and not replacement of a part. Fuses are not acceptable.

\section{Output}

The output of the convertor shall be $14.4 \mathrm{~V} \pm 1$ percent, direct current at $100 \mathrm{~A}$ maximum. Maximum ripple shall be one volt. The output voltage is to vary no more than one percent per degree $C$ change in ambient temperature.

The output of the converter shall be self-protected from a direct short of the output leads by a circuit breaker or other resettable device. Fuses are not acceptable. The convertor shall also be self-protected from a lead reversal and shorting at the output terminals. If the leads of a battery are reversed and connected to the convcrter output, no current is to flow to or from the battery.

\section{Etticiency}

The efficiency of the converter, defined as the ratio of the product of the output voltage and current to the product of the input current and voltage, shall be no less than indicated on the following table, where $P=V \times I$ and efficiency equals Pout/Pin.

$$
\begin{gathered}
\text { Percent of Rated } \\
\text { Input Power }
\end{gathered}
$$

$$
10 \%
$$$$
20 \%
$$$$
30 \%
$$$$
40 \%
$$$$
50 \%
$$$$
60 \%
$$$$
70 \%
$$

$80 \%$

$90 \%$

$\ldots 100 \%$
Ratio (efficiency)
$40 \%$
$57 \%$
$66 \%$
$72 \%$
$76 \%$
$80 \%$
$82 \%$
$84 \%$
$85 \%$
$87 \%$

* Some applications do not need weather protection (if mounted in a shed) or need only be weather resistant. 


\section{Reliability}

The converter shall be capable of meeting the specified requirements while operating in ambient-air temperatures ranging from $-20^{\circ} \mathrm{C}$ to $+50^{\circ} \mathrm{C}$. The mean time between failures (MTBF) for the converter shall be no less than 25 years. Suitabie redundancy shall be incorporated in the converter to achieve this reliability. The life of the converter shall exceed 20 years.

\section{B.IO BATTERY SPECIFICATION}

I. Design

The battery casing shall be constructed from high impact resistant plastic. The polarity of the terminals must be clearly and permanently marked. The battery shall include a means of checking electrolyte level without necessitating the removal of any components. The battery shall also include a means of adding distilled water to the electrolyte. Terminals must be protected from corrosion by suitable means and provisions must be provided to vent hydrogen gas produced by the batteries and catalytic caps must be provided which are capable of mitigating the safety hazard.

\section{Input \\ The battery charging voltage will not exceed $14.4 \mathrm{~V}$.}

\section{Output}

Battery capacity, in Ah of charge, shall be given at a 100-hour discharge rate at $-12.2^{\circ} \mathrm{C}\left(10^{\circ} \mathrm{F}\right)$. Battery output voltage must be in excess of $1.8 \mathrm{~V} /$ cell for lead-acid batteries and $1.08 \mathrm{~V} /$ cell for nickel-cadmium batteries throughout the discharge $(10.8 \mathrm{~V}$ for a nominal $12 \mathrm{~V}$ battery). The batteries must be able to deliver the peak current demand, 32.2 $\mathrm{A}$ at $-12.2^{\circ} \mathrm{C}\left(10^{\circ} \mathrm{F}\right)$ when the batteries are discharged to 60 percent of their rated capacity at $-12.2^{\circ} \mathrm{C}\left(10^{\circ} \mathrm{F}\right)$. The state of charge of the batteries shall exceed 60 percent of rated capacity at all times unless atypical weather patterns cause a discharge below the aforementioned value, where one ampere - second equals one coulomb, and one volt equals one joule per coulomb.

\section{Efficiency}

The coulombic efficiency must exceed 90 percent.

\section{Reliability}

The batteries shall provide this service for at least 5 years. Battery self-discharge must not exceed 2 percent per month at $27^{\circ} \mathrm{C}\left(80^{\circ} \mathrm{F}\right)$. I he battery must not freeze when discharged to 60 percent of its rated capacity at $-23^{\circ} \mathrm{C}\left(-10^{\circ} \mathrm{F}\right)$.

\section{B.II TEST AND ACCEPTANCE}

1. Description of the Tests

Upon completion of the entire electric power system installation, the system shall be cleaned and adjusted for proper operation. The system output at rated insolation and load shall be determined according to the test procedures described in section 3 below. All tests shall be performed by the installer under the supervision of the owner or his designee.

The contractor shall give the owner a minimum of seven (7) calendar days' notice, written or oral, before start of testing so that the operator or designee may visually inspect the final assemblies, witness the test, and review documentation to assess acceptability. 


\section{Acceptance Criteria}

The acceptance test shall be used to demonstrate whether or not the system is electrically acceptable under this specification. Note that acceptance of the system does not relieve the contractor of the responsibilities implied by the various portions of this specification, nor does it relieve the contractor of the obligations imposed by the warranties. The acceptance test is considered the minimum requirement that must be met before the owner will assume responsibility for the operation of the system and before the contractor will receive final payment. Any provisions concerning service life shall be adhered to.

The system shall be considered acceptable according to this test procedure if: (1) the total output current of the system into the load and batteries exceeds $24.0 \mathrm{~A}$ when the insolation is $0.8 \mathrm{~kW} / \mathrm{m}^{2}$ and the ambient temperature is $25^{\circ} \mathrm{C}$; and (2) when the maximum demand is placed upon the power system by the load, the current into the lond exceods $35.0 \mathrm{~A}$ at $12 \mathrm{~V}$, wlielher the source of current is from the battery or array, when the insolation is equal to $0.8 \mathrm{~kW} / \mathrm{m}^{2}$, and the ambient temperature is $25^{\circ} \mathrm{C}$.

Comment: The criterla were devised on the basis of a clean new array and system. It presumes the system meets the system-performance requirements 20 years from installation, thus, a 20-percent service life margin is included.

\section{Remedies}

If the system should be found unacceptable according to the acceptance test, modules and associated wiring shall be modified and/or added to the system by the contractor, so that the system performance is brought within the acceptance criteria.

\section{Test Procedures}

a. Measurement Accuracy

The insolation meter shall have a maximum error of \pm 2 percent of the actual reading. Either a PV detector or a thermal pyranometer, calibrated for the angle of tilt, is acceptable. The insolation metcr shall have been recently calibrated according to NBS standards. The voltages and eurrents shall be measured within + 2 percent of the actual reading, using instruments that have been recently calibrated to NBS standards.

\section{b. General Procedures}

In recognition of the fact that the insolation and temperature connot be controlled, the test can be conducted within 1.5 hours of noon at any insolation greater than $0.7 \mathrm{~kW} / \mathrm{m}^{2}$, as measured in the plane of the array, and any ambient temperature. Correction factors, described below, can be used to convert the measured performance to the standard conditions of $0.8 \mathrm{~kW} / \mathrm{m}^{2}$ at $25^{\circ} \mathrm{C}$.

The acceptance test shall be conducted with all components und subsystems connected in the system. The load can be most conveniently and controllably applied with a resistance bank that provides the same voltage/current relationship as the operating load.

The voltage and current shall be measured at the output of the array (point $A$ ); at the input to the battery (point B); and at the output of the power conditioning equipment (point $\mathrm{C}$ ). The points are illustrated in exhibit B.6. The instruments are to be attached at the points indicated on the drawings. The insolation meter is to be mounted at the same angle as the array in the plane of the array and such that the reflected and direct solar flux is the sarie as that which impinges on the array. The insolation shall be measured simultaneously with the currents and voltages at points $A, B$, and C (see exhibit B.7). 


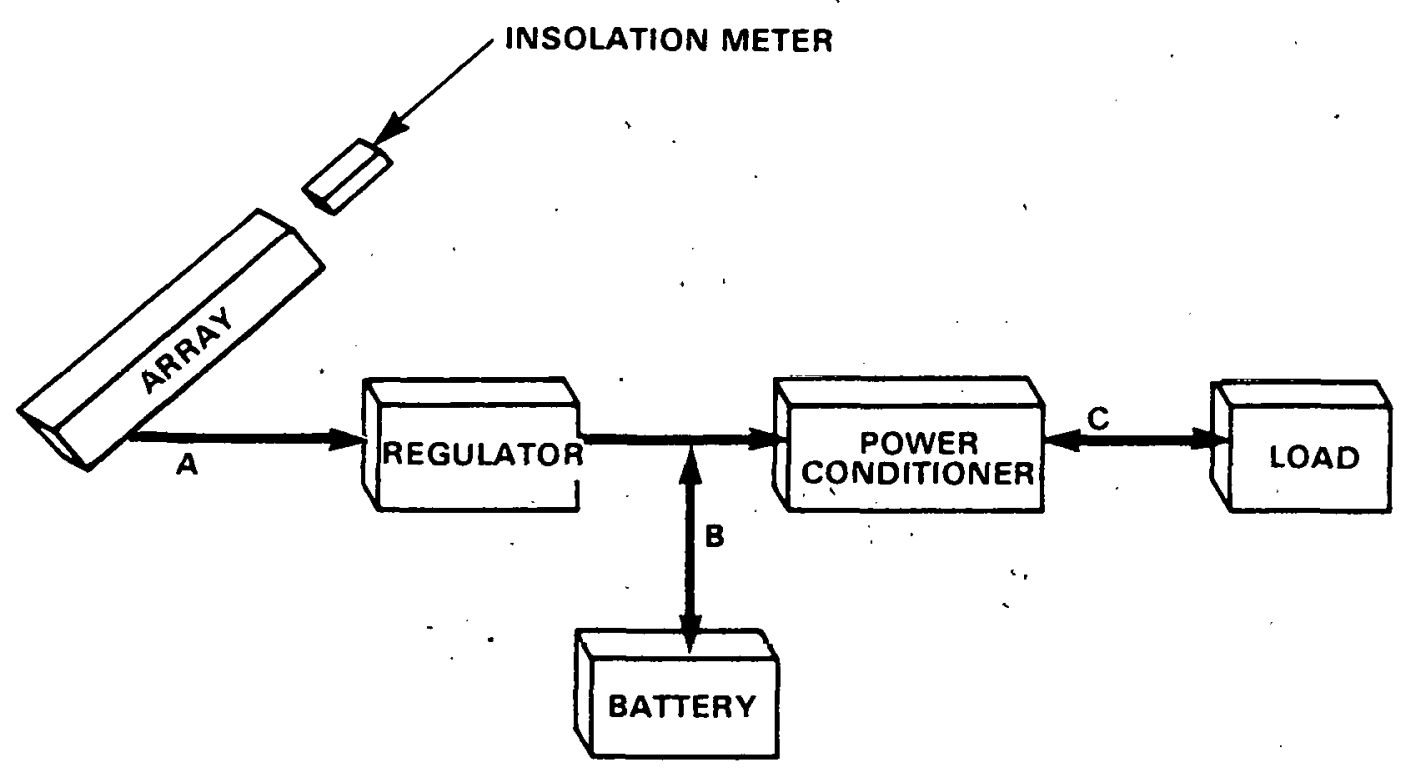

Exhibit B.6 Illustration of the Measurement Locations.

TEST AND ACCEPTANCE:

Cleaned and adjusted

Done by installer

. Data corrected to $80 \mathrm{~mW} / \mathrm{m}^{2}$ and $20^{\circ} \mathrm{C}$, cloudless Includes oversizing for degradation

POWER FROM ARRAY:

Current into load plus battery: $24 \mathrm{~A}$

Current into battery: less than $2.4 \mathrm{~A}$

POWER INTO LOAD:

Current into load: $35 \mathrm{~A}$

Current from battery: less than $10 \mathrm{~A}$

ADD MODULES UNTIL SYSTEM PASSES

\section{Exhibit B. $\overline{7}$ Test and Acceptance Procedures.}

On a cloudless day, when the insolation exceeds $0.7 \mathrm{~kW} / \mathrm{m}^{2}$, the system shall have the load applied to it, with the input to the load being $12 \mathrm{~V}$. The currents and voltages into the load and into the batteries shall be recorded, along with the insolation and ambient temperature.

c. Test with the Power from the Array only

The first test to be conducted is the test of the system output at rated insolation and ambient temperature. During this test, the current shall flow into the load and into the battery, such that the battery is being charged. If desired (and if the system operation is not affected), the battery may be removed from the circuit during this test and a resistance load may be substituted. During this test, the current into the load shall be at least 10 times that into the batteries. 
The data recorded shall be used to compute the current output at rated insolation and ambient temperature according to the following formula:

Current output at rated conditions $=$ (measured current into the load + measured current into the battery)

- $\quad \times \frac{0.8 \mathrm{~kW} / \mathrm{m}^{2}}{\text { measured insolation }} \times\left(1 .+0.004 \times\right.$ (ambient temperature $\left.-25^{\circ} \mathrm{C}\right)$ ).

The current output so computed must meet the first criterion of section 2 above. If the current output does not meet this criterion, the remedies of section 3 shall be applied. If the current at the rated conditions does meet the requirements, the system shall be deemed acceptable according to the first criterion.

J. Iesl will, Muximum Huwer inlu llie Luud

The second test to be conducted is the test of the maximum output capability of the system at rated insolation and temperature. On a cloudless day, when the insolation exceeds $0.7 \mathrm{~kW} / \mathrm{m}^{2}$, the system shall have the maximum load applied to it, with the input to the load being 12 volts. The current and voltage into both the battery and the load shall be recorded, along with the insolation and the ambient temperature. The current out of the battery shall not exceed $10 \mathrm{~A}$ during this test. The data recorded shall be used to compute the current output at rated insolation and ambient temperature according to the following formula:

Current output at rated conditions $=$ measured current + (measured current - current from battery)

$\times \quad \frac{0.8 \mathrm{~kW} / \mathrm{m}^{2}}{\text { measured insolation }} \times\left(1 .+0.004 \times\left(\right.\right.$ ambient temperature $\left.\left.-25^{\circ} \mathrm{C}\right)\right)$.

The current output so computed must meet the second criterion of section 2 above. If the current output does not meet this criterion, the remedies of section 3 shiall be upplied. If llie currenl ul the rated condltions does meet the requircment3, the $3 y 3 t e m$ shall be deemed dicejtable vicuiding lu llie securid criterion.

5. Operation and Maintenance Manual

The contractor shall prepare five copies of an operations manual and five copies of a maintenance manual, which shall include troubleshooting procedures and a checklist for checkout purposes. The operation manual shall include. descriptions of the systems and components, schematics, wiring diagrams, structure drawings, and instruction for taking operational data and acceptance data.

\section{B.12 WARRANTY}

The system shall be guaranteed by the contractor for a minimum of one (1) year from the date of final acceptance of the work by the owner in writing. The system shall be guaranteed to be free of defects of material and workmanship under the first year's operating conditions. The guarantee shall provide that any faulty materials or workmanship will be replaced or repaired, and that upon failure of this contractor to do the above, after written notice from the owner, the owner may have the work done at the expense of this contractor (see exhibit B.8). 
The contractor further warrants that the electric power system delivered under this purchase order shall conform to the requirements set forth in this specification and shall meet the system specifications for a period of 10 years from the time of delivery to the site. Performance shall be measured by the same procedures as described for the acceptance test described previously in these specifications. The contractor shall repair or replace any components or subsystems that fail to meet the conditions specified herein, with an equivalent component. Components suspected of failure shall be shipped prepaid to PRC Energy Analysis Company or NASA Jet Propulsion Laboratory for inspection and then to the contractor for repair or replacement. The contractor shall return prepaid any repaired or replaced components. Components that have been returned to the contractor as defective, but which in the opinion of the contractor are not defective, shall be reported to DOE for disposition and shipping-cost determination. This warranty does not apply to modules that DOE determines to have been subjected to gross mishandling, negligence or omissions, acts of God, tampering, lightning, hail, wind loadings in excess of the local codes, and vandalism. Cosmetic degradation which exhibits no functional degradation is not covered by this 10 year warranty.

In no event shall the contractor be liable for incidental or consequential damage. This warranty is in lieu of all other warranties whether expressed, implied or statutary, including implied warranties of merchantability and fitness for a particular purpose other than that purpose set forth in the aforementioned specification.

Placed on contractor

One year for materials and workmanship

Ten years for system performance

Remedies: repair or replace to bring into specification

Exhibit B.8 Warranty. 
Typical list of drawings:

1. Solar Photovoltaic Power System Location

2. Solar Photovoltaic System Site Plan

3. Grading Plan

4. Array Layout

5. Array Foundation System

6. Array Sections

7. Fence, Road and Trail Details

8. Gate Details

9. Battery Building Site Plan .

10. Battery Buildiriy Plun

11. Battery Building Elevations

12. Wull Secliuris und Detalls

13. Eave Detail and Sections

14. Array Subfield Assembly

15. Subframe Assembly

16. Subframe

17. Frame

18. Module

19. Battery Building Structure

20. Utilities - Water and Sewer

21. Battery Building Plumbing Plan and Details

22. Battery Building Mechanical

23. Wiring Assembly for Subarray

24. Lightning Protection and Electrical Power

25. Battery Building Lightning Protection, Grounding and Controls

26. Battery Building Electrical

27. Generator Building Electrical

28. Door and Window Details

29. Miscelluneous Detalls, Sign, Etc.

30. Visitor Overlook Plán, Audio System, Elc..

31. Electrical System Schematic

32. System Wiring Diagram

33. Distributive Systems Integration

34. Interface Control 


\section{SECTION 12 \\ PERFORMANCE CHARACTERISTICS OF TYPICAL SOLAR CELL MODULES}

\subsection{INTRODUCTION}

A convenient manufacturing package of interconnected solar cells to produce the electrical characteriștics is specified as follows:

In the package, the solar cells are:

Electrically interconnected (soldered);

Bonded onto substrate for mechanical strength; and,

Encapsulated for protection from the environment.

\subsection{CONSTRUCTION/MANUFACTURING DATA}

Cells used in the modules are round or half moon, 2 inch, 2-1/4 inch, 3 inch, and 4 inch.

Substrates and bonding materials are aluminum, silicone rubber, epoxy fiberglass, ocrylic plexiglass, and glass.

Encapsulants are glass and silica.

\subsection{TYPICAL CHARACTERISTICS}

Low voltage, 5-20 volts; low-power, 5-10 watts. The electrical circuit is isolated from the chassis only with series-connected solar cells. No diode isolation or shunt diodes are used.

An assembly of solar cell modules to produce the required electrical output at the chosen field location. Solar arrays are usually designed to produce: low voltage/low power (battery charging, portable unit, remote units); intermediate voltage/intermediate power (residential applications, small commercial complexes); and, high voltage/high power (central power stations, industrial, commercial complexes).

\subsection{ELECTRICAL PERFORMANCE}

Important performance data for users and power conditionér designers is as follows:

Electrical characteristics.

(I-V Curve, Voltage, Current, Power).

Equivalent Circuit Parameters (Impedance is a function of resistance, capacitance, inductance, and frequency)

Impedance $=$ square root $\left(\right.$ Resistance ${ }^{2}+$ Reactance $\left.^{2}\right)$, where reactance is $a$ function of capacitance, inductance, and frequency.

Factors degrading performance and amount of degradation. 


\subsubsection{ELECTRICAL CHARACTERISTICS}

$\mathrm{I}-\mathrm{V}$ of solar array is produced from the summation of all the solar cell I-Vs

$1-V$ of solar array is determined from summing the currents of all the cells at each voltage value minus the losses of the interconnects.

Assuming good matched solar cells, the $1-V$ curve at intensity $X$ and temp $Y$ is shown in exhibit 12.1.

The number of modules (hence, number of solar cells connected in series-parallel) are determined by the power requirements within the following design considerations.

Allowable number of cells in series per solar arrny:

Voltage breakdown;

Safety requirements (maintenance-code);

Reliability (loss of a single cell may result in zero output); and,

Electromagnetic Properties.

Allowable number of cells in parallel per solar array:

Cable $\mathbb{R}$ drops and size (voltage drop $=\mathbb{R}$ where $R$ is cable resistance and $I$ is current)

Rellabllity (more cells in parallel improve reliability); and,

Electromagnetic Properties.

Ways of meeting the design considerntions:

Divide array into sections (limit number of series-parallel solar cells);

Arrange sections to minimize electromagnetic properties;

Use diode isolation; and,

Use diode shunting.

Influencing parameters are solir intensity and temperaturs (exhibit 12.2)

First approximation is that intensity will vary $I_{s c}$. and that temperature will vary $V_{\text {oc }}$ and affect curve shape.

\subsubsection{CONCLUSION}

A solar array is a variable power source. 


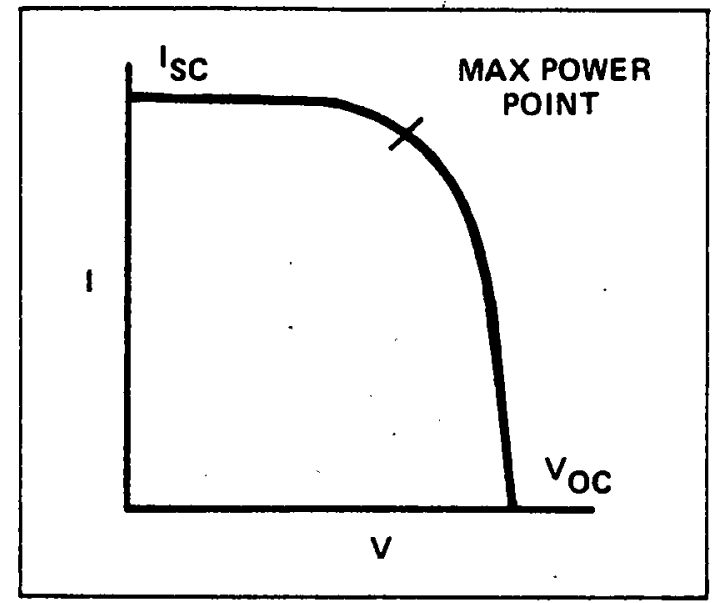

Exhibit $12.1 \mathrm{I}-\mathrm{V}$ Plot

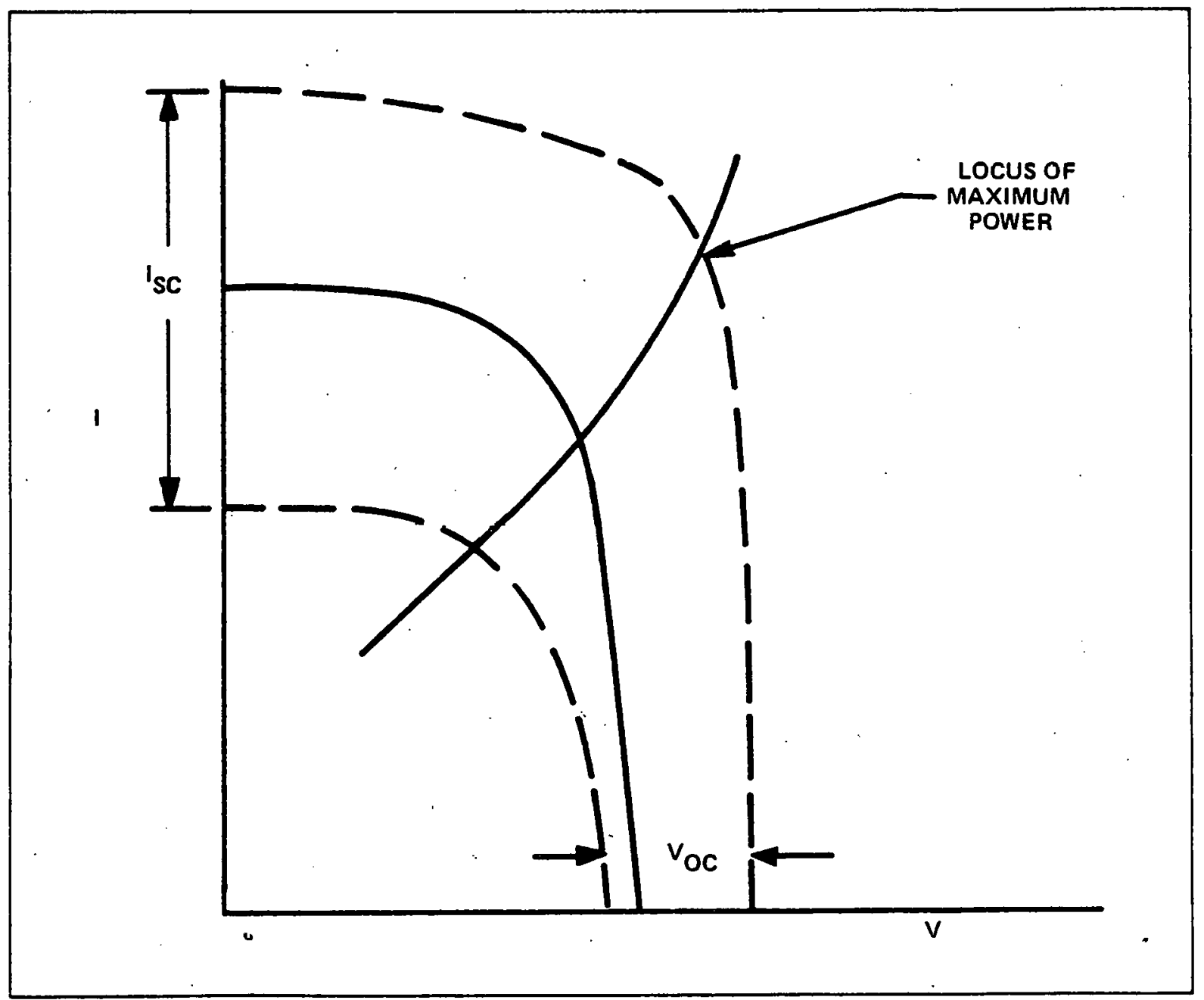

Exhibit 12.2 I-V Plot Range 


\subsection{GENERALLY ACCEPTED DC AND AC EQUIVALENT CIRCUITS}

Generally accepted DC and $A C$ equivalent circuits ore shown in exhibit 12.3.

Using a mathematical model and measurements of solar cells, the forward-biased solar cell impedance as a function of frequency for any given DC operating point has been generated as shown in exhibit 12.4 .

\subsubsection{INTERESTING OBSERVATIONS}

At lower cell illuminations, the impedance is higher at low frequencies. At higher illuminations, the impedance is relatively constant. The capacitance value varies with intensity. The typical impedance values are as follows:

$\begin{array}{ll}\text { Resistance } & 1.0 \mathrm{ohm}-\text { (small) } \\ \text { Capacitance } & 8 \mathrm{fd} / \mathrm{CELL} \text { - one } \\ (C=Q / \mathrm{V}) & \text { fd is a farad } \\ (C=\text { Capacitance, } & \text { of capacitance } \\ Q=\text { Charge, } & \text { Reactance equals } \\ V=\text { Voltage) } & -1 / \text { wc where } \\ & w=2 \pi \mathrm{f}\end{array}$

(Series resistance of solar cells and intcrconneets) Multiply by number in parallel Divide by number in series $C$ (parallel) $=C_{1}+C_{2}+C_{3} \cdots$

\subsubsection{CONCLUSION}$$
1 / C \text { (series) }=1 / C_{1}+1 / C_{2}+1 / C_{3}
$$

A solar array impedance varies with intensity, and operating point on l-V cı.rve.

\subsection{FACTORS DEGRADING PERFORMANCE}

Solar array electrical characteristics may be degraded if the solar cells are shadowed, fractured or damaged.

The amount of degradation depends on the number of shadowed, fractured or damaged solar cells and the electrical circuit design (number of solar cells in series-parallel).

\subsection{SHADOWED, FRACTURED, OR DAMAGED SOLAR CELLS}

The amount of power dissipation of a shadowed or damaged solar cell in the solar array depends upon:

(1) The reverse voltage that can be developed across the cell by the array:

a) Operating Conditions (Battery Clamp)

b) Extreme Operating Temperatures (exhibit 12.5)

(2) The amount of current forced through the cell

(3) Reverse-bios impedance (the impedance of the illuminated solar cell is greater than that of the dark cell)

Electrical characteristics of a 2-solar cell module with nne cell totally or partially shadowed and parallel connected are shown in exhibit 12.6.

\subsubsection{SINGLE SOLAR CELI}

The output of a shadowed single cell will degrade because the input energy is reduced to the cell and the internal energy losses in the non-illuminated cell portion are increased.

Typically, current output should be proportional to the illuminated areas:

$$
\begin{gathered}
I_{S C}=\left(I_{L}\right)(r) \quad \text { where } \\
r=\frac{A \text { ILL. }}{\text { A Total }} A=\text { Area, and } I_{L}=\text { illuminated current }
\end{gathered}
$$




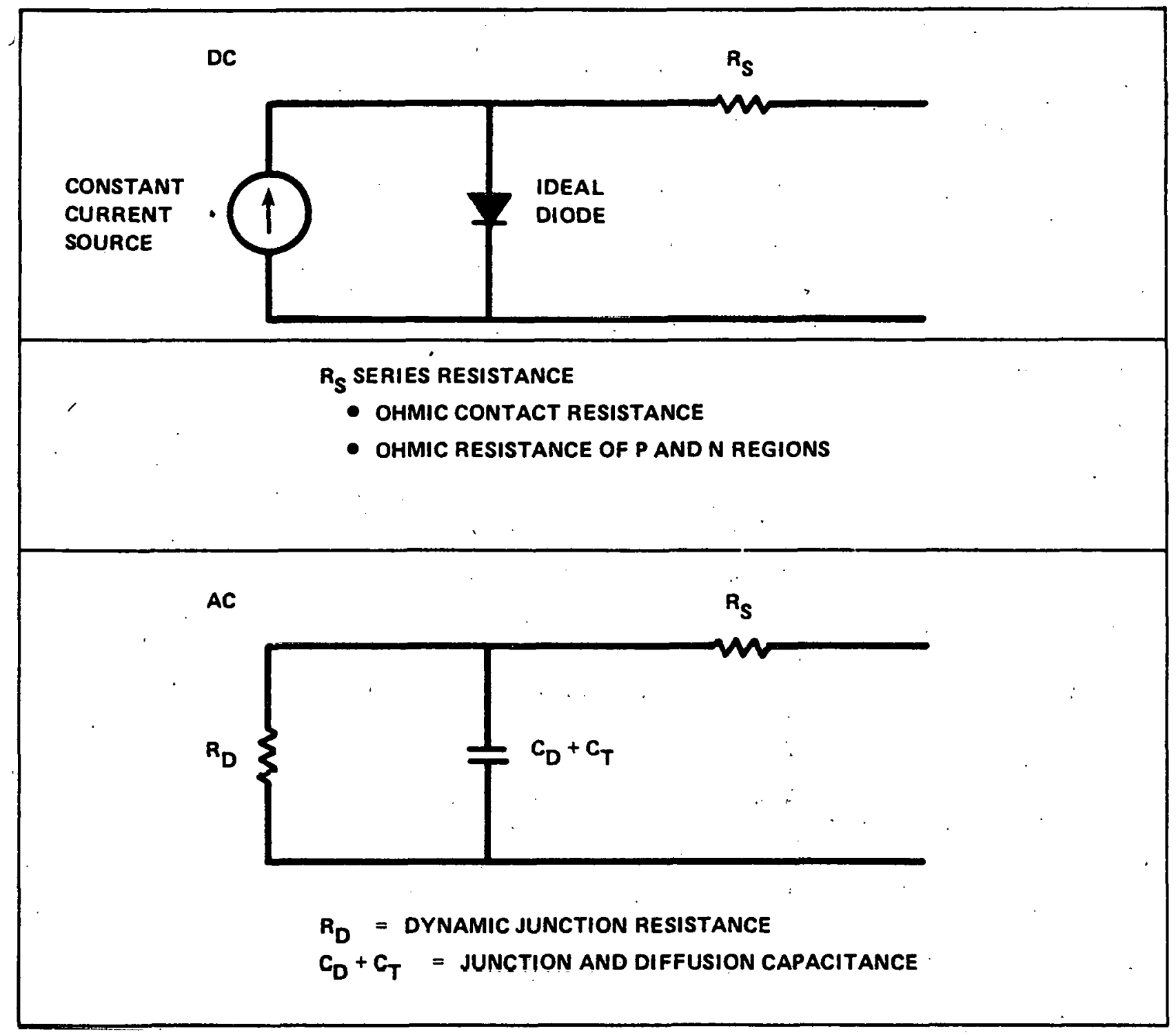

Exhibit 12.3. Generally Accepted DC and AC Equivalent Circuits 


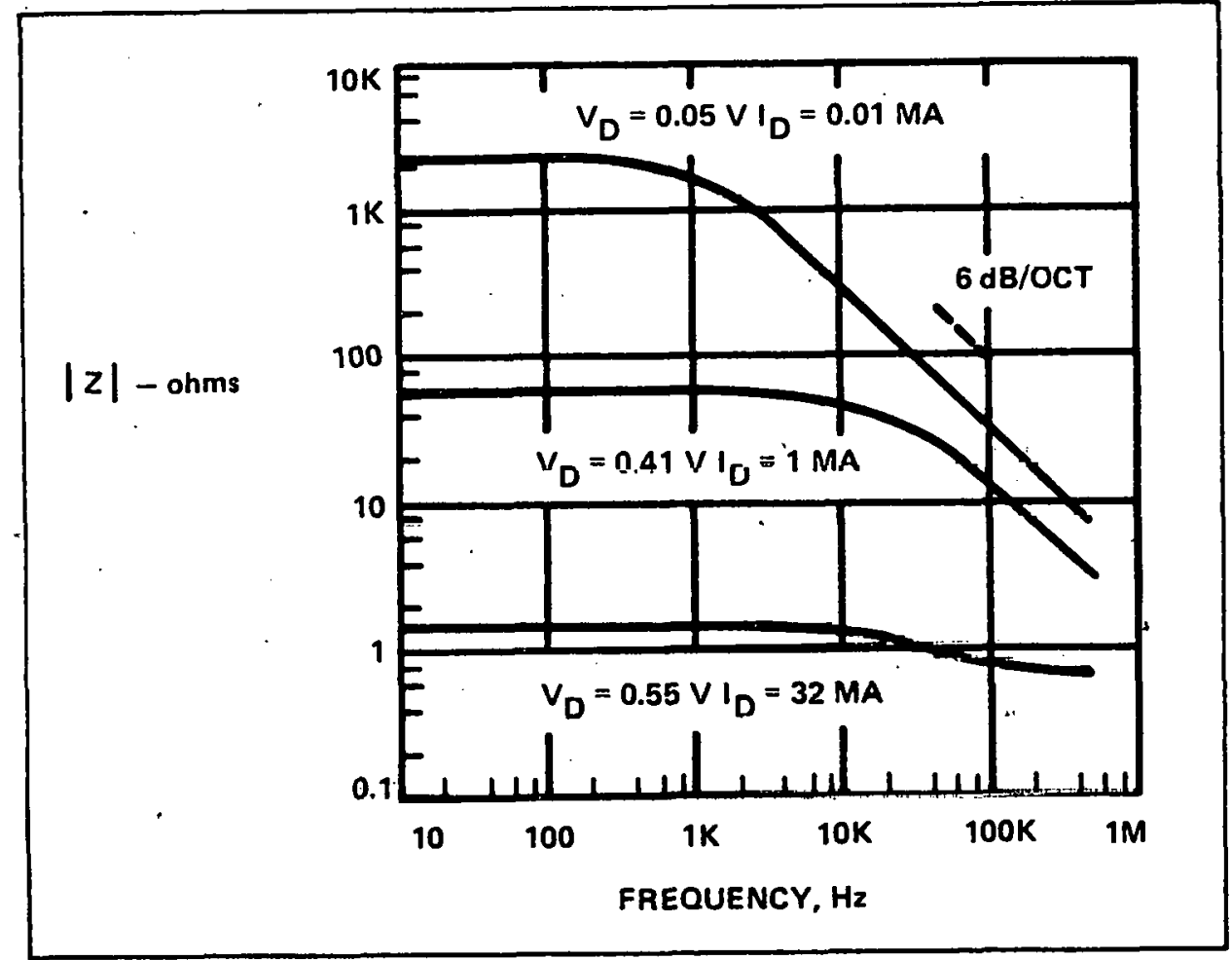

Exhibit 12.4 AC Impedance

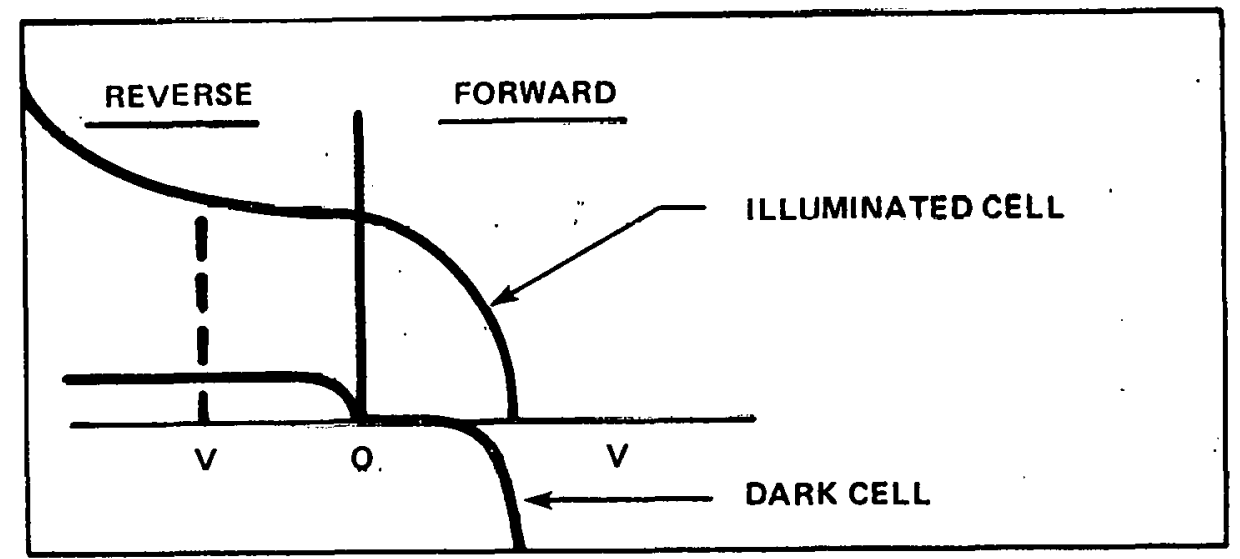

Exhibit 12.5 Reverse vs. Forward Voltage 
Exhibit 12.6 Electrical Characteristics of a 2-Solar Cell Module With One Cell Totally or Partially. Shadowed with Parallel-Connected Cells

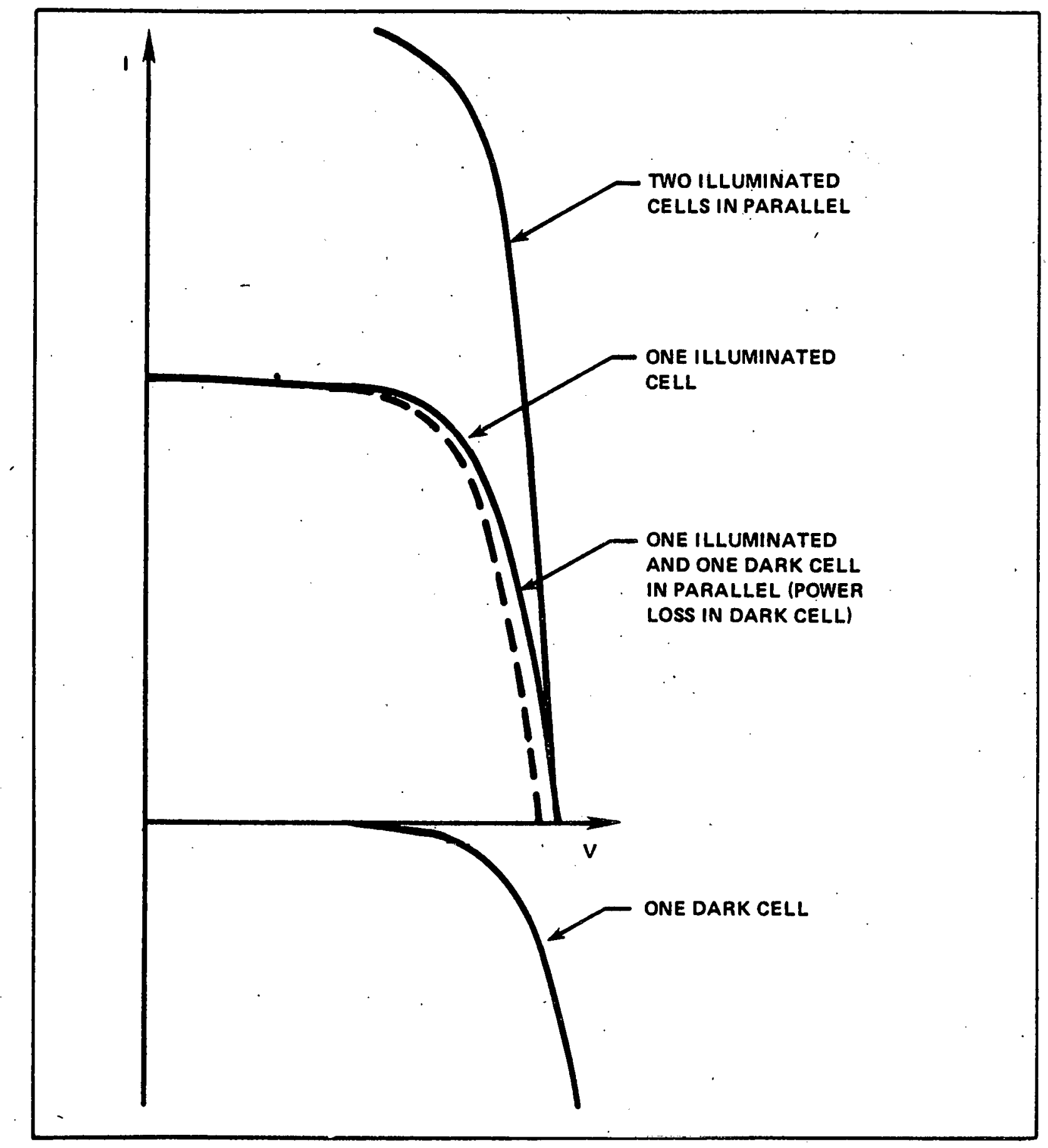


Exhibit 12:7a Series-Connected Cells (Partially Shadowed or Damaged)

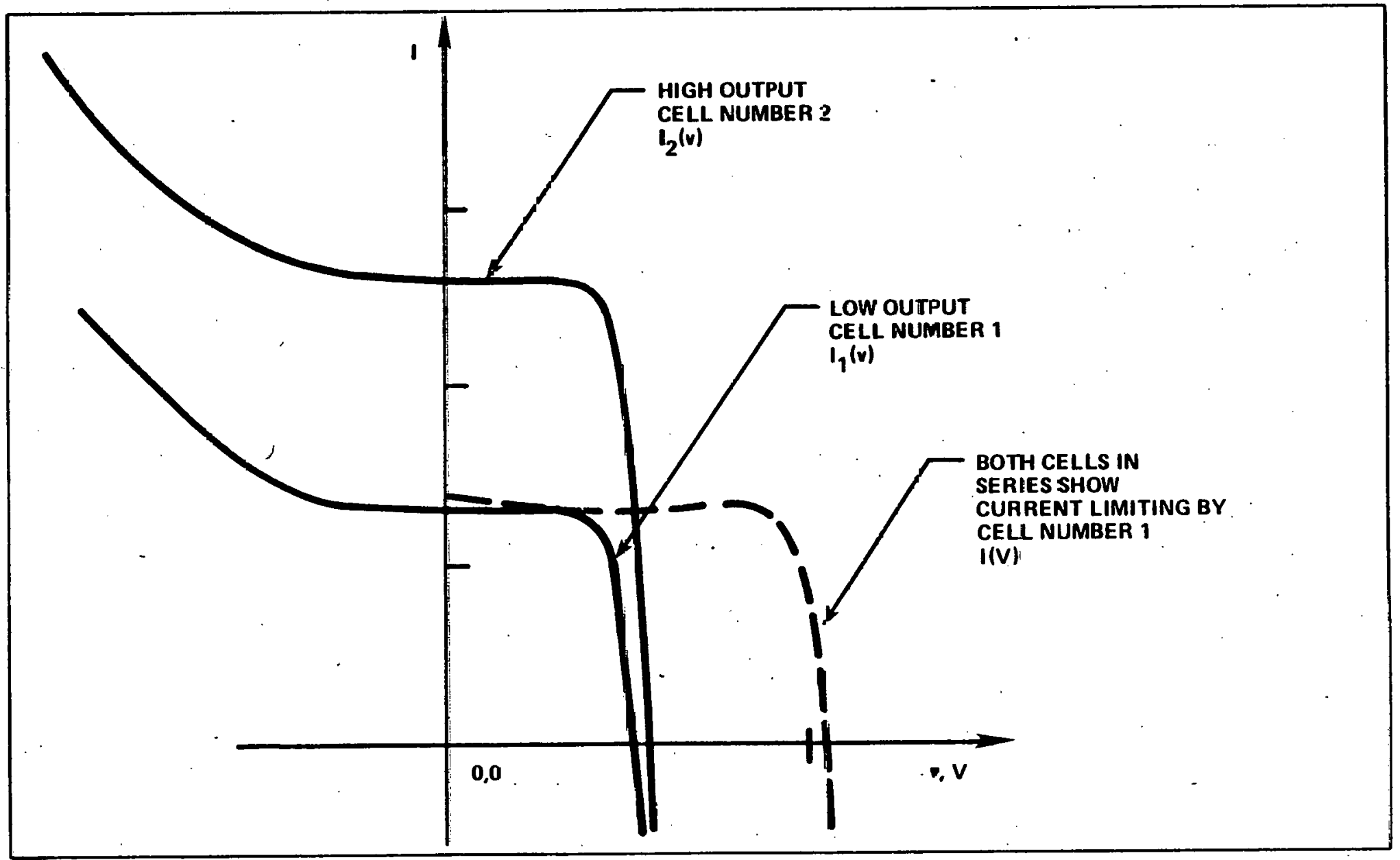




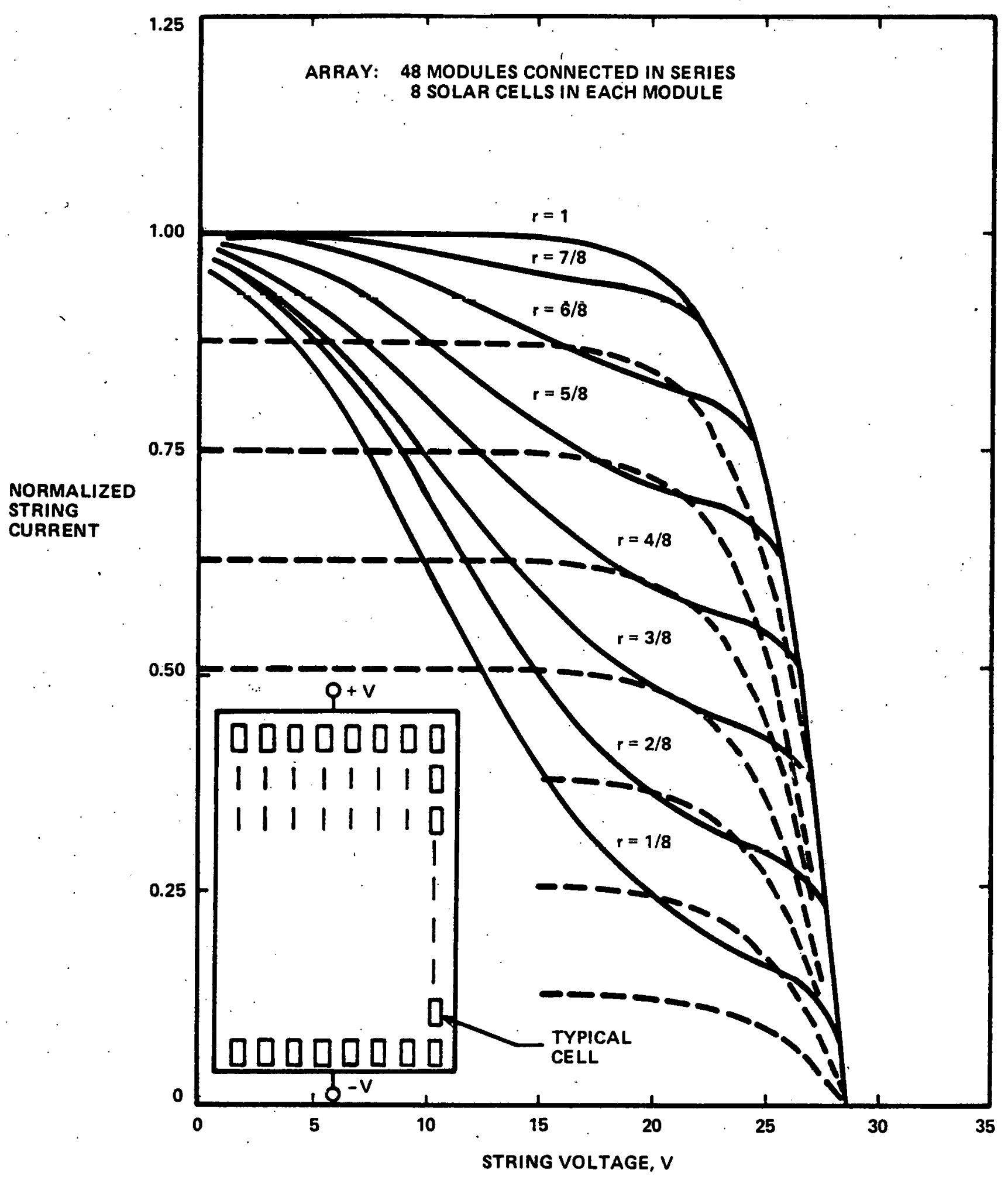

Exhibit 12.7b 


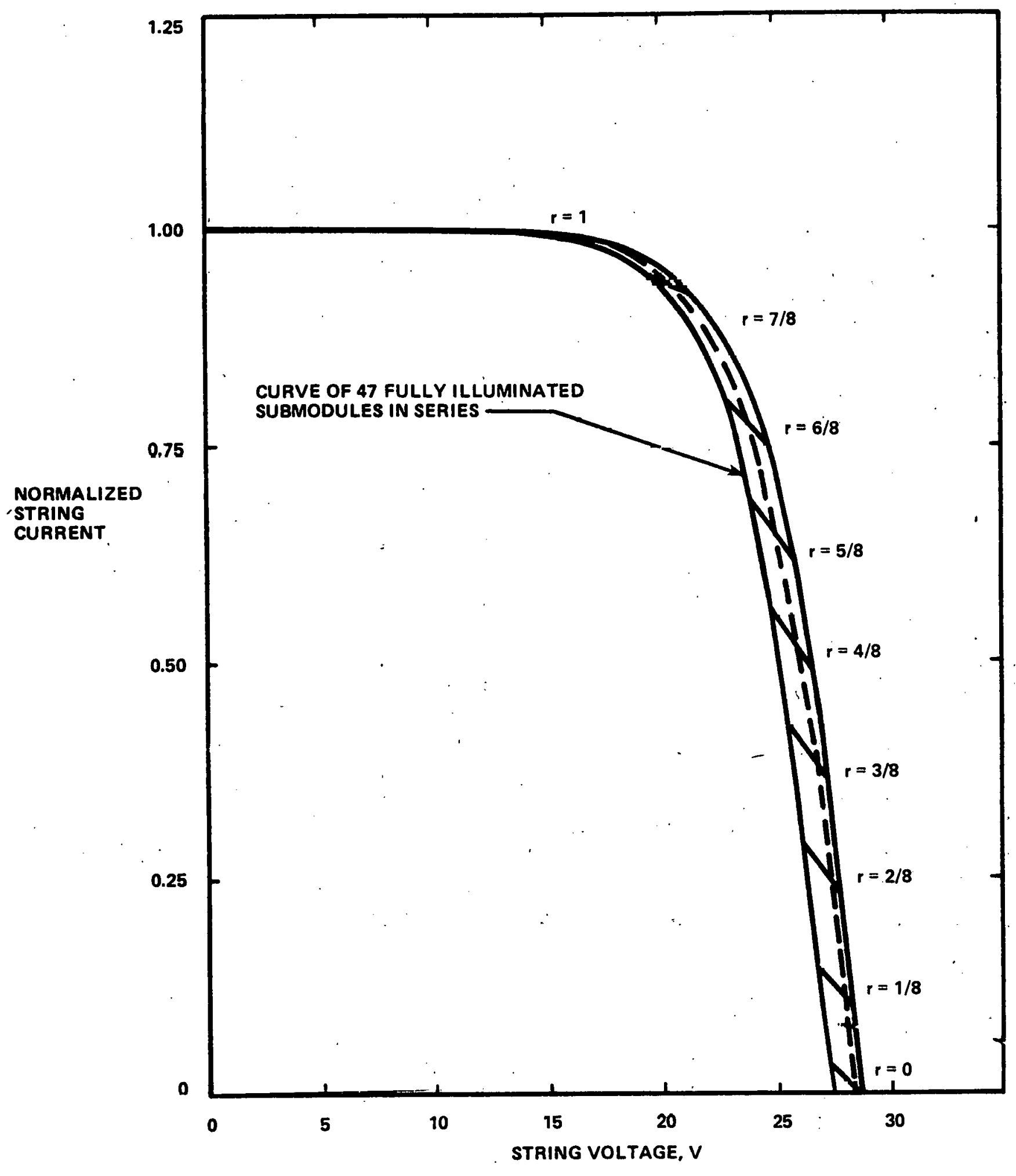

Exhibit 12.7c 
Typically, the output of a fractured or damaged solar cell is reduced by the area damaged.

\subsubsection{SOLAR ARRAY CRCUIT}

In a series string, the damaged solar cell restricts the current flow of the entire string; in a parallel string, the damaged cell will shunt part of the current of the good cells, as shown in Exhibit 12.7.

The damaged solar cell may become reverse-biased by the voltage developed across the cell and/or become a dissipator.

Power losses are usually higher than proportional to the loss of area, because in a series string, the,shadowed cells block the current flow of the illuminated cells; and in a paraltel string, the shadowed cells shunt part of the generated current of the illuminated cells. In addition, shadowed cells may become reversed-biased by the voltage developed across them; therefore, shadowed cells may become a dissipator.

\subsubsection{CONCLUSIONS}

The electrical characteristics of an array will be degraded depending on the number of shadowed, damaged, or fractured solar cells; the amount of cell area shadowed, fractured, or damaged; circuit configuration of the solar array (cells in series-parallel); the power dissipation developed, which will influence the operotional condition of other solar cells; and the use of isolation and shunt diodes.

\subsection{OPERATIONAL CONSIDERATIONS}

The solar array as a power source of a system must be compatible with the system grounding requirements, safety/maintenance requirements, electromagnetic properties (conducted and radiated interference); and lightning protection (see exhibits 12.8, 12.9).

\subsubsection{SAFETY/MAINTENANCE REQUIREMENTS}

The solar array is an active power source during illumination. To limit the solar array voltage to 60 volts (recommended by electrical code), mechanical switches should be installed between the solar cell modules (every 60 volts). The mechanical switches can be disengaged, limiting the voltage anywhere on the solar array to 60 volts for maintenance.

\subsubsection{GROUNDING REQUIREMENTS}

Center-tap grounding of the solar array electrical current via a resistor is recommended. A short. in the solar array can be detected via a sensing circuit consisting of two resistors and an ammeter. If a short occurs anywhere on the solar array, the current will flow through the current meter, which will be used to energize switch $S_{1}$ and remove the array from the system.

\subsection{ELECTRO-MAGNETIC INTERFERENCE (CONDUCTED AND RADIATED)}

\subsection{ITWO CONDITIONS}

(1) Electromognetic field generated by the electrical circuitry of the solar array as a power source.

(2) Electromagnetic field generated by the electrical circuitry of the solar array from noise fed back from the power user (power conditioners). 
Exhibit 12.8 A Typical Solar Array Farm(Conceptual Design)

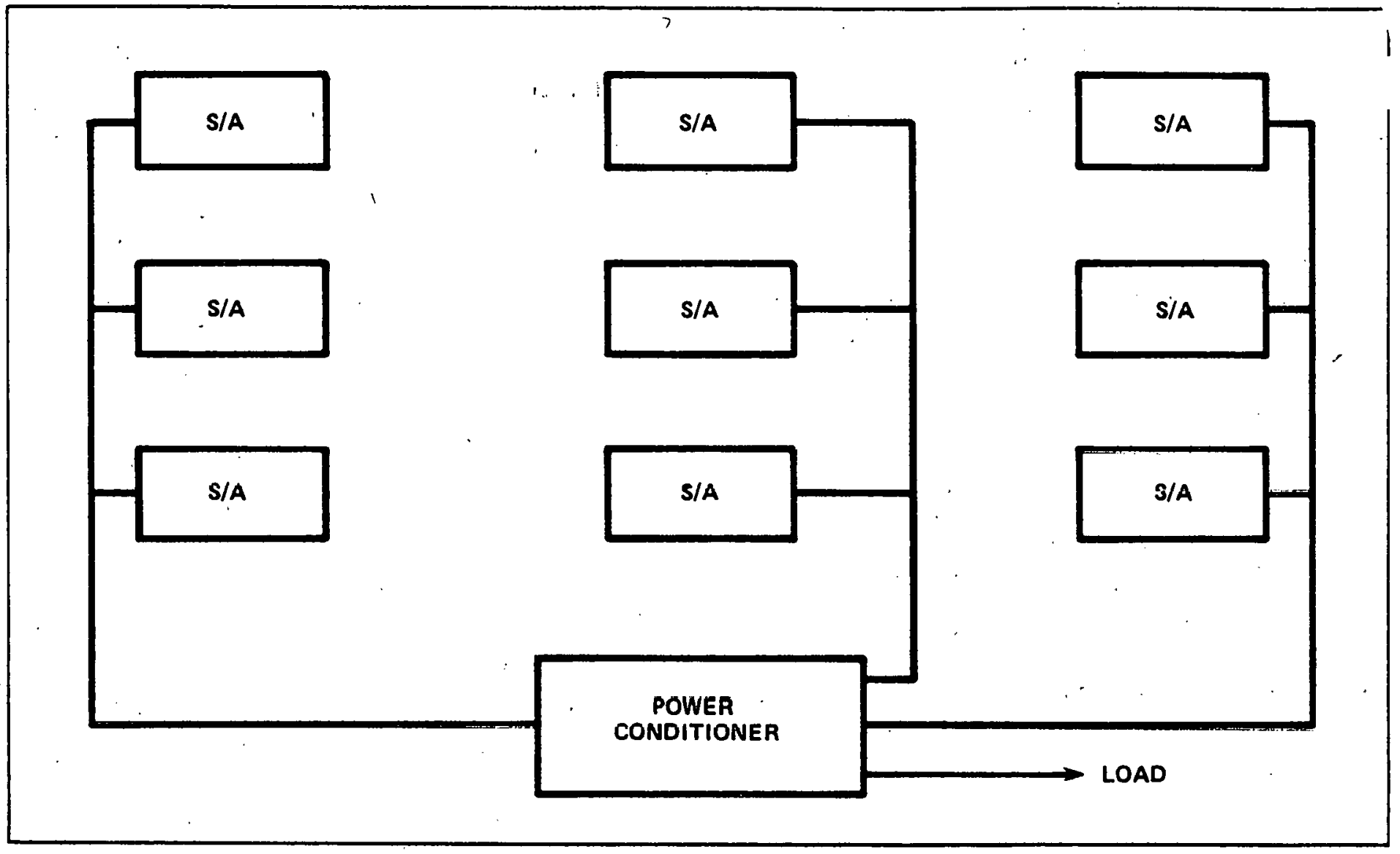

Exhibit 12.9 Terrestrial Solar Array Grounding and Safety Protection

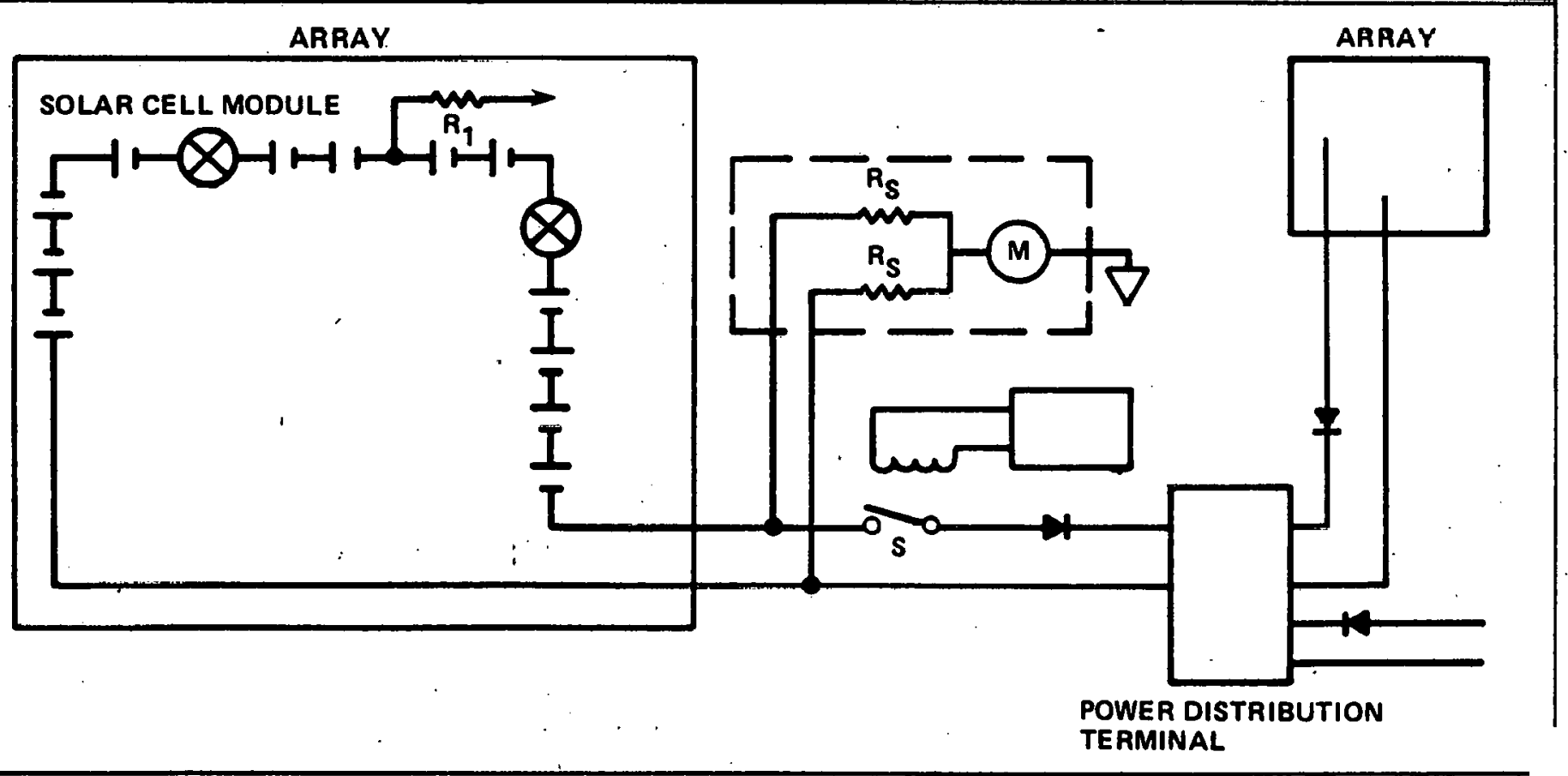




\subsubsection{SOLUTION}

(i) The internally-generated electromagnetic interference can be limited by arranging the array section and wiring to cancel the fields.

(2) The electromagnetic interference that can be generated from the power conditioners, and input ripple fed back to the solar array circuits are unknown. A study is, therefore, recommended.

\subsubsection{LIGHTNING PROTECTION}

Solar arrays, like any other power generators, are exposed to the environment and are vulnerable to lightning strikes.

\subsubsection{SOLUTION}

Lightning protection in the form of lightning arresters may be required. This will depend on location and costs.

\subsection{LOADING CONSIDERATIONS}

There are potentially two types of loads that will be connected to the solar array; stable loads and conditionally stable loads, as shown in Exhibits 12.10 and 12.11.

Stable loads (positive resistance) are resistors, batteries, shunt regulators, and series dissipative regulators.

Conditionally stable loads (negative resistance) are low-power dissipative electronic. switching regulators.

\subsection{POWER UTILIZATION CONSIDERATIONS}

There are potentially two ways of obtaining power from a solar array source; directly connected to the solar array or via maximum power-point trackers, as shown in Exhibit 12.12.

\subsubsection{MAXIMUM POWER POINT TRACKER}

Detects and tracks maximum power of the solar array and delivers it to the load. A number of approaches are reference cells, adjustable parallel load, and impedance matching. Power loss is anticipated up to $10 \%$. .

\section{'12.11.2 OBSERVATIONS}

\subsubsection{BATTERY}

Best utilization is at 12 noon.

12.11.2.2 RESISTIVE

Poor utilization at 7:30 a.m.

\subsubsection{CONSTANT POWER}

Poor utilization at 12 noon, needing other power source at 7:30 a.m.

\subsection{SUMMARY OF THE KEY SOLAR ARRAY PERFORMANCE PARAMETERS AS APPLIED TO POWER CONDITIONING}

The solar array is a variable power source. (The I-V characteristics: voltage, current and power vary with solar intensity and temperature.)

The impedance of the solar array is a variable parameter. (Varying with intensity.) 
Exhibit 12.10 Solar Array Delivering Energy into Stable Loads

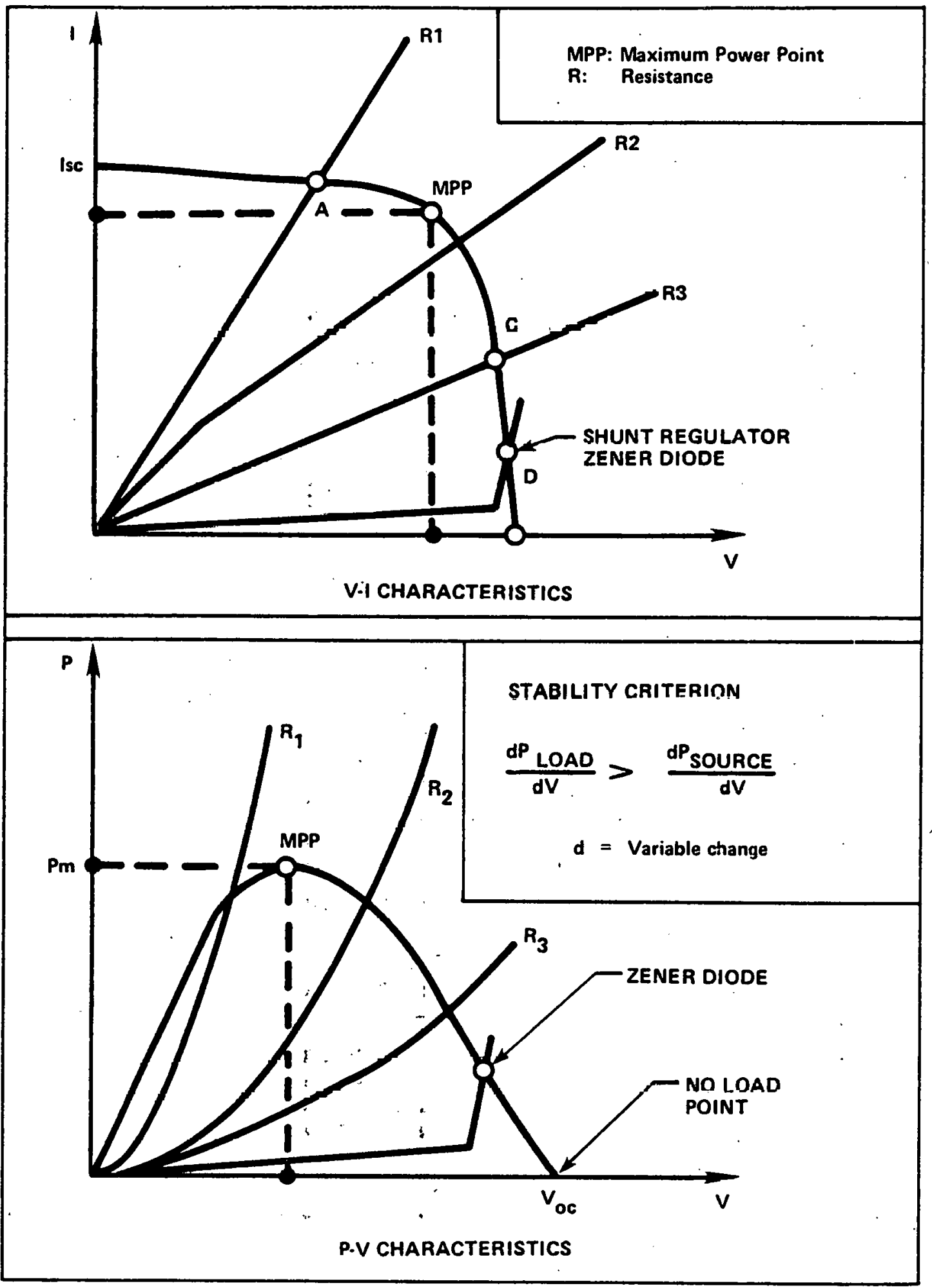




$$
\text { in }
$$

Exhibit 12.11 Solar Array Delivering Energy to a Constant Power Switching Regulator

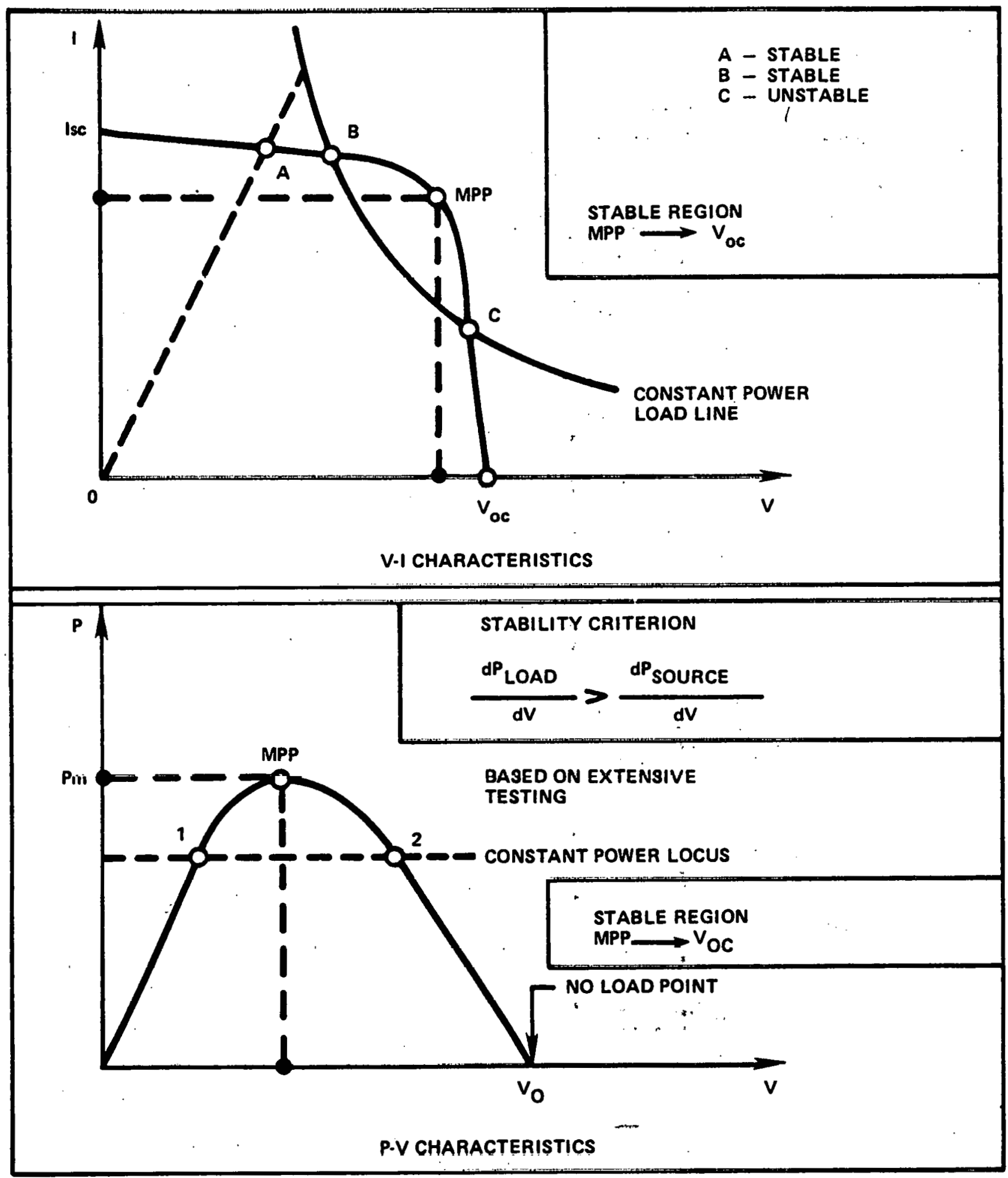


Exhibit 12.12 Power Utilization Considerations: One-Day Operation in Terrestrial Environment

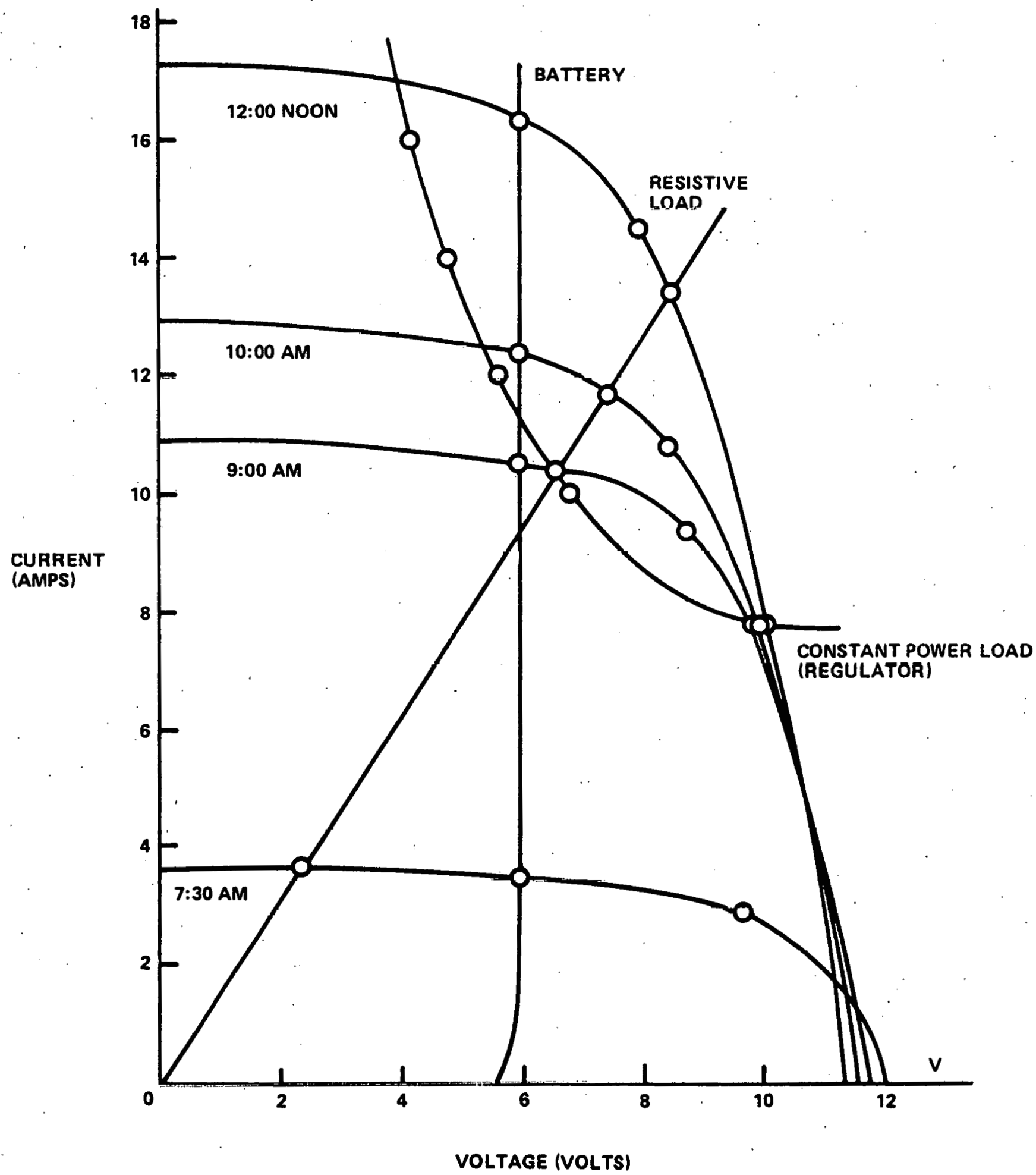


Shadowed, damaged, or fractured solar cells will affect the performance characteristics of the solar array. (Using shunt and isolation diodes can reduce the effects.)

A center-tap ground of the solar array electrical circuit is recommended for grounding of the solar array power source.

With the non-dissipotive (switching) regulators on, the stability criterion of the solar array must be observed.

Electromagnetic interference generated by ripple and noise feedback to the solar arrays from the power conditioners can be a serious problem.

Maximum power utilization considerations are essential inputs to the system and power conditioning designers.

Sensing networks for detecting shorts on the solar array and removing them from the power system circuit are recommended.

Lightning protection and safety-maintenance considerations ( $60 \mathrm{~V}$ voltage limit) as applied to the solar array source are worth remembering. 


\subsection{LIST OF REFERENCE DOCUMENTS}

\begin{tabular}{|c|c|c|}
\hline TITLE & AUTHOR & AVAILABILITY \\
\hline $\begin{array}{l}\text { SOLAR CELL ARRAY } \\
\text { DESIGN HANDBOOK }\end{array}$ & $\begin{array}{l}\text { PREPARED BY TRW FOR } \\
\text { JPL UNEER CONTRACT }\end{array}$ & TO BE RELEASED \\
\hline $\begin{array}{l}\text { ELECTRICAL OUTPUT } \\
\text { OF SHADOWED SOLAR } \\
\text { ARRAYS }\end{array}$ & $\begin{array}{l}\text { H.S. ROUSCHENBACK } \\
\text { TRW SPACE SYSTEMS }\end{array}$ & $\begin{array}{l}\text { PROCEEDINGS OF } \\
\text { THE 7TH IEEE ( } 968) \\
\text { PHOTOVOLTAIC } \\
\text { SPECIAI IST FON } \\
\text { PERENCE. }\end{array}$ \\
\hline $\begin{array}{l}\text { INVESTIGATION OF } \\
\text { SOLAR CELL INTER- } \\
\text { CONNECTION RE- } \\
\text { LIABILITY OF HIGH } \\
\text { VOLTAGE SOLAR } \\
\text { AKRAYS, THE "HOT SPOT" } \\
\text { PHENOMENON AND } \\
\text { RELATED TOPICS }\end{array}$ & $\begin{array}{l}\text { K.L. HANSON } \\
\text { F.A. BLACK } \\
\text { GENERAL ELECTRIC } \\
\text { SPACE SYSTEMS }\end{array}$ & $\begin{array}{l}\text { GE REPORT } \\
\text { 69SD } 303 \\
\text { SEPT } 69\end{array}$ \\
\hline $\begin{array}{l}\text { A BRIEF STUDY OF "HOT } \\
\text { SPOT" FAILURE MODES } \\
\text { FOR SULAR ARRAYS IN } \\
\text { TERRESTRIAL ENVIRON- } \\
\text { MENTS }\end{array}$ & $\begin{array}{l}\text { E.N. COSTOGUE } \\
\text {.IPL }\end{array}$ & $\begin{array}{l}\text { JPL DOCUM } \\
\text { EM-320 } \\
1976\end{array}$ \\
\hline $\begin{array}{l}\text { PRELIMINARY ASSESS- } \\
\text { MENT OF SOLAR CELL } \\
\text { SHORT CIRCUIT CUR- } \\
\text { RENT MEASUREMENT } \\
\text { IN A TERRESTRIAL } \\
\text { ENVIRONMENT }\end{array}$ & $\begin{array}{l}\text { E.IN. COSTOGUE } \\
\text { R. IVIUELLER } \\
\text { JPL }\end{array}$ & $\begin{array}{l}\text { JPL DOCUM } \\
\text { EMI-317 } \\
1976\end{array}$ \\
\hline $\begin{array}{l}\text { A BRIEF STUDY OF } \\
\text { LIGHTNING THEORY AND } \\
\text { PRO TECTION TECHNIQUES } \\
\text { AND PROCEDURES AND } \\
\text { THEIR APPLICABILITY } \\
\text { TO TERRESTRIAL SOLAR } \\
\text { POWER SYSTEMS, SPECI- } \\
\text { FICALLY SOLAR ARRAYS }\end{array}$ & $\begin{array}{l}\text { E.N. COSTOGUE } \\
\text { JPL }\end{array}$ & $\begin{array}{l}\text { JPL DOCUM } \\
\text { EM-330 } \\
1976\end{array}$ \\
\hline
\end{tabular}


E.N. CASTOGUE JPL

DR. Z. LINDENA EOS

D.W. ZERBEL

K.I. DECKER

TRW SYSTEMS
PROCEEDINGS

11 TH IECEC

1976

UTILIZATION

APPROACHES

AC IMPEDANCE OF

SILICON SOLAR

CELLS

PROCEEDINGS

5TH IECEC

1970 . 


\section{PAGES 13-1 to 13-9 WERE INTENTIONALLY LEFT BLANK}




\subsection{GENERAL SYSTEM CONSIDERATIONS AND CHECKLISTS}

\subsubsection{SITE SURVEY CHECKLIST}

1. Prior to visiting site
a. Calculate estimates of:
- insolation
- peak power to load
- daily energy to load.

b. Make block diagram of PV system and equipment being powered:

- array size (dimensions), including ground area required

- battery size (dimensions)

- power conditioner size (dimensions).

c. Determine required load voltage (and whether $A C$ or $D C$ ).

d. Determine in-house and contractor responsibilities for site survey, design, review, installation, operation, and maintenance.

e. Determine environmental restrictions (Federal, state, and local). Examples of such restrictions are: 'a) no digging for. cable layout, (b) no visual recognition of arrays from nature trails, (c) existing structures, special type structures, or buildings must be used.

2. At site

(Consider all information and environmental restrictions learned in above.)

a. Detemine elecir icul chumcler islics of existing equipment to be powered by $\Gamma V$ (voltage, current, hü'sepüwei', etti.).

b. Check to see if the locid or duty cycle can be reduced through more efficient use of, or replacement of, equipment.

c. Check array location for:

- shading

- foundation/structural support

- ground/roof area.

ה. Chnok possible conble locations to array and subassemblies:

- underground or overhead.

e. Check possible fence locations (if required).

f. Determine the frequency of site visits that could be uscd to check system operotion. 
g. Check installation and shipping route for site accessibility.

h. Draw sketch of site layout (indicate south), including equipment, shelters, fences, cabling, sources of array shading; give dimensions. sions.

\subsubsection{DESIGN CHECKLIST}

\section{A. Requirements}

6 - Define power and energy requirements.

6 - Can the array output be used totally or primarily during daylight hours?

6 - Is a voltage regulator needed?

6 - Is a DC/AC inverter needed?

6 - Are batteries needed? How many and what size?

10 - Is a low-power-capacity warning system needed?

$7=\quad$ Must a backup energy system be used?

10 - What instruments should be used for checkout, monitoring, and diagnosis?

B. System Design

2,4 - Determine the optimal system bus voltage, location and efficiency.

8 - Consider degradation of the array and efficiencies of the power conditioner, batteries, and connectors.

8 - Consider periodic change of the array tilt angle.

8,3 - Has arroy temperature been considered when computing instantaneous array output?

3 - Consider snow removal.

6 - Can the array output be stored in the end product, such as pumped water?

4 - Should the batteries be heated to retain capacity, prevent freezing, or permit recharge?. 
5 - Should the voltage regulator be shunt (parallel connected), partial shunt, or series?

3- is a diode provided to prevent battery discharge through the array?

8 - Has allowance been made for the voltage drop through the diode?

*8- From a sketch of the I-V characteristics of the load and the I-V characteristics of the array and batteries, can a load or powersystem mismatch be identified? Consider the effect of various operating temperatures and insolations, and degradation with time.

*5,8 - What control system, if any, is to be used?

3 - Can the control system be designed to make the power system more compatible with the load?

- 6 - Should AC or DC be used?

10,7 - Is an automatic maintenance telemetry system worthwhile?

3 - Should yandalism be considered?

7 - What provision is made for less insolation than expected?

7 - What backup power is provided (none, manual, engine, primary battery, solar-recharged emergency battery, fossil-rechnriged emergency batteryl??

8 - What spare parts should be provided?

8 - Are the costs of spare parts included in the life cycle cost anulysis?

7 - How will a malfunction be detected?

8 - Is the insolation properly computed (Aerospace/Solmet) with ground reflectance?

8 - If the energy method is used in the design computations, is the average efficiency properly chosen and justified?

* 8 - If a current method is used in the design computations, is the voltage sufficient to supply the current (eonsider termperature and low insolation)? 
8 - What is the optimal tilt, orientation, array size, and storage size?

8 - What is the projected life-cycle cost?

10 - Are sufficient permanently wired voltage meters, ammeters, and test points provided so that system performance can be readily measured?

\section{Array Design}

- From the manufacturer, obtain the I-V characteristics of the modules as a combined function of temperature and illumination.

2 - Incorporate a monitor panel with voltage and current meters to check current and voltage outputs of subarrays and possibly modules. Determine its location.

2 - Segment the array for maintenance safety from high voltage and temperature.

3 - Determine least-cost frame and cover that meets the safety requirements.

3 - Determine the least-cost packing density for the cells.

2,3 - Will the array or module withstand the environment (dust, sand, wind, temperature cycling, ice; freezing, high humidity, fog, snow, rain)?

2,3 - Does the design conform to the national and local codes: BOCA, UBC, SBC, ANSI, NBS, HUD, NEC, NFC, NEMA?

2,3 - Have the following structural loads been considered: weight, maintenance, snow, drifting, wind, earthquake, hail, ice, settlement, deflection, thermal cycling, humidity cycling, ground uplift, overturning, combinations of loods, and the probability of these events occurring?

3- Can the array be mounted on an existing structure?

3 - Are soil bor ings required?

3 - What is the least-cost foundation?

3 - What is the least-cost structural material?

3 - Should the structure be obtained from the module manufacturer or is a special design necessary? 
2- Is lightning protection provided?

3 - Is protection from falling objects (trees, sky lab, etc.) provided?

2 - How is the maintenance person protected from high voltages and temperatures associated with a sunlit array?

2 - Are dual leads provided for each cell?

D. Power Conditioner Design

5 - Does the design conform to the national and local codes: ANSI, NBS, HUD, NEC, NFC, NEMA?

9 - How is the power conditioner protected from the weather?

5 - Is there a potential instability associated with the power conditioner?

E. Energy Storage Design

4 - Does the design conform to the national and local codes: BOCA, UBC, SBC, ANSI, NBS, HUD, NEC, NEMA, NFC?

3 - Have the following structural loads been considered in the design of the housing: weight, maintenance, snow, drifting, wind, earthquake, hail, ice, settlement, deflection, thermal cycling, humidity cycling, ground uplift, overturning, combinations of loads, and the probability of these events occurring?

4- Should lead-acid (pure lead, lead-calcium, sealed, SLI), silverzinc, or nickel-codmium (pocket-plate?) batteries be used? Consider: cost, availability, depth of discharge, life (cycles, years), and capacity vs. temperature.

4 - Must the battery area be vented to prevent hydrogen buildup? (can catalytic caps or overcharge prevention be used insteod)?

4- Obtain the $1-V$ characteristics of the batteries as a combined function of temperature and state of charge.

4 - How many batteries can be charged in series?

4.- Are the batteries arranged to minimize shorting and shocking?

4 -. How rapidly will the hatteries self-discharge?

8- What is the impact of a failure of one battery? What provisions are made to offset this impact? 
F. Backup Energy System Design

7: What power source is used if photovoltaics need repair or routine maintenance?

7 - is an automatic backup power system provided (if needed)?

7 - How is the readiness of the backup power system maintained and checked?

\subsubsection{PROCUREMENT CHECKLIST}

1. Procurement Determination

a. Sole Source

b. Competitive Bid

2. Review of Bid

a. Review Sole Source price or low bid for compliance with all agency regulations.

b. Award Contract.

c. Review submittals for all equipment being furnished for project. Determine if shop drawings comply with project specifications and quantities.

d. Review manufacturers' worranties for all equipment being furnished.

e. Review contractors' bonding and warranties for all material and workmanship to complete project as specified.

\subsubsection{INSTALLATION CHECKLIST}

Tools and equipment required:

- Rachet handle

- Sockets and wrenches

- Screw driver

- Slip joint and locking pliers

- Crimping tool and terminals 
- Wire strippers

- Diagonal cutters

- Compass

- Inclinometer.

1. Handling and Unpocking

- Careful handling and unpacking of arrays is imperative. Check for shipping damage, if any and note same.

2. Mounting Structure

- Orientation

- Tilt angle

- Secure

- Lrounding and lightning protections

3. Wiring

- Proper series or parallel module interconnect

- Polarity protection/marking

- Proper wire guage

- Cable runs

- Weatherproofing

- Disconnects installed

4. Batteries.

- Proper number and size

- Correct interconnects

- Secure buse und housing

- Venting

- Safety notices

- Safety equipment (mild basic solution for lead-acid, mild acid solution for nickel-cadmium batteries to neutralize accidental spills on personnel or equipment).

\subsubsection{TEST AND ACCEPTANCE CHECKLIST}

Tools and equipment required, also see installation checklist:

- Voltage and current meters

- Insolation meter. 
1. Prior to acceptance testing insure that:

a. System has been properly cleaned and adjusted by installer.

b. Expected results are available (corrected to reference insolation, temperature, etc.).

c. Test instruments (not built into system) are available and calibrated.

- i. Mechanical Check:

= mounting in service

- grounding connected and properly installed

- lightning protection connected and properly installed

- weatherproofing installed as required

- drainage provided, if necessary

- safety codes being followed.

ii. Electrical Measurements and Record:

- insolation

- array output vol tage, current

- battery input voltage - open circuit, current - short circuit

- output of power conditioning equipment voltage, current.

\section{Documentation}

Ensure that appropriate warranty operation, maintenance, and associated documentation are supplied at time of sign-off. Representative drawings. which might be provided include:

1. Solar photovoltaic power system location

2. Solar photovoltaic system site plan

3. Grading plan

4. Array layout

5. Array foundation system

6. Array sections

7. Fence, road, and trail details

8. Gate details

9. Battery building site plan

10. Battery building plan

11. Battery building elevations

12. Wall serctions and details

13. Eave detail and sections

14. Array subfield assembly

15. Subframe assembly

16. Subframe

17. Frome 


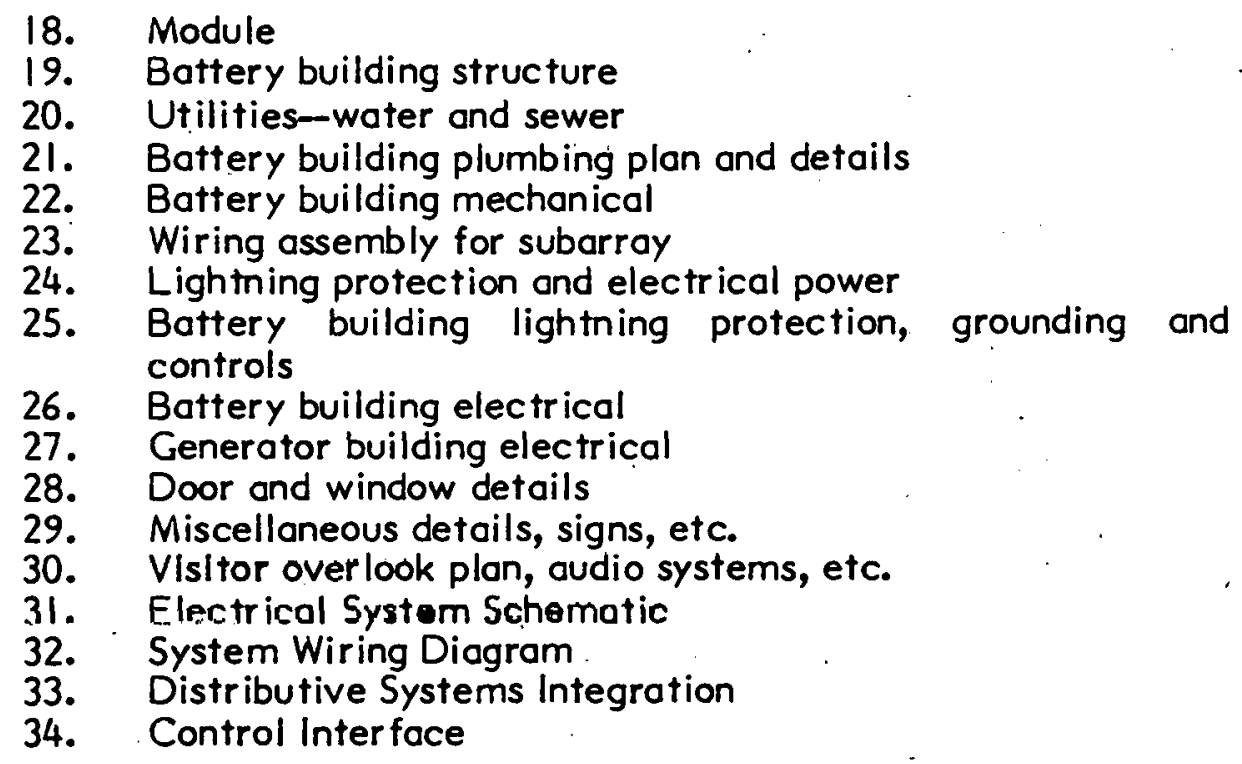

\subsubsection{OPERATIONS AND MAINTENANCE CHECKLIST}

Equipment required:
- Ammeter
- Volt meter
- Flat sturfoce thermorreter
- Hydrometer
- Immersion thermometer
- Air thermometer.
1. Battery Checks
o.: Is the electrolyte level low? Replenish with distilled water, if necessary.

b. Check the state of charge, correcting the hydrometer reading for the temperature of the electrolyte. The specific grovlty of the electrolyte should not differ by more than 0.02 between batteries.

c. Is the state of charge consistent with the season and
recent weather conditions? 


\section{Array Checks}

a. Is the array free from excessive dirt buildup? If not, wash with water or mild detergent solution. Do not use solvents, strong detergents, or abrasives.

b. Check the array for breakage, inspecting both the modules and their supporting structure.

c. Disconnect the array. Follow all safety precautions (shading the orray from the sun will reduce the possibility of injury).

d. Remove any cover on the array. Measure the open circuit voltage across the array. Is it consistent with design values?

e. Measure the short circuit current produced by the array. Is the measurement consistent with the design value (taking into account insolation conditions)? If not, procede to the troubleshooting guide.

\subsubsection{SPECIAL SAFETY CONSIDERATIONS}

All photovoltaic system designs shall comply with the safety measures set forth in the various articles of the National Electrical Code that pertain to Direct Current (DC) Systems and all other articles that are pertinent.

The following are suggestions that should be incorporated into all systems above $50 \mathrm{~V}$.

1. Provide black cloth or other suitable material to completely cover array to prevent power generation when maintenance is being performed on live electrical parts.

2. Any modules whose combined voltage exceeds $50 \mathrm{~V}$ shall be provided with a disconnecting means to facilitate maintenance and troubleshooting procedures.

3. All systems shall have total lightning protection.

4. All systems shall have a driven ground frame when system voltage exceeds $50 \mathrm{~V}$.

5. All modules shall have adequately sized, factory installed junction boxes as an integral part of the individual module.

6. Each module junction box shall have insulated stand-off terminal blocks secured firmly to the junction box with metal screws or bolts.

7. All module junction boxes shall have weathertite covers and weathertite cable entrances and exits. 
8. Provision for battery disconnecting means is required.

9. Provision for array disconnecting means is required when array and distribution panel are not within sight of each other.

10. All loads on system shall have adequate disconnecting means and branch circuit protection.

11. Adequate ventilation is imperative when batteries are in an enclosure.

12. Face mask, gloves, and acid neutralizing agent shall be provided to protect personnel servicing installations.

13. All batteries shall have flame arrestors.

\subsection{SPECIFIC SYSTEMS DESIGNS}

\subsubsection{NATIONAL ELECTRICAL CODE (NEC)}

\subsubsection{SAMPLE AREAS OF CONCERN}

The following sections of the NEC should be studied for compliance with the photovoltaic design being developed.

ARTICLÉ

Article 90-02 (b)

Article 110-17

Article $210-10$

Article 215-07

Article 250-03

Article 250-22

Article 250-93

Article 250-131

Article 280

Article 480

Article 720

\section{TITLE}

Not Covered

Guarding of Live Parts

UNGRUUNDEED Conductors Tapped from Grounded Systems

UNGROUNDED Conductors Tapped from Grounded Systems

Direct-Current Systems

Point of Connection for Direct-Current Systems

Size of Direct-Current System Grounding Conductor

Service of Less Than 1,000 Volts

Lightning Arrestors

Storage Batteries

Circuits and Equipment Operating at Less Than 50 Volts 


\section{TYPES OF BATTERIES}

\begin{tabular}{|c|c|c|c|c|}
\hline ACTIVE MATERIAL & TYPE & VOLTS/CELL & $\mathbf{w}-\mathbf{h} / \mathbf{l b}$ & FEATURES \\
\hline Zinc-Carbon & Primary & 1.5 & 35 & $\begin{array}{l}\text { Low cost, wide variety of small } \\
\text { sizes }\end{array}$ \\
\hline Alkaline-Manganese & $"$ & 1.5 & 42 & $\begin{array}{l}\text { Good low-temperature opera- } \\
\text { tion, high efficiency under high- } \\
\text { drain duty, more costly than zinc- } \\
\text { carbon }\end{array}$ \\
\hline Mencury & 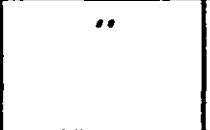 & 1.3-1.4 & 66 & $\begin{array}{l}\text { Excellent high-temperature per- } \\
\text { formance. Pelatively flat dis- } \\
\text { charge characteristics }\end{array}$ \\
\hline Lithium & 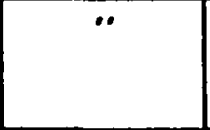 & 2.95 & 150 & $\begin{array}{l}\text { Highest energy density. temper- } \\
\text { ature range and shelf life of } \\
\text { primary cells. contains no water }\end{array}$ \\
\hline Lead Acid & Secondary & 2.0 & 12 & $\begin{array}{l}\text { Least expensive and most readily } \\
\text { available of secondary cells }\end{array}$ \\
\hline Nickel Cadmium & “ & 1.2 & 16 & $\begin{array}{l}\text { Excellent low-temperature opera- } \\
\text { tion, low weight. low mainte- } \\
\text { nance, higher initial cost than lead }\end{array}$ \\
\hline Lithium-Sulfur & .• & $\approx 1.5$ & $\approx 60$ & $\begin{array}{l}\text { Operate at high temperature. } \\
400^{\circ} \text { C. not currently com- } \\
\text { mercially available, projected } \\
\text { costs } \$ 25 / \mathrm{kWh}\end{array}$ \\
\hline Sodium, Sulfur & 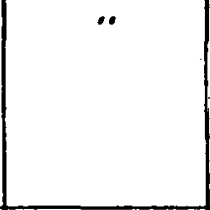 & 2.2 & $\approx 95$ & $\begin{array}{l}\text { Operate at high temperature, } \\
\approx 300^{\circ} \mathrm{C} \text {. very low self- } \\
\text { discharge, projected costs are } \\
\$ 20 / \mathrm{kWh} \text {, should be com- } \\
\text { mercially available in mid } 1980 \text { 's }\end{array}$ \\
\hline Sodium/Chloride & “ & 2.12 & $\approx 70$ & $\begin{array}{l}\text { Not commercially available, } \\
\text { projected costs } \$ 20 / \mathrm{kWh} \text { with } \\
\text { efficiencies of greater than } 90 \% \text {. } \\
\text { operates at } 200^{\circ} \mathrm{C}\end{array}$ \\
\hline
\end{tabular}




\section{WARRANTY}

It is understood and agreed that the equipment offered will be free from defects in material, workmanship and performance for a period of not less than one year after acceptance by the Government. During the guarantee period all broken or defective parts not caused by misuse or accident through fault or negligency by the Government must be replaced, and all necessary equipment adjustments occasioned by such defective parts shall be made at the contractor's expense including labor, parts and transportation costs, if any. 


\section{MANUFACTURERS PHOTOVOLTAIC MODULE/ARRAY QUALITY ASSURANCE TEST DATA}

\begin{tabular}{|c|c|}
\hline \multicolumn{2}{|c|}{ 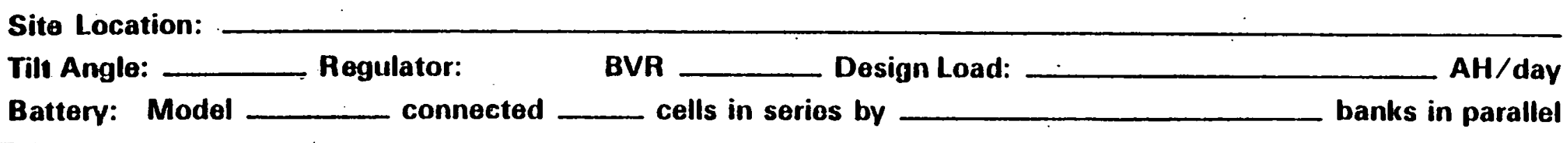 } \\
\hline & \\
\hline $\begin{array}{l}\text { Module Performance } \\
\text { Module Model: } \\
\text { Rated Output*: } \\
\text { Rated Short Circuit Current }{ }^{*}: \text { Amps at } \\
\text { No. of Series-Connected Solar Cells: } \\
\text { No. of Parallel-Connected Solar Cells: } \\
\text { Array Performance } \\
\text { No. of Separate Modules in Array: } \\
\text { Rated Stoort Circuit Current* of trame(s): }\end{array}$ & $\begin{array}{l}\text { Module Performance } \\
\text { MODEL }- \text { module } \\
\text { Rated Short Circuit Current*: } \\
\text { No. of Series-Connected PV Cells: } \\
\text { No. of Parallel-Connected PV Cells: } \\
\text { MODEL } \\
\text { Rated Short Circuit Current* } \\
\text { No. of Series-Connected PV Cells: } \\
\text { No. of Parallel-Connected PV Cells: }\end{array}$ \\
\hline Rated Short Circuit Current" of module(s): _— amps ea & $\begin{array}{l}\text { At } 100 \mathrm{~mW} / \mathrm{cm}^{2} \text { sunlight intensity and } \\
28^{\circ} \mathrm{C} \text { cell temperature. }\end{array}$ \\
\hline
\end{tabular}


Project \#00003 Sweetwater, Arizona Well \#9T216

Latitude $=36^{\circ}-40^{\prime}$

Longitude $=109^{\circ}-30^{\prime}$

\subsubsection{REQUIREMENTS}

General - The water system must supply continuous drinking water year-round for 24 people, 50 animals, and I watering point. The estimated consumption will not exceed $2,400 \mathrm{gal} / \mathrm{day}$.

The estimate was calculated in the following manner (gallonis per day):

$\begin{array}{llrr}\text { CONSUMER } & & \frac{\text { EACH }}{50} & \frac{\text { TOTAL }}{1200} \\ \text { people } & = & 12 & 600 \\ \text { animals } & = & 600 & .600 \\ \text { watering point } & & \end{array}$

Well Supply - The maximum daily consumption (2,400 gallons) must be supplied by the well. Additionally, the well must retain a depth of water necessary to prevent damage to the interior of the well, which requires that the well supply the maximum daily consumption with normal depth level retention. This well will yield $20 \mathrm{gal} / \mathrm{min}$ without pump down.

Pumping - The pump driven by photovoltaics must be capable of supplying the maximum daily consumption during daylight hours year-round.

The walking beam positive displacement pump was selected for the application. The available well is $350^{\circ}$ deep with a 2.5 in. drop pipe. The motor required is a I hp permanent majiiel. The Uriver pully, mounted on the motor, will be 4 in. in diameter. The pump will pump $648 \mathrm{gal} / \mathrm{hr}$ at 35 strokes/min.

Storage - The water storage tank must be capable of storing at least 10 days of water or 24,000 gallons.

Sensing Tank Storage - The purposes of the sensors are four-fold;

- to de-activate the pump motor when the tank is at full copacity to prevent overflow and unnecessary pumping;

- to actuate the pump motor to refill the tonk in its normal day-to-day mode;

- to actuate the pump motor to run all day, if necessary, to supply the demand water;

- to de-activate the pump motor in the event of an extreme low water condition that probably indicates a malfunction in the storage tank or distribution system. 
- Sensor at high water mark to cut off pump motor when tank has 24,000 gallons.

- Sensor (intermediate) at 19,200 gallons remaining mark to turn on pump during daylight only.

- Sensor at lower intermediate mark $(9,600$ gallons remaining mark) to turn on both battery and array.

- Sensor at critical empty (4,800 gallons remaining mark) to turn off pump motor which can then only be started by monual override.

Array and Battery Monitoring - Electrical system monitoring is required to ensure proper operation and maintenance of all components.

- Voltage meter, located in the pump house, shall isolate' and monitor voltage on each array string, which also indicates total array voltage.

- Voltage meter, located in the pump house, shall isolate and monitor voltage of each battery and total battery voltage through manual switching.

- A Ammeter shall measure total array output current via manual switching or fixed wiring.

- Ammeter shall measure pump motor current via manual switching.

Electrical Storage Batteries - Must be capable of storing sufficient energy to drive the positive displacement pump to provide 4 days' supply of water in the event of emergencies or extreme low insolation.

\subsubsection{LOAD STATISTICS AND COMPUTATIONS}

\section{Water Requirement:}

6 families +90 head of cattle

4 people $/$ family at $50 \mathrm{gal} /$ person $=200 \mathrm{gal} / \mathrm{day}$

6 families $=200 \times 6$

50 cows @ 12 gal/cow

1 watering point

Total gal/day
$=1,200 \mathrm{gal} / \mathrm{day}$

$=600 \mathrm{gal} /$ day

$=600 \mathrm{gal} / \mathrm{day}$

$=2,400$ 


$$
\begin{aligned}
\text { Existing well: } & \\
\text { well depth } & =350^{\prime} \text { pumping depth } \\
\text { pump } & =\text { walking beom positive displacement } \\
\text { drop pipe } & =2 y^{\prime \prime} \\
\text { cylinder } & =24^{\prime} \\
\text { rod } & =\# 1 \text { hollow } \\
\text { GPM } & =10.8 \\
\text { GPH } & 648 \\
\text { Motor Horsepower } & =\frac{\text { GPM } \times 1 \mathrm{~b} / \text { gal } \times \text { well depth }(\mathrm{ft})}{\text { foot pounds/horse power min. }} \\
& =\frac{10.8 \times 8.355 \times 350}{33,000} \\
& =\frac{957 \mathrm{hp}}{}
\end{aligned}
$$

Motor Drive for Pump:

use I hp permanent magnet motor

I hp motor rated at 10.6 A full load @ $90 \vee(1,725$ rpm)

Driver Pulley (Diameter):

$$
\begin{aligned}
\text { Driver Diameter } & =\frac{\text { diameter of driver } \times \text { transmission speed } \times \text { strokes } / \mathrm{min}}{\text { speed of driver motor }} \\
\text { D. D. } & =\frac{6.5^{\prime \prime} \times 30.9 \times 35}{1,725} \\
\text { D. } 0 . & =\frac{7,029.75}{1,725} \\
\text { D. D. } & =4.07^{\prime \prime} \text { dlameter (use 4" dlameter pulley) }
\end{aligned}
$$

Array Requirements (Hours per day):

$90 \mathrm{~V}$ motor being driven

batteries=45 cells@2 V=90 V 
battery storage $=156 \mathrm{Ah}$

battery selected $=3$ sets of $15 Q P 75-5$ as manufactured by $C$ and $D$ batteries.

Latitude $=30^{\circ}-40^{\prime}$

Longitude $=109^{\circ}-30^{\prime}$

Equivalent $\mathrm{hr} /$ day (with $20 \%$ safety) for south facing panel.tilted at angle to latitude := $4.54 \mathrm{hr} /$ day

Amp Demand for System: $2,400 \mathrm{gal} /$ day $\div 648 \mathrm{GPH}$ from pump

$$
=3.70 \text { hours of pumping/day }
$$

Motor demand on array $=3.70$ hours of pumping $\times 10.6 \mathrm{~A}$

$$
=39.22 \mathrm{Ah} / \mathrm{day}
$$

Battery dèmand on array $=39.22 \times 1.10=43.14 \mathrm{Ah}$

(for 90 percent efficiency of battery - multiplying factor of 1.10)

The required rated array output

$=43.14 \div 3.7$ hours of pumping

Pump Amp Draw

$$
=11.66 \text { Amps }
$$


Array Sizing (Modules)

$$
\begin{aligned}
\text { battery charging voltage } & =45 \text { cells } \times 2.6 \mathrm{~V} \text { cell } \\
& =117 \text { volts on array }
\end{aligned}
$$

each module $=16 \mathrm{~V}$

- array voltage $=117 \div 16.7 \mathrm{~V} /$ module

$=7$ modules

use 7

Optimizing Array Size (Strings)

7 modules in series $=7 \times 16.7=116.9 \mathrm{~V}$

array voltage $=116.9 \mathrm{~V} @ 1.12 \mathrm{~A} / \mathrm{s} / \mathrm{ring}$

Hours of good sun for this location $=5 \mathrm{hr} /$ day

array output $=43.14 \mathrm{Ah} \div 5 \mathrm{hr}=8.63 \mathrm{~A}$

Each string of modules $=8.63 \div 1.12 \mathrm{~A} /$ string

$$
=7.7 \text { strings }
$$

use 8

Array size $(\mathrm{Ah} /$ day $)=7$ modules series connection $=1$ string at $116.9 \mathrm{~V}$

8 strings of 7 modules

actual array output

array output (Ah/day)
$=56$ modules

$=8.96 \mathrm{~A}$

$=8.96 \times 5 \mathrm{hr}$

$=44.8 \mathrm{Ah} / \mathrm{day}$ 


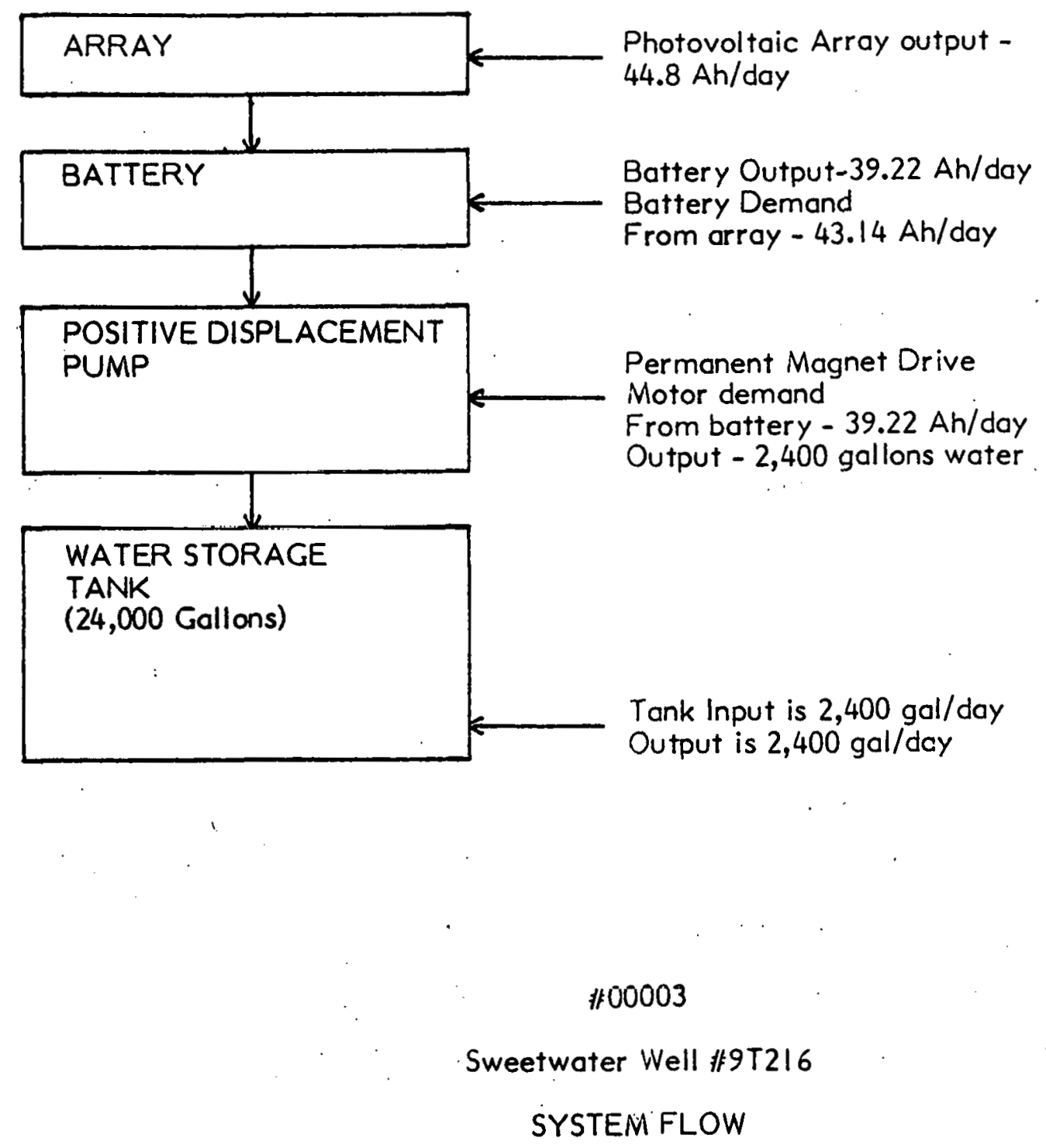




\section{GENERAL SYSTEM LAYOUT (Not to Scale)}

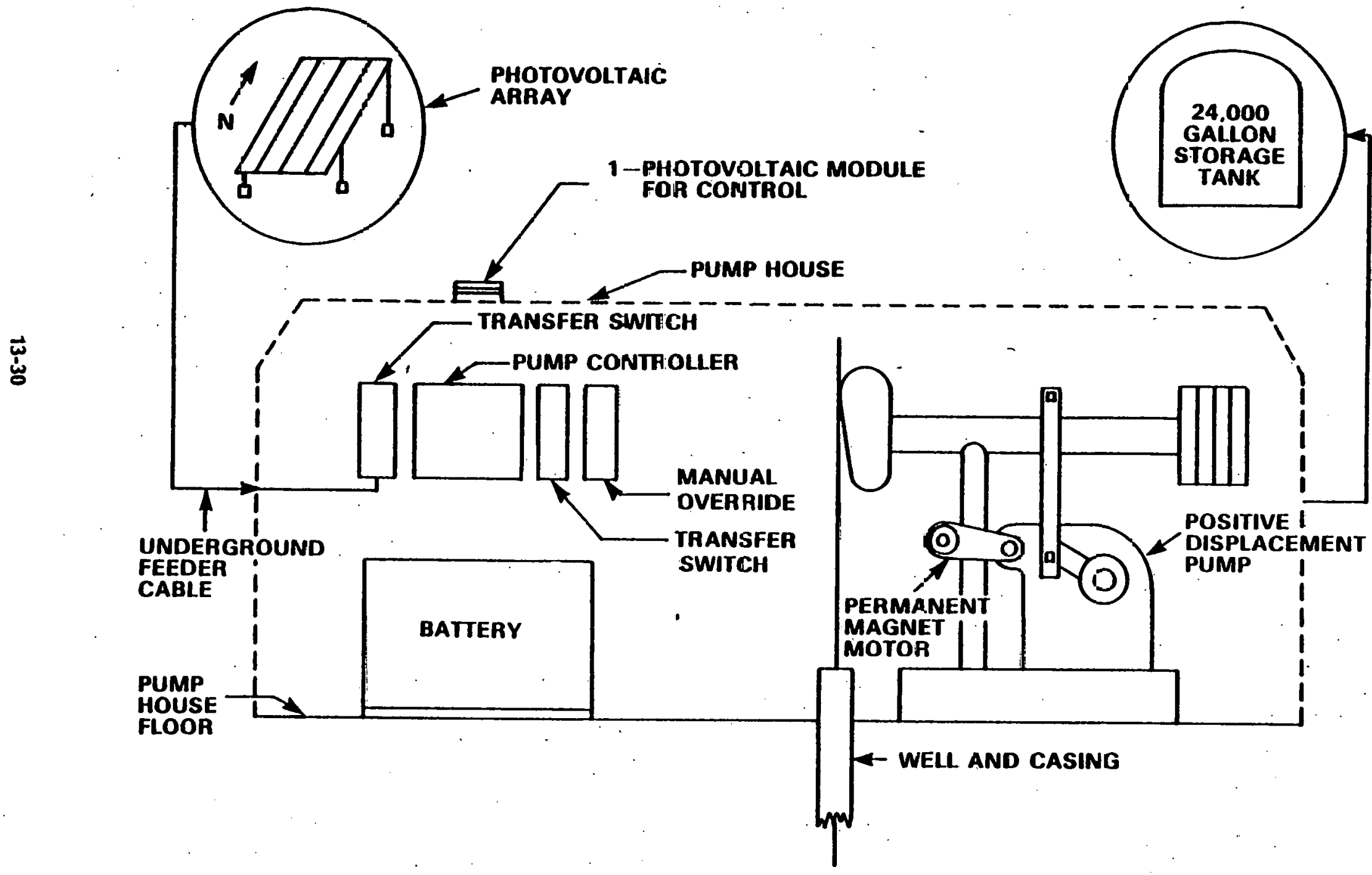




\section{SVSTEEM CONTROL}

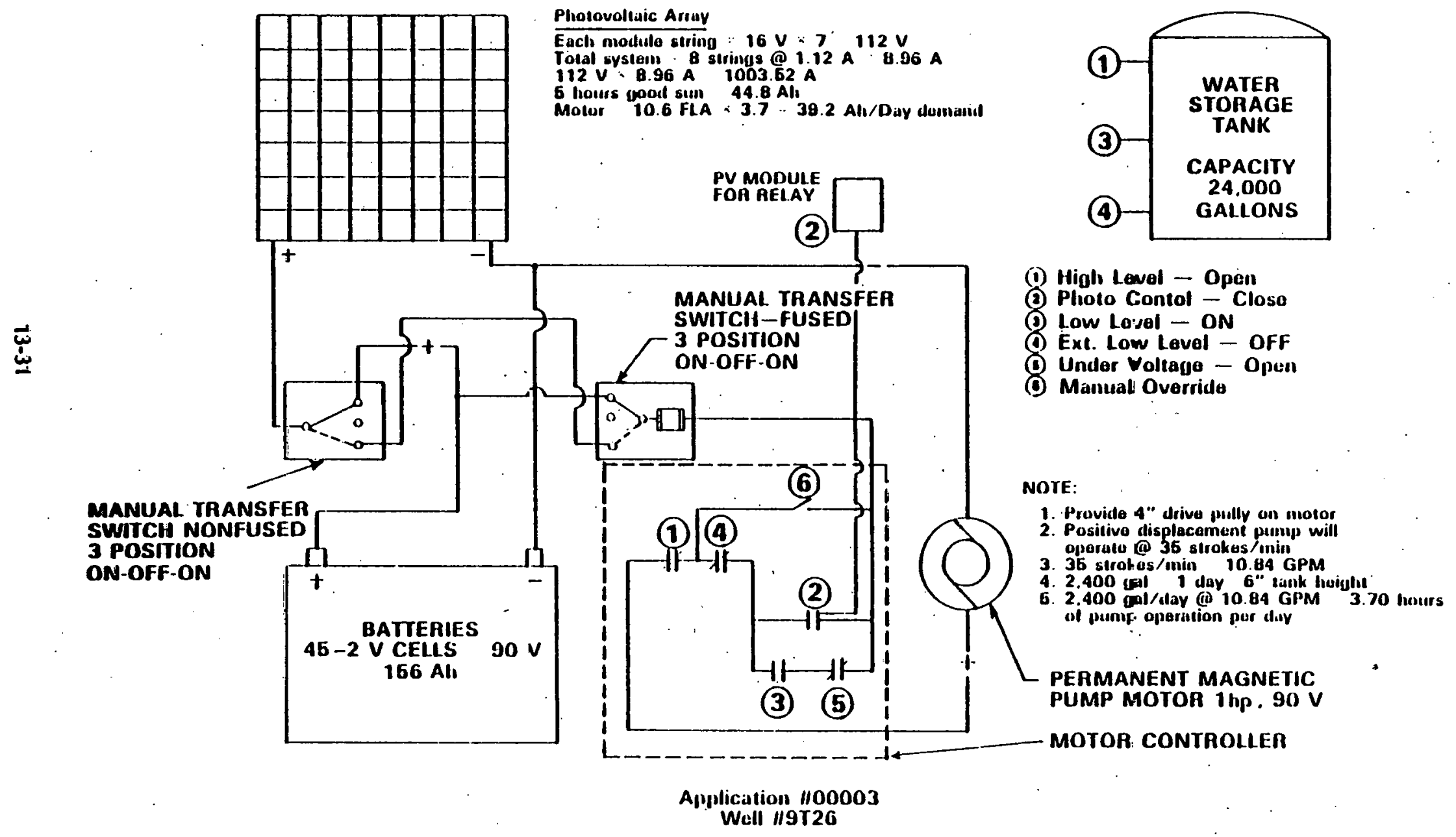




\section{STORAGE TANK CONTROL LEVELS}

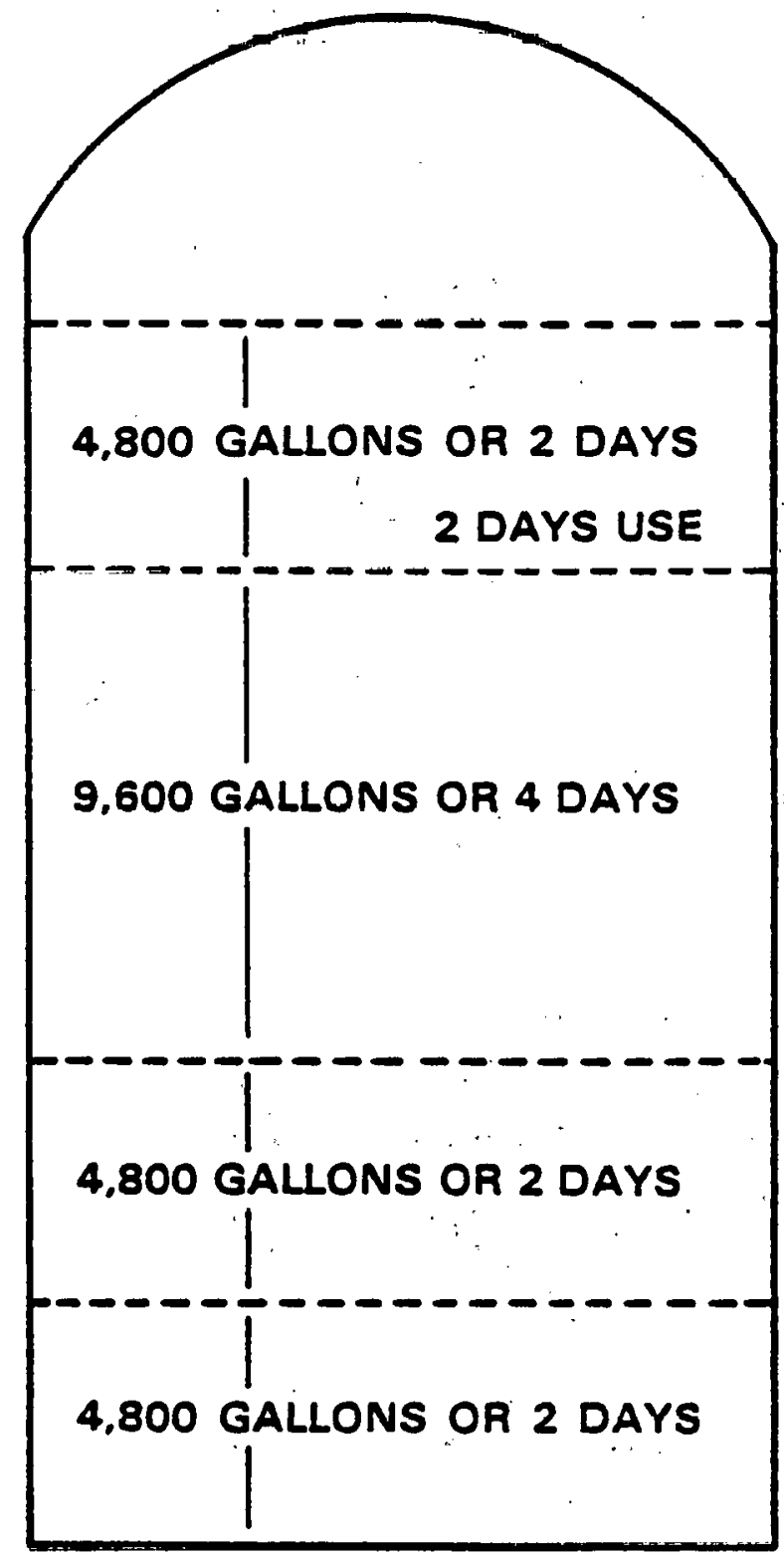

FULL OFF

NORMAL MODE PUMP DAY ONLY

PUMP DAY AND NIGHT

PUMP OFF

CAN ONLY BE

MANUALLY RESET 
MONITOR PANEL

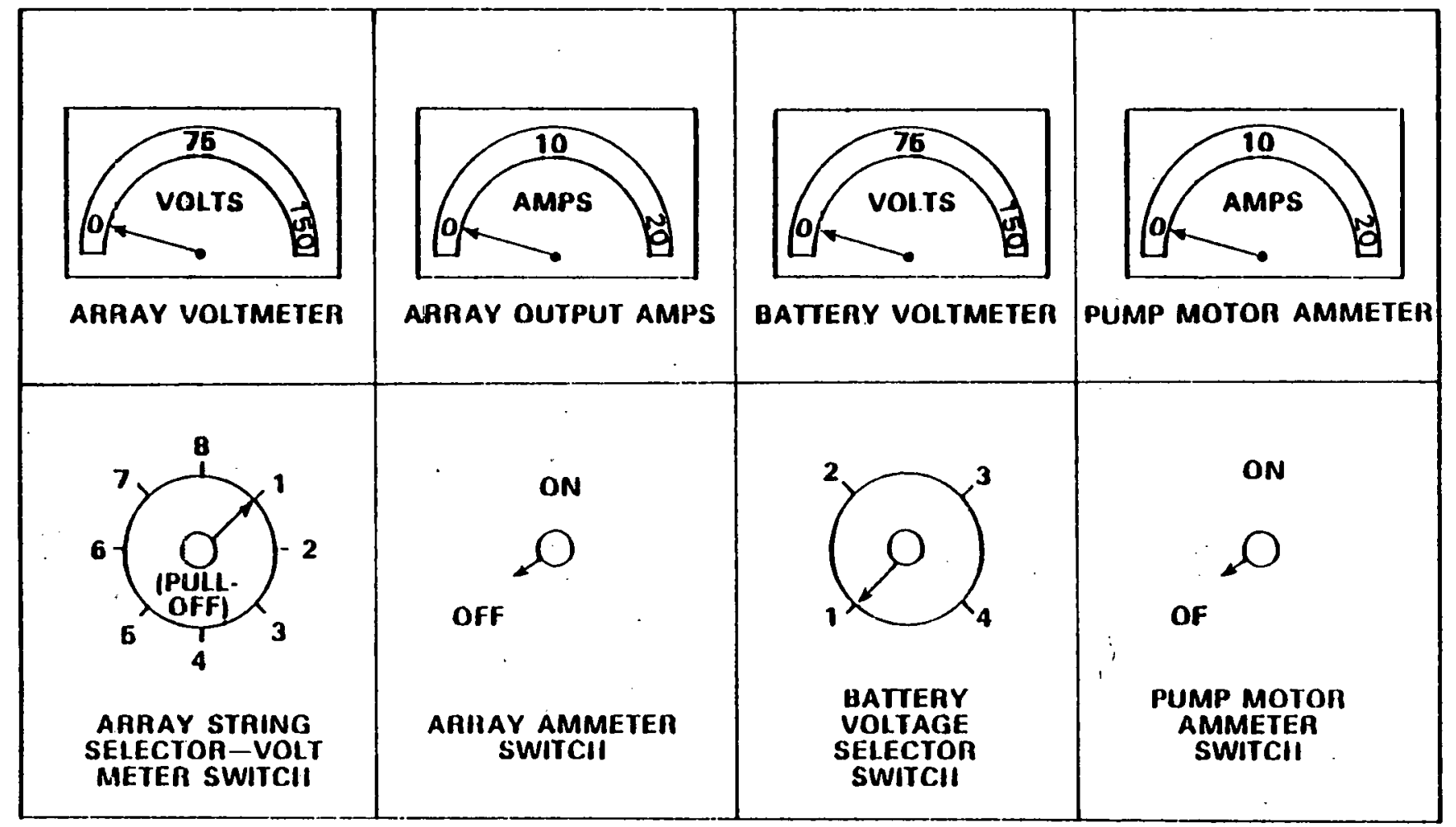

$\$ 00003$ Swoulwater Woll 97216 


\subsubsection{DOMESTIC PHOTOVOLTAIC POWER}

\subsubsection{REQUIREMENTS}

\section{General}

The photovoltaic system shall provide electricity for remotely located homes where hookup to utility grid lines is not economically feasible or where utility backup is necessary. The electricity supply shall be adequate to allow refrigeration of food, house lighting, television or radio or both, and cistern water pumping.

\section{Refrigeration}

A $6 \mathrm{cu} f t$ refrigerator shall provide cold storage for the average family. The power requirement is as follows:

$12 \vee D C$ motor, $60 \mathrm{~W}, 5 \mathrm{~A}$

25 percent duty cycle (average)

Total Ah required $=24 \mathrm{hr} \times$ duty cycle $\times 5 \mathrm{~A}=30 \mathrm{Ah}$.

\section{Lighting}

The domicile will be equipped with 4 fluorescent lights, 2-40 W and 2-10 W. A conservative estimate would be 4 hours of usage per night. The charge requirement for lighting is as follows:

$$
2 \times 40 \mathrm{~W} \div 12 \mathrm{~V} \times 4 \cdot \mathrm{hr}+2 \times 10 \mathrm{~W} \div 12 \mathrm{~V} \times 4 \mathrm{hr}=33.33 \mathrm{Ah}
$$

Television

Television usage is estimated at approximately $4 \mathrm{hr} /$ day. Charge requirements $-4 \operatorname{hr} \times 24 W \div 12 \mathrm{~V}=0.0 \mathrm{Ali}$.

Cistern Water Pump

A permanent magnet, positive displacement water pump shall be used, which draws $5 \mathrm{~A}$ under full load. The pump shall fill a pressurized tank; which shall then supply the domicile's water needs. A positive displacement pump was chosen over a centrifigal or screw type pump due to its higher efficiency $(80$ percent versus $\approx 50$ to 35 percent). An average family requires $200 \mathrm{gal} / \mathrm{day}$; the pump provides $9.4 \mathrm{gal} / \mathrm{min}$. Charge requirements $=200 \mathrm{gal} \div 9.4 \mathrm{gal} / \mathrm{min} \div$ $60 \mathrm{~min} / \mathrm{hr} \times 6 \mathrm{~A}=6.38 \mathrm{Ah}$.

\section{Food Preparation}

If . cooking cannot be done by an alternative method (i.e., LIVG, wood, etc.,) a microwave oven can be incorporated into the domicile's design. This unit will increase the electrical power needs by almost 65 percent and, as a result, increase the cost of the PV power system by several thousand dollars (33 percent). Incorporating a microwave oven into the house design (option 2) is only recommended if there is no reasonable alternative heat source available for food preparation. 
Microwave Oven (Option 2)

The microwave oven is equipped with its own inverter and transformer circuits. The size of the microwave ovén used does not appreciably alter the energy required as a larger oven would be on a proportionately shorter time (i.e., it is the number of Btu's absorbed by the food that is important). A $725 \mathrm{~W}$ unit, $60.5 \mathrm{~A}$ at $12 \mathrm{~V}$, if used for all three meals, would be used approximately $50 \mathrm{~min} /$ day. $50 \mathrm{~min} /$ day $\div 60 \mathrm{~min} / \mathrm{hr} \times 60.5$ $A=50.4 \mathrm{Ah} /$ day.

Total Charge Requirements Per Day (Average)

Option 1

$\begin{array}{ll}\text { Refrigerator } & =30.0 \mathrm{Ah} / \text { day } \\ \text { Lighting } & =33.3 \mathrm{Ah} / \text { day } \\ \text { Television } & =8.0 \mathrm{Ah} / \text { day } \\ \text { Cistern Pump } & =\frac{6.4 \mathrm{Ah} / \text { day }}{77.7 \mathrm{Ah} / \text { day }}\end{array}$

Option 2

$\begin{array}{ll}\text { Refrigerator } & =30.0 \mathrm{Ah} / \text { day } \\ \text { Lighting } & =33.3 \mathrm{Ah} / \text { day } \\ \text { Television } & =8.0 \mathrm{Ah} / \text { day } \\ \text { Cistern Pump } & =6.4 \mathrm{Ah} / \text { day } \\ \text { Microwave } & =\frac{50.4 \mathrm{Ah} / \text { day }}{\text { Total }}\end{array}$

System Component Sizing

Option 1

Array - $220 \mathrm{Wp}-13.75 \mathrm{Ap}$

$11-12 \vee$ panels

Battery 680 Ah @ 12 V

Volt regulator - yes, to prevent excessive outgassing due to overcharge

Option 2

Array - $340 W p-21.25$ Ap 
17 to 12 Volt panels

Battery=1,121 Ah@ $12 \mathrm{~V}$

Volt regulator - yes, to prevent excessive outgassing due to overcharge

\section{Control of PV Power System}

- A manual switch shall be provided to isolate the load for maintenance purposes.

- The systëm shall be fused to prevent battery drain if a short circuit develops. Standard buss (car fuses) fuses are acceptable and readily available for this pi.ırnnse.

Array and Battery Monitoring

- Voltage meter with switches located in the battery storage box that will isolate and monitor either the battery or array voltage.

- Ammeter with switches located in the battery storage box that will monitor either the total array output current or the total load current.

\section{Electrical Storage}

- Shall be provided by lead-acid batteries that exhibit low self-discharge.

- Venting or recombiner caps shall be provided to prevent dangerous hydrogen gas buildup.

- Sufficient storage shall be provided to prevent dangerous hydrogen gas buildup.

- Battery shall undérgo a maximum 60 percent depth of discharge.

\section{Sáfety Considerations}

Disconnect switches between the panels shall not be required due to the low (i.e., $12 \mathrm{~V}$ ) system voltage. An opaque cover shall be available for use during maintenance to cover the array, thereby effectively turning the array "off." . Due to the location of the battery storage in a residence, special care must be taken to ensure proper venting of gases and protection of the battery terminals against accidental shorting.

\subsubsection{SUMMARY OF DOMICILE DESIGN}

1. Schematic of Design

2. Computation of Load-Option I (no microwave)

3. Total Daily Requirement - Option I 
4. Initial Array Sizing - Option I

5. Month-by-Month Calculations - Option 1: Based on Exhibit I

Exhibit I.A - II panels (winter months used for sizing)

Exhibit I.B - 10 panels (yearly average used)

Exhibit I.C - 10 panels

6. Choice of Exhibit I.A, Exhibit I.B, or Exhibit I.C (which design is optimal?)

7. State of Charge Batteries -: Option I

8. Option II - Microwave Oven Load Computation

9. Initial Array Sizing - Option 2

10. Month-by-Month Calculations:

Exhibit 2.A - 17 panels $50^{\circ}$ Tilt

Exhibit 2.8 - 16 panels $50^{\circ}$ Tilt

11. Battery State of Charge - Option 2

12. Estimated Costs of Installed Systems - Options I and 2

\subsubsection{LOAD COMPUTATION}

Refrigeration $6 \mathrm{ft}^{3}$

12 V DC Motor, $60 \mathrm{~W}, 5 \mathrm{~A} \quad 25 \%$ duty cycle $5 \mathrm{~A} \times 24 \mathrm{hr} \div 4=30 \mathrm{Ah}$

Lighting - $4 \mathrm{FI}$ lights 4.hr/day

$$
2-10 W 12 \mathrm{~V}, 0.83 \mathrm{~A}
$$

$2 \times 3.33 \mathrm{~A} \times 4 \mathrm{hr}+2 \times 0.83 \mathrm{~A} \times 4 \mathrm{hr}=33.33 \mathrm{Ah}$

TV $-4 \mathrm{hr} /$ day $24 \mathrm{~W}, 12 \mathrm{~V}, 2 \mathrm{~A}$.

$2 \mathrm{~A} \times 4 \mathrm{hr}=8 \mathrm{Ah}$

Cistern Woter Pump -

$12 \mathrm{~V}, 72 \mathrm{~W}, 6 \mathrm{~A}$

Pump delivers $9.4 \mathrm{gal} / \mathrm{min}$

Need $200 \mathrm{gal} /$ day

$200 \mathrm{gal} /$ day $\div 9.4 \mathrm{gal} / \mathrm{min} \div 60 \mathrm{~min} / \mathrm{hr} \times 6 \mathrm{~A}=6.38 \mathrm{Ah}$ 
DAILY LOAD - OPTION I (No microwave)

\begin{tabular}{lr} 
Refrigeration & $30.0 \mathrm{Ah}$ \\
Lighting & $33.3 \mathrm{Ah}$ \\
TV & $8.0 \mathrm{Ah}$ \\
Water. Pump & 6.4 Ah \\
\hline Total & 77.7 Ah/Day
\end{tabular}

Initial Array Size (First Estimate)

A peak $=\quad \frac{\text { Average Daily Load (Ah)* }}{\text { Average Monthly Equivalent Hours Peak Sun (Winter Average) }}$

$$
A p=\frac{77.7}{\frac{(6.08+6.03+6.2)}{3}}=12.73 \text { Ap Total }
$$

Panel chosen produces $1.25 \mathrm{~A}$ at $60^{\circ} \mathrm{C}$ under an insolation of $100 \mathrm{~mW} / \mathrm{cm}^{2}$

Panels needed $=\quad \frac{12.73}{1.25}=10.18$ or 11 panels

If a yearly insolation average is used, the initial estimate would he:

$$
A p=\frac{77.7}{6.7}=11.57 \mathrm{~A}
$$

Ponels needed $=\quad \frac{11.57}{1.25}=9.26$ or 10 panels.

* Provision for degradation is not included. The calculations that follow can be used for a trade-off study regarding adding more storage capability vs. adding more modules or, if necessary, management interventions in critical design areas. 
Exhibit 1

COMPUTATION OF SYSTEM PERFORMANCE $-55^{\circ}$ Tilt, $35^{\circ}$ Latitude

1. Array size $(A h)=13.75 \mathrm{p}\left(\right.$ at 1.85 at $\left.60^{\circ} \mathrm{C}, 100 \mathrm{~mW} / \mathrm{cm}^{2}\right)$

Panels required $=\frac{12.73}{1.25}=10.18$

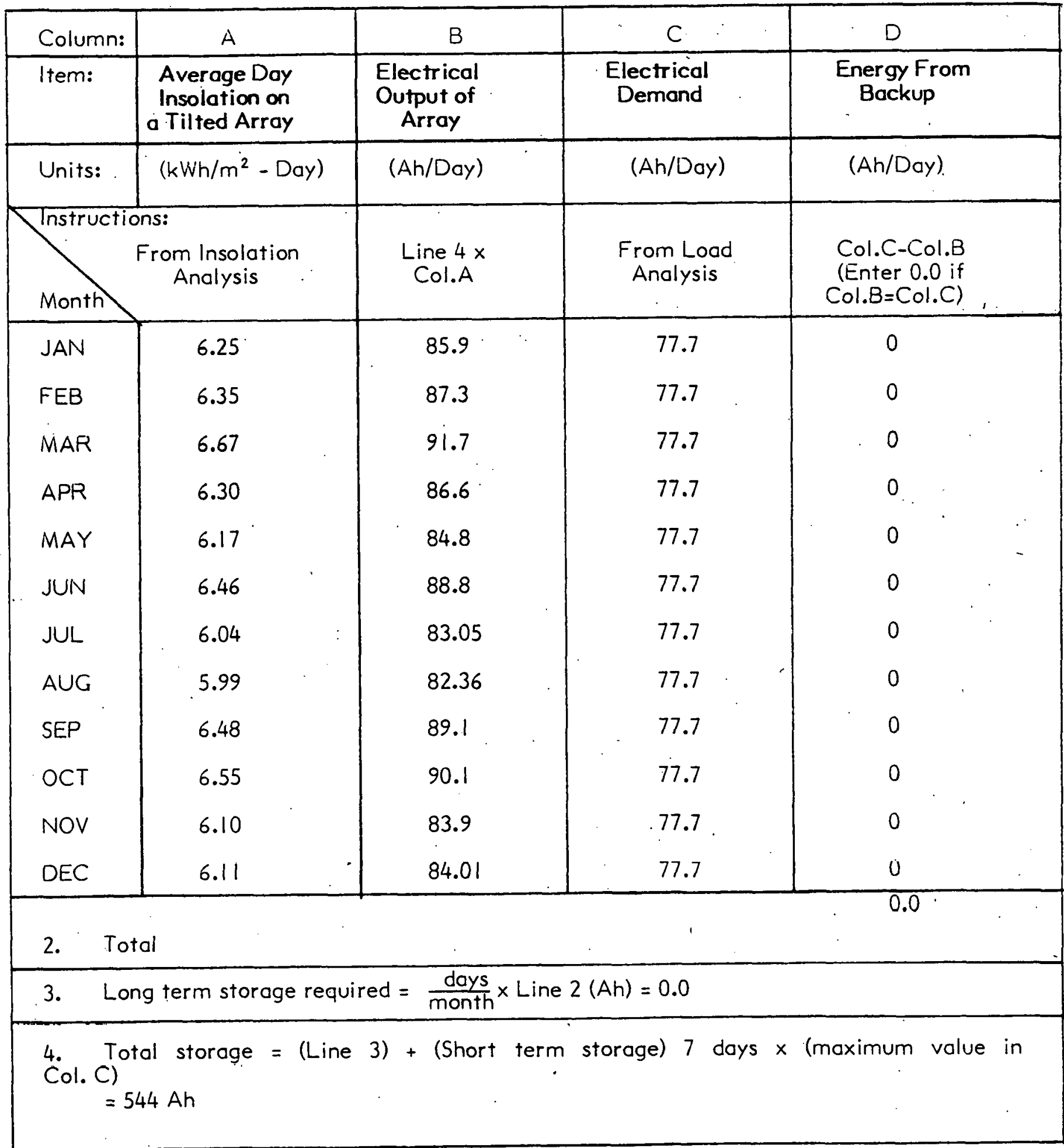


Exhibit I.A

COMPUTATION OF SYSTEM PERFORMANCE - $50^{\circ} \mathrm{Tilt}$

I. Arroy size $(A p)=13.75 \mathrm{Ap}$

$1.25 \mathrm{~A} /$ panel @ $60^{\circ} \mathrm{C} @ 100 \mathrm{~mW} / \mathrm{cm}^{2}$

$\frac{12.73}{1.73}=10.18$ panels

use II. panels $=13.75 \mathrm{~A}$

\begin{tabular}{|c|c|c|c|c|}
\hline Column: & A & 8 & r & D \\
\hline Item: & $\begin{array}{c}\text { Average Day } \\
\text { Insolation on } \\
\text { a Tilted Array }\end{array}$ & $\begin{array}{c}\text { Flectrical } \\
\text { Output of } \\
\text { Array }\end{array}$ & $\begin{array}{l}\text { Electrical } \\
\text { Demand }\end{array}$ & $\begin{array}{c}\text { Energy From } \\
\text { Backup }\end{array}$ \\
\hline Units: & $\left(\mathrm{kWh} / \mathrm{m}^{2}-\right.$ Day $)$ & (Ah/Day) & (Ah/Day) & (Ah/Day) \\
\hline Instructio & $\begin{array}{l}\text { From } \\
\text { Analysis }\end{array}$ & $\begin{array}{c}\text { Line } 1 \mathrm{x} \\
\text { Col.A }\end{array}$ & $\begin{array}{l}\text { From Load } \\
\text { Analysis }\end{array}$ & $\begin{array}{l}\text { Col.C-Col.B } \times \frac{\text { days }}{\text { (Enter } 0.0 \text { if }} \\
\text { Col.B=Col.C) }\end{array}$ \\
\hline JAN & 6.2 & 85.3 & 77.7 & 0 \\
\hline FEB & 6.37 & 87.6 & 77.7 & 0 \\
\hline MAR & 6.38 & 87.7 & 77.7 & 0 \\
\hline APR & 6.62 & 91.0 & 77.7 & 0 \\
\hline MAY & 6.57 & 90.3 & 77.7 & 0 \\
\hline JUN & 6.91 & 95.0 & 77.7 & 0 \\
\hline JUL & 6.45 & 88.7 & 77.7 & 0 \\
\hline$A \cup G$ & 6.35 & 87.3 & 77.7 & 0 \\
\hline SEP & 6.73 & 92.5 & 77.7 & 0 \\
\hline OCT & 6.63 & 91.2 & 77.7 & 0 \\
\hline NOV & 6.08 & $83.6^{\circ}$ & 77.7 & 0 \\
\hline DEC & 6.03 & 82.91 & 77.7 & 0 \\
\hline \multicolumn{5}{|c|}{ 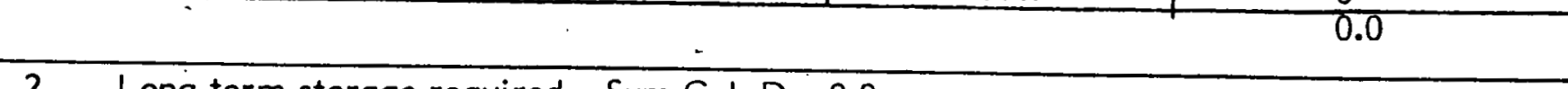 } \\
\hline \multicolumn{5}{|c|}{$\begin{array}{l}\text { 2. Long term storage required }=\text { Sum Col. } \mathrm{D}=0.0 \\
\text { 3. Total storage }=(\text { Line } 2)+(\text { Short term storage) } 7 \text { days } \times \text { (maximum value } \\
\text { in Col. C) } \\
=544 \mathrm{Ah}\end{array}$} \\
\hline
\end{tabular}


Exhibit 1.8

COMIPUTATION OF SYSTEM PERFORMANCE $-50^{\circ}$ Tilt

1. Array sizè $(A p)=12.5 \mathrm{Ap}$

10 panels.

$1.25 \mathrm{~A} /$ panel $@ 60^{\circ} \mathrm{C} @ 100 \mathrm{~mW} / \mathrm{cm}^{2}$

\begin{tabular}{|c|c|c|c|c|}
\hline Column: & $A$ & $B$ & C & $D$ \\
\hline Item: & $\begin{array}{c}\text { Average Day } \\
\text { Insolation on } \\
\text { a Tilted Array } \\
\end{array}$ & $\begin{array}{c}\text { Electrical } \\
\text { Output of } \\
\text { Array } \\
\end{array}$ & $\begin{array}{l}\text { Electrical } \\
\text { Demand }\end{array}$ & $\begin{array}{c}\text { Energy From } \\
\text { Backup }\end{array}$ \\
\hline Units: & $\left(k W h / m^{2}-D a y\right)$ & (Ah/Day) & (Ah/Day) & (Ah/Day) \\
\hline Month & $\begin{array}{l}\text { From Insolation } \\
\text { Analysis }\end{array}$ & $\begin{array}{l}\text { Line I } x \\
\text { Col.A }\end{array}$ & $\begin{array}{l}\text { From Load } \\
\text { Analysis }\end{array}$ & $\begin{array}{l}\text { Col.C-Col.B } \times \frac{\text { days }}{\text { (Enter } 0.0 \text { if }} \text { month } \\
\text { Col.B=Col.C) }\end{array}$ \\
\hline JAN & 6.2 & 77.5 & 77.7 & 6.2 \\
\hline FEB & 6.37 & 79.6 & 77.7 & 0 \\
\hline MAR & 6.38 & 79.8 & 77.7 & 0 \\
\hline APR & 6.62 & 82.8 & 77.7 & 0 \\
\hline MAY & 6.57 & 82.1 & 77.7 & 0 \\
\hline JUN & 6.91 & 86.4 & 77.7 & 0 \\
\hline JUL & 6.45 & 80.6 & 77.7 & 0 \\
\hline$A \cup G$ & 6.35 & 79.4 & $\quad 77.7$ & 0 \\
\hline SEP & 6.73 & 84.1 & 77.7 & 0 \\
\hline OCT & 6.63 & 82.9 & $\quad 77.7$ & 0 \\
\hline NOV & 6.08 & 76 & $\quad 77.7$ & 51.0 \\
\hline DEC & 6.03 & 75.4 & 77.7 & 71.3 \\
\hline & & & & 128.5 \\
\hline \multicolumn{5}{|c|}{ 2. Long term storage required $=$ Sum Col. $D=128.5$} \\
\hline $\begin{array}{r}\text { 3. To } \\
\text { in Col. } \\
=6\end{array}$ & $\begin{array}{l}\text { tal storage }=\text { (Line } \\
72 \mathrm{Ah}\end{array}$ & Short term & 7 days $\times(\operatorname{maxi}$ & nalue \\
\hline
\end{tabular}




\section{Exhibit I.C}

COMPUTATION OF SYSTEM PERFORMANCE $-40^{\circ} \mathrm{Tilt}, 35^{\circ}$, Latitude

1. Array size $(\mathrm{Ap})=12.5 \mathrm{Ap} \quad\left(1.25\right.$ at $\left.60^{\circ} \mathrm{C}, 100 \mathrm{~mW} / \mathrm{cm}^{2}\right)$

$\frac{11.6}{1.25}=9.28$

need 9.28 panels $=10$ panels

\begin{tabular}{|c|c|c|c|c|}
\hline Column: & $A$ & $B$ & $C^{\prime}$ & $D$ \\
\hline Item: & $\begin{array}{l}\text { Average Day } \\
\text { Insolation on } \\
\text { a Tilted Array }\end{array}$ & $\begin{array}{c}\text { Electrical } \\
\text { Output of } \\
\text { Array }\end{array}$ & $\begin{array}{c}\text { Electrical } \\
\text { Demand }\end{array}$ & $\begin{array}{l}\text { Energy From } \\
\text { Backup }\end{array}$ \\
\hline Units: & $\left(\mathrm{kWh} / \mathrm{m}^{2}-\right.$ Doy $)$ & (Ah/Day) & - (Ah/Day) & (Ah/Day) \\
\hline Instructic & $\begin{array}{l}\text { ons: } \\
\text { From Insolation } \\
\text { Analysis }\end{array}$ & $\begin{array}{l}\text { Line I } x \\
\text { Col.A }\end{array}$ & $\begin{array}{l}\text { From Load } \\
\text { Analysis }\end{array}$ & $\begin{array}{l}\text { Col.C-Col.B } \times \frac{\text { days }}{\text { month }} \\
\text { (Enter } 0.0 \text { if } \\
\text { Col. } B=\text { Col.C) }\end{array}$ \\
\hline JAN & 5.94 & 74.3 & 77.7 & 105.4 \\
\hline FEB & 6.28 & 78.5 & 77.7 & 0 \\
\hline MAR & 6.99 & 87.4 & 77.7 & 0 \\
\hline APR & 7.11 & 88.9 & 77.7 & 0 \\
\hline MAY & 7.22 & $y^{\prime \prime} 1.3$ & 77.7 & 0 \\
\hline JUN & 7.65 & 95.6 & 77.7 & 0 \\
\hline JUL & 7.11 & 88.9 & 77.7 & 0 \\
\hline AUG & 6.93 & 86.6 & 77.7 & 0 \\
\hline SEP & 7.06 & 88.3 & 77.7 & 0 \\
\hline OCT & 6.65 & 83.1 & 77.7 & 0 \\
\hline NOV & 5.90 & .. 73.81 & 77.7 & 117.0 \\
\hline DEC & 5.75 & $71.9^{\circ}$ & 77.7 & 179.8 \\
\hline & & & & 402.2 \\
\hline \multicolumn{5}{|c|}{$\begin{array}{l}\text { 2. Long term storage required }=\text { Sum Col. } \mathrm{D}=402 \\
\text { 3. Total storage }=(\text { Line } 2)+(\text { Short term storage) } 7 \text { days } \times \text { (maximum value } \\
\text { in Col. C) } \\
=946 \mathrm{Ah}\end{array}$} \\
\hline
\end{tabular}




\section{BATTERY DISCHARGE}

\section{Domicile}

$X$ - Min. battery storage used $\left(11\right.$ panels) $\left(50^{\circ}\right.$ Tilt)

- 10 panel Array $150^{\circ}$ Tilt)

$\vec{\omega}$
$\hat{\omega}$

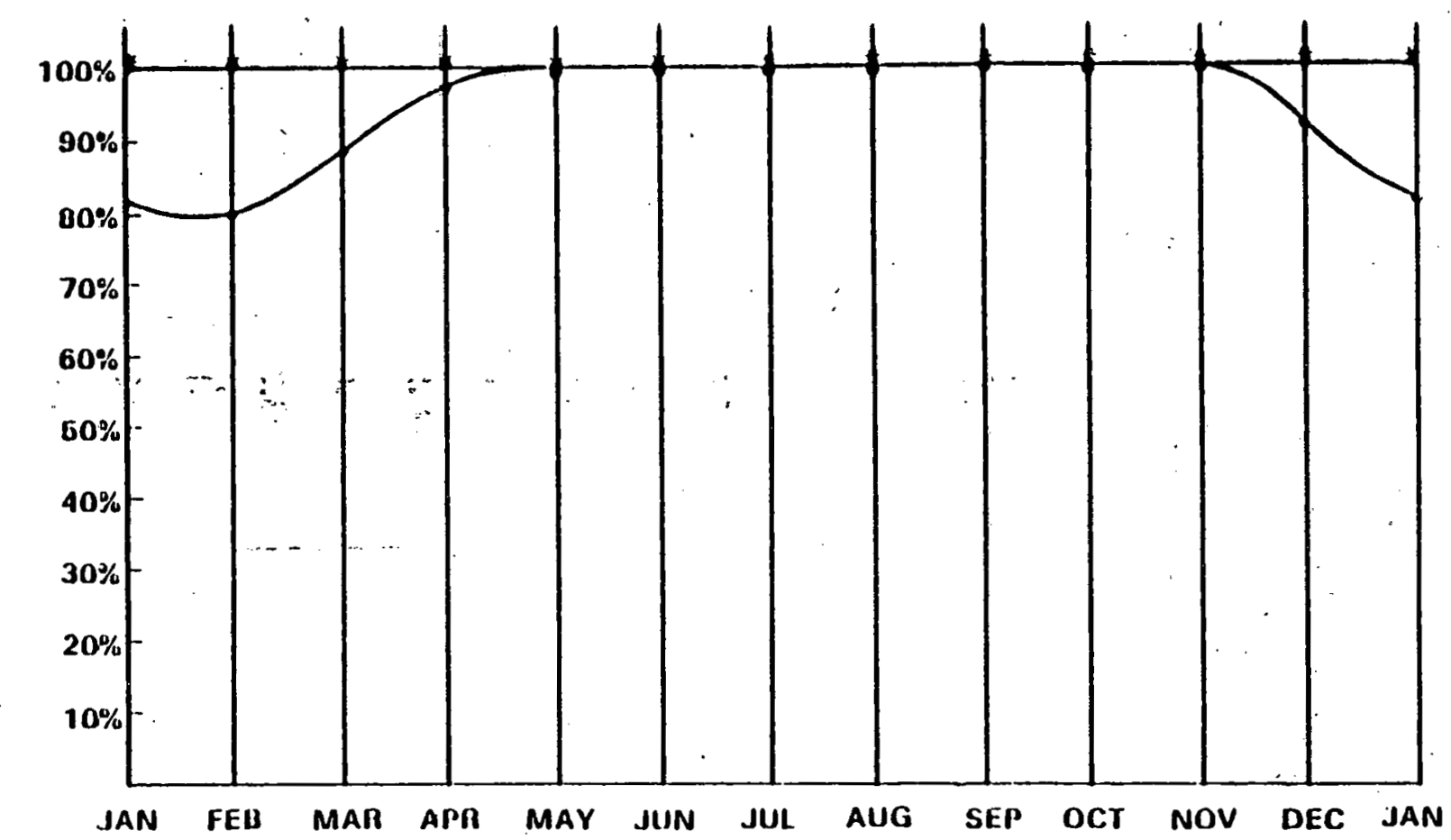




\subsubsection{DESIGN OPTIONS}

- Using 11 panels, seasonal battery storage is not needed, tilt angle used is $50^{\circ}$ (latitude $+15^{\circ}$ ).

- Using 10 panels seasonal battery storage needed is 128.5 Ah of charge with the array at $50^{\circ}$ tilt (latitude $+15^{\circ}$ ).

- Using a latitude tilt and 10 panels, the seasonal storage needs grow to 402.2 Ah.

- Optimal choice is either 1 or 2:

Choice is between I extra panel and 128.5 additional Ah of battery charge Cost of panel $(12 \mathrm{~V}, 20 \mathrm{Wp}) @ 11.25 / \mathrm{Wp}_{\mathrm{p}}=\$ 225.00$

Cost of additional lead-acid calcium grid battery storage (128.5 @ 12 V@

170/kWh@ 500 hour rate @ 60\% depth of charge) $=\$ 437$

- Difference $=\$(437-225)=\$ 212$

- Percent difference $=212 / 225=94.27$.

- Therefore use additional panel. 


\subsubsection{DOMICILE: MICROWAVE OVEN (OPTION 2)}

The microwave oven is equipped with its own inverter and transformer circuits. It requires an input of $60.5 \mathrm{~A}$ at $12 \mathrm{~V}(725 \mathrm{~W})$ and has an on time of approximately $50 \mathrm{~min} /$ day. The size of the microwave oven used does not appreciably alter the load requirement for cooking as a larger oven would be on a proportionately shorter time and a smaller oven would be on longer (i.e., it is the number of Btu's absorbed by the food that is important).

If it is assumed that the oven is used for all three meals, the on time would average approximately $50 \mathrm{~min} /$ day.

The charge per day (Ah/day) equals $60.5 \mathrm{~A} \times 50 \mathrm{~min} /$ day $\times 1 \mathrm{hr} / 60 \mathrm{~min}=50.4$.

PANELS REQUIRED: INITIAL ESTIMATE

$$
A p=\frac{128.1}{\frac{(6.08+6.03+6.2)^{*}}{3}}=20.99 \text { Ap Total }
$$

Number of panels $=\frac{20.99}{1.25}=16.8$ or 17 panels

(1.25 A@60 $\mathrm{C} @ 100 \mathrm{~mW} / \mathrm{cm}^{2}$ insolation)

* Insolation used is for a $50^{\circ}$ tilt on the array

Exhibit 2.A $\quad 50^{\circ}$. Tilt

Exhibit 2.B $\quad 50^{\circ}$ Tilt 
Exhibit 2.A

COMPUTATION OF SYSTEM PERFORMANCE $-50^{\circ}$ Tilt

1. Array size $(A p)=21.25$

$1.25 \mathrm{~A} /$ panel @ $60^{\circ} \mathrm{C}$

$\frac{20.99}{1.73}=16.8$

use 17 panels $=21.25$

\begin{tabular}{|c|c|c|c|c|}
\hline Column: & A & $B$ & C & $D$ \\
\hline Item: & $\begin{array}{l}\text { Average Day } \\
\text { Insolation on } \\
\text { a Tilted Array }\end{array}$ & $\begin{array}{l}\text { Electrical } \\
\text { Output of } \\
\text { Array }\end{array}$ & $\begin{array}{l}\text { Electrical } \\
\text { Demand }\end{array}$ & $\begin{array}{l}\text { Energy From } \\
\text { Backup }\end{array}$ \\
\hline Units: & $\left(\mathrm{kWh} / \mathrm{m}^{2}-\mathrm{Day}\right)$ & (Ah/Day) & (Ah/Day) & (Ah/Day) \\
\hline Month & $\begin{array}{l}\text { ons: } \\
\text { From Insolation } \\
\text { Anulysls }\end{array}$ & $\operatorname{Line}_{\text {Col.A }} x$ & $\begin{array}{c}\text { From Load } \\
\text { Analysis }\end{array}$ & $\begin{array}{l}\text { Col.C-Col.B } \times \frac{\text { days }}{\text { (Enter } 0.0 \text { if month }} \\
\text { Col.B=Col.C) }\end{array}$ \\
\hline JAN & 6.2 & 131.75 & 128.1 & 0 \\
\hline FEB & 6.37 & 135.4 & .128 .1 & 0 \\
\hline MAR & 6.38 & 135.6 & 128.1 & 0 \\
\hline APR & 6.62 & 140.7 & $128: 1$ & 0 \\
\hline MAY & 6.57 & 139.6 & 128.1 & 0 \\
\hline JUN & 6.91 . & 146.8 & 128.1 & 0 \\
\hline JUL & 6.45 & 137.1 & 128.1 & 0 \\
\hline AUG & 6.35 & 134.9 & 128.1 & 0 \\
\hline SEP & 6.73 & 143:0 & 128.1 & 0 . \\
\hline OCT & 6.63 & 140.9 & 128.1 & 0 \\
\hline NOV & 6.08 & 129.2 & 128.1 & 0 \\
\hline DEC & 6.03 & 128.1 & 128.1 & 0 \\
\hline & & & & 0.0 \\
\hline \multicolumn{3}{|c|}{ 2. Long term storoge required $=$ Sum Col. $D=0$} & & \\
\hline \multicolumn{5}{|c|}{$\begin{array}{l}\text { 3. Total storage }=(\text { Line } 2)+(\text { Short term storage }) 7 \text { days } \times(\text { maximum value } \\
\text { in Col. C) } \\
=897 \mathrm{Ah}\end{array}$} \\
\hline
\end{tabular}


Exhibit 2.8

COMPUTATION OF SYSTEM PERFORMANCE - $50^{\circ}$ Tilt

1. Array size $(A p)=20 \mathrm{Ap}$

Use 16 panels $=20 \mathrm{Ap}$

\begin{tabular}{|c|c|c|c|c|}
\hline Column: & A & B & C & D \\
\hline Item: & $\begin{array}{l}\text { Average Day } \\
\text { Insolation on } \\
\text { a Tilted Array }\end{array}$ & $\begin{array}{l}\text { Electrical } \\
\text { Output of } \\
\text { Array }\end{array}$ & $\begin{array}{l}\text { Electrical } \\
\text { Demand }\end{array}$ & $\begin{array}{l}\text { Energy From } \\
\text { Bockup }\end{array}$ \\
\hline Units: & $\left(k W h / m^{2}-\right.$ Day $)$ & (Ah/Day) & (Ah/Day) & (Ah/Day) \\
\hline Instructi & $\begin{array}{l}\text { From Insolation } \\
\text { Analysis }\end{array}$ & $\begin{array}{l}\text { Line } 1 x \\
\text { Col.A }\end{array}$ & $\begin{array}{c}\text { From Load } \\
\text { Analysis }\end{array}$ & $\begin{array}{l}\text { Col.C-Col.B } \\
\text { (Enter } 0.0 \text { if } \\
\text { Col.B=Col.C) }\end{array}$ \\
\hline JAN & 6.2 & $! 24.0$ & 128.1 & 124.0 \\
\hline FEB & 6.37 & 127.4 & 128.1 & 19.6 \\
\hline MAR & 6.38 & 127.6 & 128.1 & 15.5 \\
\hline APR & 6.62 & 132.4 & 128.1 & 0 \\
\hline MAY & 6.57 & 131.4 & 128.1 & 0 \\
\hline JUN & 6.91 & 138.2 & 128.1 & 0 . \\
\hline JUL & 6.45 & 128.0 & 128.1 & 0 \\
\hline$A \cup G$ & 6.35 & 127.0 & 128.1 & 34.1 \\
\hline SEP & 6.73 & 134.6 & 128.1 & 0 \\
\hline OCT & 6.63 & 132.6 & 128.1 & 0 \\
\hline NOV & 6.08 & 121.6 & 128.1 & 195.0 \\
\hline DEC & 6.03 & 120.6 & 128.1 & $\frac{232.5}{623.8}$ \\
\hline \multicolumn{5}{|c|}{ 2. Long term storage required $=$ Sum Col. $D=623.8$} \\
\hline \multicolumn{5}{|c|}{$\begin{array}{l}\text { 3. Total storage }=(\text { Line } 2)+(\text { Short term storage }) 7 \text { days } \times \text { (maximum value } \\
\text { in Col. C) } \\
=1521 \mathrm{Ah}\end{array}$} \\
\hline
\end{tabular}




\section{BATTERY DISCHARGE}

(Microwave Oven) $\quad x=17$ panels

Domicile-Option $1 \quad \bullet=16$ panels

市

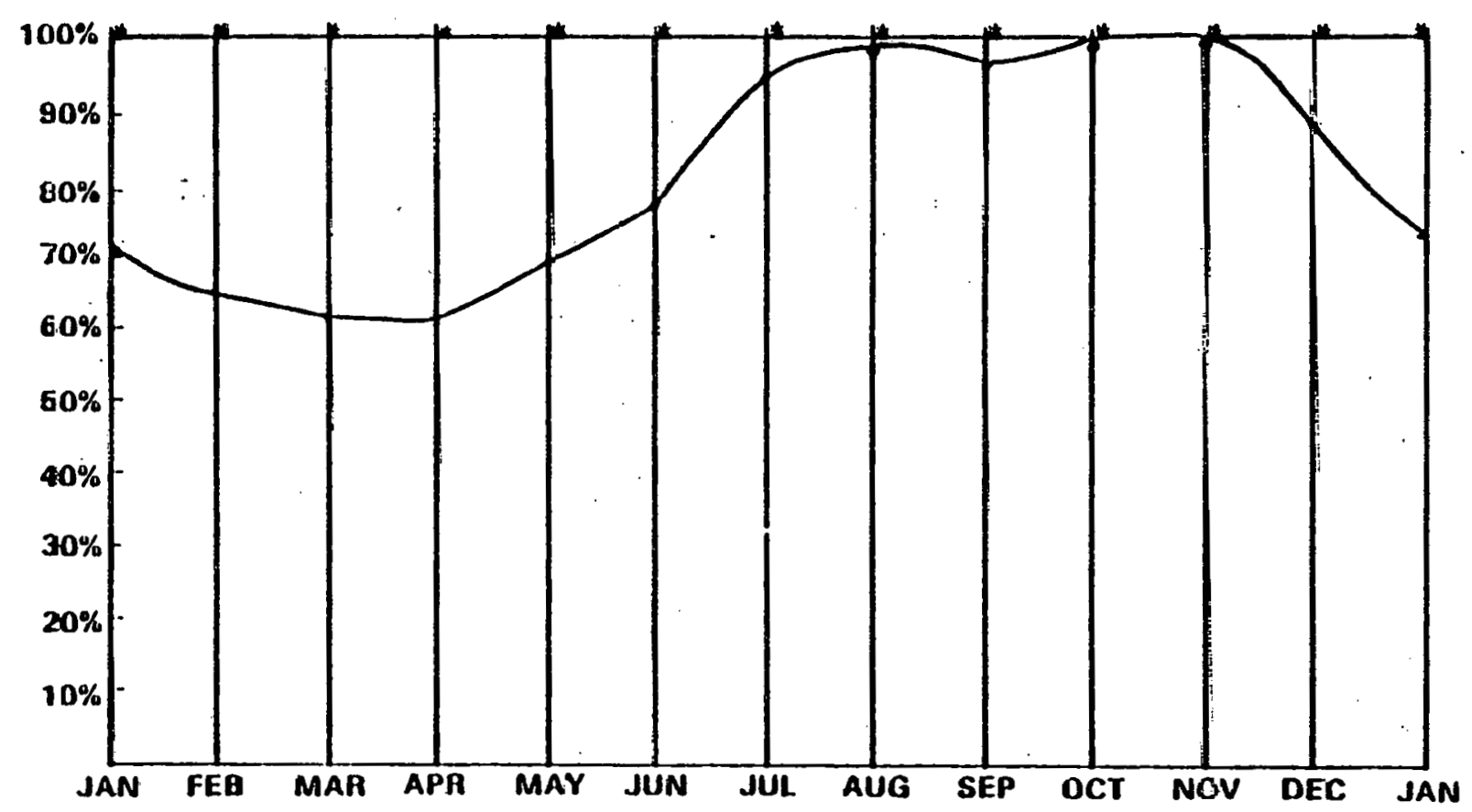




\subsubsection{ESTIMATED COST OF PV DOMICILE SYSTEM}

(7-Day No Sun Battery Storage)

\section{Component}

Photovoltaic panels

Structure

Fence* (Roof Mounted)

Power sonditinner

Storage batteries with shelter (60\% depth of discharge allowed)

Total

Total installed with fees
Basic System

$\$ 2,970(264 \mathrm{WP})$

$\$ 219$

$\$ 0$

$\$ 264$

$\$ 1,903(907 \mathrm{Ah})$

$\$ 5,356$

$\$ 8,085$
Microwave Option

$\$ 4,590(408 \mathrm{Wp})$

$\$ 262$

$\$ 0$

$\$ 408$

$\$ 3,125(1495 \mathrm{Ah})$

$\$ 8,385$

$\$ 12,662$

\subsubsection{NAVIGATIONAL AND AIRCRAFT AIDS}

\subsection{4.| REQUIREMENTS}

\section{General}

Rising battery costs and environmental concerns in disposal of spent batteries have made photovoltaics an economically viable and attrative source of power for navigational aids. Hundreds of commercial novigational aios are now operative.

\section{Specific}

The photovoltaic system shall produce sufficient power to operate a channel marker light during twilight and nighttime periods. The light operates at $24 \mathrm{VDC}$ with a current draw of 3.1 A $(74.4 \mathrm{~W})$. The power requirements of the marker buoy will peak during the winter months due to the long hours of darkness. Coast Guard regulations concerning private PV navigational aids require a minimum of 30 days of battery storage to be included in the power system.

Load - $24 \mathrm{~V}, 3.1 \mathrm{~A}$

Array Size - 12-12 V panels, $220 W_{p}(7.5$ A peak $@ 29.4 \mathrm{~V})$ 
Battery size - 1,336 Ah - $24 \mathrm{~V}$

Voltage regulator - A voltage regulator shall not be required, os the maximum charging current of $7.5 \mathrm{~A}$ is equal to 0.6 percent of the storage battery capacity and, therefore, little outgassing due to overcharge should result.

\section{Control of PV Power System}

- The light will operate only during the nighttime hours, therefore, power shall be supplied to the load by a photocell control.

Array and Battery. Monitoring

- Voltage meter located in the battery housing that will isolate and monitor either the battery or array voltage.

- Ammeter located in the battery housing that will monitor the total array output current.

\section{Electrical Storage}

Shall be provided by low self-discharge, lead-acid batteries. A minimum of 30 days of storage is required by the Coast Guard for private aids.

\section{Safety Considerations}

Disconnect switches between the panel shall not be required due to the low (i.e., $24 \mathrm{~V}$ ) system voltage. An opaque cover shall be available for use during maintenance to cover the array, thereby effectively turning the array "off."

\subsubsection{SUMMARY OF NAVIGATION AID DESIGN}

1. Schematic of Design (Either Engineering Drawing or Block Diogram),

2. Computation of Load.

3. Initial Array Sizing for Minimum Storage Design.

4. Month-by-Month Calculation for 14 Panels (Exhibit 3.A).

5. 30-Day Battery Storage Design - Initial Array Sizing.

6. Mnnth-hy-Month Calculation $40^{\circ}$ Tilt Exhibit 3.B $60^{\circ}$ Tilt Exhibit 3.C

7. Battery State of Charge (Graph) for Each Design.

8. Cost Difference Between Designs.

9. Estimated Cost of Installed System. 


\subsubsection{LOAD COMPUTATION}

Load - Channel Marker Lights

$20 \mathrm{VDC}, 3.1 \mathrm{~A}$, or $81.6 \mathrm{~W}$, battery used has a coulombic efficiency of 90 percent

$\begin{array}{lcc}\text { Month } & \frac{\text { Hours of }}{\text { Nighttime }} & \begin{array}{c}\text { Ah/Day } \\ \text { Required } \\ \text { Jan }\end{array} \\ \text { Feb } & 14.9 & 47.6 \\ \text { Mar } & 12.8 & 43.4 \\ \text { Apr } & 11.7 & 39.7 \\ \text { May } & 10.9 & 36.9 \\ \text { Jun } & 10.6 & 35.9 \\ \text { Jul } & 10.9 & 37.1 \\ \text { Aug } & 11.8 & 40.1 \\ \text { Sept } & 12.9 & 43.9 \\ \text { Oct } & 14.1 . & 27.9 \\ \text { Nov } & 15.0 & 50.9 \\ \text { Dec } & 15.3 & 52.0 \\ =\quad \text { (hours of nighttime) } \times \text { (A demand of load) } & \end{array}$

\subsubsection{MINIMUM BATTERY STORAGE DESIGN}

Initial estimate of array current $=\frac{\text { Avy. winter load }}{A \text { Avg. winter insolation }\left(60_{-}^{\circ} \text { tilt }\right)}$

$$
=\frac{50.9+52.0+50.7+47.6}{6.08+6.14+6.27+6.28}=8.12 \mathrm{~A}
$$

Panels needed @1.25 A/panel @ $60^{\circ} \mathrm{C} @ 100 \mathrm{~mW} / \mathrm{cm}^{2}$.

Panels needed $=\frac{8.12}{1.25}=6.49$

Because system is $24 \mathrm{~V}$, use $7 \times 2=14$ panels 
Exhibit 3.A

COMPUTATION OF SYSTEM PERFORMANCE $-60^{\circ} \mathrm{Tilt}, 35^{\circ}$ Latitude

i. $\quad$ Arroy size $(A p)=8.75 \mathrm{Ap}$

Panels needed minimum storage $6.49 \times 2=$ use $7 \times 2=14$ panels

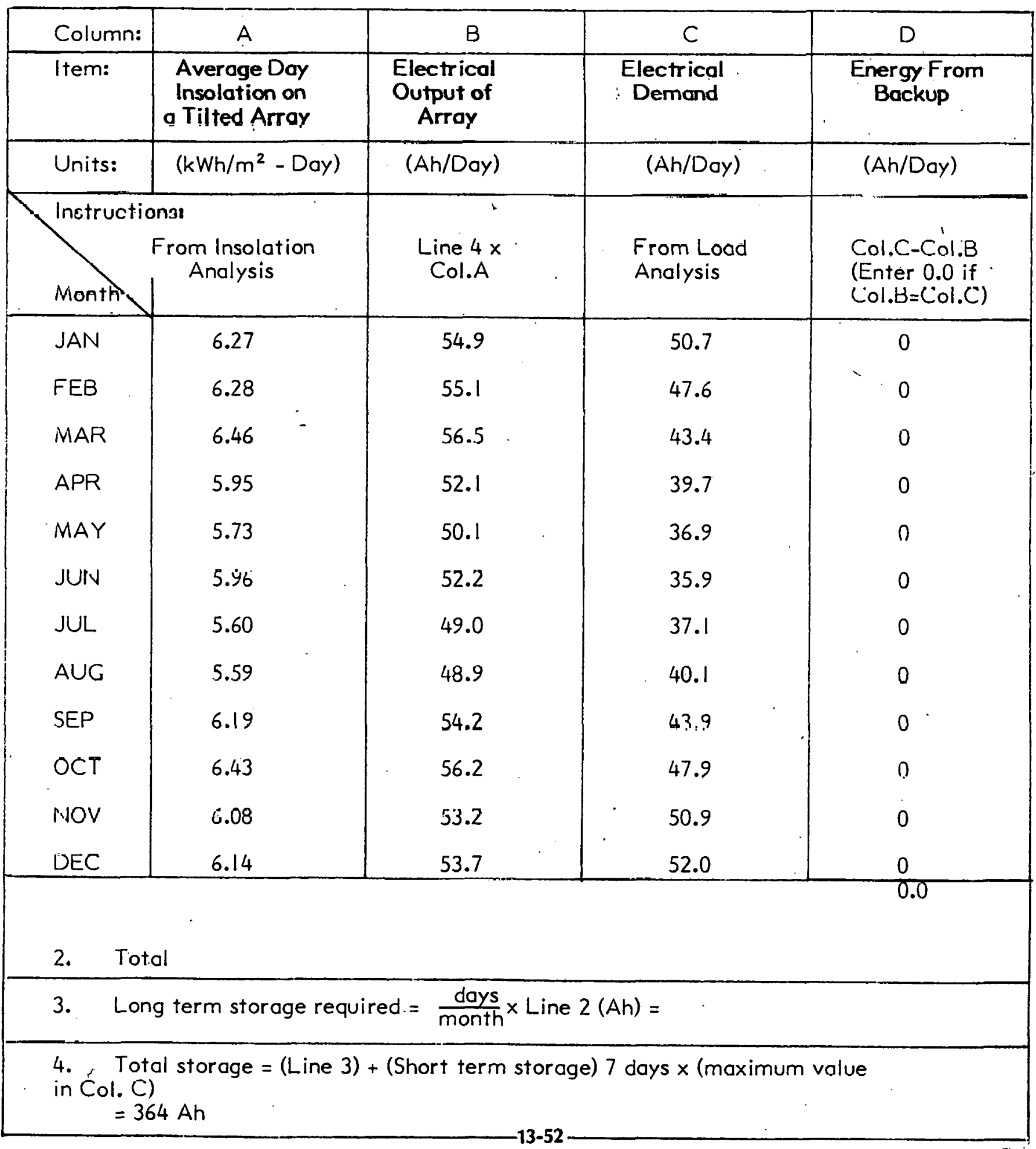




\section{Exhibit 3.B}

COMPUTATION OF SYSTEM PERFORMANCE $-40^{\circ} \mathrm{Tilt}, 35^{\circ}$ Latitude

1. Array size $(A p)=7.5 \mathrm{Ap}$

$(5.22 \times 2)$ panels needed

use $6 \times 2$ panels $=12$ panels

\begin{tabular}{|c|c|c|c|c|}
\hline Column: & A & $B$ & C & D \\
\hline Item: & $\begin{array}{l}\text { Average Day } \\
\text { Insolation on } \\
\text { a Tilted Array }\end{array}$ & $\begin{array}{l}\text { Electrical } \\
\text { Output of } \\
\text { Array }\end{array}$ & $\begin{array}{l}\text { Electrical } \\
\text { Demand }\end{array}$ & $\begin{array}{l}\text { Energy From } \\
\text { Backup }\end{array}$ \\
\hline Units: & $\left(\mathrm{kWh} / \mathrm{m}^{2}\right.$ - Day $)$ & (Ah/Day) & (Ah/Day) & (Ah/Day) \\
\hline \multicolumn{2}{|c|}{ Instructions: } & \multirow[b]{2}{*}{$\begin{array}{l}\text { Line } 4 x \\
\text { Col.A }\end{array}$} & \multirow[b]{2}{*}{$\begin{array}{l}\text { From Load } \\
\text { Analysis }\end{array}$} & \multirow[b]{2}{*}{$\begin{array}{l}\text { Col.C-Col.B } \\
\text { (Enter } 0.0 \text { if } \\
\text { Col.B=Col.C) }\end{array}$} \\
\hline Mon & $\begin{array}{l}\text { From Insolation } \\
\text { Analysis }\end{array}$ & & & \\
\hline JAN & 5.94 & 44.6 & 50.7 & 6.1 \\
\hline FEB & 6.28 & 47.1 & 47.6 & 0.5 \\
\hline MAR & 6.99 & 52.4 & 43.4 & 0 \\
\hline APR & 7.11 & 53.3 & 39.7 & 0 \\
\hline MAY & 7.22 & . 54.2 & 36.9 & 0 \\
\hline JUN & 7.65 & 57.4 & 35.9 & 0 \\
\hline JUL & 7.11 & 53.3 & 37.1 & 0 \\
\hline AUG & 6.93 & 52.0 & 40.1 & 0 \\
\hline SEP & 7.06 & 53.0 & 43.9 & 0 \\
\hline OCT & 6.65 & 49.9 & 47.9 & 0 \\
\hline NOV & 5.90 & 44.3 & 50.9 & 6.3 \\
\hline DEC & 5.75 & 43.1 & 52.0 & 8.9 \\
\hline \multicolumn{5}{|c|}{ Tot } \\
\hline & \multicolumn{4}{|c|}{ Lotal term storage required $=\frac{\text { doys }}{\text { month }} \times$ Line $2(A h)=668 \mathrm{Ah}$} \\
\hline \multicolumn{5}{|c|}{$\begin{array}{l}\text { Total storage }=(\text { Line } 3)+(\text { Short term storage }) 7 \text { days } \times \text { (maximum value } \\
\text { in Col. C) } \\
=1032 \mathrm{Ah}\end{array}$} \\
\hline
\end{tabular}


Exhibit 3.C

COMPUTATION OF SYSTEM PERFORMANCE $-60^{\circ}$ Tilt, $35^{\circ}$ Lotitude

1. Array size $(A p)=7.5 \mathrm{Ap}$

12 panels

$6 \times 2$

\begin{tabular}{|c|c|c|c|c|}
\hline Column: & $A$ & $B$ & C & $\bar{D}$ \\
\hline Item: & $\begin{array}{l}\text { Average Day } \\
\text { linsolation on } \\
\text { a Tilted Array, }\end{array}$ & $\begin{array}{c}\text { Electrical } \\
\text { Output of } \\
\text { Array }\end{array}$ & $\begin{array}{l}\text { Electrical } \\
\text { Demand }\end{array}$ & $\begin{array}{l}\text { Energy From } \\
\text { Backup }\end{array}$ \\
\hline Units: & $\left(\mathrm{kWh} / \mathrm{m}^{2}-\mathrm{Day}\right)$ & (Ah/Doy) & (Ah/Day) & (Ah/Day) \\
\hline Instructi & $\begin{array}{l}\text { From Insolation } \\
\text { Analysis }\end{array}$ & $\begin{array}{c}\text { Line } 4 x \\
\text { Col.A }\end{array}$ & $\begin{array}{l}\text { From Load } \\
\text { Analysis }\end{array}$ & $\begin{array}{l}\text { Col.C-Col. } 8 \\
\text { (Enter } 0.0 \text { if } \\
\text { Col.B=Col.C) }\end{array}$ \\
\hline JAN & 6.27 & 47.0 & 50.7 & 3.7 \\
\hline FEB & 6.28 & 47.1 & 47.6 & 0.5 \\
\hline MAR & 6.46 & 48.5 & 43.4 & 0 \\
\hline APR & 5.95 & 44.6 & 39.7 & 0 \\
\hline MAY & 5.73 & 43.0 & 36.9 & 0 \\
\hline JUN & 5.96 & 44.7 & 35.9 & 0 \\
\hline JUL & 5.60 & 42.0 & 37.1 & 0 \\
\hline$A \cup G$ & 5.59 & 41.9 & 40.1 & 0 \\
\hline SEP. & 6.19 & 46.4 & 43.9 & 0 \\
\hline ort & 6.43 & 48.2 & 27.9 & 0 \\
\hline NOV & 6.08 & 45.6 & 50.9 & 5.3 \\
\hline DEC & 6.14 & 46.1 & 52.0 & 5.9 \\
\hline & & & & 15.4 \\
\hline \\
\hline \multicolumn{5}{|c|}{$\begin{array}{l}\text { 3. Long term storage required }=\frac{\text { doys }}{\text { month }} \times \text { Line } 2(\mathrm{Ah})=470.6 \mathrm{Ah} \\
\text { 4. Total storage }=(\text { Line } 3)+(\text { Short term storage }) 7 \text { days } \times(\text { maximum value } \\
\text { in Col. } \mathrm{C}) \\
=834.6 \mathrm{Ah}\end{array}$} \\
\hline
\end{tabular}


Navigational Aid:

* - minimum battery storage used

0 - 30 day storage used $\left(40^{\circ}\right.$ Tilt)

-.. $60^{\circ}$ Tilt (30 day storage)

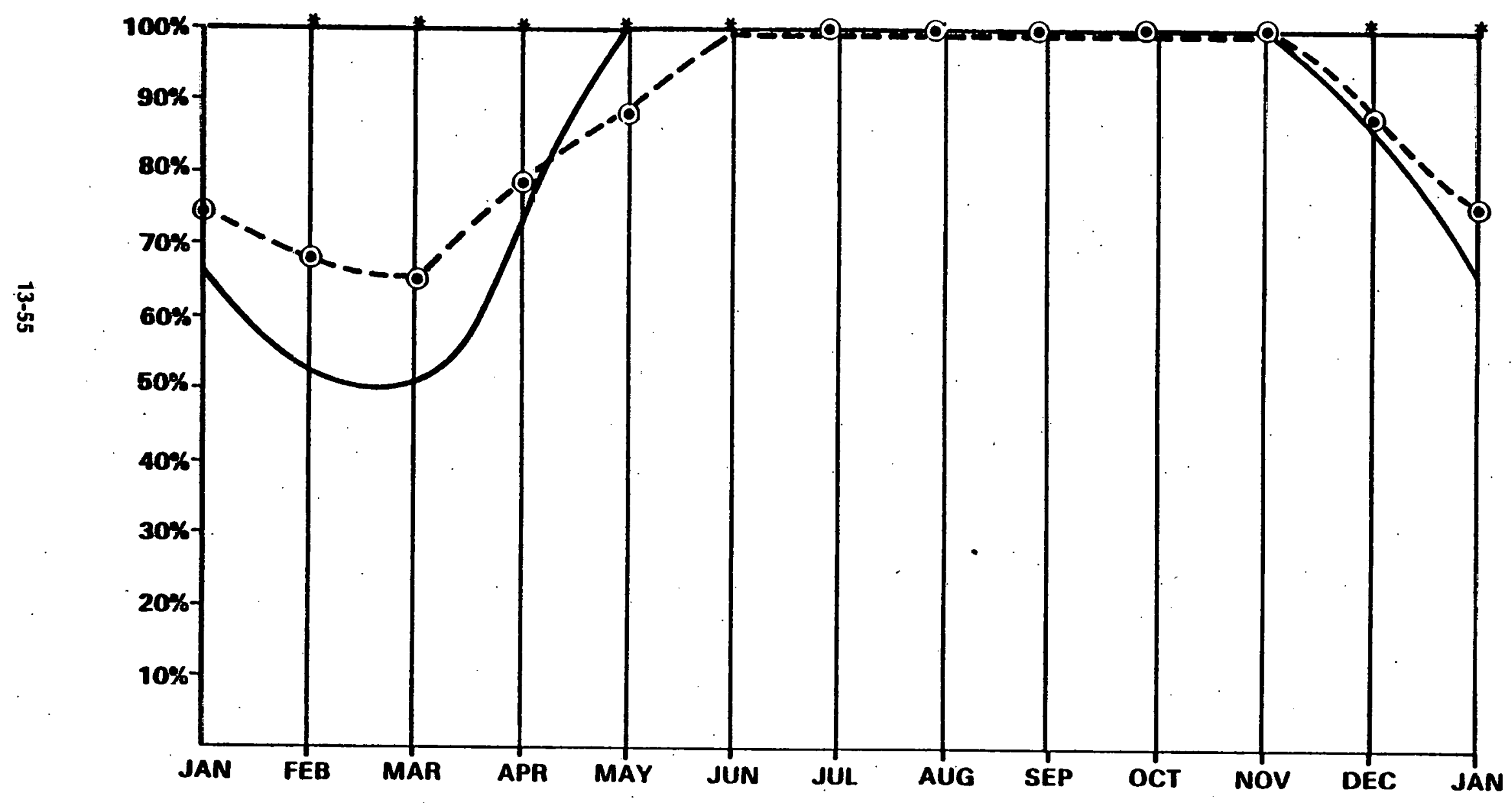




\subsubsection{DESIGNING TO COAST GUARD REQUIREMENTS}

A Coast Guard requirement states that 30 days of battery storage must be used on private navigation aids.

If one uses an average $\mathrm{Ah} /$ day requirement ( $43.8 \mathrm{Ah} /$ day), an estimate of the 30 -day storage required can be obtained.

$$
30 \times 43.8=1,314 \mathrm{Ah}
$$

One can design a system that will use this battery capacity so batteries reach a minimum of 50 percent capacity during the winter months $(657 \mathrm{Ah})$. This will minimize the array current needed.

The first estimate will use the overage load and monthly insolation to obtain an estimate of the needed array current.

$\frac{\text { Avg. load }}{\text { Avg. insolation }}=\frac{43.8}{6.7}=6.52 . \mathrm{A}$ (peak rated array output)

Panels needed @1.25 A/panel @ 60 $\mathrm{C} @ 100 \mathrm{~mW} / \mathrm{cm}^{2}$

$$
=\frac{6.25}{1.25}=5.2=\text { use } 6 \times 2=12 \text { panels }
$$

This design leads to a battery storage requirement of:

$68 \times 2$ (50 percent depth of battery discharge) $=1,336$ Ah @ $24 \mathrm{~V}$

which conforms to the Coast Guard requirement of 30 days' battery storage. 


\subsubsection{PRICE DIFFERENCE BETWEEN THE TWO DESIGNS}

$\begin{array}{ll}2 \text { panels @ \$300/panel } & =\$ 600 \\ \frac{668 \text { Ah @ 24 V@ @ } \$ 170 / \mathrm{kWh} \text { or } \$ 4.08 / \mathrm{Ah}}{\text { Difference }}= & \$ 2,725 \\ & \$ 2,125\end{array}$

The price difference is irrelevent in this case, since the first design will not meet Coast Guard requirements.

\subsubsection{ESTIMATED COST OF PV NAVIGATIONAL AIDS}

Costs of Components (Installed)

Cost of PV panels for marine environment is estimated at $\$ 15.00 / \mathrm{Wp}$.

Photovoltaic panels

Structure

Fence, power conditioner

Storage batteries

Total

Total with fees
$\$ 4,290 \quad(220 \mathrm{Wp})$

$\$ 200$

not required

$\$ 5 ; 564 \quad(1,336 \mathrm{Ah})$

$\$ 10,054$

$\$ 13,472$

\subsubsection{TELECOMMUNICATIONS}

\subsubsection{REQUIREMENTS}

\section{General}

The photovoltaic system shall provide year-round power for an unattended radio repeater. The site is sufficiently remote to preclude the extension of the existing utility grid. 


\section{Specific}

The repeater operates 7 days a week; it is active for 5 hours per day. Operating on $12 \mathrm{VDC}$, it draws $\| \mathrm{A}(132 \mathrm{~W})$ while simultaneously receiving and retransmitting (the active mode), and draws I/2 A (6 W) while listening only (the squelched mode). This load profile equates to a total power requirement of $64.5 \mathrm{Ah} /$ day. $(.774 \mathrm{kWh} /$ day $)$.

\section{Control of PV Power System}

1. Power is being supplied to the load at all times, so control hardware is not needed.

2. The system shall be fused to prevent battery drain if the repeater should fall in a short circuit mode. Standard buss fuses are available for this purpose.

Array and Battery Monitoring

1. Voltage meter with switches located in the battery storage box that will isolate

2. Ammeter with switches located in the battery storage box that will monitor either the total array output current or the total load current.

Data and Calculations

- Load - Radio Repeater

12 VDC, II A (132 W) active, I/2 A (6 W) squelched

Repeater operates $24 \mathrm{hr} /$ day

- 5 hours active

$=55.0 \mathrm{Ah}$

- 19 hours squelched

$=9.5 \mathrm{Ah}$

Total daily charge requirement

$=\frac{9.5}{64.5} \mathrm{Ah}$

Initial Estimate of Array =

$\frac{\text { average load }}{\text { average winter insolation }(50 \text { tilt })}=\frac{64.5+54.5+64.5+64.5}{6.20+6.37+6.08+6.03}=10.45$

Panels needed: (@1.25 A/panel @ $60 \mathrm{C} @ 100 \mathrm{~mW} / \mathrm{cm}^{2}$ ).

Panels needed $\quad=\frac{10.45}{1.25}=8.36 \quad$ Use 9 panels

Using 9 panels, seasonal battery storage is not needed (at $50^{\circ}$ tilt: latitude $+15)$

Providing 7 days' short term battery storage at an average Ah/day requirement of 64.5 yields 


$$
7 \times 64.5=451.5 \mathrm{Ah}
$$

Allowing for 60 percent depth of discharge

Array - $180 \mathrm{Wp}-11.25 \mathrm{Ap}$

Battery - 753 Ah @ 12 V

Voltage regulator required

Costing

\begin{tabular}{llr} 
PV panels & $=\$ 1,947$ \\
Structure & $=$ & 181 \\
Fence & $=1,281$ \\
Power conditioning & & .160 \\
Battery and shelter & & $=2,366$ \\
\hline Subtotal & & 5,935 \\
Fee (34 percent) & 2,018 \\
\hline TOTAL & $\$ 7,953$
\end{tabular}




\section{TELECOMMUNICATIONS}

COMPUTATION OF SYSTEM PERFORMANCE $-55^{\circ} \mathrm{Tilt}, 35^{\circ}$ Latitude
1. Array size $\left(A_{p}\right)=11.25 \mathrm{Ap}$
9 Panels@1.25 A/panel

\begin{tabular}{|c|c|c|c|c|}
\hline Column: & A & $\mathrm{B}$ & C & $\mathrm{D}$ \\
\hline Item: & $\begin{array}{l}\text { Average Day } \\
\text { Insolation on } \\
\text { a Tilted Array }\end{array}$ & $\begin{array}{l}\text { Electrical } \\
\text { Output of } \\
\text { Array }\end{array}$ & $\begin{array}{c}\text { Electrical } \\
\text { Demand }\end{array}$ & $\begin{array}{l}\text { Energy From } \\
\text { Backup }\end{array}$ \\
\hline Unitsi & $\left(k W h / m^{2}-D a y\right)$ & (Ah/Day) & (Ah/Day) & (Ah/Day) \\
\hline Instructic & $\begin{array}{c}\text { From Insolation } \\
\text { Analysis }\end{array}$ & $\begin{array}{c}\text { Line } 4 x \\
\text { Col.A }\end{array}$ & $\begin{array}{l}\text { From Load } \\
\text { Analysis }\end{array}$ & $\begin{array}{l}\text { Col.C-Col.B } \\
(\text { Enter } 0.0 \text { if } \\
\text { Col.B=Col.C) }\end{array}$ \\
\hline JAN & 6.25 & 70.31 & 04.5 & 0 \\
\hline FEB & 6.35 & 71.44 & 64.5 & 0 \\
\hline MAR & 6.67 & 75.04 & 64.5 & 0 \\
\hline$A P R$ & 6.30 & 70.88 & 64.5 & 0 \\
\hline MAY & 6.17 & 69.41 & 64.5 & 0 \\
\hline JUN & 6.46 & 72.68 & 64.5 & 0 \\
\hline JUL & 6.04 & 67.95 & 64.5 & 0 \\
\hline$A \cup G$ & 5.99 & 67.39 & 64.5 & 0 \\
\hline SEP & 6.48 & 72.90 & 64.5 & 0 \\
\hline OCT & 6.55 & 73.69 & 64.5 & 0 \\
\hline MOV & 6.10 & 68.13 & 64.5 & 0 \\
\hline DEC & 6.11 & 68.74 & 64.5 & 0 \\
\hline \multirow{2}{*}{\multicolumn{5}{|c|}{0.0}} \\
\hline & & & & \\
\hline \multicolumn{5}{|c|}{ 3. Long term storage required $=\frac{\text { duys }}{\text { month }} \times$ Line $2(A h)=0$} \\
\hline \multicolumn{5}{|c|}{$\begin{array}{l}\text { 4. Total storage }=(\text { Line } 3)+(\text { Short term storage }) 7 \text { days } \times \text { (maximum value } \\
\text { in Col. } \mathrm{C}) \\
=451.5 \mathrm{Ah}\end{array}$} \\
\hline
\end{tabular}




\subsection{PRACTICAL LESSONS LEARNED FROM SYSTEMS}

\subsection{EXAMPLES OF INSTALLED SYSTEMS}

EXAMPLE I

TIME: $\quad 12$ noon (local time)

LOCATION: $36^{\circ} \mathrm{N} \times 109^{\circ} \mathrm{W}$

WEATHER: Extremely clear; $4^{\circ} \mathrm{F}$

\section{System Uescription}

- Array has 108 modules; array angle $50^{\circ}$ to horizontal.

- Battery bank consisted of 14 Willard EV88's @6 V each $=84 \mathrm{~V}$.

- Pump motor $=3.5 \mathrm{hp}$ permanent magnet 25 AFL 2,750 rpm, driving $50 \mathrm{~W}$ Jensen jack and hypro pump.

- Field connections were checked and determined to be properly wired.

- System is designed to operate continuously $12 \mathrm{hr} /$ day with capacity of 5,800 gal/day, for the Jensen Jack and 7 GPM transfer water for the hypro centrifugal pump.

2. Actual Operating Description

- Control box sets voltage at high of $96 \mathrm{~V}$ and low of $78 \mathrm{~V}$. These voltages are for on and off switching.

- The longest consecutive period of time the system operated was 4 months due to high battery depth of discharge caused by failure of control box.

- System had been in operation approximately one year.

3. Initial Observation

- System appeared to be properly installed.

- Electrical connections were completed in o professional manner.

- System was permanently wired with no quick disconnects to facilitate system test measurements and maintenance.

- System did not contain a monitor test panel. 
4. Findings

- The new voltage control box failed after one day of operation.

- Visual inspection revealed no broken modules or damaged cells. When manually operated without control box, system output was excellent. 
EXAMPLE 2

TIME: $\quad 12$ noon (local time)

LOCATION: $\quad 36^{\circ} \mathrm{N} \times 109^{\circ} \mathrm{W}$

WEATHER: Extremely clear; $35^{\circ} \mathrm{F}$

1. System Description

- Array consisted of 104 modules; array angle $45^{\circ}$ to horizontal.

- Battery bank consisted of 12-8 V C \& D 非LCPSA-5.

- $\quad$ Pump motor $=1 / 2 \mathrm{hp}$ Doeer permanent magnet $4.7 \mathrm{AFL} 1,725 \mathrm{rpm}$.

- Field connections were checked and determined to be properly wired.

- System is designed to operate continuously $24 \mathrm{hr} /$ day with capacity of $2 \mathrm{gal} / \mathrm{min}$.

2. Actual Operating Description

- Pump must be manually switched on and off to prevent excessive battery discharge.

- The longest consecutive period of time the system operated was 4 hours due to high battery depth of discharge.

- System had been in operation opproximately one month. .

3. Initial Observation

- System appeared to be properly installed.

- Electrical connections were completed in a professional manner.

* System was permanently wired with no quick disconnects to facilitate system test measurements and maintenance."

* System did not contain a monitor test panel.

4. Findings

* The voltage regulutur prevented the array from charging beyond $100 \mathrm{~V}$ measured value. As a result batteries never reached their full charge of $117.6 \mathrm{~V}$ to 120 $V$ and in effect drastically reduced storage capability.

* Discrepancy 
* Visual inspection revealed that several modules contained damaged cells which reduced current output of the array.

* One of the modules electrical interconnects was miswired resulting in a string voltoge output of $4 \mathrm{~V}$. The string, which was prewired at the manufacturer's facility, should produce approximately $20 \mathrm{~V}$, apen circuit. The miswiring caused the total array open. circuit voltage to be only $135 \mathrm{~V}$ after bypassing the defective voltage regulator. After correcting the wiring, the open circuit voltage measured $150 \mathrm{~V}$.

Because miswiring occurred at the manufacturer's facility, the individual strings could not have been adequately tested prior to shipment.

* During the test, the operating point on the I-V curve at battery full cliarge was significuintly below the maximum power point of the rirve. The temperature during the test condition was approximately $1^{\circ} \mathrm{C}$; therefore, during periods of higher temperatures (i.e., late spring or summer), the voltage will drop due to temperature effects on cells, and the array will not charge the battery.

* The pump is unbalanced causing inefficient mechanical operation.

* The electrical motor (1/2 hp) driving the motor pump is oversized and should be replaced by $1 / 3 \mathrm{hp}$ motor.

5. Test Conducted

Test 1 - array + regulator + batteries - .

array voltage - $100 \mathrm{~V}$

array current - $0 \mathrm{~A}$

Test 2 - array + battery (bypass regulator with blocking diode)

array voltage - $110 \mathrm{~V}$

array current - $11 \mathrm{~A}$

Test 3 - array short circuit current - 18.5 A

array open circuit voltage - $135 \mathrm{~V}$

Test 4 - individual string tests -

String 1

String $1+2$

String $1+2+3$

** String $1+2+3+4$

* discrepancy

* abnormally low
$=19 \mathrm{~V}$

$=39 \mathrm{~V}$

$-56 \mathrm{~V}$

$=60 \mathrm{~V}$ 
Determined by above test, there was a set of conductors in the manufacturer's junction box somewhere in the fourth set of incorrectly connected modules. Removed the covers on the J-boxes of these modules and found that the positive and negative leads were indeed incorrectly connected. The leads were switched and then another test of the fourth set was done which read $75 \mathrm{~V}$. Proceeded to test the total array without going further into the manufacturer's J-boxes. This test produced $150 \mathrm{~V}$, open circuit from the array.

The next test was at the panel in the pump house:

There we read $150 \mathrm{~V}$, open circuit, and $105 \mathrm{~V}$ connected to batteries

with 13.8 A current output.

All above voltage and current tests wëre done with a new Simpson 260 meter with a Simpson DC adaptor that had a range of 25 A DC.

\subsubsection{SYSTEMS INTEGRAT́ION}

1. Design must include systems analysis and synthesis.

2. Electrical, mechanical, structural, etc., areas must be considered.

3. Safety must be designed into the system.

4. Mointenance and performance must be designed into the system.

- Quick disconnectors

- Monitor panels

- Miscellaneous items such as covering for the array during maintenance

\subsubsection{ARRAYS}

1. Bypass diodes should be used on modules.

2. Cells should not be chipped or cracked.

3. Excessive torque on panels will cause some encapsulating glass to crack.

4. Array and system should be grounded per existing codes.

5. Niaintenance considerations must be considered at system design.

6. Array should be able to be isolated and be monitored at control/test panel. 
7. Tilt angle is important but a good procedure to follow is latitude plus $15^{\circ}$.

8. Panels should be structurally supported from both sides rather than the middle.

\subsubsection{BATTERY AND STORAGE}

1. Battery capacity should be calculated not to exceed maximum discharge rate.

2. Voltage regulators must be chosen to allow batteries to charge fully which usually requires overcharging per monufacturer's specifications.

3. Gassing is important to fully recharge battery.

4. Consider venting or catalytic caps.

\subsubsection{BALANCE OF SYSTEMS SUBASSEMBLIES (Keep System SImple)}

1. Direct current

2. Minimum distance between subassemblies

3. Lightning protection

4. Grourding

5. Quick disconnects

6. Monitor panels

7. System efficiency 
SECTION 14

ADDENDUM

\subsection{PHOTOVOLTAIC SYSTEM RFP RECOMMENDED ADDENDUM}

\subsubsection{A COMPREHENSIVE NARRATIVE DESCRIPTION AND MATHEMATICAL MODEL FOR THE DETERMINATION OF THE PHOTOVOLTAIC SYSTEM DESIGN}

The contractor shall describe in detail the operation of the system and relationship of the components to the system, to the load and to the overall operation. Also included shall be mathematical computations needed to describe the system and specify all of the parameters essential in completing the system optimization including percent of oversizing necessary for degradation purposes.

\subsubsection{RESULTS OF THE MODULE AND SYSTEMS QUALITY PERFORMANCE TESTS CONDUCTED AT THE MANUFACTURERS FACILITY}

The information should be comprehensive and pertain to each photovoltaic module, total array and total system.

\subsubsection{FAILURE ANALYSIS METHODOLOGY}

A detailed description should be provided to include narrative explanation of the rationale and probability of component and system failure, the maintenance and isolation procedures which should be used in the event of component or overall system failures, the component replacement techniques including requirements for spares and the procedures to be used for the handling and analysis of malfunctioned components.

\subsubsection{COMPREHENSIVE TESII AND ACCEPTANCE PROCEDURES}

Test procedures required for system test and acceptance must be specified in detail for each of the sub-assemblies, the structure, if required, and the total system.

\footnotetext{
14.1.5 DESIGN, HARDWARE DELIVERY AND INSTALLATION SCHEDULE

Comprehensive schedules must be provided and coordinated with manufacturers, suppliers, etc.
} 


\title{
14.2 PURCHASE DESCRIPTION (5500 Watt Solar Cell Array)
}

\author{
U.S. ARMY MOBILITY EQUIPMENT \\ RESEARCH AND DEVELOPMENT COMMAND \\ Fort Belvoir, Virginia 22060 \\ 6 March 1978
}

1.0 SCOPE.

1.1 This Purchase Description covers the requirements for design, fabrication, and testing of Silicon Solar Cell Modules with high efficiency and high packaging density installed and interconnected into suitable frames. The solar cell modules shall meet the JPL Environmental Requirements No. 5-342-1, Rev B.

\subsection{Applicgble Documents.}

MIL-STD-810 Environmental Test Methöds

JPL-No-5-342-1, Rev B Der 1979 Silicon Solar Cell Module Performance, Erivironmental Tesl

Handbook H-28 Screw-thread Standards for Federal Services

MIL A-8625C Anodic Cootings for Aluminum and AL-Alloys

\subsection{Requirements.}

3.1 Module Description. The solar cell module design shall attempt to maximize the performance of the modules with respect to the following:

a. Maximize power output per module unit area. This effort will reduce solar array area requirements and thus reduce support structure and electrical interconnection costs.

b. Minimum cost per unit energy produced.

c. Service life. Maximize the service lite of the module with a goal of 10 years.

\subsection{Electrical Performance.}

\subsubsection{Module Characteristics.}

3.2.1.1 'General. The module geometry shall be such that they can be installed in subarrays of about $4 \times 8$ feet. Each subarray shall have the voltage range specified in paragraph 3.2.1.3.

3.2.1.2 Module Output Power. The average module output power, when measured according to the JPL requirements $5-342-1$, Rev B, shall not be less than $10 \mathrm{~W} / \mathrm{ft}^{2}$.

3.2.1.3 Subarray Voltage. The individual module voltage is of no consequence and is, therefore, not specified in the P.D. The subarray voltage, (a subarray consists of a number of modules installed in a $4 \times 8$ feet frame) shall be in the range of $125 \mathrm{Vdc}$ to $300 \mathrm{Vdc}$. The low voltage (125 Vdc) shall correspond to the peak power point at $50^{\circ} \mathrm{C}$ ambient temperature; the upper voltage $(300 \mathrm{Vdc})$ shall correspond to the open circuit voltage at $40^{\circ} \mathrm{C}$.

3.2.1.4 Subarray Output Power. The average subarray output power, when measured according to the JPL requirements quoted, shall not be less than 300 watts. 
3.2.2 Electrical Insulation. The electrical insulation of the module shall provide a minimum insulation resistance to ground of 100 megohms and shall withstand 1500 Vdc for one minute between circuitry and any metallic substrate, support, or frame.

3.2.3 Output Terminals and Interconnection Box. Each module shall contain redundant output terminals to permit interconnection of modules into either parallel or series arrangements. Output terminals shall be capable of carrying $5 \mathrm{~A}$, minimum. The output terminals shall be located within a waterproof interconnection box.

\subsection{Mechanical Requirements..}

3.3.1 Module Geometry. The basic module geometry shall be such that it can be mounted into a subarray nof greater than $4 \times 8$ feet, including a border for handling and fastening.

3.3.2 Interface Dimensional Tolerances. Tolerances on all external module dimensions shall be maintained at a level consistent with module interchangeability. Surfaces, mounting holes and other attachment fasteners shall be maintained with a tolerance not to exceed + $1 / 32$ inch. Surfaces not associated with the attachment interface shall be maintained with a tolerance of $+0,-1 / 8$ inch.

\subsection{Solar Cell Frames and Mounting Stands.}

3.4.I General. The solar cell modules shall be mounted and interconnected in suitable frames and associated stands. The frames shall be designed as to withstand the environmental characteristics specified herein.

\subsubsection{Panel Structure.}

3.4.2.1 Mechanical Construction. The structure shall be self-supporting, consisting of a panel frame assembly, interpanel wiring, and four legs with baseplates ánd provisions for tie-down.

3.4.2.2 Ground Clearance. Each panel structure shall be of sufficient height to prevent a build-up of wind blown debris or snow from reducing the electrical performance of the system (18 inches minimum).

3.4.2.3 Tilt Angle. The illuminated surface of the panel shall be positioned at an angle of $45^{\circ}$ from the horlzontal.

3.4.2.4 Interchangeability. The solar cell modules and the array panels shall be interchangeable.

3.4.2.5 Identification Markings. All the panel strû́ctures shall be permanently marked with identification numbers.

3.4.2.6 Materials. The panel structure shall be made of aluminum with a corrosion resisting treatment, acceptable to the C.O. Note: Anodizing according to MIL-A-8625C is an acceptable treatment.

3.4.2.7 Grounding. The panel structure shall incorporate a ground stud or $3 / 8$ hole for connecting ground cables/strips.

\subsection{System Design Requirements.}


3.5.1 Lifetime Consideration. The system shall be designed for a minimum lifetime of 10 years.

3.5.2 Transportability. The system shall withstand, without damage, shock and vibration encountered in shipment.

3.5.3 Fungus Resistance. Materials used in the fabrication of the system shall not support the growth of fungus, except that the requirement need not apply to components within hermetically sealed enclosures.

3.5.4 Environment. The solar cell array shall be designed to operate in and/or survive the following environments:

\begin{tabular}{|c|c|c|}
\hline Environment & Operate & Survive \\
\hline Ambient Temperature & $\begin{array}{l}-40^{\circ} \mathrm{C} \text { to }+50^{\circ} \mathrm{C} \\
=40^{\circ} \mathrm{F} \text { to }+122^{\circ} \mathrm{F}\end{array}$ & \\
\hline Thermal Cycling & & $\begin{array}{l}-40^{\circ} \mathrm{C} \text { to }+90^{\circ} \mathrm{C} \\
100^{\circ} \mathrm{C} / \text { hour with } \\
\text { cycle } 4 \text { hours }\end{array}$ \\
\hline Humidity & & 2 to $100 \%$ \\
\hline Cyclic Load & & $\begin{array}{l}+50 \mathrm{lb} / \mathrm{ft}_{2}^{2} \text { to } \\
-50 \mathrm{lb} / \mathrm{ft}^{2} 100 \\
\text { times }\end{array}$ \\
\hline Elevation & Sea Level to $10,000 \mathrm{ft}$ & \\
\hline Wind & & Up to 120 knots \\
\hline Hail & & $1 " D$ \\
\hline Rain & . & $\begin{array}{l}\text { Shall withstand } \\
\text { sustained rainstorms } \\
\text { at rates up to } 5 " \\
\text { per hour. Water } \\
\text { entry into connector } \\
\text { shall be considered } \\
\text { unacceptable. }\end{array}$ \\
\hline
\end{tabular}

3.5.5 Fasteners (Except Electrical). Screw threads shall conform to Handbook H-28; American National coarse threads are required where threads are provided in aluminum, magnesium or plastic parts and are preferred for all other parts. Bolts, screws, and other fasteners used on-rotating parts shall be provided with positive locking devices or means which do not depend on spring action or friction for their locking action, such as lock plates, lock wire, snap rings, castellated nuts, and cotter pins. Means for locking fasteners on all non-rotating parts shall also be provided. Swedging, peening, or stalking of threads will not be acceptable as a locking device or means for parts subject to adjustment, disassembly, or removal. The number of different sizes and types of bolts, screws, nuts, washers, etc., shall be the minimum practicable. Sheet metal screws shall not be used except in fastening of nameplates and instruction plates. 
3.5.6 Corrosion Resistance. All fasteners (bolts, screws, studs, etc.) and their associated hardware (washers, lockwashers, pins, etc.) shall be made of stainless steel, except that the following need not comply with this requirement.

a. Fasteners within hermetically sealed components.

b. Fasteners within components which operate immersed in or filled with a fluid which will form a protective film.

c. Fasteners in parts which are not subject to adjustment, disassembly, or removal for servicing, maintenance and repair during the life of the component of which they are a part.

3.5.7 Fasteners (electrical). Lock devices shall be provided each fastener used in making an electrical connection. Each fastener, locking device, and other hardware (washers, etc.) shall be made of corrosion-resisting material or shall be treated to be corrosion-resisting. Fasteners (bolts, screws, studs, etc.) shall not be depended on to carry current; they shall serve merely to hold current-carrying parts (lugs, terminals, etc.) in firm contact with each other. Where flow of current through a stud cannot be avoided, the stud and all its associated hardware (nuts, locking devices, washers, etc.) shall be made of corrosionresisting material. Pasitive means (such as pins or square shanks) shall be provided for preventing turning of studs in their mountings when nuts are tightened or loosened; lockwashers which depend on friction or spring action will not be acceptable for this purpose. Except for devices with integral studs, unused length of threads on studs (or screws used as studs) shall not exceed three threads of the stud or one-fourth inch, whichever is smaller. All threads of a nut used in making an electrical connection shall engage mating threads on the corresponding stud (i.e., length of stud is sufficient to allow complete passage through the nut).

3.5.8 Wiring. All wire shall be neatly laced into harnesses through the use of fungus resistant cord or self-locking nylon straps. Harnesses shall be so run and clamped (with insulated clamps) as to protect insulation against contact with sharp corners and edges, pinching, sharp bending and twisting, and abrasion because of vibration or contact. with moving parts. The clamps shall also serve to prevent transmittal of mechanical stress to internal connections of electrical components. Where a cable or wire is run between ports which move relative to each $c$ her, sufficient slack shall be left in the harness. Wires shall not be spliced at any point throughout the length of their runs. All harnesses used to interconnect assemblies shall terminate in qualified connectors at each end or branch. Connectors outside of enclosures shall be potted where wires exist or seals shall be used to prevent entrance of water, dust, dirt, etc. Open, exposed connections shall not be permitted. Not more than two terminal lugs shall be attached to any one stud or stud-type terminal boards. Solder terminals on electrical components shall not have more than two wires attached, unless specific written approval is obtained from the Contracting Officer's Technical Representative.

3.5.9 Wires and Cables. All wire and cable shall have conductor size not less than AWG Number 16. The following exceptions are permissible:

a. Wire used in coils and windings and wire used as short "jumpers" on printed circuit boards, etc., may be solid and smaller than AWG Number 16.

b. Wiring located within a hermetically sealed electrical component may be smaller than AWG Number 16.

c. Wire size smaller than Number 16 may be used within electrical enclosures if 
specific written opproval is obtained from the Contracting Officer's Technical Representative.

\subsection{Quality Assurance Provisions.}

4.1 Responsibility for Inspection and Test. Unless otherwise specified in the contract, the contractor is responsible for the performance of all inspection and test requirements as specified herein. Except as otherwise specified, the supplier shall utilize his own facilities or any commercial laboratory acceptable to the Government. In addition, the Government reserves the right to perform or repeat any tests or inspections set forth in this Purchase Description at any time such tests or inspections are deemed necessary by the Contracting Officer to assure equipment conforms to prescribed requirements. It is required that the contractor proposes solar cell modules equivalent in design and materials to modules which have previously met the pertinent Jet Propulsion Laboratory Environmental Requirements No. 5-342-1, Rev B, part III Test Requirements.

\subsection{Inspection and Identification.}

4.2.1 Visual Inspections. The Contracting Ufficer's Technical Representative shall make a physical inspection of all modules, frames, and stands to determine conformance with this Purchase Description, prior to the tests. The inspection shall include, but not necessarily be limited to the items of Table 1.

4.2.2 Module Identification. All modules shall be appropriately identified. 
TABLE I

VISUAL EXAMINATION

1. Workmanship

2. Safety

3. Identification

4. Treatment and Painting

5. Welds

6. Soldering

7. Electrical Wiring, Connectors and Connections

8. Output Terminals

9. Corrosion Resistant Treatment and Materials

10. Cracked or Dirty Silicon Cells

11. Encapsulation 


\subsection{Acceptance Test.}

4.3.1 General. The acceptance tests consist of the pre-manufacturing tests and the manufacturing tests. They shall be performed according to the specified JPL document, taking into consideration the different voltage and power levels of the modules. The premanufacturing tests are intended to characterize the expected module performance and to provide a high level of confidence that the modules and subarrays will function within the specified performance requirements in a terrestrial environment.

\subsubsection{Pre-manufacturing Tests.}

4.3.2.1 Electrical Performance. Perform current-voltage (I-V) characteristic tests on $5 \%$ of the modules.

4.3.2.2 Electrical Insulation Tests. Perform insulation resistance tests on $50 \%$ of the modules.

\subsubsection{Environmental Tests. Perform environmental tests on $5 \%$ of the modules.}

\subsubsection{Manufacturing Acceptance Tests.}

4.3.3.1 Electrical Performance Tests. Five percent of the solar cell modules shall be subjected to the electrical performance test.

4.3.3.2 Electrical Insulation Test. Five percent of the solar cell modules shall be subjected to the electrical insulation test.

4.3.3.3 Thermal Cycling Test. Five individual modules shall be subjected to the thermal cycling test.

4.3.3.4 Subarray Acceptance Test. All components shall be mounted on one subarray to demonstrate its ability to support same.

4.3.3.5 Compatibility Test. One subarray shall be operated and monitored for a minimum of 14 hours to verify system performance.

\subsection{Preparation for Delivery.}

5.1 Preservation and Packaging. Preservation and packaging of the solar cell modules, frames and stands shall be in accordance with good commercial practice to provide protection against deterioration and damage from the supplier to the destination.

5.2 Packaging. The solar cell modules, frames and stands shall be packed to insure corrier acceptance and safe delivery to destination at lowest rates in compliance with Uniform Freight Classification rules or National Motor Freight Classification rules.

\subsection{Notes.}

6.1 Intended Use. It is intended that the 4000 watt solar cells described herein be used for two applications, one in Yuma, AZ and the other in Dugway Proving Ground, UT.

\subsection{Definitions.}


6.2.1 Cell. A semiconductor device typically in wafer form that converts light energy to electrical energy.

6.2.2 Module. A grouping of one or more cells encapsulated into an integral, indivisible unit.

6.2.3 Subarray. A grouping of one or more modules into a free-standing unit, with module(s) electrically connected and terminals provided for connection to the array or lood.

6.2.4 Array. The entire solar cell subsystem. An array normally consists of a number of panels, although an array could consist of a single module, which, itself, could consist of a single cell. 
14.3 REJECTION CRITERIA FOR JPL LSSA MODULES

(5101-21, Rev. A)

Contents

14.3.1 OBJECTIVES

14.3.2 APPLICABLE DOCUMENTS

14.3.3 INSPECTION REQUIREMENTS

14.3.4 REJECTIOIN CRITERIA FOR MODULES

A. Module Identification

B. Module Mechanical Features

C. Solar Cells

D. Interconnects and Soldering

E. Encapsulation

F. Foreign Material

G. Hardware

H. Final Test and IV Dato

FIGURES I THROUGH 50

14-10 


\section{REJECTION CRITERIA FOR JPL LSSA MODULES}

\subsubsection{OBJECTIVE}

The objective of this document is to define the rejection criteria for silicon solar cell modules procured for the JPL Low-Cost Silicon Solar Array Project. The criteria, terminology, and illustrations are derived from the details of specific module designs. Any module design for which these criteria are inapplicable because of difference in detail shall necessitate the definition of additional or alternate rejection criteria.

\subsubsection{APPLICABLE DOCUMENTS}

The latest approved revisions of the following publications shall apply:
A. JPL Silicon Solar Cell Module Design, Performance, and Acceptance Test Requirements document.
B. Contractor interface control drawing.
C. Contractor top assembly drawing.

\subsubsection{INSPECTIONREQUIREMENTS}

Magnification: Inspection shall be performed using $6 x$ magnification. Higher magnification shall be used for evaluation or clarification.

\subsubsection{REJECTION CRITERIA FOR MODULES}

The following defects shall be cause for module rejection.

A. Module Identification

1. Module serial number location and orientation other than the position indicated on the manufacturer's drawing.

2. Required markings or identification which cannot be correctly read or interpreted.

3. Markings or identification which show signs of peeling or readily coming off.

4. Missing contractual markings or identification.

5. Incorrect markings or identification.

B. Module Mechanical Features

1. Module length, width or depth out of the tolerance specified on the manufacturer's drawing.

2. Module mounting hole size or location out of the tolerance specified in the 
manufacturer's drawing.

3. Cracked or damaged structural elements.

C. Solar Cells

1. Cracked or broken solar cells (exhibits $|-| 1$ ).

2. Cells in edge-to-edge contact (exhibit 12).

3. Cells in edge contact with metal module substrate (exhibit 13).

4. Overlapping cells (exhibit 14).

D. Interconnects and Soldering

1. Collector or interconnect delamination (exhibits 27a, 27b, 27c).

2. Alligatored contact. Silicon fracture under collector (exhibit 28).

3. Extensive collector dewetting from silicon in the interconnect-to-collector contact area (exhibit 29).

4. Less than $50 \%$ solder fillet on soldered area of interconnect-to-collector or back contact.

5. Fractured, overstressed, or damaged interconnects (exhibits 30-34).

6.' Solder joints obscured by flux.

7. Broken or nicked wire strands at solder joints in excess of these criteria:

7-strand wire: I strand broken, 2 strands nicked

9-strund wire: 2 sli urids brokken, 3 strands nieked

8. Insulation of wire buried into solder joint (exhibit 35).

9. Solder joints which lack a solder fillet to all wires or other elements contained within the solder junction (exhlblts 36, 37).

10. Broken or fractured solder joints at butput terminals (exhibits 37 and 38).

11. Split, burnt, crushed or cut insulation on any of the lead wires.

12. Cut, overstressed, or broken single strand wire.,

13. Interconnect misalignment with less than $50 \%$ of interconnect in contact with collector (exhibit 39).

14. Stress relief loop frozen by solder (exhibit 40).

15. Wire or other interconnects in or near contact with other conductors, including conductive substrates. 
16. Folded interconnects (exhibit 41).

17. Damaged flat or printed circuit paths having less than $75 \%$ of currentcarrying conductor remaining.

18. Interconnect rework, i.e., patching or solder coating, is not acceptable for exhibits 30-34.

\section{E. Encopsulation}

1. Encapsulant cracking or splitting over active elements (exhibit 42).

2. Frame seal delamination (exhibits 43,44 ).

3. Holes or air bubbles in the encapsulant, regardless of size, which could serve as a direct moisture path from the outside environment to an internal module component (exhibit 45).

4. Bubbles or delamination between output terminals or terminals to substrate (exhibits 46, 47).

5. Uncured or insufficiently cured encapsulant characterized by excessively sticky surfaces and/or streaks of liquid on the surface of the encapsulant.

6. Interlayer delamination.

7. Air bubbles or uncured encapsulant which move about under light finger pressure.

8. Delaminations and Bubbles.

a. Delamination or bubbles less than $2 \mathrm{~mm}$ across the largest dimension are acceptable at any location on the module.

b. Delamination or bubbles larger than $2 \mathrm{~mm}$ but smaller than $3 \mathrm{~mm}$ across the largest dimension are acceptable under the following constraints:

(1) There shall be none over cells, interconnects and/or terminals.

(2). There shall be no more than 10 per module.

(3) None shall be located where it can provide a path from one conductor to another or from a conductor to metallic frame or substrate (exhibits 44, 45).

(4) Delaminations or bubbles shall be at least $2 \mathrm{~cm}$ apart.

c. Delaminations or bubbles greater than $3 \mathrm{~mm}$ are not acceptable (exhibit 44).

9. Internal conductors, interconnects, or cells within $1.5 \mathrm{~mm}$ of the external surface of the encapsulant (unglassed modules only) (exhibits 48, 49). 
10. Cracked or fractured glass or other protective coating.

11. Deep scratches over $5 \mathrm{~cm}$ in length in any direction or location on module cover glass.

12. Inadequate adhesion of encapsulant in reworked areas, as indicated by the following procedure:

a. Clean the surface to be evaluated with methyl or ethyl alcohol.

b. Cut a 2 to 3 inch length of Scotch Brand Pressure Sensitive tape No. 600 or equivalent and apply immediately to the area to be tested; hold a tab end above the surface.

c. Rub tape so that air bubbles are removed.

d. Pull tape from the surface. Reject if encapsulant comes away from the module and remains on the tape.

NOTE: Do not perform this test before the encapsulant has been completely cured (i.e., a minimum of 48 hours at room temperature).

F. Foreign Material

1. Any metallic particle (including solder) resting on the cell junction (exhibit 50).

2. Any metallic particle (including solder) trapped between the interconnect and the cell junction (exhibit 50).

3. Any metallic particle trapped between an internal conductor, such as an interconnect, and a conductive substrate.

G. Hardware

I. Loose or missing hardware.

2. Damaged hardware such as threaded terminal posts and missing plated or chemically treated areas.

3. Encapsulant on external electrical contacts, mounting bosses, or mounting hardware surfaces.

4. Incorrect hardware.

H. Final Test and IV Data

1. Data missing or incorrect.

2. Data out of specification tolerances. 
FIGURES I TO 50

REJECTION CRITERIA FOR JPL LSSA MODULES 
5101-21, Rev. A

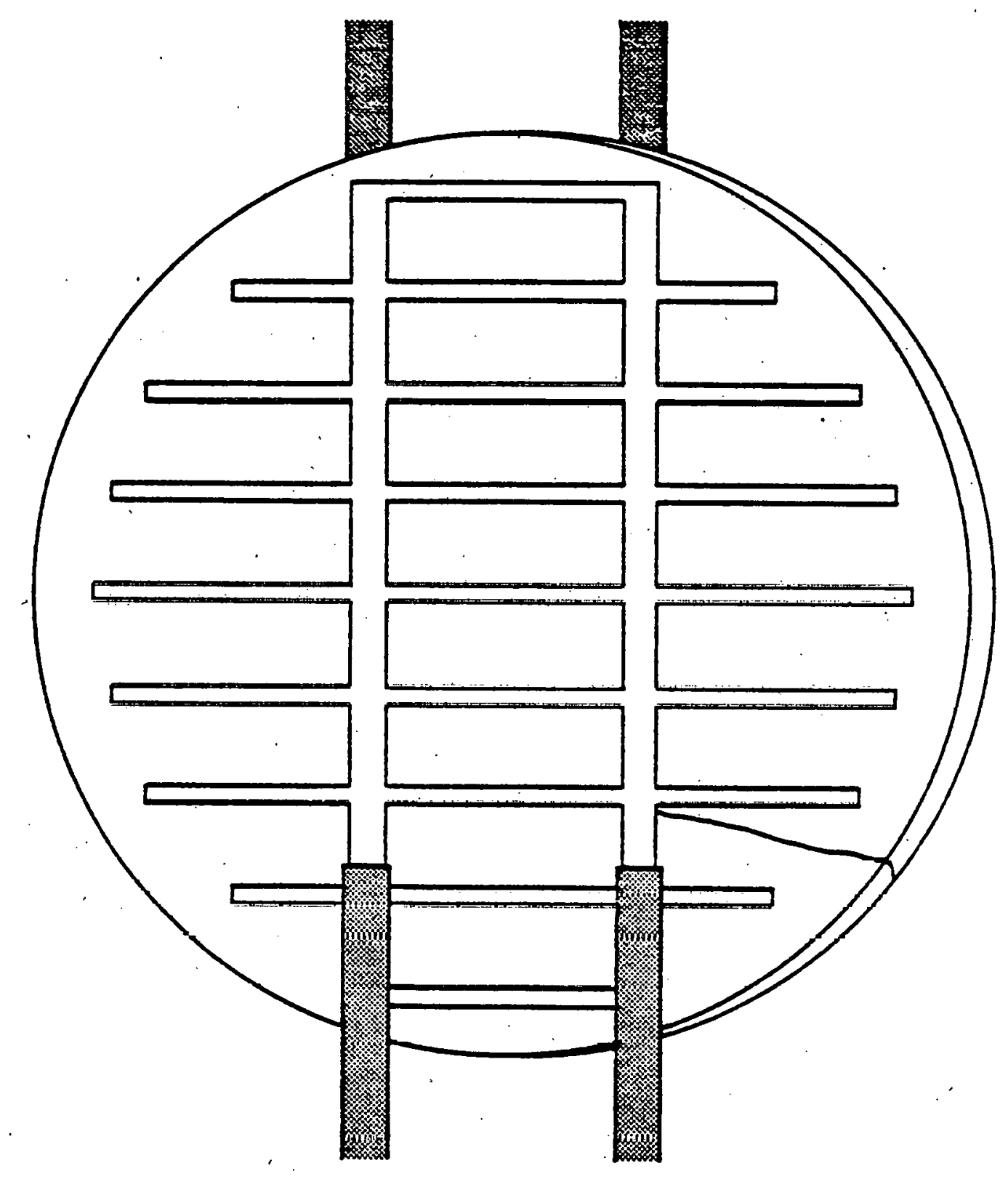

Figure 1. Terminated Crack. Reject. 
5101-21; Rev. A

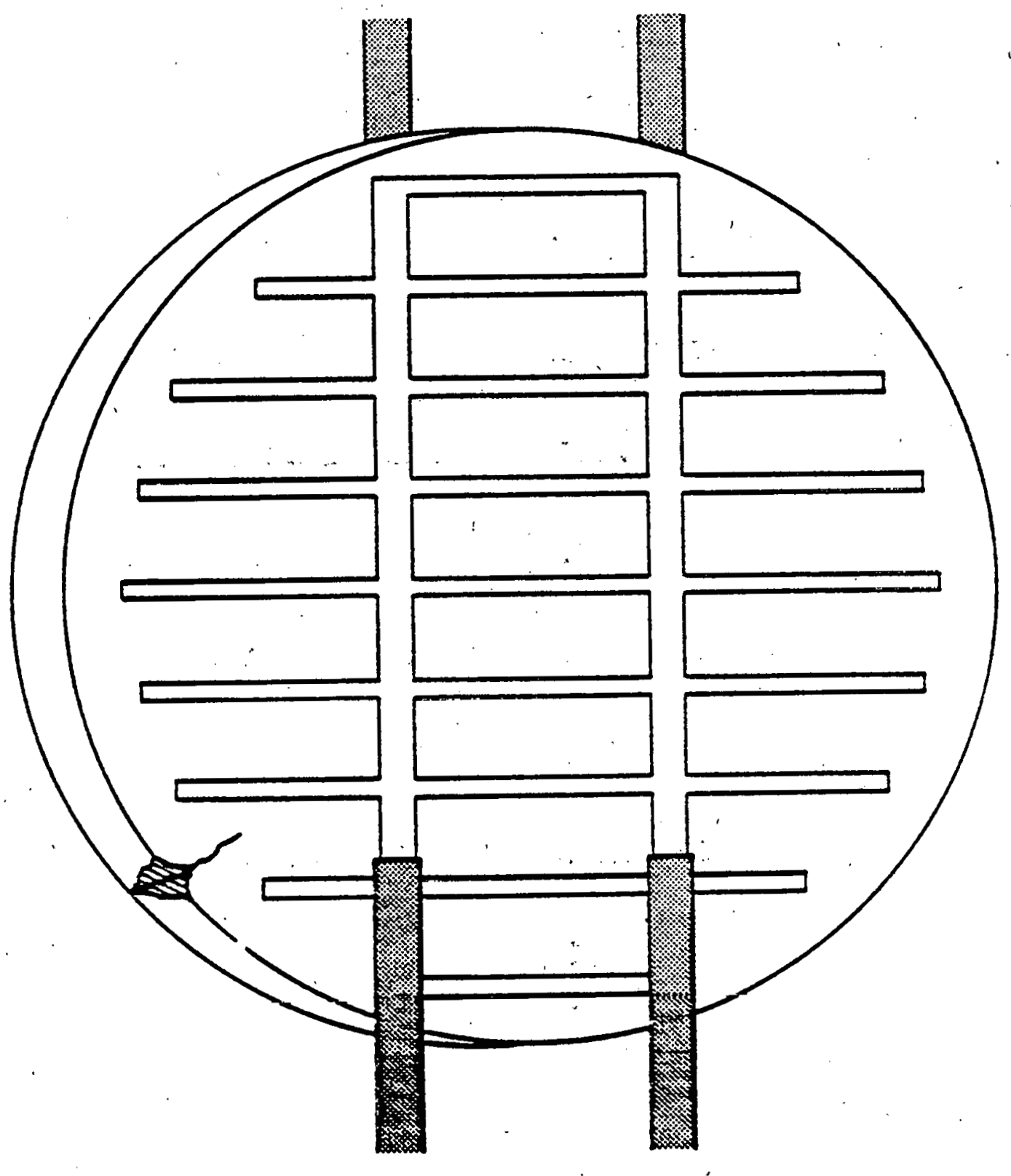

F1gure 2. Short Terminated Radial Crack Started From Edge Chip. Reject. 


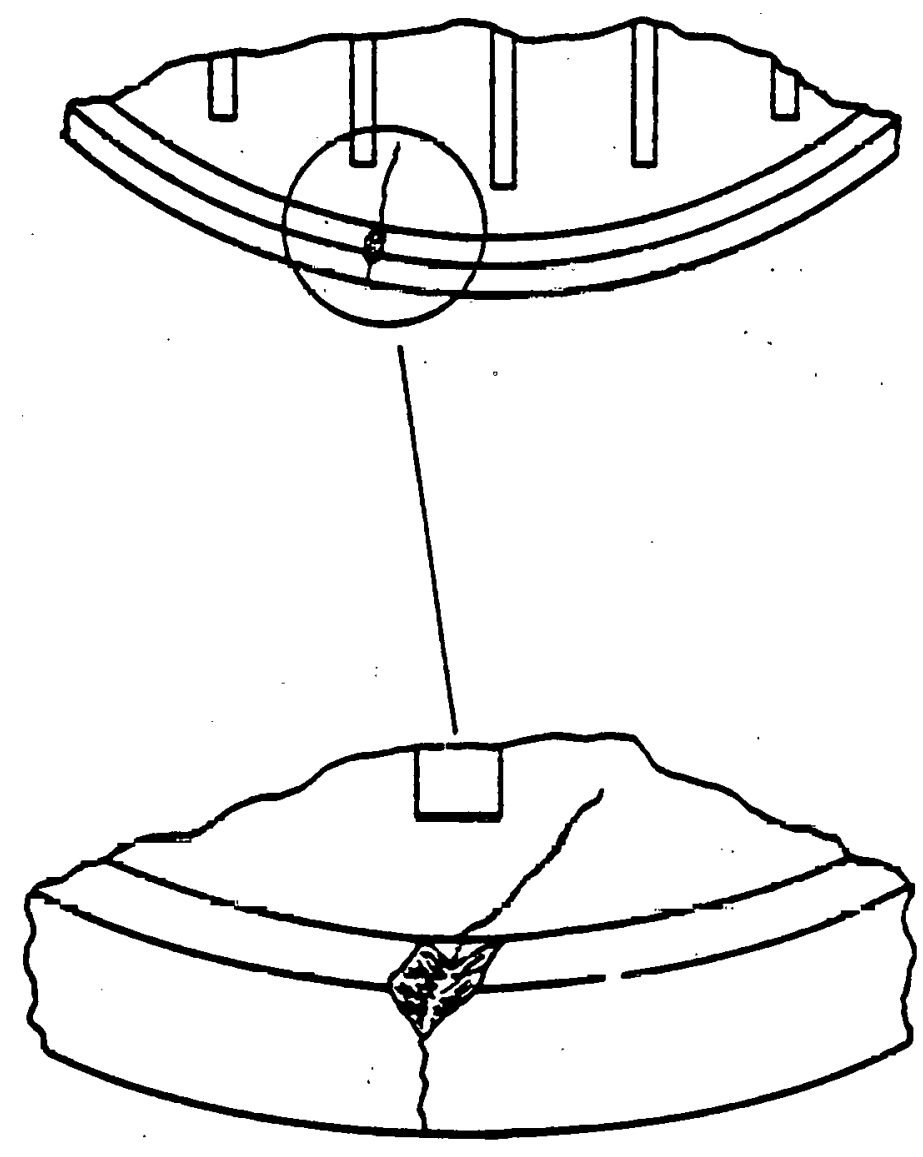

Fłgure 3. Crack Emanating From Cell RIm Deriect. Reject. 
5101-21; Rev. A

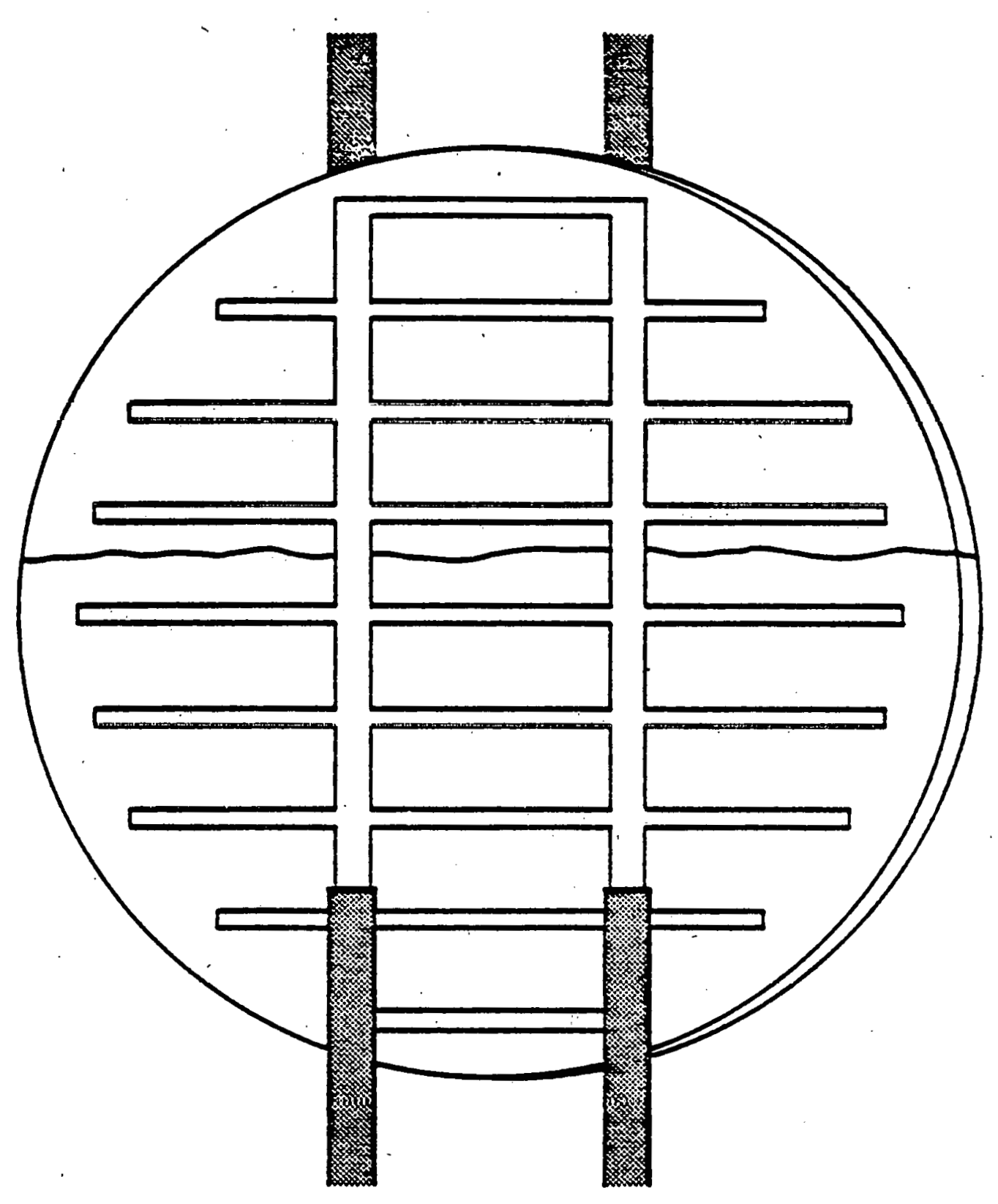

Ffgure 4. Rim to Rim Hafrline Crack. Reject. 
5101-21; Rev. A

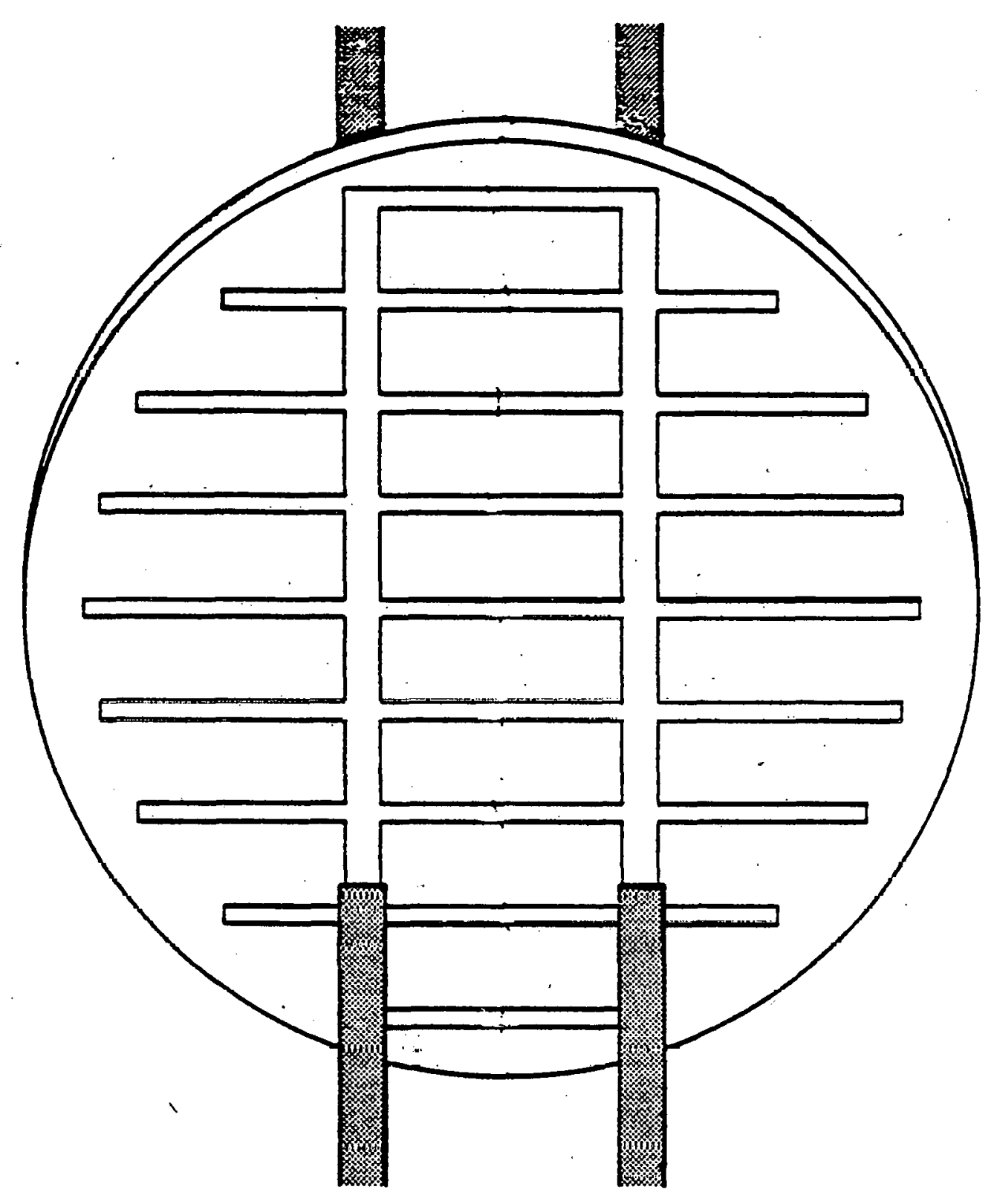

Flgure Sa. Crack Between Collectors. Acceptable. 


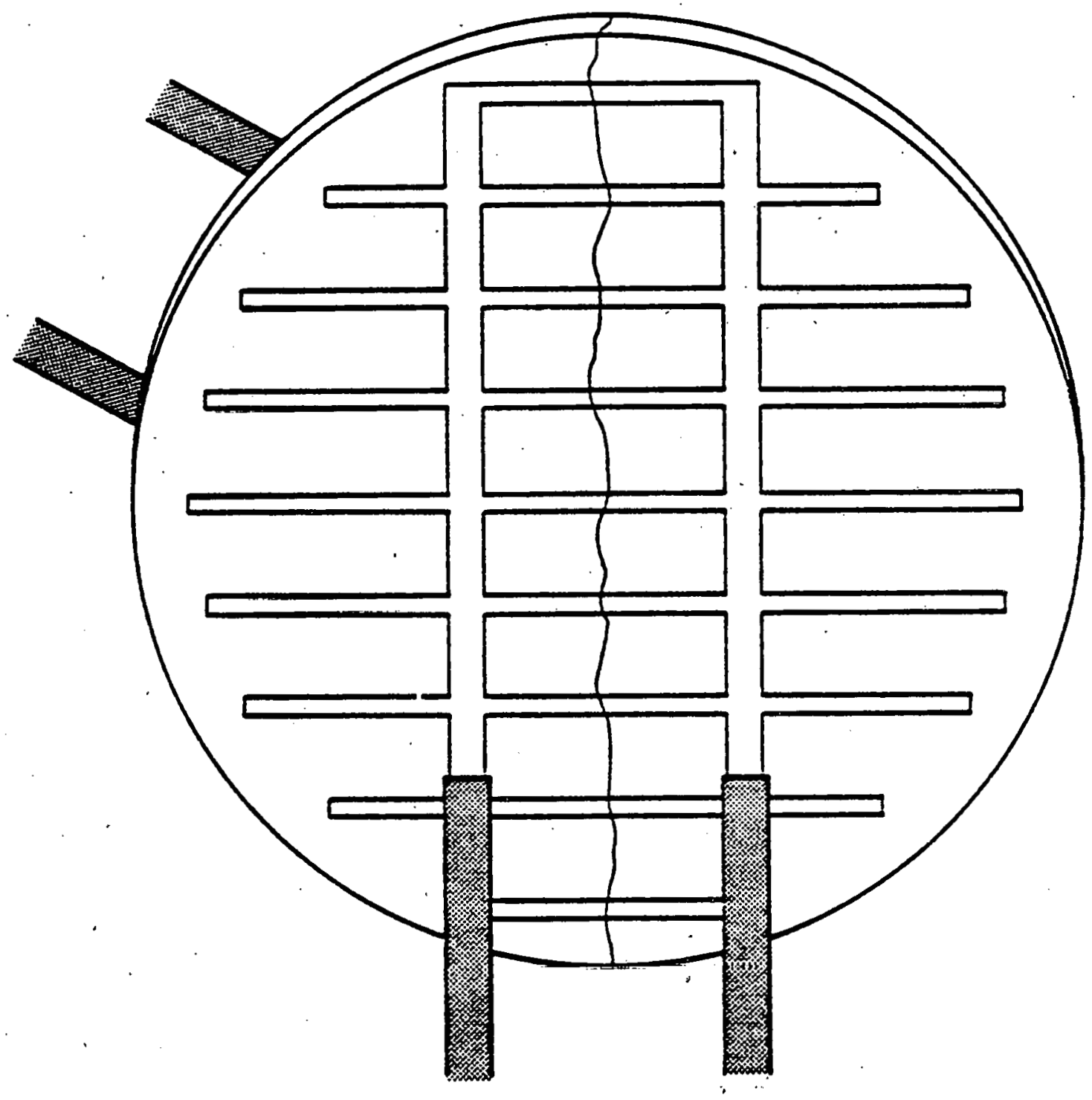

Figure 5b. Crack Isolating offset Interconnects. Reject. 
5101-21; Rev. A

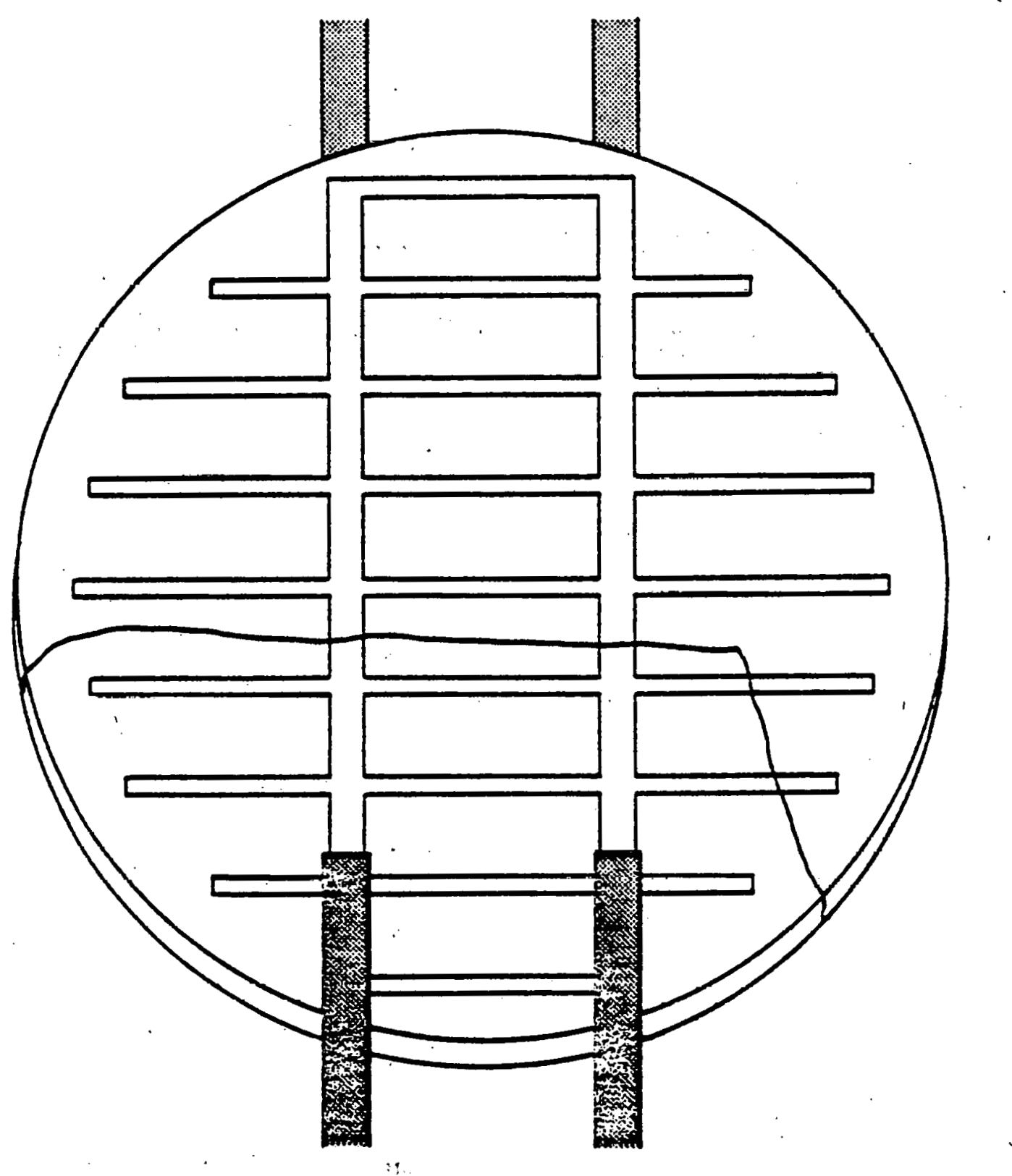

Elgure 6. Irregular Halriline Crack Through Collector. Reject. 


\section{1-21; Rev. A}

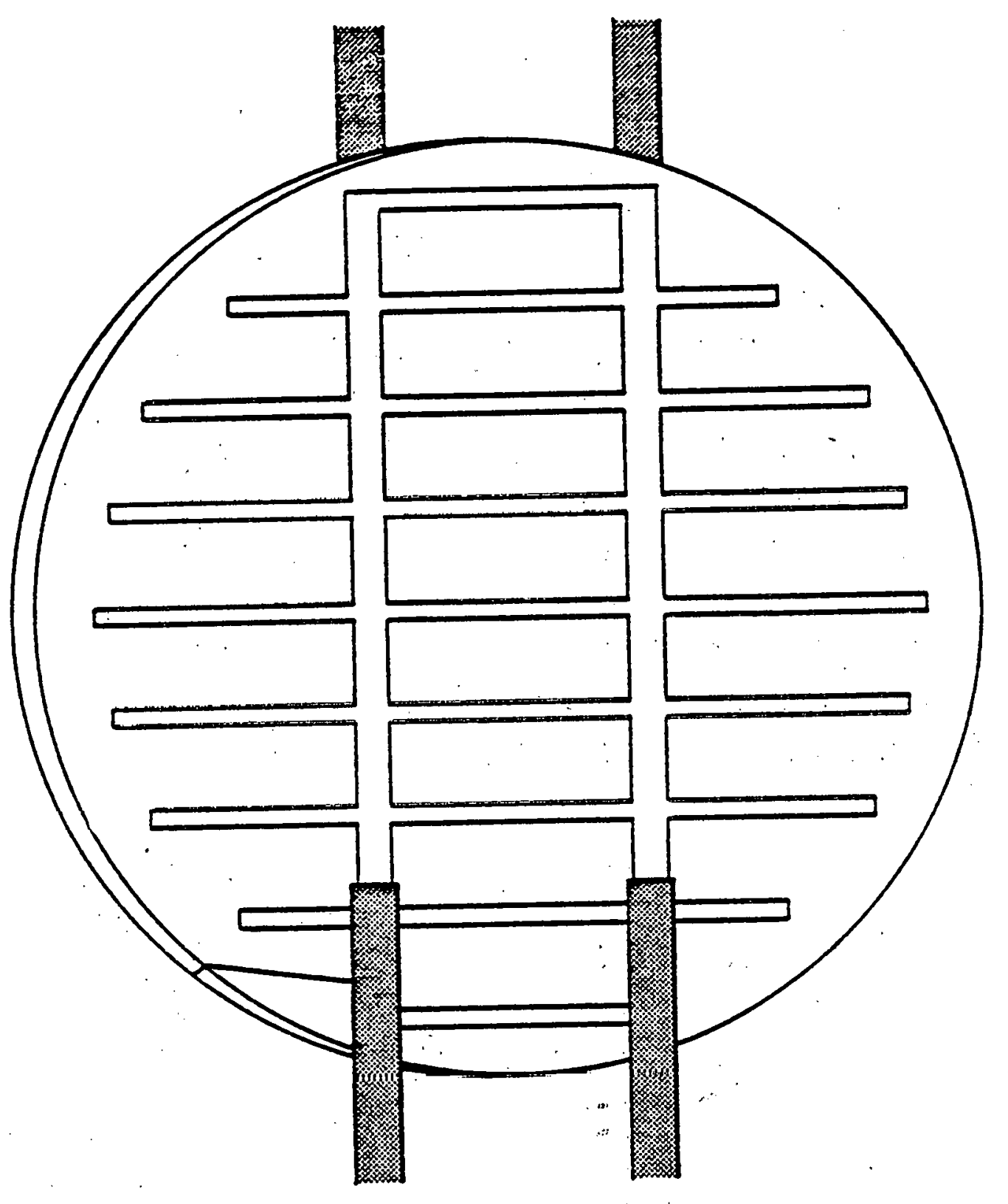

Figure 7. Edge Crack Terminated or Passing Through or Under Collector Solder Joint. Reject. 


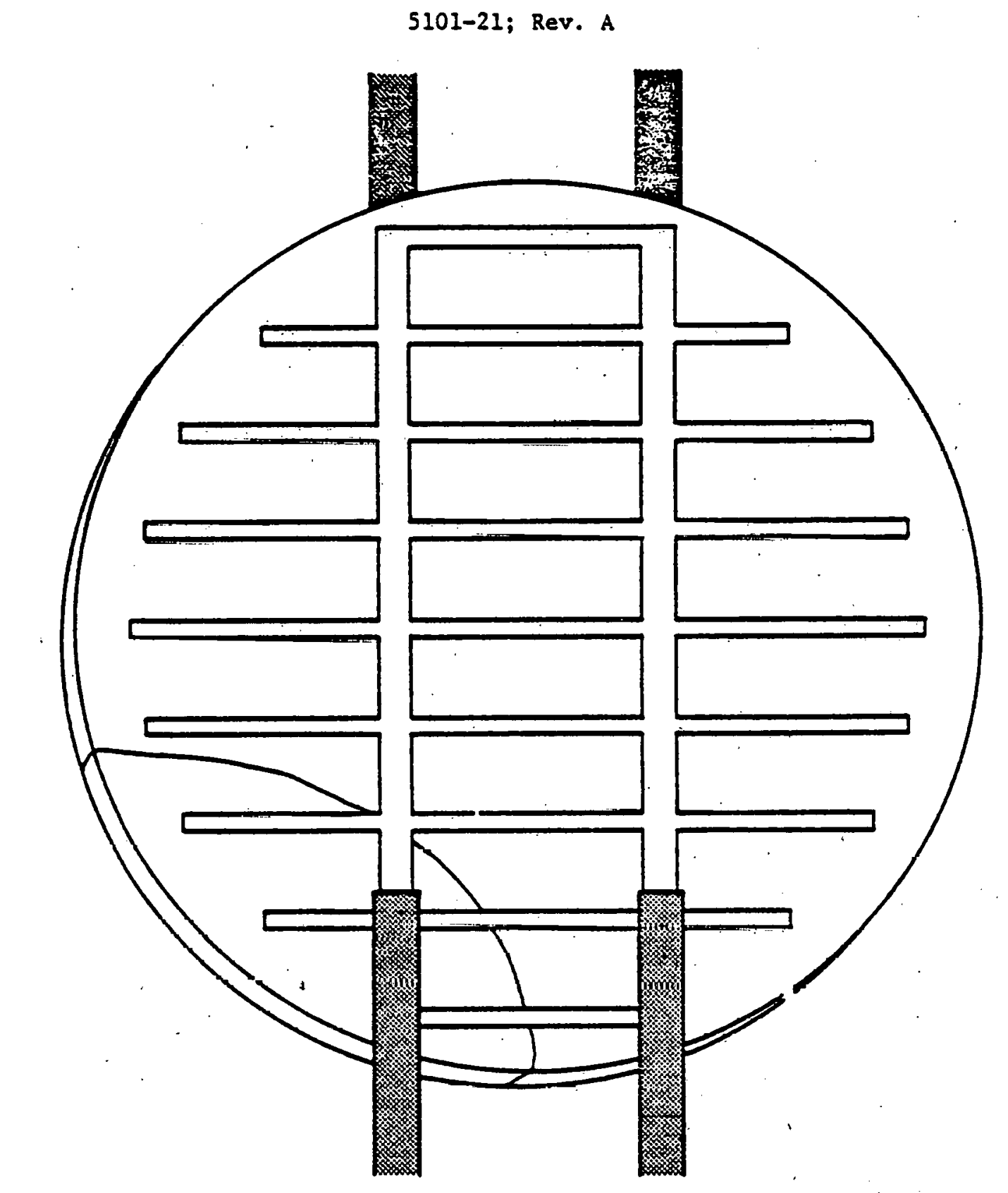

F1gure 8. Broken Portion of Cell Intersecting Collector Strip. Reject. 
5101-21; Rev. A

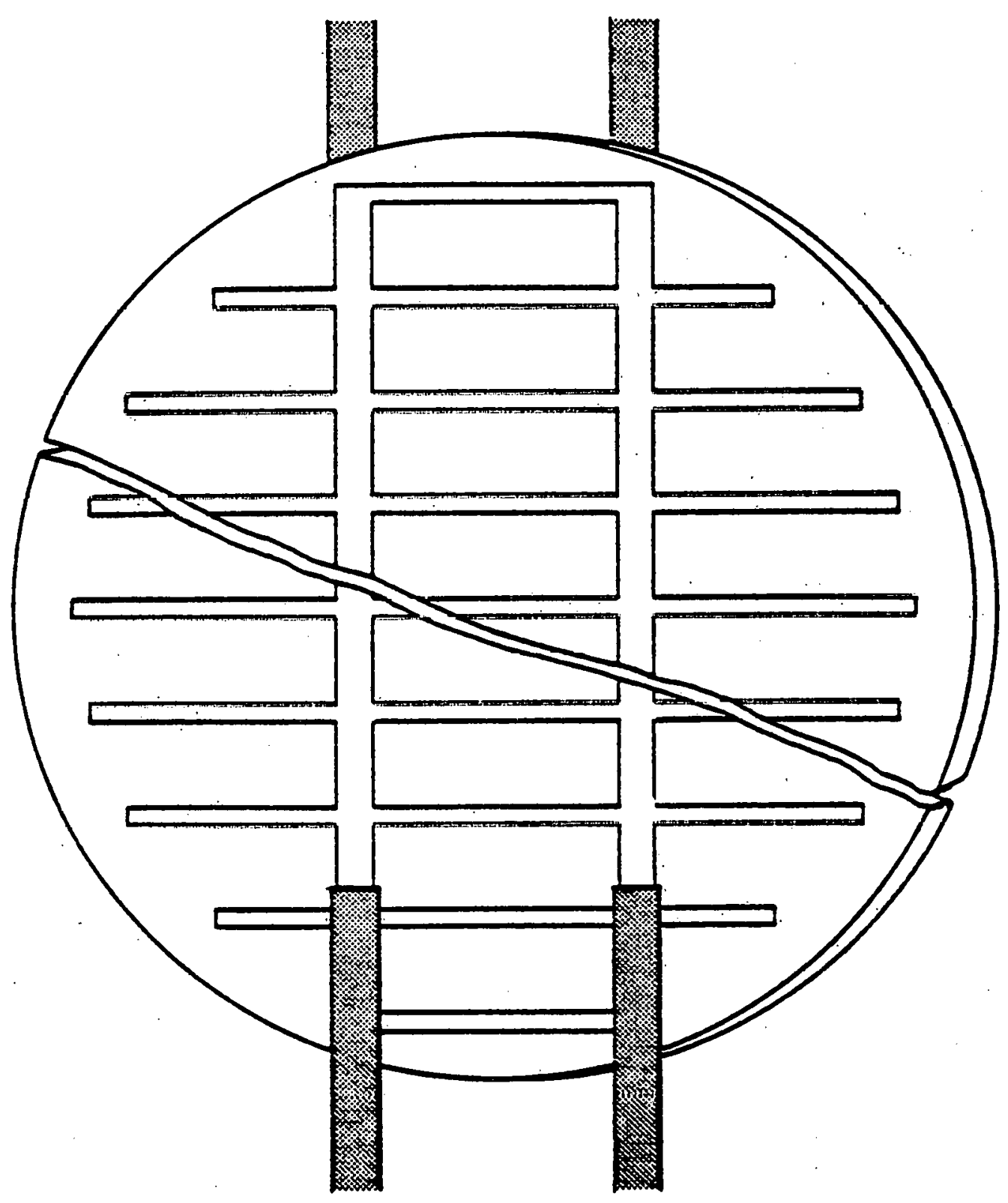

F1gure 9. Crack Causing Total Separation. Refect. 
5101-21; Rev. A

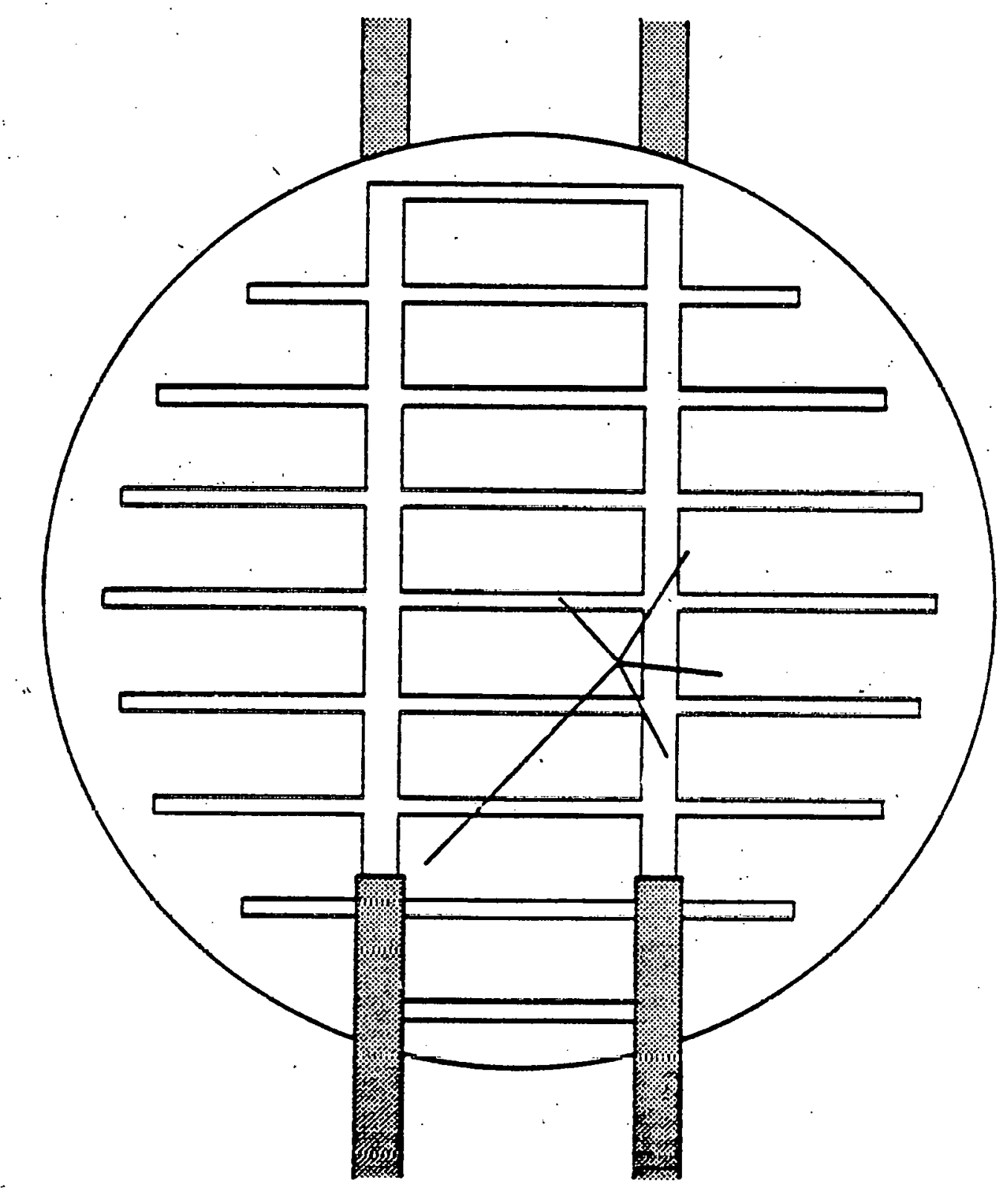

Flgure 10. Cracks Caused by Point Impact. Refect.

thiseses 


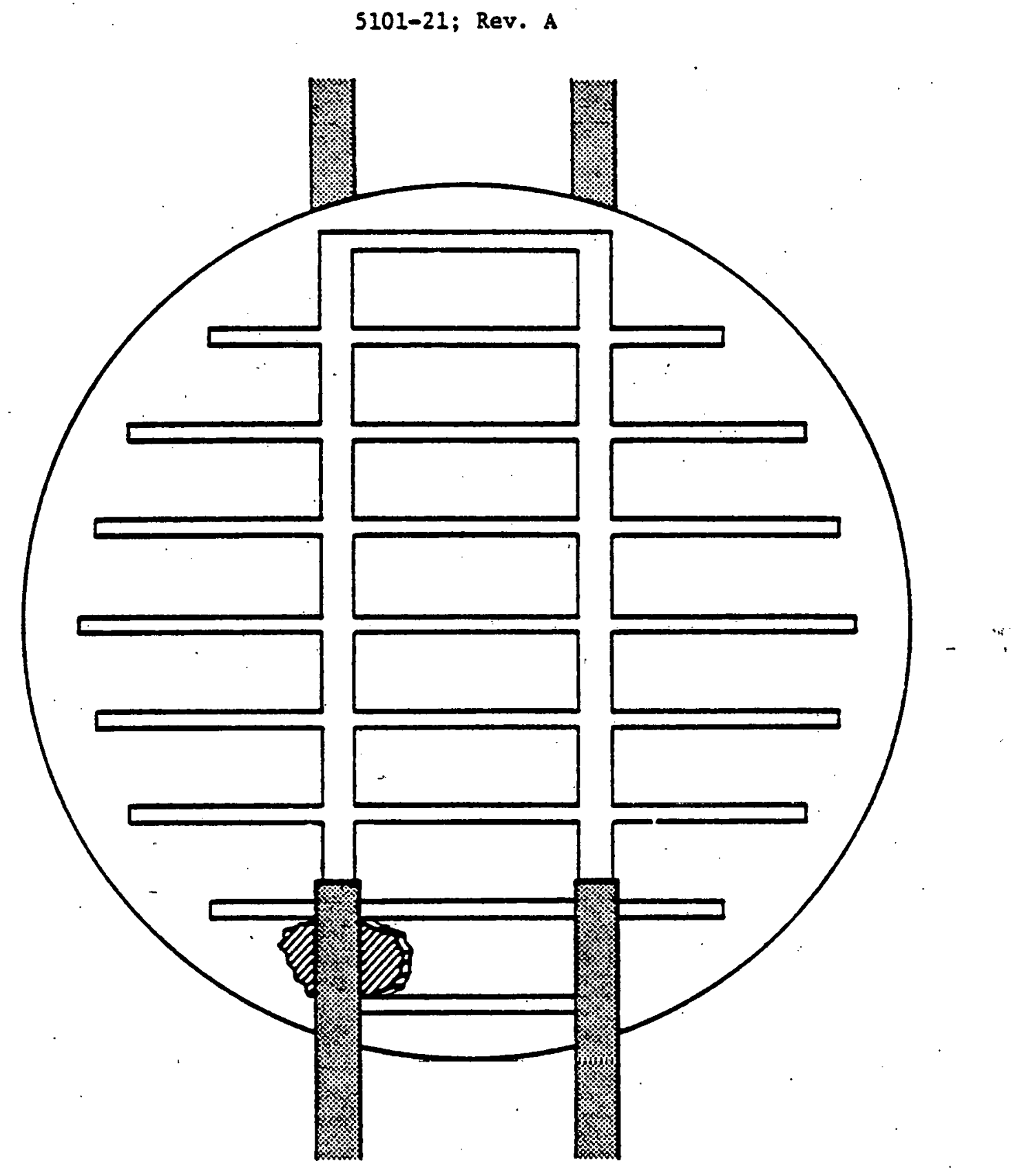

Figure 11. Spalling in Cell Surface Materlal at Collector. Refect. 
5101-21; Rev. A

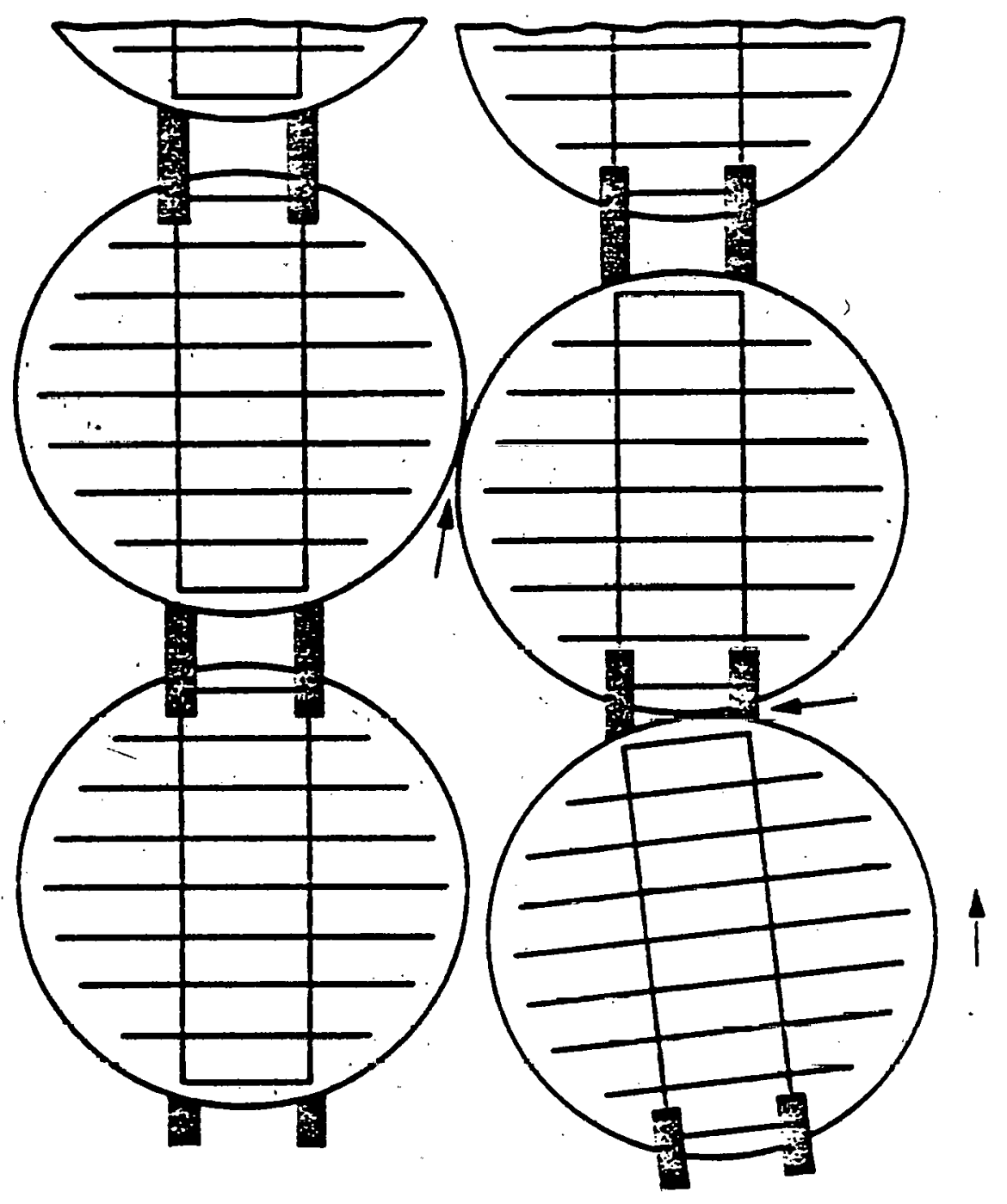

F'sure 12. Cella in Edge-to-Edge Contact. Reject: 


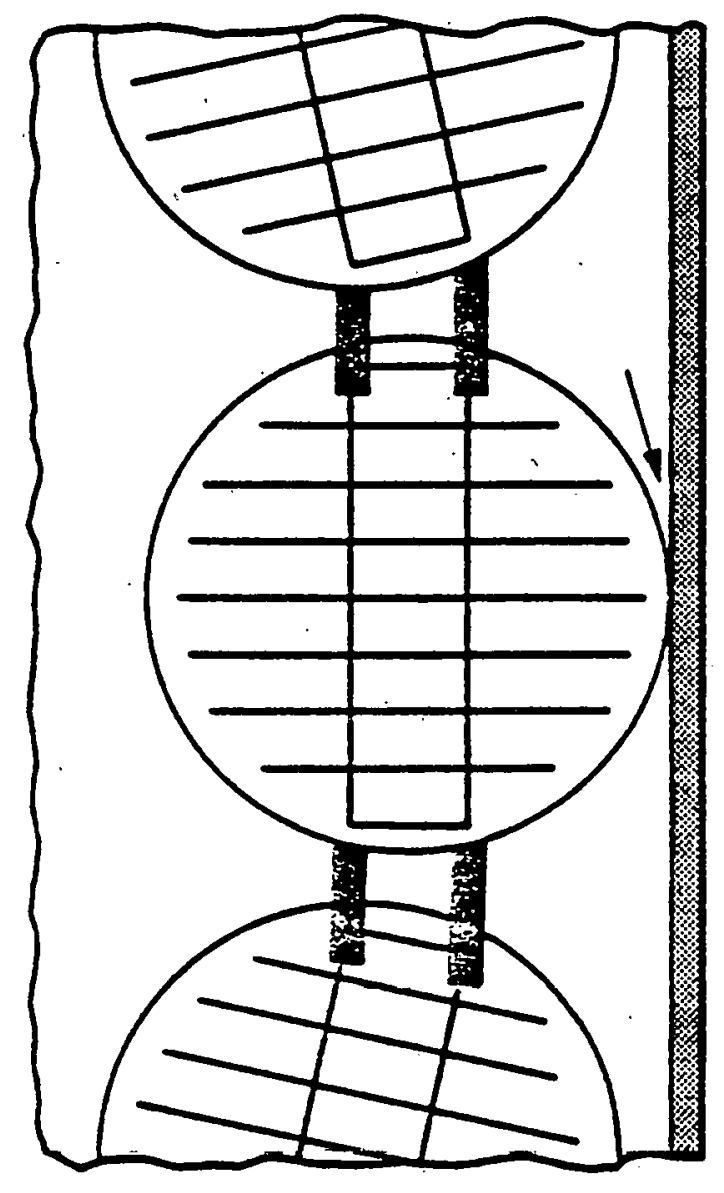

F1gure 13. Cell in Edge Contace With Metal podule Substrate. Reject. 


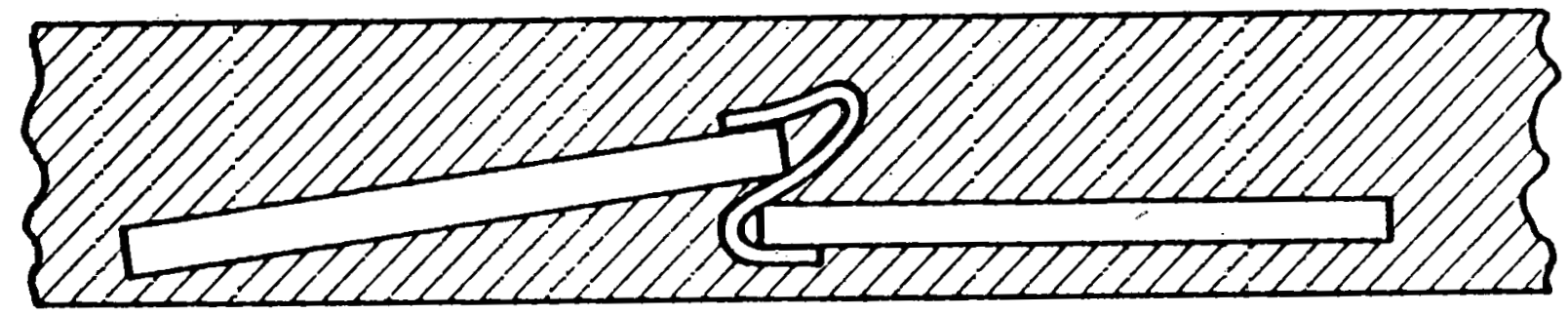

Figure 14. Overlapping Cells. Reject. 


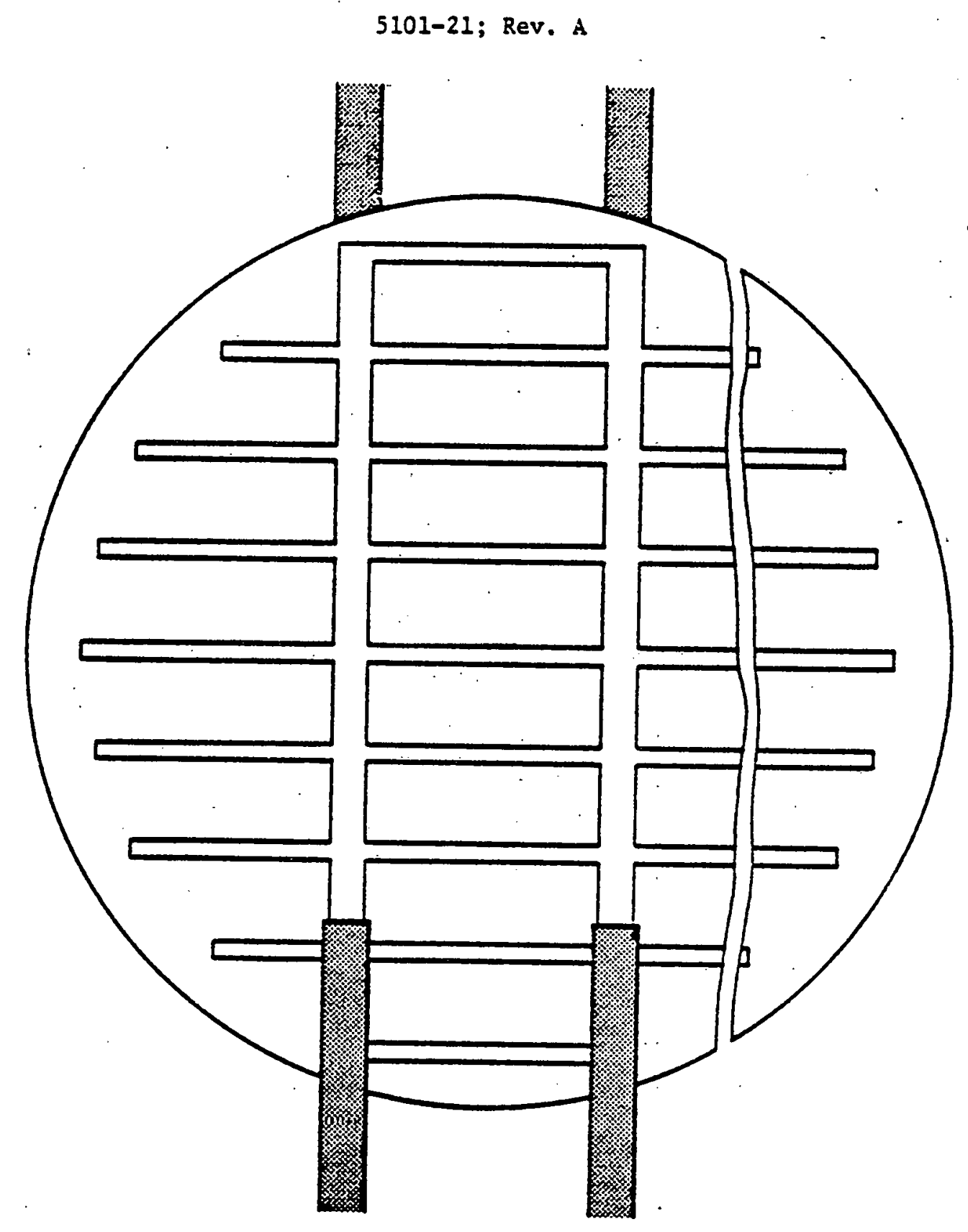

I1gure 15. Break in Cell not Intersecting Collector. Acceptable. 
5101-21; Rev. A

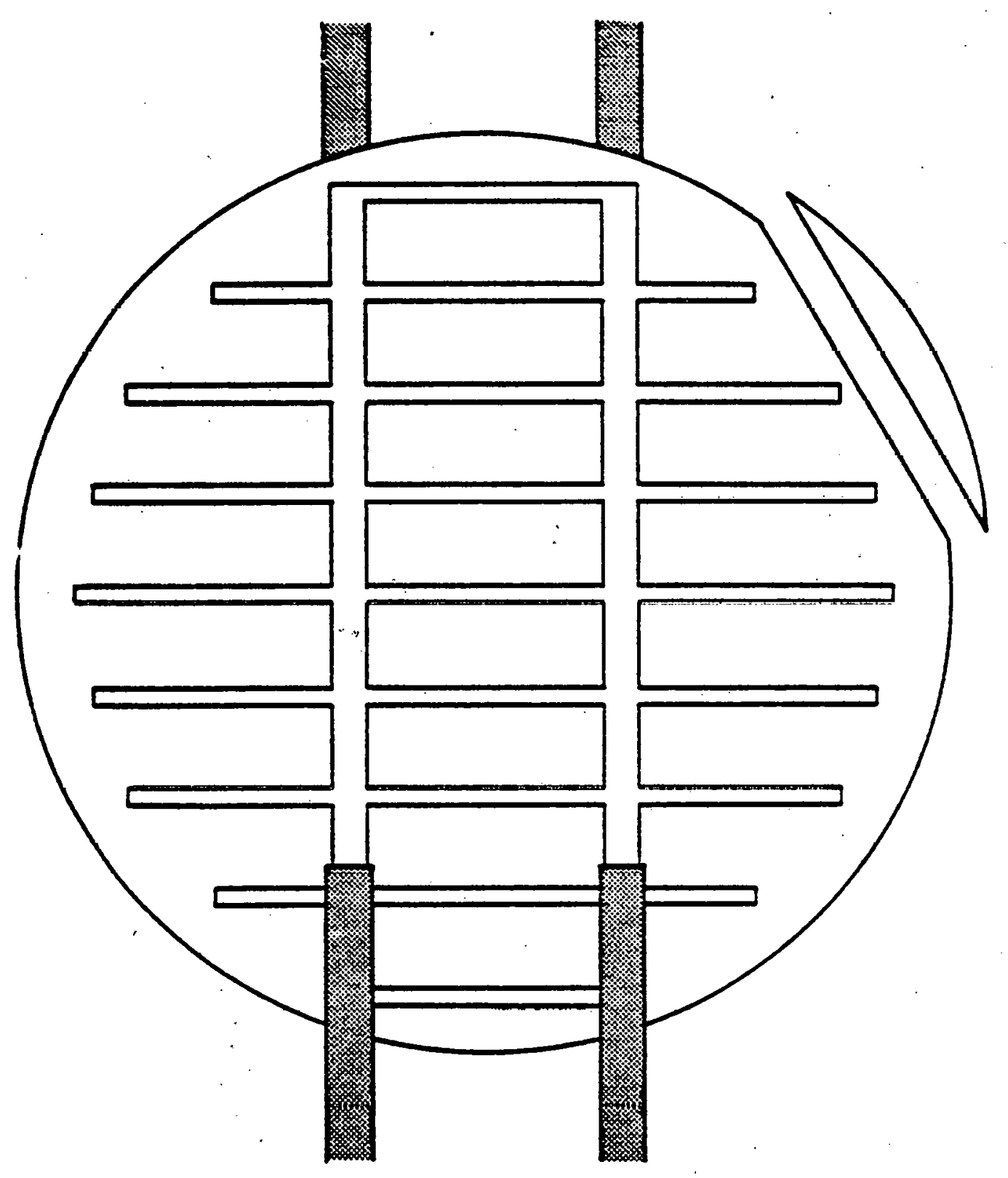

Eigure 16. Part of Cell Broken Away Without Disturbing Cell Integrity. Acceptable. 


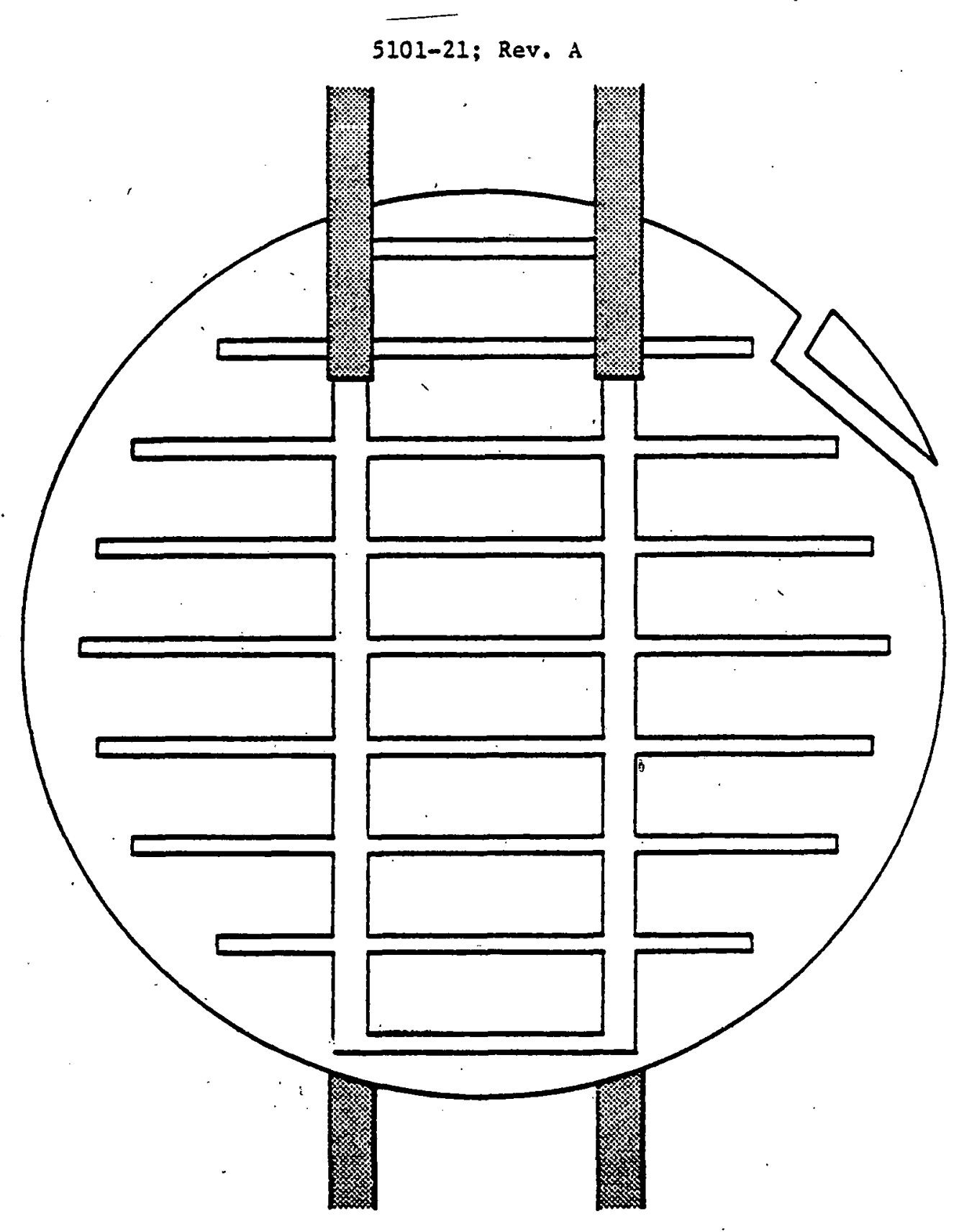

F1gure 17. Chip Broken out of Cell Body at Edge. Acceptable. 
5101-21; Rev. A

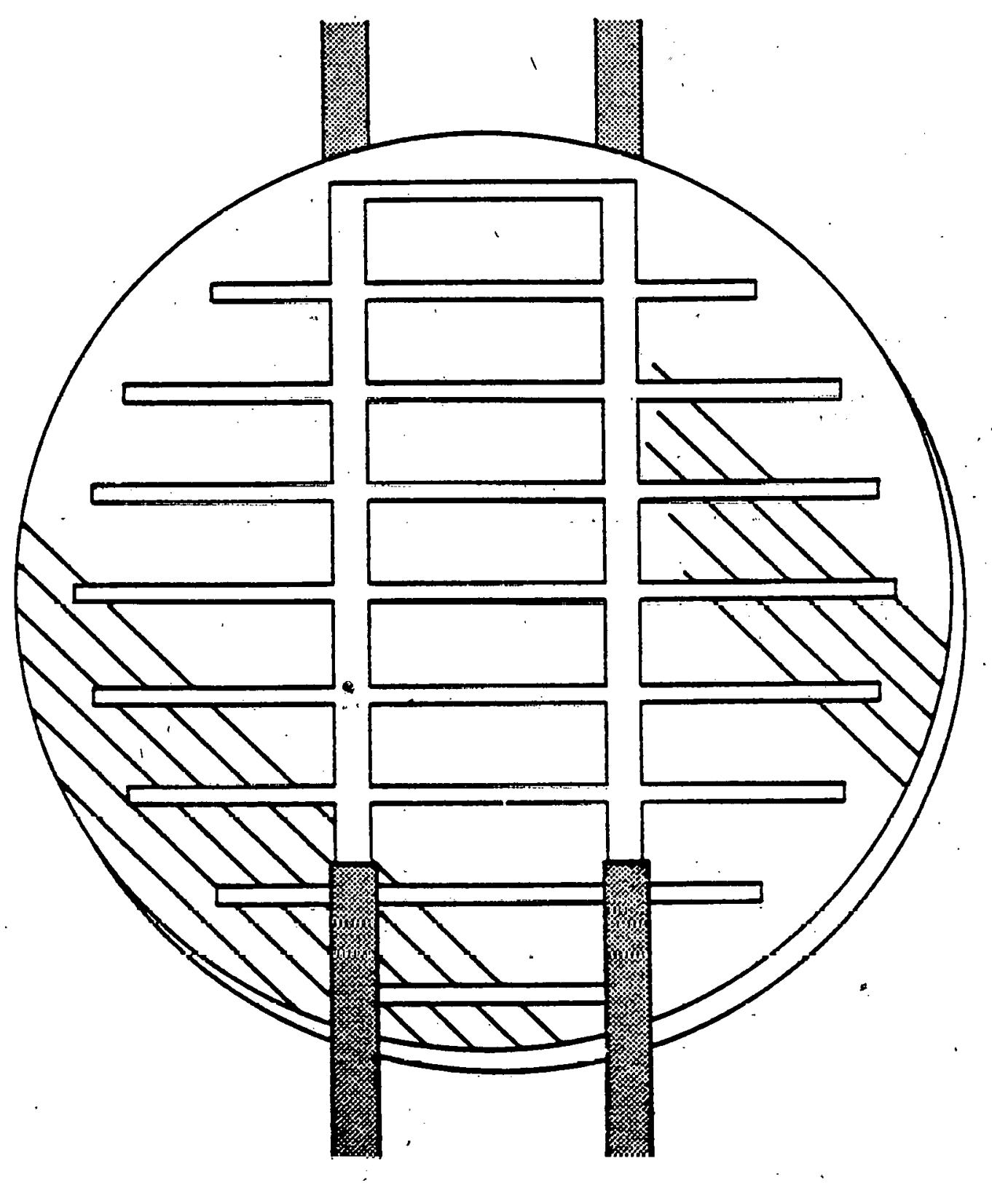

Figure 18. Parallel Saw Marks on Surface. Acceptable. 


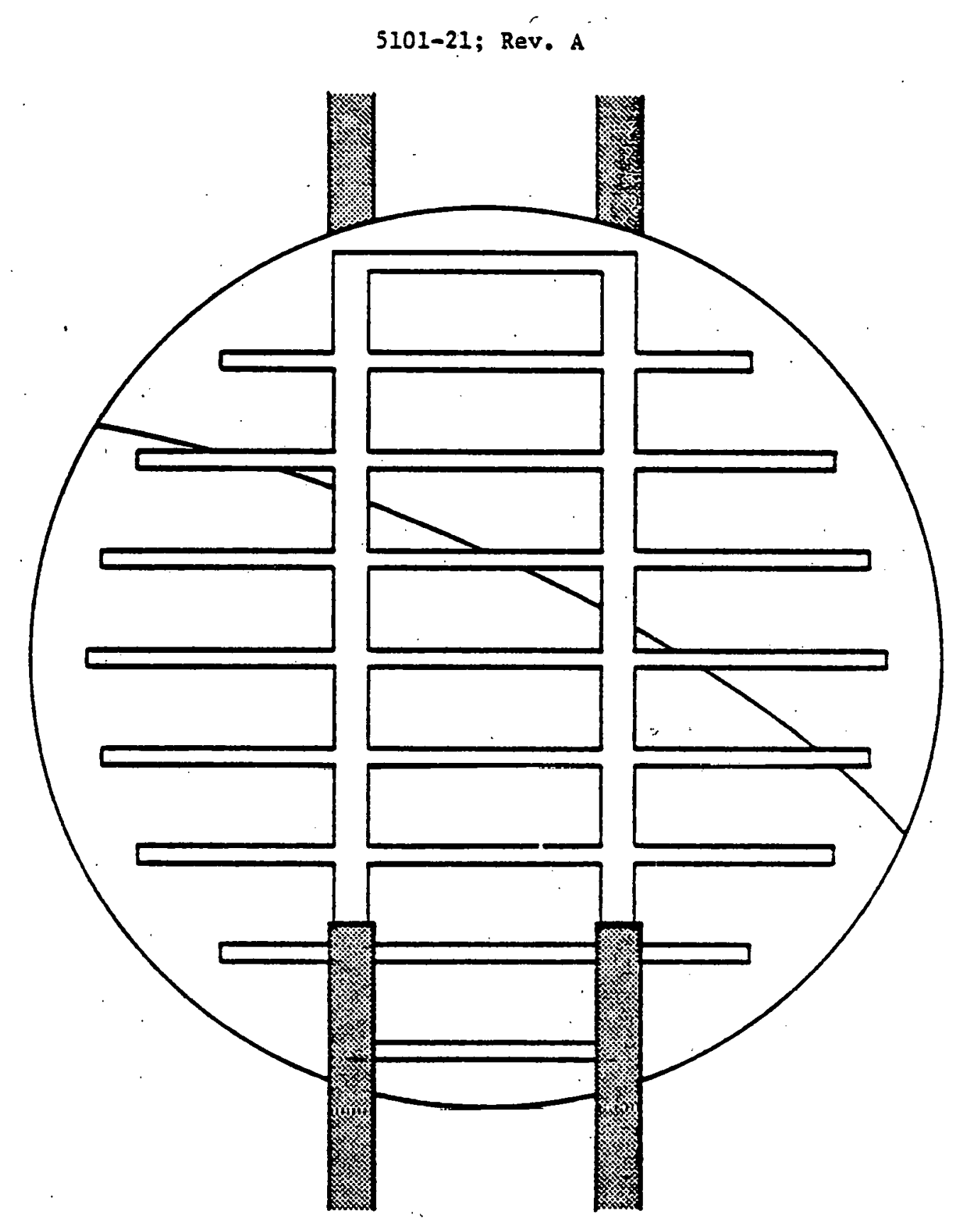

F1gure 19. Saw Mark on Cell Surface Only. May be Straighe IIne or Curved. Acceptable. 
5101-21; Rev. A

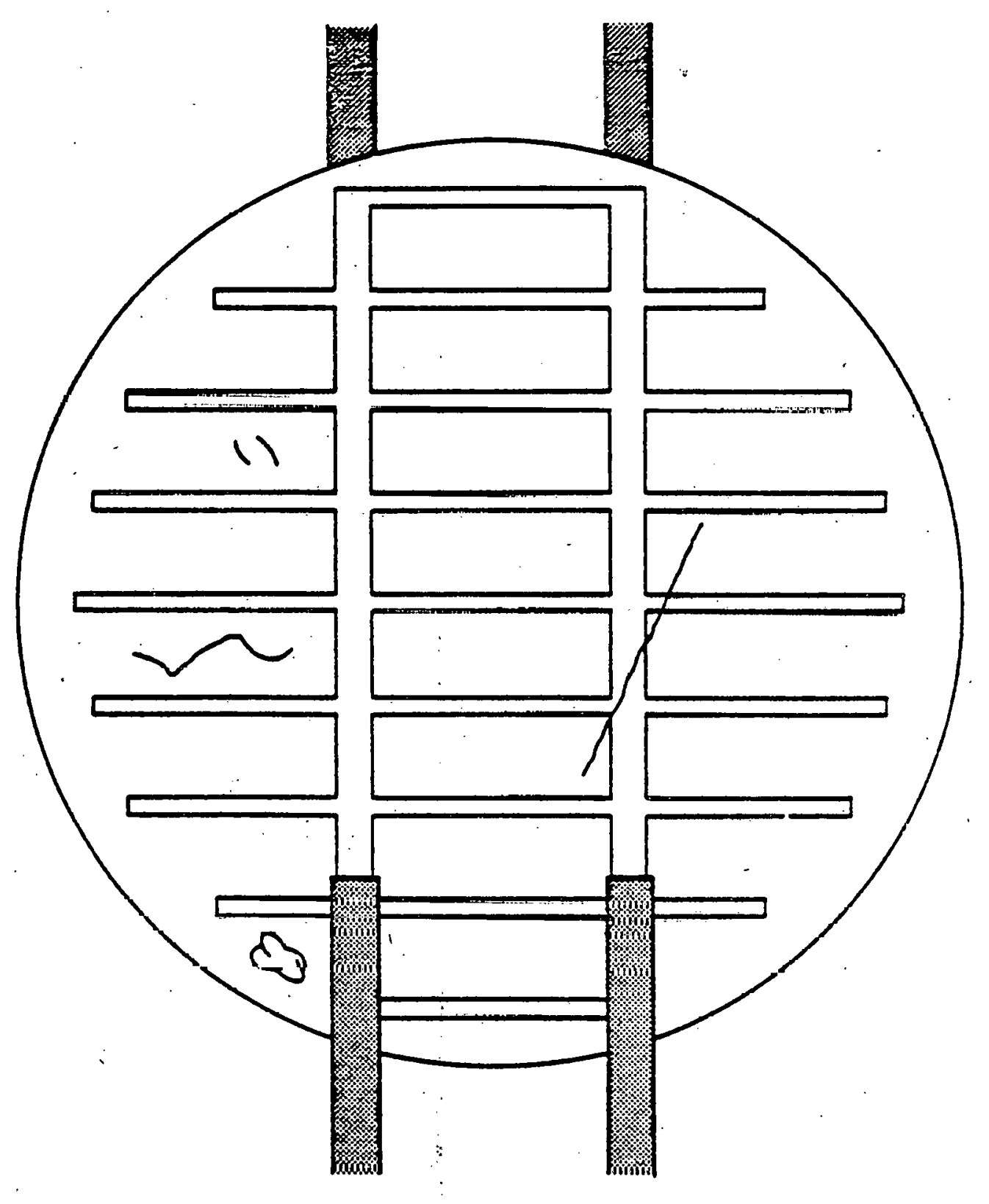

F1gura 20. Surface Scars and Scratches. Areeptable. 
5101-21; Rev. A

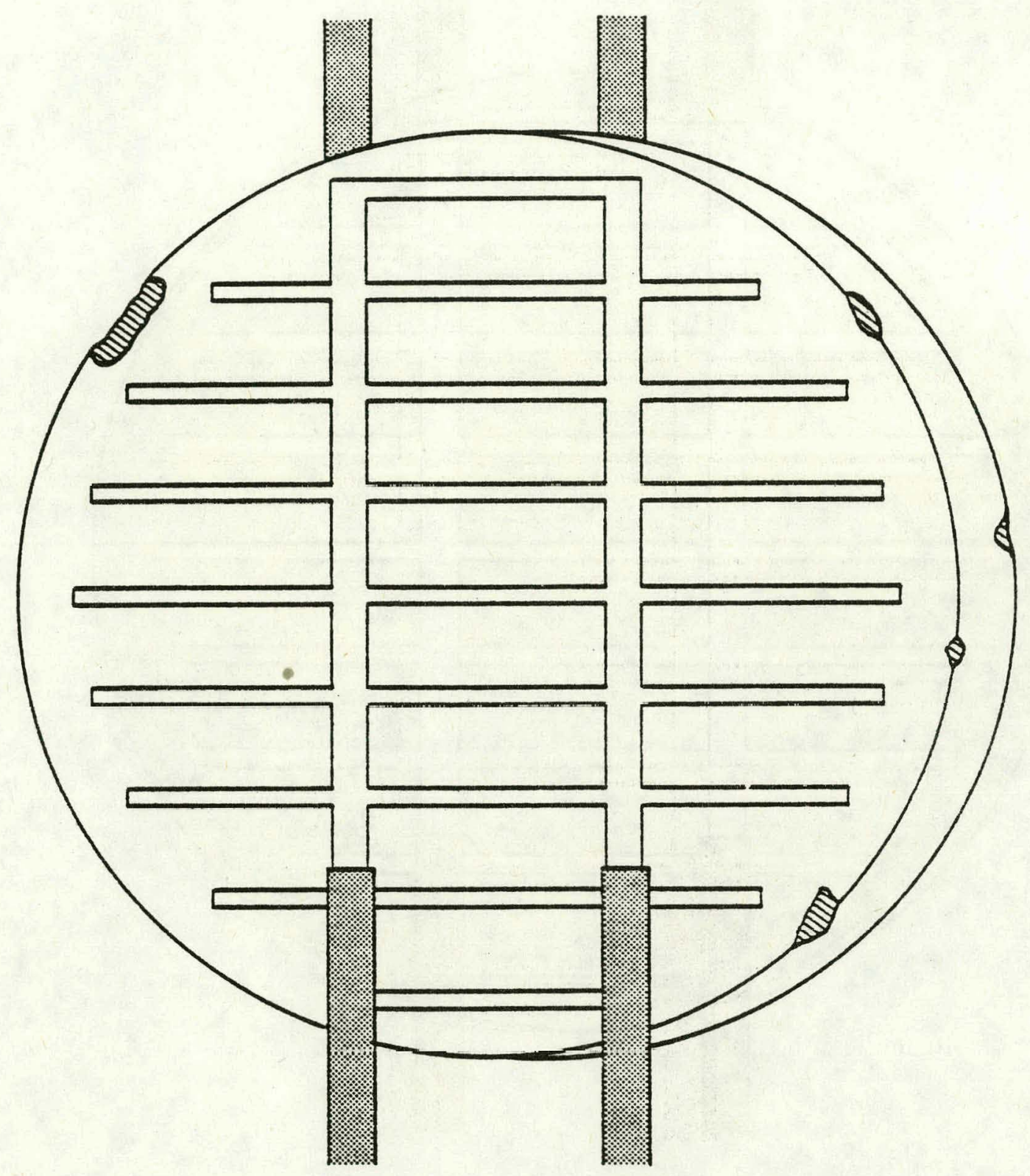

Figure 21. Edge Nicks. Acceptable. 
5101-21; Rev. A

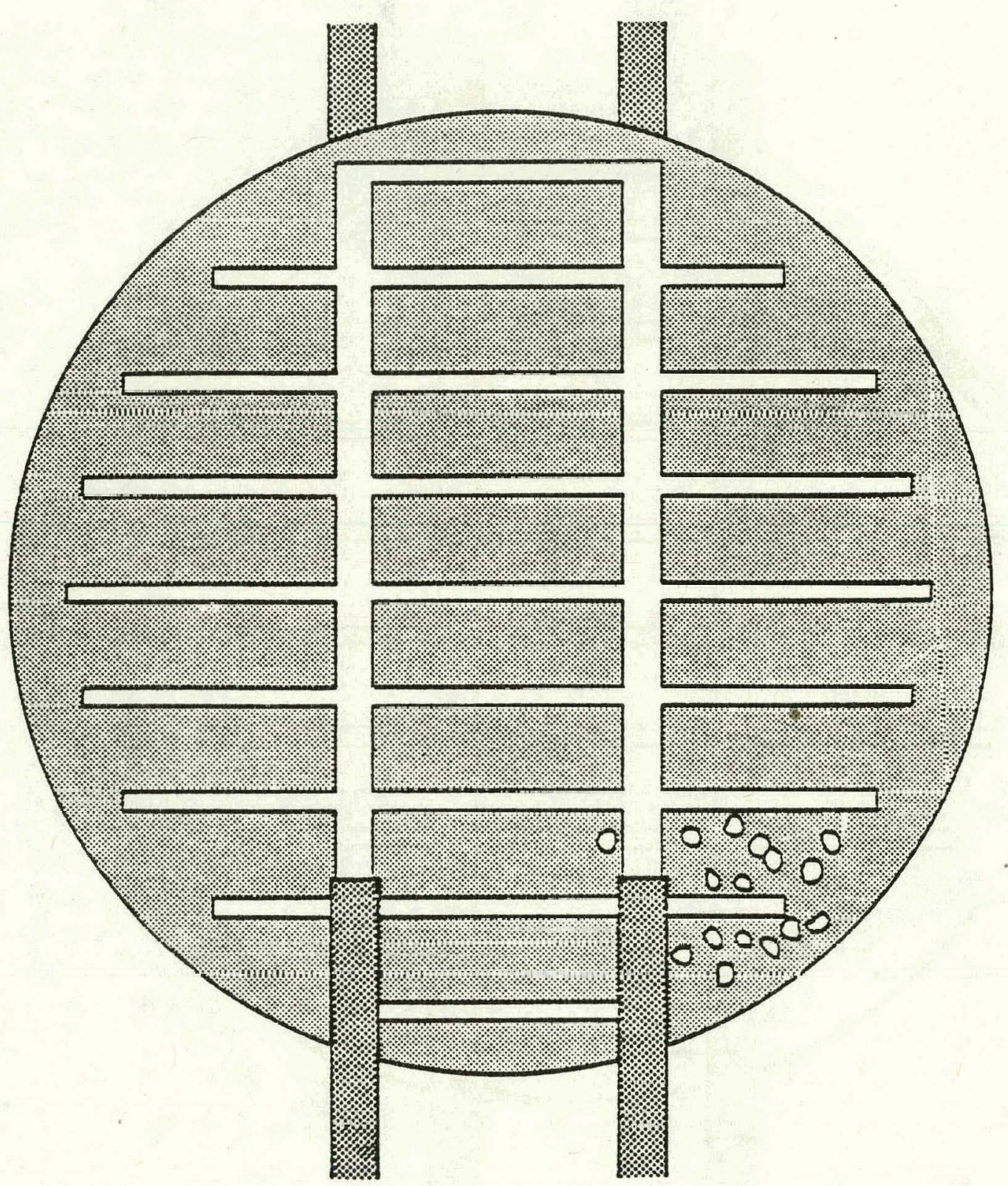

Figure 22. Excess Soldei on Cell Surface. Acceptable. 


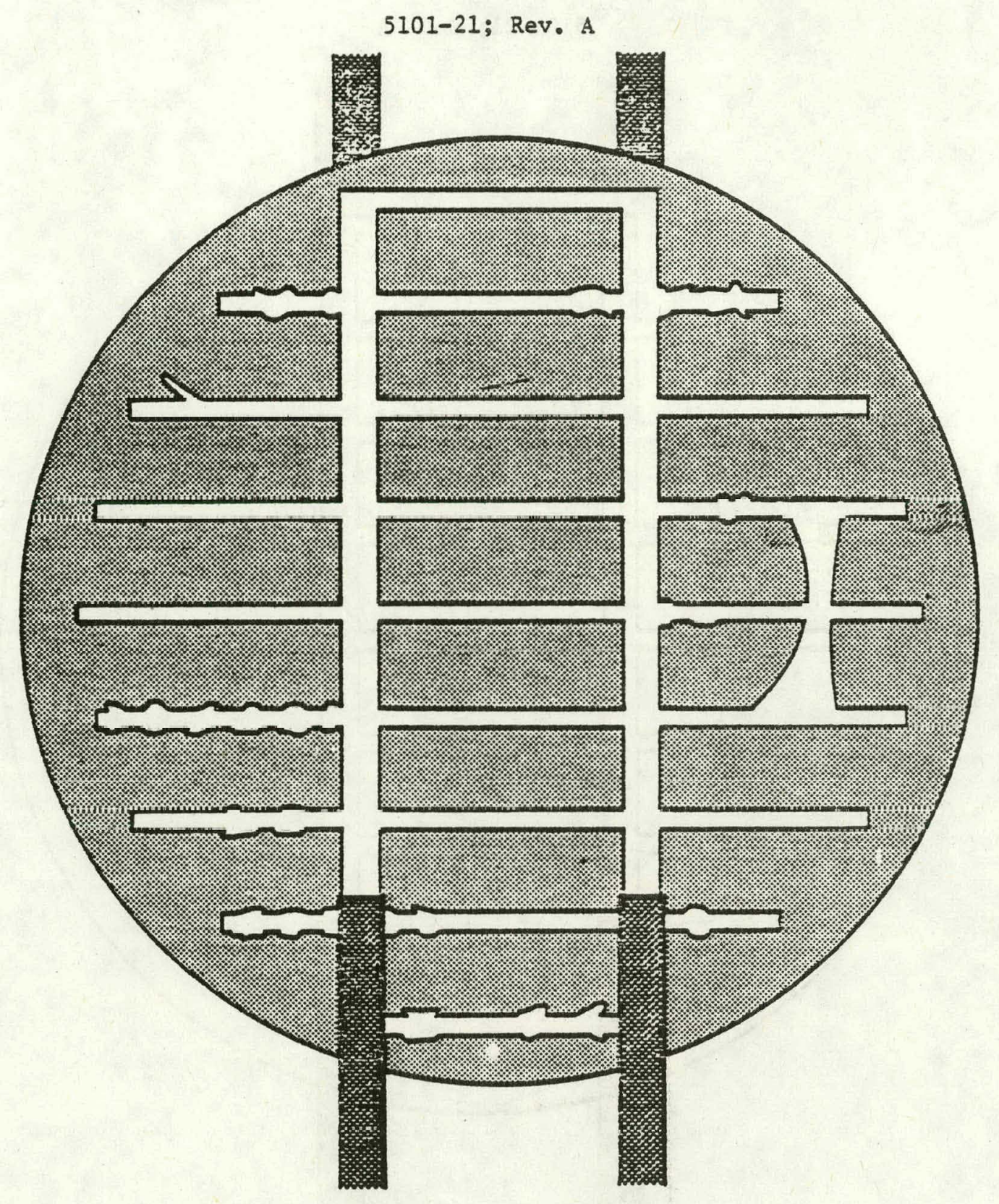

Figure 23. Excess Solder, and Solder Bridging Between Grid Lines. Acceptable. 


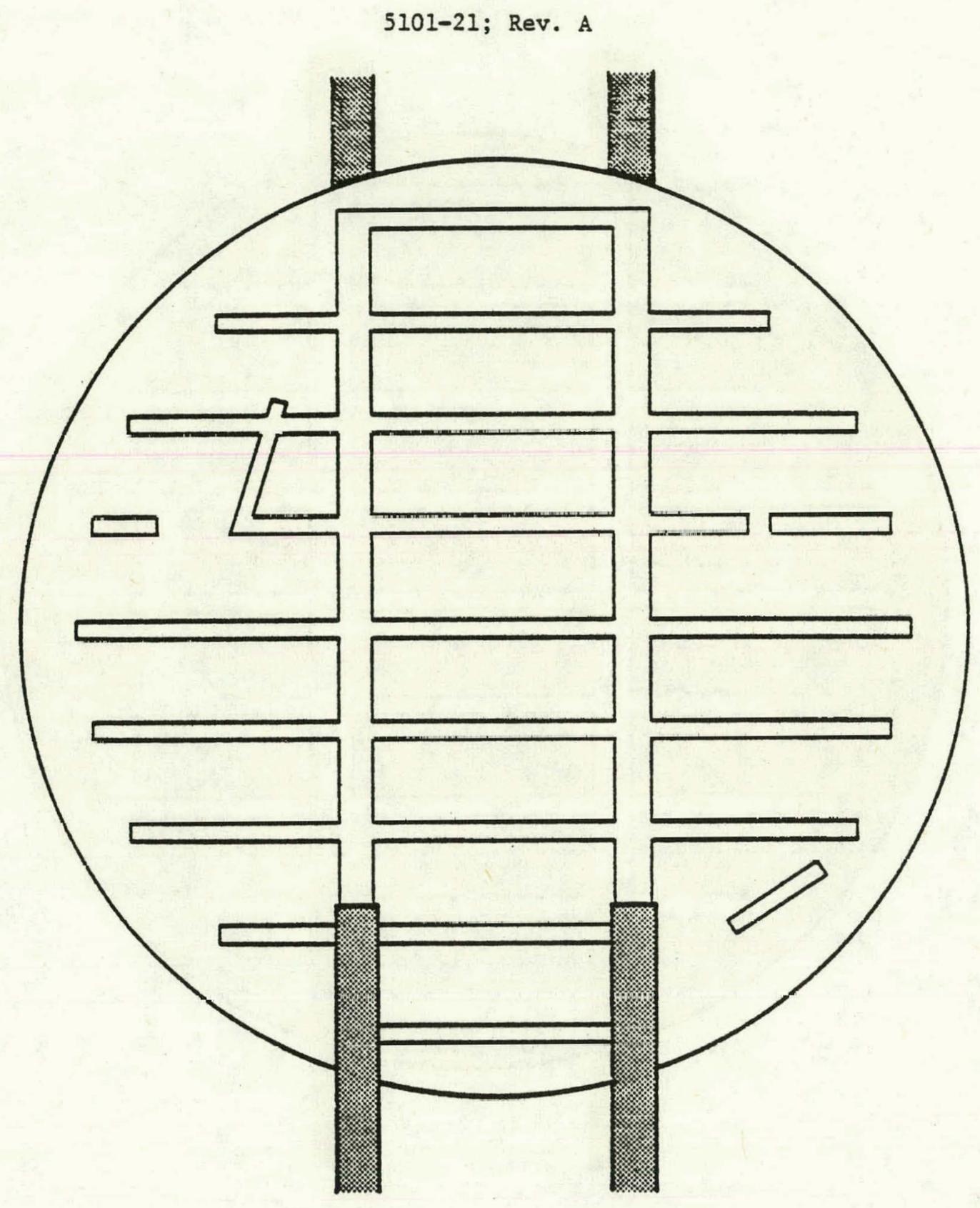

Figure 24. Broken or Missing Grid Lines With Loose Fragments of Grid Lines. Acceptable. 
5101-21; Rev. A

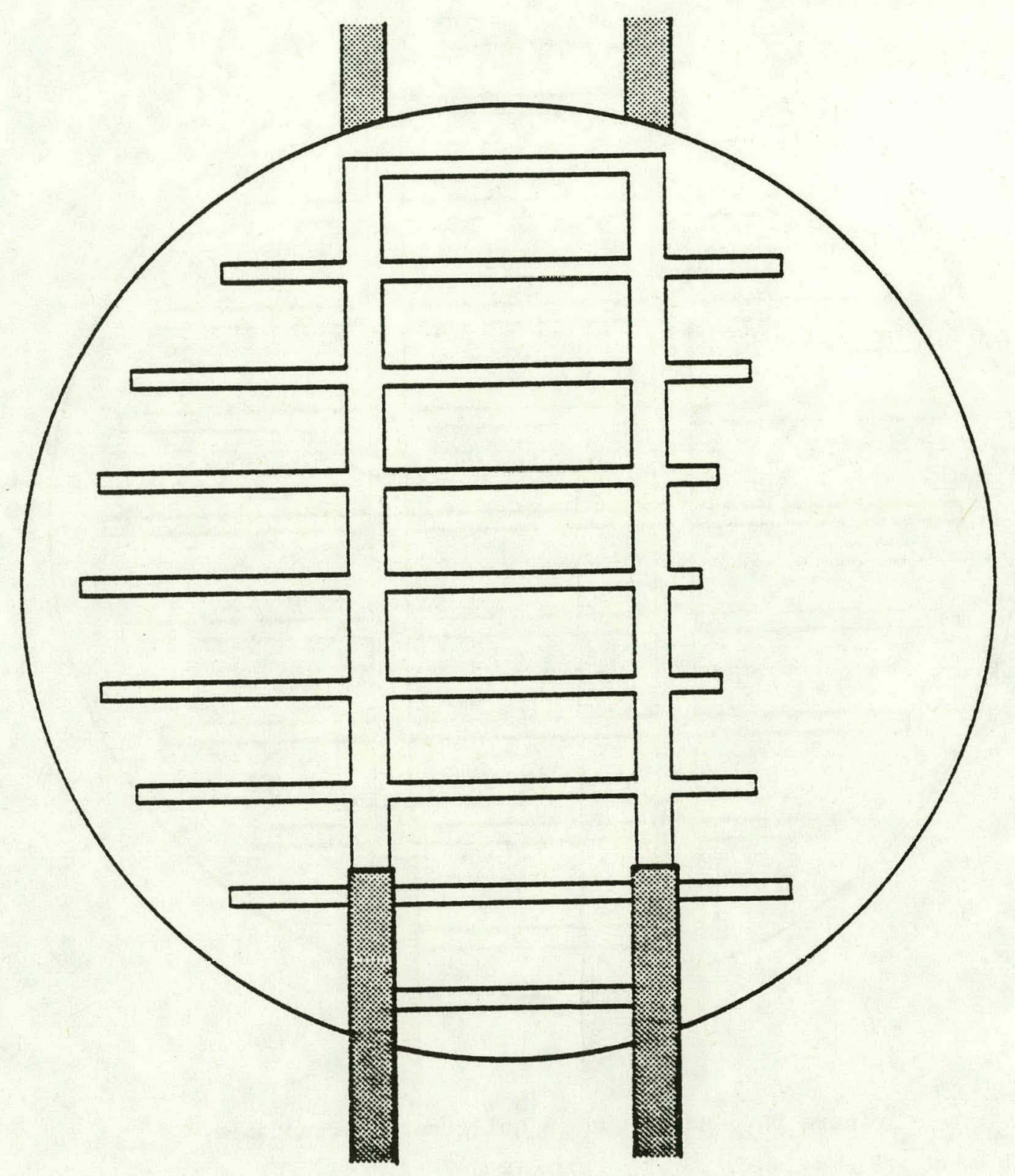

Figure 25. Missing Grid Lines. Acceptable. 
5101-21; Rev. A

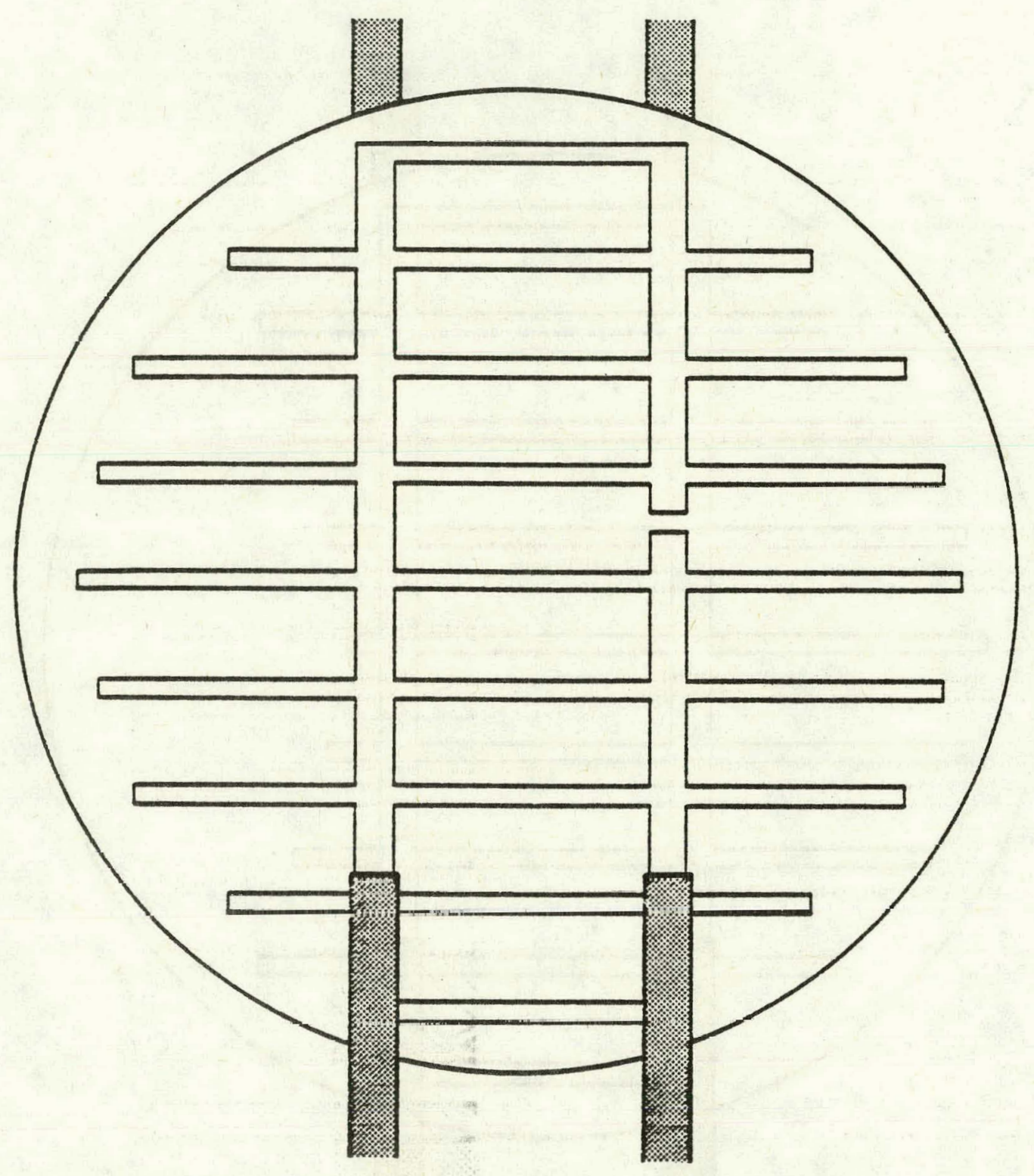

F1gure 26. Major Gap in Collector. Acceptable. 


\section{1-21, Rev. A}

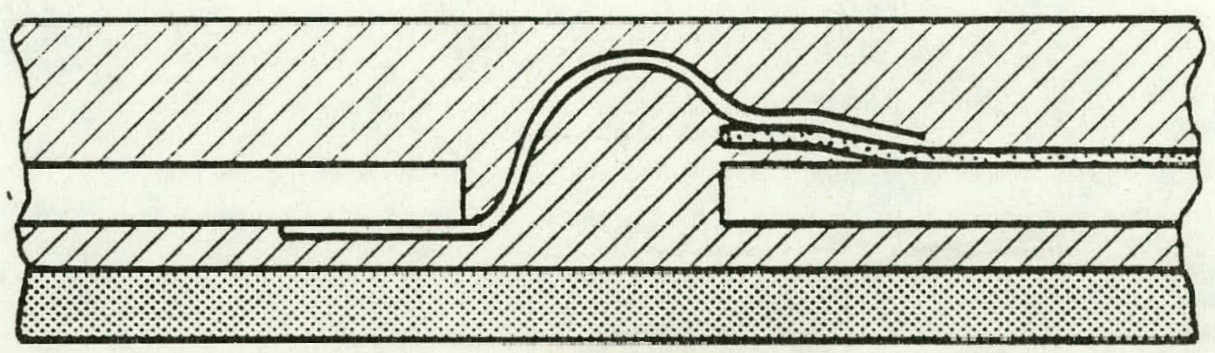

Figure 27a. Collector Delaminated at Interconnect Solder Joint. Reject.

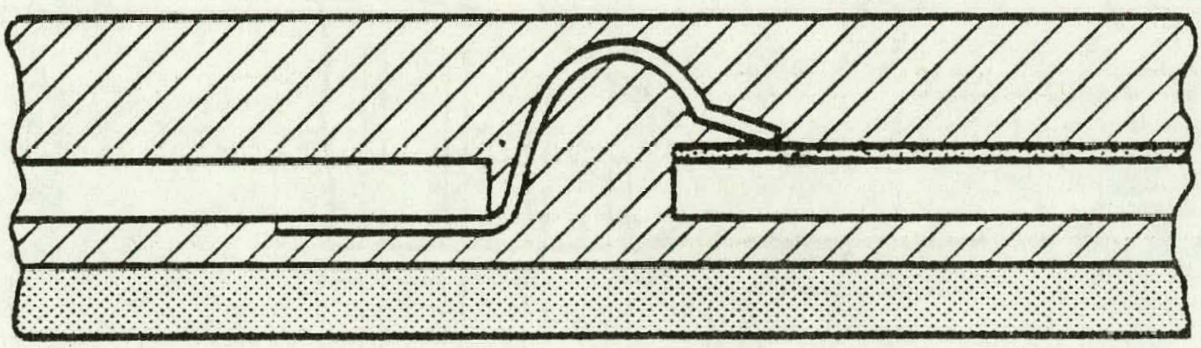

Figure 27b. Interconnect Delaminated at Solder Joint. Reject.

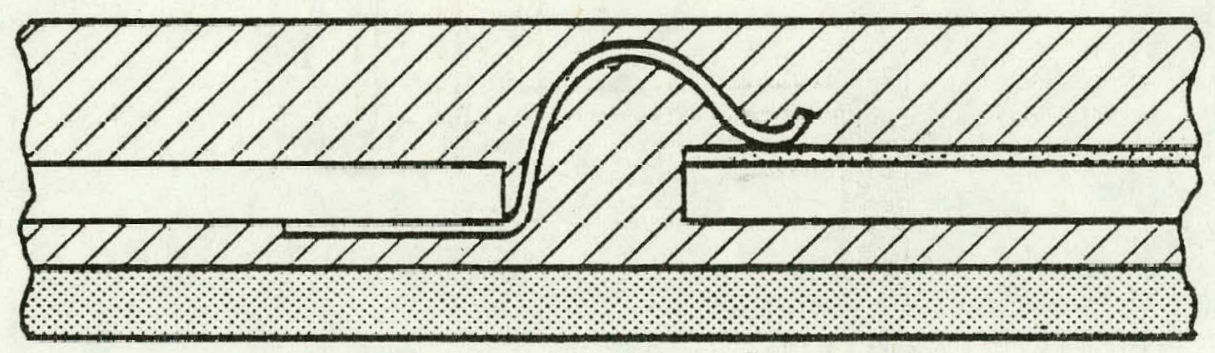

Figure 27c. Interconnect Delaminated at Solder Joint. Reject. 


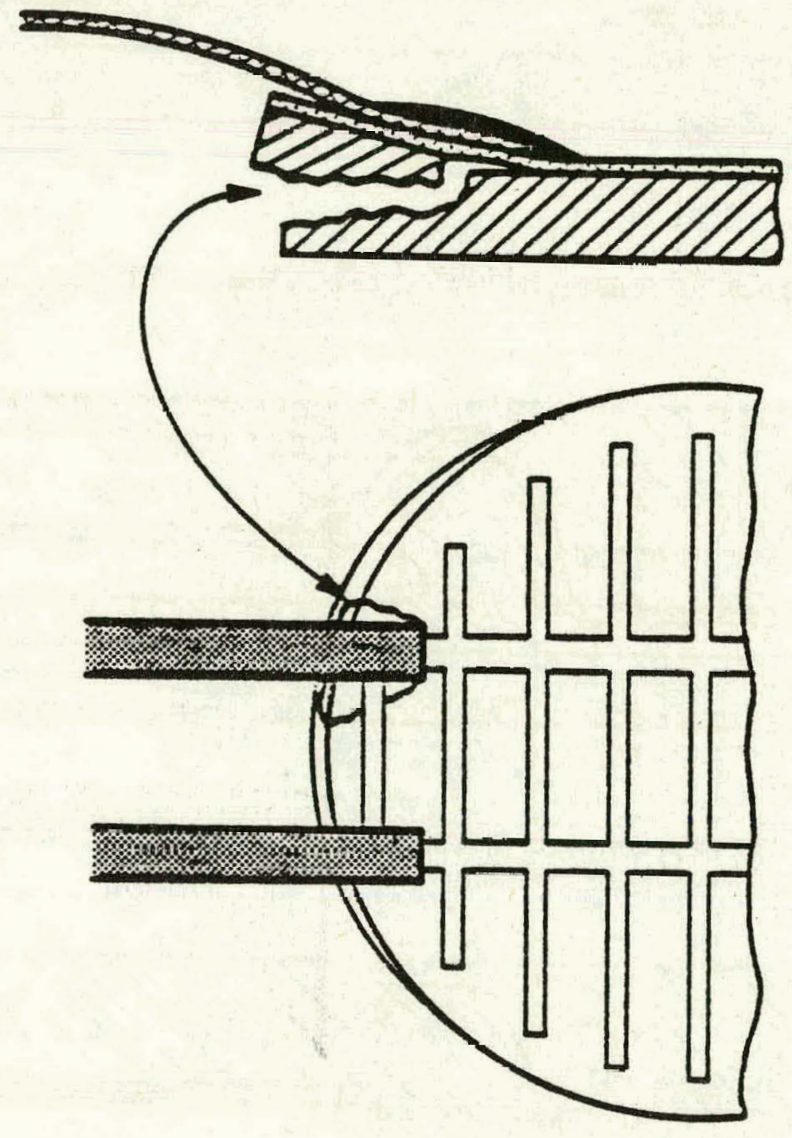

Figure 28. Alligatored Cell. Broken Material Under Collector Solder Joint Starting at Edge of Cell. Rejecr. 
5101-21; Rev. A

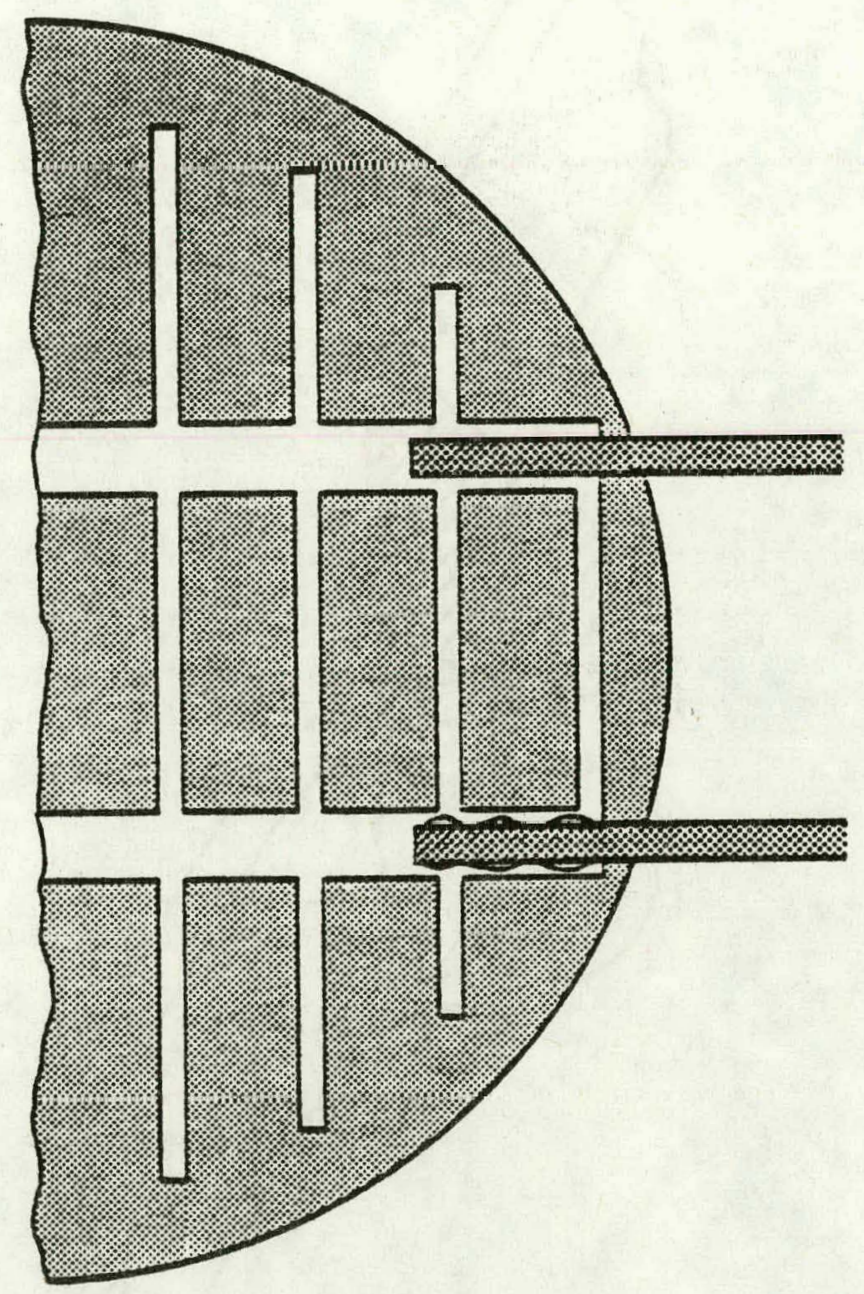

$\frac{\dot{e}}{\stackrel{\Xi}{3}}$

产

ڤ

를

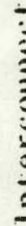

ลิ

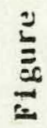




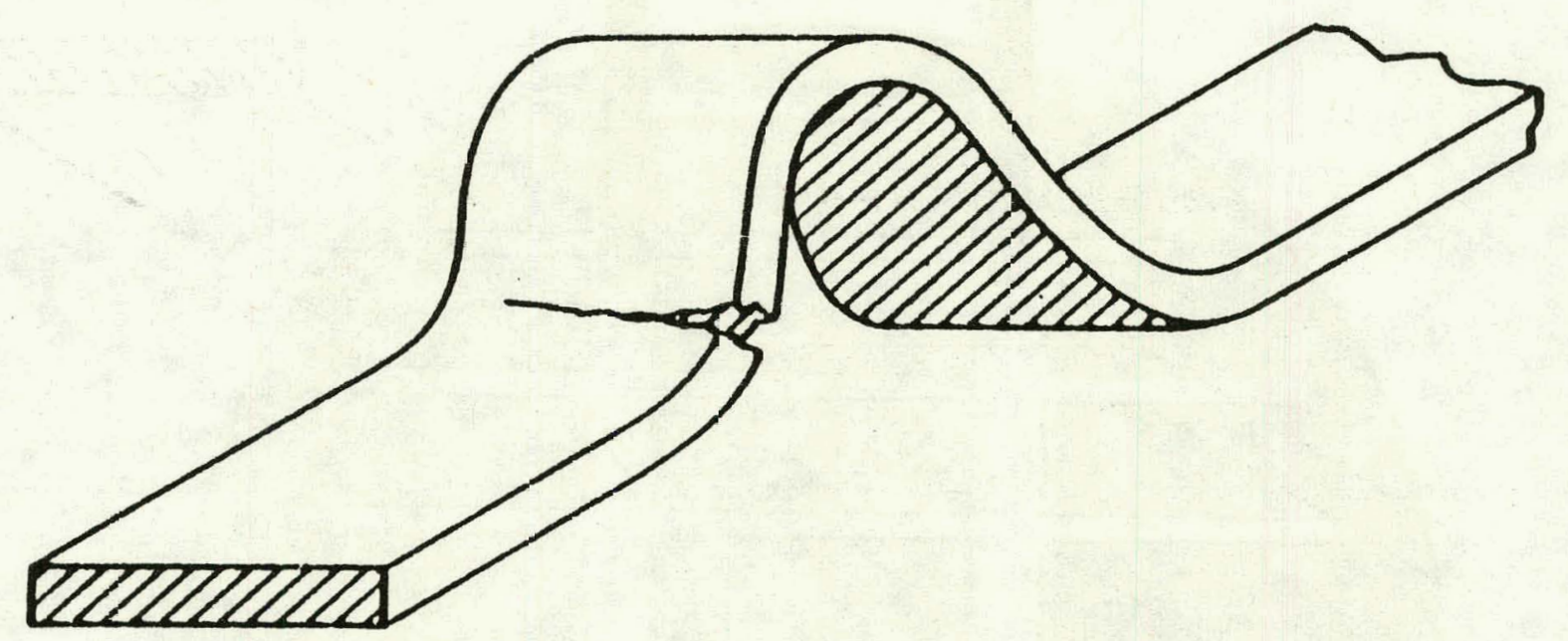

Figure 30. Solld Strip Triteromect Metal Pat Igue Practure. Reject. 


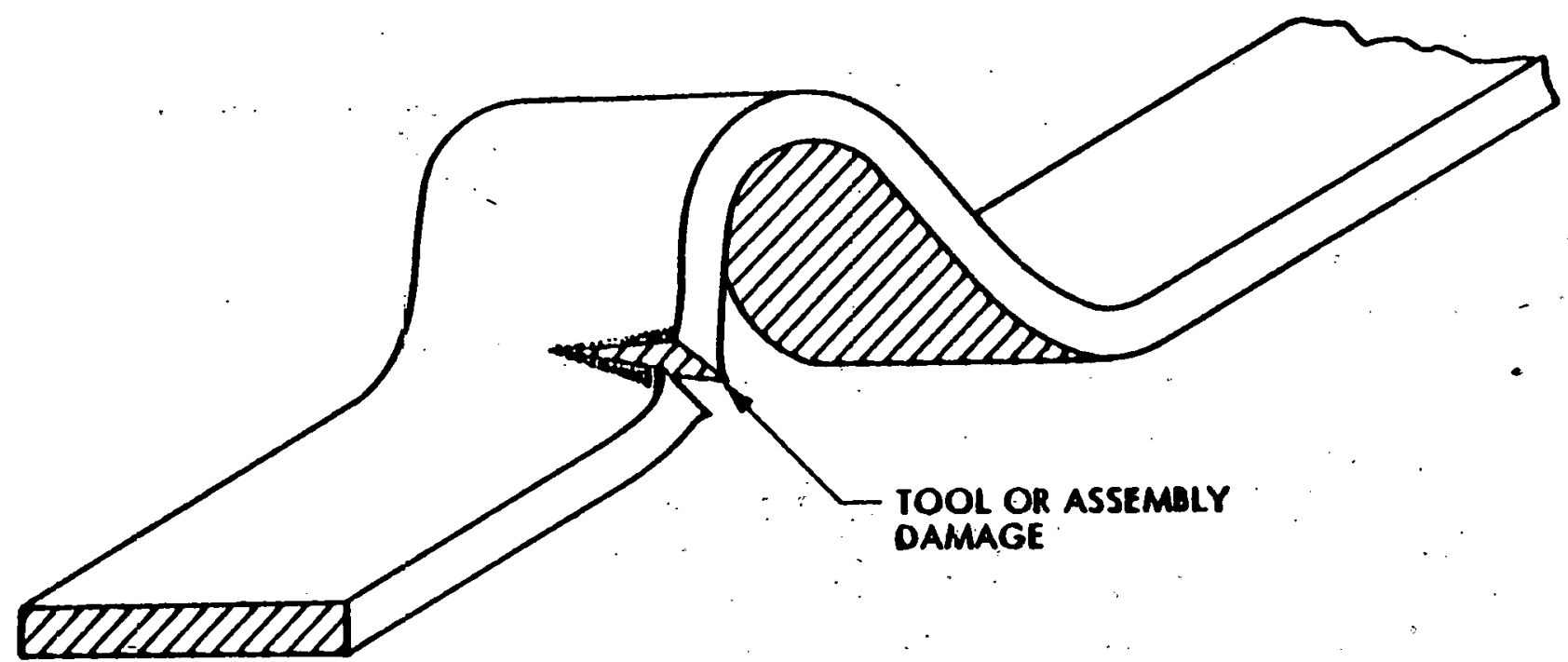

Figure 31. Solid Strip Intercomnect Discrepancles lf in Stress Relief Area. Reject if the Cross-Sectonal Area of the Strip ls Reduced hy $25 \%$ or More. 


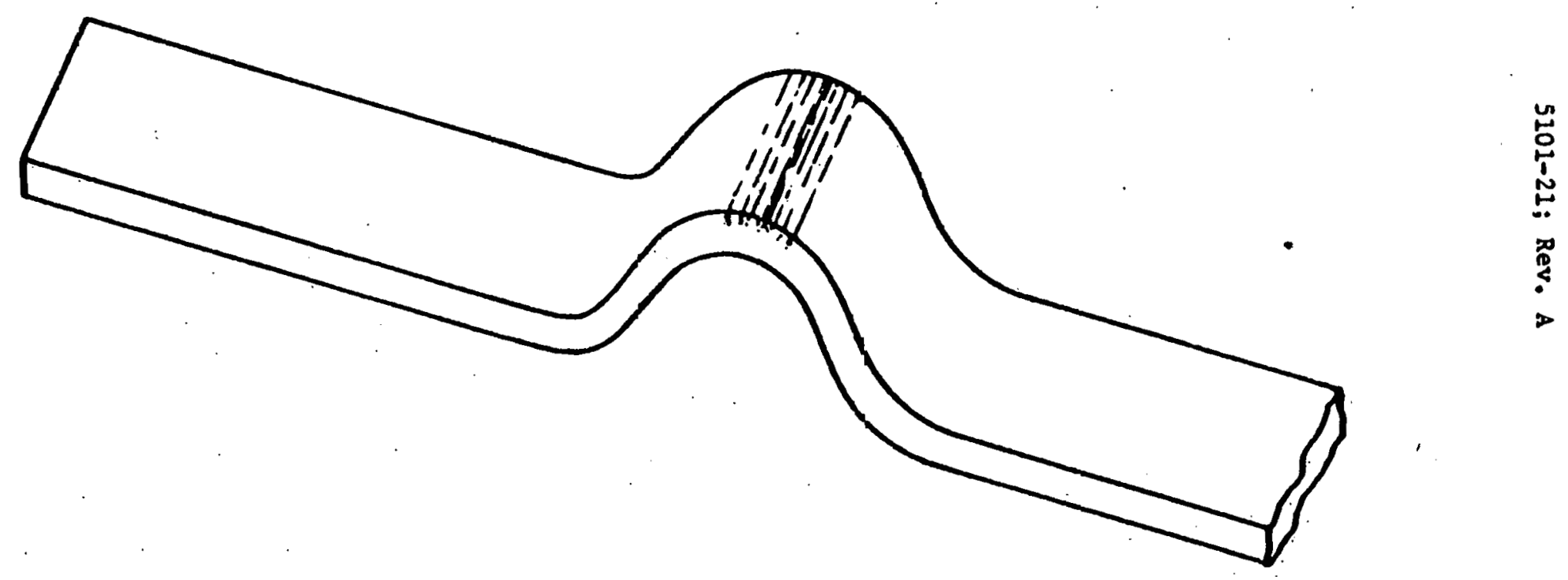

FIgure 32. Incerconnert Stress Damage. Reject. 


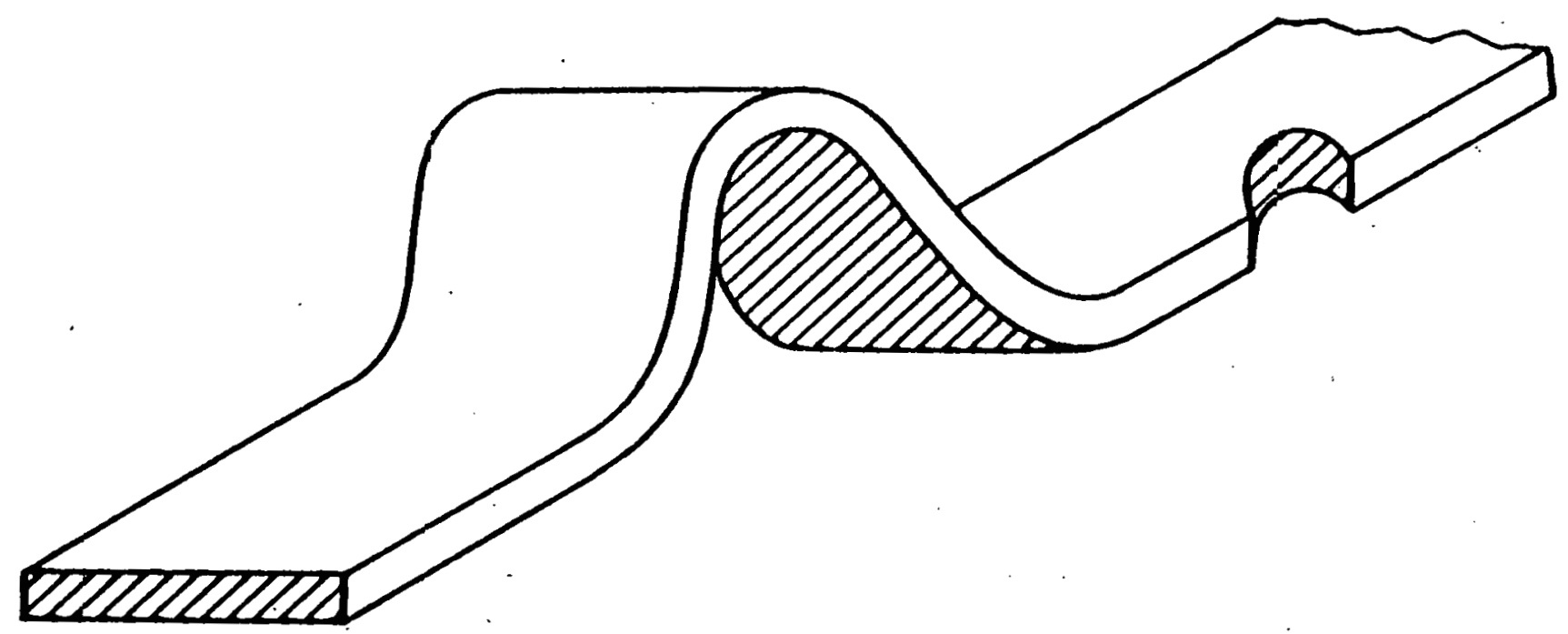

Plgure 33. Solid strip lintereomect Blst:pancies. Reject if the Cross-Sect funal Areit of the Strlp is Heduced by $25 x$ or Murte. 


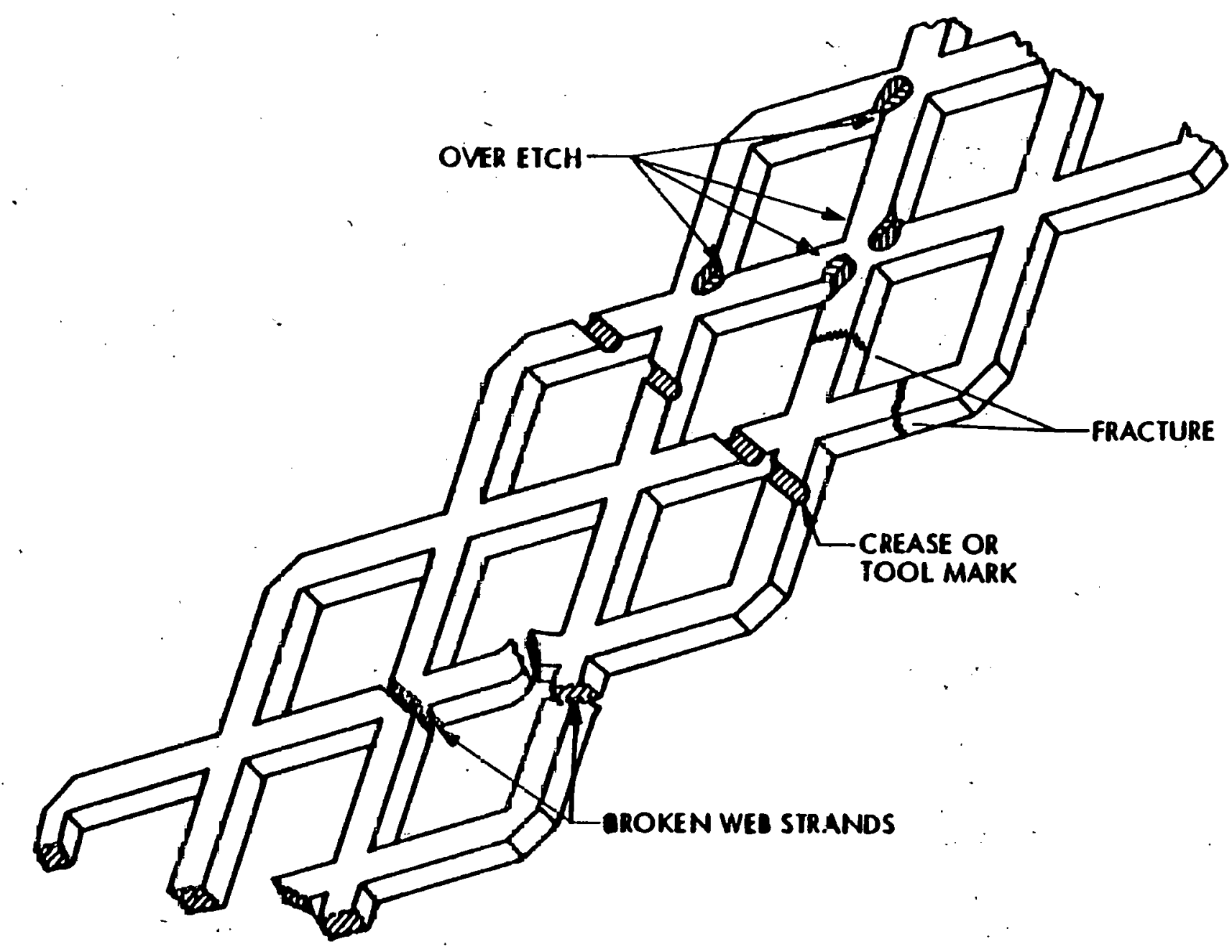

Figure 34. Neb-lype Interconnect Defects. Defect: Which Reduce the Cross-Sect Jonel Area of the Wel, Miterlal by .25\% or More are Grounds for Rejection of the UnIt. 


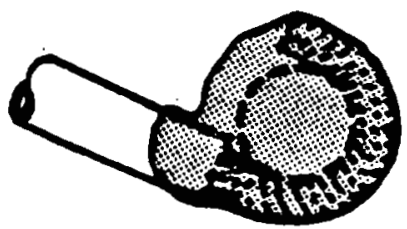

Figure 35. Insulation Burled Irto the Solder. Reject.

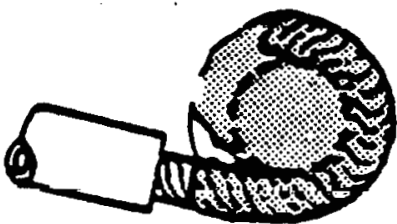

F1gure 37. Cracked Solder. Rejece.

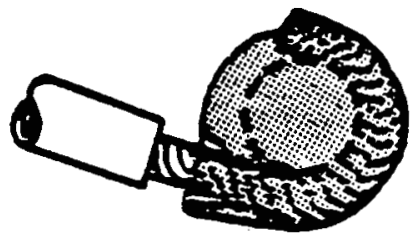

Figure 36. Poot Flow to Wire. Insufficient Heat or Contaminared Surfaces. Reject.

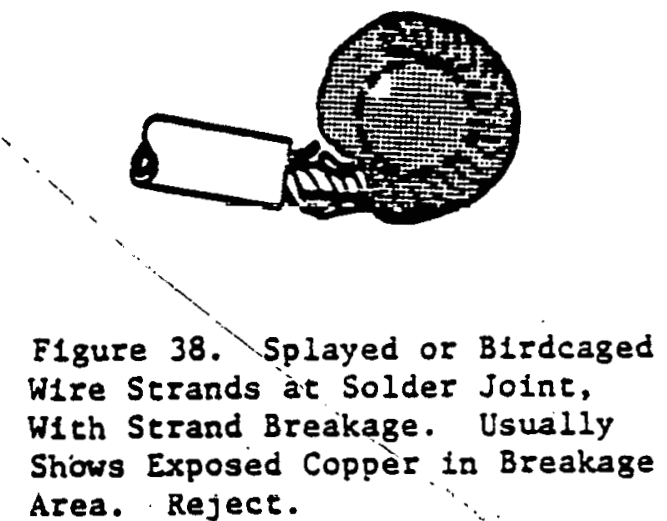




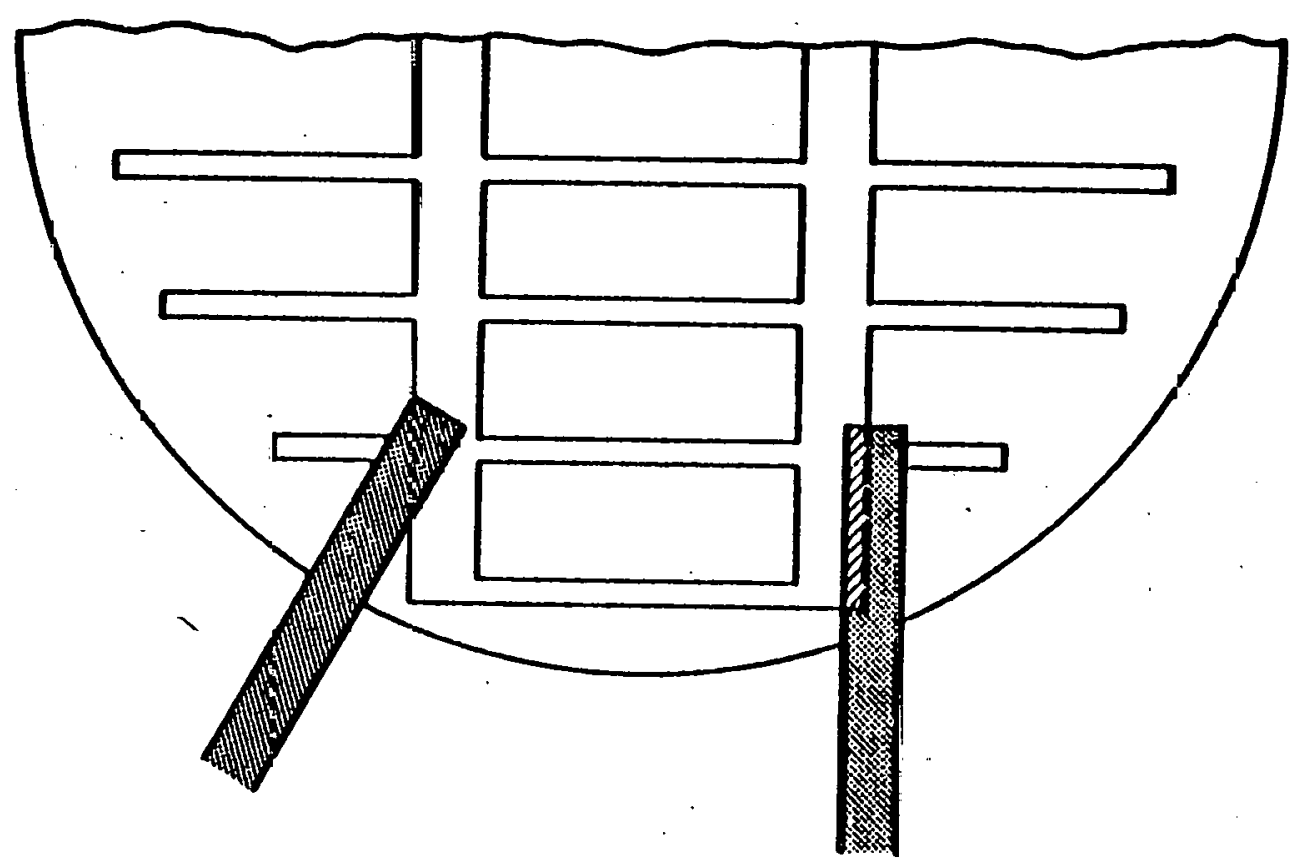

Flgure 39. Any Intercomect Tal, Misallgnment With less Than $50 \%$ Intercomect contact with. Collector., Reject. 


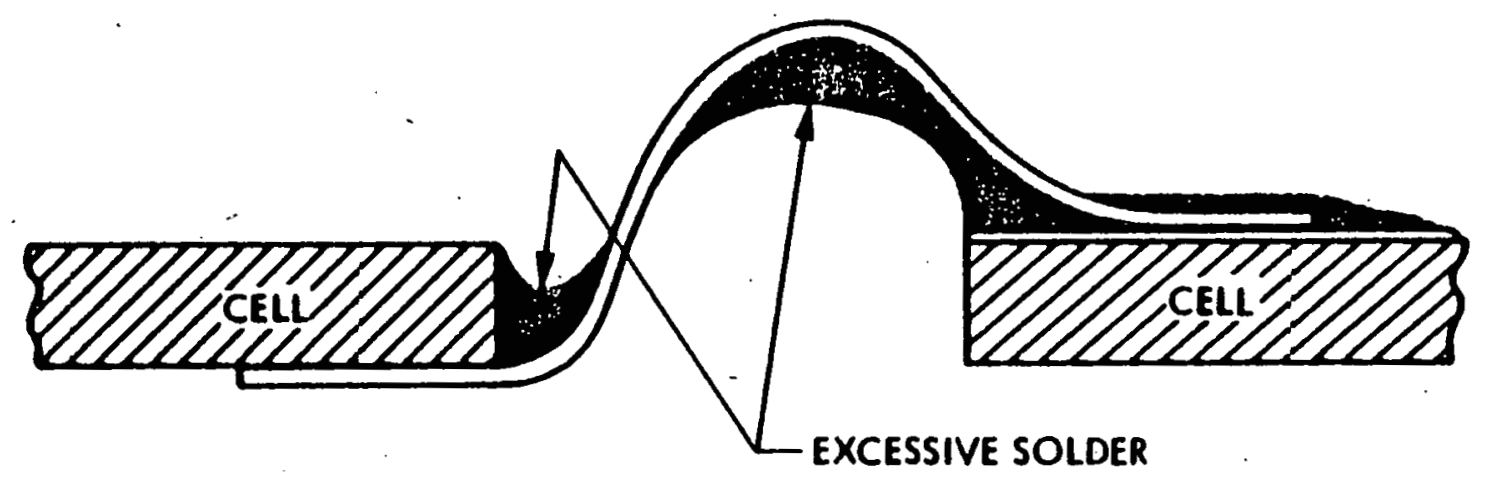

Figure 40. Stress Rellef Loop Frozen by Solder. Reject. 
5101-21; Rev. A
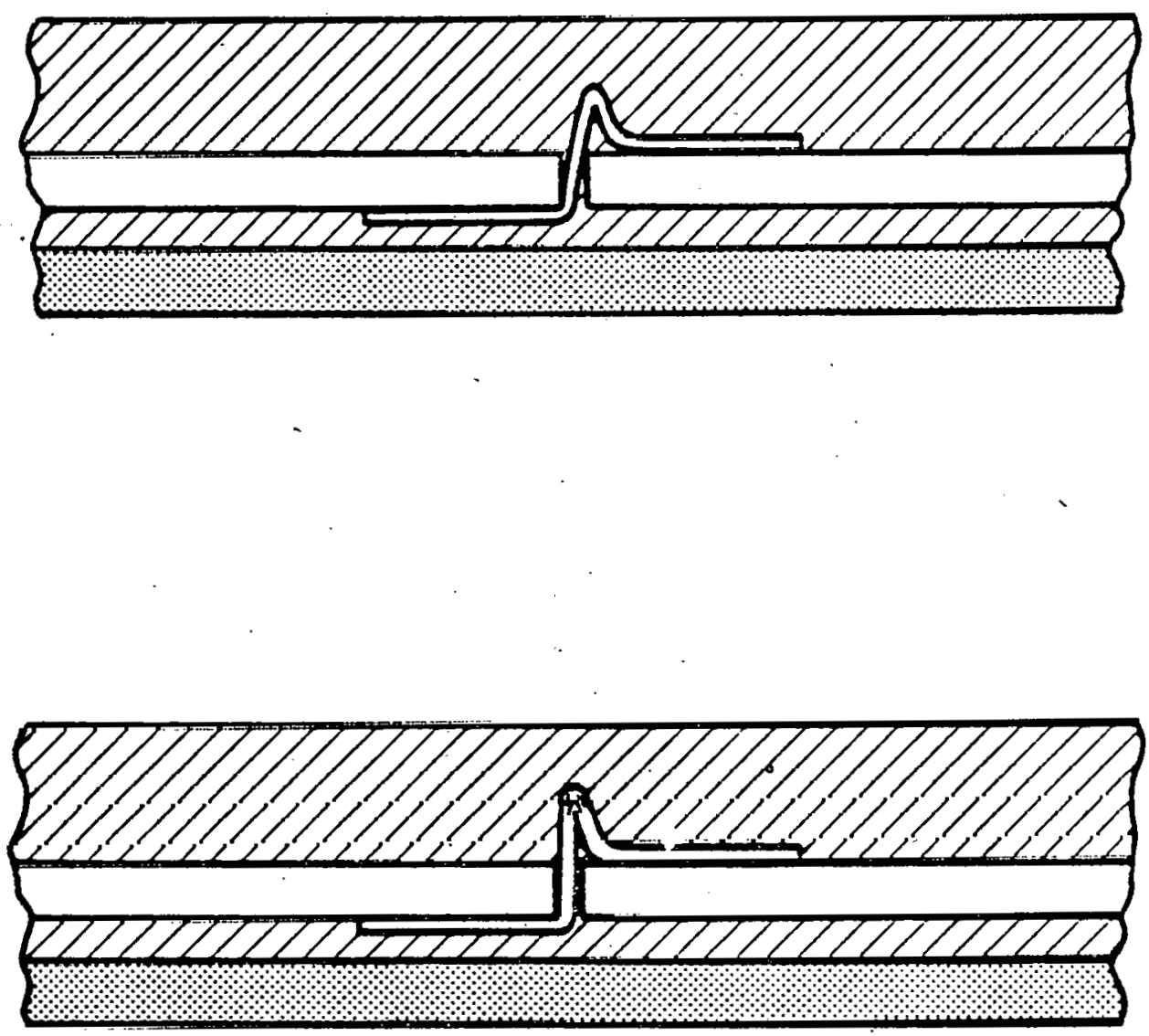

Figure 41. Interconnect Crushed Between Cell Edges and/or Distorted and Degrading Stress Relief. Reject. 


\section{1-21; Rev. A 、}

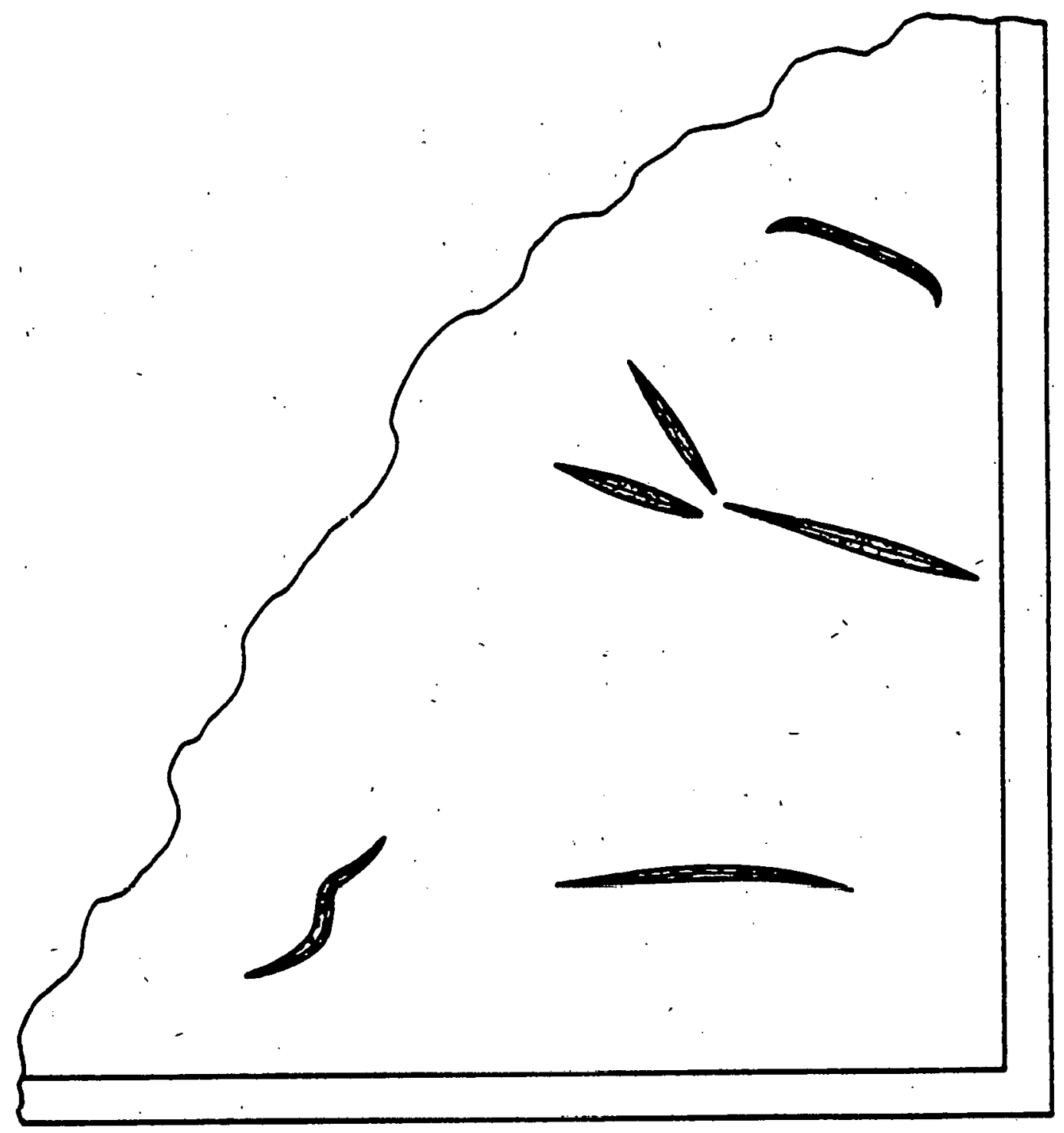

F1gure 42. Encapsulant Surface Split. Reject. 
5101-21; Rev. A

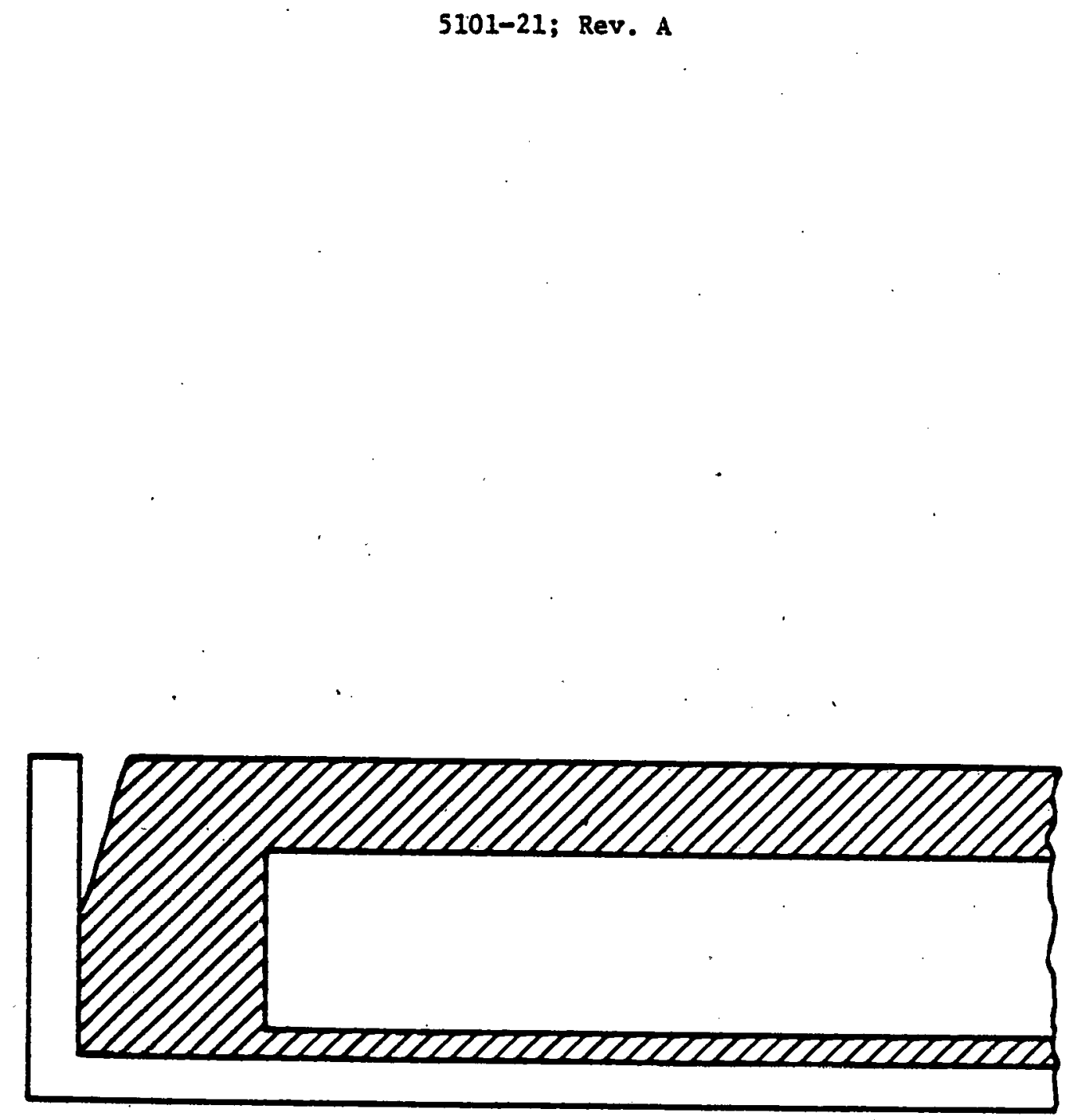

F1gure 43. Edge Seal Vold Between Substrate and Potring. (Side View) Reject. 


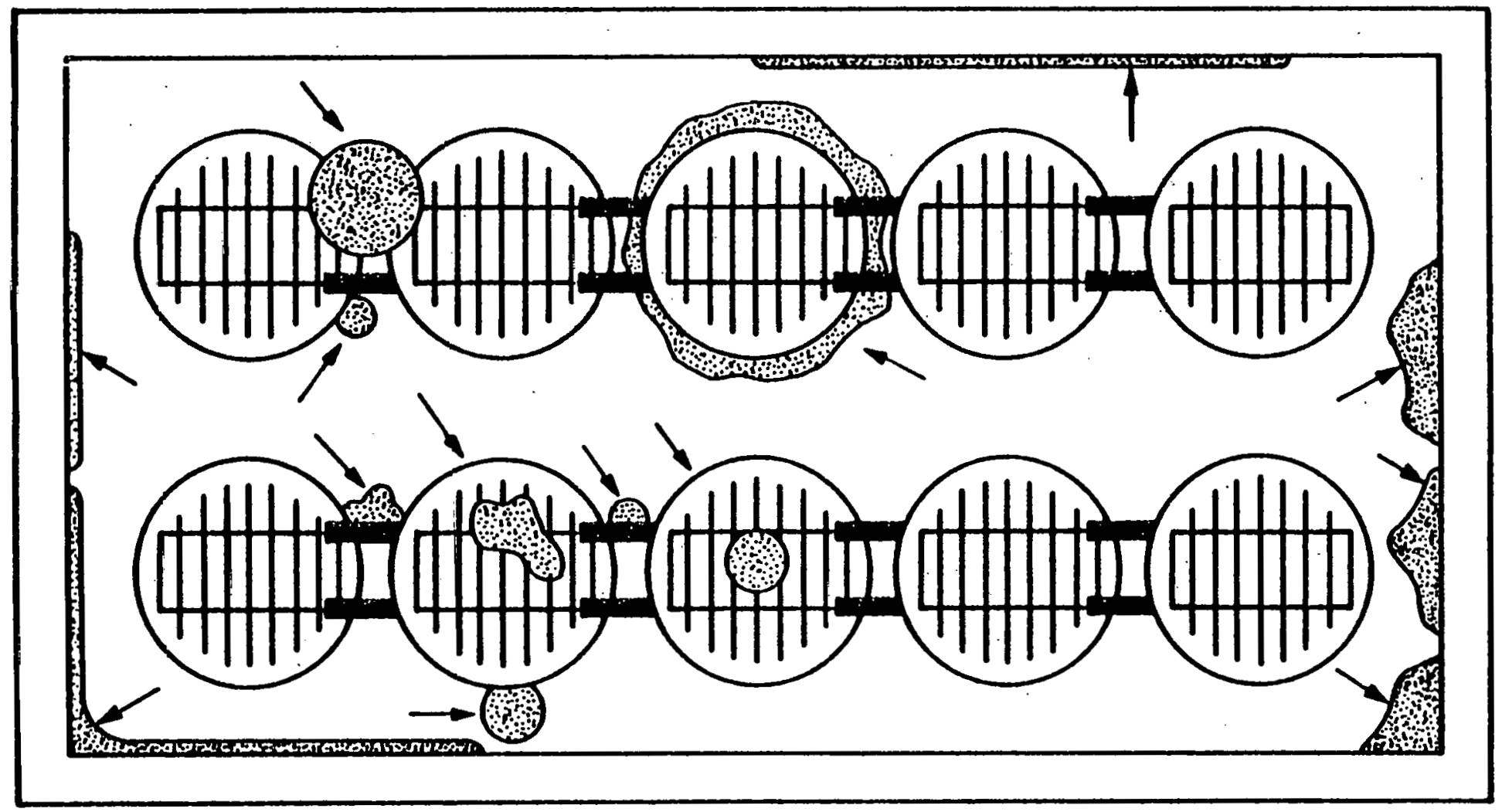

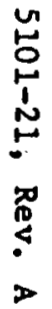

Figure 44. Any Delaminations or Bubbles Greater Than $3 \mathrm{~mm}$. Reject. 


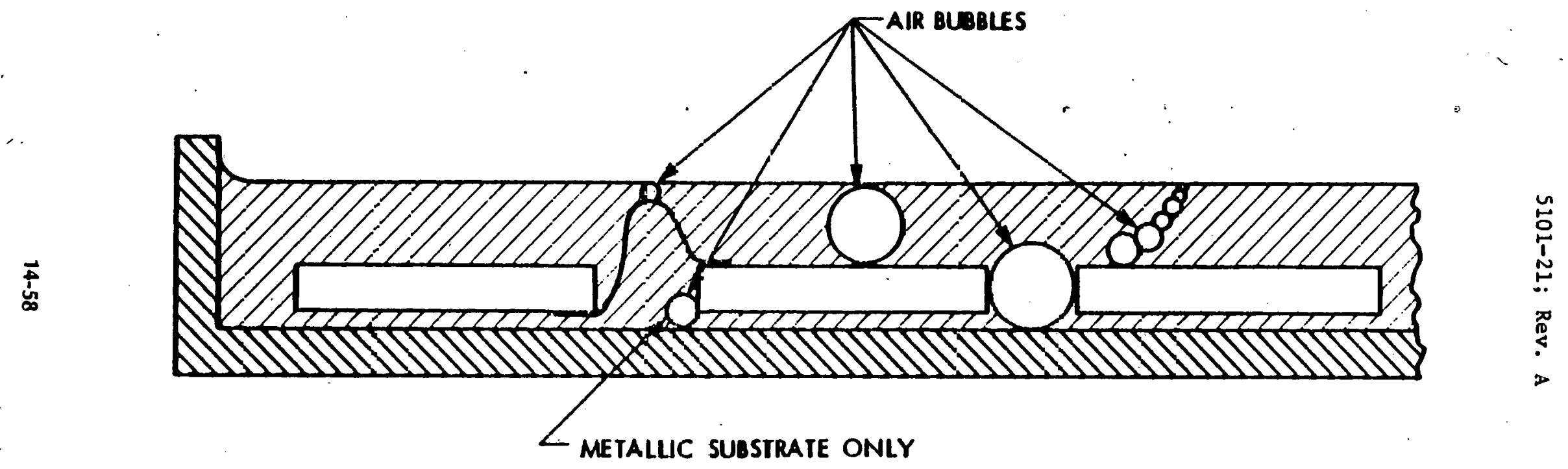

Figure 45. Alr Bubble Discrepancies. Refect. 


\section{1-21; Rev. A}

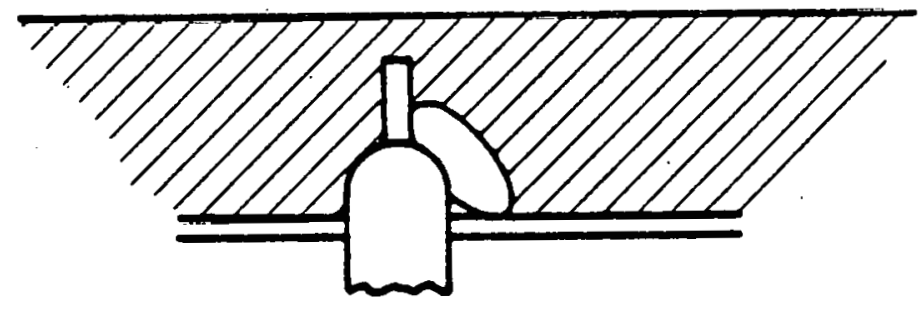

Figure 46. Void or Delamination from Output Terminal to Metal Substrate. Reject.

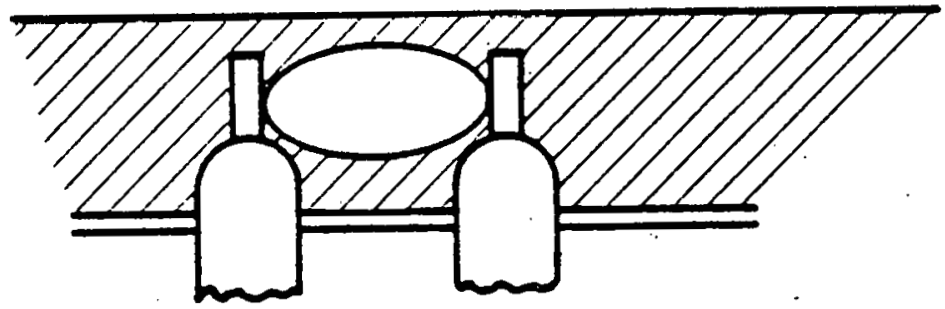

Figute 47. Void or Delamination Between Output Terminals. Reject. 

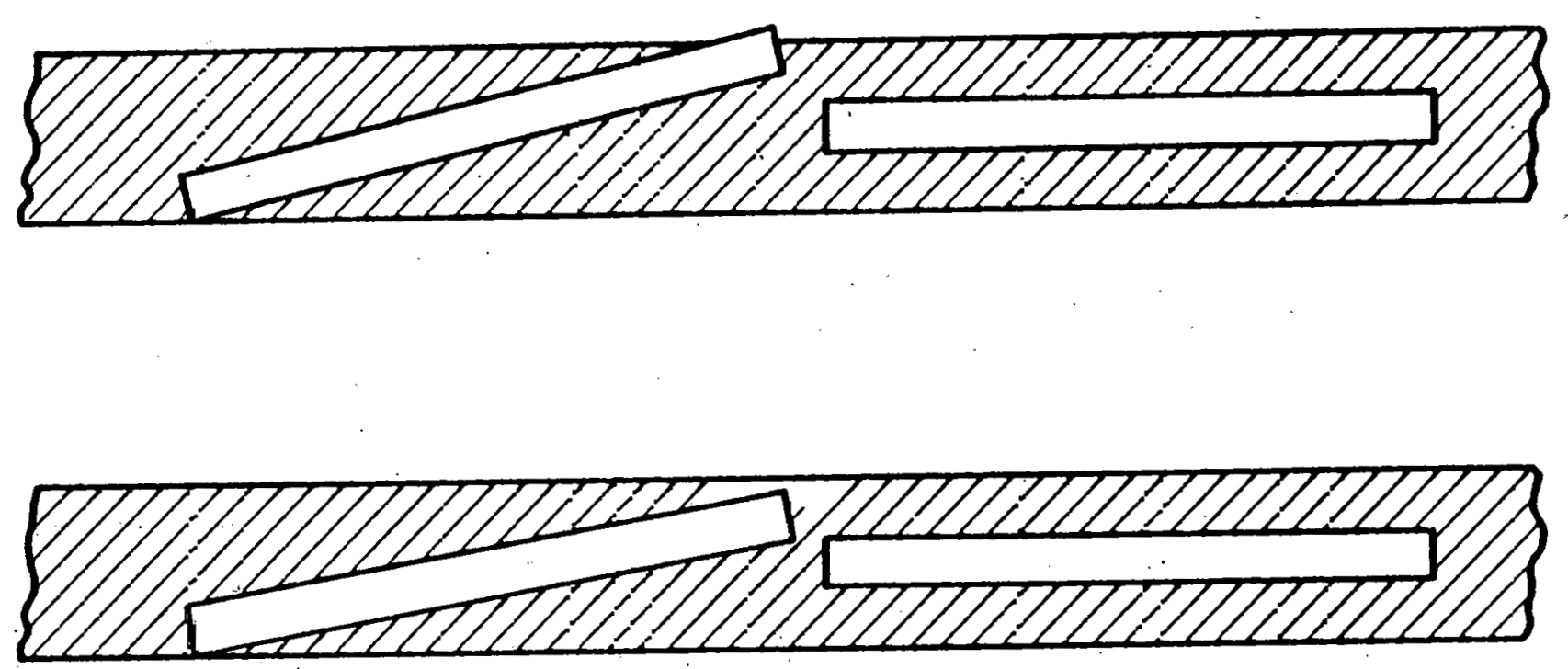

Figure 40. Cell Exposed or Less Than l.5 min Below Surface of Encapsulant. Reject. 

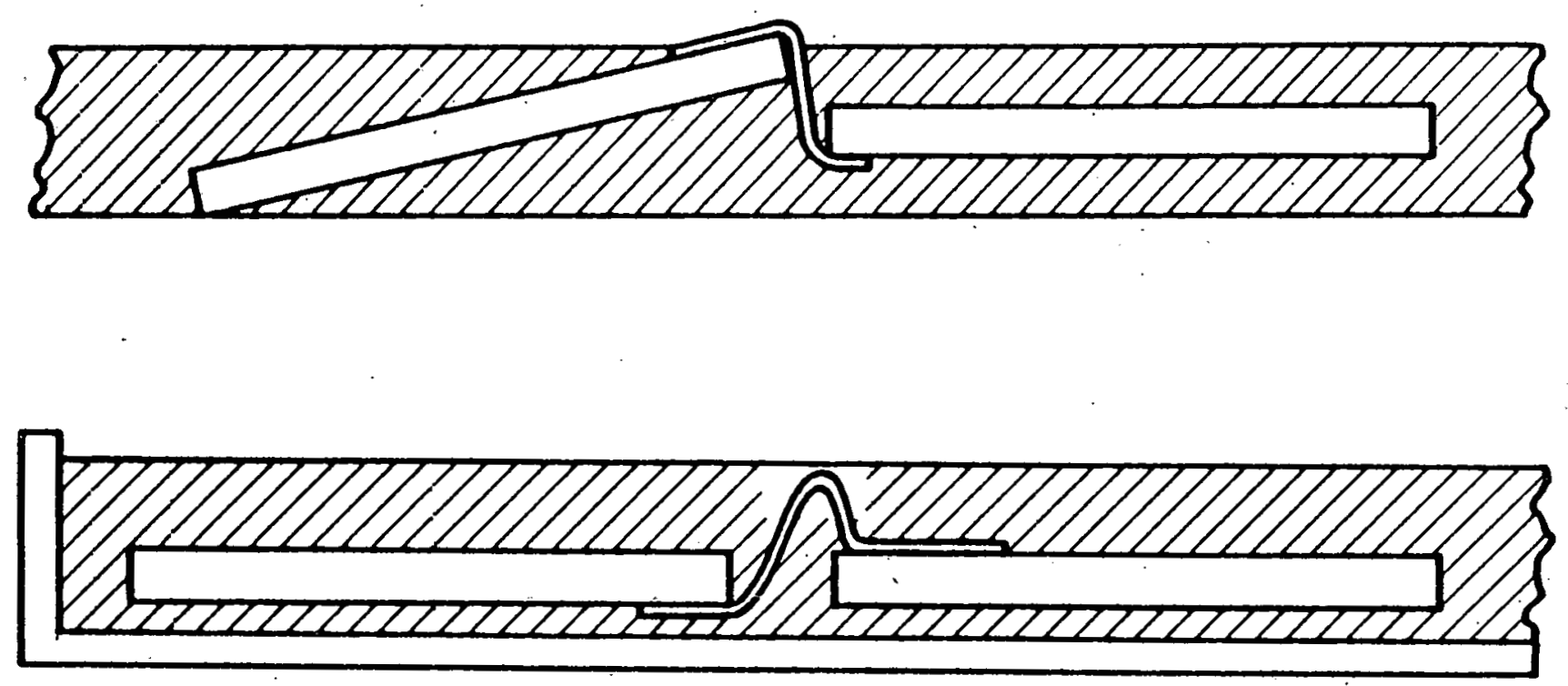

Figure 49. Interconnect Exposed or Less Than $1.5 \mathrm{~mm}$ Below Surface of Encapsulant. Reject. 


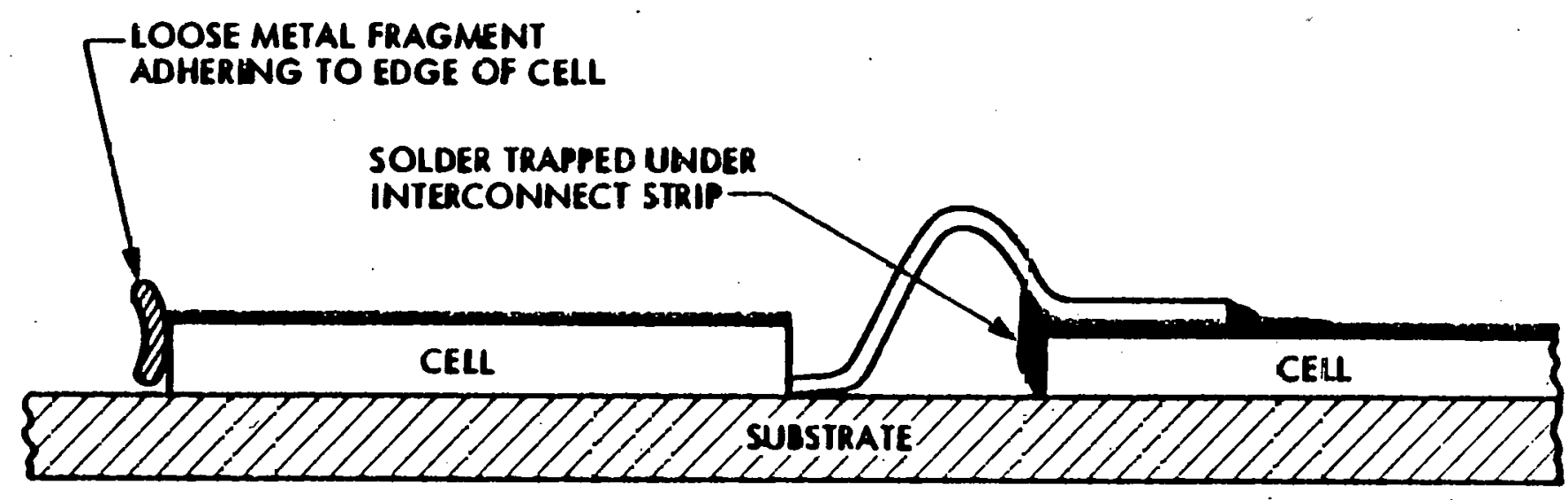

Figure 50. Possible Junction Shorts. Reject. 